\title{
URBAN/RURAL SOURCE APPORTIONMENT AND INTRAURBAN SOURCE-BASED SPATIAL ANALYSIS OF POLYCYCLIC AROMATIC HYDROCARBONS (PAH) AND ASSOCIATED TOXICITY
}

\author{
A thesis submitted to \\ the Faculty of Graduate and Postdoctoral Affairs \\ in Partial Fulfillment of the requirements for the degree \\ Doctor of Philosophy
}

by

Angelos T. Anastasopolos

M.A.Sc., B.Eng., B.A.

Department of Civil and Environmental Engineering Carleton University

Ottawa-Carleton Institute of Civil and Environmental Engineering

January 2015

(C)2015 Angelos T. Anastasopolos 


\begin{abstract}
This research identified and quantified source types contributing to ambient PAH and associated toxicity at the urban and intraurban scales, reflecting awareness of the variability in exposure toxicity and in source toxicity for PM-associated toxic pollutants.
\end{abstract}

Source apportionment analysed vapour+particle PAH time-series data (2001-2010) from central site monitoring stations at urban (Hamilton, Toronto) and rural background (Egbert) sites in Southern Ontario, Canada. Receptor modeling by Positive Matrix Factorization (PMF) identified four source types: volatilized PAH/long-range transported coal combustion, vehicle traffic exhaust, space heating, biomass combustion. At Hamilton, local industry emissions were also identified, associated with iron/steel manufacturing. Apportionment of PAH toxicity using Benzo(a)Pyrene-toxicity equivalency factors identified traffic exhaust and local industry as 'more toxic' source types, contributing comparably little to ambient $\mathrm{PAH}$ yet disproportionately to $\mathrm{PAH}-$ associated toxicity.

Intraurban investigation of $\mathrm{PAH}$ sources sampled vapour+particle $\mathrm{PAH}$ and $\mathrm{PM}_{2.5}$ from a dense network of $>30$ Hamilton sites over a two-week period in June-July and December 2009. Ambient PAH exhibited substantially greater spatial variability than $\mathrm{PM}_{2.5}$ and 'hot spots' of elevated pollutant levels were observed near/downwind of the business district and harbour-front.

A combined PMF-Chemical Mass Balance (CMB) receptor modeling approach applied factors derived from the PMF model of Hamilton central site time-series data as 'local 
source profiles' in a CMB model of spatial field sampling data, explaining spatial variability observed for PAH and PAH toxicity in terms of sources. Contributions by space heating, volatilized PAH/transported coal combustion, wood combustion showed low intraurban variability, while vehicle traffic exhaust showed moderate variability, and local industry emissions contributed significantly only near the industrial harbour-front. Vehicle traffic exhaust contributed majority of PAH toxicity at all sites, even where ambient PAH concentrations were comparably low, and local industry emissions contributed significantly only near the industrial zone, explaining 'toxicity hot spots' as high contributions of local industry in addition to vehicle traffic.

Findings recommend that efforts to reduce PAH exposures prioritize 'more toxic' source types such as vehicle traffic and local industry. PMF-CMB receptor modeling using local time-series PAH data to interpret intraurban variability in ambient PAH demonstrated a viable analysis method for other urban locations. 


\section{Acknowledgements}

I am thankful to my research partners for the opportunity to conduct this investigation. I am appreciative of the support and resources Health Canada granted to coordinate the field sampling study that generated my intraurban dataset and the enthusiastic support from the Department of Public Health at the City of Hamilton.

At Health Canada, I would like to thank Dr. Amanda Wheeler for helping give shape to my varied research interests, facilitating my interdisciplinary course work and for selflessly providing ongoing mentorship. At Environment Canada, I would like to thank Dr. Jeff Brook for serving on my advisory committee and helping clarify the central site datasets. I also thank Dr. Phil Hopke for introducing me to receptor modeling 'best practice' in a summer workshop those many years ago and for his close review of the work as external examiner.

I thank my principal advisor, Dr. Deniz Karman, for maintaining a critical perspective while encouraging me through multiple and complex research components and for his enduring patience and sustained belief in my academic capabilities through unforeseen and genuinely trying circumstances. This sentiment is felt for many other individuals at the Ottawa-Carleton Institute for Environmental Engineering, inclusive of my advisory and examination committee members and administrative staff in the Department and in the Faculty of Graduate Studies.

I dedicate this dissertation to my father, whom I can no longer thank as I would wish, and to my wife and family, whom I can. 


\section{Table of Contents}

Abstract........................................................................................................................ ii

Acknowledgements .................................................................................................................... iv

Table of Contents ................................................................................................................. v

List of Tables ............................................................................................................... xii

List of Figures....................................................................................................... xiii

List of Equations .................................................................................................................... xiv

List of Abbreviations ........................................................................................................ $\mathrm{xv}$

List of Symbols ............................................................................................................... $\mathrm{xx}$ 


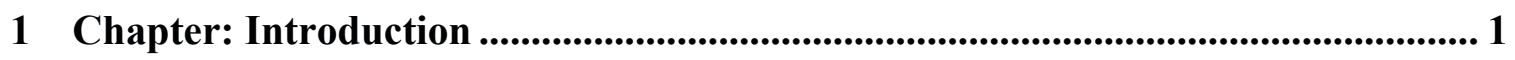

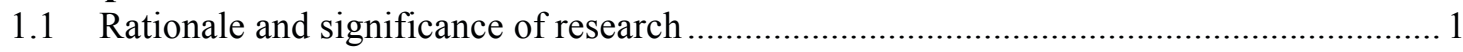

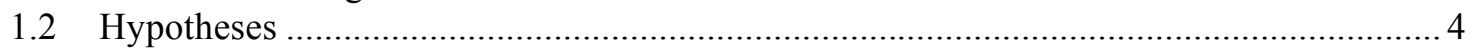

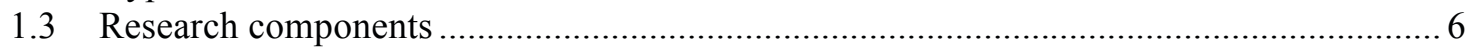

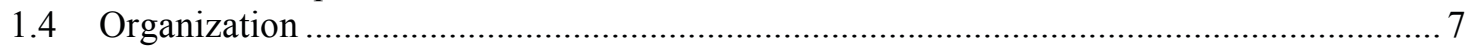

2 Chapter: Methodology.................................................................................................... 9

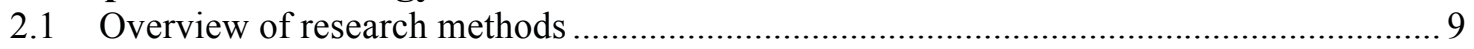

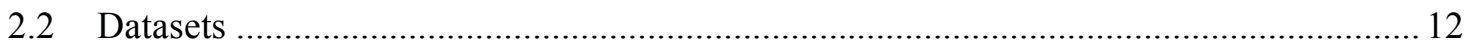

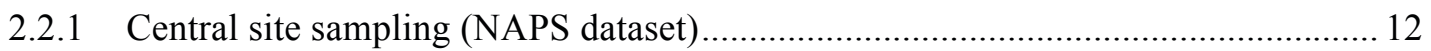

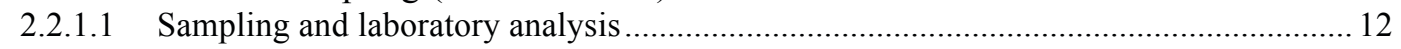

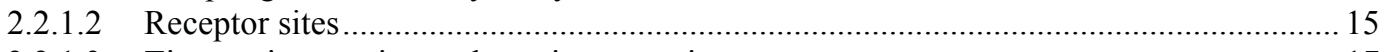

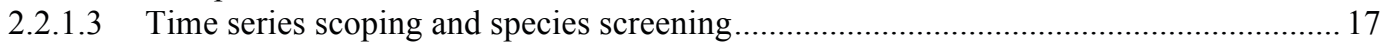

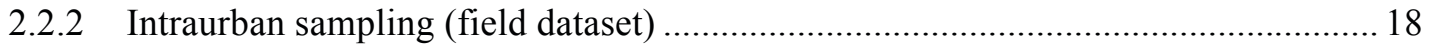

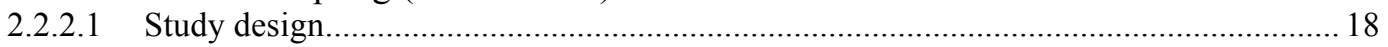

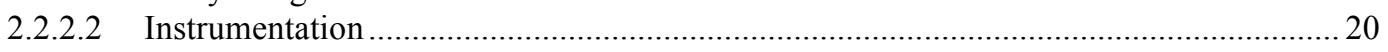

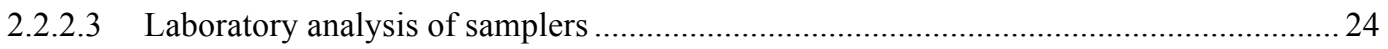

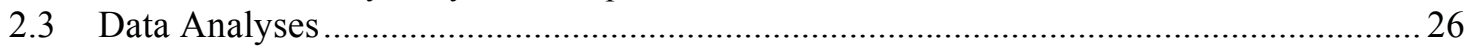

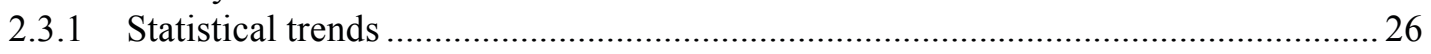

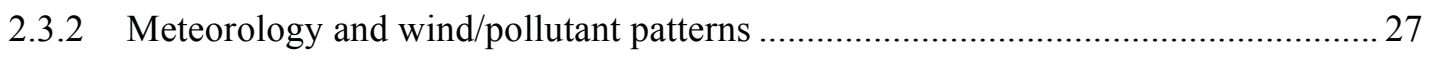

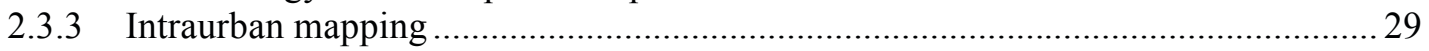

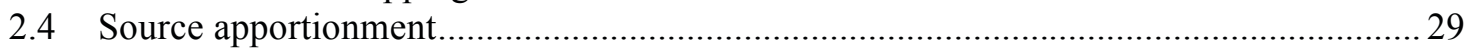

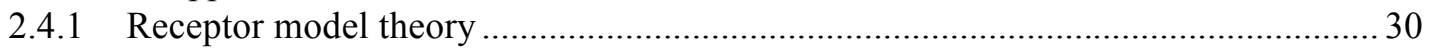

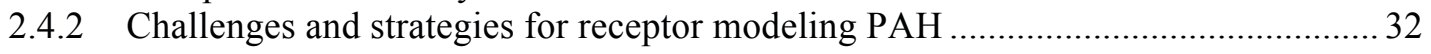

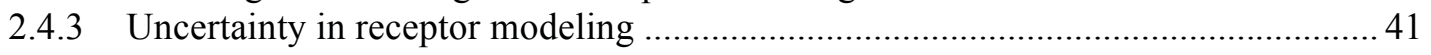

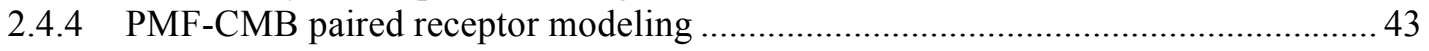

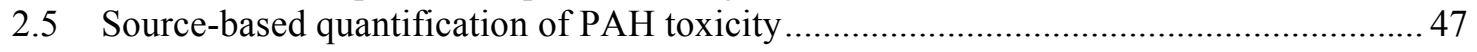




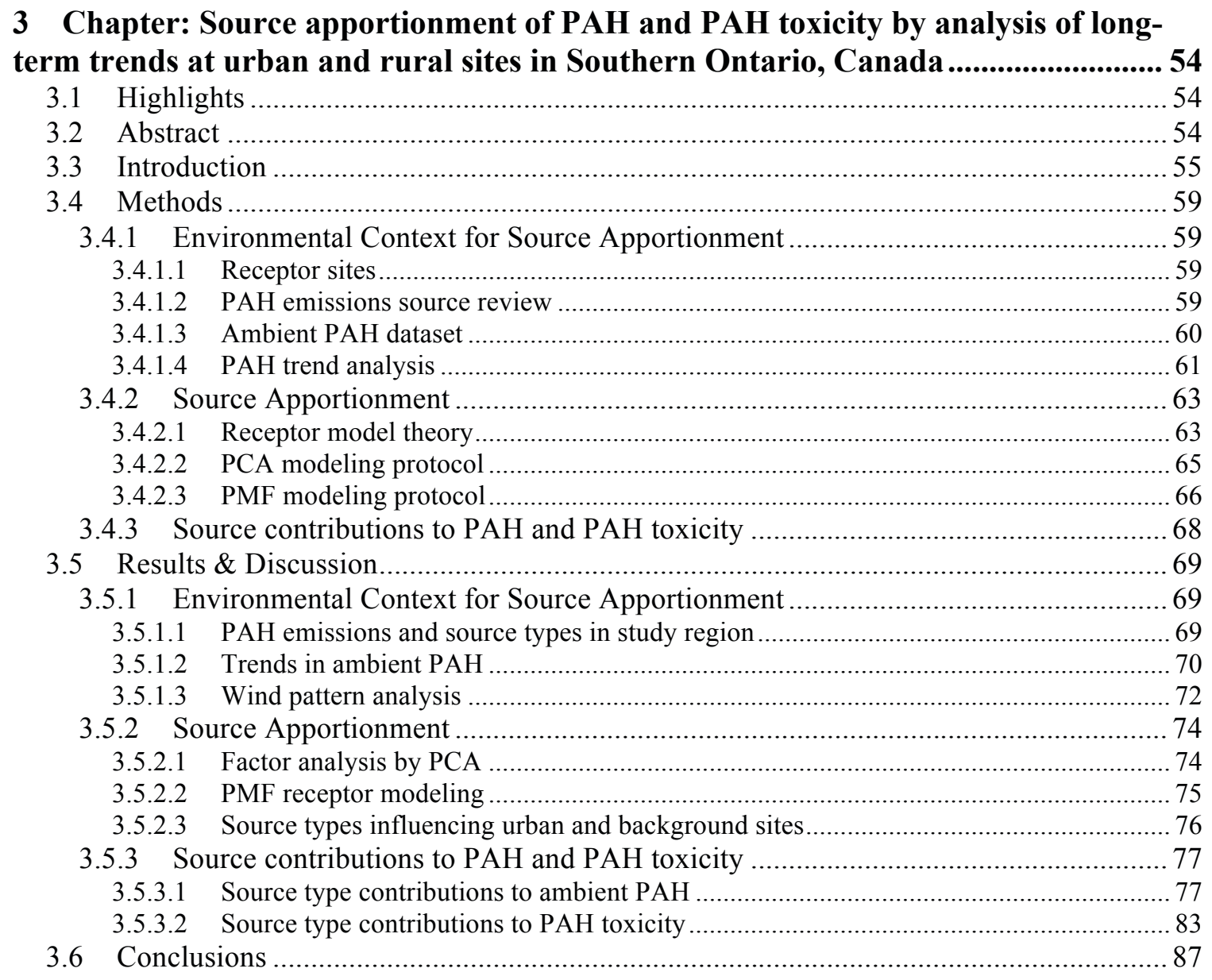




\section{Chapter: Intraurban concentrations, spatial variability and correlation of}

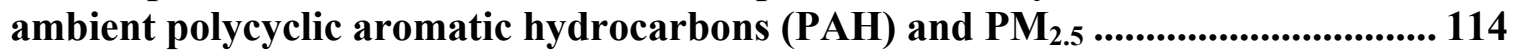

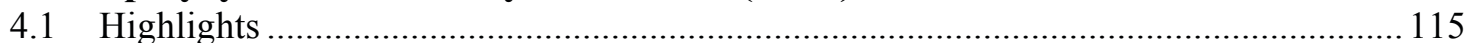

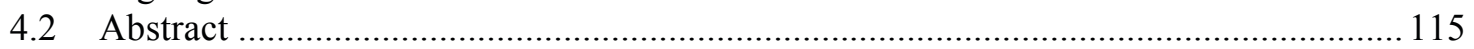

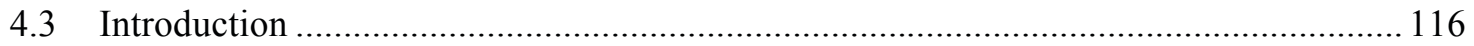

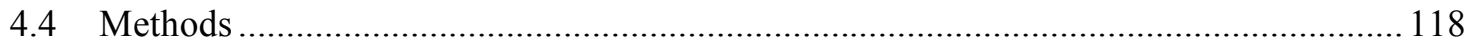

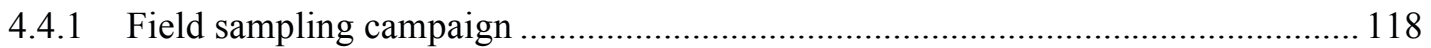

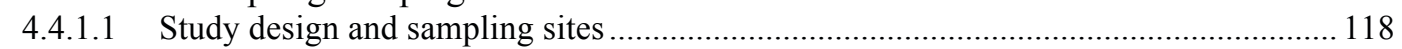

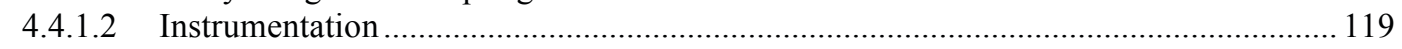

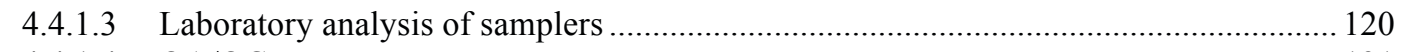

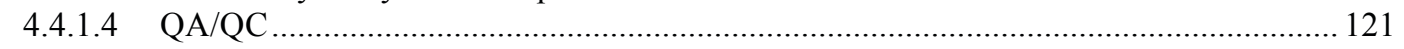

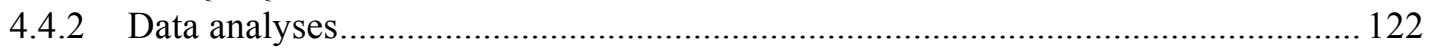

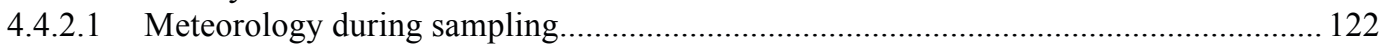

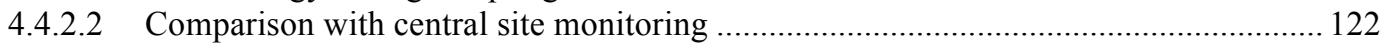

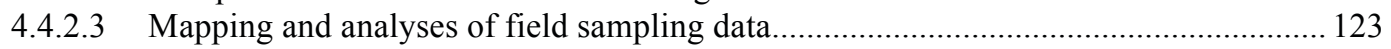

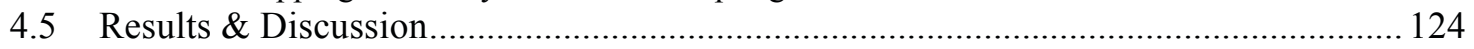

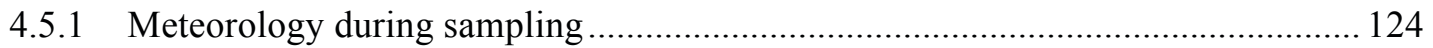

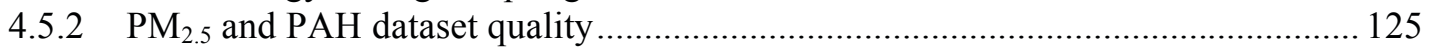

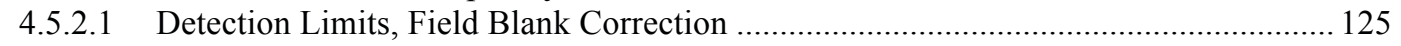

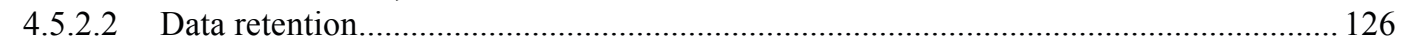

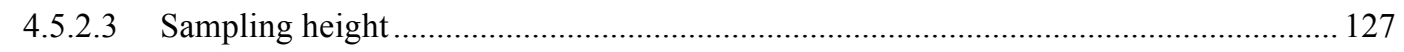

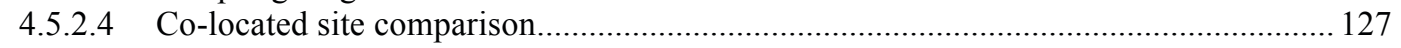

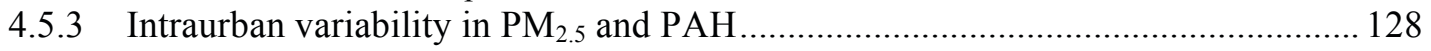

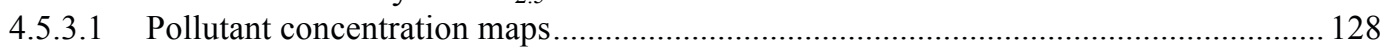

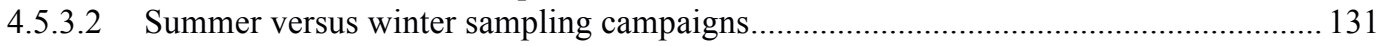

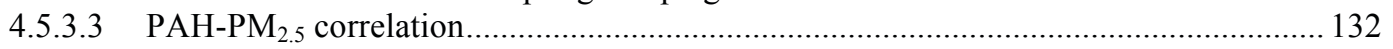

4.5.3.4 Differing PAH-PM $\mathrm{P}_{2.5}$ intraurban variability ………................................................... 133

4.5.3.5 Intraurban variability in PAH concentration versus central site monitoring .................. 134

4.5.4 Intraurban variability in Benzo(a)Pyrene-equivalent toxicity ............................... 136

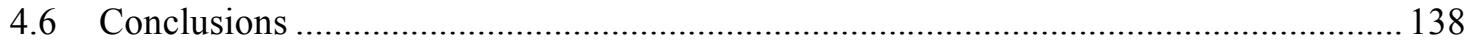




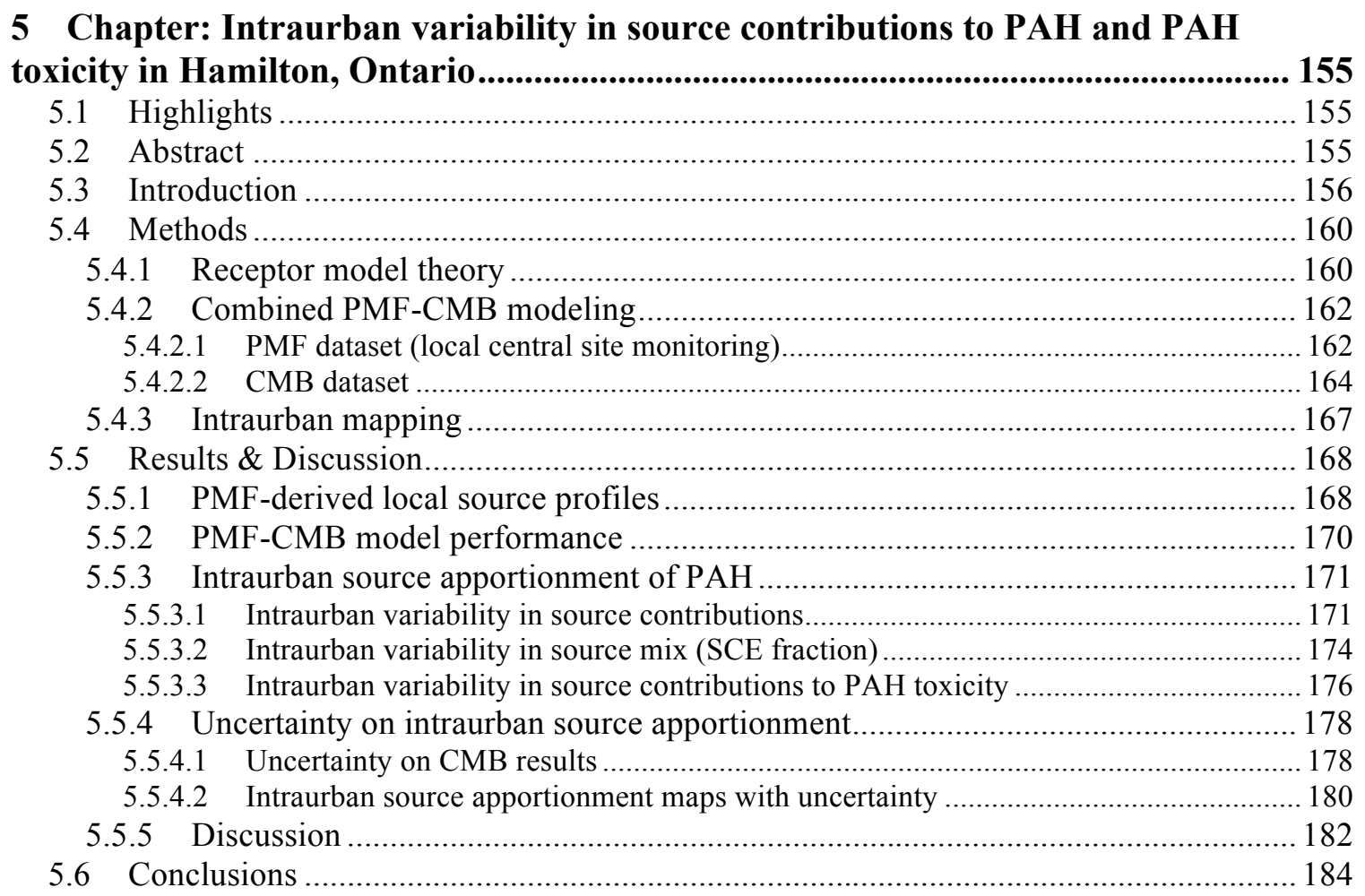


6 Chapter: Conclusions and recommendations ................................................ 201

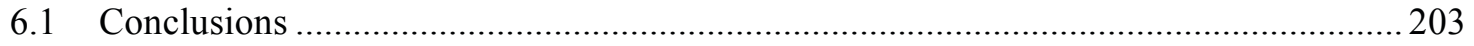

6.1.1 Urban/rural source apportionment of PAH and PAH toxicity ...............................203

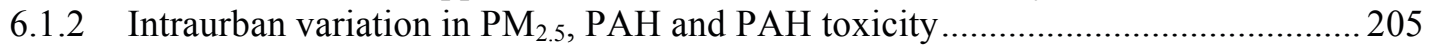

6.1.3 Intraurban source apportionment of PAH and PAH toxicity ..................................206

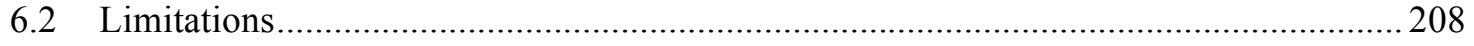

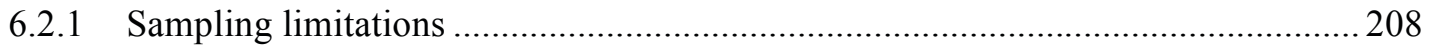

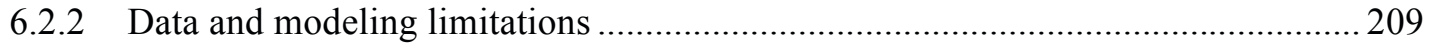

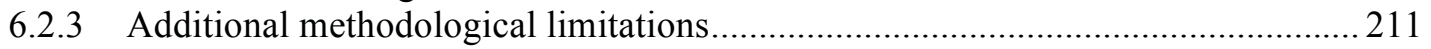

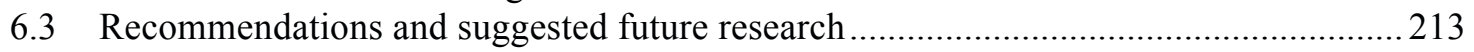

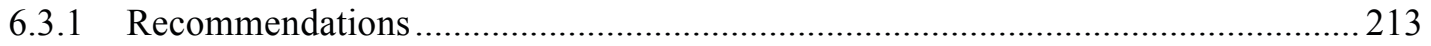

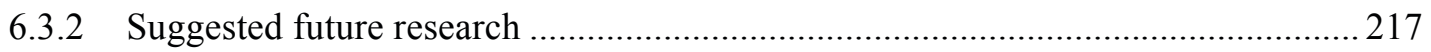


Appendices....................................................................................................................... 220

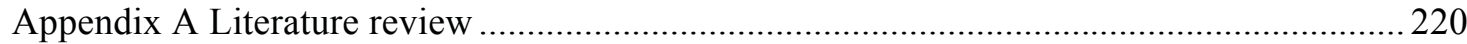

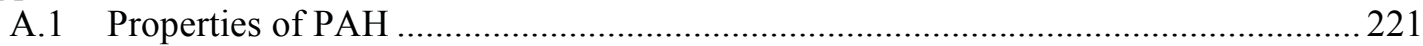

A.2 PAH exposure health effects ......................................................................... 230

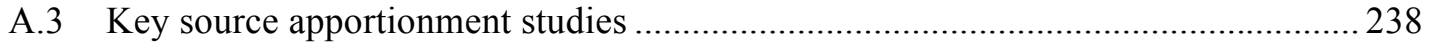

A.4 Approaches to estimating uncertainty on PMF input data ..................................... 244

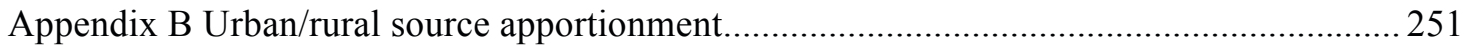

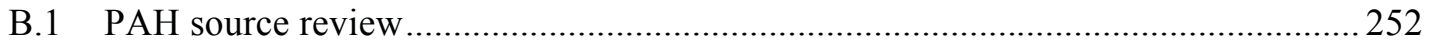

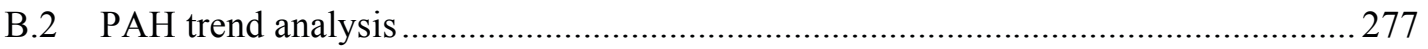

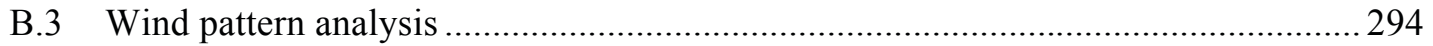

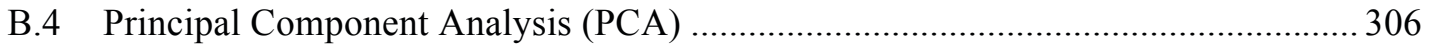

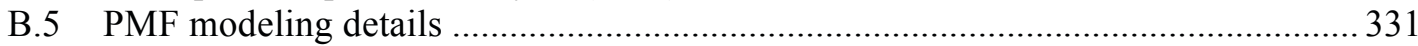

B.6 PMF Sensitivity Analyses ................................................................................. 343

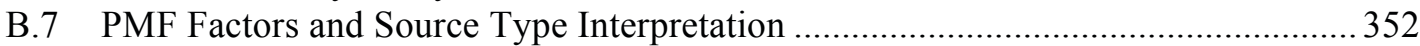

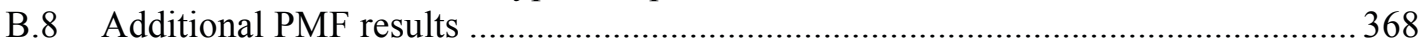

Appendix C Detailed intraurban field sampling work .........................................................38

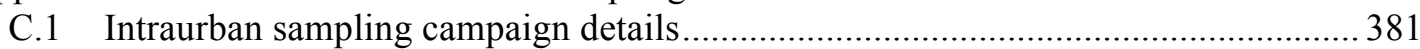

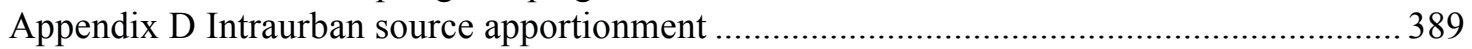

D.1 PMF-CMB model parameters and performance criteria.............................................390

D.2 Preliminary PMF modeling for generating CMB input source profiles .....................392

D.3 PMF-CMB model performance verification .............................................................. 397

D.4 Detailed results for intraurban source apportionment via PMF-CMB ........................ 401

D.5 Intraurban source apportionment using seasonal source profiles ............................... 431

Appendix E Detailed modeling methodology ................................................................... 442

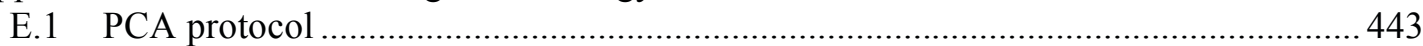

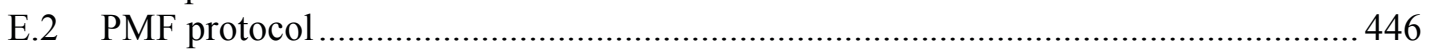

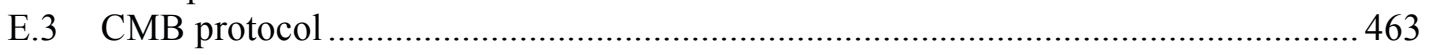

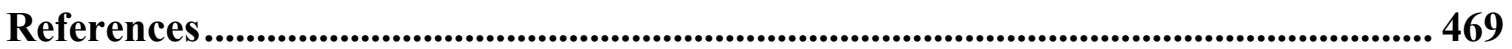




\section{List of Tables}

\section{Chapter 2}

Table 2-1. NAPS-sampled PAH detection limit and error fraction data (modeled suite).............. 14

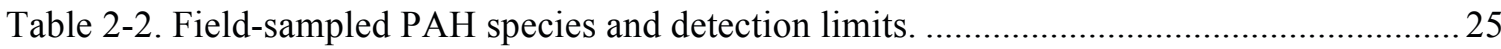

Table 2-3. NAPS monitoring, NPRI reporting, CEPA-toxicity, and US regulation of PAH. .......52

Table 2-4. Benzo(a)Pyrene-equivalent carcinogenic Toxicity Equivalency Factors (BaP-TEF) for

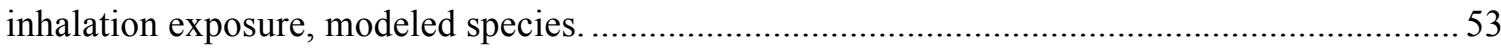

\section{Chapter 3}

Table 3-1. NAPS central site sampled PAH (modeled species), chemical formula, molecular weight grouping, and species method detection limits and error fractions.................................. 91

Table 3-2. Treatment protocols for speciated ambient receptor data used in PMF.

Table 3-3. Benzo(a)Pyrene-equivalent carcinogenic Toxicity Equivalency Factors (BaP-TEF) for

inhalation exposure, modeled species.

Table 3-4. Total aggregate source contributions to ambient PAH and toxicity (BaP-TEQ).

\section{Chapter 4}

Table 4-1. Sampled PAH species, grouping by molecular weight, sites above Lab Detection Limit.

Table 4-2. Field sampling data retention and descriptive statistics.

Table 4-3. Correlation between sampled $\mathrm{PAH}$ and $\mathrm{PM}_{2.5}$ (Spearman's rho).

Table 4-4. Descriptive statistics for field sampling data below/above (lower/upper) Hamilton escarpment

Table 4-5. $\mathrm{PAH} / \mathrm{PM}_{2.5}$ mass ratios for sites with summer and winter data $(\mathrm{ng} / \mu \mathrm{g})$.

Table 4-6. BaP-equivalent carcinogenic Toxic Equivalency Factors (BaP-TEF) for inhalation exposure.

\section{Chapter 5}

Table 5-1. Central site monitored (NAPS) PAH, detection limits, error fractions (PMF model suite).

Table 5-2. Treatment protocols for speciated ambient receptor data used in PMF and CMB.... 189

Table 5-3. Field-sampled PAH species, error fraction, detection limit, sites above DL.

Table 5-4. BaP-equivalent carcinogenic Toxic Equivalency Factors (BaP-TEF) for inhalation exposure (field-sampled species).

Table 5-5. Relative source contributions (SCE fractions) to $\Sigma$ PAH and BaP-TEQ; summary statistics. 


\section{List of Figures}

\section{Chapter 2}

Figure 2-1. Graphical abstract of research methodology.

Figure 2-2. Map of study region showing NAPS sites scoped for receptor modeling

Figure 2-3. Study area showing summer and winter campaign sites, NAPS site, meteorology

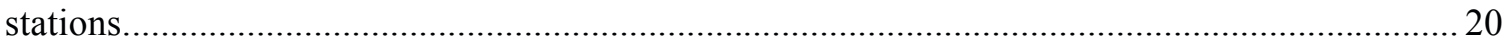

Figure 2-4. PAH and PM samplers and airflow pump inside weatherproof case .......................... 22

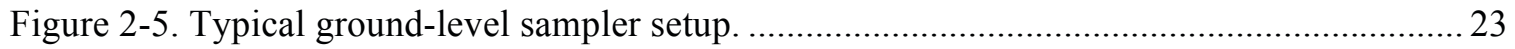

\section{Chapter 3}

Figure 3-1. Map of study region showing NAPS sites scoped for receptor modeling.................. 95

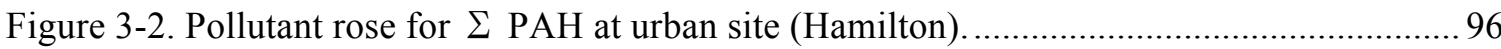

Figure 3-3. Correlation of PMF-predicted versus measured sampled mass, year-round model... 98

Figure 3-4. Bootstrap factor profiles (concentration, \% variance), year-round PMF model....... 101

Figure 3-5. Bootstrap factor profiles (concentration, \% variance) by source type, year-round

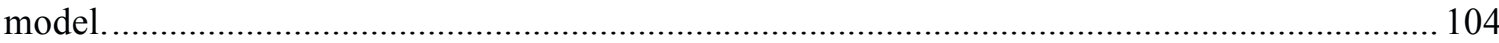

Figure 3-6. Source apportionment of PAH to PMF factors (all modeled mass).......................... 108

Figure 3-7. Inter-site comparison of PAH source apportionment (all modeled mass). ............... 110

Figure 3-8. Inter-site comparison of PAH source apportionment (Q1, median, Q3 contribution to

sample)

Figure 3-9. Inter-site comparison of PAH toxicity (BaP-TEQ) source apportionment, all modeled mass.

Figure 3-10. Source apportionment to PAH versus toxicity (BaP-TEQ), all modeled mass

\section{Chapter 4}

Figure 4-1. Study area showing summer and winter campaign sites, NAPS site, meteorology stations

Figure 4-2. PAH and PM samplers and airflow pump inside weatherproof case. ....................... 145

Figure 4-3. Typical ground-level sampler setup (i.e., sampler case on sampling tripod)........... 146

Figure 4-4. Wind rose for Hamilton study area during summer and winter campaigns.............. 147

Figure 4-5. Co-located field-sampled PAH and NAPS-sampled mean PAH (nearest bracketing

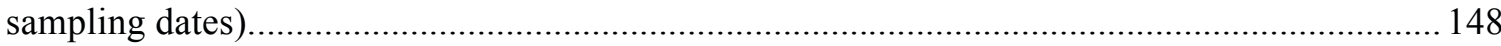

Figure 4-6. Sampled $\mathrm{PM}_{2.5}$ concentrations, by quartile, summer and winter campaigns............. 149

Figure 4-7. Sampled $\Sigma_{16} \mathrm{PAH}$ concentrations, by quartile, summer and winter campaigns....... 151

Figure 4-8. Sampled BaP-TEQ concentrations, by quartile, summer and winter campaigns...... 153 


\section{Chapter 5}

Figure 5-1. Map of study area showing escarpment, sampling sites, central monitoring station.193

Figure 5-2. Local source profiles (PR) used in CMB modeling.

Figure 5-3. Map of fractional source contribution to $\Sigma \mathrm{PAH}$, all modeled factors. .................... 195

Figure 5-4. Map of fractional source contribution to BAPTEQ, all modeled factors.................. 196

Figure 5-5. Map of source contribution to $\Sigma \mathrm{PAH}$ with uncertainty (

\section{List of Equations}

\section{Chapter 2}

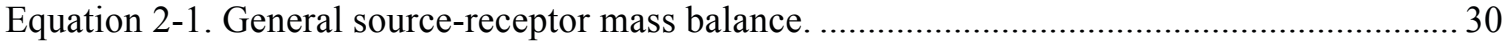

Equation 2-2. PMF minimization function for solution of source-receptor mass balance. ........... 32

Equation 2-3. Calculation of total sampled or modeled BAP-toxicity equivalent concentration. . 50

\section{Chapter 3}

Equation 3-1. General source-receptor mass balance.

Equation 3-2. Minimization function used by PMF to solve source-receptor mass balance......... 64

\section{Chapter 4}

Equation 4-1. Pollutant mass ratio for sampled $\mathrm{PM}_{2.5}$ relative to sampled $\mathrm{PAH}$.

\section{Chapter 5}

Equation 5-1. General source-receptor mass balance. 160

Equation 5-2. PMF minimization function for solution of source-receptor mass balance. 161

Equation 5-3. Species uncertainty for CMB source profiles. 


\section{List of Abbreviations}

$\mathrm{AC}$

AD

ADwPRhsw

ADwPRhw

AP-42

AQHI

ATSDR

BaP-eq

BaP-TEQ

BOOT_C

BOOT_EV

CAS

CEPA

$\mathrm{Cl}$

CMB

CSV

CV

CWS

DL

DR alternating current

Ambient Data (CMB input)

CMB model variant with winter ambient data and input source profiles derived from PMF of Hamilton central site data (year-round samples)

CMB model variant with winter ambient data and input source profiles derived from PMF of Hamilton central site data (winter month samples)

Air Pollutant-42 (emissions factor database)

Air Quality Health Index

Agency for Toxic Substances and Disease Registry

Benzo(a)Pyrene-equivalent concentration $\left[\mathrm{ng} / \mathrm{m}^{3}\right]$

Total Benzo(a)Pyrene-toxicity equivalent concentration for sampled or modeled species $\left[\mathrm{ng} / \mathrm{m}^{3}\right]$

Bootstrap factor Concentration $\left[\mathrm{ng} / \mathrm{m}^{3}\right]$

Bootstrap factor Explained Variance [\%]

Chemical Abstracts Service

Canadian Environmental Protection Act

elemental chlorine

Chemical Mass Balance (receptor model)

Comma Separated Value (file format)

Coefficient of Variation

Canada-Wide Standard

Detection Limit

Diagnostic Ratio 


\begin{tabular}{|c|c|}
\hline $\mathrm{EC}$ & Elemental Carbon \\
\hline $\mathrm{EF}$ & Emissions Factor \\
\hline EPA & Environmental Protection Agency \\
\hline ER & Emissions Rate \\
\hline ETC & Environmental Technology Center \\
\hline EU & European Union \\
\hline EV & Explained Variance $[\%]$ \\
\hline FA & Factor Analysis \\
\hline FBC & Field Blank Correction \\
\hline F_C & base run Factor Concentration $\left[\mathrm{ng} / \mathrm{m}^{3}\right]$ \\
\hline F_EV & base run Factor Explained Variance [\%] \\
\hline FIRE & Factor Information Retrieval System (emissions factor database) \\
\hline FM & Fit Measure \\
\hline Fpeak_C & F-peak factor Concentration $\left[\mathrm{ng} / \mathrm{m}^{3}\right]$ \\
\hline Fpeak_EV & F-peak factor Explained Variance [\%] \\
\hline GC-MSD & Gas Chromatography with Mass Spectrometry Detection \\
\hline GFF & Glass-Fibre Filter \\
\hline GIS & Geographic Information System \\
\hline GOF & Goodness Of Fit \\
\hline GPS & Global Positioning System \\
\hline HDV & Heavy Duty Vehicle \\
\hline HDDV & Heavy Duty Diesel Vehicle \\
\hline HMA & Hot Mix Asphalt \\
\hline
\end{tabular}




\begin{tabular}{|c|c|}
\hline hydroxy-PAH & hydroxylated Polycyclic Aromatic Hydrocarbon (OH-PAH) \\
\hline IADN & Integrated Atmospheric Deposition Network \\
\hline IQR & Interquartile Range \\
\hline LDL & Lab Detection Limit \\
\hline LDV & Light Duty Vehicle \\
\hline LRTAP & Long Range Transport of Air Pollutants \\
\hline MDL & Method Detection Limit \\
\hline me-PAH & methylated Polycyclic Aromatic Hydrocarbon $\left(\mathrm{CH}_{3}-\mathrm{PAH}\right)$ \\
\hline MOVES & Motor Vehicle Emission Simulator (emissions inventory model) \\
\hline MPIN & Modified Pseudo Inverse Matrix \\
\hline MW & molecular weight \\
\hline $\mathrm{Na}$ & elemental sodium \\
\hline $\mathrm{NH}_{4}$ & ammonium ion \\
\hline $\mathrm{NO}_{3}$ & nitrate ion \\
\hline $\mathrm{NO}_{\mathrm{x}}$ & oxides of nitrogen \\
\hline NAICS & North American Industry Classification System \\
\hline NAPS & National Air Pollution Surveillance network \\
\hline NESHAP & National Emission Standards for Hazardous Air Pollutants \\
\hline NFRAQS & Northern Front Range Air Quality Study \\
\hline nitro-PAH & nitrated Polycyclic Aromatic Hydrocarbon $\left(\mathrm{NO}_{2}-\mathrm{PAH}\right)$ \\
\hline NMIM & National Mobile Inventory Model (emissions inventory model) \\
\hline NREL & National Renewable Energy Laboratory \\
\hline NOAA & National Oceanic and Atmospheric Administration \\
\hline
\end{tabular}




\begin{tabular}{|c|c|}
\hline NPRI & National Pollutant Release Inventory \\
\hline $\mathrm{OC}$ & organic carbon \\
\hline PAH & Polycyclic Aromatic Hydrocarbon \\
\hline $\mathrm{PC}$ & Principal Component \\
\hline PCA & Principal Component Analysis (model) \\
\hline PCB & Polychlorinated Biphenyl \\
\hline PERC_MASS & percent mass (goodness of fit metric for CMB receptor model) \\
\hline PM & Particulate Matter \\
\hline $\mathrm{PM}_{10}$ & Particulate Matter of size less than $10 \mu \mathrm{m}$ (“coarse fraction") \\
\hline $\mathrm{PM}_{2.5}$ & Particulate Matter of size less than $2.5 \mu \mathrm{m}$ (“fine fraction") \\
\hline $\mathrm{PM}_{1.0}$ & Particulate Matter of size less than $1.0 \mu \mathrm{m}$ (“ultra-fine fraction) \\
\hline $\mathrm{PM}_{10-2.5}$ & Particulate Matter between $2.5 \mu \mathrm{m}$ and $10 \mu \mathrm{m}$ in size \\
\hline $\mathrm{PM}_{2.5-1.0}$ & Particulate Matter between $1.0 \mu \mathrm{m}$ and $2.5 \mu \mathrm{m}$ in size \\
\hline PMF & Positive Matrix Factorization (receptor model) \\
\hline PMF-CMB & $\begin{array}{l}\text { Positive Matrix Factorization-Chemical Mass Balance (combined receptor } \\
\text { models) }\end{array}$ \\
\hline PR & Source PRofile (CMB input) \\
\hline PRhsw & $\begin{array}{l}\text { CMB model input PRofiles derived from PMF of Hamilton central site data } \\
\text { (year-round samples) }\end{array}$ \\
\hline PRhw & $\begin{array}{l}\text { CMB model input PRofiles derived from PMF of Hamilton central site data } \\
\text { (winter samples) }\end{array}$ \\
\hline PUF & Polyurethane Foam (sampler media) \\
\hline QAQC & Quality Assurance Quality Control \\
\hline RSD & Relative Standard Deviation \\
\hline SCE & Source Contribution Estimate (CMB model output) $\left[\mathrm{ng} / \mathrm{m}^{3}\right]$ \\
\hline
\end{tabular}




\begin{tabular}{|c|c|}
\hline SCE_BaP-TEQ & $\begin{array}{l}\text { Source Contribution Estimate to Benzo(a)Pyrene-Toxicity Equivalency } \\
\text { concentration }\left[\mathrm{ng} / \mathrm{m}^{3}\right]\end{array}$ \\
\hline SCC & Source Classification Code \\
\hline $\mathrm{SD}$ & Standard Deviation \\
\hline $\mathrm{SN}$ & Signal-to-Noise \\
\hline $\mathrm{SO}_{4}$ & sulfate ion \\
\hline SP & Source Profile \\
\hline STN & Speciation Trends Network \\
\hline SVOC & Semi-Volatile Organic Compound \\
\hline TEF & $\begin{array}{l}\text { Toxicity Equivalency Factor, defined with respect to Benzo(a)Pyrene } \\
\text { [unitless] }\end{array}$ \\
\hline $\mathrm{TM}$ & Total Mass (CMB modeled mass) \\
\hline UNC & Uncertainty on Source Contribution Estimate (CMB model output) $\left[\mathrm{ng} / \mathrm{m}^{3}\right]$ \\
\hline VOC & Volatile Organic Compound \\
\hline WEBFIRE & $\begin{array}{l}\text { Web Factor Information Retrieval System (US EPA online emissions factor } \\
\text { database) }\end{array}$ \\
\hline
\end{tabular}




\section{List of Symbols}

$\mathrm{c}_{\mathrm{ij}}$

C3

$\operatorname{chi}^{2}$

$\mathrm{DL}_{\mathrm{j}}$

$\mathrm{E}$

$e_{i j}$

$\mathrm{F}$

$f_{k j}$

G

$\mathrm{g}_{\mathrm{ik}}$

$\mathrm{K}_{\mathrm{H}}$

$\mathrm{k}_{\mathrm{j}}$

$\mathrm{K}_{\text {ow }}$

n

$\mathrm{p}$

PERC_MASS

Q

$\mathrm{Q}_{1}$

$\mathrm{Q}_{2}$

$\mathrm{Q}_{3}$

Q robust sampled concentration of species $j$ in receptor sample $i$

additional modeling uncertainty as percent (PMF receptor model)

goodness of fit measure (CMB receptor model)

detection limit for species $j$

matrix of residual concentrations

model residual concentration of species $j$ in receptor sample $i$

matrix of factor concentration profiles

mass of species $j$ in source factor $k$

matrix of factor contributions

contribution of source factor $k$ to receptor sample $i$

Henry's Law Constant

error fraction for species $j$

octanol-water partition coefficient

sample size

model order

sum of source contribution estimates divided by total mass modeled, as percent (CMB receptor model)

object function for goodness of fit (PMF receptor model)

first quartile

second quartile (median)

third quartile

robust object function value for goodness of fit, calculated excluding outlier samples (PMF receptor model) 


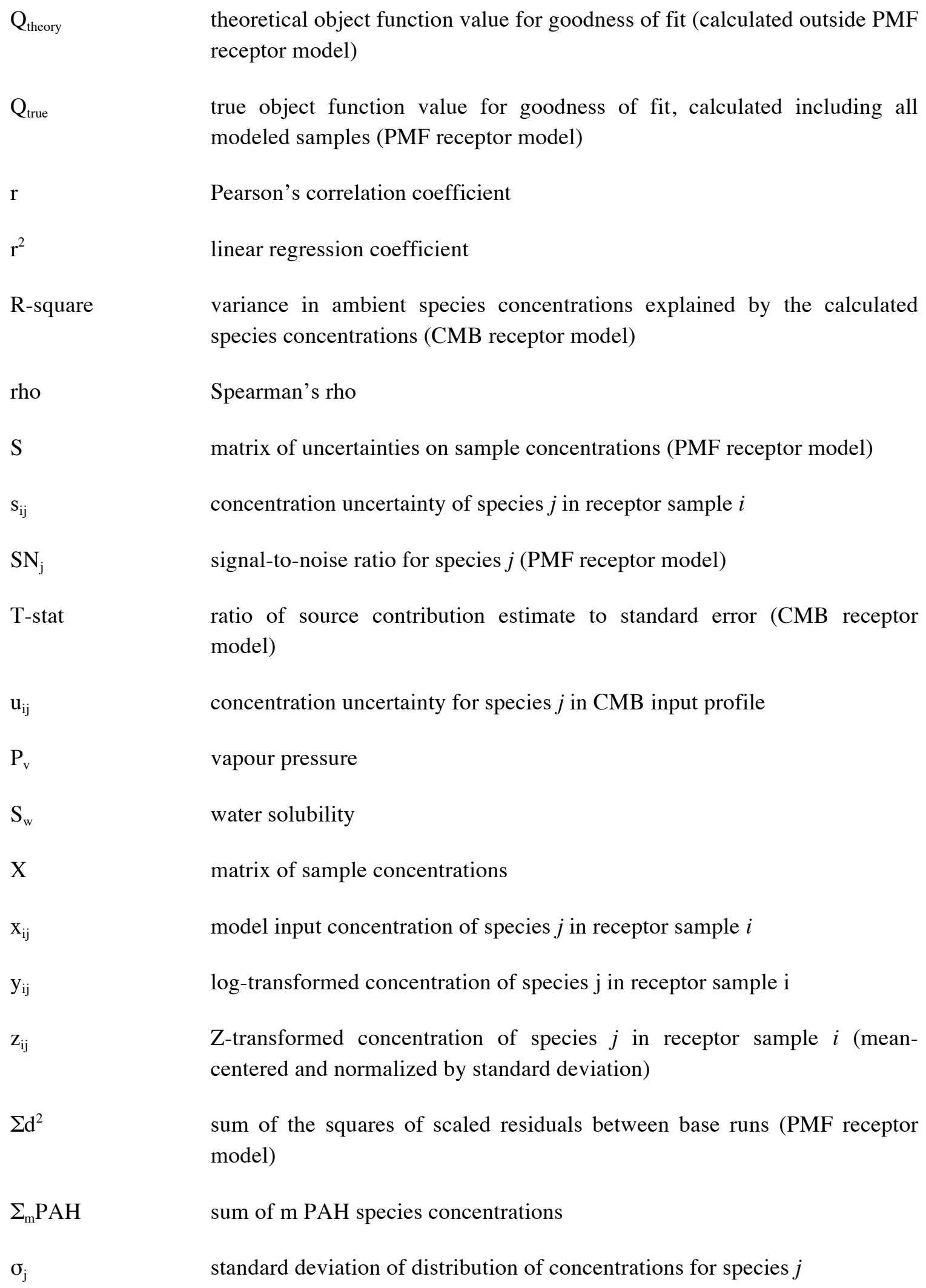

$\mathrm{Q}_{\text {true }}$

r

true object function value for goodness of fit, calculated including all modeled samples (PMF receptor model)

Pearson's correlation coefficient

linear regression coefficient

variance in ambient species concentrations explained by the calculated species concentrations (CMB receptor model)

Spearman's rho

matrix of uncertainties on sample concentrations (PMF receptor model) concentration uncertainty of species $j$ in receptor sample $i$ signal-to-noise ratio for species $j$ (PMF receptor model) ratio of source contribution estimate to standard error (CMB receptor model)

concentration uncertainty for species $j$ in CMB input profile

vapour pressure

water solubility

matrix of sample concentrations

model input concentration of species $j$ in receptor sample $i$

log-transformed concentration of species $\mathrm{j}$ in receptor sample $\mathrm{i}$

Z-transformed concentration of species $j$ in receptor sample $i$ (meancentered and normalized by standard deviation)

sum of the squares of scaled residuals between base runs (PMF receptor model)

sum of m PAH species concentrations

standard deviation of distribution of concentrations for species $j$ 
$\Sigma_{\text {LIGHT }} \mathrm{PAH} \quad$ sum of light molecular weight species concentrations

$\Sigma_{\text {HEAVY }} \mathrm{PAH} \quad$ sum of heavy molecular weight species concentrations 


\section{Chapter: Introduction}

\subsection{Rationale and significance of research}

Air quality researchers, legislators, and medical professionals are placing increased emphasis on the health risk of exposure to respirable particulate matter (PM) particularly the fine size fraction (e.g., $\mathrm{PM}_{2.5}$ ). However, as an aggregate compound, PM is not equitoxic - health risk can vary depending on aerosol size distribution as well as associated toxic compounds such as Polycyclic Aromatic Hydrocarbons (PAH). (Grahame and Schlesinger, 2007; Tolbert, 2007) Monitoring PM-associated toxics such as PAH has been a significant advance in exposure assessment, reflecting steadily growing awareness of the variability in PM toxicity.

PAHs are produced by incomplete combustion of organic material and have many anthropogenic sources (i.e., coal-fired power generation, space heating by fossil fuel combustion, vehicle exhaust, and industrial activity such as aluminum smelting and steel production) (ATSDR, 1995). As semi-volatile organic compounds (SVOCs), PAHs in ambient air are present in both vapour and particle phases; lighter molecular weight PAH species (i.e., typically 2- and 3-ring structures) are found largely in vapour phase while heavier species (i.e., typically 5-ring, molecular weight $>228$ ) are largely in particle phase and found predominantly in the smaller respirable size fraction (i.e., $\mathrm{PM}_{2.5}$ ), increasing exposure health risk (Venkataraman and Friedlander, 1994; Lu et al., 2008). PAHs have been declared toxic under the Canadian Environmental Protection Act (Environment Canada, 2011a) and a number of heavy molecular weight species are under scrutiny for their probable carcinogenicity (ATSDR, 1995) and even lighter PAHs constitute a health 
concern because they can react with sunlight or other atmospheric compounds to form transformation products (e.g., nitro-PAH, hydroxy-PAH) with greater toxicity and environmental persistence (Baek et al., 1991a).

Source-based analysis of PM-associated toxics, inclusive of PAH, was stressed by a comprehensive literature review of toxicology, epidemiology, and exposure assessment studies which found ambient monitoring to be insufficient for improving exposure assessment for health risk estimates, particularly for improving regulation of air toxics, and argued instead that "certain sources of PM emissions, by virtue of disproportionate production of PM-associated toxics such as PAH, may be considered for the purposes of health risk assessment 'more toxic' than others." (Grahame and Schlesinger, 2007) The recommendation was to better identify and quantify the sources of PM-associated air toxics such as PAH, with local sources (e.g., vehicle traffic, local industry) cited as significant contributors to variable exposure health risk (Grahame and Schlesinger, 2007; Schwartz et al, 2005; Gold et al, 2005).

This research examined ambient PAH levels from a strict source-based perspective to identify and quantify the dominant source types contributing to PAH and PAH toxicity at the urban and intraurban scales. The research findings and methodologies demonstrated in this work together provide several meaningful interdisciplinary contributions to researchers investigating PM and PAH exposure health risk.

First, by identifying the relative contribution of dominant source types to $\mathrm{PAH}$ and to PAH toxicity, the source apportionment results at urban and rural centres inform appropriate intervention strategies, both source-based (e.g., regulated emission reductions 
by industry or mobile sources) and receptor-based (e.g., zoning for greater buffer areas between industrial and residential land uses, health messaging to reduce individuals' exposures to local sources). Inter-urban and urban-rural comparison of $\mathrm{PAH}$ source apportionment results also allow environmental regulators to develop strategies that are better targeted to specific sources (i.e., based on toxicity of contributions) and sensitive to inter-urban and urban/rural differences.

Second, dense field sampling and spatial analysis of the intraurban variation in ambient $\mathrm{PAH}$ allows better characterization of $\mathrm{PM}_{2.5}$ composition and health risk, particularly for smaller urban centres where $\mathrm{PM}_{2.5}$ mass concentration may otherwise show little spatial variability (Seinfeld and Pandis, 2006; Narvaez et al, 2008; Brook et al, 2004; Fischer et al, 2000; Kinney et al, 2000). Variability in PAH and PAH toxicity is shown to be a useful indicator of relative PM composition, helping explain epidemiological studies that have demonstrated variable PM health risk at the intraurban scale (Grahame and Schlesinger, 2007; Jerrett et al, 2005; Schwartz et al, 2005; Gold et al, 2005). As one potential application, recommendation is made to include local scale PAH information in air quality metrics (e.g., health-based Air Quality Index, AQHI, currently offered in several large Canadian urban centres; Stieb et al, 2008) so as to provide messaging that is more representative of air pollution exposure health risk, particularly for sub-populations living or working in at-risk neighbourhoods.

Third, beyond identifying exposure inequalities, intraurban source apportionment of PAH permits better understanding of sources contributing to 'exposure hot spots'. This has broader application beyond the study city and will assist researchers studying intraurban 
exposure inequalities. Additionally, the explicit presentation of intraurban source apportionment results in terms of PAH toxicity will provide public health planners with vital information for prioritizing source types for effective intervention strategies (i.e., targeted to at-risk communities; scaled to local sources versus regional sources).

\subsection{Hypotheses}

As an alternative to using PM mass concentration as a proxy for health risk, the variability in fine PM composition suggests that additional qualification of PM-associated air toxics such as PAHs, and application of receptor modeling techniques to identify PAH sources and quantify spatial variability in source contributions, will prove more meaningful. To this end, the proposed research hypothesized that:

i. Inter-urban and urban/rural differences in historical ambient PAH levels will be significant and source apportionment will demonstrate these to relate to inter-site differences in dominant source types.

ii. Source apportionment will demonstrate some source types to be 'more toxic' than others (i.e., contributing relatively little to ambient PAH but significantly to PAHassociated toxicity).

iii. At the intraurban scale, $\mathrm{PAH}$ will show greater variability than $\mathrm{PM}_{2.5}$, demonstrating central monitoring as a comparatively poor proxy for estimating PAH exposure.

iv. At the intraurban scale, PAH 'exposure hot spots' (i.e., locations of elevated ambient PAH) will generally be found near concentrated local sources (e.g., 
traffic, heavy industry); 'exposure toxicity hot spots', however, will not necessarily coincide with 'exposure hot spots', indicating that some source types are contributing comparably more toxicity than others.

v. At the intraurban scale, two different receptor models (i.e., PMF-CMB) could be paired so as to make use of 'local source profiles' for meaningful source apportionment.

vi. At the intraurban scale, source contributions to PAH will show spatial variability consistent with interpreted source type and will explain previously identified 'exposure hot spots'; source apportionment of PAH toxicity will distinguish sources contributing similarly across an urban centre from those associated with 'exposure toxicity hot spots'. 


\subsection{Research components}

The research was carried out in three distinct components:

I. Source apportionment of PAH and PAH toxicity at the urban and rural scale;

II. Field-sampling for $\mathrm{PM}_{2.5}, \mathrm{PAH}$ and $\mathrm{PAH}$ toxicity at the intraurban scale; and,

III. Source apportionment of PAH and PAH toxicity at the intraurban scale.

Research component I investigated ambient PAH and contributing sources at two southern Ontario urban centers (i.e., Hamilton, Toronto) and a rural background site. Research components II and III focused on ambient PAH and contributing sources across the Hamilton urban area, selected for emphasis due to its historically highest ambient $\mathrm{PAH}$ in Ontario and location of heavy industry source types associated with PAH releases (e.g., iron/steel manufacturing).

The research components were successive and inter-linked: the source apportionment of PAH and PAH toxicity at the urban/rural scale (i.e., research component I), in addition to useful inter-site comparisons of source types and relative contributions, resolved 'local source profiles' for Hamilton that were applied to the intraurban field sampling data (i.e., research component II) to examine intraurban differences in source contributions to PAH and PAH toxicity (i.e., research component III) and better understand 'exposure hotspots' and 'exposure toxicity hotspots' (i.e., identified in research component II). 


\subsection{Organization}

This dissertation is presented in six chapters. Following the Introduction, Chapter 2 presents the methodology related to the PAH data from Environment Canada's NAPS stations in Toronto, Hamilton, and Egbert, the methodology for the intraurban field sampling in Hamilton, and the modeling approaches used in analyzing both datasets. Chapters 3, 4 and 5 are written as journal manuscripts. Due to the nature of Chapters 3, 4 and 5 there is some repetition between the presentation of the methodology in Chapter 2 and the other three chapters.

Chapter 3 presents results of the first research component: Source apportionment of PAH and PAH toxicity at the urban and rural scale.

Chapter 4 presents the results of the second research component: the collaborative work with Health Canada in intraurban field sampling in Hamilton that underlies an already published paper (Anastasopoulos et al 2012). The first author on the paper and the author of the current dissertation was in charge of the implementation of the two sessions of field sampling with 45 sampling sites, and overall coordination of the field sampling with the collaborating partners, as well as the analysis and interpretation of results. Additional details of this author's coordination and involvement in the field sampling work are presented in Appendix $C$.

Chapter 5 presents results from the third research component: Source apportionment of PAH and PAH toxicity at the intraurban scale. 
Chapter 6 concludes by presenting a synthesis of research findings across the three research components and proposing recommendations for future research.

Five Appendices complement the Chapters in the body of the thesis, presenting background material and details about experimental and modeling work. This material has been presented in the form of appendices to enable a better flowing read of the Chapters in the main body of the thesis. Appendix A presents background material on PAH and PAH literature related to source apportionment of PAH. Appendix B presents material related to research component I and is intended for submission as Supplementary Information with the journal manuscript based on Chapter 3. Appendix $C$ presents material related to research component II. Appendix $D$ is intended for submission as Supplementary Information with the journal manuscript based on Chapter 5. Appendix E addresses details of modeling with PCA, PMF and CMB to enable future work with the methodology and with comparable datasets from different locations. 


\section{Chapter: Methodology}

\subsection{Overview of research methods}

Source-based investigation of ambient PAH and PAH toxicity was conducted at two scales (i.e., urban/rural; intraurban) using two respective datasets (i.e., central site monitoring datasets from urban/rural sites; intraurban dataset from a spatial field sampling campaign).

The research applied several investigative methods and tools, itemized below for each of the three research components introduced in Chapter 1.

Research component I:

- historical and spatial trend analysis of ambient PAH at the urban/background sites (i.e., Toronto, Hamilton, background) with inter-site comparisons;

- source apportionment at the urban/background scale via Positive Matrix Factorization (PMF);

- conversion of source apportionment results to equivalent source contributions to PAH toxicity and identification of "more toxic" sources; and,

- inter-site comparisons of sources contributing to PAH and PAH toxicity.

Research component II:

- coordination and implementation of field sampling for $\mathrm{PM}_{2.5}$ and $\mathrm{PAH}$ over a dense spatial network of sites at Hamilton; 
- intraurban comparisons and spatial trends for ambient $\mathrm{PM}_{2.5}$ and $\mathrm{PAH}$ via statistical metrics and mapping with identification of 'exposure hot spots';

- conversion of ambient PAH data to PAH toxicity; and,

- $\quad$ spatial trends in ambient PAH toxicity via statistical metrics and mapping with identification of 'exposure toxicity hot spots'.

Research component III:

- source apportionment at the intraurban scale via combined application of Chemical Mass Balance (CMB) and Positive Matrix Factorization (PMF);

- conversion of source apportionment results to equivalent source contributions to PAH toxicity;

- intraurban comparisons and spatial trends in source contributions to PAH and PAH toxicity via statistical metrics and mapping, quantifying degree of intraurban variability in source contributions;

- explanation of previously identified 'exposure hot spots' in terms of contributing sources; and,

- distinguishing sources contributing to PAH toxicity similarly across the urban area from those associated with previously identified 'exposure toxicity hot spots'. 
A graphical abstract summarizing the research methodology and demonstrating how the research components are inter-linked is presented in Figure 2-1.

The subsections in the remainder of this chapter will address first the methodology related to the datasets, then the methodology related to the modeling and toxicity. Appendices $A$ through $E$ present further details to enable verification and future application to similar datasets.

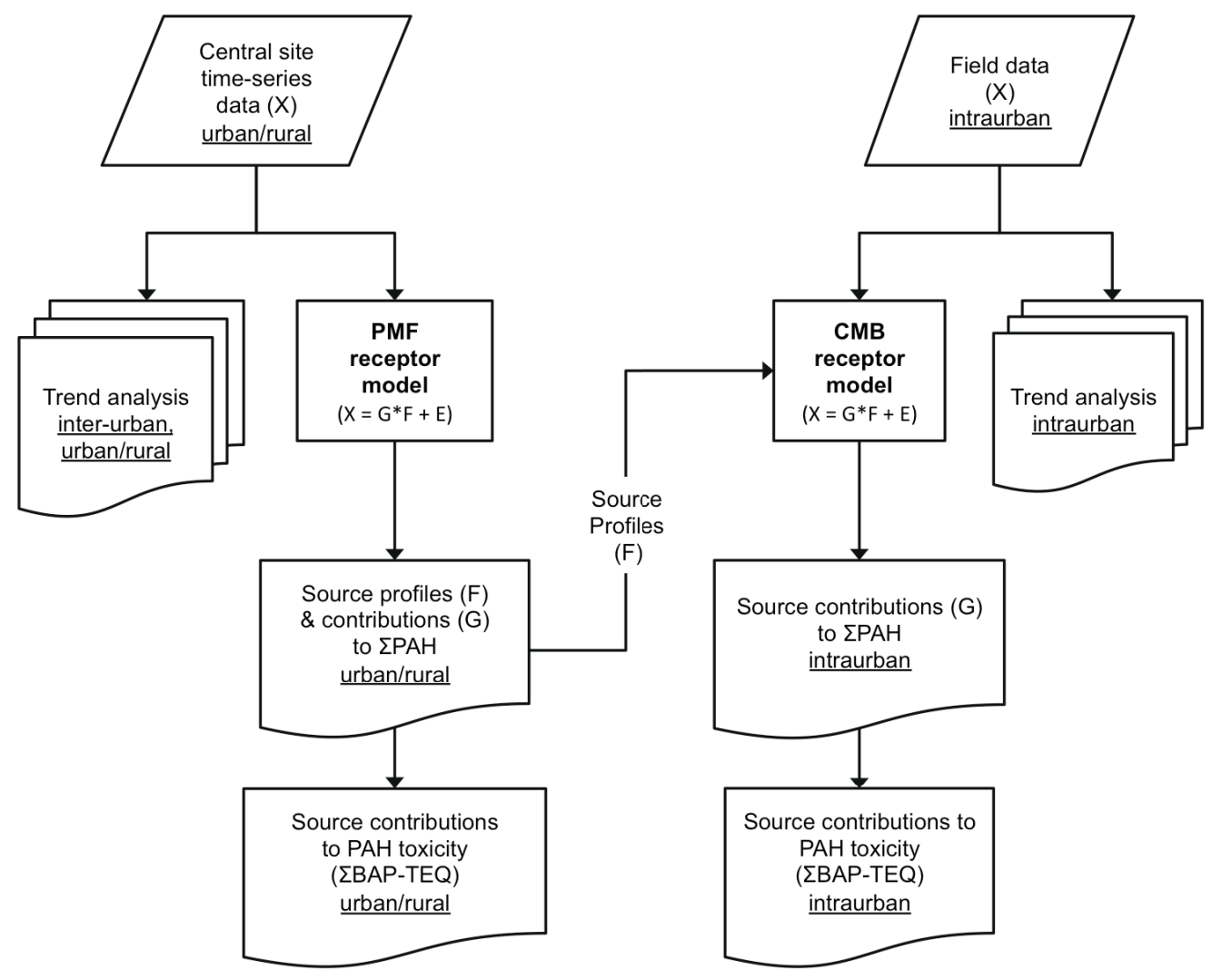

Figure 2-1. Graphical abstract of research methodology. 


\subsection{Datasets}

\subsubsection{Central site sampling (NAPS dataset)}

This author was a participant in the analysis of time series PAH data from six locations in Southern Ontario, commissioned by Environment Canada. Details of the study were previously reported to Environment Canada (Karman, Anastassopoulos, and Dogan, 2009). The analysis and modeling presented in this dissertation is based on a review of the data for three of the sites in the 2009 study (i.e., Hamilton, Toronto, Egbert).

\subsubsection{Sampling and laboratory analysis}

Ambient monitoring of PAHs in Canada is primarily through the National Air Pollution Surveillance (NAPS) network. ${ }^{1}$ In Ontario, PAH samples are collected from thirteen central site NAPS stations in urban and rural centres. (Environment Canada, 2004)

Each PAH sample is taken over a 24-hour period (i.e., 24-hr integrated sampling). Sampler analysis is coordinated and carried out at Environment Canada's Environmental Technology Center (ETC) in Ottawa, Ontario. (Environment Canada, 2007g) NAPS laboratory analysis on each sample is carried out for a total of 29 PAH species (i.e., receptor modeling species subset shown in Table 2-1; complete suite shown in Table 2-3) that range from light MW (e.g., $\leq 4-$ ring structure, MW $\leq 202$ ) to heavy MW (e.g., >4-ring structure, MW >228; light/medium/heavy MW grouping adapted from Li et al, 2005). ETC analyses PAH samples from all NAPS sites across Canada, ensuring data quality

\footnotetext{
${ }^{1}$ Additional sampling networks are operated in Ontario by the Ministry of Environment (MOE),
} often locating samplers near known stationary sources for purposes of compliance monitoring. 
control and permitting inter-site comparison of absolute levels. The sampling schedule at NAPS sites for PAHs is based on a 6-day interval (i.e., one sample taken every $6 / 12 / 18 / 24$ days as field resources and field staffing allow), to sample each day of the week an approximately equal number of times over a one-year period.

Sampling apparatus and laboratory analysis follows US EPA Method TO-13A (1999). Briefly, since ambient PAH are found in both particle and vapour phase and with phase prevalence varying by species (Venkataraman and Friedlander, 1994) a dual-media sampler is used to sample PAH from particle (i.e., glass-fibre filter, GFF) and vapour (i.e., polyurethane foam sorbent, PUF) phases at high volume flow rate. In the laboratory, the GFF and PUF samplers are co-extracted and analysed for species concentrations by three successive analytical steps (Environment Canada, 2007g), yielding total PAH concentration (i.e., sum of vapour plus particle phase): (i) extraction of PAHs from sampling media using acetone as the solvent; (ii) concentration of PAHs using nitrogen gas; and, (iii) PAH species identification and quantification by gas chromatography with mass spectrometry detection (GC-MSD).

Method detection limits (DL) for sampled species were obtained from a representative laboratory Quality Assurance Quality Control (QAQC) dataset made available by Environment Canada (2005) and are shown in Table 2-1. 
Table 2-1. NAPS-sampled PAH detection limit and error fraction data (modeled suite).

\begin{tabular}{|c|c|c|c|c|}
\hline PAH Species & Label & $\begin{array}{r}\text { MW } \\
\text { Grouping }\end{array}$ & $\begin{array}{r}\text { Method Detection } \\
\text { Limit (MDL) } \\
{\left[\mathrm{ng} / \mathrm{m}^{3}\right]}\end{array}$ & $\begin{array}{r}\text { error fraction }\left(\mathbf{k}_{\mathrm{j}}\right) \\
\text { [fraction] }\end{array}$ \\
\hline acenaphthylene & $A C Y$ & light & 0.001 & 48.7 \\
\hline acenaphthene & ACE & light & 0.002 & 19.8 \\
\hline fluorene & FLU & light & 0.003 & 25.7 \\
\hline anthracene & ANT & light & 0.002 & 27.1 \\
\hline phenanthrene & PHE & light & 0.004 & 10.9 \\
\hline 2-methyl-fluorene & MFLU & light & 0.004 & 27.6 \\
\hline fluoranthene & FLT & light & 0.002 & 9.0 \\
\hline pyrene & PYR & light & 0.001 & 9.2 \\
\hline 1-methyl-pyrene & MPYR & med & 0.001 & 15.2 \\
\hline benzo(a)fluorene & BaFLU & med & 0.002 & 17.1 \\
\hline benzo(b)fluorene & $\mathrm{BbFLU}$ & med & 0.002 & 24.0 \\
\hline benzo(g,h,i)fluoranthene & BghiFLT & med & 0.002 & 14.1 \\
\hline chrysene & $\mathrm{CHR}$ & heavy & 0.001 & 10.5 \\
\hline triphenylene & TRIP & heavy & 0.002 & 13.3 \\
\hline benzo(a)anthracene & BAA & heavy & 0.004 & 11.7 \\
\hline retene & RET & heavy & 0.005 & 36.7 \\
\hline benzo(a)pyrene & BAP & heavy & 0.003 & 12.5 \\
\hline benzo(e)pyrene & BEP & heavy & 0.002 & 10.4 \\
\hline benzo(b)fluoranthene & BbFLT & heavy & 0.003 & 11.3 \\
\hline benzo(k)fluoranthene & BkFLT & heavy & 0.003 & 15.2 \\
\hline benzo(g,h,i)perylene & BghiP & heavy & 0.004 & 14.4 \\
\hline indeno(1,2,3-cd)pyrene & IPYR & heavy & 0.003 & 16.5 \\
\hline
\end{tabular}

Notes:

1. Method Detection Limit (MDL) data taken from a 2005 QAQC dataset of NAPS data made available by Environment Canada; calculated as: $\mathrm{DL}_{\mathrm{j}}=$ (t-statistic) * $\mathrm{SD}\left(\mathrm{x}_{\mathrm{ij}}\right)$ where $\mathrm{SD}_{\mathrm{j}}=$ calculated as standard deviation of 7 replicate samples analysed for respective PAH species (due to outlier species sample concentrations, 5 samples used for ACE, FLU, PHE; 6 samples used for FLT, PYR); t-value = Student-t test statistic at $95 \%$ confidence interval, for 5-7 samples (4-6 degrees of freedom), dependent on species.

2. Error fraction $\left(k_{j}\right)$ data from Method 3.03/5.1, Appendix B - "Uncertainty estimates by species, not recovery corrected" (Environment Canada, 2011f). Error fraction data are not recovery corrected, consistent with NAPS and field sampling data. Species error fractions account for major sources of uncertainty in PAH sample preparation and measurement and are determined via: $\mathrm{k}_{\mathrm{j}}=\mathrm{k}$ * RSD where RSD = relative standard deviation = $\mathrm{SD} /$ mean of 30 replicate samples; $\mathrm{k}=\mathrm{t}$-value for $95 \%$ confidence interval on 30 replicate samples $=2.0$

3. Retene also known as 1-methyl-7-isopropylphenanthrene. 


\subsubsection{Receptor sites}

This research selected two large Canadian urban centers (Toronto, population 6M; Hamilton, population 700,000; Statistics Canada, 2012a, 2012b) and a rural background site (Egbert) for source apportionment (Figure 2-2) and related analysis. The centers chosen for study were selected because: (i) Toronto is the most populated city in Canada with a dense transportation infrastructure so ambient PAH are expected to be strongly influenced by vehicle traffic emissions; (ii) Hamilton is a medium-sized city with historically very high levels of ambient PAH and is expected to be strongly influenced by industry source emissions from local heavy industry facilities (i.e., coking and sintering operations in iron/steel manufacturing); and, (iii) Egbert was chosen as a background site due to sufficient distance upwind (i.e., prevailing westerly) from the urban sites (i.e., 90km NW of Toronto; 140km N of Hamilton).

NAPS central monitors at each location had available long term (i.e., >10 years) historical ambient PAH data records. The Toronto central monitoring site (i.e., Gage Institute: $43.658417 \mathrm{~N}, 79.397139 \mathrm{~W})$ is located in the central business district with surrounding land uses classified as urban-commercial; the site is characterized by traffic, particularly during peak commuting periods via adjacent surface streets and nearby expressways. The Hamilton central monitoring site (i.e., Beasley Park: 43.257722N, $79.861611 \mathrm{~W})$ is also located near the central business district; surrounding land use is urban-commercial and industrial land use is proximal (e.g., iron and steel manufacturing and other heavy industry at the harbour-front). Surrounding land uses at the background 
site (i.e., Egbert: $44.232500 \mathrm{~N}, 79.781389 \mathrm{~W}$ ) are classified as rural-agricultural. Images of land use near sampling sites are presented in Appendix B.1.

Due to its combination of very high historical PAH levels, concentration of heavy industry sources alongside other PAH source types typical of urban areas (e.g., space heating, vehicle traffic), and escarpment topography, the city of Hamilton was selected for source-based study at the intraurban scale (i.e., additional field sampling and source apportionment analysis; Anastasopoulos et al, 2012 - Chapter 4; Chapter 5).

\section{southern Ontario (satellite view)}

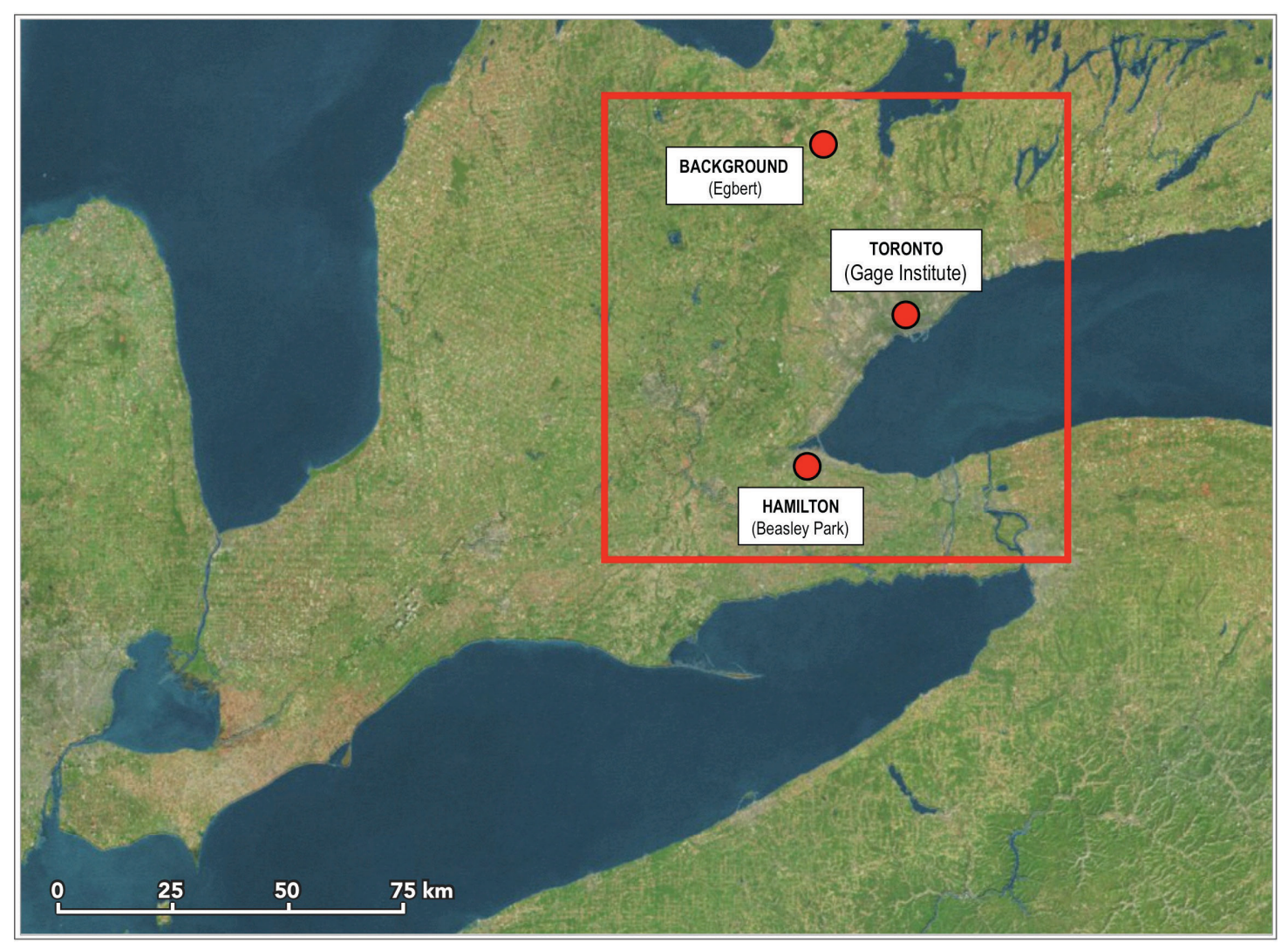

Figure 2-2. Map of study region showing NAPS sites scoped for receptor modeling. 


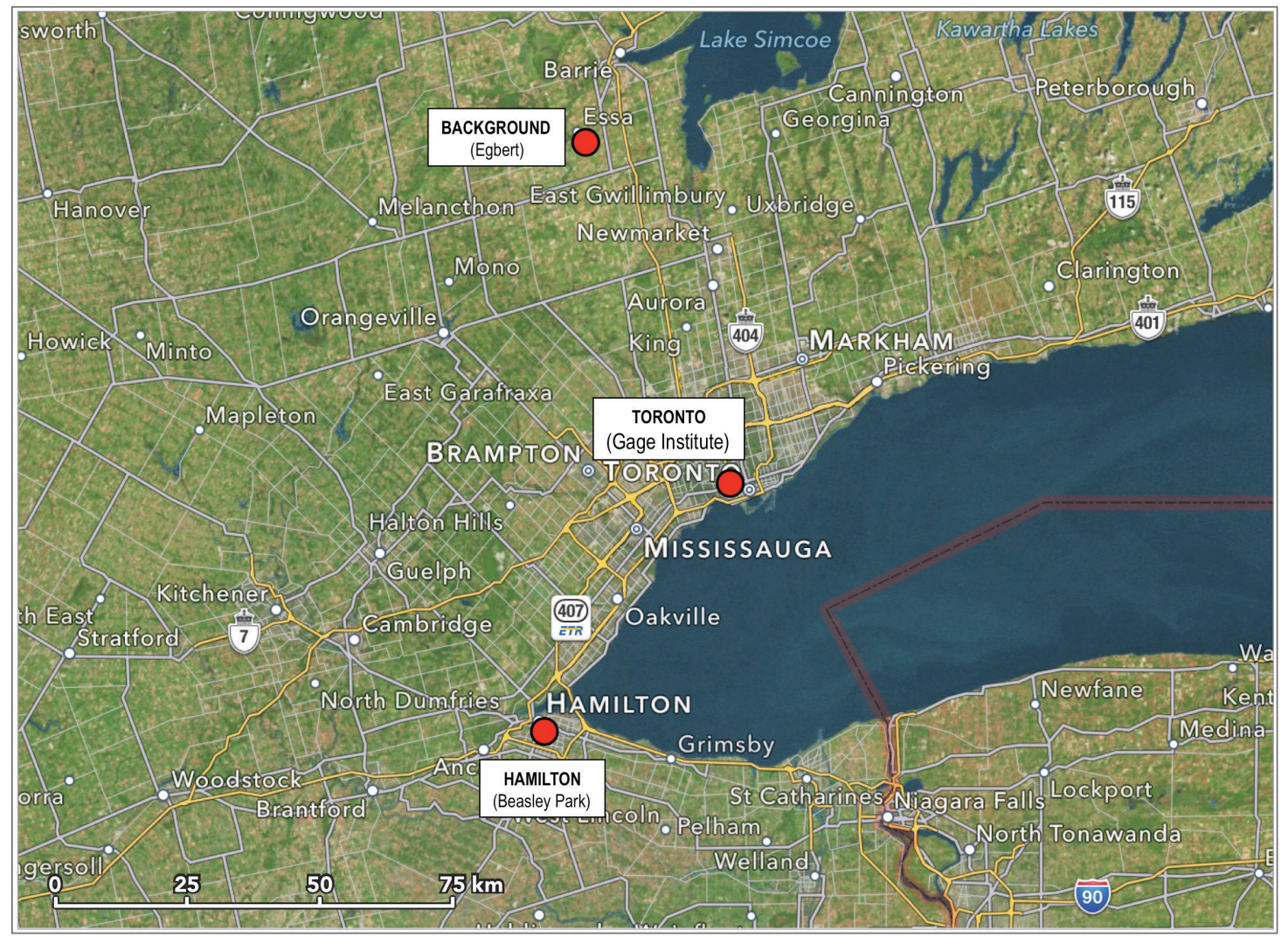

Figure 2-2 (continued)

\subsubsection{Time series scoping and species screening}

The historical central site PAH datasets were scoped to a sufficiently long and recent time series (2001-2010), avoiding an overly long period where changes in relative source contribution may have occurred due to regulatory or economic changes while maintaining sufficient dataset size for receptor modeling (i.e., bolstering model power). Modeled years had generally comparable annual and seasonal sample densities to ensure good representation in the receptor model. Years with significantly fewer samples were 
2010 at all sites and 2003 at Hamilton; these were omitted when inferring overall annual trends in PAH levels or source contributions.

Species were screened for inclusion in receptor modeling by several data quality criteria: excluding species with very high $(>90 \%)$ portion of time series missing or below detection limit $\left(\mathrm{DL}_{\mathrm{j}}\right)$; excluding species with very low time series variance $(\mathrm{CV}<0.3)$; excluding species with known sampling or sample analysis issues; but including marker species with appropriately conservative error estimates when otherwise suggested for exclusion by other criteria (US EPA, 2008; Zhao et al, 2006; Hopke et al, 2003b; Reff et al, 2007). Ultimately, species screening retained a common species suite across all three sites to facilitate inter-site comparisons of PAH trends and source apportionment results (Table 2-1).

\subsubsection{Intraurban sampling (field dataset)}

This author coordinated an intraurban field sampling campaign in Hamilton, Ontario for Health Canada in collaboration with the City of Hamilton. Details of the study were previously reported to Health Canada (Karman and Anastassopoulos, 2010) and also published in the open literature (Anastasopoulos et al, 2012).

\subsubsection{Study design}

Two-week integrated samples of PM and speciated PAH (i.e., combined vapour and particle phase) were collected in summer (24 June - 8 July 2009) and winter (2 - 16 December 2009) campaigns. Sampling campaigns were six months apart to capture any seasonality and were selected by review of National Air Pollution Surveillance (NAPS) 
PAH data (1999-2005; Environment Canada, 2011c) using the methods of Henderson et al., (2007).

The study area and sampling sites are shown in Figure 2-3. Samplers were deployed at 43 and 46 sites in summer and winter campaigns, respectively; summer campaign sites were repeated in the winter campaign with some additional winter sites. Sampling locations were selected based on results of a location-allocation model, a formal methodology for locating air pollution monitors to capture micro-scale variations (Kanaroglou et al., 2005), ensuring representative coverage of population density.

Hamilton Public Health Services and the city's Geographic Information System (GIS) group provided an inventory of available municipal sites, which were screened as candidate surrogates for the location-allocation model sites (i.e., screening criteria included proximity to location-allocation model sites and inter-site distance). Final site selection was then confirmed by on-site visits (i.e., screening criteria included availability of electrical power, equipment security, possibility of sample bias from on-property sources). Sampling sites provided good coverage of the city and suburbs above and below the escarpment with a spatial distribution of approximately 1 sampler per $5 \mathrm{~km}^{2}$ and an average distance between adjacent sites of approximately $1.5 \mathrm{~km}$. A field sampler was also co-located with the NAPS central site monitor (i.e., indicated by 'star' symbol on site map in Figure 2-3). Additional details of study design are provided in Appendix C.1. 


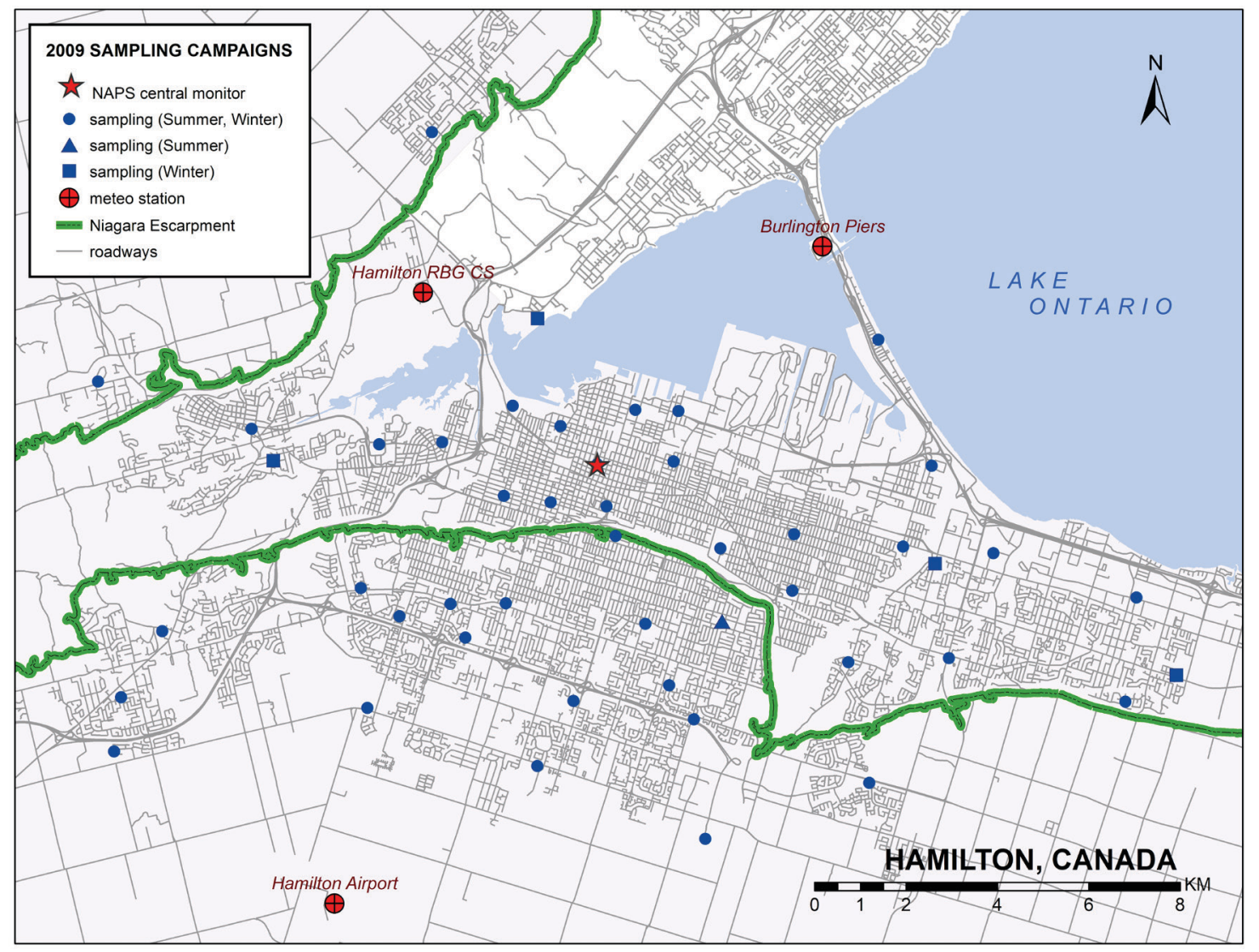

Figure 2-3. Study area showing summer and winter campaign sites, NAPS site, meteorology stations.

\subsubsection{Instrumentation}

PAH were sampled using personal pesticide samplers with an inlet cut-point of $2.5 \mu \mathrm{m}$ (Model URG-2000-25AG, URG, Chapel Hill, NC) at a nominal flow rate of 2Lpm. Sampling was from both particle and vapour phase, employing a Teflon filter in-line with a polyurethane foam (PUF) plug (i.e., particle phase collected on Teflon filter, vapour phase on PUF). Co-located with the PAH samplers, PM was sampled in three PM size fractions (i.e., particles with aerodynamic diameter between 2.5 and $10 \mu \mathrm{m}, \mathrm{PM}_{10-2.5}$; particles between 1.0 and $2.5 \mu \mathrm{m}, \mathrm{PM}_{2.5-1.0}$; particles less than $1 \mu \mathrm{m}, \mathrm{PM}_{1.0}$ ) using a three- 
stage Harvard Cascade Impactor (Harvard School of Public Health, Boston, MA) at a nominal flow rate of 5.0 L per minute $(\mathrm{Lpm})$. Additional sampler description is provided by Miller et al (2010).

PAH and PM sampling airflow was maintained by an SKC Leland Legacy field sampling pump (SKC Inc., Eighty Four, PA) connected to on-site AC power. Flows were verified at sampling setup and takedown using a DryCal DC-Lite Model "M" primary standard airflow meter (optimum flow range 100mLpm - 7Lpm, Bios International Corp., Butler, $\mathrm{NJ})$. Initial flow rates were set within $+-5 \%$ of nominal; at takedown, sites with greater than $20 \%$ deviation from nominal were excluded from further analysis.

PAH and PM samplers were arranged inside a weatherproof sampling case (Pelican Products, Torrance, California), oriented vertically with the sampling inlet facing downwards to minimize contamination (i.e., precipitation, insects) and deployed at approximately $1.5 \mathrm{~m}$ height, mounted on a survey-type tripod or appropriate on-site infrastructure (e.g., fence post). For a small number of sites where ground-level sampling was not possible, elevated or rooftop sampling was used. Portable Global Positioning System (GPS) devices were used to record sampling site location coordinates. The PM and PAH samplers are shown inside the sampling case in Figure 2-4; a typical ground level sampling setup is shown in Figure 2-5. 


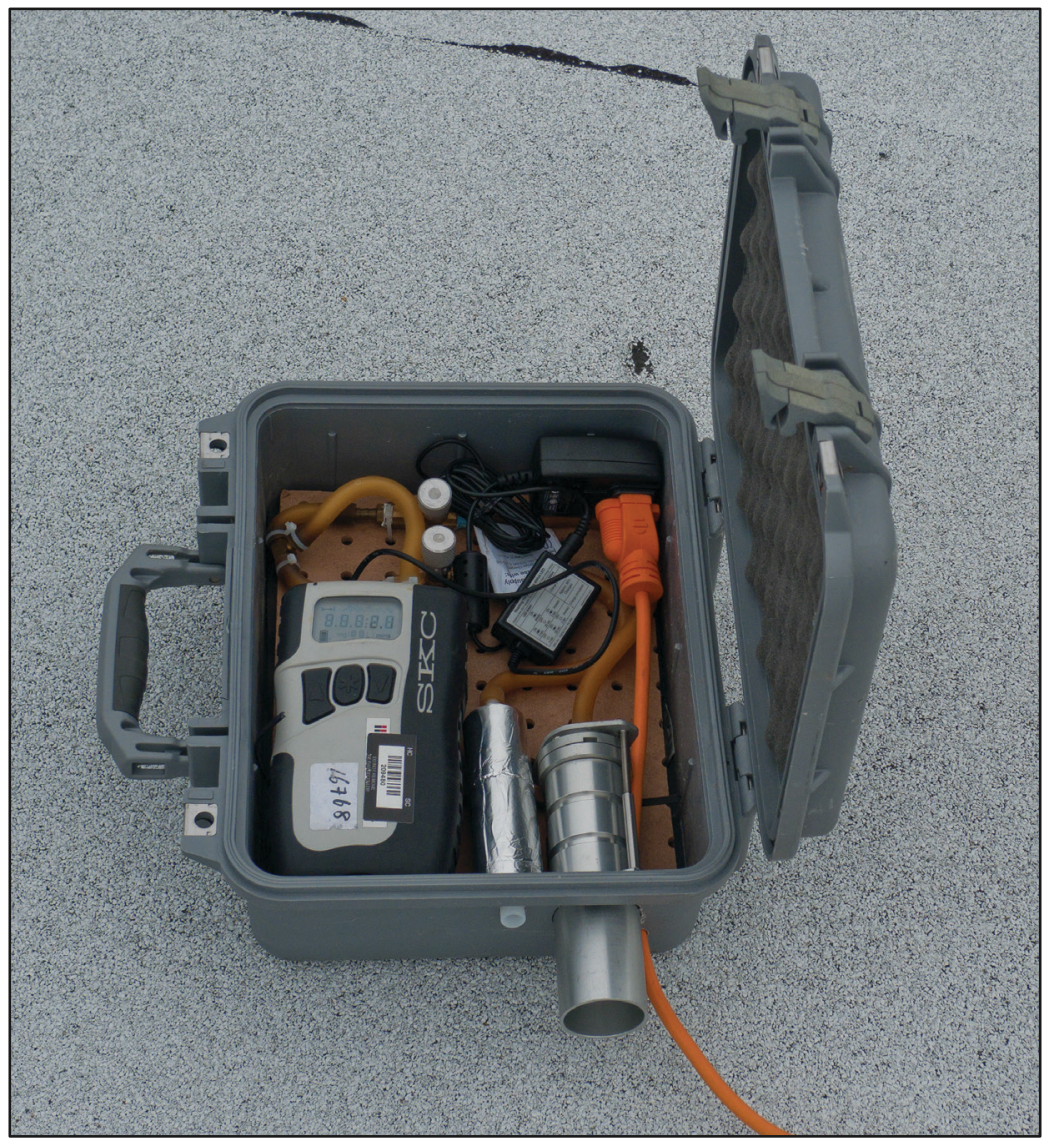

Figure 2-4. PAH and PM samplers and airflow pump inside weatherproof case. 


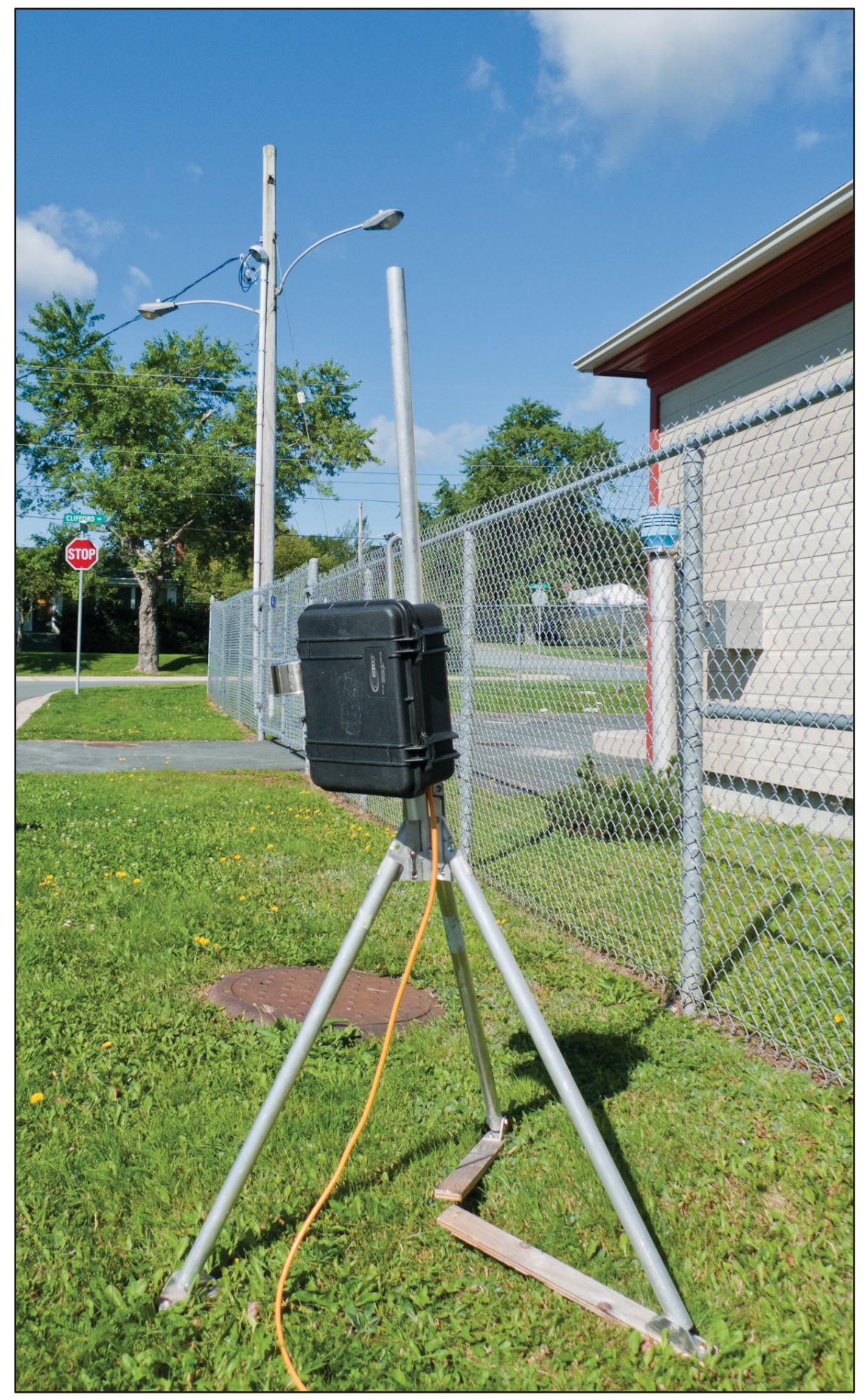

Figure 2-5. Typical ground-level sampler setup. 


\subsubsection{Laboratory analysis of samplers}

Laboratory analysis of PAH samplers followed US EPA Method TO-13A (1999), consistent with analysis of central site NAPS samplers. Teflon and PUF filters were coextracted and analysed for total PAH concentration (i.e., sum of vapour plus particle phase) of 16 PAH species (Table 2-2). Species concentrations were determined by gas chromatography with mass selective detection (GC-MSD) and included analysis of lab blanks, standards and species-dependent recovery efficiencies. PM and PAH filter masses reported as below Lab Detection Limit (LDL) were substituted with LDL/2.

PM sample filters were conditioned before and after sampling and filter masses were determined by gravimetric analysis, following EPA quality assurance guidelines (US EPA, 1998a).

Two-week (14-d) integrated mass concentrations for PM $\left(\mu \mathrm{g} / \mathrm{m}^{3}\right)$ and PAH $\left(\mathrm{ng} / \mathrm{m}^{3}\right)$ were calculated by dividing the respective filter masses by the volume of air sampled. Speciated method detection limits $\left(\mathrm{DL}_{\mathrm{j}}\right)$ for the 14-day field samples were obtained from the analyzing laboratory (Airzone One, Mississauga) and are shown in Table 2-2.

Field blanks for PAH and PM samplers were deployed in summer and winter sampling campaigns at approximately $10 \%$ of the sampling sites and analysed to quantify the active sampler mass attributed to handling and transportation. Field Blank Correction (FBC) was determined as follows: field blanks below LDL were treated by substitution of LDL/2 and screened for outliers; field blank datasets from the two campaigns were checked for statistical difference in means and pooled where no statistical difference was 
shown; finally, to determine whether an FBC should be applied to the active pollutant sampler masses, separate or pooled field blank filter masses were compared to the LDL. Where greater than half the field blanks were above LDL, FBC was calculated as the median of the field blank filter masses and subtracted from active pollutant sampler masses; no FBC was applied where more than half of the field blanks were below LDL (Wheeler et al., 2011).

Table 2-2. Field-sampled PAH species and detection limits.

\begin{tabular}{|c|c|c|c|}
\hline PAH Species & Label & $\begin{array}{r}\text { MW } \\
\text { group }\end{array}$ & $\begin{array}{r}\text { 14-day } \\
D L \\
{\left[\mathrm{ng} / \mathrm{m}^{3}\right]}\end{array}$ \\
\hline naphthalene & NAP & light & 0.124 \\
\hline acenaphthylene & $\mathrm{ACY}$ & light & 0.142 \\
\hline acenapthene & ACE & light & 0.134 \\
\hline fluorene & FLU & light & 0.102 \\
\hline anthracene & ANT & light & 0.099 \\
\hline phenanthrene & PHE & light & 0.076 \\
\hline fluoranthene & FLT & light & 0.068 \\
\hline pyrene & PYR & light & 0.054 \\
\hline chrysene & $\mathrm{CHR}$ & heavy & 0.110 \\
\hline benzo(a)anthracene & BAA & heavy & 0.112 \\
\hline benzo(a)pyrene & BAP & heavy & 0.189 \\
\hline benzo(b)fluoranthene & $\mathrm{BbFLT}$ & heavy & 0.181 \\
\hline benzo(k)fluoranthene & BkFLT & heavy & 0.178 \\
\hline dibenz $(a, h)$ anthracene & DBA & heavy & 0.215 \\
\hline benzo(g,h,i)perylene & BghiP & heavy & 0.207 \\
\hline indeno(1,2,3-cd)pyrene & IPYR & heavy & 0.229 \\
\hline
\end{tabular}

Note:

1. Detection Limit (DL) for intraurban field sampling dataset as reported by analyzing lab (i.e., 14day field sampling period at nominal flow rate). 


\subsection{Data Analyses}

\subsubsection{Statistical trends}

$\mathrm{PAH}$ species in the central site and intraurban datasets were grouped by MW to facilitate analysis and discussion of concentration trends and source profiles (i.e., light: $\leq 4$ rings, MW $\leq 202$; heavy: >4rings, MW>228; following Li et al, 2005a). Statistical analyses employed several statistics packages (i.e., SYSTAT 13, Systat Software Inc., Chicago, IL, USA; MINITAB 14, MINITAB Inc., State College, Pennsylvania, USA; Microsoft Excel 2011, Redmond, CA, USA). Tests of statistical difference used the non-parametric Kruskal-Wallis or Mann-Whitney tests at the 5\% significance level.

For the central site data, descriptive statistics were calculated for individual PAH and species groupings (i.e., total sampled PAH, $\Sigma_{29} \mathrm{PAH} ; \Sigma_{\mathrm{LIGHT}} \mathrm{PAH} ; \Sigma_{\mathrm{HEAVY}} \mathrm{PAH}$ ) and reviewed for data density and sample-to-sample variability to confirm amenability to receptor modeling. Species abundances (i.e., species concentration $/ \Sigma_{29} \mathrm{PAH}$ ) were ranked to determine dominant species and confirm general consistency in source mix over the modeled time period (Kuntasal, 2005; Gaga, 2004). Annual and seasonal (i.e., winter: October - April; summer: May - September) trends were reviewed across sites and reconciled with differences in site characteristics and expected general source types.

For the intraurban data, descriptive statistics were calculated for individual sampled PAH and MW groups (i.e., total sampled PAH, $\Sigma_{15} \mathrm{PAH} ; \Sigma_{\mathrm{LIGHT}} \mathrm{PAH} ; \Sigma_{\mathrm{HEAVY}} \mathrm{PAH}$ ) and for $\mathrm{PM}_{2.5}$. Normality of all pollutant datasets was examined using the Anderson-Darling test statistic alongside probability plots (normal distribution) and kurtosis and the seasonal pollutant datasets (i.e., summer, winter) were tested for statistical difference. Intraurban 
variabilities in pollutant datasets were quantified using Coefficient of Variation (CV) and relationships among sampled pollutants were quantified using Spearman's rank correlation and Pearson's correlation.

A subset of $15 \mathrm{PAH}$ species were common between the intraurban field sampling dataset and the larger NAPS central site monitoring dataset (i.e., field-sampled dibenz(ah)anthracene isomer was compared with combined dibenz(ac) and (ah)anthracene isomers reported by NAPS). While noting sampling differences (i.e., duration, PM phase size fraction), samplers and lab analytical methods were sufficiently similar between NAPS central site monitoring and field sampling to allow reasonable data comparison for common PAH species, verified by consistency between ambient concentrations sampled at the co-located site during the intraurban field campaigns with nearest coincident dates in NAPS central site monitor data (Chapter 4), and to support combined receptor modeling at the intraurban scale (Chapter 5).

\subsubsection{Meteorology and wind/pollutant patterns}

For the urban/rural analysis, historical meteorological data were downloaded from Environment Canada's online searchable database (2011e) for the meteorology station most proximal to the central site monitor (i.e., Toronto airport $43.68 \mathrm{~N}, 79.63 \mathrm{~W}$; Hamilton airport 43.17 N, $79.93 \mathrm{~W}$; background co-located with NAPS sampler). Data downloaded included hourly ambient temperature, relative humidity, barometric pressure, and wind speed/direction, and precipitation.

Wind roses were constructed using WRPlot 7 software (Lakes Environmental Software Inc., Waterloo, Ontario, Canada) for the year-round dataset (2000-2010) and for winter 
and summer months (2000-2010) to determine seasonal differences in wind patterns. Predominant wind directions were compared across sites and assessed against the location of local PAH source areas (e.g., stationary or mobile sources); 24-hr and 48-hr wind roses were also constructed for days with the highest and lowest sampled PAH concentrations to better identify the probable influence of local source areas.

Since Hamilton was to be analyzed more closely via intraurban field sampling (Anastasopoulos et al, 2012 - Chapter 4) and receptor modeled at the intraurban scale (Chapter 5), wind data were also combined with the central site (NAPS) PAH sampling data to construct a pollutant rose for $\Sigma_{29} \mathrm{PAH}$ (i.e., by concentration, by quartiles; WRPlot software, Lakes Environmental Inc.); the pollutant rose more directly indicated local source areas associated with high ambient PAH (i.e., upwind of central monitor during periods of high ambient PAH).

For the intraurban analysis, the broad spatial extent of the field sampling sites across Hamilton and complex topography, inclusive of the escarpment and lake harbour, precluded adequately representing meteorological conditions at all sites using only a single weather station. Instead, historical meteorological data were downloaded from three Environment Canada stations that spanned the city's extent, as shown in Figure 2-3. Hourly meteorological data (i.e., ambient temperature, relative humidity, barometric pressure, wind speed/direction, and precipitation) were downloaded for the summer and winter sampling dates (summer: 24 June - 8 July 2009; winter: 2-16 December 2009) and averaged to generate a single meteorological dataset representative of the study area for each seasonal campaign. Comparison was made with historic climate normals (1971- 
2000; Environment Canada, 2011d) to determine if sampling weather conditions were typical during each campaign. Precipitation data were reviewed to indicate potential washout of aerosol pollutants over the summer and winter sampling periods. Wind roses were prepared (WRPlot 7) to indicate predominant wind direction in the summer and winter sampling periods.

\subsubsection{Intraurban mapping}

Maps used in the spatial analysis of the Hamilton field data were prepared using ArcGIS 10 (ESRI Inc., Redlands, California, USA) and enabled review of intraurban trends as well as presentation of results. Maps were prepared for sampled ambient pollutant concentrations $\left(\mathrm{PAH}, \mathrm{PM}_{2.5}\right), \mathrm{PAH}$ source apportionment results (i.e., contributions to total sampled PAH and to PAH toxicity) and receptor model performance metrics and associated uncertainties on source apportionment results.

\subsection{Source apportionment}

The primary interest in the ambient PAH data discussed in Section 2.2 are the sources that could possibly be contributing to the observed ambient concentrations, and their relative contributions. This research applied three specific models in seeking answers to these questions: Principal Component Analysis (PCA), Positive Matrix Factorization (PMF), and Chemical Mass Balance (CMB). The underlying principles of these models

are presented in the next section while the details of their implementation with the datasets described above is presented in Appendix E. 


\subsubsection{Receptor model theory}

Receptor models attempt to identify and quantify the relative contribution of different sources to observed ambient concentrations of air pollutants, based on the principle of mass balance of pollutants between sources and receptor (Equation 2-1; matrix and indexed forms). In Equation 2-1, $\boldsymbol{X}$ is the sample matrix (i.e., speciated mass concentrations at the receptor; $x_{i j}$ is measured concentration of species $j$ in receptor sample $i$ ), $\boldsymbol{F}$ is the factor profile matrix (i.e., speciated profiles to be interpreted as physically meaningful sources or source types; $f_{k j}$ is concentration of species $j$ in the pollutant profile for source factor $k$ ), $\boldsymbol{G}$ is the matrix of factor contributions to each sample (i.e., $g_{i k}$ is contribution of source factor $k$ to receptor sample $i$ ), and $\boldsymbol{E}$ is the model residual (i.e., mass unexplained by model; $e_{i j}$ is unexplained mass of species $j$ in receptor sample $i$ ). (Hopke, 2003a)

Equation 2-1. General source-receptor mass balance.

$$
\boldsymbol{X}=\boldsymbol{G} \cdot \boldsymbol{F}+\boldsymbol{E}
$$

$$
\begin{aligned}
& \begin{array}{l}
\boldsymbol{X} \\
\boldsymbol{G}
\end{array}=\text { sample concentration matrix } \\
& \boldsymbol{F}=\text { factor profile matrix } \\
& \boldsymbol{E}=\text { model residual matrix } \\
& \qquad x_{i j}=\sum_{k=1}^{p} g_{i k} f_{k j}+e_{i j} \\
& x_{i j}=\text { concentration species } j \text { in receptor sample } i\left(\mathrm{ng} / \mathrm{m}^{3}\right) \\
& g_{i k}=\text { contribution of source factor } k \text { to receptor } \mathrm{sample} i \\
& f_{k j}=\text { concentration of species } j \text { to source factor } k\left(\mathrm{ng} / \mathrm{m}^{3}\right) \\
& e_{i j}=\text { model residual species } j \text { in receptor sample } i\left(\mathrm{ng} / \mathrm{m}^{3}\right)
\end{aligned}
$$


PCA is a basic model that resolves a set of independent principal components (PC) that together explain a maximum of the total variance of the sample matrix $\boldsymbol{X}$; the components explain which species are co-varying at the receptor and thus are likely being emitted from a common source, although source contributions associated with each component are not quantified. (Hopke, 2006; Mudge, 2007) Details of the PCA modeling protocol applied in the research are presented in Appendix E.1.

CMB solves the source-receptor mass balance (Equation 2-1) directly by using as input source profiles determined from source measurements independently of the ambient data (i.e., factor profile matrix, $\boldsymbol{F}$ ) for source types expected to be contributing to observations at the receptor (i.e., sample matrix, $\boldsymbol{X}$ ). This leaves only the factor contributions $(\boldsymbol{G})$ unknown, which $\mathrm{CMB}$ solves using an effective-variance least-squares approach to minimize the residuals. (US EPA, 2004; Hopke, 2003a) Details of the CMB modeling protocol applied in the research are presented in Appendix E.3.

Similar to general factor analysis models, such as PCA, PMF applies statistical analysis of trends in a speciated pollutant dataset to yield factors explaining the data variation but resolved factor profiles $(\boldsymbol{F})$ can be interpreted as physical meaningful source types and source contributions $(\boldsymbol{G})$ are directly quantified. (Hopke, 2004; Brook et al, 2004) PMF solves the source-receptor mass balance (Equation 2-1) by using an iterative algorithm to populate the $\boldsymbol{F}$ and $\boldsymbol{G}$ matrices simultaneously while seeking to minimize an objective function Q (Equation 2-2), subject to the constraint that elements in $\boldsymbol{G}$ and $\boldsymbol{F}$ be nonnegative. The objective function $\mathrm{Q}$ is the sum of the squares of the model residual $\left(\boldsymbol{E}, e_{i j}\right.$; calculated from Equation 2-1) weighted by the measurement uncertainty $\left(\boldsymbol{S}, s_{i j}\right)$. 
Weighting of the model residual by the measurement uncertainty is the key distinguishing advantage of the PMF model from other factor analysis models, ensuring the model solution is influenced predominantly by data with lower associated uncertainty, while data with greater associated uncertainty are prevented from unduly influencing the solution. (Hopke, 2003a; Paatero and Tapper, 1994; Lee, 2003) Details of the CMB modeling protocol applied in the research are presented in Appendix E.2.

Equation 2-2. PMF minimization function for solution of source-receptor mass balance.

$$
\begin{aligned}
& Q=\sum \sum\left(\frac{\boldsymbol{E}}{\boldsymbol{S}}\right)^{2} \\
& \boldsymbol{E}=\text { model residual matrix } \\
& \boldsymbol{S}=\text { uncertainty matrix } \\
& Q=\sum_{i=1}^{n} \sum_{j=1}^{m}\left(\frac{e_{i j}}{s_{i j}}\right)^{2}
\end{aligned}
$$

$e_{i j}=$ model residual of species $j$ in receptor sample $i\left(\mathrm{ng} / \mathrm{m}^{3}\right)$, see Equation 2-1

$s_{i j}=$ uncertainty of species $j$ in receptor sample $i\left(\mathrm{ng} / \mathrm{m}^{3}\right)$

\subsubsection{Challenges and strategies for receptor modeling PAH}

As with all environmental modeling, receptor modeling has fundamental assumptions that should be met for results to be physically meaningful, broadly summarized as:

(i) source specificity (i.e., unique source profile for each source type);

(ii) source consistency (i.e., source characteristics do not change significantly over the period for which data are being analyzed); and,

(iii) conservation of relative species mass between source and receptor. 
Applying receptor model techniques to $\mathrm{PAH}$ poses several challenges to these assumptions due to the properties of this compound class (i.e., properties summarized and tabulated and compound structures presented in Appendix A.1).

A comprehensive review of PAH source apportionment literature (Galarneau, 2008) applied meta-analysis to studies that used source marker 'diagnostic ratios' (i.e., ratio of two PAH species traditionally considered to be unique identifier of a source type) as well as, to a lesser degree, on source profiles (i.e., larger suite of PAH species). Galarneau found that the assumption of source specificity for PAH emissions is often violated when simple species diagnostic ratios are used as these were shown to vary significantly between locations or even among varying source operating conditions (e.g., diesel engine drive cycles). This suggests that receptor location-specific source profiles are preferable for a source apportionment study, but these are not often available to researchers.

The meta-analysis (Galarneau, 2008) also demonstrated that the assumption of species conservation may be significantly affected by several transformative processes in the atmosphere, including inter-species differences in partitioning (i.e., between vapour and particle phases), reactivity (i.e., due to oxidation, solar radiation), species removal (i.e., due to air-water exchange, particle washout/deposition), and sampling losses. Other associated challenges include a lack of a priori knowledge of the number of sources impacting a receptor.

In this research, several tools and methods were applied to address the challenges of source apportioning PAH using receptor models, summarized below. 


\section{Source specificity}

For the challenge of source specificity, use was made of multi-species reference source profiles rather than diagnostic ratio species pairs. The existing limited compilation of PAH sources (e.g., US EPA 1998, 2003, 2004) was augmented by source characterization studies that report PAH, particularly for expected key sources.

Further, rather than rely solely on reference source profile to identify and interpret model factor results, additional 'layers of evidence' were used. These included: temporal trends in factor contributions (e.g., seasonal, weekday/weekend), comparison of source apportionment estimates with emissions inventories, inter-site spatial trends (e.g., differences in urban versus rural data to help discriminate local sources from transported sources; Brook et al, 2004). Validation of model results was further augmented by wind pattern analysis for identification of local sources. Overall, source types were interpreted from model factors based on a preponderance of contextual evidence (Reff et al, 2007).

\section{Source consistency}

Several preliminary and semi-quantitative data analyses were used to verify that base assumption of source consistency was suitably met or to suggest procedurally rational methods for doing so. These included review of data quality (e.g., length of time series dataset, degree of missing data), examination of temporal trends using time-series plots (i.e., monthly, seasonal), understanding of prevailing wind directions (Brook et al, 2004). Review of environmental context for receptor modeling was also completed (Reff et al, 
2007) inclusive of identifying expected source types, source facility locations, and any significant changes in source activity within the scoped study region.

A key decision addressing the challenge of source consistency was the scoped length of the modeled time series; good modeling strategy avoids an overly long time period due to the likelihood of significant changes in source emission intensity or characteristics (i.e., due to changes in regulations, emissions reduction technologies, or economic and developmental factors). This was first investigated by examining historical ambient PAH trends for large changes and then further investigated by model sensitivity analyses to time series length (i.e., modeling early year and later year subsets of the scoped time series to verify that model results remained consistent).

\section{Source-receptor conservation of mass}

To address vapour-particle partitioning, modeling used total ambient PAH sample data (i.e., sum of particle and vapour phase), which has been shown to be more suitable for receptor modeling than PAH sampled from only the particle phase (Mari et al, 2010; Harrison et al, 1996). Similarly, preference was generally given to reference source profiles based on total PAH data (e.g., source sampling studies or receptor modeling studies). Where reference profiles were only available for particle phase PAH, responsible use of these as reference for interpretation of model factors considered preferential species partitioning (i.e., as a general rule of thumb, lighter MW PAH with 3ring structures partition predominantly to vapour phase, heavier MW PAH with 5- and 6ring structures partition predominantly to particle phase; Venkataraman and Friedlander, 1994); as an example, interpreting a model factor profile enriched in heavy MW species 
could quite meaningfully reference source profiles that restricted sampling to particle phase PAH since this would have accounted for essentially all the species mass for heavy MW species. Additional details of vapour-particle partitioning are presented in Appendix A.1.

The issue of inter-species differences in reactivity challenging PAH receptor modeling was explicitly investigated by a sampling and receptor modeling study conducted by Mari et al (2010) who concluded that atmospheric transformations have little confounding effect. Mari et al (2010) also cite the similar results of the Canadian study by Sanderson et al (2004), which found that PAH source releases age to a stable profile during sourcereceptor transport and consequently demonstrated relatively long atmospheric lifetimes for PAH. Nevertheless, to address inter-species differences in reactivity in the receptor modeling in this research, uncertainty estimates on ambient data were species-specific and ensured that higher relative uncertainty was assigned to more reactive species (e.g., ACY, ACE, ANT, RET) in the PMF and CMB receptive models. Further, in the PMF receptor model, variants of the species suite excluded or down-weighted the importance of more reactive species; sensitivity analyses confirmed the factor apportionment of more reactive species to ensure that source type interpretations were not contradicted. As an example, ACY was generally excluded from receptor models due to known sampling issues (US EPA, 1999); PMF sensitivity analysis included it to confirm that it generally apportioned alongside other volatile species such as ACE.

To address potential air-water exchange, wind rose and pollutant rose analysis was used to reconcile source-receptor transport directions with potential residence time over large 
water bodies. To address potential species mass loss due to particle deposition or washout by precipitation, specifically for the intraurban field sampling (i.e., low flow rate and two-week integrated sampling time), meteorological records were reviewed to identify potentially significant conditions or events that could lead to species mass loss.

\section{Seasonal effects}

Seasonal differences in PAH concentrations could be expected a priori due to combination of: (i) presence and contribution of source types (e.g., heating combustion sources present largely in fall/winter months with low ambient temperatures; Sun et al, 2006); and, (ii) temperature-related atmospheric processes affecting PAH (e.g., changes in vapour/particle partitioning of light and medium MW species; Venkataraman and Friedlander, 1994). Sources that are present/absent depending on season do not pose a problem for receptor modeling time series data, such as via PMF, since contributions would be increased or decreased accordingly by season; in fact, presence/absence of seasonal trends are a significant aid in interpreting receptor model factors as physically meaningful source types. However, seasonal changes in temperature-related atmospheric processes can challenge receptor modeling where these changes affect modeled species differentially, potentially confounding interpretation of the resolved factor profiles. To investigate potential seasonal confounding, central site PAH data and associated meteorology data were examined for the 2000-2010 time period at Toronto, selected as the site with the greatest number of samples available.

First, seasonal differences in temperature, wind speed and sampled ambient PAH were confirmed statistically (Kruskal-Wallis non-parametric test of fall/winter and 
spring/summer medians at 0.05 significance level). This found that seasonal trends were as expected and would not challenge reasonable interpretation of receptor model results. Temperatures were statistically higher in summer and wind speeds were statistically higher in winter, as is typical for the region (Environment Canada, 2011d). For the light MW species groups, PAH levels were statistically higher in summer, readily understandable as being driven by temperature effects increasing partitioning to vapour phase (i.e., Venkataraman and Friedlander, 1994; potential confounding controlled by sampling and modeling PAH that is total of vapour and particle phase) and increasing volatilization from paved surfaces (i.e., Dimashki et al, 2001; minimal potential confounding due to strong seasonality, PMF capable of resolving a separate factor representing PAH volatilization emissions that would reflect seasonal trend in contribution). For the heavy MW species group, PAH levels were statistically higher in winter, understandable as being driven primarily by lower mixing height and presence of space heating source emissions (i.e., Sun et al, 2006; no confounding to receptor model since space heating emissions are seasonal source type that PMF is capable of resolving with appropriate seasonal trend in contribution).

Next, the correlation between ambient PAH and temperature was calculated for the winter and summer season sample datasets for total PAH $\left(\Sigma_{29} \mathrm{PAH}\right)$ and the light and heavy MW species groupings $\left(\Sigma_{\text {light }} \mathrm{PAH}, \Sigma_{\text {heavy }} \mathrm{PAH}\right)$. For winter season samples, ambient PAH was not found to correlate to temperature for any species grouping, indicating that changes in sampled PAH were independent of temperature changes for winter samples for all sampled species. For summer season samples, ambient PAH correlated positively 
with ambient temperature for the light MW species group but remained uncorrelated for the heavy MW species group. The inter-species difference in PAH correlation with $\mathrm{T}$ in summer samples could be reasonably controlled within PMF by incorporating greater uncertainty on species concentrations for the most volatile species (i.e., via greater assigned species error fraction or, if further uncertainty were to be required, by downweighting species fit in model via species categorization to 'weak').

Last, the potential for seasonal difference in photochemical decomposition was checked by applying the method of Park et al $(2011,2002)$ to compare the relative concentrations of $\mathrm{BaP}$ (i.e., highly reactive species) to $\mathrm{BeP}$ (i.e., highly stable species) between winter and summer seasons of the Toronto 2000-2010 NAPS data. Test of statistical difference between seasons showed no difference in medians between the BaP-to-BeP ratio (i.e., Kruskal-Wallis test of medians, 5\% significance level) indicating that any seasonal variation in $\mathrm{PAH}$ concentrations were not due to significant photochemical decomposition, and thus the receptor modeling could be reasonably applied to the yearround dataset rather than requiring seasonal stratification.

Finally, while these several investigations of seasonality demonstrated that the NAPS central site PAH dataset was amenable to receptor modeling as a year-round dataset (i.e., without need for seasonal stratification), the research included a sensitivity analysis of all model results (i.e., PCA, PMF, CMB) as a final check that factors and contributions were insensitive to seasonal stratification of the PAH time series data. 


\section{Number of sources}

The number of distinct factors contributing $\mathrm{PAH}$ to the receptor sites that could be explained from the receptor data was determined by investigating $3-7$ factor solutions in PMF and the optimal model order provided defensible interpretation of factors as physically meaningful source types. Optimal model order was also informed by preliminary application of the PCA factor analysis model, which was used to reveal data structure by extracting factor loading patterns of co-varying species and to indicate a lower bound on the number of reasonably distinct factors that could be expected from application of PMF.

The information on optimal model order determined from PCA and PMF was then used to guide expected model order for the CMB model. Several model orders were run in the CMB model, with allowance made for reduced model power due to the smaller number of species in the intraurban field sampling dataset being modeled.

Further insight into number of sources contributing to ambient PAH at the scoped receptor sites was gained during the first phase of the research, 'environmental context for source apportionment', and included: review of source types from industry pollutant reporting databases; review of local source facilities from maps and local knowledge; and, review of relevant and recent PAH emissions inventories (e.g., Galarneau et al, 2007). 


\subsubsection{Uncertainty in receptor modeling}

Uncertainty estimates on the input data (i.e., ambient PAH samples) are required for the $\mathrm{PMF}$ and $\mathrm{CMB}$ receptor models. For $\mathrm{CMB}$, uncertainty on input concentrations is applied to estimating uncertainty on model output (i.e., source apportionment estimates); for PMF, uncertainty on input concentrations is used to distinguish between more and less precise data when solving the mass balance. The advantages of including uncertainty in either receptor model are realized only where uncertainty on input data has been estimated realistically and thus various approaches to estimating uncertainty have been applied in the receptor modeling literature (i.e., summarized in Reff et al, 2007; comprehensive literature review provided in Appendix A.4).

The receptor modeling carried out in this research was mindful of PAH being a broad pollutant class (i.e., ranging from 2- and 3-ring light MW species to 4- and 5-ring heavy MW species) and its constituent compounds having varying properties (i.e., PAH properties and structures presented in Appendix A.1) with related inter-species differences in sampling (e.g., breakthrough risk of lightest and most volatile species in high volume sampling; US EPA, 1999) and lab analysis (e.g., recovery efficiency). To this end, species-specific error fractions $\left(\mathrm{k}_{\mathrm{j}}\right)$ on lab-reported concentrations were obtained from an appropriate Methods document (Environment Canada, 2011f; see Table 2-1), quantifying cumulative uncertainty for major sources of uncertainty in lab analysis as a fraction of the lab-reported concentration that is uncertain (i.e., uncertainty estimate is proportional to concentration). Major sources of uncertainty represented by the error fraction data are listed in the Methods document and include uncertainty in the matrix sample preparation 
(i.e., sample preparation, spike preparation, instrument response, preparation method, matrix interference, purity), bias in standard preparation, and uncertainty in sample size measurement (Environment Canada, 2011f).

In addition to proportional uncertainty quantified by the species error fraction, labreported ambient PAH concentrations have additional uncertainty related to the species method detection limit (i.e., concentrations below DL cannot be distinguished from zero). Method detection limits were specific to the laboratory analysis methods used for each ambient dataset and were obtained from the respective analyzing labs: for the NAPS dataset, DL values referenced a representative QAQC dataset (Environment Canada, 2005; Table 2-1); for the field dataset, DL values were reported with the ambient data (Airzone One, Mississauga; Table 2-2.).

Conservative sample and species-specific uncertainty estimates were calculated for the ambient PAH datasets modeled by PMF and CMB by applying the species-specific error fractions $\left(\mathrm{k}_{\mathrm{j}}\right)$ and detection limits $\left(\mathrm{DL}_{\mathrm{j}}\right)$. (Details in Appendix E.)

This research also placed strict importance on quantifying uncertainty on source apportionment results (i.e., receptor model outputs). For PMF, uncertainty was reported for source profiles and for source contributions to PAH. For CMB, uncertainty was reported for source contributions to $\mathrm{PAH}$.

In addition to merely reporting uncertainty, interpretations (e.g., factor profile interpretations as source types) and implications (e.g., relative importance of factors by relative contribution to ambient $\mathrm{PAH}$ ) were drawn from source apportionment results 
only where these remained consistent with uncertainty estimates. PMF factor interpretation was mindful that 'characteristic species' remained enriched in the factor profile even with uncertainty estimates taken into consideration; PMF source apportionment results presented and discussed a statistical range of source contribution estimates rather than limiting inferences to a single aggregate contribution estimate. For CMB intraurban source apportionment results, high-uncertainty sites were screened from the analysis to confirm that spatial trends were preserved.

\subsubsection{PMF-CMB paired receptor modeling}

There have been few studies that have combined the PMF and CMB receptor models, motivating the current work in undertaking a PMF-CMB methodology for PAH source apportionment. This section will present a brief review of these studies, leading to the approach presented graphically in Figure 2-1.

The majority of studies that have applied both PMF and CMB to the same dataset have done so for purposes of comparison or validation. Jaeckels et al (2007) used PMF and compared results with $\mathrm{CMB}$ in analyzing source contributions for approximately 100 organic compounds including 20 PAHs measured over a two year period at a receptor in St. Louis, IL, USA. A more integrated model comparison was shown in Viana et al (2008), who applied PMF and CMB models to a single ambient dataset of 24-hr $\mathrm{PM}_{10}$ samples analyzed for elemental constituent species. Samples were taken in the vicinity of an industrial area for which source sampling profiles were available to allow validating of the receptor models. Both $\mathrm{PMF}$ and $\mathrm{CMB}$ were able to explain the ambient $\mathrm{PM}_{10}$ data well (i.e., correlation between modeled and measured data: $\mathrm{PMF}, \mathrm{R}^{2}=0.93 ; \mathrm{CMB}, \mathrm{R}^{2}=$ 
0.83). With respect to source identification, PMF was found to reliably resolve five sources from the ambient data, whereas seven a priori known source profiles were input to $\mathrm{CMB}$ (i.e., three from local source sampling, three literature reference profiles, one by combining a locally sampled profile with a reference profile). When the authors compared PMF profiles (i.e., model output) to $\mathrm{CMB}$ profiles (i.e., model input) good agreement was found for the four common sources contributing the majority of the $\mathrm{PM}_{10}$ mass. The strongest correlation was found between PMF profiles corresponding to CMB input profiles determined from local source sampling. The authors thus recommended combining the PMF and CMB models for source apportionment: PMF to identify source types impacting a receptor and provide initial quantification of contributions, followed by CMB (i.e., ideally with source profiles derived from local source sampling) to then more reliably quantify relative contributions. The finding that PMF-resolved profiles agreed well with locally measured source profiles suggests that, in the absence of such locally measured source profiles (i.e., a common reality of exposure assessment research), PMFresolved profiles could be preferred over reference profiles from literature.

Formal 'pairing' of the receptor models (i.e., PMF-CMB: PMF applied to identify receptor sources and resolve locally representative profiles followed by CMB to quantify relative source contributions) has been suggested as a better method for identifying and characterizing source types that can be considered unique to a local study area. Duvall et al (2007) presented a PMF-CMB methodology for a $\mathrm{PM}_{2.5}$ dataset speciated by inorganics, EC, and OC). Two source types were identified as unique to the local study area, with no suitable source profile available in reference literature (i.e., vehicle 
emissions; local industry emissions); for these source types, PMF modeling of local central-site ambient data would be used to resolve more locally representative source profiles which could then be used in the CMB model.

Shi et al (2009) proposed a PMF-CMB protocol for source apportionment of coarse PM (i.e., synthetic $\mathrm{PM}_{10}$, speciated by elements): the authors suggested applying PMF to time series ambient data to resolve source profiles (i.e., classed by the authors as either "Extracted Simplex" sources, interpreted as a single source type, or "Extracted Complex sources, interpreted as a mixed source type) and then to retain "Extracted Simplex" sources for direct input to $\mathrm{CMB}$ and replace "Extracted Complex" sources with constituent sub-profiles prior to CMB input (i.e., ideally with source profiles from local source sampling, alternately from literature).

Park and Kim (2005) proposed PMF-CMB as a means of addressing the severely limited availability of 'local source profiles' (i.e., not available at most receptor locations without additional time-consuming and cost-prohibitive source sampling, particularly for less commonly sampled pollutants of interest such as organic air toxics): for an urban receptor site in South Korea and a $\mathrm{PM}_{2.5}$ dataset (i.e., speciation by elements and organics inclusive of $\mathrm{PAH}$ ) the authors found that $\mathrm{CMB}$ results were improved when PMFresolved source profiles from local ambient data were used as CMB input, compared with the more typical practice of inputting only reference profiles from literature. The authors recommend this combined PMF-CMB approach for urban areas where source profiles have not otherwise been developed; such is the case for PAH source apportionment as proposed in this research. 
The case for the use of 'local source profiles' in lieu of reference profiles is particularly clear for source types such as vehicle emissions and local industry emissions, were profiles are expected to vary from one study area to another (Duvall et al, 2007; Shi et al, 2009; Park and Kim, 2005); 'local source profiles' are also highly relevant for source apportionment of PAH due to the inherent difficulties of otherwise obtaining sufficiently representative reference profiles for this pollutant class (i.e., source specificity concern, see Section 2.4.2). Thus, this research extended previous limited PMF-CMB source apportionment efforts to PAH and PAH toxicity at the intraurban scale by:

i. Applying the PMF model to central monitoring site data to resolve 'local source profiles' (i.e., source types contributing to Hamilton ambient PAH).

ii. Applying the CMB model to quantify the contributions of major source types to PAH and PAH toxicity across an urban area (i.e., source apportion the Hamilton intraurban field dataset), permitting source-based spatial analysis.

In the interest of developing the methodology, several PMF models (i.e., differences in model order, species suite) and variants of PMF output (i.e., base run, base run with Fpeak, bootstrap run) were compared as CMB input source profiles. The coupled PMFCMB modeling process was thus iterative and ultimately yielded a recommended method of PMF modeling and $\mathrm{CMB}$ input file preparation where the intent is to apply PMF factor profile output as a set of distinct source profiles in CMB modeling. The details of the methodology are presented in Appendix E. 


\subsection{Source-based quantification of PAH toxicity}

PAHs are on the Canadian Toxic Substances List (Environment Canada, 2007b, 2007e) and Schedule 1 of the Canadian Environmental Protection Act 1999 (Environment Canada, 2006; CEPA, 1999). Several PAH species have been declared toxic under CEPA (i.e., Section 64; CEPA, 1999) because they are entering the environment in a quantity or concentration or under conditions that: have or may have an immediate or long-term harmful effect on the environment or its biological diversity; and, constitute or may constitute a danger to the environment on which life depends (i.e., toxicity class "a" and "c", respectively). Additional information on PAH exposure health effects is presented in Appendix A.2 .

There is currently no Canada-Wide Standard (CWS) for PAHs. This is also the case at the national level in the United States and the European Union (EU). However, some EU states have set a voluntary ambient air quality standard for $\mathrm{BaP}$ of between 0.1 and 1.3 $\mathrm{ng} / \mathrm{m}^{3}$ (Integaire, 2005). Several occupational exposure limits for PAHs have been set,

regulating $\mathrm{BaP}$ exposure specifically as well as exposure to coal tar pitch volatiles (US EPA, 2007)

Although there are more than 100 different PAHs, a subset is typically chosen for study and regulation based on prevalence in the environment, likelihood of human exposure, and demonstrated or suspected toxicity. In Canada, a total of 17 PAH are classified as CEPA-toxic; the United States uses a largely similar listing under its regulatory definition (ATSDR, 1995). PAH species classified as toxic are summarized and presented alongside the large suite of monitored species in Table 2-3. 
The assessed toxicity of PAHs is based on studies of several PAH species; although the chemical structure and properties of PAH species varies across the family of compounds (see Appendix A.1 for table of properties and compound structures for species analysed in this research), toxicity profiles are expected to be similar for most PAHs. Thus, benzo(a)pyrene $(\mathrm{BaP})$ has been used as an indicator of other PAHs, with the relative concentrations of other $\mathrm{PAH}$ species reported as a concentration ratio to $\mathrm{BaP}$. $\mathrm{BaP}$ is also used as the indexing species for quantifying exposure toxicity associated with other PAH, following the work of Nisbet and Lagoy (1992) who reviewed epidemiological and toxicological literature and presented a set of Toxicity Equivalence Factors (TEF) for a broad suite of PAH species, listed in Table 2-4. Additional information on the TEF data used in this research is presented in Appendix A.2.

In this research, all key results (i.e., field sampling results, source apportionment results) are reported in terms of 'PAH toxicity' (i.e., for carcinogenic potential of inhalation exposure) based on the BaP-toxicity equivalent concentration. The conversion from sampled ambient PAH concentration to 'BaP-toxicity equivalent concentration' is shown in 
Equation 2-3. Individual species concentrations (C) are converted to BaP-toxicity equivalent species concentrations (BaP-eq), multiplying by their respective TEF (Table 2-4); summation of individual BaP-eq yields total sampled or modeled BaP-toxicity equivalent concentration (BaP-TEQ). (Jung et al, 2010) 
Equation 2-3. Calculation of total sampled or modeled BAP-toxicity equivalent concentration.

$$
\begin{aligned}
& B A P-T E Q=\sum_{j=1}^{m}\left(B A P-e q_{j}\right)=\sum_{j=1}^{m} C_{j} * T E F_{j}\left[n g / m^{3}\right] \\
& B a P-T E Q=\text { Total BAP-toxicity equivalent concentration for m summed species }\left(\mathrm{ng} / \mathrm{m}^{3}\right) \\
& B a P-e q_{j}=\text { BAP-toxicity equivalent concentration of species } \mathrm{j}\left(\mathrm{ng} / \mathrm{m}^{3}\right) \\
& C_{j}=\text { concentration of species } j \\
& T E F_{j}=\text { Toxicity Equivalency Factor for species } \mathrm{j} \text { (unitless) }
\end{aligned}
$$

This research also explicitly quantified source contributions to PAH toxicity (i.e., source contributions to BaP-TEQ), converting PMF and CMB source apportionment results by extending the methods of Jung et al (2010); calculation is entirely analogous to 
Equation 2-3 but for factor contributions to species concentrations $\left(\mathrm{ng} / \mathrm{m}^{3}\right)$ rather than sampled ambient concentrations.

Reporting of the source apportionment results in terms of PAH toxicity was devised in response to the fundamental concern of differing source toxicity (Grahame and Schlesinger, 2007) and permitted significant additional analysis of the source apportionment results, inclusive of identifying sources contributing disproportionately to toxicity at the urban/ rural scale and, at the intraurban scale, further distinguishing between sources contributing similar toxicity across an urban centre from those associated with 'hot spots' of exposure toxicity near local sources. 
Table 2-3. NAPS monitoring, NPRI reporting, CEPA-toxicity, and US regulation of PAH. (Environment Canada, 2007a, 2007c, 2007d; ATSDR, 1995)

\begin{tabular}{|c|c|c|c|c|c|}
\hline Count & PAH & $\begin{array}{c}\text { NAPS } \\
\text { Monitoring }\end{array}$ & $\begin{array}{c}\text { NPRI } \\
\text { Reporting }\end{array}$ & $\begin{array}{c}\text { CEPA-toxic } \\
\text { Status } \\
\end{array}$ & $\begin{array}{l}\text { US ATSDR } \\
\text { Regulation }\end{array}$ \\
\hline 1 & PAHs (total) & $\bullet$ & $\bullet$ & $\mathrm{N} / \mathrm{A}$ & N/A \\
\hline 2 & acenaphthylene & $\bullet$ & $\bullet$ & & \\
\hline 3 & acenapthene & $\bullet$ & $\bullet$ & & \\
\hline 4 & anthanthrene & $\bullet$ & & & \\
\hline 5 & anthracene & $\bullet$ & $\bullet$ & & \\
\hline 6 & benzo(a)anthracene & $\bullet$ & $\bullet$ & $\bullet$ & $\bullet$ \\
\hline 7 & benzo(a)phenanthrene & & $\bullet$ & $\bullet$ & \\
\hline 8 & benzo(a)pyrene & $\bullet$ & $\bullet$ & $\bullet$ & $\bullet$ \\
\hline 9 & benzo(b)chrysene & - & & & \\
\hline 10 & benzo(b)fluoranthene & $\bullet$ & $\bullet$ & $\bullet$ & $\bullet$ \\
\hline 11 & benzo(b)fluorene & $\bullet$ & & & \\
\hline 12 & benzo(e)pyrene & $\bullet$ & $\bullet$ & $\bullet$ & \\
\hline 13 & benzo( $g, h, i) f l u o r a n t h e n e$ & $\bullet$ & & & \\
\hline 14 & benzo(g,h,i)perylene & - & $\bullet$ & $\bullet$ & $\bullet$ \\
\hline 15 & benzo(j)fluoranthene & & $\bullet$ & $\bullet$ & \\
\hline 16 & benzo(k)fluoranthene & $\bullet$ & $\bullet$ & $\bullet$ & $\bullet$ \\
\hline 17 & chrysene & $\bullet$ & & & - \\
\hline 18 & coronene & & & & $\bullet$ \\
\hline 19 & dibenz $(a, j)$ acridine & & $\bullet$ & $\bullet$ & \\
\hline 20 & dibenzo(a,h)anthracene & $\bullet$ & $\bullet$ & $\bullet$ & \\
\hline 21 & dibenzo(a,h)perylene & & & & $\bullet$ \\
\hline 22 & dibenzo(a,i)pyrene & & $\bullet$ & $\bullet$ & \\
\hline 23 & fluoranthene & $\bullet$ & $\bullet$ & $\bullet$ & $\bullet$ \\
\hline 24 & fluorene & $\bullet$ & $\bullet$ & & $\bullet$ \\
\hline 25 & indeno(1,2,3-c,d)fluoranthene & - & & & \\
\hline 26 & indeno $(1,2,3-\mathrm{c}, \mathrm{d})$ pyrene & $\cdot$ & $\bullet$ & $\bullet$ & $\bullet$ \\
\hline 27 & napthalene & & $\bullet$ & & $\bullet$ \\
\hline 28 & perylene & $\bullet$ & $\bullet$ & $\bullet$ & \\
\hline 29 & phenanthrene & $\bullet$ & $\bullet$ & $\bullet$ & $\bullet$ \\
\hline 30 & pyrene & $\bullet$ & $\bullet$ & $\bullet$ & $\bullet$ \\
\hline 31 & retene & $\cdot$ & & & \\
\hline 32 & triphenylene & $\bullet$ & & & \\
\hline 33 & 1-Me-pyrene & $\bullet$ & & & \\
\hline 34 & 2-Me-Fluorene & $\bullet$ & & & \\
\hline 35 & 3-me-cholanthrene & $\bullet$ & & & \\
\hline 36 & 7H-Dibenzo(c,g)carbazole & & $\bullet$ & $\bullet$ & \\
\hline 37 & 7-Me-Benz(a)anthracene & - & & & \\
\hline
\end{tabular}

Notes:

1. PAH species ordered alphabetically.

2. NAPS monitoring, NPRI reporting, and CEPA-toxic status data sourced from Environment Canada, 2007a, 2007c, 2007d, respectively.

3. US regulation sourced from ATSDR, 1995. 
Table 2-4. Benzo(a)Pyrene-equivalent carcinogenic Toxicity Equivalency Factors (BaP-TEF) for inhalation exposure, modeled species.

\begin{tabular}{llr}
\hline Species ID & Species name & BaP-TEF \\
\hline 01_ACY & acenaphthylene & 0.001 \\
02_ACE & acenapthene & 0.001 \\
03_FLU & fluorene & 0.001 \\
04_ANT & anthracene & 0.01 \\
05_PHE & phenanthrene & 0.001 \\
06_MFLU & 2-me-fluorene & $0.001^{\mathrm{a}}$ \\
07_FLT & fluoranthene & 0.001 \\
08_PYR & pyrene & 0.001 \\
09_MPYR & 1-me-pyrene & $0.001^{\mathrm{a}}$ \\
10_BaFLU & benzo[a]fluorene & $0.001^{\mathrm{b}}$ \\
11_BbFLU & benzo[b]fluorene & $0.001^{\mathrm{b}}$ \\
12_BghiFLT & benzo[g,h,i]fluoranthene & $0.001^{\mathrm{c}}$ \\
13_CHRY & chrysene & 0.01 \\
14_TRIPH & triphenylene & $0.01^{\mathrm{d}}$ \\
15_BaA & benzo[a]anthracene & 0.1 \\
16_RET & retene (1-methyl-7- & 0.1 \\
18_BaP & isopropylphenanthrene) & $0.001^{\mathrm{e}}$ \\
19_BeP & benzo[a]pyrene & 1 \\
20_BbFLT & benzo[e]pyrene & $0.01^{\mathrm{f}}$ \\
21_BkFLT & benzo[b]fluoranthene & 0.1 \\
25_BghiPE & benzo[k]fluoranthene & 0.1 \\
26_IPYR & benzo[g,h,i]perylene & indeno[1,2,3-cd]pyrene \\
\hline
\end{tabular}

Note: Data from Nisbet and Lagoy, 1992 and ATSDR, 1995 except where noted:

(a) Assigned same TEF as parent compound.

(b) Assigned same TEF as FLU (i.e., toxicity classified equivalent to FLU in report to Health Canada, PAH PEF Toxicity Update, 2006).

(c) Assigned TEF value as per Nielsen et al, 1996 (i.e., grouped with structurally similar B[c]PHEN) and recommendation to Health Canada (i.e., recommends TEF value for $\mathrm{B}[\mathrm{c}] \mathrm{PHEN}$ that is one order of magnitude lower than TEF for CHRY; Equilibrium Environmental, 2006).

(d) Assigned same TEF as CHRY (i.e., Nielsen et al, 1996 show single value for CHRY+TRIP).

(e) No toxicity data available since retene less commonly sampled; conservative assumption is that compound has degree of toxicity since an alkylated variant of phenanthrene therefore assigned same TEF as phenanthrene (i.e., lowest toxicity value in modeled species suite; also see Scott, 2009).

(f) Assigned TEF as per Malcolm and Dobson, 1994 and Nielsen et al, 1996. 


\section{Chapter: Source apportionment of PAH and PAH toxicity by analysis of long-term trends at urban and rural sites in Southern Ontario, Canada}

\subsection{Highlights}

- Wind/pollutant rose analysis found PAH levels consistent with local source regions.

- Key PAH source types and contributions to PAH and toxicity were found at all sites.

- Vehicle traffic exhaust contributed majority of PAH toxicity at all sites.

- Iron/steel manufacture contributed significant PAH toxicity at the industrial site.

- Source apportionment to PAH toxicity is meaningful for regulation/health intervention.

\subsection{Abstract}

Central site time series data for speciated vapour plus particle phase ambient PAH were analysed at two urban centers (Hamilton, Toronto) and a rural background site in Ontario, Canada. Urban ambient total sampled $\left(\Sigma_{29} \mathrm{PAH}\right)$ levels were several times higher than background with highest levels at Hamilton, site of significant local industry (iron/steel manufacturing). Ambient PAH showed meaningful inter-site differences in seasonality and no consistent seasonality at Hamilton due to influence of local industry. Inter-site differences in individual and heavy MW species concentrations indicated differences in source mix. Wind direction analysis confirmed high/low ambient PAH consistent with local source regions and pollutant rose analysis at Hamilton confirmed highest ambient $\mathrm{PAH}$ aligned with industrial harbour-front and towards the escarpment. Preliminary modeling by Principle Component Analysis (PCA) indicated data suitability for advanced receptor modeling. Positive Matrix Factorization (PMF) identified four source types: volatilized PAH and long-range transported coal combustion, vehicle traffic exhaust, space heating, and biomass combustion. At Hamilton, local industry emissions were also identified, associated with iron/steel manufacturing. PAH volatilization and transported coal combustion contributed the majority of modeled ambient PAH emissions at all sites. Vehicle traffic exhaust contributions were highest at Toronto, location of most intense transportation infrastructure. Coal-related PAH emissions were greatest at Hamilton due to addition of local emissions from iron/steel production. Space heating and biomass combustion together contributed significant ambient $\mathrm{PAH}$ at all sites. Source apportionment of PAH toxicity using B(a)P Toxicity Equivalency Factors (BaP-TEF) identified traffic exhaust as a 'more toxic' source type, contributing little to ambient PAH yet dominating PAH toxicity at all sites and quantified significant contributions to PAH toxicity from local industry emissions. 


\subsection{Introduction}

Monitoring air toxics associated with respirable particulate matter (PM), such as Polycyclic Aromatic Hydrocarbons (PAH), has been a significant advance in exposure assessment, reflecting steadily growing awareness by air quality researchers, legislators, and medical professionals of the variability in toxicity of respirable PM. (Grahame and Schlesinger, 2007; Tolbert, 2007) A next step in better understanding exposures is to examine inter-urban and urban-rural differences in ambient PAH levels and identify and quantify the dominant source types contributing to PAH and PAH toxicity.

PAHs are produced by incomplete combustion of organic material and have many anthropogenic sources, including coal-fired power generation, space heating by fossil fuel combustion, vehicle exhaust, and industrial activity such as aluminum smelting and steel production (Ravindra, 2008). PAHs are semi-volatile organic compounds (SVOCs) present in vapour and particle phases in ambient air. Generally, lighter molecular weight (MW) species with 2 and 3-ring structures (MW $\leq 202$ ) are found largely in vapour phase while heavier MW species with 5-ring and 6-ring structures (MW >228) are largely in particle phase and predominantly in the smaller respirable size fraction (i.e., $\mathrm{PM}_{2.5}$ ), increasing exposure risk (Venkataraman and Friedlander, 1994; Lu et al., 2008; additional information on vapour-particle partitioning and predominant particulate size fractions in Appendix A.1). PAHs have been declared toxic under the Canadian Environmental Protection Act (Environment Canada, 2011a) and a number of heavy MW species classed for probable carcinogenicity (ATSDR, 1995). PAHs may also react with sunlight or other atmospheric compounds to form transformation products, such as nitro- 
$\mathrm{PAH}$ and hydroxy-PAH, with greater toxicity and environmental persistence (Baek et al., 1991a).

The need for source-based PAH assessment was stressed by comprehensive review of toxicology, epidemiology, and exposure assessment studies, finding that monitoring was insufficient for improving exposure assessment for health risk estimates or regulation of air toxics, and argued instead that "certain sources of PM emissions, by virtue of disproportionate production of PM-associated toxics such as PAH, may be considered for the purposes of health risk assessment 'more toxic' than others." (Grahame and Schlesinger, 2007). Source identification and quantification for PAH and other PMassociated toxics is recommended, and local sources such as vehicle traffic and heavy industry have been cited as significant contributors to variable exposure health risk (Schwartz et al, 2005; Gold et al, 2005).

Various multi-step procedural frameworks have been proposed for receptor modeling (Watson et al, 2008, 2002; Reff et al, 2007; Brook et al, 2004) and 'best practice' guidelines are readily available (US EPA, 2008; Sonoma, 2008). However, even with continued model support and development by US EPA, many studies lack adequate and transparent reporting of assumptions made and model parameters used, rendering the methods difficult for other researchers to replicate or apply correctly to other datasets (Watson et al, 2008; Reff et al, 2007). The inherent degree of subjectivity in iterative modeling protocols and in interpreting modeled factors as physically meaningful source types is an additional challenge. Uncertainty on model solutions, both source profiles and source contribution estimates, is also frequently not reported or is otherwise inadequately 
estimated, restricting inter-study comparisons. (Reff et al, 2007) Last, sufficient environmental context for receptor modeling is often lacking or not reported, impeding corroboration of receptor modeling results by independent observations.

Studies that have attempted to identify and quantify PAH source contributions by receptor modeling methods often do not adequately address challenges associated with modeling this semi-volatile class (Galarneau, 2008). Studies have often been limited by time series length, number of species measured, and availability of combined vapour and particle phase PAH concentrations (i.e., required to better represent total ambient PAH exposures and avoid phase partitioning challenges associated with single-phase measurements; ATSDR, 1995). Further, uncertainties estimates on ambient data have not always been sufficiently conservative or recognized inter-species differences in sampling and laboratory analysis uncertainty inherent to PAH suites encompassing a broad MW range (US EPA, 1999; Venkataraman and Friedlander, 1994).

This research analysed long time-series central site monitoring data for total ambient PAH (vapour+particle phase) at two urban centers and a rural background site in Ontario, Canada and applied receptor modeling techniques to identify dominant source types contributing to ambient PAH. Key pre- and post-modeling analyses recommended for responsible interpretation of PAH sources and contributions (Watson et al, 2008, 2002; Reff et al, 2007; Brook et al, 2004) are presented, including: historical trend analysis; more than one study site and inclusion of a suitable background site to facilitate interpretation, ensure reasonable inter-site agreement and yield useful insight on inter-site trends; comprehensive a priori source review of study area using emissions inventory 
data, land use classification and local knowledge; and, wind pattern analysis relative to local source locations. Modeling was conducted with a rigor intended to address methodological challenges and provide checks on results and several new sensitivity analyses are presented in the interest of reducing subjectivity in the PMF modeling process (Reff et al, 2007) and advancing modeling practice for practitioners. Finally, uncertainties are estimated and reported for all key source apportionment results.

This research is comparatively rare among receptor model studies in explicitly addressing fundamental concerns of differing source toxicity (Grahame and Schlesinger, 2007) by presenting source apportionment results in terms of PAH toxicity, thereby identifying which sources are contributing disproportionately to toxicity and investigating how a health-based source apportionment can accentuate meaningful differences between urban population centers. Findings will assist environmental regulators in developing exposure intervention strategies better targeted to specific sources and sensitive to inter-urban differences. Furthermore, findings enable development of receptor modeling methods for the analysis of spatial variability in source contributions at the intraurban scale (Chapter 5), using a spatial field dataset available for one of the studied urban centers (Anastasopoulos et al, 2012; Chapter 4). To ensure clarity and facilitate application to other datasets, methods and results adapted general procedural frameworks (Watson et al, 2008 , 2002) to PAH and are presented in three parts: (i) environmental context for source apportionment; (ii) source apportionment modeling; and, (iii) source type contributions to PAH and PAH toxicity. 


\subsection{Methods}

\subsubsection{Environmental Context for Source Apportionment}

\subsubsection{Receptor sites}

Two large Canadian urban centers (Toronto, population 6M; Hamilton, population 700,000; Statistics Canada, 2012a, 2012b) and a rural background site were selected for PAH source apportionment (Figure 3-1). The Toronto central monitoring site (Gage Institute: $43.658417 \mathrm{~N}, 79.397139 \mathrm{~W})$ is in a central business district and is characterized by traffic on adjacent surface streets and nearby expressways, particularly during peak commuting periods. The Hamilton central monitoring site (Beasley Park: 43.257722N, $79.861611 \mathrm{~W})$ is also near a central business district with proximal industrial land use (e.g., iron/steel manufacturing and other harbour-front heavy industry). Hamilton ambient PAH levels were frequently highest among monitored Canadian central sites so additional field sampling and source apportionment analysis was completed at the intraurban scale in related papers (Anastasopoulos et al, 2012; Chapter 5). The rural site (Egbert: $44.232500 \mathrm{~N}, 79.781389 \mathrm{~W}$ ) is approximately $90 \mathrm{~km} \mathrm{NW}$ of Toronto and $140 \mathrm{~km}$ $\mathrm{N}$ of Hamilton and is upwind by prevailing westerly wind direction and so was a suitable background site. (Aerial photos of sampling sites in Supplementary Information, Appendix B.1.)

\subsubsection{PAH emissions source review}

Broad Canadian and provincial context for PAH source types was established by review of National Pollutant Release Inventory (NPRI; Environment Canada, 2009) data and a relevant regional emissions inventory (Galarneau et al, 2007). Industrial stationary 
sources located near the study sites and reporting airborne PAH emissions were identified by review of 2009 NPRI data (Environment Canada, 2009), the year selected to correspond to related intraurban field sampling completed in 2009 (Anastasopoulos et al, 2012). Additional stationary sources (institutional, residential) and areas/routes with concentrated mobile source emissions were identified by aerial maps of the study area along with local knowledge. For major source types identified as influencing the study area, a literature review of emissions databases and source sampling studies yielded multi-species PAH emissions profiles and identified source-characteristic species that could inform receptor model factor interpretation. Comprehensive discussion of the emissions source review is presented in Supplementary Information, Appendix B.1.

\subsubsection{Ambient PAH dataset}

The ambient PAH concentration data used for the modeling are from central monitoring sites that are part of the NAPS Network (Environment Canada, 2004), sampling particulate $\mathrm{PAH}$ on glass-fibre filters (GFF) and vapour phase $\mathrm{PAH}$ on polyurethane foam (PUF) sorbent over 24-hr, once every six-days for a broad suite of species (Table $3-1){ }^{2}$ Species identification and quantification is via gas chromatography with mass spectrometry detection (GC-MSD) after co-extraction of GFF and PUF media, following US EPA Method TO-13A (1999) to determine total ambient PAH concentrations (i.e., sum of particle+vapour phase; $\mathrm{ng} / \mathrm{m}^{3}$ ). Total ambient PAH data has been shown to be

${ }^{2}$ NAPS-monitored species suite compared with regulatory classification (CEPA, US EPA) in Appendix B.2. 
suitable for receptor modeling (Mari, 2010) due to the partitioning behaviour of light and medium MW species (Venkataraman and Friedlander, 1994; Galarneau, 2008).

Historical central site PAH datasets were scoped to a recent time series (2001-2010) to avoid an overly long period where changes in relative source contribution may have occurred (i.e., regulatory, economic changes) while maintaining sufficient dataset size for modeling. Annual and seasonal sample densities were comparable within each dataset to ensure good model representation of all years. ${ }^{3}$ Species screening applied data quality criteria, excluding species with very high $(>90 \%)$ portion of time series missing or below detection limit $\left(\mathrm{DL}_{\mathrm{j}}\right)$, very low time series variance $(\mathrm{CV}<0.3)$, or known sampling or sample analysis issues; marker species were generally included, with appropriately conservative error estimates for those with lower data quality (US EPA, 2008; Zhao et al, 2006; Hopke et al, 2003b; Reff et al, 2007). A common species suite was modeled to facilitate inter-site comparisons of source apportionment results (Table 3-1). ${ }^{4}$

\subsubsection{PAH trend analysis}

A comprehensive review of the $\mathrm{PAH}$ datasets was carried out prior to source apportionment and compared across sites, inclusive of: descriptive statistics (i.e., individual PAH, $\left.\Sigma_{29} \mathrm{PAH} ; \quad \Sigma_{\mathrm{LIGHT}} \mathrm{PAH} ; \quad \Sigma_{\mathrm{HEAVY}} \mathrm{PAH}\right)$; dataset amenability to receptor

${ }^{3} 2010$ (all sites) and 2003 (Hamilton) were years with significantly fewer samples and were omitted when inferring annual trends in PAH levels or source contributions.

${ }^{4}$ Dibenz(ah)anthracene (DBA) omitted due to large number of missing and below $\mathrm{DL}_{\mathrm{j}}$ samples at background site (US EPA PMF, 2008; Sonoma, 2008; Reff et al, 2007) despite sufficient data quality for modeling at Hamilton. 
modeling (i.e., data density, sample-to-sample variability); annual and seasonal trends (i.e., 'winter', October - April; 'summer', May - September); species abundance (normalized to $\Sigma_{29} \mathrm{PAH}$ ) ranking to determine dominant species and confirm general consistency in source mix over the modeled time period (Kuntasal, 2005; Gaga, 2004).

Year-round and seasonal wind roses were prepared (WRPlot software, Lakes Environmental Inc.) from historical (2000-2010) hourly wind data (i.e., weather station nearest each site: Toronto airport 43.68 N, 79.63 W; Hamilton airport 43.17 N, 79.93 W; background co-located; data from Environment Canada, 2011e). Predominant wind directions were compared across sites and assessed against local PAH source areas; 24-hr and 48-hr wind roses were also constructed for days with highest and lowest sampled PAH concentrations to better identify probable influence of local source areas. Since Hamilton was to be analyzed more closely via intraurban field sampling (Anastasopoulos et al, 2012) and receptor modeled at the intraurban scale (Chapter 5), a pollutant rose combined wind data with central site sampling data for $\Sigma_{29} \mathrm{PAH}$ to indicate local source areas frequently associated with high ambient PAH (i.e., upwind of central monitor during periods of high ambient $\mathrm{PAH})$. 


\subsubsection{Source Apportionment}

\subsubsection{Receptor model theory}

Receptor models attempt to identify and quantify the relative contribution of different sources to observed ambient concentrations of air pollutants, based on the principle of mass balance of pollutants between sources and receptor. As presented in Equation 3-1, $\boldsymbol{X}$ is the sample matrix, $\boldsymbol{F}$ is the factor profile matrix, $\boldsymbol{G}$ is the matrix of factor contributions to each sample and $\boldsymbol{E}$ is the model residual. (Hopke, 2003a)

\section{Equation 3-1. General source-receptor mass balance.}

$$
\boldsymbol{X}=\boldsymbol{G} \cdot \boldsymbol{F}+\boldsymbol{E}
$$

$$
\begin{aligned}
& \boldsymbol{X}=\text { sample concentration matrix } \\
& \boldsymbol{G}=\text { factor contribution matrix } \\
& \boldsymbol{F}=\text { factor profile matrix } \\
& \boldsymbol{E}=\text { model residual matrix } \\
& \qquad x_{i j}=\sum_{k=1}^{p} g_{i k} f_{k j}+e_{i j}
\end{aligned}
$$

$$
\begin{aligned}
& x_{i j}=\text { concentration species } j \text { in receptor sample } i\left(\mathrm{ng} / \mathrm{m}^{3}\right) \\
& g_{i k}=\text { contribution of source factor } k \text { to receptor sample } i \\
& f_{k j}=\text { concentration of species } j \text { to source factor } k\left(\mathrm{ng} / \mathrm{m}^{3}\right) \\
& e_{i j}=\text { model residual species } j \text { in receptor sample } i\left(\mathrm{ng} / \mathrm{m}^{3}\right)
\end{aligned}
$$

Prior to receptor modeling, a Principal Component Analysis (PCA) was applied to resolve a set of independent components that together explained a maximum of the total variance of the sample matrix $\boldsymbol{X}$ (i.e., components explain which species are co-varying at the receptor and thus are likely being emitted from a common source but source contributions associated with each component are not quantified; Hopke, 2006; Mudge, 2007). The research then applied Positive Matrix Factorization (PMF; US EPA PMF 3), 
an advanced receptor model (i.e., model development described by Paatero and Tapper, 1994; Paatero, 1997) with key advantage that knowledge of potential sources contributing to ambient concentration at a receptor is not required a priori. PMF input requires only sufficient series of speciated ambient measurements $(\boldsymbol{X})$ and an estimate of associated uncertainty $(\boldsymbol{S})$. Statistical analysis of trends in the speciated pollutant data yields factors explaining the data variation that may be interpreted as physical meaningful source types. (Hopke, 2004; Brook et al, 2004) PMF solves the source-receptor mass balance (Equation 3-1) by using an iterative algorithm to populate $\boldsymbol{F}$ and $\boldsymbol{G}$ matrices simultaneously, subject to the constraint that matrix elements be non-negative, while seeking to minimize an objective function Q (Equation 3-2). The objective function's weighting of the model residual $(\boldsymbol{E})$ by the measurement uncertainty $(\boldsymbol{S})$ ensures the solution is influenced predominantly by data with lower associated uncertainty, down-weighting data with greater associated uncertainty. (Hopke, 2003a; Paatero and Tapper, 1994; Lee, 2003)

Equation 3-2. Minimization function used by PMF to solve source-receptor mass balance.

$$
\begin{aligned}
& Q=\sum \sum\left(\frac{\boldsymbol{E}}{\boldsymbol{S}}\right)^{2} \\
& \boldsymbol{E}=\text { model residual matrix } \\
& \boldsymbol{S}=\text { uncertainty matrix } \\
& Q=\sum_{i=1}^{n} \sum_{j=1}^{m}\left(\frac{e_{i j}}{s_{i j}}\right)^{2}
\end{aligned}
$$

$e_{i j}=$ model residual of species $j$ in receptor sample $i\left(\mathrm{ng} / \mathrm{m}^{3}\right)$, see Equation 3-1

$s_{i j}=$ uncertainty of species $j$ in receptor sample $i\left(\mathrm{ng} / \mathrm{m}^{3}\right)$ 
Source type interpretation was reserved for PMF due to the model's several functional advantages over simpler factor analysis models (e.g., PCA), which assist more complex interpretation of factors as source types (Hopke, 2006). These include incorporating sample uncertainty, which permits data weighting to differ between species and between samples, and estimates of uncertainty on factor profiles, which allows comparisons with reference profiles to be made within uncertainty bounds. PMF also directly quantifies factor contributions and this enables factor interpretation to extend beyond simplistic comparisons of factor profile: overall relative factor contribution can be compared with a priori expected contributions, such as available from inventory estimates, and seasonal and weekday/weekend trends can be compared with expected source type trends, such as seasonal versus year-round source releases.

\subsubsection{PCA modeling protocol}

PCA used as input data the same PAH dataset and species suite prepared for PMF modeling and revealed data 'structure' in the urban and background $\mathrm{PAH}$ datasets, principal components (PC) indicating patterns of co-varying PAH species. PCA was also used to indicate the number of factors that could be reasonably extracted from the dataset, providing a lower bound on the number of physically meaningful factors expected from application of the PMF receptor model, and to explore data sensitivity to seasonal stratification. The PCA was run using SYSTAT 13 (Systat Software Inc., Chicago, IL, USA) with a correlation matrix and Varimax rotation. Details of the PCA modeling protocol and results are presented in Supplementary Information, Appendix B.4 (see also Appendix E.1). 


\subsubsection{PMF modeling protocol}

The urban and background site PAH datasets were modeled using PMF3 to identify major source types contributing to ambient PAH at the three sites and quantify source type contributions. The PMF data treatment protocol for input concentrations $\left(x_{i j}\right)$ and uncertainties $\left(s_{i j}\right)$ reflected best practice guidelines (Reff et al, 2007; US EPA, 2000) and is summarized in Table 3-2. Effort was made to apply sample uncertainty $\left(s_{i j}\right)$ that would adequately capture, estimate and aggregate the uncertainties associated with laboratory analysis of PAH samples, thereby ensuring appropriate weighting of individual samples in the PMF receptor model. Species uncertainty data were calculated using the 'equationbased' subroutine available in PMF3 and were consistent with species concentration data treatment (Table 3-2). Species error fraction data were obtained from the appropriate laboratory methods document (Environment Canada, 2011f) and more conservative species were assigned less uncertainty than highly volatile and more reactive species, varying from approximately $10 \%$ to $50 \%$ (Table $3-1$ ). Since PMF down-weights outliers (Hopke, 2003a), and because efforts were made to retain model power and adequate sample-to-sample variability, a conservative protocol was applied for excluding outliers: samples were excluded only if they contained statistically extreme outlier species concentrations (i.e., higher or lower than three times IQR) and outlier status was corroborated by poor model performance for that sample date and exclusion would not meaningfully alter source profiles or trends in contribution.

PMF modeling followed best practice guidelines (US EPA, 2008; Sonoma, 2008) and recommendations in the comprehensive literature review of PMF studies completed by 
Reff et al (2007). Briefly: explored 3 to 7 factor solutions and selected optimal model order with respect to model performance and to ensure physical meaning of solution; verified solution unique and stable (i.e., converged to local minimum with narrow range in Q; further validated using random seed); verified solution converged to theoretical global minimum (i.e., compared $\mathrm{Q}_{\text {robust }}$ to $\mathrm{Q}_{\text {theory }}$ ) and not subject to outlier influence (i.e., compared $\mathrm{Q}_{\text {robust }}$ to $\mathrm{Q}_{\text {true }}$ ); verified all fitted species modeled well (e.g., high $\mathrm{r}^{2}$, low species residuals, normally distributed species residuals); and, verified solution was reasonably free from rotational ambiguity (i.e., scatter in inter-factor contribution plots, 'G-space'; exploration of F-peak solution rotation).

Most critically, model solutions were verified for robustness via bootstrapping analysis, the PMF3 subroutine that repeatedly models differing subsets of the original concentration dataset and statistically summarizes their solutions (US EPA, 2008). This ensured that interpreted solutions were statistically representative results and that factors were sufficiently distinct from each other (i.e., bootstrap factors being mapped consistently to the same base factor) to merit interpreting as a distinct source type. Bootstrapping analysis also provided a procedurally rational method of quantifying uncertainty on the PMF solution (Reff et al, 2007; Watson et al, 2008), via interquartile range (IQR) statistics (i.e., narrower IQR quantifying less uncertainty). Additional details of the PMF modeling protocol are presented in Supplementary Information, Appendix B.5 (also see Appendix E.2). 


\subsubsection{Source contributions to PAH and PAH toxicity}

Relative factor contributions (\%) to ambient PAH were first calculated as 'total aggregate contributions' of source types to total modeled PAH (i.e., sum of modeled PAH aggregated over all samples; 2001-2010, $\Sigma_{21} \mathrm{PAH}$ at urban sites; 2005-2010, $\Sigma_{20} \mathrm{PAH}$ at background). The overall source apportionment results were useful for comparison with emissions inventory data (i.e., emissions inventories similarly aggregate total PAH releases over a given time period) or with other receptor modeling results. Since source contributions can vary significantly from sample-to-sample and some source types may show greater variability in relative contribution (e.g., due to dependence on wind direction), source type contributions to modeled PAH (\%) were also calculated for each sample and statistically summarized by median and IQR (i.e., adapting mean contribution calculation of Larsen and Baker, 2003). Thus, this research reported the range of source contributions expected at the modeled sites, facilitating comparison with other source apportionment results (Watson et al, 2008; Reff et al, 2007).

Source apportionment results also explicitly quantified the relative toxicity of source contributions (i.e., carcinogenic potential of inhalation exposure). PMF factor contributions to total modeled PAH ( $\mathrm{PAH})$ were converted to equivalent factor contributions to total modeled BaP-toxicity equivalent concentration (BaP-TEQ, ng/m $/ \mathrm{m}^{3}$ ) by multiplying factor contributions to individual species concentrations by their respective Toxicity Equivalency Factor (TEF; Table 3-3), which quantify species toxicity relative to BaP (Nisbet and Lagoy, 1992), and summing over all species, adapting the method described in Jung et al (2010). 


\subsection{Results \& Discussion}

\subsubsection{Environmental Context for Source Apportionment}

\subsubsection{PAH emissions and source types in study region}

The fundamental premise driving source apportionment at the urban and background sites was that PAH source types comprising a small portion of overall annual anthropogenic Canadian or Ontario releases could be significant sources at a given receptor location due to their exposing a specific population continuously or disproportionately (e.g., living near the source or exposed due to work location). Broad Canadian and provincial context for PAH source types is presented in Supplementary Information, Appendix B.1.

Within the study area, industrial (e.g., iron/steel emissions, chemical and petrochemical manufacturing emissions) and institutional facilities (i.e., space heating emissions) reporting airborne PAH emissions (Environment Canada, 2009) or otherwise identified as relevant were mapped alongside the scoped sites (Supplementary Information, Appendix B.1). Private residences are found in very high density in the greater urban areas around the central monitoring sites and are associated with space heating combustion (i.e., natural gas, oil) and biomass combustion (i.e., wood-burning fireplaces/supplemental heating). In addition to stationary sources (i.e., industrial, institutional, residential), mobile sources (i.e., vehicle exhaust emissions) are distributed throughout the study region and concentrated in the urban locations. Additional details of the source review are presented in Supplementary Information, Appendix B.1. 


\subsubsection{Trends in ambient PAH}

$\Sigma_{29} \mathrm{PAH}$ was found to be most variable at Hamilton over the model time period (i.e., coefficient of variation, $\mathrm{CV}=1.1)$, next-highest at background $(\mathrm{CV}=0.7)$, and least variable at Toronto $(\mathrm{CV}=0.5)$, also confirming each dataset's amenability to receptor modeling (i.e., CV > 0.3 suitable for PMF; Sonoma, 2008). Inter-site differences in CV were consistent with site characteristics: Hamilton has significant local industry sources and an escarpment topography so high variability in ambient PAH was expected; the Toronto site is located in a central business district expected to be subject to consistent patterns of emission from adjacent traffic and commercial/residential land use therefore so less variability was expected; the background site necessitates source-receptor transport over larger distances and greater dependence on meteorology and so moderate variability was reasonably expected.

Ambient PAH levels were relatively consistent over the modeling period at all sites and thus amenable to receptor modeling while preserving sufficient data density. As expected, urban sites had monitored ambient levels of $\Sigma_{29} \mathrm{PAH}$ several times higher than background (Supplementary Information, Appendix B.2). Highest levels PAH levels were seen at Hamilton, a trend consistent with the station's long-term monitoring records (Dann, 1997). Comparison of background site data to an even more remote monitoring site (i.e., Pt. Petre; 43.856611N, 77.150000W) showed similar $\Sigma_{29} \mathrm{PAH}$ and BAP levels, confirming it as a suitable background site for the study area.

As the most highly urbanized site, Toronto followed expected seasonal trends over majority of the scoped time series. Median sampled $\Sigma_{\text {HEAVY }} \mathrm{PAH}$ levels (i.e., 
predominantly particle phase; Venkataraman and Friedlander, 1994) were generally higher in winter and so were typical of particle phase ambient PAH in urban centres (Sun et al, 2006); median $\Sigma_{29} \mathrm{PAH}$ levels (i.e., including lighter $\mathrm{MW}$ species found predominantly in vapour phase; Venkataraman and Friedlander, 1994) were higher in summer. This was consistent with factors characterizing the densely urbanized central business district location around the monitoring site, including: PAH volatilization from uncombusted liquid fossil fuels (e.g., fuel storage and handling) and re-volatilization from built surfaces higher in summer months due to high temperatures (Dimashki et al, 2001); addition of PAH emissions from summer road maintenance and related construction (e.g., asphalt paving; Dann, 1997); and, high levels of particle phase PAH expected during smog episodes where PM in the fine size fraction is elevated, which most frequently occur in summer weather conditions (Environment Canada, 2004). Hamilton urban site data showed less consistent seasonality for $\Sigma_{29} \mathrm{PAH}$ and $\Sigma_{\mathrm{HEAVY}} \mathrm{PAH}$ (i.e., some years higher seasonal median in winter, others in summer). This suggested influence from local sources tending to attenuate seasonal differences (i.e., industry emissions, traffic emissions) and a more variable source mix (i.e., CV indicated highest sample-to-sample variability for $\Sigma_{29} \mathrm{PAH}$ and most individual species). At the background site, median sampled concentrations of $\Sigma_{29} \mathrm{PAH}$ and $\Sigma_{\mathrm{HEAVY}} \mathrm{PAH}$ were consistently higher in winter, suggesting that ambient PAH was primarily due to transported regional source emissions rather than from appreciable local sources.

By broad species grouping, light MW species contributed between approximately $85 \%$ (Hamilton) and $90 \%$ (Toronto) of sample mass at the urban sites and $89 \%$ at background. 
This is typical of total ambient PAH data; vapour phase concentrations are much higher than particle phase concentrations and so species predominantly found apportioned to vapour phase will dominate sample mass (Venkataraman and Friedlander, 1994; Lu et al., 2008). Results are also consistent with recent PAH field sampling studies with vapour and particle phase sampled together (Anastasopoulos et al, 2012; Miller et al, 2010). The majority of the contribution to $\Sigma_{29} \mathrm{PAH}$ was concentrated among relatively few dominant species (PHEN, FLU, FLT, PYR, ACE, MFLU, ACY). The most abundant species held relatively constant trends in annual median abundance, suggesting no significant change in source mix over the monitoring period and corroborating time series suitability for receptor modeling. Broad comparison between sites showed statistically significant intersite differences in median abundance and in $\sum_{\mathrm{HEAVY}} \mathrm{PAH}$, however, suggesting inter-site differences in source mix consistent with differences in local sources known a priori (i.e., significant heavy industry at Hamilton, significant traffic at Toronto).

Descriptive statistics for the urban and background ambient PAH datasets and additional details of the various trend analyses (i.e., time series variability, annual trends, seasonal trends, species abundance) are presented in Supplementary Information, Appendix B.2.

\subsubsection{Wind pattern analysis}

Westerly (i.e., NW/W/SW) winds were predominant at all sites, year-round (i.e., wind roses in Supplementary Information, Appendix B.3). To identify potential source areas, predominant wind directions were reviewed alongside surrounding land use. At Hamilton, predominant westerly winds indicated the site most often downwind of mobile sources and moderately dense residential/commercial land use and often upwind of the 
city's industrial harbour-front (i.e., heavy and medium industry inclusive of two iron/steel manufacturing facilities). At Toronto, predominant westerly winds indicated the site most often downwind of mobile sources and dense residential/commercial land use. At background, NW winds indicated the site often downwind of a sparsely populated forested region while $\mathrm{S}$ winds indicated lightly trafficked roadways as potential sources.

Review of wind directions and speeds for days with highest and lowest sampled PAH (i.e., statistically extreme outlier days; wind rose constructed for sampling day and prior day) at Hamilton (i.e., results for other sites in Supplementary Information, Appendix B.3) confirmed that high PAH days were typically characterized by NE winds (i.e., industrial harbour-front and downtown source region rendered upwind) and low wind speeds (i.e., reduced pollutant dispersion); conversely, low PAH days were typically characterized by westerly low-speed winds. A pollutant rose for $\Sigma_{29} \mathrm{PAH}$ (Figure 3-2, $\mathrm{ng} / \mathrm{m}^{3}$ and quartiles) at Hamilton confirmed the highest ambient PAH levels (third-quartile and greater concentrations) occurred when winds were from the NE direction, aligned with the city's industrial harbour-front. This occurred in summer and winter periods, consistent with industrial source emissions being significant year-round. High PAH levels also occurred frequently for $\mathrm{N}$ and $\mathrm{E}$ winds suggesting that, in addition to upwind sources, the escarpment could increase ambient concentrations by impeding further dispersion (i.e., central site monitor is located below the escarpment; N/NW/E winds are "towards the escarpment'). Westerly winds (i.e., predominant wind direction) were more frequently coincident with low ambient PAH (i.e., first and second-quartile concentrations), 
suggesting less local PAH sources in that direction and generally less contribution from sources above the escarpment.

\subsubsection{Source Apportionment}

\subsubsection{Factor analysis by PCA}

Detailed PCA results for the three sites are presented and discussed in Supplementary Information, Appendix B.4. Four factors explained the most data variance at Hamilton, least at the background site (i.e., lowest data quality and greatest source-receptor distances). Factor profile patterns were strongly similar between sites, consistent with major PAH source types tending to influence all sites as local or proximal transported sources (e.g., vehicle traffic exhaust emissions, residential heating) and corroborated by similar findings by previous PCA studies of multiple sites (Mari et al, 2004). Accordingly, PMF modeling was expected to find PAH source types influencing the sites differing more by degree (i.e., relative contribution) than by kind (i.e., source identification). At Hamilton, however, distinctions between PCA factor loadings were less sharp than at Toronto for several abundant species (FLU, MFLU, FLT, PYR) and so the more advanced PMF model was reasonably expected to resolve a local source type unique to this highly industrialized urban center. PCA results on seasonally stratified variants of the datasets showed little seasonal sensitivity, corroborating modeling the NAPS central site data in PMF without seasonal stratification, particularly since restricting modeling to a winter-only time series would severely reduce modeling power and impede corroborating factor interpretations by seasonality in contribution. 


\subsubsection{PMF receptor modeling}

The decision process for selecting a PMF model order for interpretation was complex and balanced good model performance with need for physically meaningful factors interpretable as source types. (Reff et al, 2007) On the balance of these analyses, a fivefactor PMF solution proved optimal at the urban sites and a four-factor solution proved optimal at background.

The PMF receptor model predicted the original sampled ambient PAH well at all sites (Figure 3-3-a), explaining the original ambient dataset best at Hamilton $\left(r^{2}=0.995\right)$, nextbest at Toronto $\left(r^{2}=0.985\right)$, and slightly less well at the background site $\left(r^{2}=0.952\right)$. Model results were also regressed (Figure 3-3-b) against the total of all 29 NAPSsampled species $\left(\Sigma_{29} \mathrm{PAH}\right)$ to confirm the larger species suite was adequately being predicted by the subset of fitting species (i.e., 21 species at the urban sites, 20 species at background site) and good correlation was confirmed at all sites (i.e., Hamilton, $\mathrm{r}^{2}=$ 0.989; Toronto, $\mathrm{r}^{2}=0.976$; background, $\left.\mathrm{r}^{2}=0.956\right)$. As an additional confirmation of overall PMF model performance, a plot of PMF-predicted versus sampled average species concentration (Figure 3-3-c) showed excellent overall correlation at all sites (i.e., Hamilton, $\mathrm{r}^{2}=0.998$; Toronto, $\mathrm{r}^{2}=0.999$; background, $\mathrm{r}^{2}=0.997$ ).

The PMF model solutions met PMF best practice requirements (i.e., stable, good fit, robust, rotationally unique) and, most important, resolved distinct factor profiles that could be reasonably interpreted as PAH source types. Model performance metrics are summarized and discussed further in Supplementary Information, Appendix B.5. In the interests of methodological rigor, several post-modeling checks verified consistency of 
the interpreted results, including: sensitivity to time series, model stability and robustness, sensitivity to F-peak rotation and exclusion of fitting species. Several new sensitivity analyses were also conducted to reduce subjectivity in the PMF modeling process (Reff et al, 2007) and advance modeling practice for practitioners. These were sensitivity of model performance and interpreted factors to input uncertainty, outlier exclusion, and base run choice. Model checks and sensitivity analyses are discussed in detail in Supplementary Information, Appendix B.6.

The set of factors resolved by PMF at each of the modeled sites are shown in Figure 3-4 (i.e., bootstrap results: concentration profile, F_C; explained variance profile, F_EV). Factor profile patterns were labeled according to salient species in the F_EV profile. Variants of five basic profile patterns were resolved across the sites: "light", "heavy", "volatile", "trimodal", and "retene". Factors could be grouped into broadly similar profile patterns (Figure 3-5), anticipated by the PCA (Supplementary Information, Appendix B.4; see also Mari et al, 2004) and the general similarity in ambient PAH profile for many Canadian urban centers (Sanderson et al, 2004). Factor profile patterns were stable with model order, generally remaining consistent at a model order one lower or one higher than retained for interpretation.

\subsubsection{Source types influencing urban and background sites}

Source types interpreted from the five factor profile variants were: PAH from volatilization of fossil fuels and re-volatilization from built surfaces plus coal combustion emissions via long range transport of air pollutants (i.e., abbreviated for convenience to "PAH volatilization and LRTAP coal combustion"; "light" factor profile); vehicle traffic 
exhaust ("heavy"); space heating ("volatile"); biomass combustion ("retene"); and, at Hamilton, local industry (iron/steel manufacturing; "trimodal"). Interpretation of model factors as physically meaningful source types was based on a preponderance of contextual evidence (e.g., factor enrichment in known source marker or sourcecharacteristic species, evaluation of seasonal or other temporal trends in factor contributions; Reff et al, 2007; Brook et al, 2004), with inter-site comparison corroborating similar source types or confirming site-unique local source types. For transparency (Reff et al, 2007; Watson et al, 2008; Ioannidis et al, 2005; Moonesinghe et al, 2007) and reducing subjectivity in PMF modeling practice, interpretive evidence is presented in entirety in Supplementary Information, Appendix B.7.

\subsubsection{Source contributions to PAH and PAH toxicity}

\subsubsection{Source type contributions to ambient PAH}

Inter-urban, urban-background differences in source contributions

Source type contributions to ambient PAH (i.e., sum of modeled PAH aggregated over all samples $^{5}$ ) are presented in Table 3-4 (i.e., see also pie charts in Figure 3-6). Combining traffic factors at Toronto and space heating and wood combustion factors at all sites (Figure 3-7), source type ranking was generally similar between urban sites; differences were significant contributions from local heavy industry at Hamilton and the greater traffic contribution at Toronto. At the Toronto site, contributions from urban mode mixed

\footnotetext{
${ }^{5}$ Total aggregate contributions to $\Sigma_{21} \mathrm{PAH}$ at urban sites and $\Sigma_{20} \mathrm{PAH}$ at background site.
} 
traffic were greater than they were for free-flowing highway mode mixed traffic (Figure 3-6-b), consistent with high emissions associated with congested urban conditions and high PM emissions associated with commercial heavy duty vehicles, particularly when operating in the urban traffic mode.

Relative source type contributions to ambient PAH differed most at the background site, expected partially due to model differences (i.e., lower model order, higher uncertainty in data). However, urban-rural differences remained reasonable given differences in population, land use, and local source activity: volatilized PAH + transported coal combustion lower than at urban sites (i.e., less volatilization component due to few local fuel storage facilities and less paved surfaces); space heating high relative to other background source types (i.e., oil combustion more common in rural residential areas due to limited natural gas network and due to use in agricultural activity); biomass combustion contributions higher than at urban sites (i.e., expected for rural residential and agriculture land use; open biomass burning is uncontrolled combustion and releases a disproportionate amount of PAH; Ontario - Environment Canada, 2009; Canada + NE USA - Galarneau, 2008); vehicle traffic exhaust least relative to other source types yet remain significant (i.e., nearby highways, agricultural vehicles/equipment).

\section{Contributions by source type}

PAH volatilization and transported coal combustion ("light" factor) contributed the majority of modeled ambient PAH emissions at both urban sites and showed a very similar contribution $(\sim 40 \%)$ as expected for a source type representing a local source present at both sites (volatilized PAH) and a regional component (LRTAP) affecting both 
sites similarly (i.e., accentuated by relatively close site proximity). This source type also contributed the majority of ambient PAH at the rural site $(\sim 30 \%)$ indicating that volatilized PAH and transported coal combustion emissions together dominated the background PAH signal. This significant combined contribution was expected. Background ambient $\mathrm{PM}_{2.5}$ and associated particle-phase $\mathrm{PAH}$ at Ontario sites have been consistently attributed to long range transport, with the heavily industrialized (i.e., concentrated coal-fired power generation facilities) American Midwest and Ohio Valley Region, being frequent source regions (Ontario Ministry of Environment, 2013; Anastassopoulos et al, 2004). Additional relevant (i.e., frequently upwind or proximal) Ontario coal-fired power plants operating during 2000-2010 were Nanticoke Generating Station near Simcoe, Lambton Generating Station south of Sarnia, and Lakeview Generating Station in Mississauga ${ }^{6}$. Regional coal combustion (i.e., not including proximal industry emissions) was found by receptor modeling to account for nearly half the ambient PAH in Chicago (Simcik et al, 1999). Regional inventories have shown coal combustion emissions to dominate industry releases (Ontario - Environment Canada, 2009; Canada + NE USA - Galarneau, 2008). The significant added component of PAH volatilization, contributing more in 'summer' season due to higher temperatures promoting greater volatilization and contributing more at the urban sites, is also consistent with facilities identified prior to receptor modeling, including oil refining and other industry facilities within the study area (Supplementary Information, Appendix B.1)

\footnotetext{
${ }^{6}$ Minor influence expected since Lakeview Generation Station was operated only as needed to supplement peak demand and closed in spring 2005.
} 
and a large number of fueling stations and paved surfaces associated with urban infrastructure.

Traffic exhaust ("heavy" factor variants) contributions were found at all sites and were lowest at the background site (11\%) and highest at the large urban site of Toronto (20\%). This was consistent with emissions inventories: while regional and provincial scale emissions inventories show on-road traffic to contribute a relatively small portion $(\sim 5 \%)$ of Canadian (Galarneau, 2008) and Ontario (Environment Canada, 2009) heavy MW $\mathrm{PAH}$ emissions, the percentage of $\mathrm{PAH}$ emissions apportioned to traffic is typically significantly higher at the local scale, particularly for urban centres (Ontario Ministry of Environment, 2013; Ravindra, 2008).

Local industry emissions ("trimodal" factor) contributed a significant portion (16\%) of modeled ambient PAH emissions at Hamilton, as expected for industrial operations from the city's two harbour-front iron and steel industry facilities. The majority of emissions are releases from coke ovens rather than iron sintering stack releases and thus influence the city, particularly below the escarpment. Coal-related PAH emissions were greatest at Hamilton as expected due to the combined impact of regional (i.e., $440 \%$ regional contribution of PAH volatilization and LRTAP source, similar to Toronto) plus local emissions (i.e., 16\% for local industry source). Contributions were also consistent with receptor modeling results for Chicago, a North American city with comparable iron/steel industry facilities (Simcik et al, 1999). 
Space heating ("volatile" variants) and biomass combustion ("retene" variants) together ${ }^{7}$ contributed significantly to ambient PAH at all sites as expected from regional emissions inventory estimates (Environment Canada, 2009; Galarneau, 2008). Stronger seasonality at Toronto suggests "volatile+retene" factor was largely representing space heating with a higher contribution compared to Hamilton consistent with the city's much greater population density. Provincial and larger regional inventories (Environment Canada, 2009; Galarneau, 2008) show biomass combustion emissions as the largest single source type by total annual release. However, at the modeled urban sites local biomass combustion was expected to have a tertiary contribution due to the episodic nature of burning and wood typically being only a supplementary heating fuel, particularly relative to near-continuous releases from traffic and proximal industry sources (Ontario Ministry of Environment, 2013; Ravindra, 2008). Primary heating fuel was reported as natural gas/electric/oil/propane in more than $95 \%$ of Ontario homes; approximately $8 \%$ of Ontario homes reported using wood burning for supplementary heating/aesthetics (Natural Resources Canada, 2007; Statistics Canada, 2011), Recent receptor modeling studies which sampled both particle and vapour phase PAH have found the contribution from factors identified as space heating to be significant and modeling studies have found a wide range of contributions for factors identified as biomass combustion. While the apportionment of biomass combustion is reasonable, it inherently represented greater

\footnotetext{
${ }^{7}$ Although space heating and biomass combustion source types are not necessarily coincident, overlap noted in Toronto model results (i.e., wood combustion marker species retene co-extracted with oil combustion marker species) made it conservative to discuss the source types together when comparing against emissions inventories or other receptor model studies.
} 
uncertainty than other source type contribution estimates due to the high uncertainty assigned to RET. Recent studies have also showing RET to be an imperfect marker for biomass combustion (Shen et al, 2012).

Sample-to-sample variability in contributions

Relative source type contributions to modeled PAH were calculated for each sample and statistically summarized (median, IQR) to quantify sample-to-sample variability (Figure 3-8; also see Supplementary Information, Appendix B.8). Median contributions were consistent with total aggregate contributions at all sites (i.e., within 5\%-10\% for all source types). Overall, median source apportionment results can be considered more statistically meaningful estimates of 'typical' or 'frequent' source contributions and are recommended as a supplement to total aggregate contribution data typically reported by receptor models or determined via emission inventories. The IQR in relative source type contributions quantified the range in source contributions that can be expected at each receptor due to combined variance in source releases and meteorological conditions and related factors influencing source-receptor transport. With this understanding, the IQR can be considered a conservative estimate of uncertainty on the source apportionment results and facilitate reconciling with other source apportionment results (Watson et al, 2008; Reff et al, 2007).

Additional salient observations on sample-to-sample variability in contribution are:

- Hamilton local industry contributions varied more than other source types due to the variability in industry coke oven releases, the upwind location of heavy 
industry from sampling the sampling site, and the effects of the escarpment topography);

- Toronto urban mode vehicle traffic exhaust contributions varied more than freeflow/highway mode vehicle traffic exhaust;

- At the background (Egbert) station there was generally low variability in factor contributions with the exception of vehicle traffic exhaust due to the sporadic traffic patterns on nearby highways and limited local rural roads.

\subsubsection{Source type contributions to PAH toxicity}

\section{Contribution by source type}

Total aggregate contributions (i.e., sum of modeled PAH aggregated over all samples ${ }^{8}$ ) to ambient PAH toxicity (BaP-TEQ) are summarized in Table 3-4 and compared across sites in Figure 3-9 which combines the traffic factors at Toronto, and the space heating and wood combustion factors at all sites). (Sample-to-sample variability in contributions to PAH toxicity in Supplementary Information, Appendix B.8.)

Vehicle traffic exhaust dominated contributions to ambient PAH-associated toxicity at all sites. Toronto showed highest total traffic contributions to PAH toxicity (68\%), consistent with it being the most heavily populated site with highest traffic contributions to ambient PAH. In comparison to traffic exhaust contributions to PAH toxicity, all other

\footnotetext{
${ }^{8}$ Total aggregate contributions to $\Sigma_{21} \mathrm{PAH}$ at urban sites and $\Sigma_{20} \mathrm{PAH}$ at background site.
} 
source types were secondary in importance and showed greater inter-site differences. At Hamilton, after traffic exhaust, contributions to PAH toxicity were significant for coal related emissions (i.e., 14\% from local industry in "trimodal" factor and additional contributions from transported coal combustion component in "light"). Since space heating and biomass combustion had partial overlap in source fuel (i.e., wood) and in source activity (i.e., typically higher in winter) their total contributions to PAH toxicity were combined and found to be substantial at Toronto (24\%) and background (26\%).

\section{Source types with disproportionate contributions to PAH toxicity}

Review of source contributions to $\mathrm{PAH}$ alongside corresponding toxicity revealed a principal research finding: source types contributing relatively little mass to total ambient PAH could contribute significantly to toxicity of ambient PAH; these source types can be considered comparatively 'more toxic' due to their disproportionate contribution to PAH toxicity and thus to chronic health risk (Grahame and Schlesinger, 2007). In this research, the source type contributing most disproportionately to PAH toxicity was vehicle traffic exhaust emissions, at all sites, clearly seen in Figure 3-10. At Hamilton, local industry emissions may also be cited for significant contribution to PAH toxicity (14\%) relative to contribution to PAH (16\%).

This key research finding is consistent with health effects studies examining source type contributions to chronic health risk and citing traffic and local industry as significant contributors to exposure health risk (Schwartz et al, 2005; Gold et al, 2005). Results are also consistent with a comprehensive intraurban sampling study carried out at Hamilton, which found that sites near concentrated industry and traffic sources tended to be 
enriched in heavier and more toxic PAH species, and thus had disproportionately high sampled PAH toxicity relative to sampled ambient PAH (Anastasopoulos et al, 2012; Chapter 4). A related intraurban scale source apportionment study completed for the city of Hamilton similarly found traffic emissions to be the predominant source of PAH exposure toxicity throughout the urban area despite contributing little to $\Sigma \mathrm{PAH}$, and local industry emissions to contribute significantly to exposure toxicity near the city's industrial zone (Chapter 5).

\section{Implications}

Where previous studies have inferred or indirectly apportioned PAH toxicity to source type, this research has explicitly quantified the degree to which source types can contribute to PAH-associated chronic health risk due to inhalation exposure ${ }^{9}$.

Several implications follow from the source apportionment findings. First, since vehicle traffic exhaust emissions were found to dominate contributions to PAH toxicity at both urban sites and even at the rural background site, this source type can be considered "for the purposes of health risk assessment 'more toxic' than others." (Grahame and Schlesinger, 2007) At Hamilton, local industry emissions can also be considered 'more toxic' since they contribute significantly to PAH toxicity relative to contribution to PAH; this latter finding confirmed that sources of concern may differ even between nearby urban sites, reflecting the importance of local source contributions and other

${ }^{9}$ Individual personal health risk will depend on individual exposure and possible synergistic effects between pollutants and other risk factors. 
characteristics affecting source-receptor transport. This underscored the importance of a thorough review of environmental context prior to receptor modeling, as exampled in this paper. It also emphasized the importance of selecting a scale of study appropriate to local exposed populations, such as by urban center (this paper), or at the intra-urban scale (Chapter 4, Chapter 5).

Next, care should be taken to report and analyse emissions inventories not only on the more common basis of $\Sigma \mathrm{PAH}$ but also on the basis of BaP-TEQ, the latter being more meaningful where source inventories mean to inform and guide emissions regulations and health policy. This is the case even where a source inventory is limited to particle phase PAH data since species toxicities vary widely (Table 3-3), even between heavy MW species where species mass is essentially entirely in particle phase.

Last, interventions intended to reduce PAH source emissions (e.g., emissions control regulation) would best consider contribution to PAH toxicity so that sources contributing disproportionately to toxicity are identified as source types yielding best reductions in health effects rather than in ambient PAH levels. It is understood that this requires educating stakeholders designing regulatory tools for air toxics to consider both amount and toxicity of source emissions rather than only amount, despite the latter having worked well in past for single compound criteria pollutants. Analogously, interventions intended to reduce PAH exposures (e.g., central site monitoring and public health messaging) would benefit from using language stressing toxicity and focusing awareness on 'more toxic' source types such as vehicle traffic. More nuanced health messaging tools, such as Canada's health-based AQI (AQHI; Stieb et al, 2008), are a step in the right direction and 
consideration should be given to analogous aggregate indicators for chronic health risk, incorporating PM-associated PAH and other key air toxics.

\subsection{Conclusions}

\section{Implications}

This research has provided a comprehensive analysis of ambient PAH and contributing sources based on long time series data at two distinctly differing urban sites and a rural background site. The identification of dominant source types contributing to ambient PAH concentrations and PAH toxicity can assist epidemiologists and other health researchers further understand inter-urban and urban-rural differences in exposure toxicity and help regulators target interventions to reduce health effects of PAH exposure.

\section{Recommendations and possible improvements in methodology}

Three main limitations are acknowledged. First, central monitoring site samplers are subject to risk of sample loss for highly volatile PAH species (i.e., acenaphthylene, acenaphthene) due to breakthrough from PUF at high flow rate and high temperatures (US EPA, 1999). Sample degradation due to potential reactions of other species with ambient oxidants such as ozone, hydroxyl radical, or nitrogen oxides is another potential loss mechanism (e.g., BAP; Jung et al, 2010). Further, nitrated/oxygenated PAH species were not analysed in this study. Thus, sampled PAH concentrations likely represent lower values. Second, dibenz(ah)anthracene (DBA) was excluded from analysis due to a large number of missing and below $\mathrm{DL}_{\mathrm{j}}$ samples at the background site but had sufficient data quality to permit inclusion in the Hamilton receptor model. There is incentive for including DBA in future receptor modeling efforts since DBA is more toxic than BAP 
(i.e., typically used as indexing species for equivalent toxicity). Meanwhile, it is reasonably expected from review of other receptor modeling studies and source sampling studies that DBA would apportion to factors interpreted as traffic exhaust emissions (Park et al, 2011) and local heavy industry emissions (Fang, 2006; Yang, 1998) further increasing the health risk contribution of these source types. Third, while BaP-TEQ as presented indicates potential chronic health risk, individual health risk will depend on individual exposure and possible synergistic effects between pollutants and other risk factors. Additionally, given there are several TEF datasets available in the PAH toxicology literature, choice of individual TEF values will increase or decrease estimations of PAH toxicity.

\section{Inter-site trends in ambient $P A H$}

Urban sites had monitored ambient levels of $\Sigma_{29} \mathrm{PAH}$ several times higher than background with highest levels at Hamilton, site with significant local industry (iron/steel manufacturing). Seasonality in ambient PAH differed by site: at Hamilton, there was no consistent seasonality due to year-round operations of local industry; at Toronto, median $\Sigma_{29} \mathrm{PAH}$ was higher in summer, median $\Sigma_{\mathrm{HEAVY}} \mathrm{PAH}$ higher in winter; at Egbert (background), median $\Sigma_{29} \mathrm{PAH}$ and $\Sigma_{\mathrm{HEAVY}} \mathrm{PAH}$ were higher in the winter. At all sites, the majority of PAH sample mass $(85 \%$ - 90\%) was light MW species. Contributions of individual species and of heavy MW species differed between sites, indicating interurban differences in relative source mix. Wind rose analysis confirmed high and low PAH concentrations were consistent with locations of local source regions. Pollutant rose 
analysis at Hamilton confirmed highest ambient PAH occurred when winds aligned with the industrial harbour-front and blew towards the escarpment.

\section{Receptor modeling protocol}

Rigorous receptor modeling of long time series central site PAH data by PMF resolved distinct and interpretable factor profiles and met all 'best practice' performance criteria, yielding a stable, well-fit, robust and rotationally unique solution. Model checks and sensitivity analyses confirmed that the PMF model results retained for source type interpretation and reporting were resolved consistently, including sensitivity to input uncertainty, outlier exclusion, seasonal stratification, and base run choice. Sensitivity analyses reduced procedural subjectivity and generally facilitate replication of results.

\section{Uncertainty on factor profiles and variability in source contributions}

Presenting factor profiles with appropriate uncertainty estimates facilitated comparison between profiles and sites and encourages comparison with profile results obtained by other receptor modeling studies or via source sampling. Rather than a single source apportionment estimate for each source type (i.e., total aggregate over modeled time series), sample-to-sample variability in contributions was also estimated by median and IQR statistics, facilitating comparison with other source apportionment results.

\section{Source types contributing to ambient PAH}

Major source types contributing to ambient PAH at the modeled sites were: $P A H$ from volatilization (e.g., fossil fuel, pavement, built surfaces) plus coal combustion emissions (power generation) via long range transport of air pollutants (LRTAP), vehicle traffic exhaust emissions, space heating (oil, wood), biomass combustion and, at Hamilton, 
local industry emissions from iron/steel manufacturing. PAH volatilization and transported coal combustion contributed the majority of modeled ambient PAH emissions at all sites. Traffic exhaust contributions were found at all sites and were lowest at the background site and highest at Toronto, the urban site with highest population, densest urban development and heaviest traffic patterns. Coal-related PAH emissions were greatest at Hamilton due to significant local emissions from iron/steel manufacturing and regional transported emissions. Space heating and biomass combustion together also contributed significantly to ambient PAH at all sites.

\section{Source type contributions to ambient PAH toxicity}

Vehicle traffic exhaust emissions were found to dominate contributions to PAH toxicity at all sites. Traffic exhaust emissions and local industry emissions at Hamilton were comparatively 'more toxic' source types due to significant contributions to total ambient PAH toxicity (BaP-TEQ), despite relatively low contributions to total ambient PAH, findings in agreement with comprehensive chronic health effects studies. Source apportionment to aggregate $\mathrm{PAH}$ toxicity is a more meaningful basis for quantifying relative source contributions, whether by receptor modeling, as in this study, or by emissions inventories. Source apportionment to PAH toxicity is also valuable for design of interventions intended to reduce health effects of ambient PAH exposures, and can usefully inform source regulation policy and public health messaging. 
Table 3-1. NAPS central site sampled PAH (modeled species), chemical formula, molecular weight grouping, and species method detection limits and error fractions.

\begin{tabular}{|c|c|c|c|c|c|c|c|}
\hline PAH Species & Label & $\begin{array}{l}\text { Chem. } \\
\text { Formula }\end{array}$ & $\begin{array}{r}\# \\
\text { rings }\end{array}$ & $\begin{array}{r}\text { Mol. } \\
\text { Wt. } \\
(\mathrm{MW})\end{array}$ & $\begin{array}{l}\text { MW } \\
\text { group }\end{array}$ & $\begin{array}{r}\text { Method } \\
\text { Detection } \\
\text { Limit } \\
(\mathrm{MDL}) \\
{\left[\mathrm{ng} / \mathrm{m}^{3}\right]}\end{array}$ & $\begin{array}{r}\text { error } \\
\text { fraction } \\
\left(\mathbf{k}_{\mathbf{j}}\right)\end{array}$ \\
\hline acenaphthylene & $A C Y$ & $\mathrm{C}_{12} \mathrm{H}_{8}$ & 3 & 152.2 & light & 0.001 & 48.7 \\
\hline acenaphthene & ACE & $\mathrm{C}_{12} \mathrm{H}_{10}$ & 3 & 154.2 & light & 0.002 & 19.8 \\
\hline fluorene & FLU & $\mathrm{C}_{13} \mathrm{H}_{10}$ & 3 & 166.2 & light & 0.003 & 25.7 \\
\hline anthracene & ANT & $\mathrm{C}_{14} \mathrm{H}_{10}$ & 3 & 178.2 & light & 0.002 & 27.1 \\
\hline phenanthrene & PHE & $\mathrm{C}_{14} \mathrm{H}_{10}$ & 3 & 178.2 & light & 0.004 & 10.9 \\
\hline 2-methyl-fluorene & MFLU & $\mathrm{C}_{14} \mathrm{H}_{12}$ & 3 & 180.3 & light & 0.004 & 27.6 \\
\hline fluoranthene & FLT & $\mathrm{C}_{16} \mathrm{H}_{10}$ & 4 & 202.3 & light & 0.002 & 9.0 \\
\hline pyrene & PYR & $\mathrm{C}_{16} \mathrm{H}_{10}$ & 4 & 202.3 & light & 0.001 & 9.2 \\
\hline 1-methyl-pyrene & MPYR & $\mathrm{C}_{17} \mathrm{H}_{12}$ & 4 & 216.2 & med & 0.001 & 15.2 \\
\hline benzo(a)fluorene & BaFLU & $\mathrm{C}_{17} \mathrm{H}_{12}$ & 4 & 216.2 & med & 0.002 & 17.1 \\
\hline benzo(b)fluorene & BbFLU & $\mathrm{C}_{17} \mathrm{H}_{12}$ & 4 & 216.2 & med & 0.002 & 24.0 \\
\hline benzo(g,h,i)fluoranthene & BghiFLT & $\mathrm{C}_{18} \mathrm{H}_{10}$ & 5 & 226.3 & med & 0.002 & 14.1 \\
\hline chrysene & $\mathrm{CHR}$ & $\mathrm{C}_{18} \mathrm{H}_{12}$ & 4 & 228.3 & heavy & 0.001 & 10.5 \\
\hline triphenylene & TRIP & $\mathrm{C}_{18} \mathrm{H}_{12}$ & 4 & 228.3 & heavy & 0.002 & 13.3 \\
\hline benzo(a)anthracene & BAA & $\mathrm{C}_{18} \mathrm{H}_{12}$ & 4 & 228.3 & heavy & 0.004 & 11.7 \\
\hline retene & RET & $\mathrm{C}_{18} \mathrm{H}_{18}$ & 3 & 234.3 & heavy & 0.005 & 36.7 \\
\hline benzo(a)pyrene & BAP & $\mathrm{C}_{20} \mathrm{H}_{12}$ & 5 & 252.3 & heavy & 0.003 & 12.5 \\
\hline benzo(e)pyrene & BEP & $\mathrm{C}_{20} \mathrm{H}_{12}$ & 5 & 252.3 & heavy & 0.002 & 10.4 \\
\hline benzo(b)fluoranthene & $\mathrm{BbFLT}$ & $\mathrm{C}_{20} \mathrm{H}_{12}$ & 5 & 252.3 & heavy & 0.003 & 11.3 \\
\hline benzo(k)fluoranthene & BkFLT & $\mathrm{C}_{20} \mathrm{H}_{12}$ & 5 & 252.3 & heavy & 0.003 & 15.2 \\
\hline benzo(g,h,i)perylene & BghiP & $\mathrm{C}_{22} \mathrm{H}_{12}$ & 6 & 276.3 & heavy & 0.004 & 14.4 \\
\hline indeno(1,2,3-cd)pyrene & IPYR & $\mathrm{C}_{22} \mathrm{H}_{12}$ & 6 & 276.3 & heavy & 0.003 & 16.5 \\
\hline
\end{tabular}

Notes:

1. Data from ATSDR, 1995 and Mackay, 2006.

2. Retene also known as 1-methyl-7-isopropylphenanthrene.

3. Molecular weight grouping adapted from Li et al, 2005. 
Table 3-2. Treatment protocols for speciated ambient receptor data used in PMF.

\begin{tabular}{|l|l|l|}
\hline $\begin{array}{l}\text { Lab-reported sample } \\
\text { concentration }\left(c_{i j}\right)\end{array}$ & $\begin{array}{l}\text { Treated sample } \\
\text { concentration }\left(x_{i j}\right)\end{array}$ & Treated sampled uncertainty $\left(\mathrm{s}_{\mathrm{ij}}\right)$ \\
\hline $\mathrm{C}_{\mathrm{ij}}>\mathrm{DL}_{\mathrm{j}}$ & $\mathrm{x}_{\mathrm{ij}}=\mathrm{c}_{\mathrm{ij}}$ & $\mathrm{s}_{\mathrm{ij}}=\left[\left(\left(\mathrm{k}_{\mathrm{j}}\right)\left(\mathrm{x}_{\mathrm{ij}}\right)\right)^{2}+\left(D \mathrm{D}_{\mathrm{j}}\right)^{2}\right]^{(1 / 2)}$ \\
\hline $\mathrm{C}_{\mathrm{ij}}<\mathrm{DL}_{\mathrm{j}}$ & $\mathrm{x}_{\mathrm{ij}}=(1 / 2) \mathrm{DL}$ & $\mathrm{s}_{\mathrm{ij}}=(5 / 6) \mathrm{DL}$ \\
\hline $\begin{array}{l}\text { missing or invalid data } \\
\mathrm{C}_{\mathrm{ij}}=-999\end{array}$ & $\mathrm{x}_{\mathrm{ij}}=$ median $\left(\mathrm{x}_{\mathrm{ij}}\right)$ & $\mathrm{s}_{\mathrm{ij}}=(4)$ median $\left(\mathrm{x}_{\mathrm{ij}}\right)$ \\
\hline
\end{tabular}

Notes:

1. Method Detection Limit (MDL) data taken from a 2005 QAQC dataset of NAPS data made available by Environment Canada; calculated as: $\mathrm{DL}_{\mathrm{j}}=\left(\mathrm{t}\right.$-statistic) ${ }^{*} \mathrm{SD}\left(\mathrm{x}_{\mathrm{ij}}\right) \quad$ where $\mathrm{SD}_{\mathrm{j}}=$ calculated as standard deviation of 7 replicate samples analysed for respective PAH species (due to outlier species sample concentrations, 5 samples used for ACE, FLU, PHEN; 6 samples used for FLT, PYR); t-value = Student-t test statistic at 95\% confidence interval, for 5-7 samples (4-6 degrees of freedom), dependent on species.

2. Error fraction $\left(k_{j}\right)$ error fraction data from Method 3.03/5.1, Appendix B - "Uncertainty estimates by species, not recovery corrected" (Environment Canada, 2011f). Error fraction data are not recovery corrected, consistent with NAPS and field sampling data. Species error fractions account for major sources of uncertainty in PAH sample preparation and measurement and are determined via: $\mathrm{k}_{\mathrm{j}}=\mathrm{k}{ }^{*} \mathrm{RSD}$ where RSD = relative standard deviation = $\mathrm{SD} /$ mean of 30 replicate samples; $\mathrm{k}=\mathrm{t}$-value for $95 \%$ confidence interval on 30 replicate samples $=2.0$ 
Table 3-3. Benzo(a)Pyrene-equivalent carcinogenic Toxicity Equivalency Factors (BaP-TEF) for

inhalation exposure, modeled species.

\begin{tabular}{llr}
\hline Species ID & Species name & BaP-TEF \\
\hline 01_ACY & acenaphthylene & 0.001 \\
02_ACE & acenapthene & 0.001 \\
03_FLU & fluorene & 0.001 \\
04_ANT & anthracene & 0.01 \\
05_PHE & phenanthrene & 0.001 \\
06_MFLU & 2-me-fluorene & $0.001^{\mathrm{a}}$ \\
07_FLT & fluoranthene & 0.001 \\
08_PYR & pyrene & 0.001 \\
09_MPYR & 1-me-pyrene & $0.001^{\mathrm{a}}$ \\
10_BaFLU & benzo[a]fluorene & $0.001^{\mathrm{b}}$ \\
11_BbFLU & benzo[b]fluorene & $0.001^{\mathrm{b}}$ \\
12_BghiFLT & benzo[g,h,i]fluoranthene & $0.001^{\mathrm{c}}$ \\
13_CHRY & chrysene & 0.01 \\
14_TRIPH & triphenylene & $0.01^{\mathrm{d}}$ \\
15_BaA & benzo[a]anthracene & 0.1 \\
16_RET & retene (1-methyl-7- & $0.001^{\mathrm{e}}$ \\
18_BaP & isopropylphenanthrene) & 1 \\
19_BeP & benzo[a]pyrene & $0.01^{\dagger}$ \\
20_BbFLT & benzo[e]pyrene & 0.1 \\
21_BkFLT & benzo[b]fluoranthene & 0.1 \\
25_BghiPE & benzo[k]fluoranthene & 0.01 \\
26_IPYR & benzo[g,h,i]perylene & 0.1 \\
\hline & indeno[1,2,3-cd]pyrene &
\end{tabular}

Note: Data from Nisbet and Lagoy, 1992 and ATSDR, 1995 except where noted:

(a) Assigned same TEF as parent compound.

(b) Assigned same TEF as FLU (i.e., toxicity classified equivalent to FLU in report to Health Canada, PAH PEF Toxicity Update, 2006).

(c) Assigned TEF value as per Nielsen et al, 1996 (i.e., grouped with structurally similar B[c]PHEN) and recommendation to Health Canada, 2006 (i.e., recommends TEF value for $\mathrm{B}[\mathrm{c}] \mathrm{PHEN}$ that is one order of magnitude lower than TEF for CHRY; Equilibrium Environmental, 2006).

(d) Assigned same TEF as CHRY (i.e., Nielsen et al, 1996 show single value for CHRY+TRIP).

(e) No toxicity data available since retene less commonly sampled; conservative assumption is that compound has degree of toxicity since an alkylated variant of phenanthrene therefore assigned same TEF as phenanthrene (i.e., lowest toxicity value in modeled species suite; also see Scott, 2009).

(f) Assigned TEF as per Malcolm and Dobson, 1994 and Nielsen et al, 1996. 
Table 3-4. Total aggregate source contributions to ambient PAH and toxicity (BaP-TEQ).

\begin{tabular}{|c|c|c|c|}
\hline \multirow[b]{2}{*}{ SOURCE TYPE } & \multicolumn{3}{|c|}{$\begin{array}{c}\text { SOURCE APPORTIONMENT OF PAH and TOXICITY (BaP-TEQ) } \\
\text { approximate } \% \mathrm{G} \text { to } \\
\Sigma_{\text {modeled }} \mathrm{PAH}\left(\Sigma_{\text {modeled }} \mathrm{BaP} \text {-eq }\right)\end{array}$} \\
\hline & $\begin{array}{l}\text { Urban - } \\
\text { Hamilton }\end{array}$ & $\begin{array}{l}\text { Urban - } \\
\text { Toronto }\end{array}$ & background \\
\hline volatized PAH + LRTAP (coal) & $40 \%(14 \%)$ & $39 \%(8 \%)$ & $29 \%(11 \%)$ \\
\hline local industry (iron/steel) & $16 \%(14 \%)$ & -- & -- \\
\hline traffic exhaust & $13 \%(59 \%)$ & $20 \%(68 \%)$ & $11 \%(62 \%)$ \\
\hline space heating (oil + wood) & $16 \%(8 \%)$ & $33 \%(9 \%)$ & $35 \%(16 \%)$ \\
\hline biomass combustion & $15 \%(6 \%)$ & $8 \%(15 \%)$ & $25 \%(10 \%)$ \\
\hline
\end{tabular}

Note: Source type contribution data is percent of all modeled PAH mass (2001-2010 for urban sites, 2005-2010 for background site). 


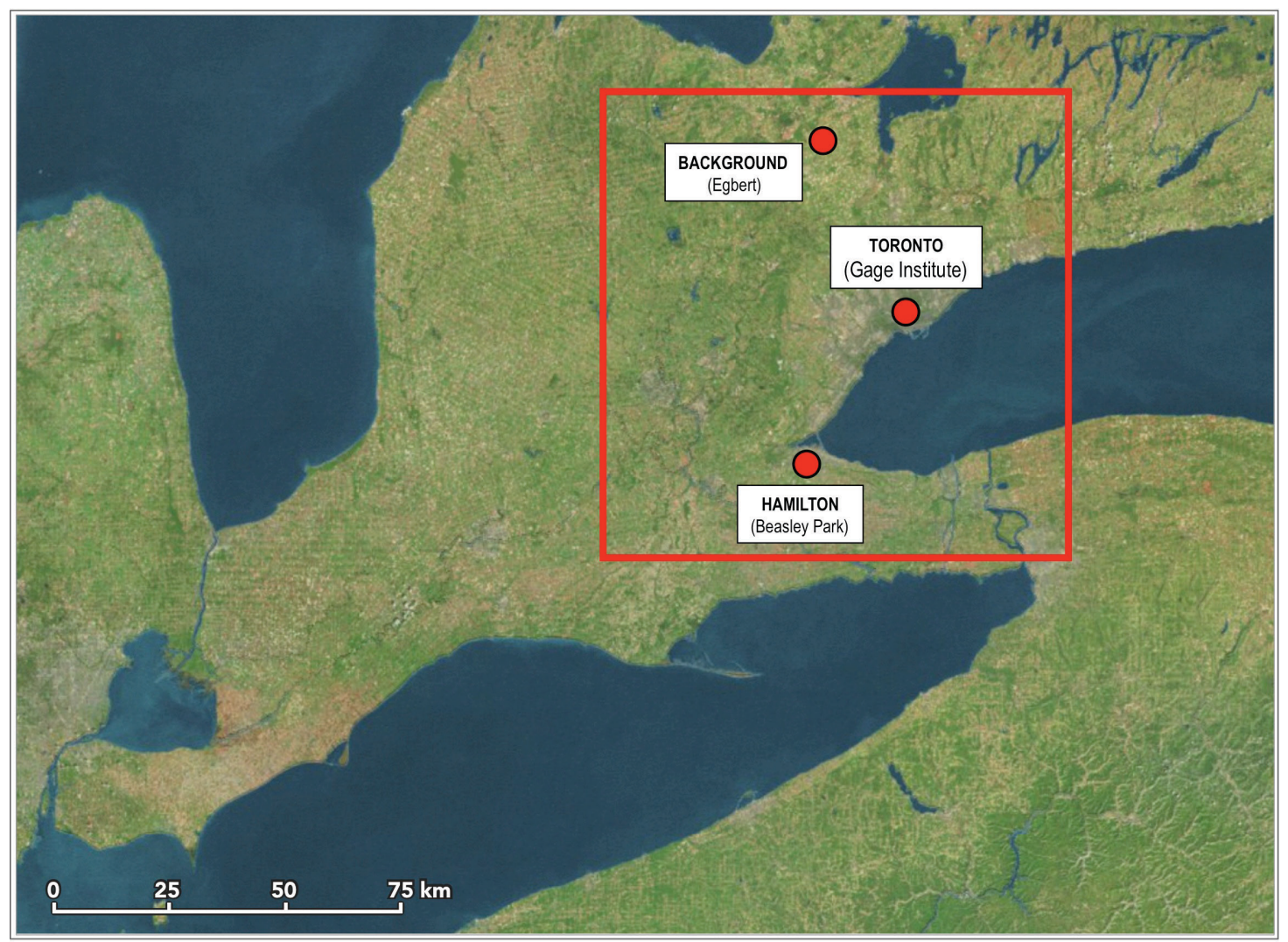

Figure 3-1. Map of study region showing NAPS sites scoped for receptor modeling. 
$\Sigma$ PAH concentration $\left(\mathrm{ng} / \mathrm{m}^{3}\right)$

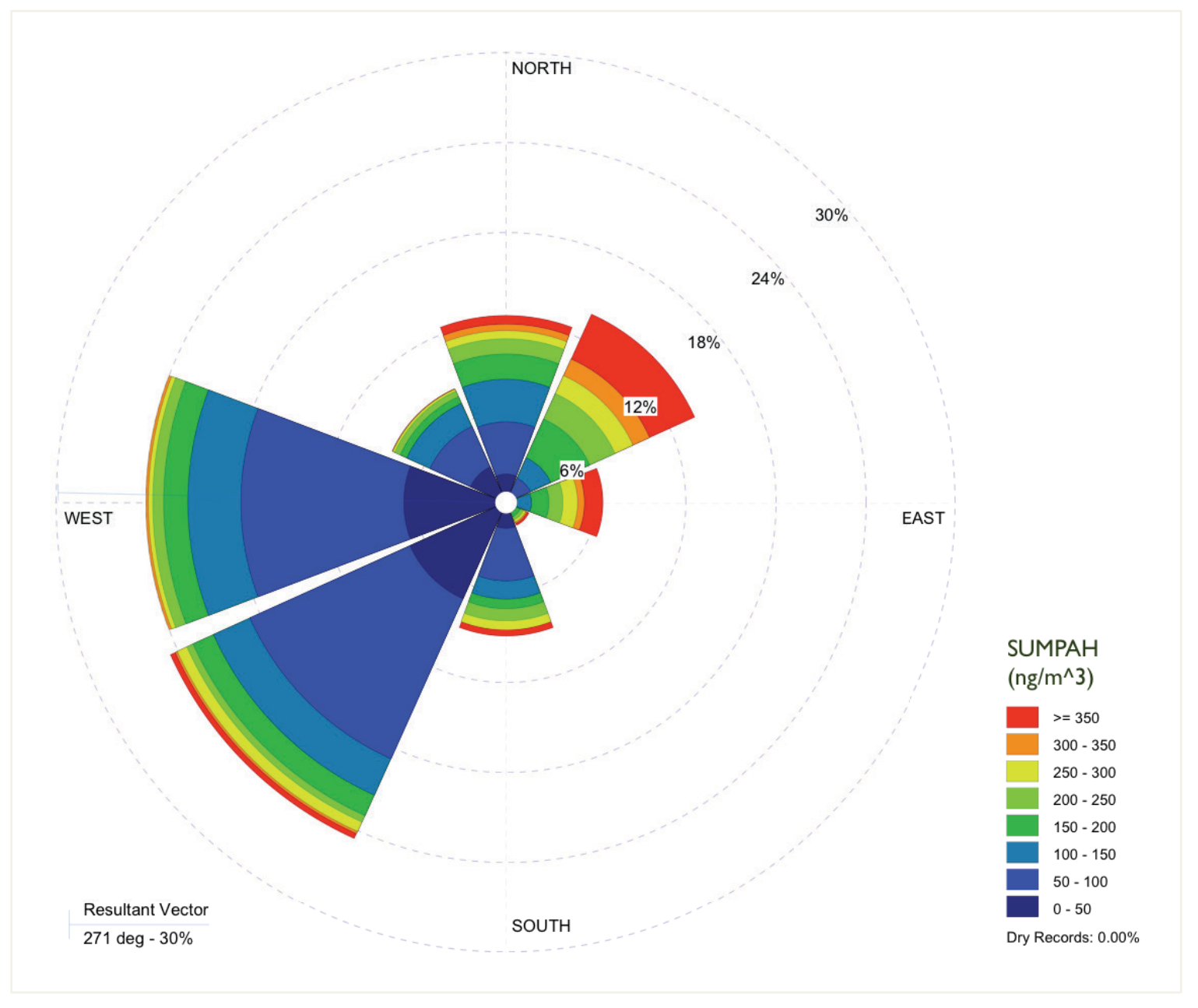

Figure 3-2. Pollutant rose for $\Sigma$ PAH at urban site (Hamilton). 


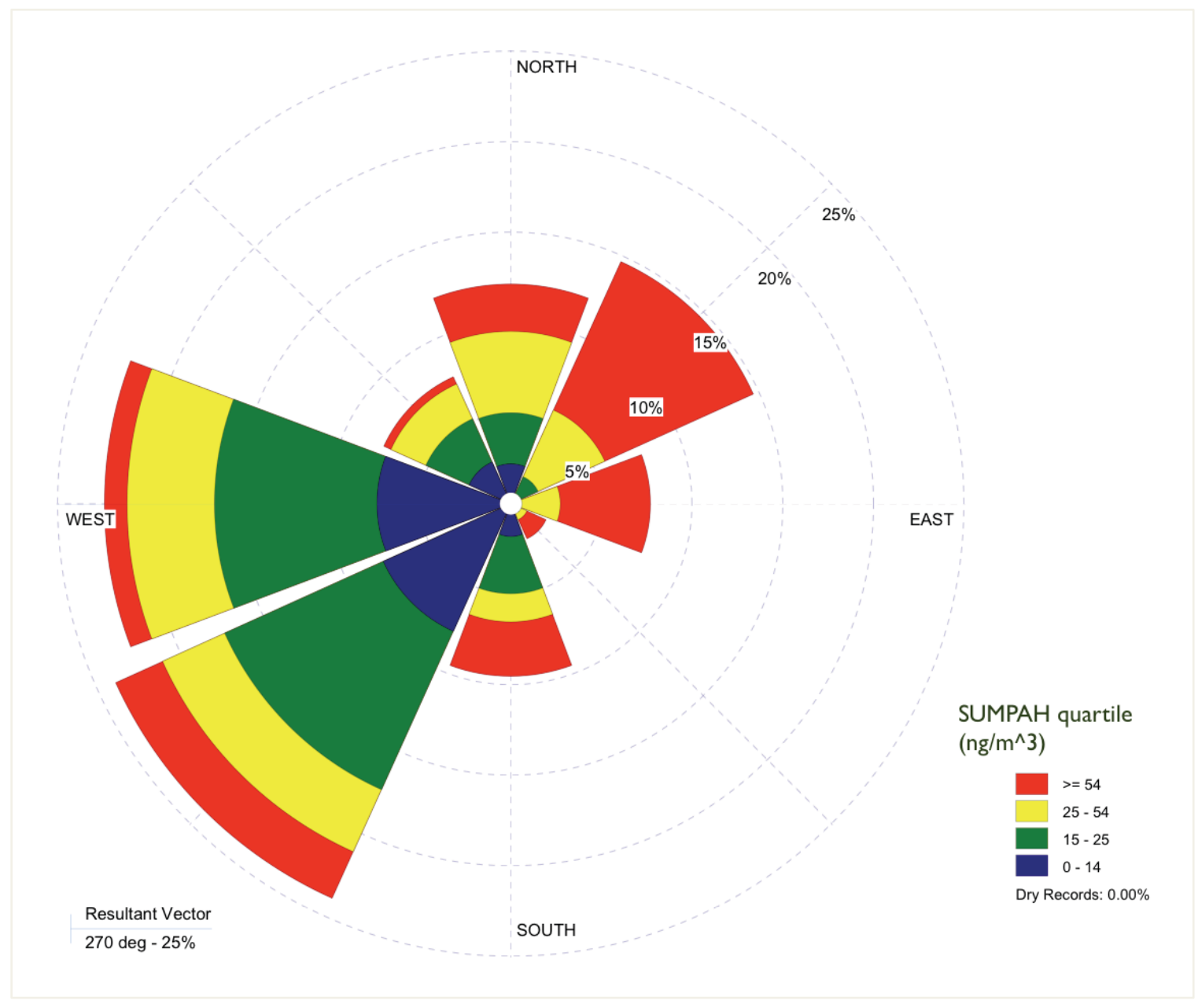

Figure 3-2 (continued) 
(a) predicted vs. sampled, modeled species (urban, $\Sigma_{2 I} P A H$; background, $\Sigma_{20} P A H$ )
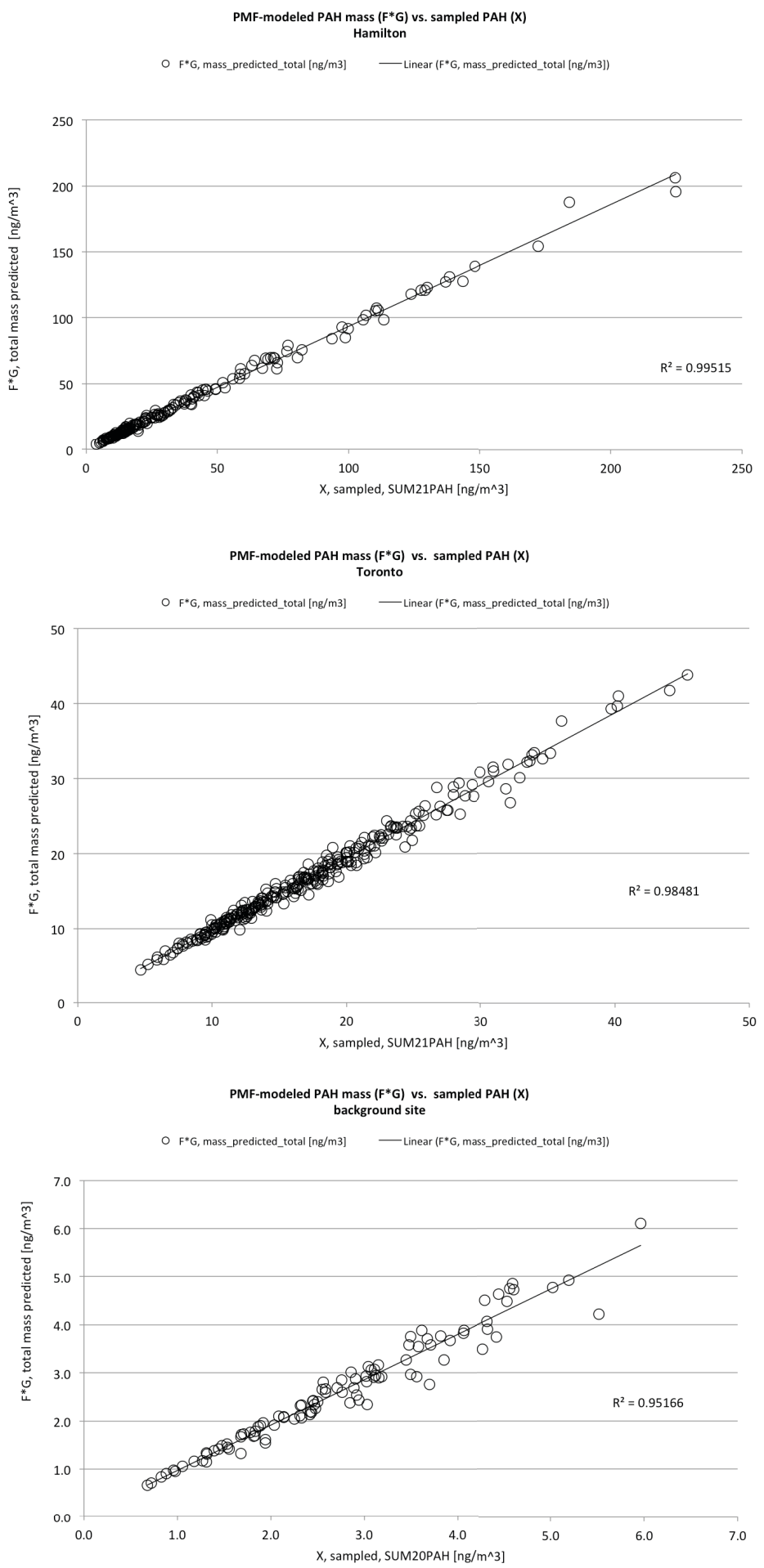

Figure 3-3. Correlation of PMF-predicted versus measured sampled mass, year-round model. 
(b) predicted versus sampled, all sampled species ( $229 P A H)$
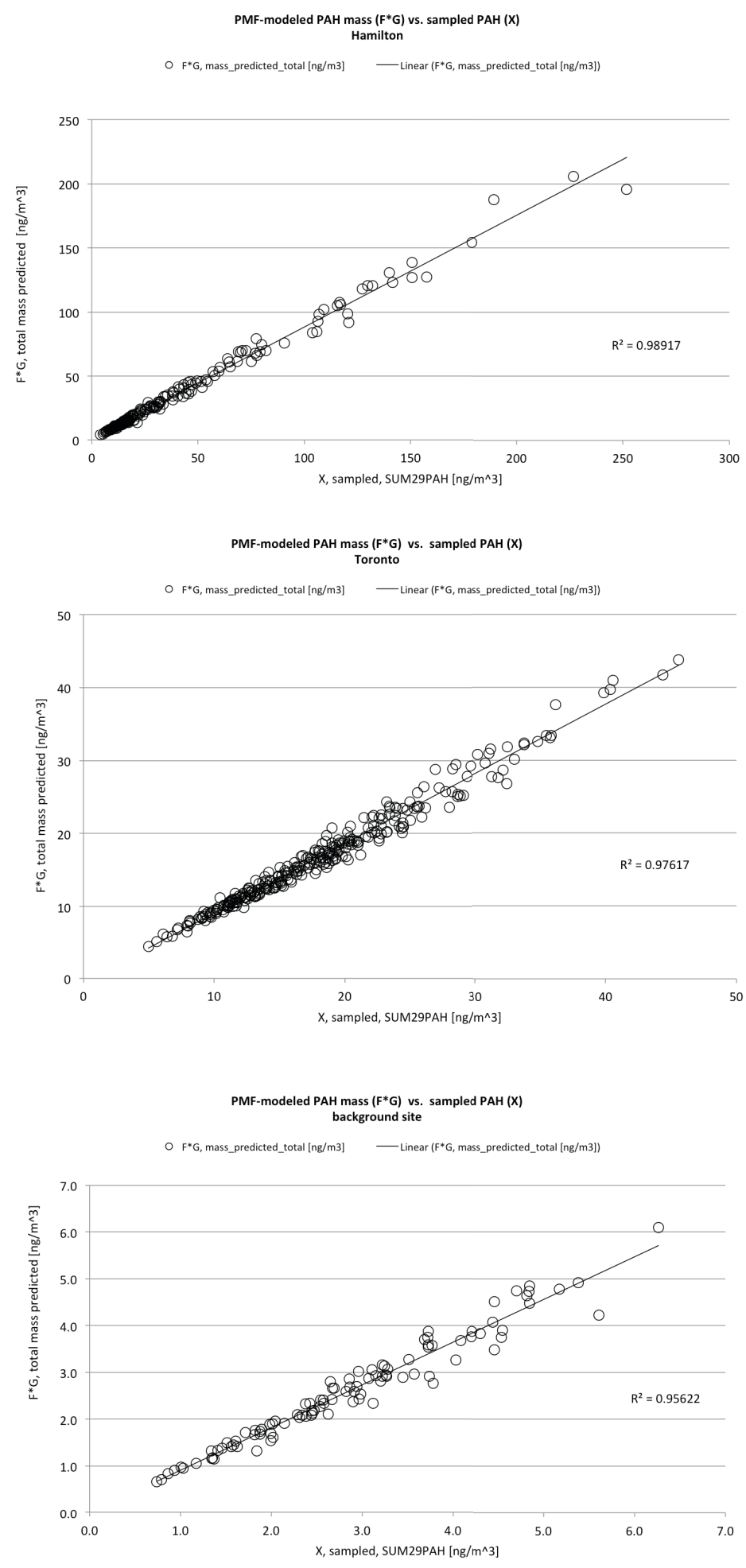

Figure 3-3 (continued) 
(c) predicted versus sampled, average species concentration
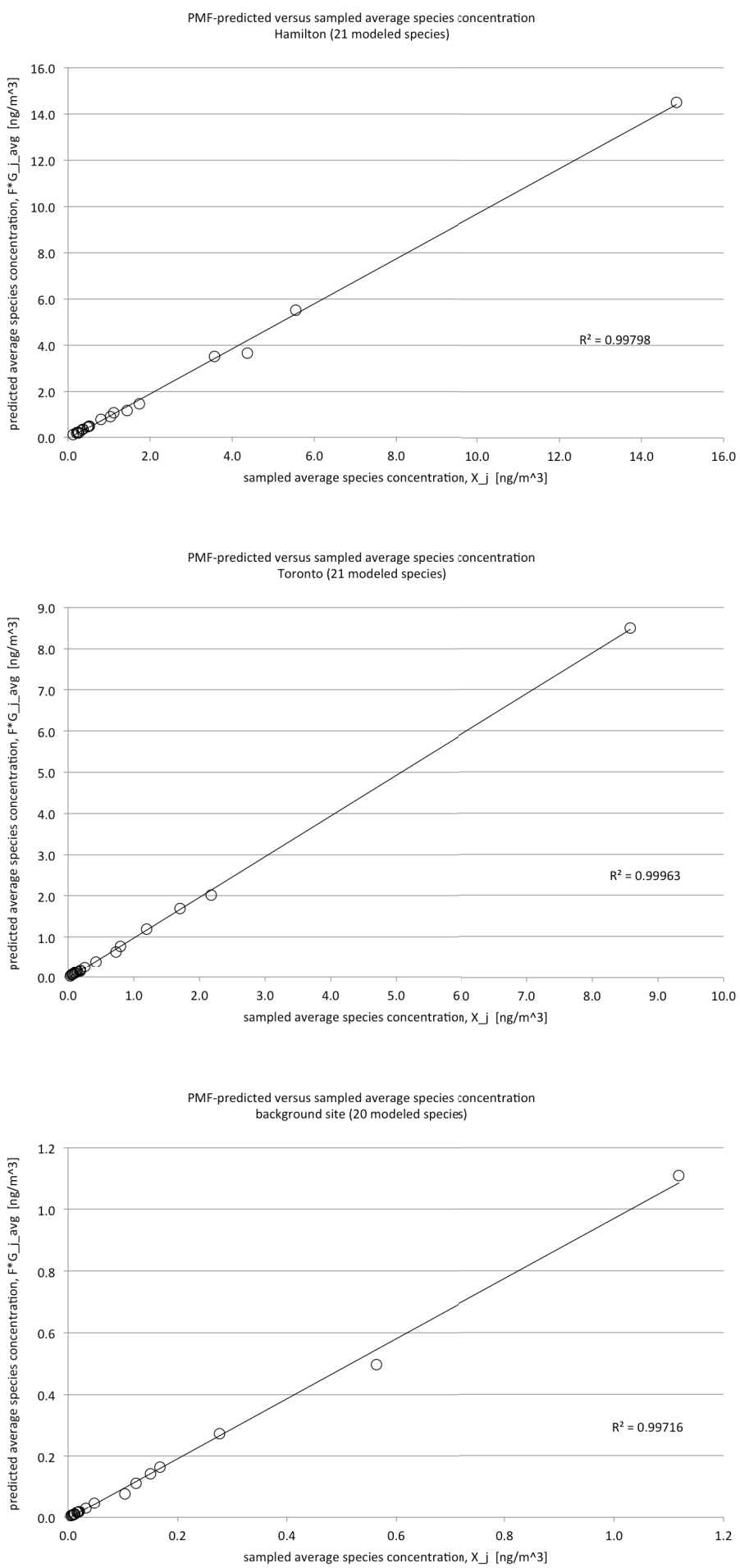

Figure 3-3 (continued) 


\section{Hamilton}
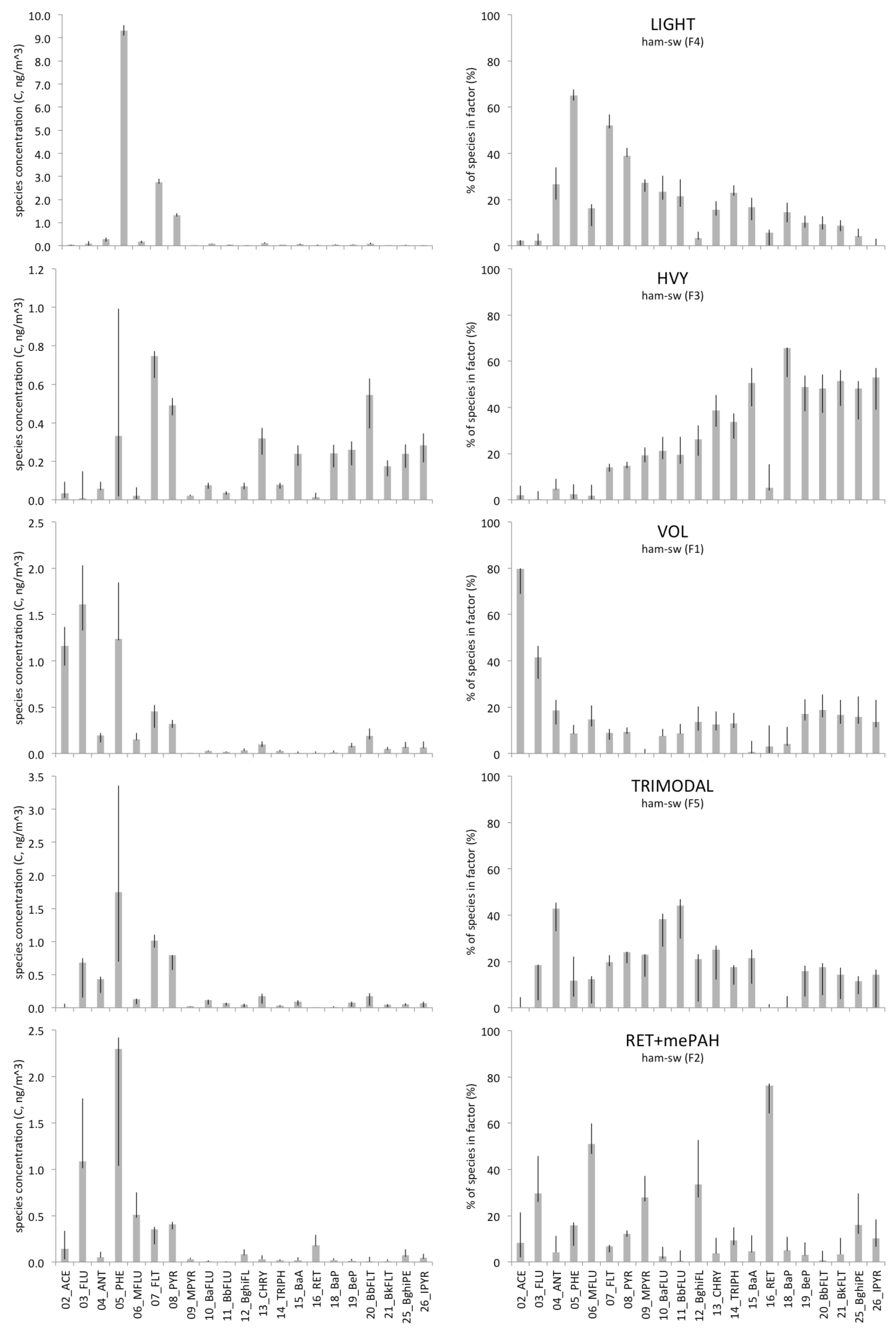

Figure 3-4. Bootstrap factor profiles (concentration, \% variance), year-round PMF model. 


\section{Toronto}

(a)
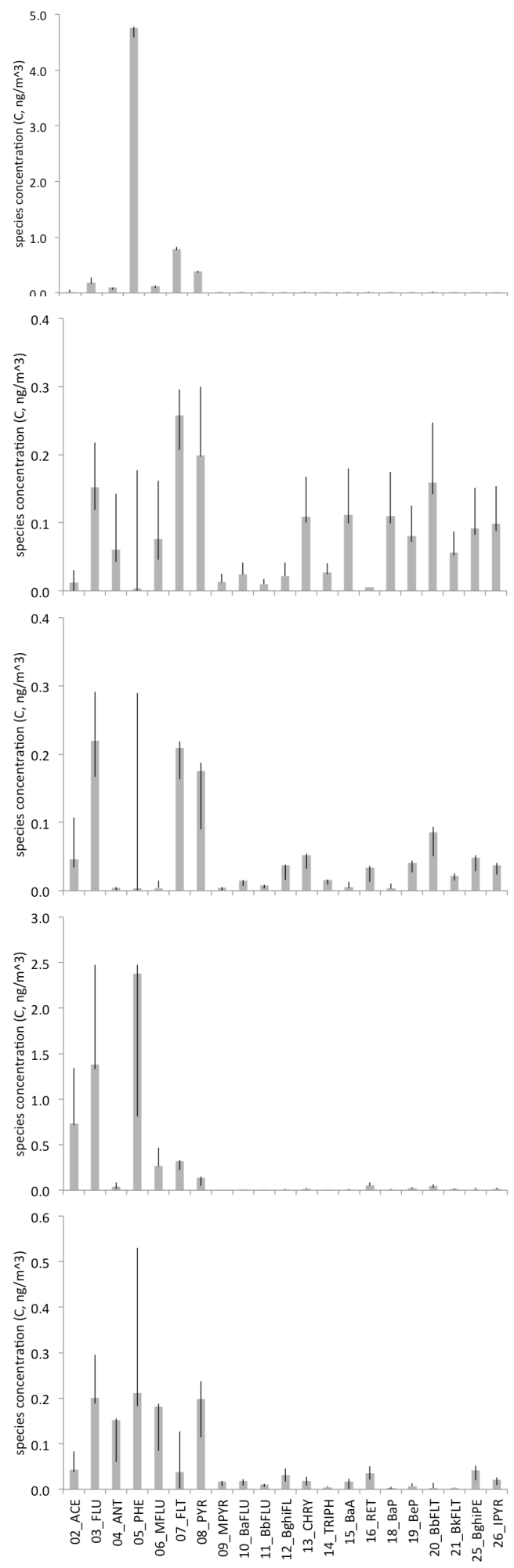
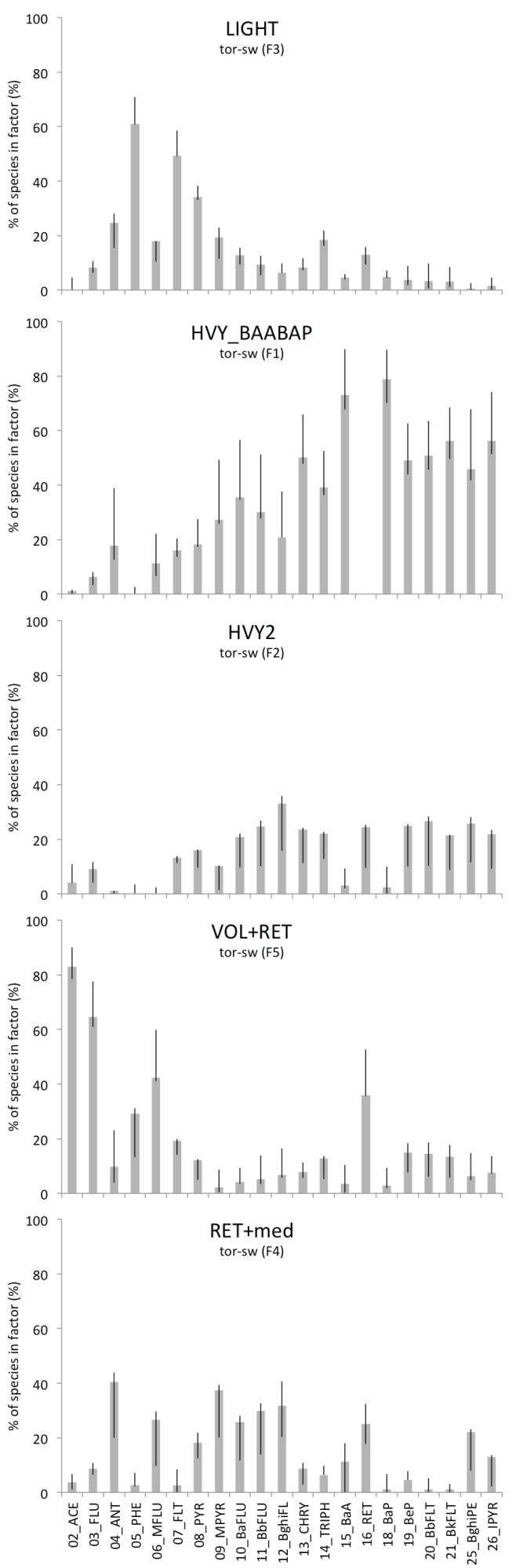

Figure 3-4 (continued) 
background (Egbert)
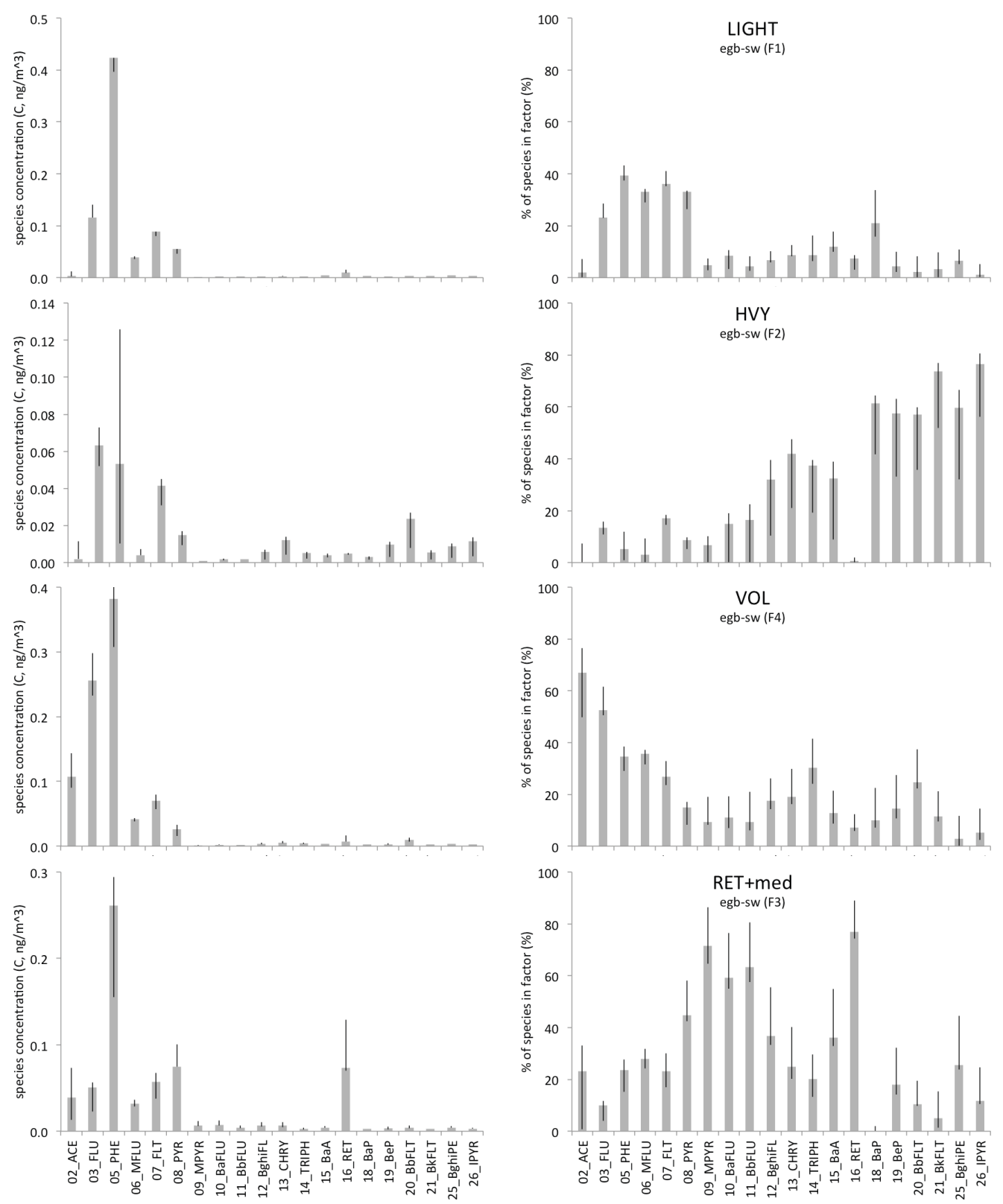

Figure 3-4 (continued) 


\section{PAH volatilization + LRTAP}
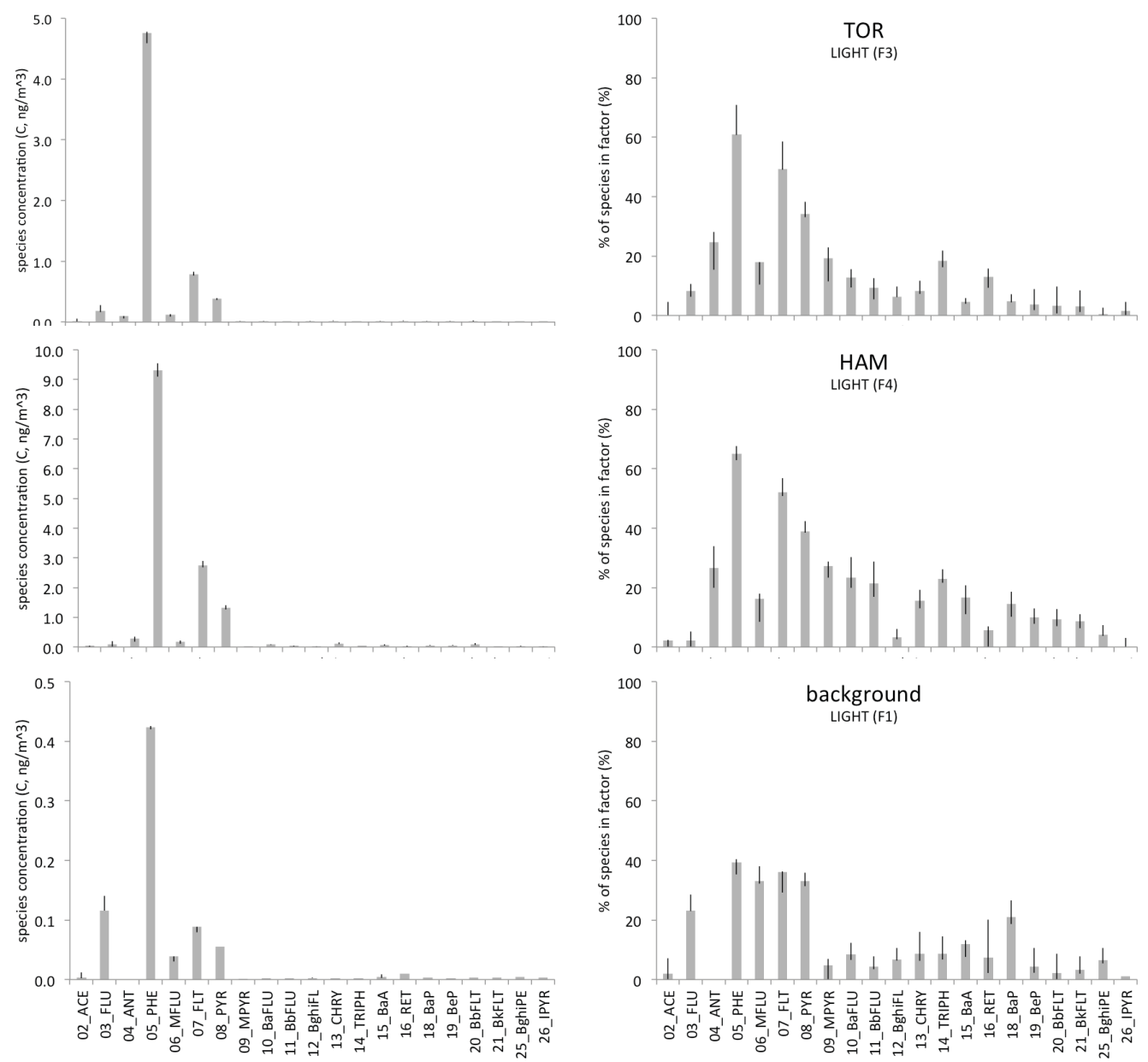

Note: ANT not modeled at background (Egbert) site due to poor data quality.

Figure 3-5. Bootstrap factor profiles (concentration, \% variance) by source type, year-round model. 
traffic emissions
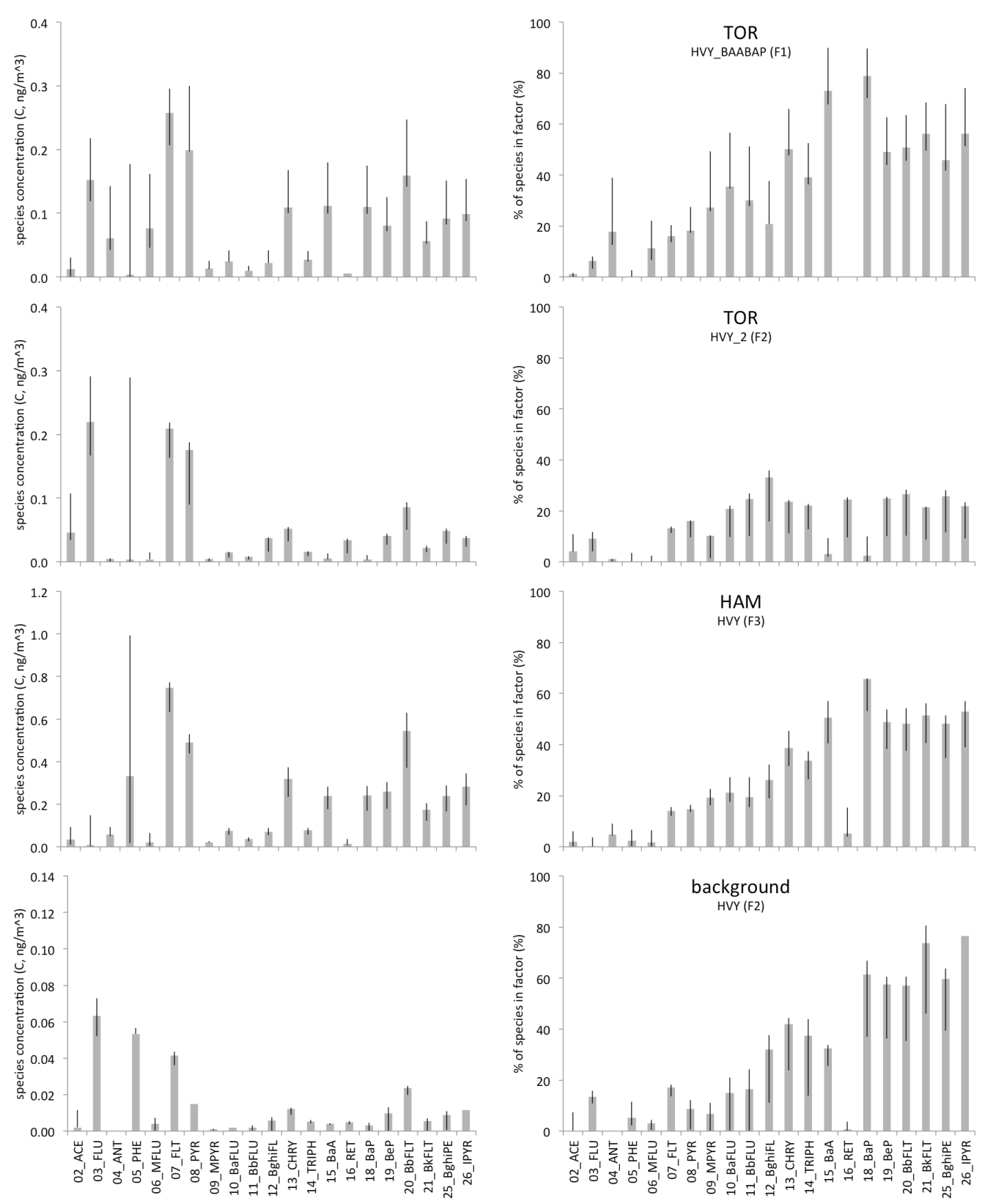

Note: ANT not modeled at background (Egbert) site due to poor data quality.

Figure 3-5 (continued) 
space heating - oil and natural gas combustion
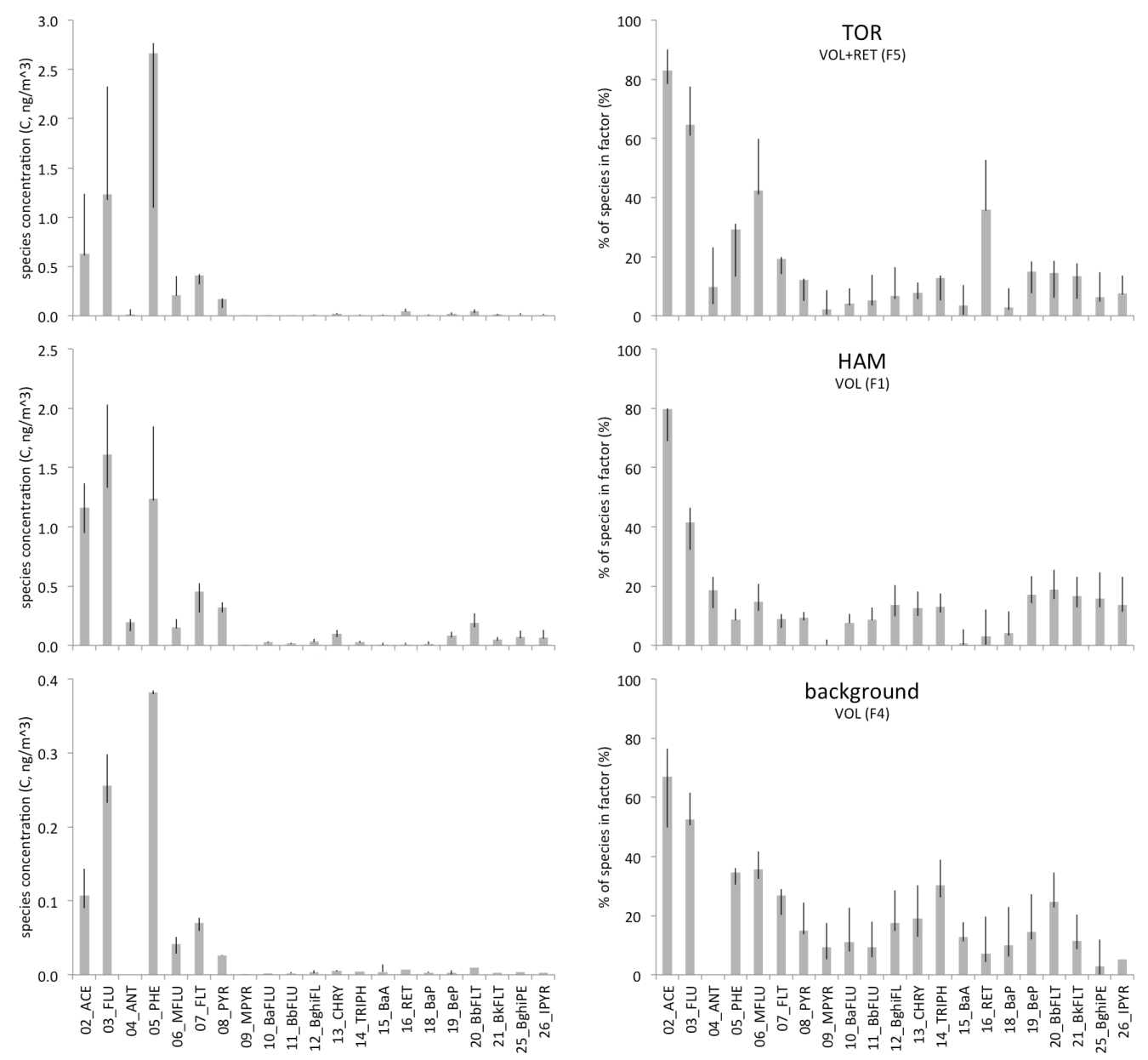

Note: ANT not modeled at background (Egbert) site due to poor data quality.

Figure 3-5 (continued) 


\section{local industry}
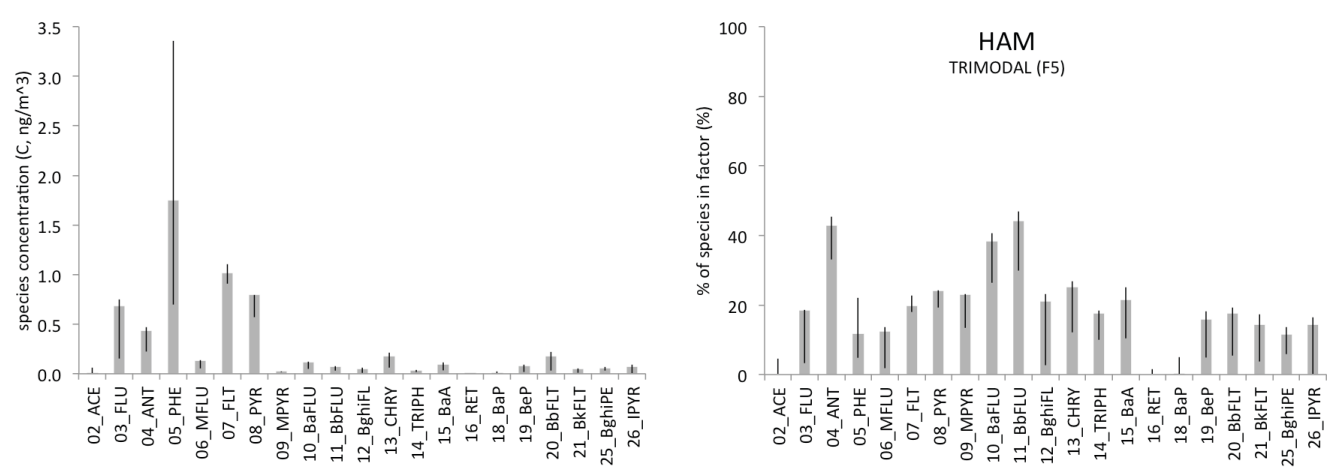

space heating - wood combustion
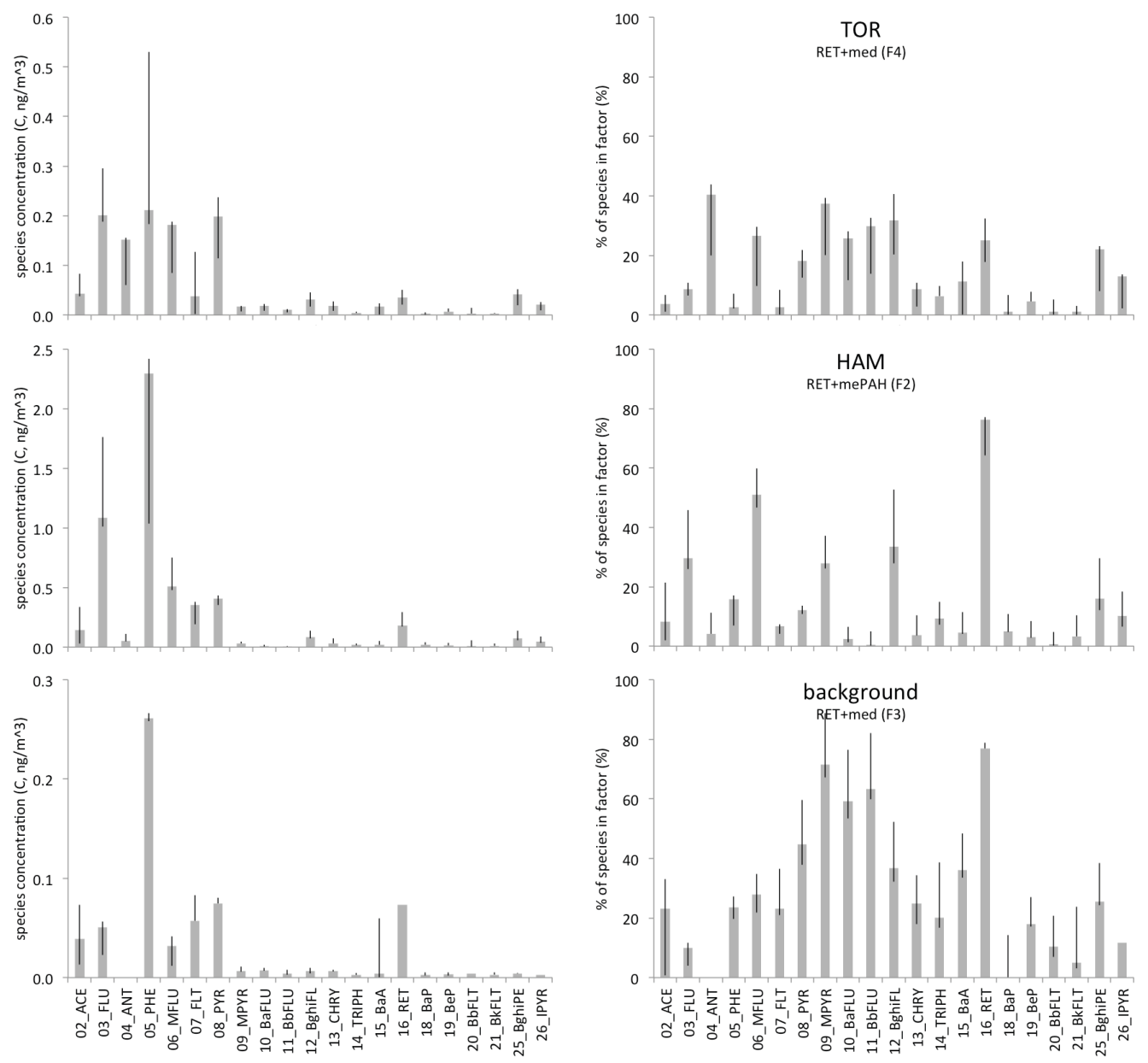

Note: ANT not modeled at background (Egbert) site due to poor data quality.

Figure 3-5 (continued) 


\section{Hamilton}

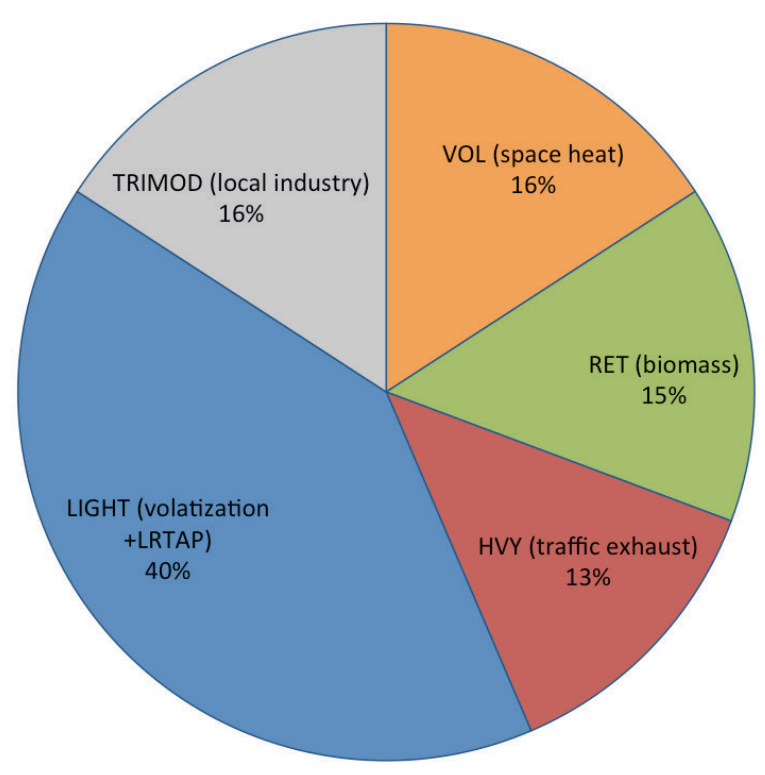

Toronto

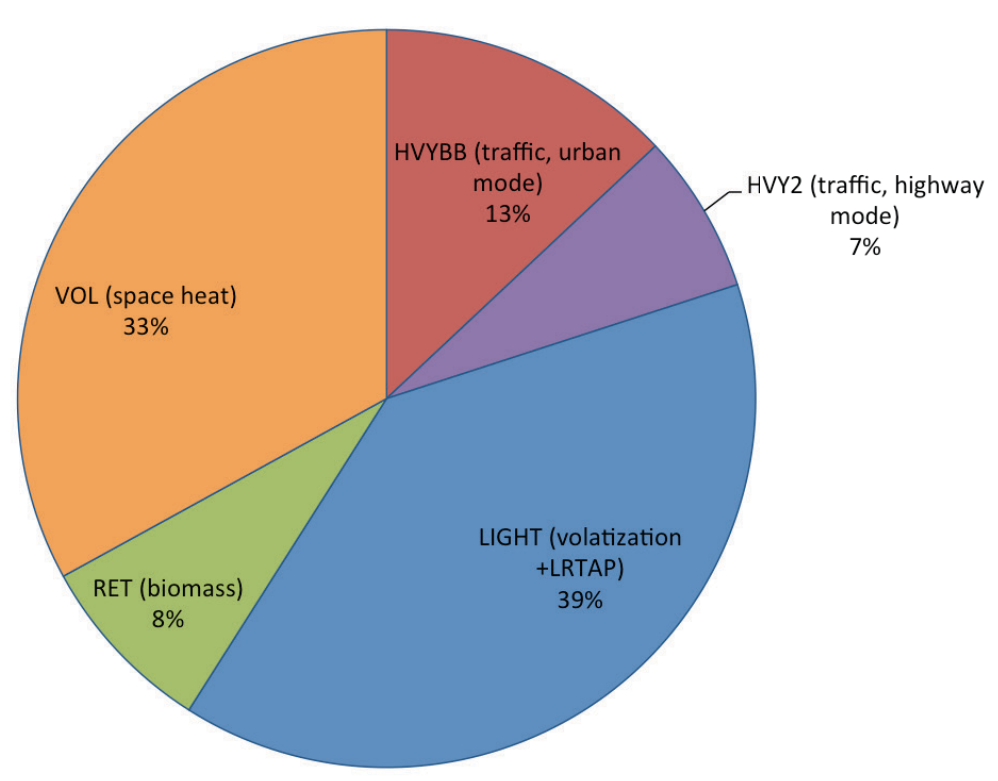

Figure 3-6. Source apportionment of PAH to PMF factors (all modeled mass). 
background (Egbert)

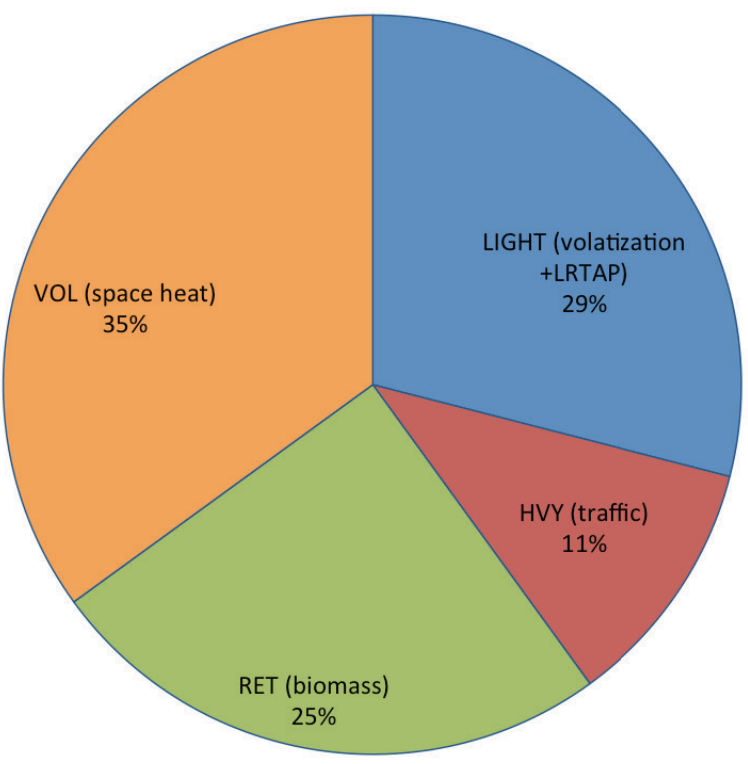

Figure 3-6 (continued) 


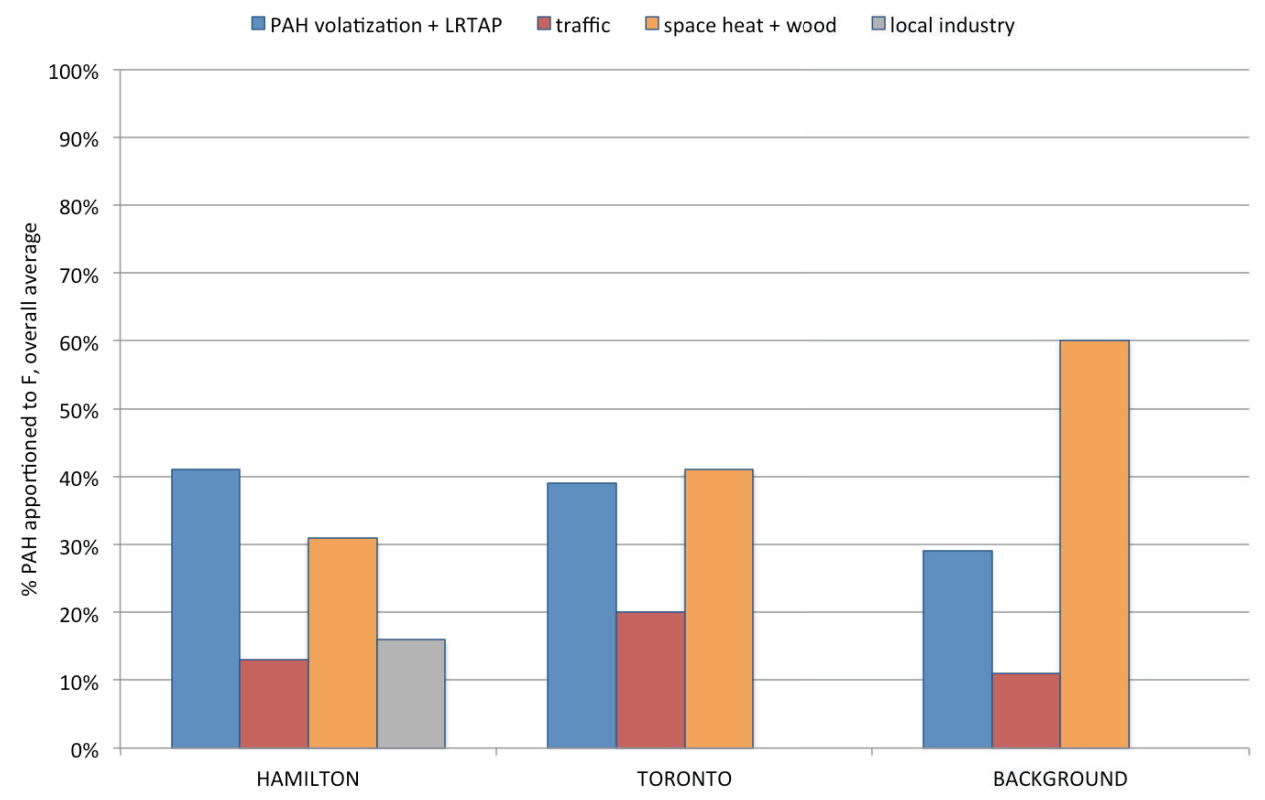

Note: Traffic factors grouped at Toronto; space heating and wood combustion factors grouped at all sites.

Figure 3-7. Inter-site comparison of PAH source apportionment (all modeled mass).

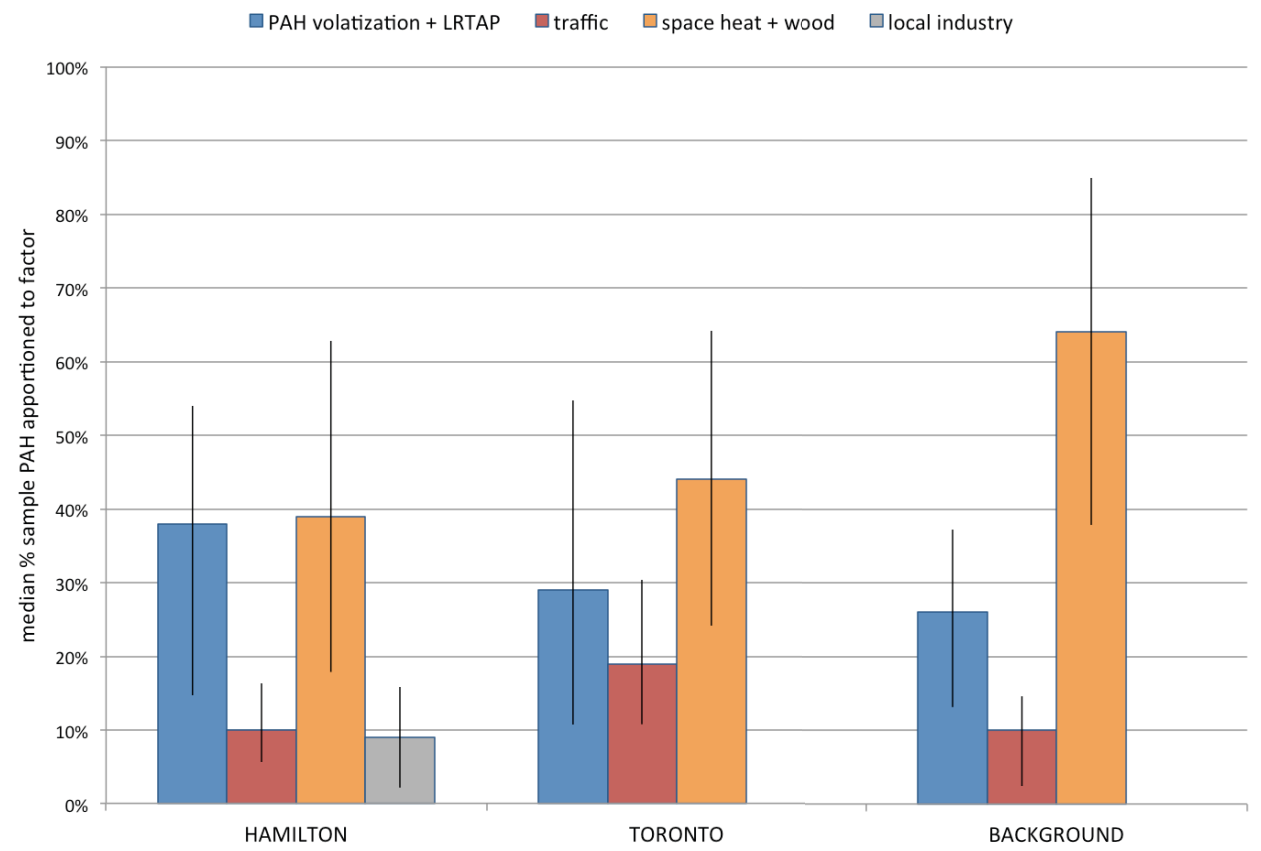

Note: Traffic factors grouped at Toronto; space heating and wood combustion factors grouped at all sites.

Figure 3-8. Inter-site comparison of PAH source apportionment (Q1, median, Q3 contribution to sample). 


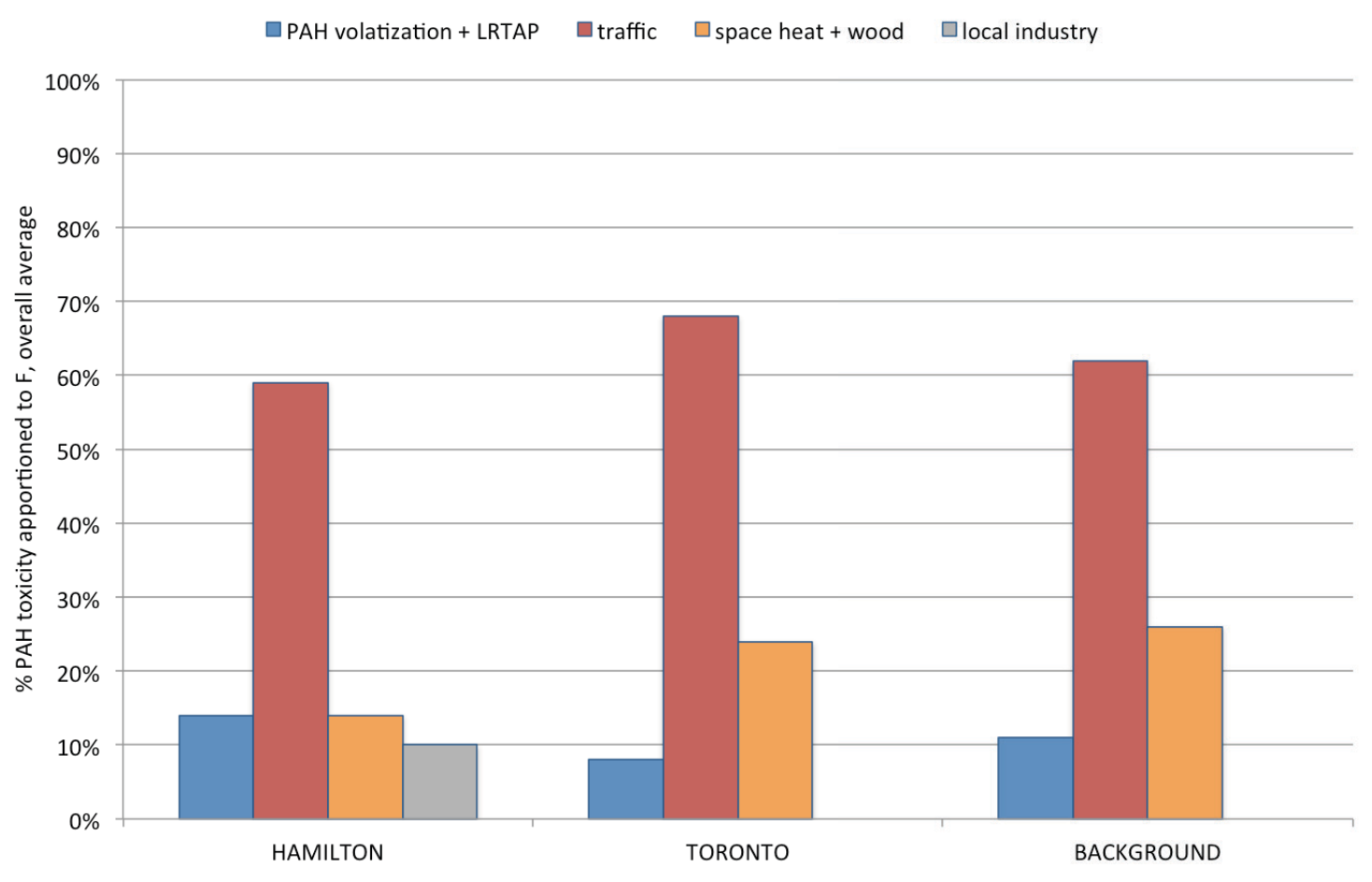

Note: Traffic factors grouped at Toronto; space heating and wood combustion factors grouped at all sites.

Figure 3-9. Inter-site comparison of PAH toxicity (BaP-TEQ) source apportionment, all modeled mass. 

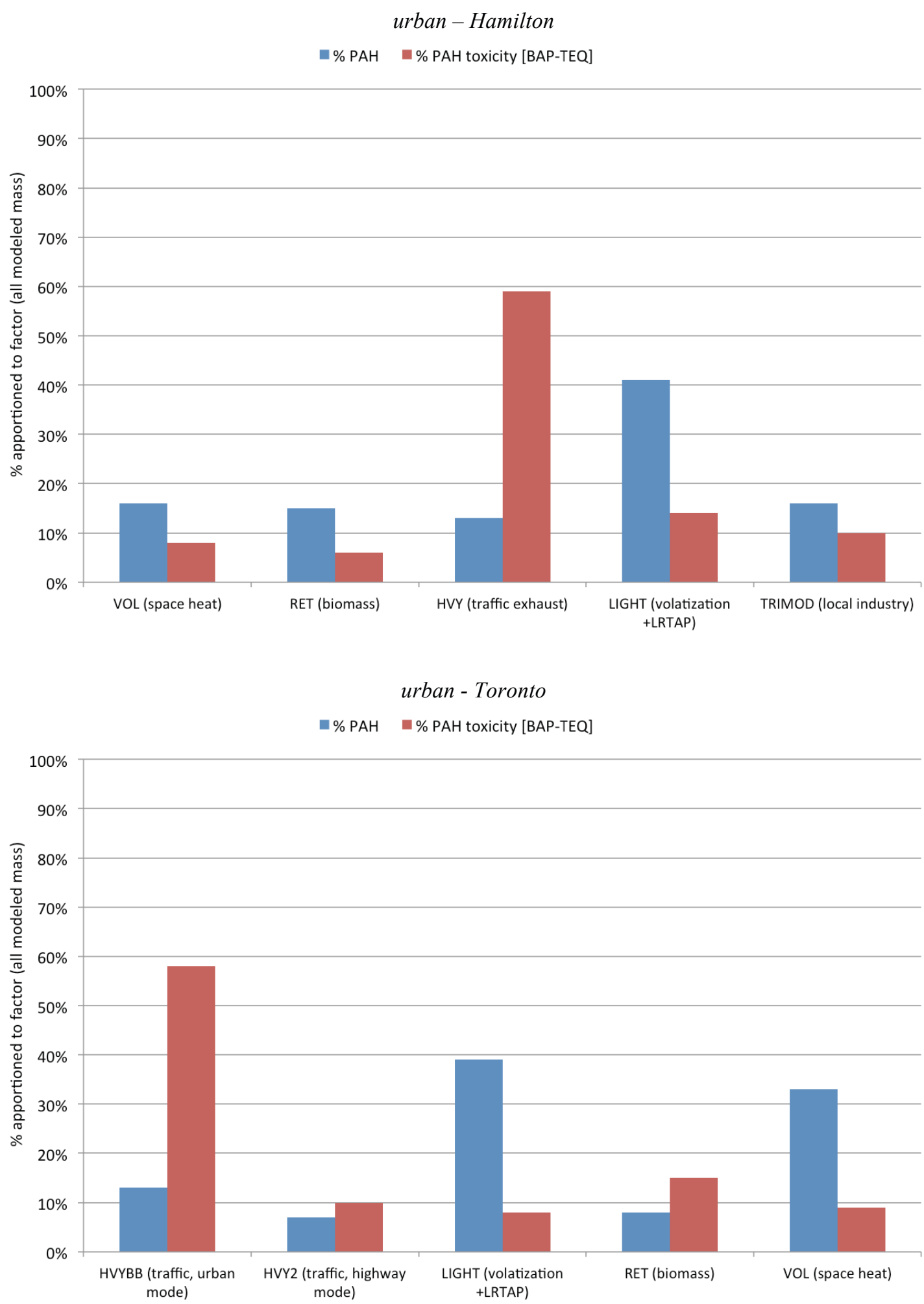

Figure 3-10. Source apportionment to PAH versus toxicity (BaP-TEQ), all modeled mass. 


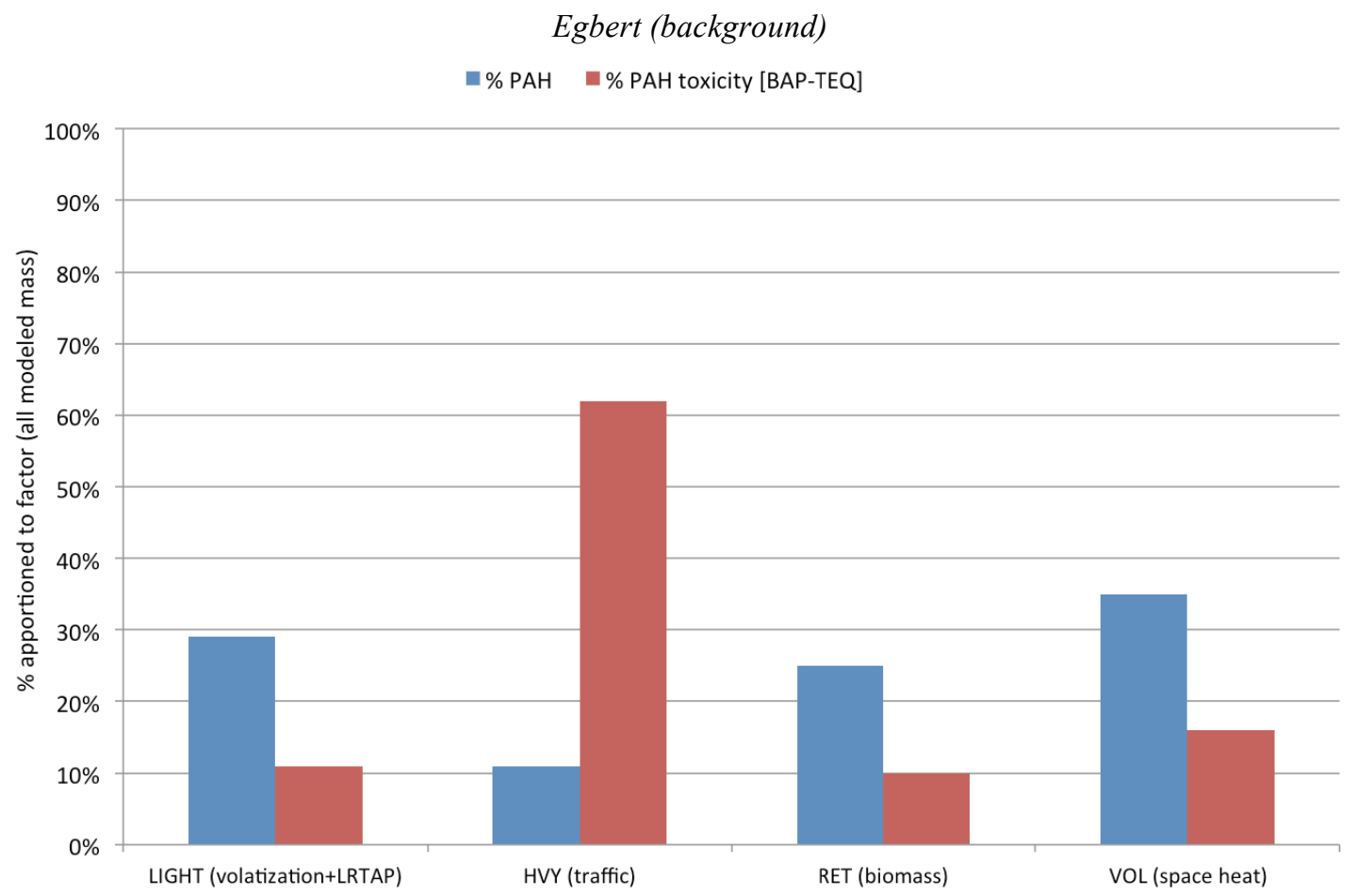

Figure 3-10 (continued) 


\section{Chapter: Intraurban concentrations, spatial variability and correlation of ambient polycyclic aromatic hydrocarbons (PAH) and PM 2.5}

The pages in this chapter reproduce a copyrighted article with permission from the coauthors and the journal, Atmospheric Environment:

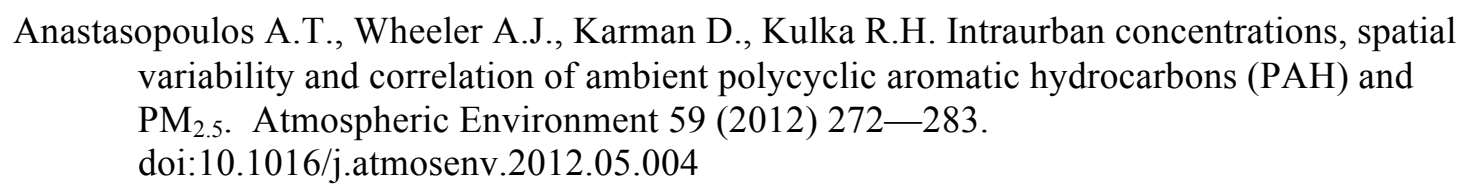
variability and correlation of ambient polycyclic aromatic hydrocarbons $(\mathrm{PAH})$ and $\mathrm{PM}_{2.5}$. Atmospheric Environment 59 (2012) 272-283.

doi:10.1016/j.atmosenv.2012.05.004

The first author on the paper and the author of the current dissertation was in charge of the implementation of the two sessions of field sampling with $\sim 50$ sampling sites, and overall coordination of the field sampling with the collaborating partners, as well as the analysis and interpretation of results.

Figure 4-2 and Figure 4-3 have been added to the text in the dissertation and do not appear in the above-cited article. Supplementary details of the intraurban sampling campaign are presented in Appendix C.I (e.g., site selection protocol, fieldwork instructions, sampling equipment and documentation). 


\subsection{Highlights}

- Ambient $\mathrm{PM}_{2.5}$ and 16 PAH were sampled in a dense intraurban network in two seasons.

- PAH showed much greater intraurban variability than $\mathrm{PM}_{2.5}$ in both seasons.

- Central monitoring was a poor intraurban proxy for $\mathrm{PM}_{2.5}$ and especially for PAH.

- $\quad \Sigma \mathrm{PAH} / \mathrm{PM}_{2.5}$ and $\mathrm{BaP}-\mathrm{TEQ}$ indicated potential exposure hot spots near local sources.

- Local scale differences are important in exposure assessment of light and heavy PAH.

\subsection{Abstract}

To investigate the intraurban spatial variability of air toxics associated with respirable particulate matter (PM), ambient $\mathrm{PM}_{2.5}$ and 16 polycyclic aromatic hydrocarbons (PAH) species (vapour phase plus $2.5 \mu \mathrm{m}$ particle phase) were sampled over a dense network of sites in Hamilton, Ontario, Canada in June/July 2009 and December 2009. $\mathrm{PM}_{2.5}$ levels ranged from $2.46-11.0 \mu \mathrm{g} / \mathrm{m}^{3}$ in the summer campaign and $6.52-13.4 \mu \mathrm{g} / \mathrm{m}^{3}$ in the winter campaign. Total sampled PAH $\left(\sum_{16} \mathrm{PAH}\right)$ levels ranged from $10.2-83.7 \mathrm{ng} / \mathrm{m}^{3}$ in the summer campaign and $8.31-52.1 \mathrm{ng} / \mathrm{m}^{3}$ in the winter campaign. Ambient $\mathrm{PM}_{2.5}$ and $\mathrm{PAH}$ concentrations were greater below the city's escarpment with a below/above escarpment difference in concentration much greater for $\mathrm{PAH}$ than for $\mathrm{PM}_{2.5}$ in both summer and winter sampling campaigns. Elevated levels of both pollutants were observed to occur near or downwind of the central business district and industrialized harbour-front area, suggesting the contribution of local sources. Ambient PAH exhibited a substantially greater degree of intraurban variability than $\mathrm{PM}_{2.5}$ (coefficient of variation approximately three times greater in summer campaign, four times greater in winter campaign) both above and below the escarpment, particularly for heavy molecular weight species found predominantly in the particle phase. Benzo(a)Pyrene-equivalent toxicity (BaP-TEQ) associated with ambient PAH showed a generally similar spatial distribution to $\Sigma_{16} \mathrm{PAH}$; however, several sites with relatively low $\Sigma_{16} \mathrm{PAH}$ had high BaP-TEQ (enriched in more toxic heavy molecular weight species), indicating potential hot spots for elevated PAH exposures and local source contributions. Co-located field sampling data showed that central site monitoring was a poor proxy for $\mathrm{PM}_{2.5}$ and particularly for $\mathrm{PAH}$ and associated toxicity (BaP-TEQ) across the urban centre, underestimating levels at many sites, likely due to the significant number of locally distributed sources and mixed land use. The much greater intraurban variability of PAH relative to $\mathrm{PM}_{2.5}$, particularly for toxic heavy molecular weight species predominantly in particle phase, demonstrated variability in $\mathrm{PM}_{2.5}$ composition and confirmed the importance of the local scale for PAH exposure health risk assessment. 


\subsection{Introduction}

Health risk from exposure to respirable particulate matter of aerodynamic diameter less than $2.5 \mu \mathrm{m}\left(\mathrm{PM}_{2.5}\right)$ has been shown to vary at the intraurban scale (Jerrett et al., 2005a). The determinants of $\mathrm{PM}_{2.5}$ toxicity are complex due to $\mathrm{PM}$ being an aggregate compound, with associated toxic compounds such as polycyclic aromatic hydrocarbons (PAH) varying from one aerosol to another, even within a single size fraction (Grahame and Schlesinger, 2007). Particularly since PAHs are expected to vary more than $\mathrm{PM}_{2.5}$ at the local scale (Narvaez et al., 2008; Fischer et al., 2000), comprehensive epidemiology studies of particulate air pollution require improved information on the distribution of PAHs in the urban setting (Samet and Pope, 2003).

PAHs are produced by incomplete combustion of organic material; anthropogenic sources include coal-fired power generation, space heating (i.e., wood, fossil), mobile source emissions (i.e., gasoline, diesel), and industrial source emissions (e.g., aluminum smelting, steel production) (ATSDR, 1995). As semi-volatile organic compounds (SVOCs), PAHs in ambient air are present in both vapour and particle phases; lighter PAH species (i.e., typically 2 and 3-ring structures) are found largely in vapour phase while heavier species (i.e., typically 5-ring, molecular weight $>228$ ) are largely in particle phase and found predominantly in the smaller respirable size fraction (i.e., $\mathrm{PM}_{2.5}$ ), increasing exposure risk (Venkataraman and Friedlander, 1994; Lu et al., 2008).

PAHs have been declared toxic under the Canadian Environmental Protection Act (Environment Canada, 2011a) and a number of heavy molecular weight species are under scrutiny for their probable carcinogenicity (ATSDR, 1995). Even lighter molecular 
weight PAHs constitute a health concern because they can react with sunlight or other atmospheric compounds to form transformation products (e.g., nitro-PAH, hydroxyPAH) with greater toxicity and environmental persistence (Baek et al., 1991a).

To support future epidemiology and health impact research, a spatial monitoring campaign was conducted to collect ambient airborne $\mathrm{PAH}$ and $\mathrm{PM}_{2.5}$ concentrations from a dense intraurban network of observation sites in two seasons. Sampling was carried out in the city of Hamilton, Ontario, Canada due to its historically high levels of ambient PAHs and diverse combination of local air pollution sources expected to contribute to intraurban variability in ambient air pollutants (i.e., steel mills and steel-related manufacturing, chemical manufacturing, paving material manufacturing, cement and concrete product manufacturing, urban traffic inclusive of several expressways, industrial harbour-front) (Environment Canada, 2011b). The study area's topography includes an approximately $100 \mathrm{~m}$ escarpment that topographically separates the city into lower and upper regions and was also expected to influence air pollutant concentrations (Wallace et al, 2010).

Ambient sampling for PM alongside PAH has been previously carried out only in a few recent studies in Hamilton (Sofowote et al., 2010; Somers et al., 2004; Legzdins et al., 1994) or nearby urban centres (Windsor, Ontario / Detroit, Michigan, USA; Miller et al., 2010); most earlier studies used a limited number of sites and did not sample $\mathrm{PM}_{2.5}$. Additionally, where previous studies report only particle-phase PAH, the combined vapour/particle phase PAH data collected in this study allows a better total estimate of intraurban variations in ambient exposures. Finally, the comprehensive dataset collected 
in this study enables quantitation of intraurban variability in PAH toxicity as well as the examination of PAHs as local-scale indicators of varying $\mathrm{PM}_{2.5}$ composition, with results applicable to other urban centres.

\subsection{Methods}

\subsubsection{Field sampling campaign}

\subsubsection{Study design and sampling sites}

Health Canada collaborated with Carleton University and City of Hamilton to conduct ambient PM and speciated PAH sampling at multiple intraurban sites in Hamilton, Ontario. Two-week integrated samples of PM and speciated PAH (combined vapour and particle phase) were collected in summer (24 June - 8 July 2009) and winter (2 - 16 December 2009) campaigns. Sampling campaigns were six months apart to capture any seasonality and were selected by review of National Air Pollution Surveillance (NAPS) PAH data (1999-2005; Environment Canada, 2011c) using the methods of Henderson et al., (2007).

The study area and sampling sites are shown in Figure 4-1. Samplers were deployed at 43 and 46 sites in summer and winter campaigns, respectively; summer campaign sites were repeated in the winter campaign with some additional winter sites. Sampling locations were selected based on results of a location-allocation model, a formal methodology for locating air pollution monitors to capture micro-scale variations (Kanaroglou et al., 2005), ensuring representative coverage of population density. 
Hamilton Public Health Services and the city's Geographic Information System (GIS) group provided an inventory of available municipal sites, which were screened as candidate surrogates for the location-allocation model sites (i.e., screening criteria included proximity to location-allocation model sites and inter-site distance). Final site selection was then confirmed by on-site visits (i.e., screening criteria included availability of electrical power, equipment security, possibility of sample bias from on-property sources). Sampling sites provided good coverage of the city and suburbs above and below the escarpment with a spatial distribution of approximately 1 sampler per $5 \mathrm{~km}^{2}$ and an average distance between adjacent sites of approximately $1.5 \mathrm{~km}$.

\subsubsection{Instrumentation}

The PM and PAH samplers used were identical to those described by Miller et al., (2010). At each sampling site, three PM size fractions (i.e., particles with aerodynamic diameter between 2.5 and $10 \mu \mathrm{m}, \mathrm{PM}_{10-2.5}$; particles between 1.0 and $2.5 \mu \mathrm{m}, \mathrm{PM}_{2.5-1.0}$; particles less than $1 \mu \mathrm{m}, \mathrm{PM}_{1.0}$ ) were sampled using a three-stage Harvard Cascade Impactor (Harvard School of Public Health, Boston, MA) at a nominal flow rate of 5.0 L per minute (Lpm). PAH were sampled using URG personal pesticide samplers with an inlet cut-point of 2.5 $\mu \mathrm{m}$ (Model URG-2000-25AG, URG, Chapel Hill, NC) at a nominal flow rate of $2 \mathrm{Lpm}$; these had a Teflon filter in-line with a polyurethane foam (PUF) plug (i.e., particle phase collected on Teflon filter, vapour phase on PUF).

PM and PAH sampling airflow was maintained by an SKC Leland Legacy field sampling pump (SKC Inc., Eighty Four, PA) connected to on-site AC power. Flows were verified at sampling setup and takedown using a DryCal DC-Lite Model "M" primary standard 
airflow meter (optimum flow range 100mLpm - 7Lpm, Bios International Corp., Butler, $\mathrm{NJ})$. Initial flow rates were set within $+-5 \%$ of nominal; at takedown, sites with greater than $20 \%$ deviation from nominal were excluded from further analysis.

PM and PAH samplers were arranged inside a weatherproof sampling case (Pelican Products, Torrance, California), oriented vertically with the sampling inlet facing downwards to minimize contamination (i.e., precipitation, insects) and deployed at approximately $1.5 \mathrm{~m}$ height, mounted on a survey-type tripod or appropriate on-site infrastructure (e.g., fence post). For a small number of sites where ground-level sampling was not possible, elevated or rooftop sampling was used. Portable Global Positioning System (GPS) devices were used to record sampling site location coordinates. The PM and PAH samplers are shown inside the sampling case in Figure 4-2; a typical ground level sampling setup is shown in Figure 4-3.

\subsubsection{Laboratory analysis of samplers}

PM sample filters were conditioned before and after sampling and filter masses were determined by gravimetric analysis, following EPA quality assurance guidelines (US EPA, 1998a). For PAH samplers, laboratory analysis co-extracted the particle phase (Teflon) and vapour phase (PUF) filters and reported PAH species concentrations as a total of the two sampled phases. The 16 analysed PAH species are listed in Table 4-1; species concentrations were determined by gas chromatography/mass selective detection (GC-MSD) and included analysis of lab blanks, standards and species-dependent recovery efficiencies, following US EPA Method TO-13A (US EPA, 1999). PM and PAH filter masses reported as below Lab Detection Limit (LDL) were substituted with 
LDL/2. Two-week integrated mass concentrations for PM $(\mu \mathrm{g} / \mathrm{m} 3)$ and $\mathrm{PAH}\left(\mathrm{ng} / \mathrm{m}^{3}\right)$ were calculated by dividing the respective filter masses by the volume of air sampled.

Speciated PAH mass concentrations were grouped by molecular weight (Table 4-1; Li et al., 2005a) into three summed mass concentrations:

i. $\quad$ total sampled $\mathrm{PAH}\left(\Sigma_{16} \mathrm{PAH}\right)$;

ii. sum of light molecular weight $\mathrm{PAH}$ species $\left(\Sigma_{\text {light }} \mathrm{PAH}, \mathrm{PAH}_{1-8}\right.$, naphthalene through pyrene); and,

iii. sum of heavy molecular weight $\mathrm{PAH}$ species $\left(\Sigma_{\text {heavy }} \mathrm{PAH}, \mathrm{PAH}_{9-16}\right.$, benzo[a]anthracene through benzo[ghi]perylene).

\subsubsection{QA/QC}

PM and PAH field blanks were deployed in both sampling campaigns at approximately $10 \%$ of the sampling sites and analysed to quantify the active sampler mass attributed to handling and transportation. Field Blank Correction (FBC) was determined as follows: field blanks below LDL were treated by substitution of LDL/2 and screened for outliers; field blank datasets from the two campaigns were checked for statistical difference in means and pooled where no statistical difference was shown; finally, to determine whether an FBC should be applied to the active pollutant sampler masses, separate or pooled field blank filter masses were compared to the LDL (i.e., where greater than half the field blanks were above LDL, FBC was calculated as the median of the field blank filter masses and subtracted from active pollutant sampler masses; no FBC was applied where more than half of the field blanks were below LDL) (Wheeler et al., 2011). 


\subsubsection{Data analyses}

\subsubsection{Meteorology during sampling}

Meteorological data (i.e., hourly ambient temperature, relative humidity, barometric pressure, and wind speed/direction) were obtained from three Environment Canada stations (Figure 4-1) and averaged to generate a single meteorological dataset representative of the study area for each campaign. Wind roses were prepared using WRPlot View 7 (Lakes Environmental Software Inc., Waterloo, Ontario, Canada). Precipitation data were reviewed to indicate potential washout of aerosol pollutants. Comparison was made with historic weather data (1971-2000; Environment Canada, 2011d) to determine if sampling weather conditions were typical during each campaign.

\subsubsection{Comparison with central site monitoring}

A field monitor was co-located with Hamilton's regulatory NAPS station currently measuring ambient $\mathrm{PM}_{2.5}$ and PAH (Figure 4-1). NAPS PAH samples are taken over a 24-hour period, scheduled on a six-day interval, and analyzed for 29 species, 15 of which are common to the current study (i.e., field-sampled dibenz[ah]anthracene isomer was compared with combined dibenz[ac] and [ah]anthracene isomers reported by NAPS). While noting sampling duration difference between NAPS and field sampling, samplers and lab analytical methods were sufficiently similar to allow data comparison for common PAH species (i.e., $\Sigma_{15} \mathrm{PAH}$, individual species). 


\subsubsection{Mapping and analyses of field sampling data}

$\mathrm{PM}_{2.5}$ and PAH concentrations at the sampling sites were mapped over the study area using ArcGIS 10 (ESRI Inc., Redlands, California, USA). All statistical analyses were completed using MINITAB 14 (MINITAB Inc., State College, Pennsylvania, USA) at a 5\% significance level. Normality of sampling datasets was examined using the AndersonDarling test statistic alongside probability plots (normal distribution) and kurtosis. Statistical difference was tested using the non-parametric Mann-Whitney test statistic. Intraurban variabilities in pollutant datasets were quantified using Coefficient of Variation (CV). Relationships among sampled pollutants were quantified using Spearman's rank correlation and Pearson's correlation.

A pollutant mass ratio (Equation 4-1) was defined at each site as an indicator of intraurban variability in $\mathrm{PM}_{2.5}$ relative to sampled $\mathrm{PAH}$ levels, using $\Sigma_{16} \mathrm{PAH}$ and $\Sigma_{\text {heavy }}$ PAH.

Equation 4-1. Pollutant mass ratio for sampled $\mathbf{P M}_{2.5}$ relative to sampled PAH.

$$
\text { pollutant mass ratio }=\frac{\sum P A H}{P M_{2.5}} \quad\left[\frac{n g}{\mu g}\right]
$$

The speciated ambient PAH data from the two sampling campaigns was also analysed on the basis of carcinogenic potential of inhalation exposure, referencing Toxicity Equivalence Factors (TEF) of each sampled species relative to benzo(a)pyrene (BaP) (Table 4-6; Nisbet and Lagoy, 1992). For each sampling site, the speciated PAH concentration was multiplied by its respective BaP-TEF and summed over all sampled 
species to yield the BaP-equivalent concentration (BaP-TEQ, ng/m $\mathrm{m}^{3}$, adapting the method described in Jung et al (2010) to this study's larger suite of 16 PAH species.

\subsection{Results \& Discussion}

\subsubsection{Meteorology during sampling}

Temperatures over the sampling campaigns were historically typical (1971-2000; Environment Canada, 2011d) for Hamilton (summer campaign, mean $19.3^{\circ} \mathrm{C}$, standard deviation $3.7^{\circ} \mathrm{C}$; winter campaign, mean $0.1^{\circ} \mathrm{C}$, standard deviation $4.1^{\circ} \mathrm{C}$ ). Wind directions during each campaign were also typical (Figure 4-4), with westerly prevailing winds in the summer campaign (mean wind direction $\mathrm{W}, 272^{\circ}$ ) and more frequent southwesterly winds in the winter campaign (mean wind direction WSW, $247^{\circ}$ ), classifying east and northeast Hamilton as downwind of central business district and industrial / harbour-front sources. Wind speeds were historically typical as well: higher in the winter campaign and generally calmer in lower Hamilton in both sampling campaigns, indicating some microclimate effects.

Precipitation during the summer campaign was observed as short rainfall events on approximately half the sampling days (daily mean, $5.5 \mathrm{~mm}$; standard deviation, $9.7 \mathrm{~mm}$ ). Since rainfall occurred only $28 \mathrm{~h}$ of the two-week campaign ( $8 \%$ of sampling hours), washout of airborne pollutants was considered to be minor. During the winter campaign, precipitation was observed as predominantly rainfall (90\% rainfall, 10\% snowfall) on approximately half the sampling days, with a daily mean of $3.2 \mathrm{~mm}$ (standard deviation, $6.4 \mathrm{~mm}$ ); pollutant washout was judged to be moderate (rainfall $36 \%$ of sampling hours) 
and is representative of winter conditions in Hamilton (i.e., winter campaign precipitation levels were historically typical, 1971-2000).

\subsection{2 $\quad \mathrm{PM}_{2.5}$ and PAH dataset quality}

\subsubsection{Detection Limits, Field Blank Correction}

The laboratory detection limit (LDL) for PM was $4 \mu \mathrm{g} /$ filter. PAH LDL were speciesspecific, varying from $2.14 \mathrm{ng} / \mathrm{sample}$ to $9.18 \mathrm{ng} / \mathrm{sample}$ (Table 1). $\mathrm{PM}_{2.5}$ concentrations were above LDL at all sites in summer and winter sampling campaigns. PAH concentrations were generally above LDL at the majority of sites for species in the light molecular weight grouping in summer and winter campaigns (Table 1). Heavier molecular weight species (exception of chrysene) were generally below LDL in the summer campaign; however, several heavier molecular weight species had concentrations above LDL at a large number of sites in the winter campaign: benzo[a]anthracene, chrysene, benzo[k]fluoranthene, benzo[a]pyrene, benzo[ghi]perylene. Although the general trend in summer campaign PAH species concentrations was similar to that noted by Miller et al., (2010) in Windsor (i.e., late summer sampling campaign with comparable mean temperature of $19.1^{\circ} \mathrm{C}$ ), the additional winter campaign deployed in Hamilton sampled heavy molecular weight species in higher concentrations.

PM and PAH field blank concentrations did not differ statistically $(\mathrm{p}<0.05)$ between campaigns and were pooled for use in Field Blank Correction (FBC) calculations. All three PM size fractions had field blank filter masses below LDL for more than $50 \%$ of pooled field blanks, precluding FBC of PM samplers; similarly, more than $50 \%$ of pooled 
PAH field blanks were below LDL for the majority of species, precluding FBC with exception of phenanthrene and pyrene.

\subsubsection{Data retention}

Retention of field sampling data for $\mathrm{PM}_{2.5}$ and $\mathrm{PAH}$ are summarized in Table 4-2. In the summer campaign, valid data was retained from more than $80 \%$ of deployed sites; in the winter campaign, valid data was retained at approximately $70 \%$ of deployed sites. The majority of missing data were due to field issues (e.g., power failure, pump failure, weather event), invalid lab results (e.g., negative filter mass reported by lab), and at a small number of sites, confirmed outliers (i.e., statistical outliers differing from the mean by more than three times interquartile range and corroborated by field issues).

In the summer campaign, sites with missing $\mathrm{PM}_{2.5}$ data were split evenly between upper and lower Hamilton and were not concentrated in any one area of the sampling network; sites with missing PAH data were largely below the escarpment but were similarly not concentrated in any one area. Thus, bias due to missing sites is expected to be minimal in the summer campaign, with good representation of the entire study region for $\mathrm{PM}_{2.5}$ and PAH. In the winter campaign, sites with missing $\mathrm{PM}_{2.5}$ data were nearly all below the escarpment, the majority concentrated in northeastern Hamilton or north of the harbour; these areas were more poorly represented compared with central business district / harbour-front and western sampling areas. As a result, intraurban variability of $\mathrm{PM}_{2.5}$ quantified for lower Hamilton (CV) is expected to be somewhat greater than captured. Sites with missing PAH data were also largely below the escarpment but were more evenly distributed, preserving good representation of the study region for PAH. 


\subsubsection{Sampling height}

Ambient $\mathrm{PM}_{2.5}$ and heavy molecular weight $\mathrm{PAH}$ have been shown to attenuate with increasing height ( $\mathrm{Li}$ et al., 2005b). In the retained $\mathrm{PM}_{2.5}$ and $\mathrm{PAH}$ datasets, four sites (two above and two below escarpment) were sampled at an elevated height ( $>1$ storey above ground level). Rooftop-sampled $\Sigma_{16} \mathrm{PAH}$ concentrations were expected to be similar to equivalent ground-level measurements since heavy PAH species contributed minimally to total sampled mass and because levels were consistent with $\Sigma_{16} \mathrm{PAH}$ at nearby ground-level sites. In comparison, review of rooftop $\mathrm{PM}_{2.5}$ sampling data against nearby ground-level sites suggested possible non-conservative bias (i.e., ground-level $\mathrm{PM}_{2.5}$ expected to be greater). However, rooftop $\mathrm{PM}_{2.5}$ data is reported as sampled because rooftop sites with $\mathrm{PM}_{2.5}$ data were few (four in summer campaign, two in winter campaign) and application of an ad hoc correction factor to equivalent ground-level concentration would introduce additional error.

\subsubsection{Co-located site comparison}

PAH field samplers and lab analytical methods were sufficiently similar to NAPS central site monitoring to allow co-located data comparison for the 15 commonly sampled species. In both summer and winter campaigns, NAPS-monitored and field-sampled concentrations were of comparable magnitudes for $\Sigma_{15} \mathrm{PAH}$ and the majority of individual species, with field sampling data between the levels measured by NAPS on the two nearest bracketing sampling dates: summer campaign, field-sampled $\Sigma_{15} \mathrm{PAH}$ was 26.9 $\mathrm{ng} / \mathrm{m}^{3}$, NAPS mean $\Sigma_{15} \mathrm{PAH}$ was $25.1 \mathrm{ng} / \mathrm{m}^{3}$ (6/18/2009, 7/12/2009); winter campaign, field-sampled $\Sigma_{15}$ PAH was $28.2 \mathrm{ng} / \mathrm{m}^{3}$, NAPS mean $\Sigma_{15} \mathrm{PAH}$ was $31.4 \mathrm{ng} / \mathrm{m}^{3}(12 / 3 / 2009$, 
12/21/2009). PAH species profile was also similar between NAPS and co-located field sampling (Pearson correlation: summer campaign, $\mathrm{r}^{2}=0.95$; winter campaign, $\mathrm{r}^{2}=0.81$ ). Plots comparing field-sampled and NAPS-sampled PAH concentrations are included in Figure 4-5. The general agreement between NAPS and co-located field sampled concentrations suggests that intraurban differences in PAH observed in the sampling campaigns reflect differing source contributions across Hamilton.

\subsubsection{Intraurban variability in $\mathrm{PM}_{2.5}$ and $\mathrm{PAH}$}

\subsubsection{Pollutant concentration maps}

Descriptive statistics for the $\mathrm{PM}_{2.5}$ and PAH datasets are shown in Table 4-2 and mapped by quartile in Figure 4-6 $\left(\mathrm{PM}_{2.5}\right)$ and Figure 4-7 ( $\left.\Sigma_{16} \mathrm{PAH}\right)$. In both sampling campaigns, sites with fourth quartile (highest) $\mathrm{PM}_{2.5}$ and $\Sigma_{16} \mathrm{PAH}$ concentrations were largely below the escarpment and concentrated in the central business district and the heavily industrialized harbour-front area as well as to the east of it (i.e., downwind, consistent with prevailing westerly winds, Figure 4-4). Conversely, first quartile (lowest) concentrations were largely at sites above the escarpment or on the outer perimeter of the study area in both campaigns.

Hamilton $\mathrm{PM}_{2.5}$ (summer campaign: $2.46-11.0 \mu \mathrm{g} / \mathrm{m}^{3}$; winter campaign: $6.52-13.4$ $\mu \mathrm{g} / \mathrm{m}^{3}$ ) has decreased notably from historical levels (approximately $8 \mu \mathrm{g} / \mathrm{m}^{3}$ at perimeter locations above escarpment to $20 \mu \mathrm{g} / \mathrm{m}^{3}$ at industrial centre / harbour-front, 1985-1994 data; Jerrett et al., 2005a). The decreasing temporal trend was verified by review of historical $\mathrm{PM}_{2.5}$ data at Hamilton's NAPS and Ontario Ministry of Environment central 
monitoring sites, which show a decrease of approximately 35\% since 1999 both below and above the escarpment (Clean Air Hamilton, 2011), consistent with historical decreases reported for other Canadian urban sites (27\% decrease in mean annual ambient $\mathrm{PM}_{2.5}$ between 1990-2001; Environment Canada, 2004). The decrease in ambient $\mathrm{PM}_{2.5}$ in Hamilton can be largely attributed to reductions in local and regional source emissions: Canada-wide NPRI-reported PM $_{2.5}$ emissions between 1990 and 2010 decreased 54\% for industrial sources, $15 \%$ for non-industrial sources, and $35 \%$ for mobile sources (Environment Canada, 2011b). Examining NPRI emissions inventory data for local industrial sources, reported $\mathrm{PM}_{2.5}$ emissions from Hamilton harbour-front's two iron and steel manufacturing sites decreased by approximately $16 \%$ over the 2002 - 2010 reporting period (Environment Canada, 2011b). Despite the overall reduction in ambient $\mathrm{PM}_{2.5}$ from historical levels (Jerrett et al, 2005a), the Hamilton sampling campaigns confirmed that site-to-site variability in concentration remains significant, reflecting the ongoing contribution of local sources (e.g., heavy and light industry, traffic, commercial/institutional and residential space heating) to ambient exposures.

Comparing to nearby Windsor-Detroit urban data (Miller et al., 2010; summer campaign with $\mathrm{PM}_{2.5}$ and $\mathrm{PAH}$ sampling methods identical to present study), mean summer campaign $\mathrm{PM}_{2.5}$ in Hamilton was slightly lower (Hamilton: $6.7 \mu \mathrm{g} / \mathrm{m}^{3}$; Windsor: $7.2 \mu \mathrm{g} / \mathrm{m}^{3}$; Detroit: $8.0 \mu \mathrm{g} / \mathrm{m}^{3}$ ). Comparing ambient levels of the six common PAH species sampled by Miller et al., (2010), mean summer campaign total PAH (i.e., $\Sigma_{6}$ PAH, six commonly sampled species) was several times higher in Hamilton than in Windsor (Hamilton: $25.6 \mathrm{ng} / \mathrm{m}^{3}$; Windsor: $\left.9.5 \mathrm{ng} / \mathrm{m}^{3}\right)$ and approached the Detroit mean $\left(26.9 \mathrm{ng} / \mathrm{m}^{3}\right)$, 
a large urban centre with concentrated industry and traffic-related PAH sources. Hamilton PAH concentrations also compared well with an earlier study by Legzdins et al., (1994), considering that Legzdins sampled particle phase PAH at a single Hamilton location while this study sampled vapour and particle phase PAH at multiple locations (i.e., seven commonly sampled species, $\Sigma_{7} \mathrm{PAH}$, mean: Legzdins et al., $12.0 \mathrm{ng} / \mathrm{m}^{3}$; this study, $\left.18.7 \mathrm{ng} / \mathrm{m}^{3}\right)$.

The $\mathrm{PM}_{2.5}$ and $\mathrm{PAH}$ datasets were also divided into lower/upper subsets based on sampling site location relative to the escarpment, with descriptive statistics shown in Table 4-4. Median pollutant levels were statistically greater (Mann-Whitney, p < 0.05) below the Hamilton escarpment for $\mathrm{PM}_{2.5}$ in the winter campaign and for $\mathrm{PAH}$ in both summer and winter campaigns. Higher pollutant levels in lower Hamilton were expected and consistent with previous sampling in the city (Somers et al., 2004; Jerrett et al., 2005a). The statistically significant difference between lower and upper Hamilton concentrations was large for $\sum_{16} \mathrm{PAH}$ and $\sum_{\text {light }} \mathrm{PAH}$, with the lower site median approximately $70 \%$ to $80 \%$ greater than the upper site median in both sampling campaigns; in the winter campaign, where heavy PAH species were found in greater concentrations, the difference in $\Sigma_{\text {heavy }} \mathrm{PAH}$ was even larger, with the median concentration of the lower sites more than twice that of the upper sites. In comparison, the lower/upper difference in ambient concentrations was small for $\mathrm{PM}_{2.5}$, with no significant difference in the summer campaign and less than $20 \%$ difference in the winter campaign. 


\subsubsection{Summer versus winter sampling campaigns}

Median $\mathrm{PM}_{2.5}$ concentration was significantly higher in the winter campaign than in the summer campaign (Mann-Whitney, $\mathrm{p}<0.05$ ). Although $\mathrm{PM}_{2.5}$ is typically seasonally

highest in summer (Environment Canada, 2004), the higher $\mathrm{PM}_{2.5}$ levels sampled during the winter campaign in Hamilton were consistent with observations at nearby southern Ontario locations (Blanchard et al., 2002), likely due to weather conditions favourable for particle buildup (i.e., reduced mixing height, higher wind speeds and more frequent southwesterly winds transporting emissions to the study area from proximal expressway traffic sources). Our study thus confirmed that southern Ontario experiences winter episodes of elevated $\mathrm{PM}_{2.5}$, corroborating Blanchard et al., (2002).

PAH levels in the Great Lakes region are typically reported as higher in winter (Sun et al., 2006), which can be attributed to added emissions from combustion heating sources and factors associated with winter conditions (i.e., reduced mixing height, decreased atmospheric reactivity). In the Hamilton sampling, median $\Sigma_{16} \mathrm{PAH}$ and $\sum_{\text {light }} \mathrm{PAH}$ concentrations were statistically similar in the summer and winter campaigns $(p=0.197)$, consistent with urban sampling studies showing higher summer concentrations of light molecular weight PAH species due to significant volatilization from surfaces (e.g., roads) at high temperatures (Dimashki et al., 2001). The $\Sigma_{\text {heavy }}$ PAH levels, however, were shown to be statistically higher in the winter campaign $(\mathrm{p}<0.05)$, following the expected seasonal trend; this was consistent with the observed higher winter campaign $\mathrm{PM}_{2.5}$ levels, as these PAH species are found largely in the particulate phase. 


\subsubsection{PAH-PM $\mathbf{M}_{2.5}$ correlation}

The site-to-site $\mathrm{PAH}-\mathrm{PM}_{2.5}$ relationship was investigated by assessing correlations (Spearman's rank, $\mathrm{p}<0.05)$ for each sampling campaign (Table 4-3). Correlation between $\Sigma_{16} \mathrm{PAH}$ and $\mathrm{PM}_{2.5}$ was weak $(\varrho \leq 0.3)$ and not significant for the summer campaign; conversely, winter data correlation was moderately strong ( $\varrho=0.75)$ and highly significant. In both sampling campaigns, correlation of $\mathrm{PM}_{2.5}$ with the light and heavy PAH species groupings was similar to that with $\Sigma_{16} \mathrm{PAH}$.

In sampling comparable to the Hamilton summer campaign, Miller et al., (2010) found no correlation between $\Sigma_{6} \mathrm{PAH}$ and $\mathrm{PM}$ in Windsor for any of their three sampled size fractions although reporting a slightly greater correlation for the Detroit portion of their dataset. Elsewhere, Ohura et al., (2004) show weak PAH-PM 2.5 correlation (i.e., PAH sampling restricted to particle phase) but stronger correlation in winter conditions. In light of this, the moderate increase in $\mathrm{PAH}-\mathrm{PM}_{2.5}$ correlation seen in the Hamilton winter campaign may be due to the effect of reduced mixing height promoting a more uniform distribution among sites (lower CV of both pollutants in winter campaign, Table 4-2), and lower atmospheric reactivity of lighter $\mathrm{PAH}$ species. The higher $\mathrm{PAH}^{\mathrm{P}} \mathrm{PM}_{2.5}$ correlation in the winter campaign is also subject to the previously noted spatial bias introduced by the more limited $\mathrm{PM}_{2.5}$ sampling network (i.e., reduced site density in east and north areas below the escarpment). 


\subsubsection{Differing PAH-PM ${ }_{2.5}$ intraurban variability}

All three PAH metrics $\left(\Sigma_{16} \mathrm{PAH}, \Sigma_{\text {light }} \mathrm{PAH}, \Sigma_{\text {heavy }} \mathrm{PAH}\right)$ showed greater site-to-site variability than $\mathrm{PM}_{2.5}$ : intraurban variability $(\mathrm{CV}$, Table $4-2)$ was approximately three times greater for the summer campaign and four times greater in the winter campaign. This finding is consistent with previous studies in other urban centres (Windsor-Detroit: Miller et al., 2010; New York City, USA: Narvaez et al., 2008; Netherlands: Fischer et al., 2000).

The greater intraurban variability of $\mathrm{PAH}$ relative to $\mathrm{PM}_{2.5}$ was observed both above and below the escarpment in both campaigns (Table 4-4), with $\Sigma_{16} \mathrm{PAH}$ levels varying much more across lower Hamilton sites than they varied across upper Hamilton sites. The greater variation in land use, stationary sources, and road network in lower Hamilton (heavy industrial, light industrial, commercial, institutional, residential land use zones) compared with upper Hamilton (largely residential, retail, commercial land uses) suggests that local PAH sources are a significant component of the intraurban variability in PAH. The generally lower intraurban variability of $\mathrm{PM}_{2.5}$ may indicate that transported $\mathrm{PM}_{2.5}$ from regional sources is a larger component of ambient levels.

Sites with high $\Sigma \mathrm{PAH} / \mathrm{PM}_{2.5}$ (Equation 1) indicate potential hot spots for elevated PAH exposures, possibly from local PAH sources upwind and in close proximity to the sampling site. Comparing $\sum_{16} \mathrm{PAH} / \mathrm{PM}_{2.5}$ across the study area, sites with the highest PAH relative to $\mathrm{PM}_{2.5}$ were observed to occur below the escarpment and near or downwind of the central business district / harbour-front area in both sampling campaigns. Median $\Sigma_{16} \mathrm{PAH} / \mathrm{PM}_{2.5}$ was significantly higher (Mann-Whitney, $\left.\mathrm{p}<0.05\right)$ below the escarpment 
by approximately $80 \%$ and $60 \%$ in summer and winter campaigns, respectively (Table 4-5); examining PAH species found largely in particle phase, $\Sigma_{\text {heavy }} \mathrm{PAH} / \mathrm{PM}_{2.5}$ was also significantly higher at lower Hamilton sites in the winter campaign (Table 4-5). Site-tosite variability in $\Sigma_{16} \mathrm{PAH} / \mathrm{PM}_{2.5}$ and $\Sigma_{\text {heavy }} \mathrm{PAH} / \mathrm{PM}_{2.5}$ was also greater below the escarpment (CV, Table 5). Comparing to Windsor-Detroit sampling data (Miller et al., 2010), Hamilton mass ratios were consistently higher than the mean mass ratio in Windsor (Hamilton mean mass ratio four times and twice greater for lower and upper Hamilton sites, respectively) and also higher than mean mass ratio in Detroit (three times and nearly twice greater for lower and upper Hamilton sites, respectively). Pollutant mass ratios indicated that $\mathrm{PM}_{2.5}$ composition, relative to $\mathrm{PAH}$, can differ greatly at the intraurban scale and between relatively proximal urban centres.

\subsubsection{Intraurban variability in PAH concentration versus central site monitoring}

Ambient concentration at the field sampling site co-located with NAPS (Figure 4-1) was not a good proxy for $\mathrm{PM}_{2.5}$ or $\mathrm{PAH}$ across Hamilton, generally overestimating levels at sites above the escarpment and, more critically and despite being located below the escarpment, underestimating levels at sites below the escarpment (Figure 4-6, Figure 4-7). For $\mathrm{PM}_{2.5}$, this was primarily observed in the summer campaign since the range of $\mathrm{PM}_{2.5}$ concentrations in the winter campaign was relatively narrow. For $\mathrm{PAH}$, however, numerous sites had significantly higher levels than the NAPS location in both campaigns, with even proximal sites differing greatly from the PAH level sampled at the central site. Further, the pollutant mass ratios $\left(\Sigma \mathrm{PAH} / \mathrm{PM}_{2.5}\right)$ at the site co-located with NAPS were 
below the mean and median mass ratio for lower Hamilton sites in both campaigns (Table 4-5), demonstrating that central site monitoring was a poor intraurban estimate of $\mathrm{PM}_{2.5}$ composition relative to $\mathrm{PAH}$.

The large variability between central site and spatially distributed PAH concentrations, particularly for particulate-associated heavy molecular weight PAH, is likely due to the significant number of local sources in Hamilton (e.g., coke oven emissions from steel industry, exhaust emissions from gas and diesel fueled vehicles, heating combustion, wood combustion). As such, these findings are expected to apply to many urban centres containing mixed land uses (i.e., industrial, commercial, residential).

Our comparison of central site and spatially distributed field monitors in Hamilton agrees with previous research citing limitations of central site monitoring for $\mathrm{PM}_{2.5}$ exposure assessment (Kinney et al., 2006; Jerrett et al., 2005b; Pinto et al., 2004) and further demonstrates that central site monitoring is a poor proxy for PM toxicity, since particleassociated PAH concentrations were shown to vary greatly across the intraurban study area, relative to $\mathrm{PM}_{2.5}$ concentrations (i.e., $\Sigma_{\text {heavy }} \mathrm{PAH} / \mathrm{PM}_{2.5}, \Sigma_{16} \mathrm{PAH} / \mathrm{PM}_{2.5}$ ). This emphasizes that reliance on central site monitors in urban areas with multiple and spatially distributed local PAH sources carries potential for exposure misclassification for $\mathrm{PAH}$ as well as exposure health risk misclassification for $\mathrm{PM}_{2.5}$. 


\subsubsection{Intraurban variability in Benzo(a)Pyrene-equivalent toxicity}

The BaP-equivalent toxicity (BaP-TEQ, $\mathrm{ng} / \mathrm{m}^{3}$ ) dataset provided a more direct indication of particle-associated PAH toxicity. Descriptive statistics are shown in Table 4-2 and Table 4-4 and mapped by quartile in Figure 4-8. For both field campaigns, BaP-TEQ levels were statistically higher below the escarpment (Mann-Whitney, $\mathrm{p}<0.05$ ). The spatial distribution of BaP-TEQ was similar to that of $\sum_{16} \mathrm{PAH}$ : third and fourth quartile BaP-TEQ were generally at sites below the escarpment and first and second quartile BaPTEQ were at sites above the escarpment or on the study area's perimeter; sites with the highest carcinogenic potential were concentrated near the heavily industrialized harbourfront and east of it (downwind). Intraurban variability (CV, see Table 4-4) was approximately three to four times greater below the escarpment, suggesting that local sources and more diverse land use in lower Hamilton were contributing to higher BaPTEQ levels and greater variability between sites.

Since BaP-TEQ is calculated from the sum of TEF-weighted PAH concentrations, the general similarities observed in their spatial distribution (Figure 4-6, Figure 4-7) was expected. However, several sites classified as having relatively low ambient $\Sigma_{16} \mathrm{PAH}$ (first or second quartile) had high (third and fourth quartile) BaP-TEQ (see Figure 4-8, indicated with '+'). Closer examination of the speciated PAH data at these sites showed them to be enriched in the heavier molecular weight and more toxic (greater BaP-TEF) species. The comparisons of $\Sigma_{16} \mathrm{PAH}$ concentrations with BaP-TEQ suggests that $\Sigma_{16} \mathrm{PAH}$ should not be used as a sole proxy for chronic health risk, as doing so may result in sites 
being misclassified with respect to their toxicity; this is of greater concern where PAH toxicity is underestimated.

Correlation between field-sampled $\Sigma_{16} \mathrm{PAH}$ and BaP-TEQ (Spearman's rho, $\mathrm{p}<0.05$ ) was moderate in the summer campaign $(r=0.558)$ and strong in the winter campaign $(r=$ 0.923); correlation between $\Sigma_{\text {heavy }} \mathrm{PAH}$ and BaP-TEQ was strong in both datasets (summer campaign, $r=0.665$; winter campaign, $r=0.908$ ), since heavy molecular weight species tend to have much higher toxicity factors than lighter molecular weight species (Table 4-6). These results demonstrate that sampling could be restricted to heavy molecular weight species where a study's primary objective is to quantify PAH toxicity, potentially benefiting lab analysis resources for dense intraurban studies with a large number of PAH samplers requiring analysis (e.g., Jung et al, 2010). However, targeting a larger suite of PAH species that includes light molecular weight species permits more analyses options (e.g., source apportionment modeling).

Finally, BaP-TEQ concentrations across the study area also correlated well with the $\Sigma \mathrm{PAH} / \mathrm{PM}_{2.5}$ pollutant mass ratio (Equation 1) in both sampling campaigns (Spearman's rho: summer campaign, $\mathrm{r}=0.668$; winter campaign, $\mathrm{r}=0.916$ ). This demonstrated that the $\Sigma \mathrm{PAH} / \mathrm{PM}_{2.5}$ ratio can identify potential hotspots of elevated particle-associated $\mathrm{PAH}$, as previously noted, but can also be used as an indicator of intraurban locations with elevated exposure toxicity. 


\subsection{Conclusions}

This field study has provided a much denser sampling dataset of co-located $\mathrm{PM}_{2.5}$ and PAH than previously available. The information on the spatial distribution of PAH and BaP-TEQ in an urban setting can assist epidemiologists and other health researchers attempting to understand how intraurban variability can influence particle toxicity.

We acknowledge limitations inherent in the field sampling study. Sampling network spatial distribution was constrained by availability of equipment but achieved good siting density ( $\sim 1$ sampler $/ 5 \mathrm{~km}^{2} ; 1.5 \mathrm{~km}$ average inter-site distance). The two-week duration of each sampling campaign was not necessarily seasonally representative of summer and winter, although the observed data generally aligned well with expected seasonal trends. Potential sampler breakthrough of lightest molecular weight and most volatile PAH species naphthalene, acenaphthylene, and acenaphthene has been demonstrated for PUF sorbent particularly at high flow rates and high temperatures (US EPA, 1999), though PUF collection efficiencies of light molecular weight compounds have been shown to improve when sampling flow rate is reduced (Ward and Smith, 2004). Sampling flow rate used in our study was significantly less than previous studies and is reasonably expected to attenuate sorbent breakthrough of the lightest molecular weight PAH. However, since vapour phase breakthrough was not quantified, higher ambient temperatures in the summer campaign suggest that collection efficiencies for the lightest molecular weight species may have been reduced slightly compared to winter sampling campaign data. Sample degradation of other PAH species (e.g., BaP) is an additional inherent limitation due to potential reactions with ambient oxidants such as ozone, hydroxyl radical, or 
nitrogen oxides (Jung et al, 2010); further, nitrated/oxygenated PAH species were not analysed in this study. Thus, sampled PAH concentrations likely represent lower values. Finally, while BaP-TEQ as presented indicates variation in potential chronic health risk across the study area, individual health risk will depend on individual exposure and possible synergistic effects between pollutants and other risk factors.

Pollutant mapping and statistical analysis of the Hamilton dataset revealed ambient $\mathrm{PM}_{2.5}$ and PAH concentrations to be significantly greater below the city's escarpment with a below/above escarpment difference in concentration much greater for $\mathrm{PAH}$ than for $\mathrm{PM}_{2.5}$ in both summer and winter campaigns. Elevated levels of both pollutants were observed near or downwind of the central business district and industrialized harbour-front area, suggesting that contribution of local sources (e.g., industry, traffic, space heating, wood combustion) can be important when assessing exposure. PAH concentrations sampled at the central site monitor were often exceeded at other locations in the city and hence could not be considered conservative estimates of PAH levels for the city as a whole. This is likely due to the significant number of locally distributed sources, findings that are expected to apply to many urban centres containing mixed land uses (i.e., industrial, commercial, residential).

Ambient PAH exhibited a substantially greater degree of intraurban variability than $\mathrm{PM}_{2.5}$, particularly for heavy molecular weight species found predominantly in the particle phase. Calculation of BaP-TEQ provided a better indicator of the chronic health risk (i.e., carcinogenicity) associated with field-sampled ambient PAH levels. Although the BaP-TEQ levels showed less intraurban variability than PAH $\left(\sum_{16} \mathrm{PAH}\right)$ and a 
generally similar spatial distribution, several sites classified as having relatively low ambient $\Sigma_{16} \mathrm{PAH}$ had high BaP-TEQ and were enriched in more toxic and heavier molecular weight PAH species.

Overall, the spatially dense dataset demonstrated the potential for large variability in $\mathrm{PM}_{2.5}$ composition at the local scale, underscoring that reliance on central site monitors in urban areas with multiple and spatially distributed local PAH sources carries potential for exposure misclassification for $\mathrm{PAH}$ as well as exposure health risk misclassification for $\mathrm{PM}_{2.5}$. Pollutant mass ratios $\left(\Sigma \mathrm{PAH} / \mathrm{PM}_{2.5}\right)$ provided a simple indicator metric for $\mathrm{PM}_{2.5}$ composition with respect to PAH. These correlated well with BaP-TEQ concentrations, identifying potential exposure and toxicity 'hot spots' for local PAH source contributions and could be applied to future studies in other urban centres. As toxic air pollutants are increasingly studied and regulated, future exposure assessment work can benefit from quantification of local scale differences in ambient PAH concentration, particularly for heavy molecular weight species demonstrating greater toxicity. 
Table 4-1. Sampled PAH species, grouping by molecular weight, sites above Lab Detection Limit.

\begin{tabular}{lrrrrrrr}
\hline PAH Species & $\begin{array}{r}\text { Chemical } \\
\text { Formula }\end{array}$ & \# rings & $\begin{array}{r}\text { Molecular } \\
\text { Weight } \\
\text { (MW) }\end{array}$ & $\begin{array}{r}\text { MW } \\
\text { Grouping }\end{array}$ & $\begin{array}{r}\text { Lab Detection } \\
\text { Limit (LDL) } \\
\text { [ng/sample] }\end{array}$ & $\begin{array}{r}\text { Summer } \\
\text { sites above } \\
\text { LDL (\%) }\end{array}$ & $\begin{array}{r}\text { Winter } \\
\text { sites above } \\
\text { LDL (\%) }\end{array}$ \\
\hline naphthalene & $\mathrm{C}_{10} \mathrm{H}_{8}$ & 2 & 128.2 & light & 4.94 & $89 \%$ & $0 \%$ \\
acenaphthylene & $\mathrm{C}_{12} \mathrm{H}_{8}$ & 3 & 152.2 & light & 5.67 & $69 \%$ & $3 \%$ \\
acenapthene & $\mathrm{C}_{12} \mathrm{H}_{10}$ & 3 & 154.2 & light & 5.34 & $97 \%$ & $100 \%$ \\
fluorene & $\mathrm{C}_{13} \mathrm{H}_{10}$ & 3 & 166.2 & light & 4.09 & $100 \%$ & $100 \%$ \\
phenanthrene & $\mathrm{C}_{14} \mathrm{H}_{10}$ & 3 & 178.2 & light & 3.04 & $100 \%$ & $100 \%$ \\
anthracene & $\mathrm{C}_{14} \mathrm{H}_{10}$ & 3 & 178.2 & light & 3.97 & $100 \%$ & $100 \%$ \\
fluoranthene & $\mathrm{C}_{16} \mathrm{H}_{10}$ & 4 & 202.3 & light & 2.72 & $100 \%$ & $100 \%$ \\
pyrene & $\mathrm{C}_{16} \mathrm{H}_{10}$ & 4 & 202.3 & light & 2.14 & $100 \%$ & $100 \%$ \\
benzo[a]anthracene & $\mathrm{C}_{18} \mathrm{H}_{12}$ & $>4$ & 228.3 & heavy & 4.50 & $19 \%$ & $61 \%$ \\
chrysene & $\mathrm{C}_{18} \mathrm{H}_{12}$ & $>4$ & 228.3 & heavy & 4.39 & $47 \%$ & $100 \%$ \\
benzo[b]fluoranthene & $\mathrm{C}_{20} \mathrm{H}_{12}$ & $>4$ & 252.3 & heavy & 7.25 & $3 \%$ & $0 \%$ \\
benzo[k]fluoranthene & $\mathrm{C}_{20} \mathrm{H}_{12}$ & $>4$ & 252.3 & heavy & 7.12 & $3 \%$ & $100 \%$ \\
benzo[a]pyrene & $\mathrm{C}_{20} \mathrm{H}_{12}$ & $>4$ & 252.3 & heavy & 7.56 & $3 \%$ & $39 \%$ \\
indeno[1,2,3-cd]pyrene & $\mathrm{C}_{22} \mathrm{H}_{12}$ & $>4$ & 276.3 & heavy & 9.18 & $0 \%$ & $15 \%$ \\
dibenz[a,h]anthracene & $\mathrm{C}_{22} \mathrm{H}_{14}$ & $>4$ & 278.4 & heavy & 8.58 & $0 \%$ & $0 \%$ \\
benzo[g,h,i]perylene & $\mathrm{C}_{22} \mathrm{H}_{12}$ & $>4$ & 276.3 & heavy & 8.27 & $0 \%$ & $42 \%$ \\
\hline
\end{tabular}

Note: Molecular weight data from ATSDR, 1995.

Table 4-2. Field sampling data retention and descriptive statistics.

\begin{tabular}{|c|c|c|c|c|c|c|c|c|}
\hline Pollutant & $\begin{array}{r}\text { \# sites } \\
\text { deployed } \\
\text { (lost) }\end{array}$ & $\begin{array}{r}\text { \# sites } \\
\text { invalid } \\
\text { (outliers) }\end{array}$ & $\begin{array}{r}\text { retained, } \\
\text { n (\%) }\end{array}$ & kurtosis & $\begin{array}{r}\text { mean } \\
\text { (std.dev) }\end{array}$ & $\begin{array}{r}\text { CV, } \\
\%\end{array}$ & $\min -\max$ & Q1 / median / Q3 \\
\hline \multicolumn{9}{|c|}{ SUMMER SAMPLING } \\
\hline $\mathbf{P M}_{2.5}$ & $43(3)$ & $5(1)$ & $35(81 \%)$ & 3.2 & $6.71(1.44)$ & 21.5 & $2.46-11.0$ & $6.10 / 6.43 / 6.95$ \\
\hline$\Sigma_{16}$ PAH & $43(4)$ & $3(2)$ & $36(84 \%)$ & 2.6 & $27.5(17.5)$ & 63.6 & $10.2-83.7$ & $16.6 / 21.2 / 31.4$ \\
\hline$\Sigma_{\text {light }} \mathbf{P A H}$ & - & - & $36(84 \%)$ & 2.7 & $26.6(17.2)$ & 64.6 & $9.39-82.9$ & $15.9 / 20.5 / 30.7$ \\
\hline$\Sigma_{\text {heavy }}$ PAH & - & - & $36(84 \%)$ & 29.8 & $0.937(0.740)$ & 79.0 & $0.663-5.07$ & $0.698 / 0.746 / 0.843$ \\
\hline BaP-TEQ & - & - & $36(84 \%)$ & 24.4 & $0.716(0.102)$ & 14.2 & $0.587-1.26$ & $0.673 / 0.693 / 0.731$ \\
\hline \multicolumn{9}{|c|}{ WINTER SAMPLING } \\
\hline $\mathbf{P M}_{2.5}$ & $46(6)$ & $9(1)$ & $31(67 \%)$ & 2.3 & $10.7(1.33)$ & 12.4 & $6.52-13.4$ & $10.1 / 10.7 / 11.9$ \\
\hline$\Sigma_{16}$ PAH & $46(7)$ & $6(0)$ & $33(72 \%)$ & 0.4 & $22.3(11.6)$ & 51.7 & $8.31-52.1$ & $14.0 / 18.6 / 28.0$ \\
\hline$\Sigma_{\text {light }}$ PAH & - & - & $33(72 \%)$ & 0.5 & $20.1(10.3)$ & 51.4 & $7.30-47.4$ & $12.8 / 16.7 / 25.6$ \\
\hline$\Sigma_{\text {heavy }}$ PAH & - & - & $33(72 \%)$ & 0.1 & $2.21(1.25)$ & 56.6 & $1.02-5.29$ & $1.24 / 1.71 / 2.73$ \\
\hline BaP-TEQ & - & - & $33(72 \%)$ & -0.3 & $0.837(0.193)$ & 23.1 & $0.634-1.29$ & $0.685 / 0.756 / 0.973$ \\
\hline
\end{tabular}

Note: $\mathrm{PM}_{2.5}\left[\mu \mathrm{g} / \mathrm{m}^{3}\right]$, PAH $\left[\mathrm{ng} / \mathrm{m}^{3}\right]$, BaP-TEQ $\left[\mathrm{ng} / \mathrm{m}^{3}\right]$. 
Table 4-3. Correlation between sampled $P A H$ and $P_{2.5}$ (Spearman's rho).

\begin{tabular}{lrr}
\hline Pollutant & $\begin{array}{r}\text { SUMMER } \\
\text { PM }_{2.5}\end{array}$ & $\begin{array}{r}\text { WINTER } \\
\mathbf{P M}_{2.5}\end{array}$ \\
\hline $\boldsymbol{\Sigma}_{\mathbf{1 6}} \mathbf{P A H}$ & 0.293 & $0.751 *$ \\
$\boldsymbol{\Sigma}_{\text {light }} \mathbf{P A H}$ & 0.289 & $0.758 *$ \\
$\boldsymbol{\Sigma}_{\text {heavy }} \mathbf{P A H}$ & 0.295 & $0.711 *$ \\
\hline
\end{tabular}

Note: Spearman's rho, significance denoted by $*(\mathrm{p}<0.05)$

Table 4-4. Descriptive statistics for field sampling data below/above (lower/upper) Hamilton escarpment.

\begin{tabular}{|c|c|c|c|c|c|c|c|}
\hline Pollutant & $\begin{array}{l}\text { lower / } \\
\text { upper }\end{array}$ & $\begin{array}{r}\text { sites, } \\
\mathbf{n}\end{array}$ & mean & std.dev & $\mathrm{CV}, \%$ & $\min -\max$ & Q1/median/Q3 \\
\hline \multicolumn{8}{|c|}{ SUMMER SAMPLING } \\
\hline \multirow[t]{2}{*}{$\mathbf{P M}_{2.5}$} & lower & 19 & 7.03 & 1.24 & 17.6 & $4.89-9.40$ & $6.26 / 6.57 / 7.81$ \\
\hline & upper & 16 & 6.33 & 1.61 & 25.4 & $2.46-11.0$ & $5.95 / 6.27 / 6.60$ \\
\hline \multirow[t]{2}{*}{$\Sigma_{16}$ PAH } & lower & 19 & 36.9 & 19.6 & 53.2 & $13.9-83.7$ & $21.7 / \mathbf{2 9 . 8 *} / 52.2$ \\
\hline & upper & 17 & 17.1 & 4.14 & 24.3 & $10.2-22.3$ & $13.2 / \mathbf{1 7 . 5} * / 21.0$ \\
\hline \multirow[t]{2}{*}{$\Sigma_{\text {light }}$ PAH } & lower & 19 & 35.8 & 19.2 & 53.8 & $13.2-82.9$ & $21.0 / \mathbf{2 9 . 0} * / 51.5$ \\
\hline & upper & 17 & 16.3 & 4.14 & 25.4 & $9.39-21.5$ & $12.4 / \mathbf{1 6 . 7} * / 20.3$ \\
\hline \multirow[t]{2}{*}{$\Sigma_{\text {heavy }}$ PAH } & lower & 19 & 1.11 & 0.995 & 89.4 & $0.670-5.07$ & $0.712 / \mathbf{0 . 8 0 7} * / 1.07$ \\
\hline & upper & 17 & 0.741 & 0.0713 & 9.63 & $0.663-0.907$ & $0.682 / \mathbf{0 . 7 3 2} * / 0.779$ \\
\hline \multirow[t]{2}{*}{ BaP-TEQ } & lower & 19 & 0.750 & 0.128 & 17.1 & $0.672-1.26$ & $0.691 / \mathbf{0 . 7 2 5} * / 0.769$ \\
\hline & upper & 17 & 0.678 & 0.034 & 5.04 & $0.587-0.732$ & $0.662 / \mathbf{0 . 6 7 4} * / 0.706$ \\
\hline \multicolumn{8}{|c|}{ WINTER SAMPLING } \\
\hline \multirow[t]{2}{*}{$\mathbf{P M}_{2.5}$} & lower & 14 & 11.7 & 0.987 & 8.47 & $10.3-13.4$ & $10.8 / \mathbf{1 1 . 9}$ */ 12.5 \\
\hline & upper & 17 & 10.0 & 1.12 & 11.2 & $6.52-11.3$ & $9.63 / \mathbf{1 0 . 1} * / 10.8$ \\
\hline \multirow[t]{2}{*}{$\Sigma_{16}$ PAH } & lower & 19 & 28.4 & 11.8 & 41.5 & $12.0-52.1$ & $19.0 / \mathbf{2 5 . 5}$ * / 39.4 \\
\hline & upper & 14 & 14.1 & 2.94 & 20.9 & $8.31-19.1$ & $12.0 / 14.4 * / 16.5$ \\
\hline \multirow[t]{2}{*}{$\Sigma_{\text {light }}$ PAH } & lower & 19 & 25.5 & 10.6 & 41.4 & $10.7-47.4$ & $17.4 / \mathbf{2 2 . 8 *} / 35.1$ \\
\hline & upper & 14 & 12.8 & 2.70 & 21.1 & $7.30-17.1$ & $10.9 / \mathbf{1 3 . 1} * 14.9$ \\
\hline \multirow[t]{2}{*}{$\Sigma_{\text {heavy }} \mathbf{P A H}$} & lower & 19 & 2.87 & 1.27 & 44.3 & $1.17-5.29$ & $1.93 / 2.67 * / 4.17$ \\
\hline & upper & 14 & 1.31 & 0.299 & 22.8 & $1.02-2.03$ & $1.06 / \mathbf{1 . 2 6} * / 1.46$ \\
\hline \multirow[t]{2}{*}{ BaP-TEQ } & lower & 19 & 0.945 & 0.191 & 20.2 & $0.677-1.29$ & $0.769 / \mathbf{0 . 9 4 1} * / 1.14$ \\
\hline & upper & 14 & 0.690 & 0.033 & 4.79 & $0.634-0.756$ & $0.663 / \mathbf{0 . 6 9 3} * / 0.705$ \\
\hline
\end{tabular}

Note: $\mathrm{PM}_{2.5}\left[\mu \mathrm{g} / \mathrm{m}^{3}\right]$, PAH $\left[\mathrm{ng} / \mathrm{m}^{3}\right]$, BaP-TEQ $\left[\mathrm{ng} / \mathrm{m}^{3}\right]$. Significant difference between lower and upper median concentrations indicated by * (Mann-Whitney, $\mathrm{p}<0.05$ ). 
Table 4-5. PAH/PM $\mathbf{P M}_{2.5}$ mass ratios for sites with summer and winter data $(\mathrm{ng} / \mu \mathrm{g})$.

\begin{tabular}{|c|c|c|c|c|c|c|c|}
\hline $\begin{array}{l}\text { Pollutant Mass } \\
\text { Ratio }[n g / \mu g]\end{array}$ & $\begin{array}{l}\text { lower / } \\
\text { upper }\end{array}$ & $\begin{array}{r}\text { sites, } \\
\text { n }\end{array}$ & mean & median & std. dev. & $\mathrm{CV}, \%$ & $\min -\max$ \\
\hline \multicolumn{8}{|c|}{ SUMMER SAMPLING } \\
\hline \multirow[t]{2}{*}{$\Sigma_{16} \mathbf{P A H} / \mathbf{P M}_{2.5}$} & lower & 11 & 6.07 & 5.11* & 2.84 & 46.8 & $2.70-12.73$ \\
\hline & upper & 10 & 2.72 & 2.82* & 0.85 & 31.1 & $1.31-4.14$ \\
\hline \multicolumn{8}{|c|}{ WINTER SAMPLING } \\
\hline \multirow[t]{2}{*}{$\Sigma_{16} \mathbf{P A H} / \mathbf{P M}_{2.5}$} & lower & 11 & 2.46 & $2.26 *$ & 0.89 & 36.3 & $1.12-4.29$ \\
\hline & upper & 10 & 1.46 & $1.43^{*}$ & 0.24 & 16.6 & $1.12-1.94$ \\
\hline \multirow[t]{2}{*}{$\Sigma_{\text {heavy }} \mathbf{P A H} / \mathbf{P M}_{2.5}$} & lower & 11 & 0.243 & $0.230 *$ & 0.095 & 39.0 & $0.107-0.418$ \\
\hline & upper & 10 & 0.135 & $0.122 *$ & 0.034 & 25.0 & $0.101-0.206$ \\
\hline
\end{tabular}

Note: Pollutant mass ratio $[\mathrm{ng} / \mu \mathrm{g}]$; summer $\Sigma_{\text {heavy }} \mathrm{PAH} / \mathrm{PM}_{2.5}$ not reported due to majority of heavy MW species levels below LDL at most sites. Significant difference between lower and upper median values indicated by * (Mann-Whitney, $p$ $<0.05)$.

Table 4-6. BaP-equivalent carcinogenic Toxic Equivalency Factors (BaP-TEF) for inhalation exposure.

\begin{tabular}{lr}
\hline PAH Species & BaP-TEF \\
\hline naphthalene & 0.001 \\
acenaphthylene & 0.001 \\
acenapthene & 0.001 \\
fluorene & 0.001 \\
phenanthrene & 0.001 \\
anthracene & 0.01 \\
fluoranthene & 0.001 \\
pyrene & 0.001 \\
benzo[a]anthracene & 0.1 \\
chrysene & 0.01 \\
benzo[b]fluoranthene & 0.1 \\
benzo[k]fluoranthene & 0.1 \\
benzo[a]pyrene & 1 \\
indeno[1,2,3-cd]pyrene & 0.1 \\
dibenz[a,h]anthracene & 5 \\
benzo[g,h,i]perylene & 0.01 \\
\hline
\end{tabular}

Note: Data from Nisbet and Lagoy, 1992; ATSDR, 1995. 


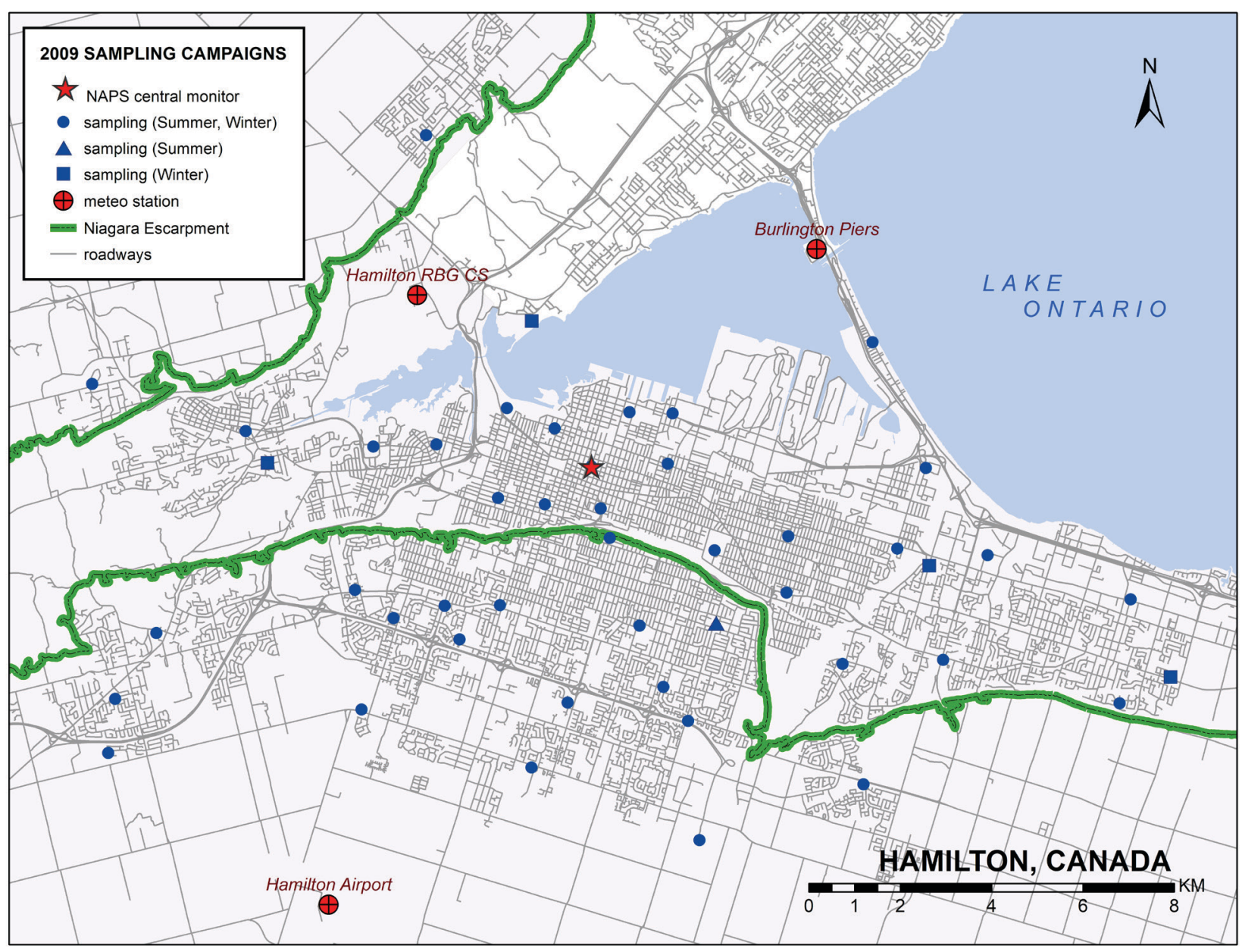

Figure 4-1. Study area showing summer and winter campaign sites, NAPS site, meteorology stations. 


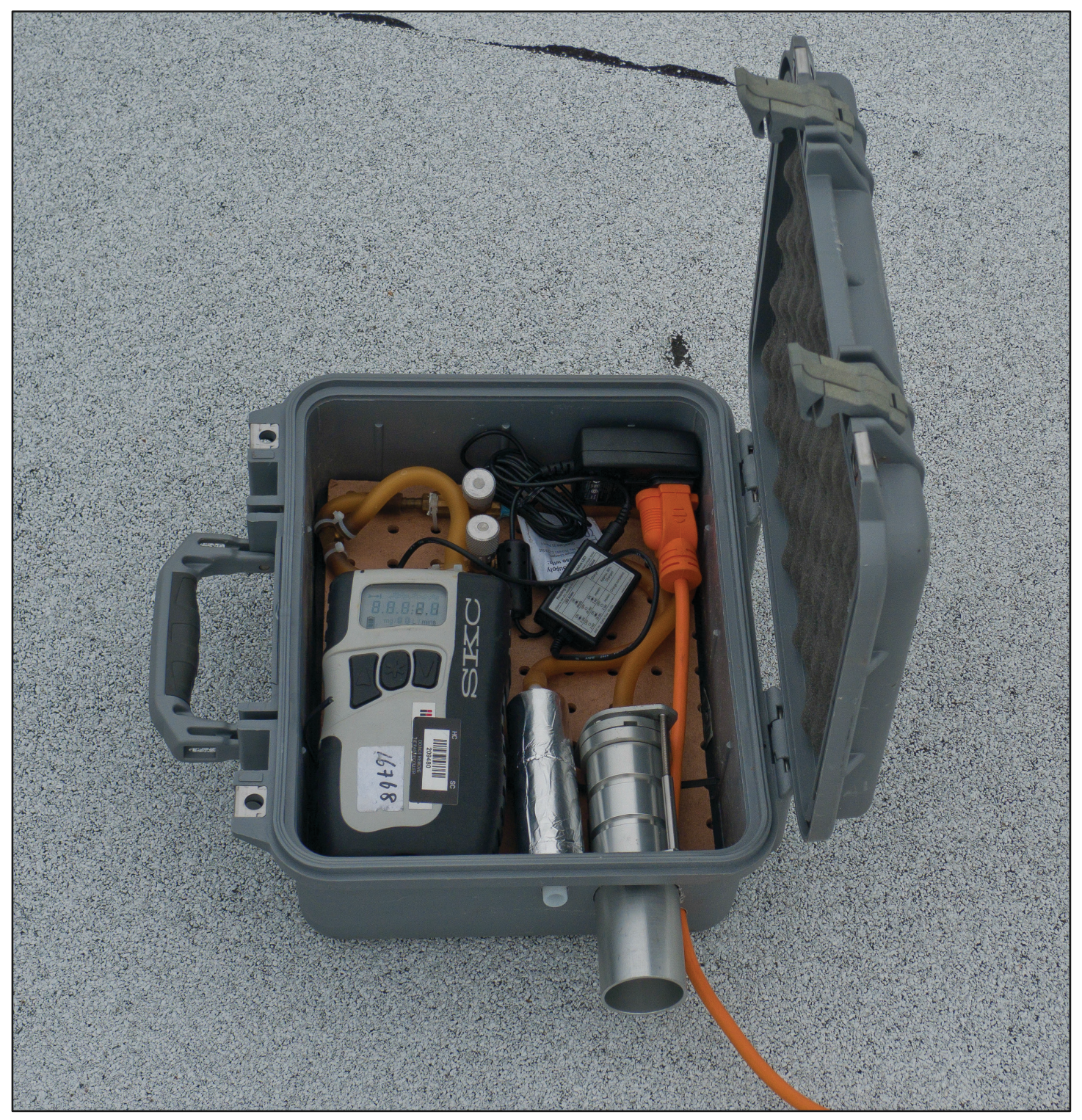

Figure 4-2. PAH and PM samplers and airflow pump inside weatherproof case. 


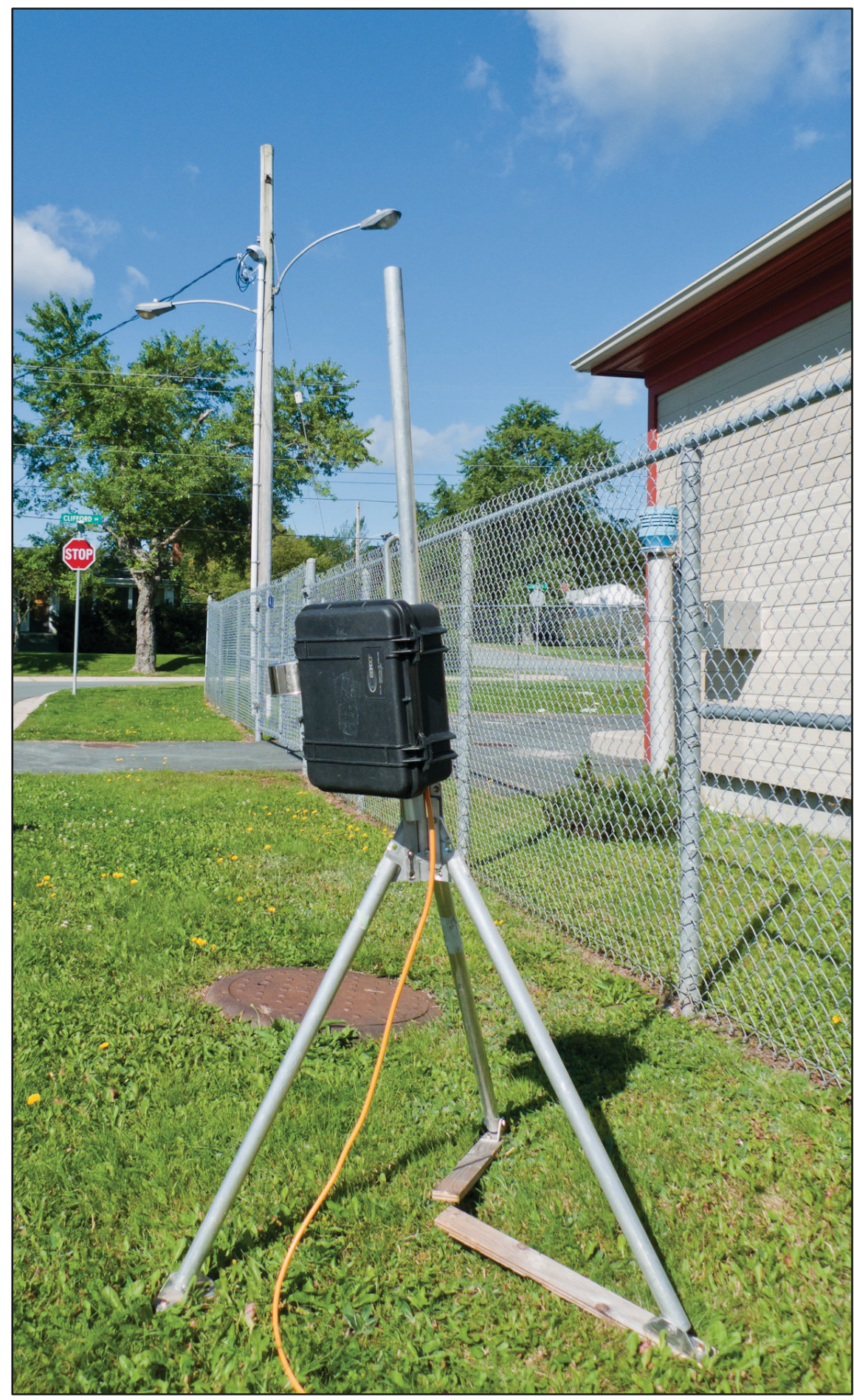

Figure 4-3. Typical ground-level sampler setup (i.e., sampler case on sampling tripod). 


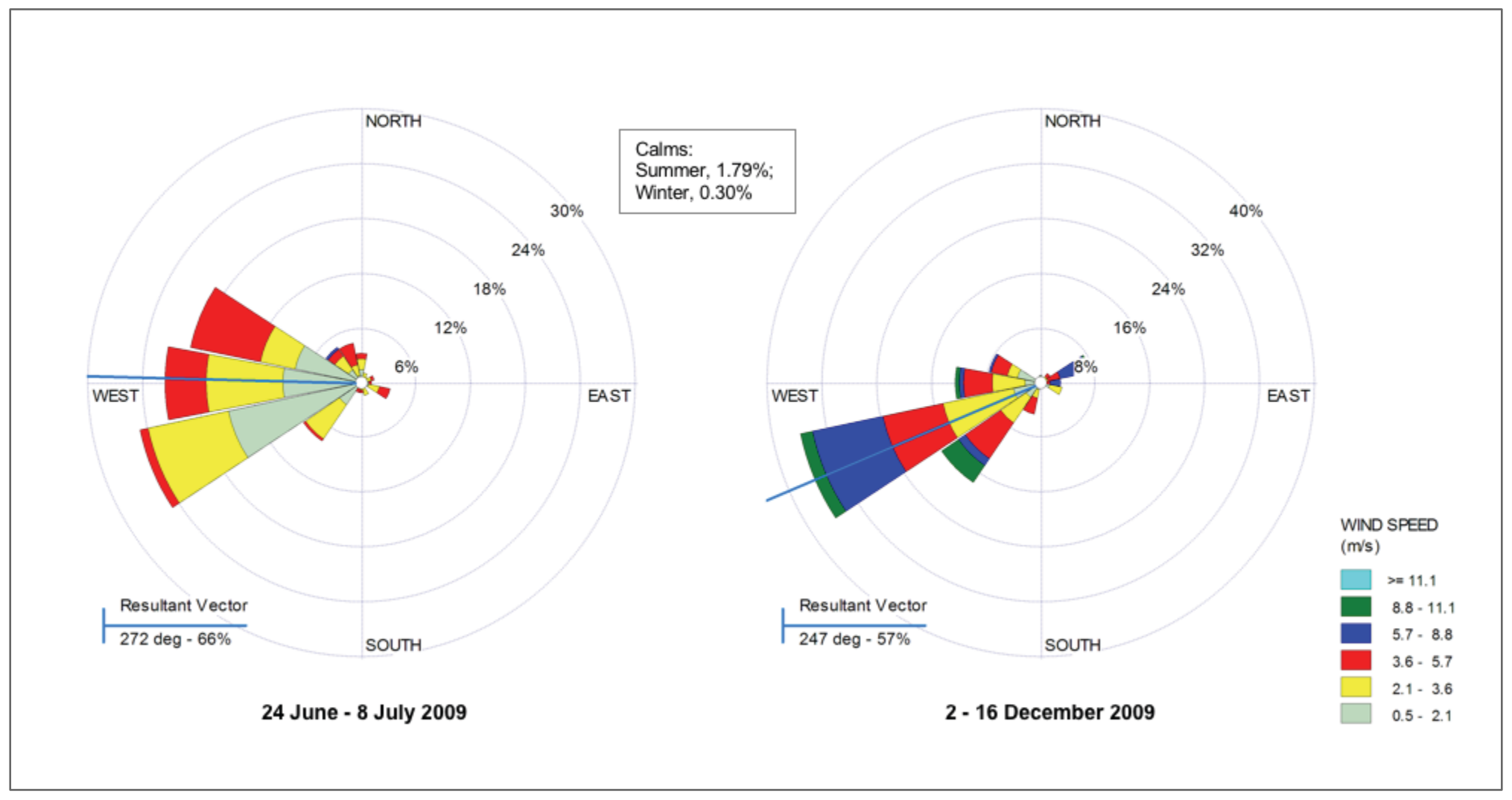

Figure 4-4. Wind rose for Hamilton study area during summer and winter campaigns. 
summer campaign

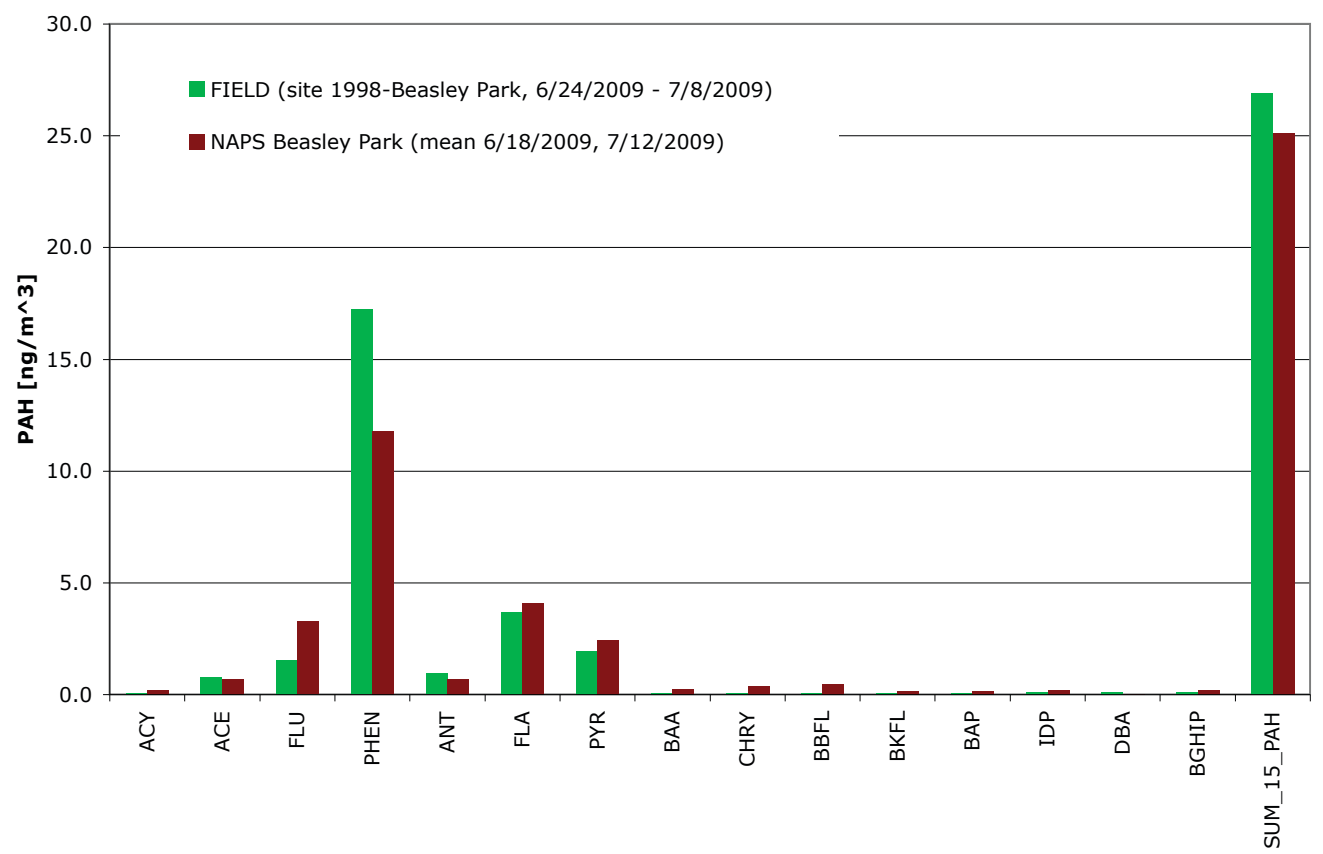

winter campaign

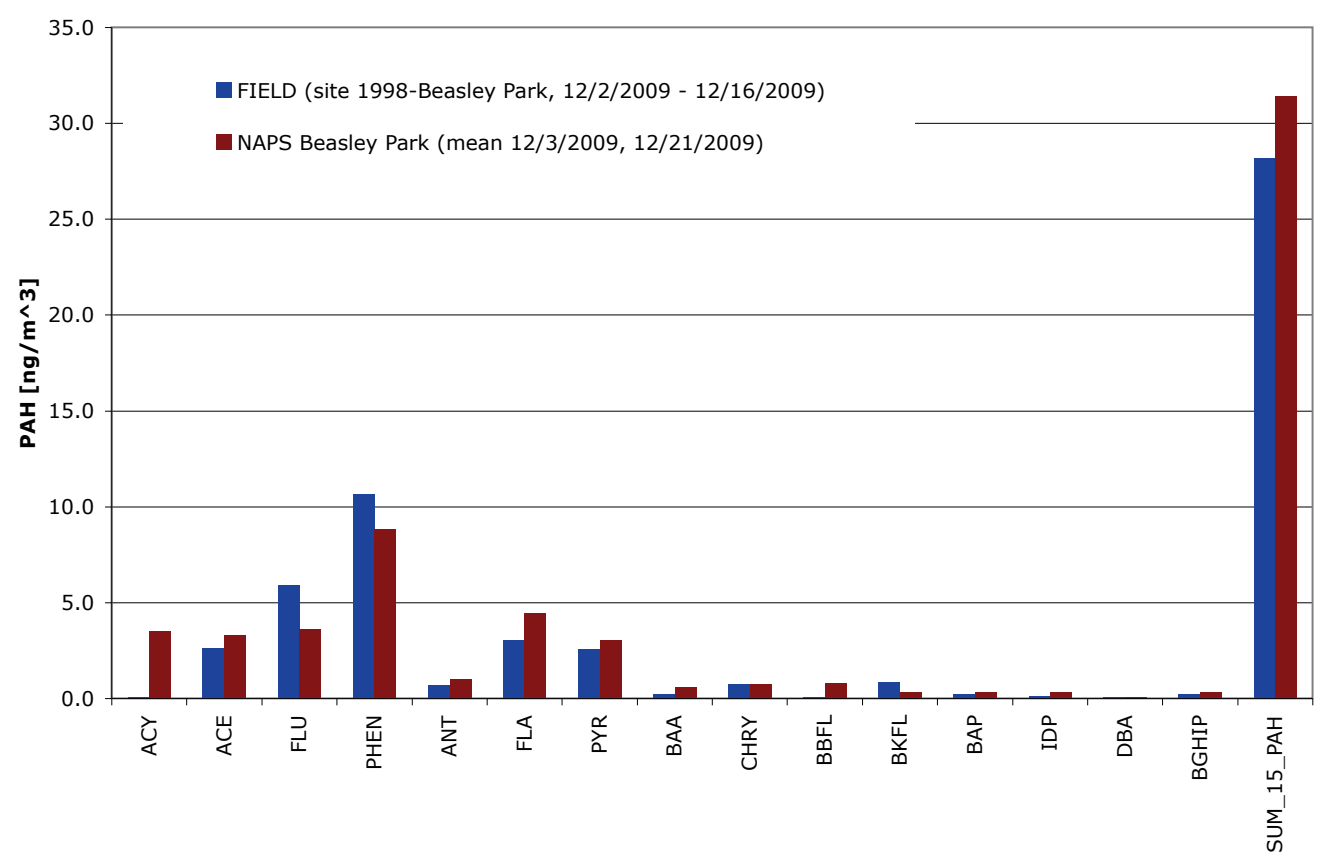

Figure 4-5. Co-located field-sampled PAH and NAPS-sampled mean PAH (nearest bracketing sampling dates). 


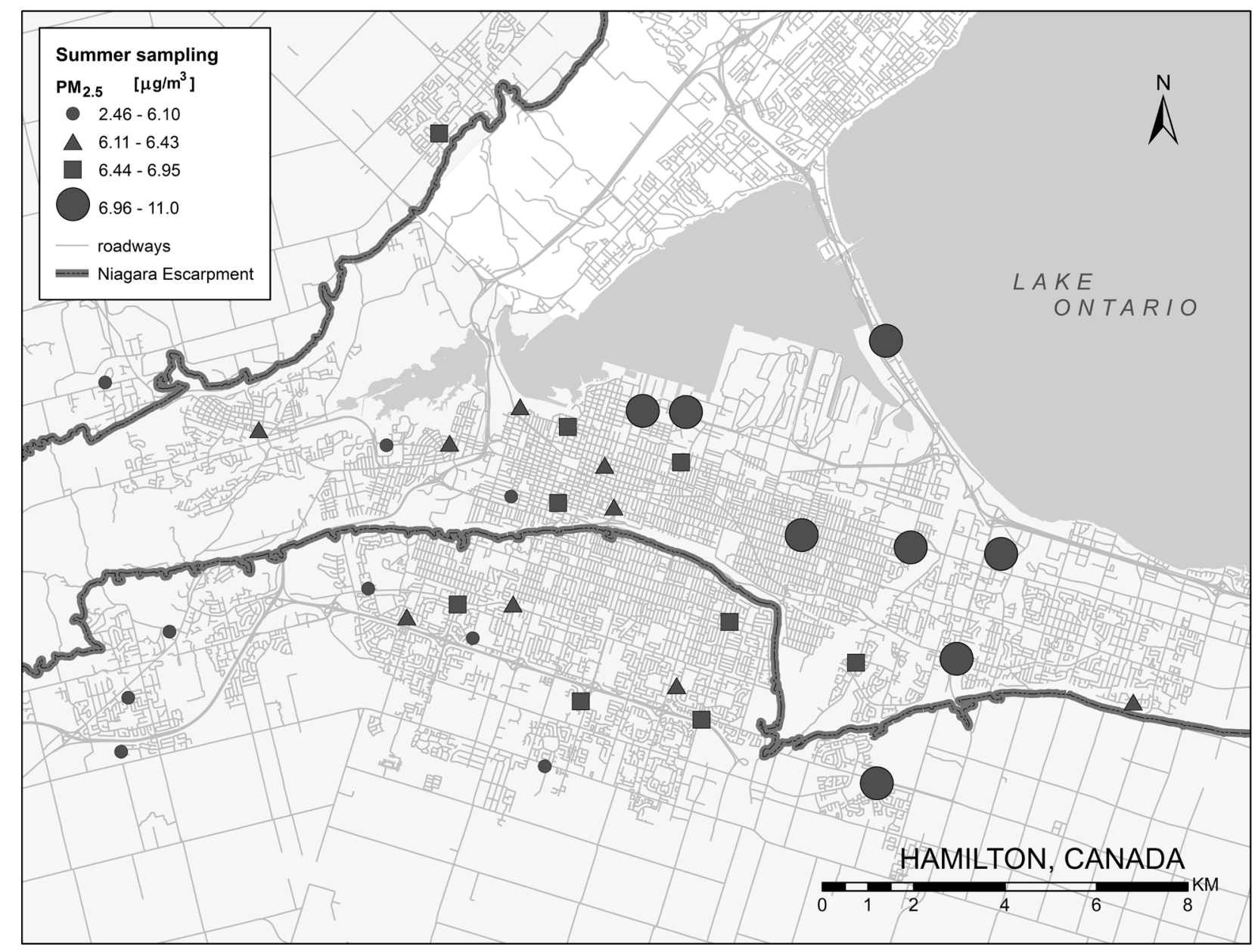

Figure 4-6. Sampled $\mathrm{PM}_{2.5}$ concentrations, by quartile, summer and winter campaigns. 


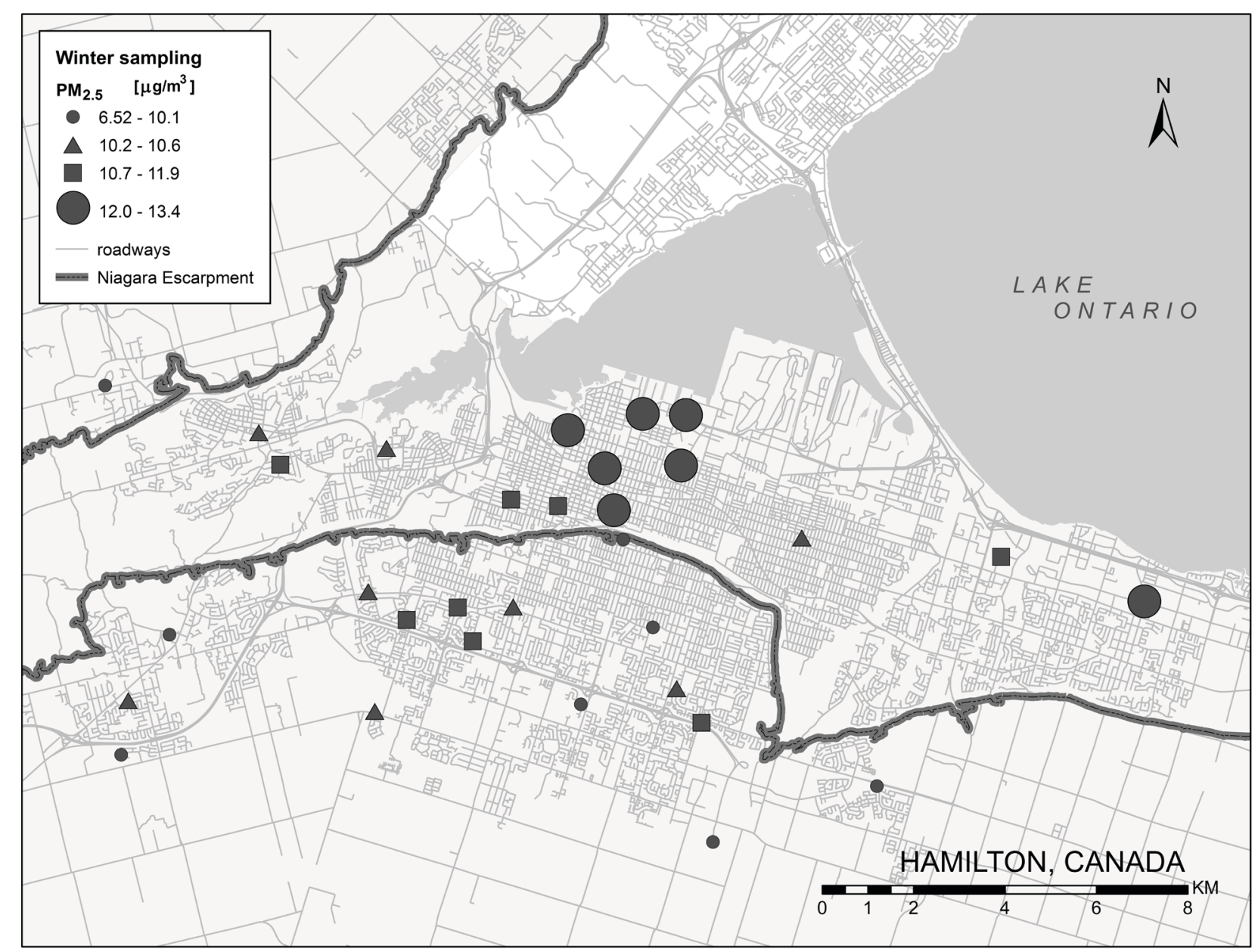

Figure 4-6 (continued) 


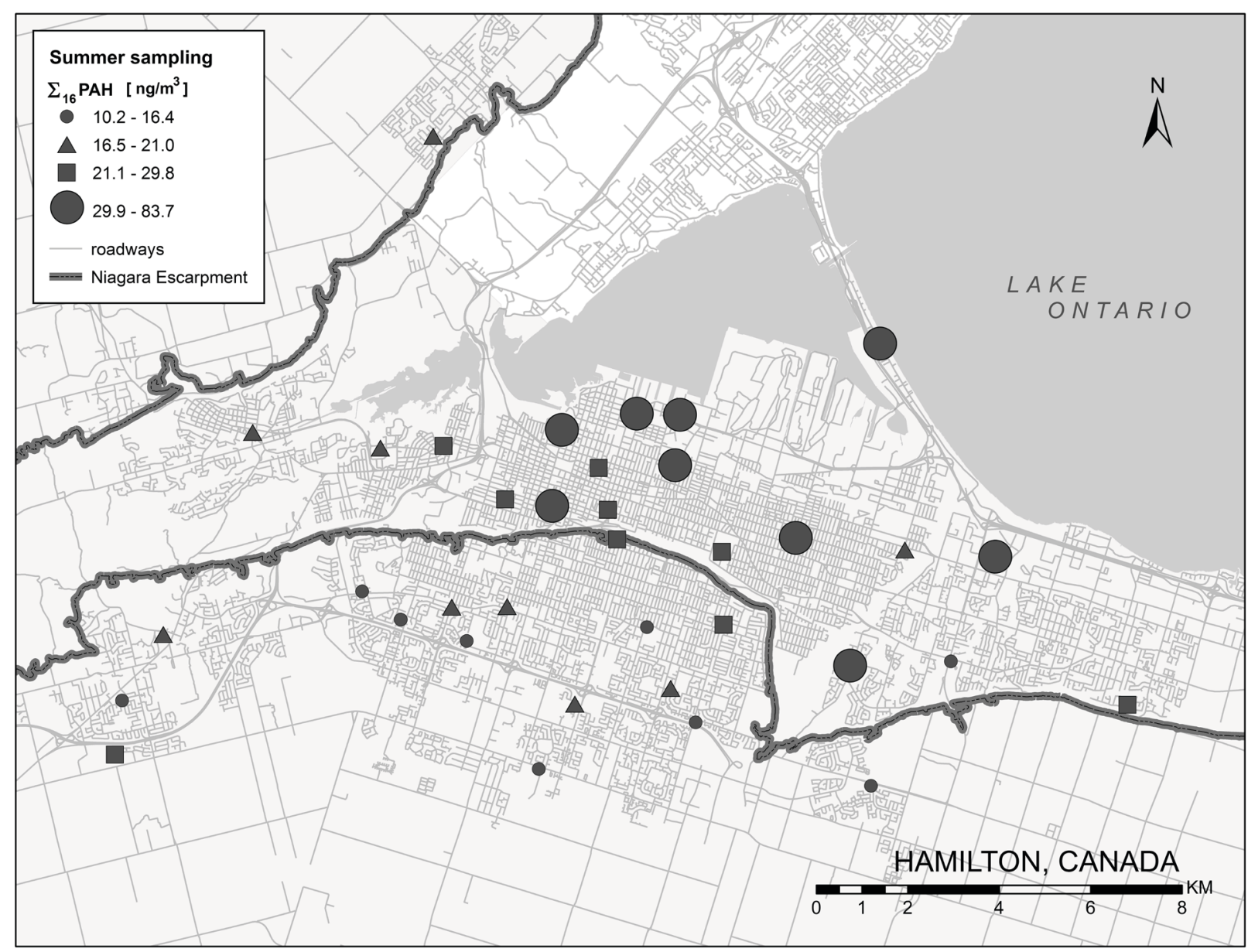

Figure 4-7. Sampled $\Sigma_{16} \mathrm{PAH}$ concentrations, by quartile, summer and winter campaigns. 


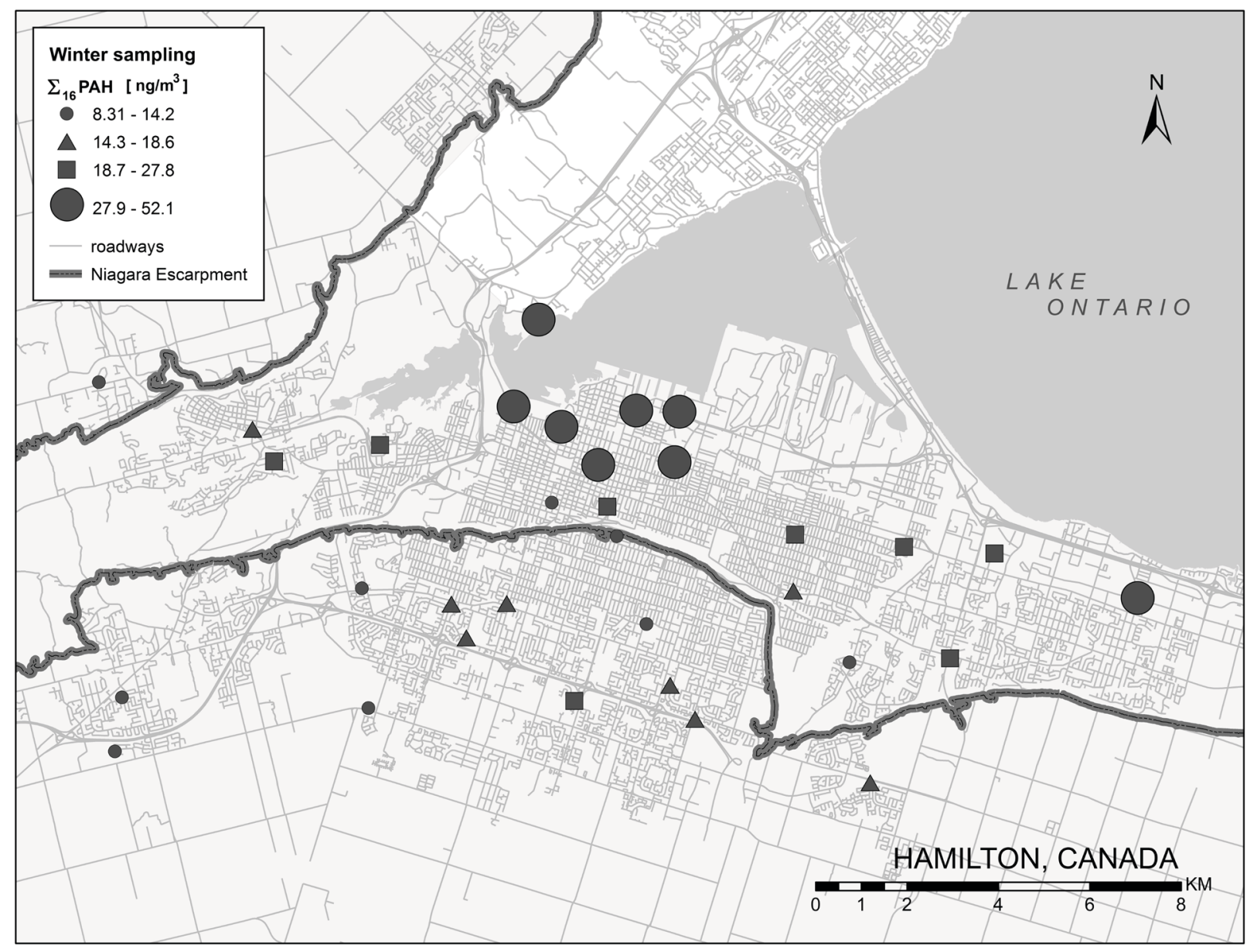

Figure 4-7 (continued) 


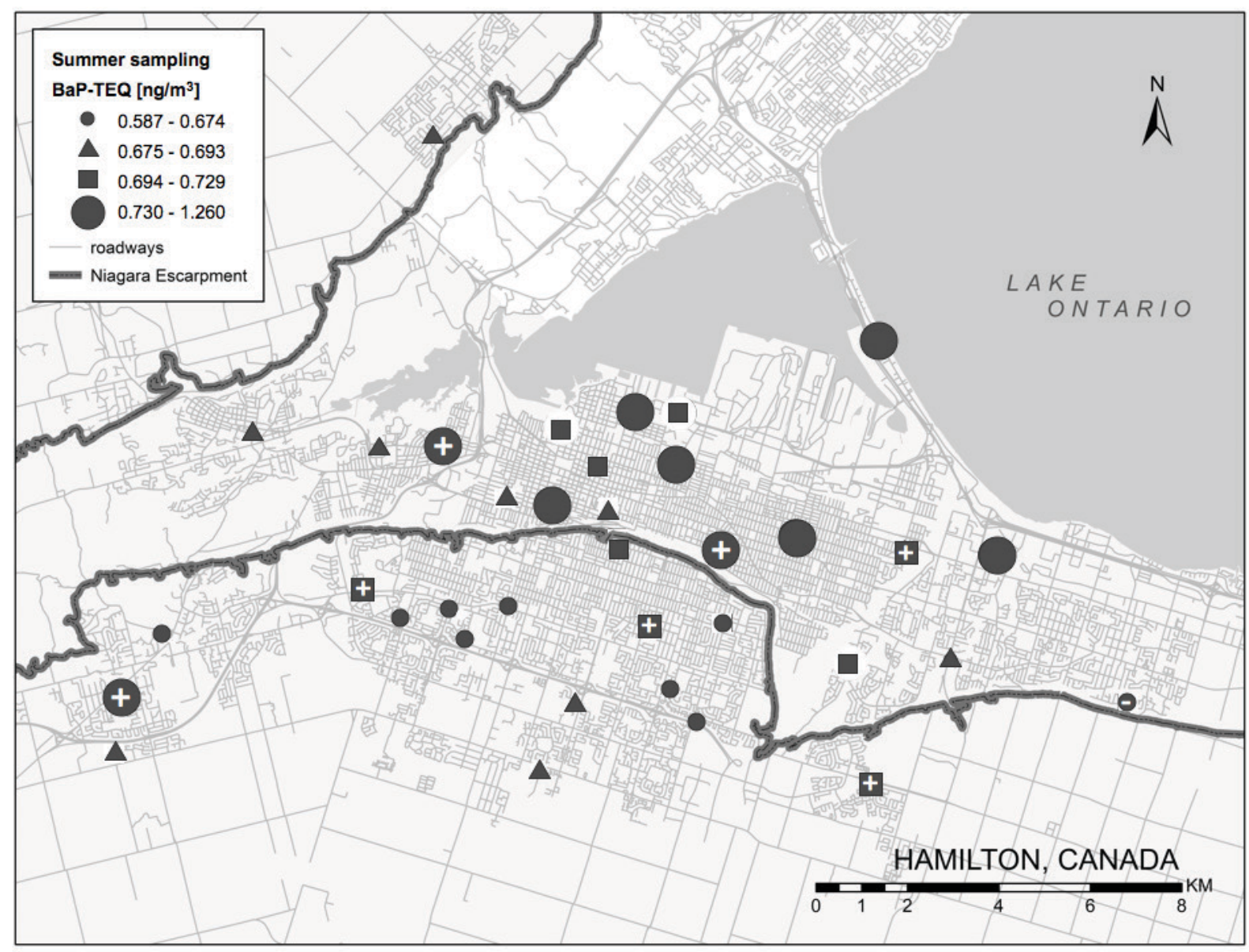

Note: + indicates BaP-TEQ $>\Sigma_{16} \mathrm{PAH}$ by one or more quartiles; - indicates BaP-TEQ $<\Sigma_{16} \mathrm{PAH}$ by two quartiles.

Figure 4-8. Sampled BaP-TEQ concentrations, by quartile, summer and winter campaigns. 


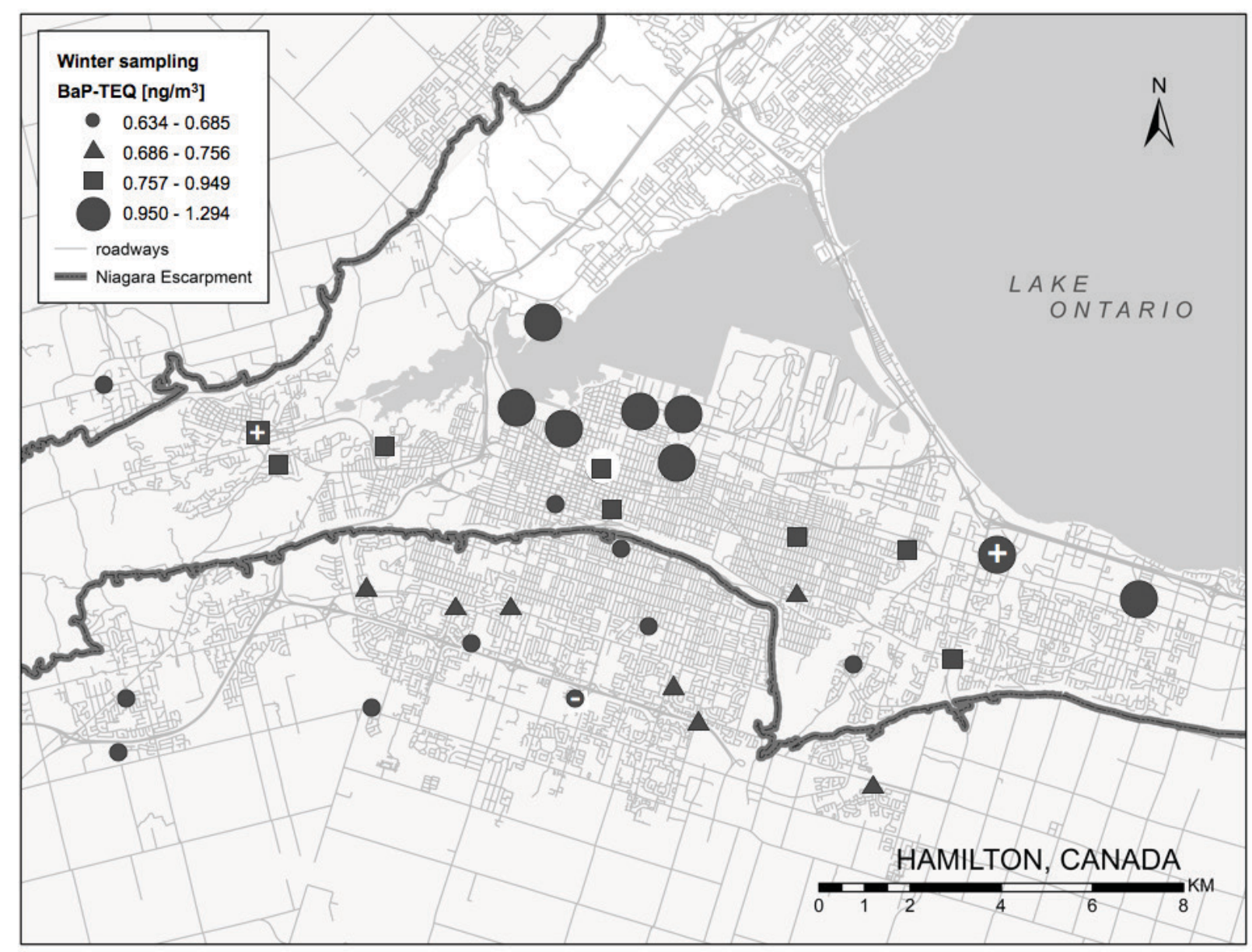

Note: + indicates BaP-TEQ $>\Sigma_{16} \mathrm{PAH}$ by one or more quartiles; - indicates BaP-TEQ $<\Sigma_{16} \mathrm{PAH}$ by two quartiles.

Figure 4-8 (continued) 


\section{Chapter: Intraurban variability in source contributions to PAH and PAH toxicity in Hamilton, Ontario}

\subsection{Highlights}

- PMF-CMB quantified intraurban variability in source contributions to PAH and PAH toxicity.

- Intraurban variability in contribution to sampled $\Sigma \mathrm{PAH}$ differed by PAH source type.

- Contributions to $\mathrm{PPAH}$ and BaP-TEQ confirmed source toxicity differences.

- Traffic exhaust contributions to $\Sigma \mathrm{PAH}$ were low yet dominated PAH exposure toxicity at all sites.

- Local industry contributions varied widely but were significant near the industrial zone.

\subsection{Abstract}

To identify and quantify intraurban differences in source contributions to PAH and PAH exposure toxicities, a combined PMF-CMB (US EPA PMF 3; US EPA CMB 8.2) receptor model approach was used with speciated $\mathrm{PAH}$ data from a dense intraurban sampling campaign in Hamilton, Canada. Local source profiles were obtained in lieu of proxies by applying the PMF receptor model to central site speciated PAH time-series data and a fourth-order PMF model resolved profiles representing PAH volatilization Itransported coal and wood combustion, oil and wood combustion, traffic exhaust, and local industry. The PMF-resolved local source profiles were then applied to a CMB model to determine their relative contributions at 33 intraurban sampling sites. Significant differences in source contributions were observed between the city's two geographically distinct regions, above and below the $\sim 100 \mathrm{~m}$ high escarpment, and within these regions. Source contributions to total Benzo(a)Pyrene-toxicity equivalent concentration, BaP-TEQ, were determined at each sampling site using Benzo(a)Pyrene Toxicity Equivalency Factors (TEF) for modeled PAH species. Source contributions to PAH toxicity did not necessarily follow the same spatial gradients as contributions to total PAH mass ( $\mathrm{P} \mathrm{PAH})$ because of differing source toxicity. Vehicle traffic exhaust emissions were the predominant source of PAH exposure toxicity throughout the urban area, despite contributing little to $\mathrm{P} A H$. Local industry emissions were found to be contributing significantly to exposure toxicity only near the city's industrial zone. 


\subsection{Introduction}

Exposure health risk has been shown to vary at the intraurban scale for respirable particulate matter $\left(\mathrm{PM}_{2.5}\right)$ (Jerrett et al, 2005a) with a need for more information on the distribution of PM components contributing to these differences, such as polycyclic aromatic hydrocarbons (PAH). This is especially the case in urban settings where differences in ambient PAH concentrations are believed to be due to the influence of local sources (Grahame and Schlesinger, 2007; Maciejczyk et al, 2004; Li et al, 2005).

PAHs are produced by incomplete combustion of organic material with many anthropogenic sources, including coal-fired power generation, space heating by fossil fuel combustion, vehicle exhaust, and industrial activity such as aluminum smelting and steel production (Ravindra, 2008). PAHs are semi-volatile organic compounds (SVOCs) and present in both vapour and particle phases in ambient air. Generally, lighter molecular weight (MW) species with 2 and 3-ring structures (MW $\leq 202$ ) are found largely in vapour phase while heavier MW species with 5-ring and 6-ring structures (MW >228) are largely in particle phase and predominantly in the smaller respirable size fraction $\left(\mathrm{PM}_{2.5}\right)$, increasing exposure risk (Venkataraman and Friedlander, 1994; Lu et al., 2008; additional information on vapour-particle partitioning and predominant particulate size fractions in Appendix A.l). PAHs have been declared toxic under the Canadian Environmental Protection Act (Environment Canada, 2011a) and a number of heavy MW species are under scrutiny for their probable carcinogenicity (ATSDR, 1995). PAHs may also react with sunlight or other atmospheric compounds to form transformation products 
such as nitro-PAH and hydroxy-PAH, with greater toxicity and environmental persistence (Baek et al., 1991a).

In response to growing awareness of varying PM toxicity, monitoring PM-associated toxics such as PAH has been a significant advance in exposure assessment. Field sampling research has shown significantly greater intraurban variability for ambient PAH than for $\mathrm{PM}_{2.5}$ (Jerrett et al, 2005a; Fischer et al, 2000). Comprehensive field sampling in Hamilton, Canada (Anastasopoulos et al, 2012) at more than 30 sites representing differing land use types demonstrated this for summer and winter season $\mathrm{PM}_{2.5}$ and speciated PAH. The next step in better understanding intraurban variability in PAH levels is a source-based investigation to quantify source contributions to ambient PAH, examine intraurban differences in source contributions, and assess source types by relative contributions to PAH toxicity. Need for source-based assessment of intraurban PAH exposures was stressed by a comprehensive literature review of toxicology, epidemiology and exposure assessment studies which found that monitoring PM and its toxic components was insufficient for improving exposure assessment for health risk estimates or regulation of air toxics, and argued instead that "certain sources of PM emissions, by virtue of disproportionate production of PM-associated toxics such as PAH, may be considered for the purposes of health risk assessment 'more toxic' than others." (Grahame and Schlesinger, 2007) Quantification of PM-associated toxics, such as PAH, and source contributions was recommended, with local sources such as traffic and local industry cited as significant contributors to variable exposure health risk (Grahame and Schlesinger, 2007; Schwartz et al, 2005; Gold et al, 2005). 
Despite such strong recommendations for source-based assessment of air toxics, intraurban sampling studies that include PAH alongside criteria pollutants (e.g., $\mathrm{PM}_{2.5}$, $\mathrm{NO}_{\mathrm{x}}$ ) are comparatively few, typically sample only particle phase $\mathrm{PAH}$ underestimating total particle +vapour phase ambient exposures, sample few PAH species, or have used limited sampling networks. Further, most intraurban studies of PAH have typically associated higher PAH concentrations with local sources by proximity or upwind/downwind analysis but do not quantify source contributions to ambient PAH and to PAH exposure toxicity (Schnelle-Kreis, 2001; Maciejczyk et al, 2004; Li, 2005; Rehwagen, 2005).

This research advances the state of the science by presenting a source-based approach to assessing PAH exposures at the intraurban scale by applying combined receptor model to quantify source contributions at numerous urban receptor locations, permitting quantification of the differences in source mix between urban zones. The combined receptor model applies 'local source profiles' derived from local central site monitoring data, rather than reference profiles from source sampling or receptor modeling at other locations, which have significant challenges in their application as proxy profiles (Galarneau, 2008). The intraurban source apportionment of PAH was carried out for a spatial network much denser than previously available to better identify micro-variability in source contributions and better understand previously identified 'exposure hotspots' (Anastasopoulos et al, 2012) in terms of contributing sources. Intraurban source apportionment results are also presented in terms of PAH toxicity, identifying sources contributing disproportionately to toxicity and distinguishing sources contributing 
similarly across an urban centre from those associated with 'hot spots' of exposure toxicity near a local source, thereby building on previous work that confirmed total sampled PAH toxicity (BaP-TEQ) may show greater intraurban variability than total sampled PAH ( $\mathrm{PAH})$ or than individual 'source marker' species (Anastasopoulos et al, 2012; Ravindra, 2006; Olson and McDow, 2009).

The research hypothesized that:

(i) use of 'local source profiles', derived by a PMF receptor model from local time-series data, in a CMB receptor model will allow meaningful source apportionment of field-sampled speciated PAH;

(ii) source contributions to PAH will show intraurban variability that differs by source type; and,

(iii) source contributions to PAH toxicity will differ from source contributions to PAH.

Results are of interest to researchers studying local exposure inequalities and to public health planners seeking to meaningfully identify source types most suitable for intervention strategies to reduce exposure to PM-associated toxics. 


\section{$5.4 \quad$ Methods}

\subsubsection{Receptor model theory}

Receptor models attempt to identify and quantify the relative contribution of different sources to observed ambient concentrations of air pollutants, based on the principle of mass balance of pollutants between sources and receptor (Equation 5-1; matrix, indexed forms). In Equation 5-1, $\boldsymbol{X}$ is the sample matrix, $\boldsymbol{F}$ is the factor profile matrix, $\boldsymbol{G}$ is the matrix of factor contributions to each sample and $\boldsymbol{E}$ is the model residual. (Hopke, 2003a)

Equation 5-1. General source-receptor mass balance.

$$
\boldsymbol{X}=\boldsymbol{G} \cdot \boldsymbol{F}+\boldsymbol{E}
$$

$$
\begin{aligned}
& \boldsymbol{X}=\text { sample concentration matrix } \\
& \boldsymbol{G}=\text { factor contribution matrix } \\
& \boldsymbol{F}=\text { factor profile matrix } \\
& \boldsymbol{E}=\text { model residual matrix } \\
& \qquad x_{i j}=\sum_{k=1}^{p} g_{i k} f_{k j}+e_{i j} \\
& x_{i j}=\text { concentration species } j \text { in receptor sample } i\left(\mathrm{ng} / \mathrm{m}^{3}\right) \\
& g_{i k}=\text { contribution of source factor } k \text { to receptor } \mathrm{sample} i \\
& f_{k j}=\text { concentration of species } j \text { to source factor } k\left(\mathrm{ng} / \mathrm{m}^{3}\right) \\
& e_{i j}=\text { model residual species } j \text { in receptor sample } i\left(\mathrm{ng} / \mathrm{m}^{3}\right)
\end{aligned}
$$

The Chemical Mass Balance receptor model (CMB; US EPA CMB 8.2) solves the source-receptor mass balance (Equation 5-1) directly by applying user-input profiles $(\boldsymbol{F})$ for source types expected to be contributing to observations at the receptor $(\boldsymbol{X})$ and solving factor contributions $(\boldsymbol{G})$ using an effective-variance least-squares approach. (US EPA, 2004; Hopke, 2003a) 
Positive Matrix Factorization (PMF; US EPA PMF 3) is an advanced receptor model whose development has been described in detail elsewhere (Paatero and Tapper, 1994; Paatero, 1997) with key advantage that knowledge of potential sources contributing to ambient concentration at a receptor is not required a priori. PMF input requires only sufficient series of speciated ambient measurements $(\boldsymbol{X})$ and an estimate of associated uncertainty $(\boldsymbol{S})$. Statistical analysis of trends in the speciated pollutant data yields factors explaining the data variation that may be interpreted as physical meaningful source types. (Hopke, 2004; Brook et al, 2004) PMF solves the source-receptor mass balance (Equation 5-1) by using an iterative algorithm to populate $\boldsymbol{F}$ and $\boldsymbol{G}$ matrices simultaneously, subject to the constraint that matrix elements be non-negative, while seeking to minimize an objective function Q (Equation 5-2). The objective function's weighting of the model residual $(\boldsymbol{E})$ by the measurement uncertainty $(\boldsymbol{S})$ ensures the solution is influenced predominantly by data with lower associated uncertainty, down-weighting data with greater associated uncertainty. (Hopke, 2003a; Paatero and Tapper, 1994; Lee, 2003)

Equation 5-2. PMF minimization function for solution of source-receptor mass balance.

$$
\begin{aligned}
& Q=\sum \sum\left(\frac{\boldsymbol{E}}{\boldsymbol{S}}\right)^{2} \\
& \boldsymbol{E}=\text { model residual matrix } \\
& \boldsymbol{S}=\text { uncertainty matrix } \\
& Q=\sum_{i=1}^{n} \sum_{j=1}^{m}\left(\frac{e_{i j}}{s_{i j}}\right)^{2}
\end{aligned}
$$

$e_{i j}=$ model residual of species $j$ in receptor sample $i\left(\mathrm{ng} / \mathrm{m}^{3}\right)$, see Equation $5-1$

$s_{i j}=$ uncertainty of species $j$ in receptor sample $i\left(\mathrm{ng} / \mathrm{m}^{3}\right)$ 


\subsubsection{Combined PMF-CMB modeling}

Limited research efforts have shown the viability of combining PMF with CMB. The models have been applied to a common ambient dataset for purposes of comparison or validation and found to agree well (Jaeckels et al, 2007; Viana et al, 2008). Formal 'pairing' of the models (PMF-CMB), with PMF to identify sources and resolve their profiles followed by CMB to quantify relative source contributions, has been suggested to apportion source types considered unique to a local study area, inclusive of vehicle and local industry emissions (Duvall et al, 2007; Shi et al, 2009; Park and Kim, 2005).

Following from previous and limited PMF-CMB source apportionment of PM, this work applied PMF-CMB to identify and quantify intraurban differences in source contributions to PAH, a pollutant type (i.e., SVOC) and scale of study (i.e., intraurban) for which obtaining sufficiently representative reference profiles would be otherwise challenged (Galarneau, 2008). 'Local source profiles' were obtained in lieu of proxies by applying the PMF receptor model to speciated PAH time-series data from the local central site monitor and then input to a CMB model of intraurban field-sampled ambient PAH. Modeled datasets are described below and modeling parameters and performance criteria applied in PMF and CMB are presented in Supplementary Information (Appendix D.I; also see Appendices E.2, E.3).

\subsubsection{PMF dataset (local central site monitoring)}

PMF input referenced historical central site monitoring data from Environment Canada's National Air Pollution Surveillance network (NAPS) for the city of Hamilton (population 700,000; Statistics Canada, 2012b) an urban center with very high historical levels of 
ambient PAH (Dann, 1997). The monitoring site is located near the central business district (i.e., Beasley Park: 43.257722N, 79.861611W; Figure 5-1) with proximal urbancommercial and industrial land use, inclusive of iron and steel manufacturing and other heavy industry at the harbour-front.

Ambient PAH were sampled in particle phase, on glass-fibre filters (GFF), and in vapour phase, using polyurethane foam (PUF) sorbent over 24-hr, following a schedule of once every six days. Species identification and quantification via gas chromatography with mass spectrometry detection (GC-MSD) followed US EPA Method TO-13A (US EPA, 1999), with co-extraction of GFF and PUF media to determine total PAH concentration (i.e., sum of particle and vapour phase) for a broad suite of species (Table 5-1). Total ambient PAH concentration data has been shown to be more suitable for receptor modeling (Mari, 2010) than particle-only PAH data due to the partitioning behaviour of light and medium MW species (Venkataraman and Friedlander, 1994; Galarneau, 2008). PMF modeled data were scoped to a recent time period (2001-2010) with sufficient sample size and good representation of all seasons.

The PMF data treatment protocol for input concentrations $\left(\mathrm{x}_{\mathrm{ij}}\right)$ and uncertainties $\left(\mathrm{s}_{\mathrm{ij}}\right)$ reflected best practice guidelines (Reff et al, 2007; US EPA, 2000) and is summarized in

Table 5-2. Species detection limits $\left(\mathrm{DL}_{\mathrm{j}}\right)$ and error fraction data were obtained from a laboratory Quality Assurance Quality Control (QAQC) dataset (Environment Canada, 2005) and laboratory methods document (Environment Canada, 2011f), respectively (Table 5-1). Generally, more conservative species were assigned less uncertainty than 
highly volatile and more reactive species, with error fractions varying from approximately $10 \%$ to $50 \%$.

\subsubsection{CMB dataset}

$\underline{\text { Local source profiles }}$

CMB model input requires source profile data $(\mathrm{PR})$, comprising a species mass fraction and an associated uncertainty on the species mass fraction, for source types expected to be contributing to the ambient data (AD) observations at the receptor. $\mathrm{CMB}$ input PR were constructed from the PMF bootstrap results (i.e., median of bootstrap concentration profile), as a statistically representative and probable PMF solution generally recommended for best practice interpretation and reporting (Watson et al, 2008; Reff et al, 2007). PR uncertainties were estimated by referencing the analogous bootstrap IQR statistics to meaningfully assign uncertainty to all species (Equation 5-3). ${ }^{10}$

Equation 5-3. Species uncertainty for CMB source profiles.

$$
\begin{aligned}
& u_{j}=\frac{\left(Q_{3_{j}}-Q_{1_{j}}\right)}{2} \text { where } E V_{Q 1_{j}} \geq 5 \% \\
& u_{j}=\frac{\left(Q_{3_{j}}-Q_{2_{j}}\right)}{2} \text { where } E V_{Q 1_{j}}<5 \%
\end{aligned}
$$

$u_{j}=$ uncertainty for species $\mathrm{j}$ in PMF bootstrap concentration profile $\left(\mathrm{ng} / \mathrm{m}^{3}\right)$

$Q_{1 j}, Q_{2 j}, Q_{3 j}=$ first, second, third quartile for species $j$ in PMF bootstrap concentration profile $\left(\mathrm{ng} / \mathrm{m}^{3}\right)$

${ }^{10}$ Equation 5-3 makes distinction between 'characteristic' and 'non-characteristic' by species' first quartile $\left(\mathrm{Q}_{1}\right)$ explained variance value. It was observed that 'non-characteristic' species with very low EV can show near-zero factor profile concentrations in some bootstrap runs, possibly skewing $\mathrm{Q}_{1}$ concentrations unrealistically below $\mathrm{DL}_{\mathrm{j}}$ and thus exaggerating the uncertainty estimate. 
$E V_{Q 1 j}=$ explained variance first quartile for species $j$ (PMF bootstrap profile; \%)

Preparation of the PR input file converted PMF bootstrap factor median concentrations $\left(\mathrm{ng} / \mathrm{m}^{3}\right)$ and corresponding uncertainties (Equation $5-3 ; \mathrm{ng} / \mathrm{m}^{3}$ ) to mass fractions (i.e., dividing each factor's species mass concentration by sum total of species mass concentrations in factor) and ordered species by increasing MW for more meaningful review of factor profiles and analysis of model results.

$\underline{\text { Intraurban ambient data }}$

$\mathrm{CMB}$ ambient data (AD) input referenced spatially distributed receptor data from a comprehensive intraurban field sampling campaign. The detailed methodology for field sampling and analysis was reported in Anastasopoulos et al (2012). Briefly, 16 PAH species (Table 5-3) were sampled $\left(\mathrm{PM}_{2.5}\right.$ particle phase, plus vapour phase) over a dense network of sites in Hamilton (Figure 5-1) over a two-week period in June/July 2009 (summer session, 43 sites, 36 sites with valid PAH data) and December 2009 (winter session, 46 sites, 33 sites with valid PAH data). Sampling provided good coverage of the city below and above the $100 \mathrm{~m}$ escarpment (a unique feature of Hamilton) with a sampling density of $\sim 1$ per $5 \mathrm{~km}^{2}$; inter-site distance $\sim 1.5 \mathrm{~km}$; locations selected by a location-allocation model (Kanaroglou et al, 2005) and included one site co-located with the NAPS central site monitor. Field PAH samplers were lab-analysed following US EPA Method TO-13A (1999) for consistency with NAPS central site data and appropriate QAQC protocols were applied for field and lab blank corrections (Wheeler et al, 2011). While noting differences in sampling (i.e., duration, PM phase size fraction), samplers 
and lab analytical methods were sufficiently similar between NAPS and intraurban field sampling for combined receptor modeling of commonly sampled species.

Winter campaign field sampling data proved highly amenable to CMB modeling with most sampled species sampled above $\mathrm{DL}_{\mathrm{j}}$ at majority of sites and with good intraurban variation in sampled concentrations (coefficient of variation, $\mathrm{CV}=0.52,0.57$ for $\Sigma_{\text {light }} \mathrm{PAH}, \Sigma_{\text {heavy }} \mathrm{PAH}$; Anastasopoulos et al, 2012; MW groupings as per Li et al, 2005a, light: $\leq 4$ rings, $M W \leq 202$; heavy: $>4$ rings, $M W>228$ ). The winter period was also preferred for representation of local source types, due to more frequent southwesterly winds favouring transport of proximal expressway traffic sources (Anastasopoulos et al, 2012), and seasonal source types such as space heating. These considerations were in addition to inherent advantages of receptor modeling winter season PAH data, including increased source-receptor conservation due to decreased atmospheric reactivity at lower temperatures and increased PM-associated heavy MW PAH concentrations due to seasonal meteorological factors such as reduced mixing height and higher wind speeds (Anastasopoulos et al, 2012; Sun et al, 2006). ${ }^{11}$

\footnotetext{
${ }^{11}$ Summer intraurban field dataset was not modeled due to concentrations sampled below $\mathrm{DL}_{\mathrm{j}}$ at majority of sites for many heavy MW species (Anastasopoulos et al, 2012). The absence of sufficient sampled mass to capture intraurban variability in heavy MW PAH is a significant limitation since these species are required to adequately characterize traffic exhaust emissions (Park, 2011; Ravindra, 2008; Larsen and Baker, 2003) as well as to adequately estimate PAH toxicity (i.e., heavier MW species exhibit greater toxicity; Nisbet and Lagoy, 1992).
} 
Species screening of the intraurban field sampling data for CMB modeling retained 13 species (Table 5-3) ${ }^{12}$ and the AD input file used lab-reported concentrations $\left(\mathrm{ng} / \mathrm{m}^{3}\right)$ identical to prior published analysis (Anastasopoulos et al, 2012) with data treatment consistent between PMF and CMB receptor models (

Table 5-2). Treatment of below detection limit sampled concentrations was to avoid introducing 'false intraurban variability' for species with low concentrations, since intersite differences in concentration could not be meaningfully distinguished below detection limits. Species detection limits $\left(\mathrm{DL}_{\mathrm{j}}\right)$ for the 14-day field samples were obtained from the analyzing laboratory (Table 5-3) and error fractions $\left(\mathrm{k}_{\mathrm{j}}\right)$ were species-specific and consistent with PMF modeling (Environment Canada, 2011f; Table 5-3). As in the PR file, the AD input files ordered species by increasing MW.

\subsubsection{Intraurban mapping}

To investigate spatial differences in source contributions, maps were prepared for the CMB source apportionment results (i.e., contributions to total sampled PAH and to PAH toxicity), model performance metrics, and for associated uncertainties on the source apportionment results. All maps were prepared using ArcGIS 10 (ESRI Inc., Redlands, California, USA).

\footnotetext{
${ }^{12}$ Naphthalene (NAP), Acenaphthylene (ACY) excluded due to incompatibility with NAPS data (i.e., NAP sampled as VOC rather than PAH) and known volatility-related negative sampling artifacts (i.e., US EPA, 1999) and sampled below $\mathrm{DL}_{\mathrm{j}}$ at nearly all winter sites. Dibenz(ah)anthracene (DBA) excluded due to sampling below $\mathrm{DL}_{\mathrm{j}}$ at all intraurban sites and a large number central site time series samples missing and below $\mathrm{DL}_{\mathrm{j}}$ (US EPA, 2008) at background site in prior PMF modeling (Chapter 3).
} 


\subsection{Results \& Discussion}

\subsubsection{PMF-derived local source profiles}

A 4-factor PMF model order with 13 fitting species was run on the Hamilton NAPS central site data (i.e., 2001-2010, year-round samples) to resolve local CMB source profiles that reflected the resolution of the available field data (AD). ${ }^{13}$ The optimal PMF model met 'best practice' requirements of a good solution (i.e., stable, good fit, robust, rotationally unique; US EPA, 2004; Reff et al, 2007; model solution summarized in Supplementary Information, Appendix D.4).

Interpretation of PMF model factors as physically meaningful source types applied several layers of evidence as detailed in Chapter 3:

- corroboration with alternate model results;

- factor enrichment in known source marker or source-characteristic species;

- comparison with reference profiles;

- evaluation of seasonal or other temporal trends in factor contributions; and,

- comparison of source apportionment estimates with emissions inventories.

13 Preliminary modeling iterations considered model order, species suite, and seasonal stratification and are presented as Supplementary Information, Appendix D.2. 
The identified factors were:

- "light-r" = PAH volatilization and LRTAP coal combustion + biomass combustion;

- "trimodal" = local industry;

- "volatile-r" = space heating (oil + natural gas + biomass combustion $)$; and,

- "heavy" = vehicle traffic exhaust.

Retene, which has been used as a convenient biomass combustion marker by previous researchers (Jang et al, 2013; Menezes, 2012; Fang, 2006; Larsen and Baker, 2003; Simcik, 1999; Shen et al, 2012), was not available in the field sampling data. However, 4factor PMF results using the larger species suite from the central site dataset (including retene), indicated biomass combustion emissions to be accounted for between the "light" factor attributed to PAH volatilization, and the "volatile" factor attributed to space heating. This is a reasonable result considering the high volatility of retene, and the use of wood for supplemental space heating.

Overall, the source profiles resolved by the 4-factor 13-species PMF model were meaningfully representative of their respective source types and were thus applied to CMB with confidence. The PMF-derived local source profiles used in CMB modeling are shown in Figure 5-2. (PMF output used to construct CMB input source profiles presented in Supplementary Information, Appendix D.4.) 


\subsubsection{PMF-CMB model performance}

Viability of the PMF-CMB model was verified by comparing CMB results with previous PMF source apportionment results (Chapter 3) at the central monitoring site for the $\sim 60$ NAPS sampling days in 2009. The CMB goodness of fit criteria (i.e., $\mathrm{r}^{2}, \%$ mass, $\mathrm{chi}^{2}$ ) were good for the majority of 2009 dates and source contributions estimates generally consistent with previous PMF source apportionment results at the central site. Seasonal trends in CMB source contributions estimates were also consistent with trends observed in the PMF model. The strong CMB model performance and inter-model consistency in source apportionment results, inclusive of seasonal trends in source contributions, verified the PMF-CMB methodology.

Verification also examined the viability of PMF-CMB to apply source profiles derived from local NAPS data to source apportionment of the intraurban field campaign dataset (Anastasopoulos et al, 2012), referencing the field site co-located with the NAPS central monitor (Figure 5-1) and comparing source contribution estimates between datasets (i.e., apportionment of NAPS data versus apportionment of co-located field data). The NAPS monitoring site data are for 24 -hr samples while field sampling data are two-week integrated concentrations, precluding direct comparison. However, comparing the source contribution estimates for the two or three NAPS sampling days coinciding with the field sampling sessions with source contribution estimates for the two-week integrated field sampling results offers confidence in the approach and the results. Estimated source contributions were consistent in relative magnitude for similar source types, despite the reduced modeling power due to the smaller field sampling suite and ambient sampling 
differences. (Detailed verification results presented in Supplementary Information, Appendix D.3.)

After the preliminary checks on the applicability of the combined PMF-CMB modeling to the NAPS monitoring site data and the co-located field sampling data, the modeling was extended to all sites in the winter field sampling dataset (i.e., 33 sites). The CMB goodness of fit criteria were good at the majority of sites, above and below escarpment, indicating that profiles derived by PMF from historical local central site monitoring data could explain ambient observations in intraurban field sampling data and thus be used for spatial analysis of relative source contributions. (Detailed performance metrics presented by site in Supplementary Information, Appendix D.4.)

\subsubsection{Intraurban source apportionment of PAH}

\subsubsection{Intraurban variability in source contributions}

As noted in prior work (Anastasopoulos et al, 2012), higher ambient PAH concentrations were generally observed below the escarpment $\left(14.4 \mathrm{ng} / \mathrm{m}^{3}\right.$ upper sites, $25.5 \mathrm{ng} / \mathrm{m}^{3}$ lower sites, $77 \%$ higher median below escarpment) and above/below-escarpment difference was significant (Mann-Whitney, $\mathrm{p}<0.05)$. CMB-estimated source contributions (SCE) to ambient PAH now allowed examining above/below-escarpment differences by modeled source type. This showed a small above/below-escarpment difference in median

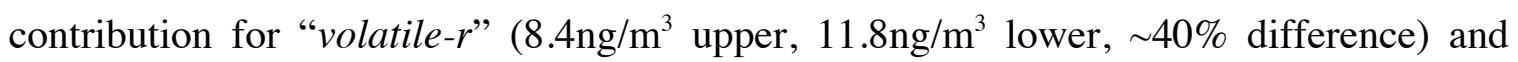

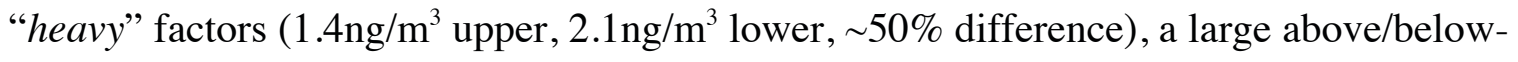
escarpment difference for the "light-r" factor $\left(3.5 \mathrm{ng} / \mathrm{m}^{3}\right.$ upper, $7.7 \mathrm{ng} / \mathrm{m}^{3}$ lower, $\sim 120 \%$ 
difference), and a very large above/below-escarpment difference for the "trimodal" factor $\left(0.5 \mathrm{ng} / \mathrm{m}^{3}\right.$ lower, $3.1 \mathrm{ng} / \mathrm{m}^{3}$ upper, $520 \%$ difference). (Supplementary Information, Appendix D.4, Figure D.4-9.)

Over all sites, intraurban variability in contributions from the "volatile-r" and "heavy" factors was generally low (i.e., low CV, evident in SCE maps, Appendix D.4, Figure D.49). This was consistent with source type interpretations: space heating emissions ("volatile-r") would be prevalent throughout the city but likely higher in lower Hamilton due to denser residential zoning, greater concentration of commercial and industrial buildings, and added oil combustion associated with industrial processes; traffic exhaust emissions ("heavy") are also present throughout the city but with varying traffic intensity between zones such as the central business district compared with suburban neighbourhoods. Somewhat higher traffic exhaust contributions were seen in lower Hamilton near the harbour-front and also at two of the lower-east sites, consistent with high traffic counts of heavy duty vehicles (i.e., gasoline and diesel) associated with the industrial sector and close site proximity to freeways (i.e., downwind of freeways by westerly winds prevailing over sampling period; Anastasopoulos et al, 2012).

The "light-r" factor also showed generally low intraurban variability in source contributions (Appendix D.4, Figure D.4-9), consistent with the local volatilization component, which can be considered an area source. A pronounced increase in "light-r" contributions (i.e., higher contributions of volatile light MW species) was observed at sites near the harbour-front and was physically meaningful: fuel storage facilities are associated with heavy industry sites and shipping activity; asphalt production and 
handling facilities are located in this zone and associated with release of light MW PAH; and, industry sources are expected to be contributing to greater levels of adsorbed PAH on proximal infrastructure (i.e., surfaces as temporary 'sink' subject to re-volatilization).

In contrast to the other three factors, the "trimodal" factor was generally found as an estimable source only below the escarpment (Appendix D.4, Figure D.4-9) and showed large site-to-site differences in contribution ( $\mathrm{CV}$ of 0.8 below escarpment). $\mathrm{CMB}$ apportioned significant PAH mass to the "trimodal" factor at sites near the iron and steelworks facilities, consistent with the factor's interpretation. ${ }^{14}$ High intraurban variability in contribution observed is consistent with a local industry source, with source-receptor dispersion strongly subject to weather patterns, particularly for sites upwind of the industrial harbour-front.

A related observation serves a good example of how receptor modeling can augment understanding of intraurban differences in ambient pollutant levels: local industry factor contributions were apportioned nearly exclusively below the escarpment by the CMB model, suggesting that higher ambient PAH concentrations sampled in this part of the city (Anastasopoulos et al, 2012) can be partially attributed to emissions inputs from concentrated harbour-front local industry facilities.

\footnotetext{
${ }^{14}$ Higher contributions were also resolved at two sites in lower-east Hamilton which are downwind of the industrial harbour-front but in close proximity to an elevated expressway cited by local knowledge as a significant emissions concern; thus, some cross-identification between the traffic and local industry source types cannot be ruled out for these sites. Review of uncertainty (Figure 5-5) corroborated the finding that local industry contributions were most significant (i.e., low relative uncertainty) near the industrial harbour-front.
} 


\subsubsection{Intraurban variability in source mix (SCE fraction)}

Intraurban trends in absolute source contribution (i.e., $\mathrm{SCE}, \mathrm{ng} / \mathrm{m}^{3}$ ) were more closely assessed on a relative basis, as fractional contributions to 'source mix' at each site (i.e., SCE fraction or $\%$ of total modeled mass; Table 5-5; tabulated and mapped by site in Supplementary Information, Appendix D.4, Figure D.4-6). Since above/belowescarpment differences were found to be meaningful for ambient sampled PAH (Anastasopoulos et al, 2012) and for absolute source contribution, fractional SCE were also examined for above/below-escarpment differences (Table 5-5) and mapped across the study area (Figure 5-3; pie charts at each site scaled to sampled $\Sigma \mathrm{PAH}$ to allow normalized comparison of 'source mix' across the study area).

The above/below-escarpment difference in fractional contribution was minimal for "light-r" (5\% median difference) and "heavy" (3\%) while a much larger above/belowescarpment difference in fractional contribution was seen for "volatile-r" (13\%) and "trimodal" factors (10\%). Above/below-escarpment difference was most pronounced for "trimodal" since the factor was essentially not resolved above the escarpment but was found to be contributing significantly to ambient PAH at a clustered group of sites in lower Hamilton where its relative contribution was greater than that of the "heavy" factor (median SCE fraction below escarpment 10\% "trimodal", 8\% "heavy").

Over the two-week sampling period median PAH levels for field sampling stations was $14.4 \mathrm{ng} / \mathrm{m}^{3}$ above the escarpment, $25.5 \mathrm{ng} / \mathrm{m}^{3}$ below the escarpment, and $28.3 \mathrm{ng} / \mathrm{m}^{3}$ at the co-located site 1998 (Anastasopoulos et al, 2012). Thus, one might infer that the NAPS monitoring station data may also be more representative of the levels below the 
escarpment. However, the results of the combined PMF-CMB model confirm that the central site monitor was able to capture the key source types impacting Hamilton, since the four modeled source types were effective in estimating relative contributions throughout the city as well as at the co-located field site (Figure 5-3)

CMB found fractional contributions to PAH across all sites to be greatest for "volatile-r" (56\%; median SCE fraction, Table 5-5), followed by "light-r" (28\%), "heavy" (9\%), and "trimodal" (2\%). More meaningfully, individual mapped SCE fractions (Supplementary Information, Appendix D.4, Figure D.4-7) demonstrated the significant degree to which PAH source mix could change across the urban area. The relative contribution of "light$r$ " and "volatile- $r$ " factors varied little across the study area $(\mathrm{CV}$, all sites $=0.2)$; traffic exhaust ("heavy") contributions to overall source mix varied moderately $(\mathrm{CV}=0.4)$, consistent with variation in road network density and traffic patterns. In contrast to other source types, the "trimodal" factor showed that a source type that may contribute minimally across much of an urban area could be a significant component of the source mix at some sites (i.e., below the escarpment near the industrial harbour-front) with large spatial variability even over smaller areas of the city $(\mathrm{CV}$, lower sites, 0.8$)$. This finding underscored the importance of the intraurban scale for source apportionment, particularly where intent is to inform health messaging that is relevant to local receptor subpopulations self-identifying at the neighbourhood scale. 


\subsubsection{Intraurban variability in source contributions to PAH toxicity}

Building on previous analysis, the source apportionment results were also examined from the perspective of quantifying source contributions to PAH toxicity, defined in this research as carcinogenic potential of inhalation exposure ${ }^{15}$ and referencing Toxicity Equivalency Factors (TEF) of each sampled species relative to $\mathrm{BaP}$ (

Table 5-4; Nisbet and Lagoy, 1992). This was done by converting CMB factor contributions to total modeled $\mathrm{PAH}$ (SCE to $\Sigma \mathrm{PAH}, \mathrm{ng} / \mathrm{m}^{3}$ ) to equivalent factor contributions to total modeled BaP-toxicity equivalent concentrations (SCE to BaP-TEQ, $\mathrm{ng} / \mathrm{m}^{3}$ ); specifically, for each sampling site and factor, speciated SCE were multiplied by respective TEF and summed over all species, adapting the method of Jung et al, 2010.

As noted in previous research (Anastasopoulos et al, 2012), median sampled PAH $(\Sigma \mathrm{PAH})$ and PAH toxicity (BaP-TEQ) were both significantly higher below the escarpment (Mann-Whitney, $\mathrm{p}<0.05)$ but above/below-escarpment differences were much less pronounced for PAH toxicity (BaP-TEQ, $0.693 \mathrm{ng} / \mathrm{m}^{3}$ upper, $0.941 \mathrm{ng} / \mathrm{m}^{3}$ lower, $36 \%$ higher below escarpment compared with $\Sigma$ PAH $14.4 \mathrm{ng} / \mathrm{m}^{3}$ upper, $25.5 \mathrm{ng} / \mathrm{m}^{3}$ lower, $77 \%$ higher below escarpment). ${ }^{16}$

${ }^{15}$ Individual personal health risk will depend on individual exposure and possible synergistic effects between pollutants and other risk factors.

${ }^{16} \Sigma \mathrm{PAH}, \mathrm{BaP}-\mathrm{TEQ}$ values in Anastasopoulos et al (2012) included 16 sampled species; CMB results include 13 species (i.e., NAP, ACY, DBA not modeled). Difference between $\Sigma_{16} \mathrm{PAH}$ and $\Sigma_{13} \mathrm{PAH}$ is nominal but difference in $\Sigma_{16} \mathrm{BaP}-\mathrm{TEQ}$ and $\Sigma_{13} \mathrm{BaP}$-TEQ is larger due to high toxicity equivalency factor for DBA (Nisbet and Lagoy, 1992). 
Review of the absolute source contributions to sampled PAH toxicity (Supplementary Information, Appendix D.4, Figure D.4-9) showed significant difference from source contributions to ambient PAH. Over all sites, factor contributions to sampled PAH toxicity were ranked by decreasing median contribution as: "heavy" $\left(0.144 \mathrm{ng} / \mathrm{m}^{3}\right)$, "volatile-r" $\left(0.092 \mathrm{ng} / \mathrm{m}^{3}\right)$, "trimodal" $\left(0.034 \mathrm{ng} / \mathrm{m}^{3}\right)$ and "light-r" $\left(0.028 \mathrm{ng} / \mathrm{m}^{3}\right)$. It is immediately seen that this differed significantly from the ranking by median factor contribution to PAH (i.e., "volatile-r" > "light-r" > "trimodal" > "heavy"). The implication was that source types contributing relatively little mass to total ambient PAH were contributing disproportionately to toxicity of ambient PAH.

Relative factor contributions to PAH toxicity (Table 5-5; fractional SCE_BAPTEQ) were meaningful. At most sites, the "heavy" factor dominated contributions to sampled PAH toxicity (median SCE_BaP-TEQ fraction, all sites, 53\%) followed by significant contributions to toxicity by "volatile-r" (30\%); median contributions to sampled toxicity were comparably small for "light-r" (10\%) and minor for the "trimodal" factor (2\%). The factor ranking with respect to contribution to PAH toxicity (i.e., "heavy" > "volatile-r" > "light-r" > "trimodal") was similar below and above the escarpment with the "heavy" factor contributing an even greater $58 \%$ of modeled PAH toxicity above the escarpment.

To better assess spatial variability in source contributions to toxicity, a 'source toxicity mix' map was prepared (Figure 5-4; individual factor maps in Supplementary Information, Appendix D.4, Figure D.4-8). Similar to their fractional contributions to $\Sigma \mathrm{PAH}$, "light-r" and "volatile-r" factors showed relatively little intraurban variability in fractional contributions to BaP-TEQ (CV, all sites, 0.3 - 0.4) and relatively little 
above/below escarpment difference. For the "heavy" factor, fractional contributions to BaP-TEQ varied even less ( $\mathrm{CV}$, all sites, 0.2$)$. This observation is consistent with the association of this factor with traffic emissions which have previously demonstrated shallow concentration gradients across urban areas (Hamilton - Anastasopoulos et al, 2012; Windsor-Detroit - Wheeler et al, 2008; Miller et al, 2010; New York, USA Narvaez et al, 2008). In sharp comparison, the "trimodal" factor showed very high siteto-site difference in fractional contribution to PAH toxicity $(\mathrm{CV}$, all sites, $1.3 ; 0.8$ below escarpment), analogous to its spatial trend in fractional contribution to PAH (Figure 5-3) and again consistent with local industry emissions (i.e., clustering at harbour-front).

\subsubsection{Uncertainty on intraurban source apportionment}

\subsubsection{Uncertainty on $\mathrm{CMB}$ results}

Comparatively little emphasis has been placed on reporting uncertainty in receptor modeling results (Reff et al, 2007), so this paper sought to quantify the uncertainty associated with intraurban source contributions resolved by the combined PMF-CMB model. The CMB model quantifies uncertainty (UNC) for all source contribution estimates (SCE), reflecting uncertainty in input (i.e., factor profiles, ambient data) and inter-factor collinearity (US EPA, 2004). Inter-factor collinearity was not strictly found at any of the modeled sites (i.e., no estimable factor pairings indicated by model), suggesting minimal contribution to model uncertainty and verifying the PMF-derived source profiles as being appropriately distinct.

Conservative uncertainty estimates were used in preparation of CMB model inputs for both ambient data and source profiles. Species with comparably high uncertainty 
included: PHEN and BAA (Figure 5-2); ANT (Table 5-3); and some heavy MW species (Table 5-3). From this, it could reasonably be expected a priori that factors more dependent on these species for their characterization, namely "heavy" and "trimodal", would show larger uncertainty in the CMB-calculated SCE particularly where characteristic species were sampled below DL.

Factor T-stat values were classified by relative magnitude and tallied across sites (Supplementary Information, Appendix D.4, Figure D.4-5), confirming the model generally resolved greater uncertainty for "heavy" and "trimodal" contributions than for "light-r" and "volatile-r" contributions. The "light-r" and "volatile-r" factors were found as estimable sources at all 33 modeled sites with low associated uncertainty (“light-r" Tstat $>2$ at 30 sites, $>1.5$ at 3 remaining sites; "volatile-r" T-stat $>2$ at 33 sites). The "heavy" factor SCE were also generally assigned low uncertainty at most sites (T-stat $>1.5$ at 22 sites) but indicated comparably higher uncertainty than other factors (i.e., Tstat values typically lower for “heavy").

Conversely, the SCE for the "trimodal" factor indicated low uncertainty at few sites (Tstat $>1.5$ at 8 sites, 11 sites $>1$ at 11 sites, $<1$ at remaining sites), as expected. However, the small number of sites at which the factor was resolved and the smaller subset of these with 'good' T-stat values (>1.5) suggested that uncertainty in SCE was also due to the inherent spatial variability of local industry emissions. As a rule, "trimodal" contributions were resolved with low relative uncertainty at sites with high ambient sampled concentrations. Conversely, sites with sampled concentrations closer to median levels showed no "trimodal" contribution or, at some sites, minor contribution with very high 
uncertainty. This model result corroborated previous inference that sites with high sampled ambient PAH were partially due to local source contributions increasing PAH over median levels (Anastasopoulos et al, 2012).

\subsubsection{Intraurban source apportionment maps with uncertainty}

To confirm intraurban trends in the $\mathrm{CMB}$ results in the context of uncertainty, estimated factor contributions to ambient PAH were mapped alongside their estimated uncertainties (Supplementary Information, Appendix D.4, Figure D.4-11; discussion of uncertainty for source contribution estimates to PAH toxicity also in Appendix D.4). The "light-r" and "volatile-r" factor contributions had low comparative uncertainty at all sites and showed generally little spatial variation, indicating that these factors were fit well by the CMB model at majority of sites. At the majority of sites, the "heavy" factor showed similarly good model fit, with low uncertainty on source contributions. Review of sites with high uncertainty (i.e., low T-stat) was consistent with a traffic source: these sites tended to be private homes or municipal sites characterized by low traffic intensity in their immediate surroundings. In comparison to other factors, "trimodal" factor contributions showed significant intraurban variation in relative uncertainty. At sites where local industry emissions were expected to be significantly contributing to ambient $\mathrm{PAH}, \mathrm{CMB}$ source contribution estimates had low associated uncertainty. Conversely, sites located above the escarpment or at greater distance from the iron/steelworks facilities at the industrial harbour-front showed high uncertainty, effectively indicating CMB source contributions that could not be distinguished from zero. 
Care was required inferring spatial trends from sites where factor contribution estimates were assigned high comparative uncertainty (i.e., defined by study methodology as T-stat $<1.5)$. Thus, an additional set of source apportionment maps were prepared by censoring the contribution estimates at high-uncertainty sites (Figure 5-5; sites with T-stat $<1.5$ denoted by ' $x$ '). Review of the censored source contribution maps showed that spatial trends in contributions previously noted were largely preserved and key trends were, in fact, accentuated. No sites were censored by the T-stat $<1.5$ criterion for "light-r" and "volatile-r" factors, so intraurban trends in source contributions and inferences of source type were unchanged. For traffic exhaust ("heavy") contributions, a number of sites were censored by the $\mathrm{T}$-stat $<1.5$ criterion but intraurban trends in source contributions and inferences of source type remained clearly evident. For local industry ("trimodal") contributions, only 8 sites remained with viable estimates of factor contribution to modeled PAH after censoring by T-stat $<1.5$; all were located below the escarpment and seven of the eight were clustered at or near the harbour-front. The remaining site was located immediately downwind of the harbour-front by predominant westerly winds observed for the sampling period (Anastasopoulos et al, 2012). A more stringent map censoring sites with $\mathrm{T}$-stat $<2$ identified just two sites where CMB estimated high factor contributions, both located nearest the harbour-front and iron/steel manufacturing facilities. 


\subsubsection{Discussion}

Intraurban source apportionment of PAH and PAH toxicity at Hamilton contributed significantly to understanding previously identified 'exposure hotspots' (Anastasopoulos et al, 2012) in terms of sources. The source apportionment was able to quantify variability in source contributions and found that this varies by source type. Briefly, space heating emissions ("volatile-r") contributed to ambient PAH similarly across the urban area, with little above/below escarpment difference. Emissions from PAH volatilization and transported coal combustion ("light-r") contributed similarly across the urban area with exception of the industrial harbour-front, consistent with the location of related source facilities, such as fuel storage and asphalt production/handling. Vehicle traffic exhaust ("heavy") contributions were also generally similar across the urban area, with higher contributions observed in zones associated with greater commercial vehicle traffic and near high-density roadways. Local industry emissions ("trimodal") were found to contribute significantly to ambient PAH only below the escarpment and were greatest near the iron/steelworks at the harbour-front, contributing a significant $11 \%$ of modeled $\mathrm{PAH}$ at the central site monitor and thus helping quantify the degree to which this key stationary source type was contributing to the higher ambient PAH levels sampled in lower Hamilton (Anastasopoulos et al, 2012).

The CMB source apportionment to PAH toxicity extended previous inter-urban source apportionment (Chapter 3) to the intraurban scale and found traffic emissions to contribute majority of modeled PAH toxicity at all field sites, indicating traffic emissions as a source type of concern for the entire urban area rather than only in densely developed 
zones (e.g., central business district). Local industry contributions to PAH toxicity varied greatly across the study area, with high contributions below the escarpment and near the industrial zone and generally minimal contribution above the escarpment. The CMB source apportionment results withstood considerations of uncertainty; maps censoring high-uncertainty sites (T-stat <1.5) preserved - or for local industry accentuated observed spatial trends in contributions.

The research explicitly demonstrated that source types contributing relatively little mass to total ambient PAH could contribute disproportionately to ambient PAH toxicity and thereby be considered comparatively 'more toxic' (Grahame and Schlesinger, 2007). Sources found to contribute disproportionately to PAH toxicity were traffic exhaust emissions and local industry emissions, results consistent with source apportionment of long time-series (2001-2010) central site data at Hamilton and a second urban site using the PMF receptor model (Chapter 3). Findings are also in agreement with health effects studies that examined source type contributions to chronic health risk and specifically cited traffic and local industry such as iron/steel manufacturing, as significant contributors to exposure health risk (Schwartz et al, 2005; Gold et al, 2005).

The large degree of disproportionality demonstrated for some source types recommends that emissions inventory efforts include BaP-TEQ where intent is to inform and guide emissions regulations and health policy, thereby determining which source types could best yield meaningful reductions in health effects. This is the case even for source inventories limited to particle phase PAH data since species toxicities vary widely even between species found predominantly in particle phase. 


\subsection{Conclusions}

Source-based approach to intraurban exposure assessment

This study presented a source-based approach to assessing PAH exposures at the intraurban scale. A combined PMF-CMB receptor model was applied to local central site time-series data to resolve locally representative source profiles for subsequent use in CMB source apportionment of field-sampled ambient data. This made it possible to quantify the differences in 'source mix' between urban zones. In the specific application at Hamilton, Canada, a fourth-order PMF model of year-round samples resolved a set of local source profiles representing major PAH source types: volatilized PAH and transported coal combustion + wood combustion, space heating, vehicle traffic exhaust, and local industry (including iron/steel manufacturing). Application of these factors as contributing sources in $\mathrm{CMB}$ analysis of an intraurban field dataset was effective in resolving individual source contributions to ambient $\mathrm{PAH}$ levels observed at the field sampling sites. Source contribution estimates to total BaP-equivalent PAH toxicity were also quantified and proved meaningful for discussion of source types with respect to PAH-associated health risk.

\section{Combined PMF-CMB receptor model for 'local source profiles'}

PAH emission sources have been the subject of attempts to adequately characterize them with speciated PAH measurements for a reasonably long time (Ravindra et al, 2008) but source sampling profiles have significant challenges in their application as 'reference' profiles to $\mathrm{CMB}$ receptor models (Galarneau, 2008) if they are based on limited data or are obtained from locations different from a desired study area or locally-specific source 
type. The current research demonstrated that 'local source profiles', empirically determined from PMF modeling of local central monitoring site data, may be preferable as input to a $\mathrm{CMB}$ model seeking to explain source contributions to urban receptors.

\section{Recommendations and Possible improvements in methodology}

The combined PMF-CMB methodology was demonstrated for a single location with specific characteristics that necessarily influenced results. It would be desirable to apply PMF-CMB modeling to an urban centre comparable in scale to Hamilton but sufficiently different in contributing local source types and geographic characteristics so as to further support or challenge the observations about the effectiveness of the methodology.

Differences in the available species suite for the central site monitoring data and the field sampling campaign data reduced the explanatory power of the modeling and necessitated iterations in the PMF modeling for improved CMB modeling performance. For optimal PMF-CMB modeling it is desirable to field-sample the same PAH species suite as in long-term central monitoring station data; larger numbers of viable species are further expected to improve model performance.

The explanatory power of the factors identified at the central monitoring station via PMF modeling and the source contribution estimates of these factors at the field sampling sites are strongly affected by ambient data uncertainties. While sampler media were similar, lab analysis methods identical, and co-located concentration data demonstrated to be generally compatible, there remained a significant difference in sampling airflow rates and duration between the central monitoring station data and the field sampling campaign data. For future field sampling studies, it is desirable to increase sampling airflow rates 
and decrease sampling periods to obtain field samples with reduced measurement uncertainties and ensure higher sample mass, ensuring more species data are sampled above method detection limits. This would further increase compatibility between PMF and CMB datasets and optimize combined PMF-CMB modeling.

While the effect of stratifying the central site historical time-series data by season did not improve $\mathrm{CMB}$ explanatory power in this work, complementary seasonally stratified analysis can be generally recommended for other locations (i.e., differing sources and seasonal characteristics).

\section{Intraurban variability in source contributions to PAH and PAH toxicity}

The CMB source apportionment of PAH showed source mix to vary significantly across the urban area, with some source types demonstrating greater spatial variability, emphasizing need for source apportionment at the intraurban scale. Source apportionment of PAH toxicity (BaP-TEQ) showed that source contributions to total PAH toxicity do not necessarily follow the same gradient as source contributions to total PAH mass. Traffic emissions were found to be the predominant source of PAH toxicity and to be contributing to exposure toxicity similarly throughout the urban area. Meanwhile, the relative contribution to toxicity of the local industry factor varied greatly across the urban area and was found to be contributing significantly to exposure toxicity in one portion of the study area, the industrial harbour-front. Health interventions intended to reduce PAH exposures (e.g., neighbourhood-scale ambient monitoring, public health messaging) may benefit by focusing awareness on such 'more toxic' source types. 
Spatial trends observed in CMB source contributions withstood considerations of uncertainty and were maintained or accentuated by censoring sites with excessive uncertainty. Overall, the $\mathrm{CMB}$ findings underscored the importance of carrying out source apportionment at the intraurban scale where the intent is to identify and provide health messaging to neighbourhoods with greater exposure health risk or sub-populations spending significant time in high-exposure health risk areas. 
Table 5-1. Central site monitored (NAPS) PAH, detection limits, error fractions (PMF model suite).

\begin{tabular}{|c|c|c|c|c|c|c|c|}
\hline PAH Species & Label & $\begin{array}{l}\text { Chemical } \\
\text { Formula }\end{array}$ & $\begin{array}{r}\# \\
\text { rings }\end{array}$ & $\begin{array}{r}\text { Mol. } \\
\text { Weight } \\
(\mathrm{MW})\end{array}$ & $\begin{array}{l}\text { MW } \\
\text { group }\end{array}$ & $\begin{array}{r}\text { Method } \\
\text { Detection } \\
\text { Limit }(D L) \\
{\left[\mathrm{ng} / \mathrm{m}^{3}\right]}\end{array}$ & $\begin{array}{r}\text { error } \\
\text { fraction } \\
\left(\mathrm{k}_{\mathrm{j}}\right)\end{array}$ \\
\hline acenaphthylene & $\mathrm{ACY}$ & $\mathrm{C}_{12} \mathrm{H}_{8}$ & 3 & 152.2 & light & 0.001 & 48.7 \\
\hline acenaphthene & ACE & $\mathrm{C}_{12} \mathrm{H}_{10}$ & 3 & 154.2 & light & 0.002 & 19.8 \\
\hline fluorene & FLU & $\mathrm{C}_{13} \mathrm{H}_{10}$ & 3 & 166.2 & light & 0.003 & 25.7 \\
\hline anthracene & ANT & $\mathrm{C}_{14} \mathrm{H}_{10}$ & 3 & 178.2 & light & 0.002 & 27.1 \\
\hline phenanthrene & PHE & $\mathrm{C}_{14} \mathrm{H}_{10}$ & 3 & 178.2 & light & 0.004 & 10.9 \\
\hline 2-methyl-fluorene & MFLU & $\mathrm{C}_{14} \mathrm{H}_{12}$ & 3 & 180.3 & light & 0.004 & 27.6 \\
\hline fluoranthene & FLT & $\mathrm{C}_{16} \mathrm{H}_{10}$ & 4 & 202.3 & light & 0.002 & 9.0 \\
\hline pyrene & PYR & $\mathrm{C}_{16} \mathrm{H}_{10}$ & 4 & 202.3 & light & 0.001 & 9.2 \\
\hline 1-methyl-pyrene & MPYR & $\mathrm{C}_{17} \mathrm{H}_{12}$ & 4 & 216.2 & med & 0.001 & 15.2 \\
\hline benzo(a)fluorene & BaFLU & $\mathrm{C}_{17} \mathrm{H}_{12}$ & 4 & 216.2 & med & 0.002 & 17.1 \\
\hline benzo(b)fluorene & BbFLU & $\mathrm{C}_{17} \mathrm{H}_{12}$ & 4 & 216.2 & med & 0.002 & 24.0 \\
\hline benzo(g,h,i)fluoranthene & BghiFLT & $\mathrm{C}_{18} \mathrm{H}_{10}$ & 5 & 226.3 & med & 0.002 & 14.1 \\
\hline chrysene & $\mathrm{CHR}$ & $\mathrm{C}_{18} \mathrm{H}_{12}$ & 4 & 228.3 & heavy & 0.001 & 10.5 \\
\hline triphenylene & TRIP & $\mathrm{C}_{18} \mathrm{H}_{12}$ & 4 & 228.3 & heavy & 0.002 & 13.3 \\
\hline benzo(a)anthracene & BAA & $\mathrm{C}_{18} \mathrm{H}_{12}$ & 4 & 228.3 & heavy & 0.004 & 11.7 \\
\hline retene & RET & $\mathrm{C}_{18} \mathrm{H}_{18}$ & 3 & 234.3 & heavy & 0.005 & 36.7 \\
\hline benzo(a)pyrene & BAP & $\mathrm{C}_{20} \mathrm{H}_{12}$ & 5 & 252.3 & heavy & 0.003 & 12.5 \\
\hline benzo(e)pyrene & BEP & $\mathrm{C}_{20} \mathrm{H}_{12}$ & 5 & 252.3 & heavy & 0.002 & 10.4 \\
\hline benzo(b)fluoranthene & $\mathrm{BbFLT}$ & $\mathrm{C}_{20} \mathrm{H}_{12}$ & 5 & 252.3 & heavy & 0.003 & 11.3 \\
\hline benzo(k)fluoranthene & BkFLT & $\mathrm{C}_{20} \mathrm{H}_{12}$ & 5 & 252.3 & heavy & 0.003 & 15.2 \\
\hline benzo(g,h,i)perylene & BghiP & $\mathrm{C}_{22} \mathrm{H}_{12}$ & 6 & 276.3 & heavy & 0.004 & 14.4 \\
\hline indeno(1,2,3-cd)pyrene & IPYR & $\mathrm{C}_{22} \mathrm{H}_{12}$ & 6 & 276.3 & heavy & 0.003 & 16.5 \\
\hline
\end{tabular}

Notes:

1. Retene also known as 1-methyl-7-isopropylphenanthrene.

2. Molecular weight data from ATSDR, 1995 and Mackay, 2006.

3. Molecular weight grouping adapted from Li et al, 2005.

4. Acenaphthylene excluded from PMF ('bad' categorization) due to known sampling issues (US EPA, 1999; confirmed by very high species uncertainty estimate).

5. Method Detection Limit (DL) data taken from a 2005 QAQC dataset of NAPS data made available by Environment Canada; calculated as: $\mathrm{DL}_{\mathrm{j}}=(\mathrm{t} \text {-statistic })^{*} \mathrm{SD}\left(\mathrm{x}_{\mathrm{ij}}\right)$ where $\mathrm{SD}_{\mathrm{j}}=$ calculated as standard deviation of 7 replicate samples analysed for respective PAH species (due to outlier species sample concentrations, 5 samples used for ACE, FLU, PHE; 6 samples used for FLT, PYR); t-value = Student-t test statistic at $95 \%$ confidence interval, for 5-7 samples (4-6 degrees of freedom), dependent on species.

6. Error fraction $\left(k_{j}\right)$ data from Method 3.03/5.1, Appendix B - "Uncertainty estimates by species, not recovery corrected" (Environment Canada, 2011f). Error fraction data are not recovery corrected, consistent with NAPS and field sampling data. Species error fractions account for major sources of uncertainty in PAH sample preparation and measurement and are determined via: $k_{j}=k{ }^{*} R S D$ where RSD = relative standard deviation $=S D /$ mean of 30 replicate samples; $\mathrm{k}=\mathrm{t}$-value for $95 \%$ confidence interval on 30 replicate samples $=2.0$ 
Table 5-2. Treatment protocols for speciated ambient receptor data used in PMF and CMB.

\begin{tabular}{|c|c|c|}
\hline $\begin{array}{l}\text { Lab-reported sample } \\
\text { concentration }\left(\mathrm{c}_{\mathrm{ij}}\right)\end{array}$ & $\begin{array}{l}\text { Treated sample concentration } \\
\left(\mathrm{PMF}, \mathrm{CMB}, \mathrm{x}_{\mathrm{ij}}\right)\end{array}$ & $\begin{array}{l}\text { Treated sampled uncertainty } \\
\left(\mathrm{PMF}, \mathrm{s}_{\mathrm{ij}} ; \mathrm{CMB}, \mathrm{u}_{\mathrm{ij}}\right)\end{array}$ \\
\hline$c_{i j}>D L_{j}$ & $\begin{array}{l}\frac{P M F}{x_{i j}=c_{i j}} \\
\frac{C M B}{x_{i j}=c_{i j}}\end{array}$ & $\begin{array}{l}\frac{P M F}{S_{i j}=\left[\left(\left(k_{j}\right)\left(x_{i j}\right)\right)^{2}+\left(D L_{j}\right)^{2}\right]^{(1 / 2)}} \\
\frac{\text { CMB }}{u_{i j}=\left(k_{j}\right)\left(x_{i j}\right)+(1 / 3)\left(D L_{j}\right)}\end{array}$ \\
\hline $\mathrm{c}_{\mathrm{ij}}<\mathrm{DL}_{\mathrm{j}}$ & $\begin{array}{l}\frac{P M F}{x_{i j}=(1 / 2) D L_{j}} \\
\frac{C M B}{x_{i j}=(1 / 2) D L_{j}}\end{array}$ & $\begin{array}{l}\frac{P M F}{S_{i j}=(5 / 6) D L_{j}} \\
\frac{C M B}{u_{i j}=D L_{j}}\end{array}$ \\
\hline $\begin{array}{l}\text { missing or invalid data } \\
c_{i j}=-999\end{array}$ & $\begin{array}{l}\frac{P M F}{x_{i j}=\text { median }\left(x_{i j}\right)} \\
\frac{\text { CMB }}{\text { no missing field sampling data }}\end{array}$ & $\begin{array}{l}\frac{\mathrm{PMF}}{\mathrm{S}_{\mathrm{ij}}=(4) \text { median }\left(\mathrm{x}_{\mathrm{ij}}\right)} \\
\frac{\mathrm{CMB}}{\text { no missing field sampling data }}\end{array}$ \\
\hline
\end{tabular}

Notes:

1. Method Detection Limit (DL).

For PMF, DL data taken from a 2005 QAQC dataset of NAPS data made available by Environment Canada; calculated as: $\mathrm{DL}_{\mathrm{j}}=\left(\mathrm{t}\right.$-statistic) ${ }^{*} \mathrm{SD}\left(\mathrm{x}_{\mathrm{ij}}\right)$ where $\mathrm{SD}_{\mathrm{j}}=$ calculated as standard deviation of 7 replicate samples analysed for respective PAH species (due to outlier species sample concentrations, 5 samples used for ACE, FLU, PHEN; 6 samples used for FLT, PYR); $t$-value = Student-t test statistic at 95\% confidence interval, for 5-7 samples (4-6 degrees of freedom), dependent on species.

For $\mathrm{CMB}, \mathrm{DL}$ data as reported by analyzing lab for the 14-day field sampling period at nominal flow rate.

2. Error fraction $\left(\mathrm{k}_{\mathrm{j}}\right)$.

For PMF and CMB, error fraction data from Method 3.03/5.1, Appendix B - "Uncertainty estimates by species, not recovery corrected" (Environment Canada, 2011f). Error fraction data are not recovery corrected, consistent with NAPS and field sampling data. Species error fractions account for major sources of uncertainty in PAH sample preparation and measurement and are determined via: $\mathrm{k}_{\mathrm{j}}=\mathrm{k}{ }^{*} \mathrm{RSD}$ where RSD $=$ relative standard deviation $=$ $\mathrm{SD} /$ mean of 30 replicate samples; $\mathrm{k}=\mathrm{t}$-value for $95 \%$ confidence interval on 30 replicate samples $=2.0$ 
Table 5-3. Field-sampled PAH species, error fraction, detection limit, sites above DL.

\begin{tabular}{llrrrrr}
\hline & & & error fraction \\
PAH Species & Label & $\begin{array}{r}\text { MW } \\
\text { group }\end{array}$ & $\begin{array}{r}\text { 14-day } \\
\text { [fraction] }\end{array}$ & $\begin{array}{r}\text { Summer } \\
\text { DL } \\
{\left[\mathrm{ng} / \mathrm{m}^{3}\right]}\end{array}$ & $\begin{array}{r}\text { sites } \\
>\text { DL }[\%]\end{array}$ & $\begin{array}{r}\text { Winter sites } \\
>\text { DL [\%] }\end{array}$ \\
\hline naphthalene & NAP & light & 41.7 & 0.124 & $89 \%$ & $0 \%$ \\
acenaphthylene & ACY & light & 48.7 & 0.142 & $69 \%$ & $3 \%$ \\
acenapthene & ACE & light & 19.8 & 0.134 & $97 \%$ & $100 \%$ \\
fluorene & FLU & light & 25.7 & 0.102 & $100 \%$ & $100 \%$ \\
anthracene & ANT & light & 27.1 & 0.099 & $100 \%$ & $100 \%$ \\
phenanthrene & PHE & light & 10.9 & 0.076 & $100 \%$ & $100 \%$ \\
fluoranthene & FLT & light & 9.0 & 0.068 & $100 \%$ & $100 \%$ \\
pyrene & PYR & light & 9.2 & 0.054 & $100 \%$ & $100 \%$ \\
chrysene & CHR & heavy & 10.5 & 0.110 & $47 \%$ & $100 \%$ \\
benzo(a)anthracene & BAA & heavy & 11.7 & 0.112 & $19 \%$ & $61 \%$ \\
benzo(a)pyrene & BAP & heavy & 12.5 & 0.189 & $3 \%$ & $39 \%$ \\
benzo(b)fluoranthene & BbFLT & heavy & 11.3 & 0.181 & $3 \%$ & $0 \%$ \\
benzo(k)fluoranthene & BkFLT & heavy & 15.2 & 0.178 & $3 \%$ & $100 \%$ \\
dibenz(a,h)anthracene & DBA & heavy & 13.1 & 0.215 & $0 \%$ & $0 \%$ \\
benzo(g,h,i)perylene & BghiP & heavy & 14.4 & 0.207 & $0 \%$ & $42 \%$ \\
indeno(1,2,3-cd)pyrene & IPYR & heavy & 16.5 & 0.229 & $0 \%$ & $15 \%$ \\
\hline
\end{tabular}

1. Method Detection Limit (DL) for intraurban field sampling dataset as reported by analyzing lab for the 14-day field sampling period at nominal flow rate.

2. Error fraction $\left(k_{j}\right)$ for PMF and CMB from Method 3.03/5.1, Appendix B - "Uncertainty estimates by species, not recovery corrected" (Environment Canada, 2011f). Error fraction data are not recovery corrected, consistent with NAPS and field sampling data. Species error fractions account for major sources of uncertainty in PAH sample preparation and measurement and are determined via: $\mathrm{k}_{\mathrm{j}}=\mathrm{k}$ * RSD where RSD = relative standard deviation = $\mathrm{SD} /$ mean of 30 replicate samples; $\mathrm{k}=\mathrm{t}$-value for $95 \%$ confidence interval on 30 replicate samples $=2.0$ 
Table 5-4. BaP-equivalent carcinogenic Toxic Equivalency Factors (BaP-TEF) for inhalation exposure (field-sampled species).

\begin{tabular}{llr}
\hline Species name & Species ID & BaP-TEF \\
\hline naphthalene & NAP & 0.001 \\
acenaphthylene & ACY & 0.001 \\
acenapthene & ACE & 0.001 \\
fluorene & FLU & 0.001 \\
anthracene & ANT & 0.01 \\
phenanthrene & PHE & 0.001 \\
fluoranthene & FLT & 0.001 \\
pyrene & PYR & 0.001 \\
chrysene & CHR & 0.01 \\
benzo[a]anthracene & BAA & 0.1 \\
benzo[a]pyrene & BAP & 1 \\
benzo[b]fluoranthene & BbFLT \\
benzo[k]fluoranthene & BkFLT & 0.1 \\
dibenz[a,h]anthracene & DBA & 0.1 \\
benzo[g,h,i]perylene & BghiP & 1.1 \\
indeno[1,2,3-cd]pyrene & IPYR & 0.01 \\
\hline
\end{tabular}

Note: Data from Nisbet and Lagoy, 1992 and ATSDR, 1995. 
Table 5-5. Relative source contributions (SCE fractions) to $\Sigma$ PAH and BaP-TEQ; summary statistics.

\begin{tabular}{|c|c|c|c|c|c|c|c|c|c|}
\hline & & \multicolumn{4}{|c|}{ SCE to SUMPAH (fraction) } & \multicolumn{4}{|c|}{ SCE to SUMBAPTEQ (fraction) } \\
\hline & & $\begin{array}{l}\text { 皇 } \\
\text { 喜 }\end{array}$ & $\stackrel{2}{\circ}$ & 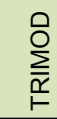 & $\gtreqless$ & 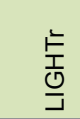 & 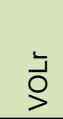 & 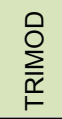 & $\gtreqless$ \\
\hline \multirow{8}{*}{ 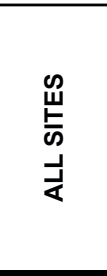 } & $\min$ & 0.21 & 0.31 & 0.00 & 0.01 & 0.06 & 0.22 & 0.00 & 0.09 \\
\hline & mean & 0.30 & 0.55 & 0.06 & 0.10 & 0.12 & 0.32 & 0.05 & 0.51 \\
\hline & $\max$ & 0.51 & 0.71 & 0.23 & 0.16 & 0.24 & 0.55 & 0.22 & 0.67 \\
\hline & Q1 & 0.25 & 0.47 & 0.00 & 0.07 & 0.09 & 0.26 & 0.00 & 0.44 \\
\hline & median & 0.28 & 0.56 & 0.02 & 0.09 & 0.10 & 0.30 & 0.02 & 0.53 \\
\hline & Q3 & 0.33 & 0.62 & 0.11 & 0.12 & 0.14 & 0.36 & 0.10 & 0.60 \\
\hline & SD & 0.07 & 0.11 & 0.07 & 0.03 & 0.04 & 0.08 & 0.07 & 0.12 \\
\hline & $\mathrm{CV}=\mathrm{SD} /$ mean & 0.2 & 0.2 & 1.2 & 0.4 & 0.4 & 0.3 & 1.3 & 0.2 \\
\hline \multirow{8}{*}{ 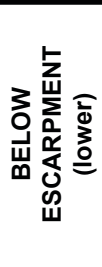 } & $\min$ & 0.21 & 0.31 & 0.00 & 0.01 & 0.07 & 0.22 & 0.00 & 0.09 \\
\hline & mean & 0.32 & 0.50 & 0.10 & 0.08 & 0.13 & 0.31 & 0.09 & 0.47 \\
\hline & $\max$ & 0.51 & 0.70 & 0.23 & 0.14 & 0.24 & 0.51 & 0.22 & 0.63 \\
\hline & Q1 & 0.26 & 0.42 & 0.03 & 0.06 & 0.09 & 0.25 & 0.02 & 0.42 \\
\hline & median & 0.31 & 0.49 & 0.10 & 0.08 & 0.12 & 0.28 & 0.09 & 0.48 \\
\hline & Q3 & 0.37 & 0.58 & 0.15 & 0.11 & 0.17 & 0.32 & 0.13 & 0.57 \\
\hline & SD & 0.08 & 0.12 & 0.07 & 0.03 & 0.05 & 0.08 & 0.08 & 0.13 \\
\hline & $\mathrm{CV}=\mathrm{SD} /$ mean & 0.2 & 0.2 & 0.8 & 0.4 & 0.4 & 0.3 & 0.8 & 0.3 \\
\hline \multirow{8}{*}{ 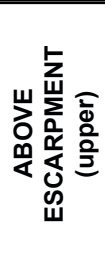 } & $\min$ & 0.22 & 0.48 & 0.00 & 0.04 & 0.06 & 0.25 & 0.00 & 0.33 \\
\hline & mean & 0.27 & 0.61 & 0.01 & 0.11 & 0.10 & 0.34 & 0.00 & 0.56 \\
\hline & $\max$ & 0.41 & 0.71 & 0.04 & 0.16 & 0.15 & 0.55 & 0.03 & 0.67 \\
\hline & Q1 & 0.24 & 0.57 & 0.00 & 0.09 & 0.09 & 0.27 & 0.00 & 0.52 \\
\hline & median & 0.26 & 0.62 & 0.00 & 0.11 & 0.10 & 0.32 & 0.00 & 0.58 \\
\hline & Q3 & 0.29 & 0.65 & 0.00 & 0.12 & 0.10 & 0.38 & 0.00 & 0.60 \\
\hline & SD & 0.05 & 0.06 & 0.01 & 0.03 & 0.02 & 0.08 & 0.01 & 0.09 \\
\hline & $\mathrm{CV}=\mathrm{SD} /$ mean & 0.2 & 0.1 & 2.4 & 0.3 & 0.2 & 0.2 & 2.4 & 0.2 \\
\hline
\end{tabular}




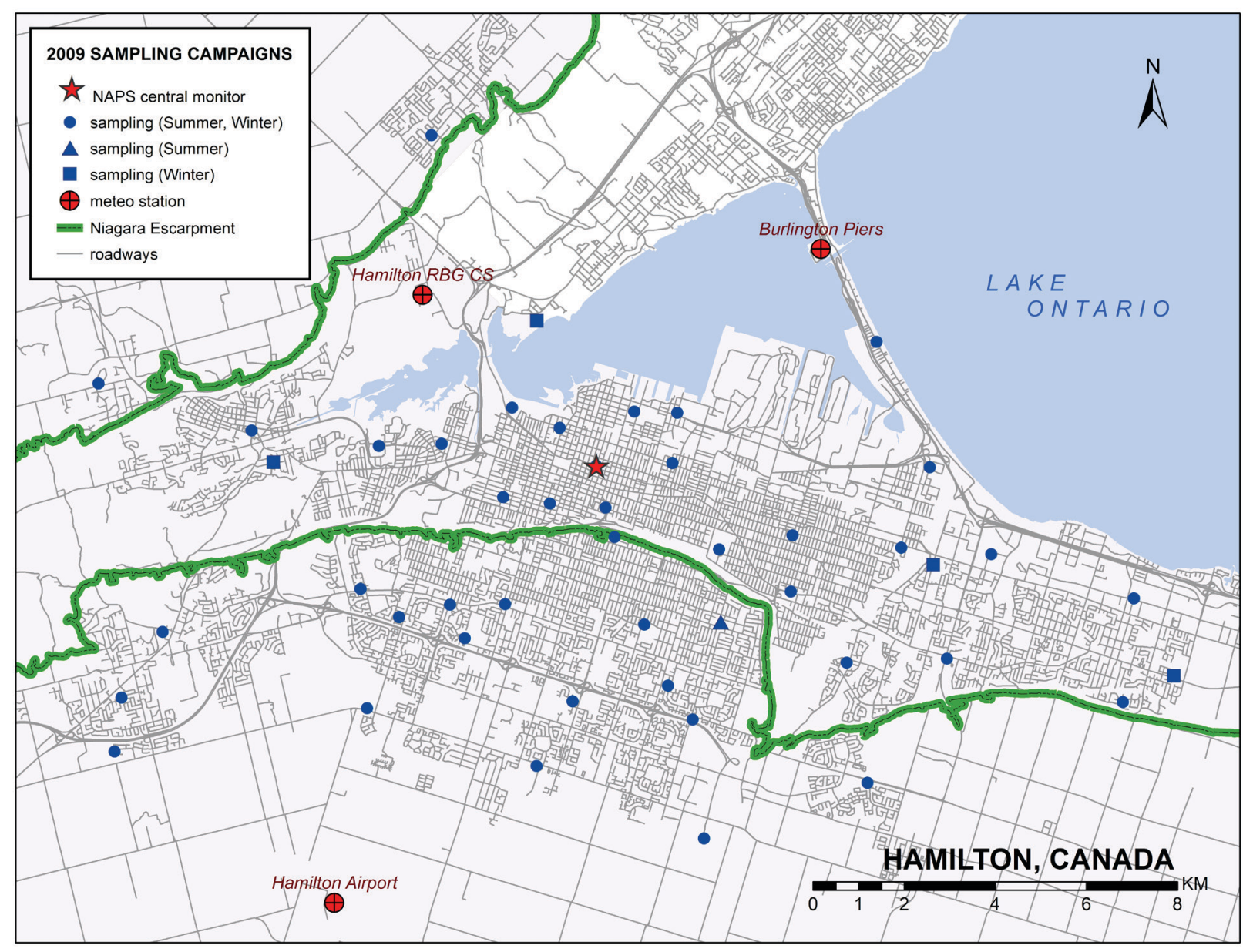

Figure 5-1. Map of study area showing escarpment, sampling sites, central monitoring station. 


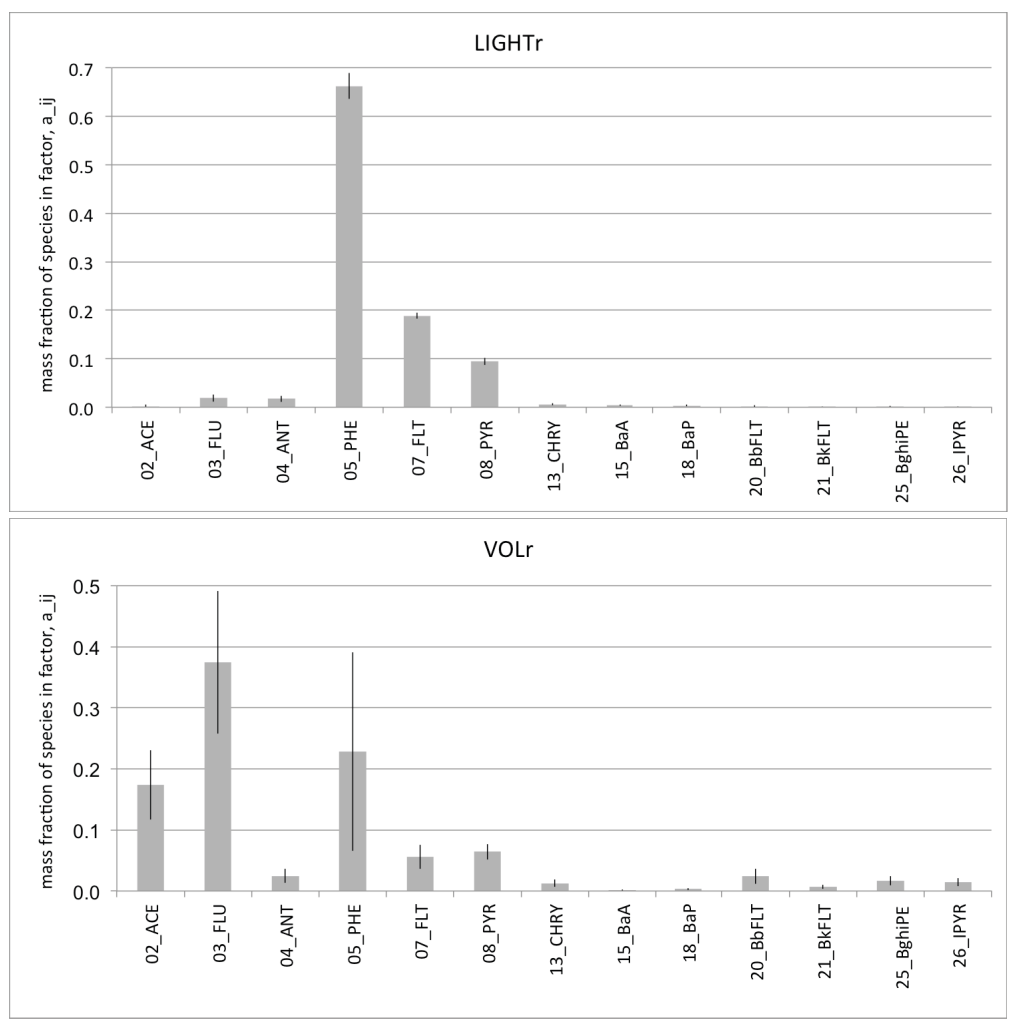

Figure 5-2. Local source profiles (PR) used in CMB modeling.
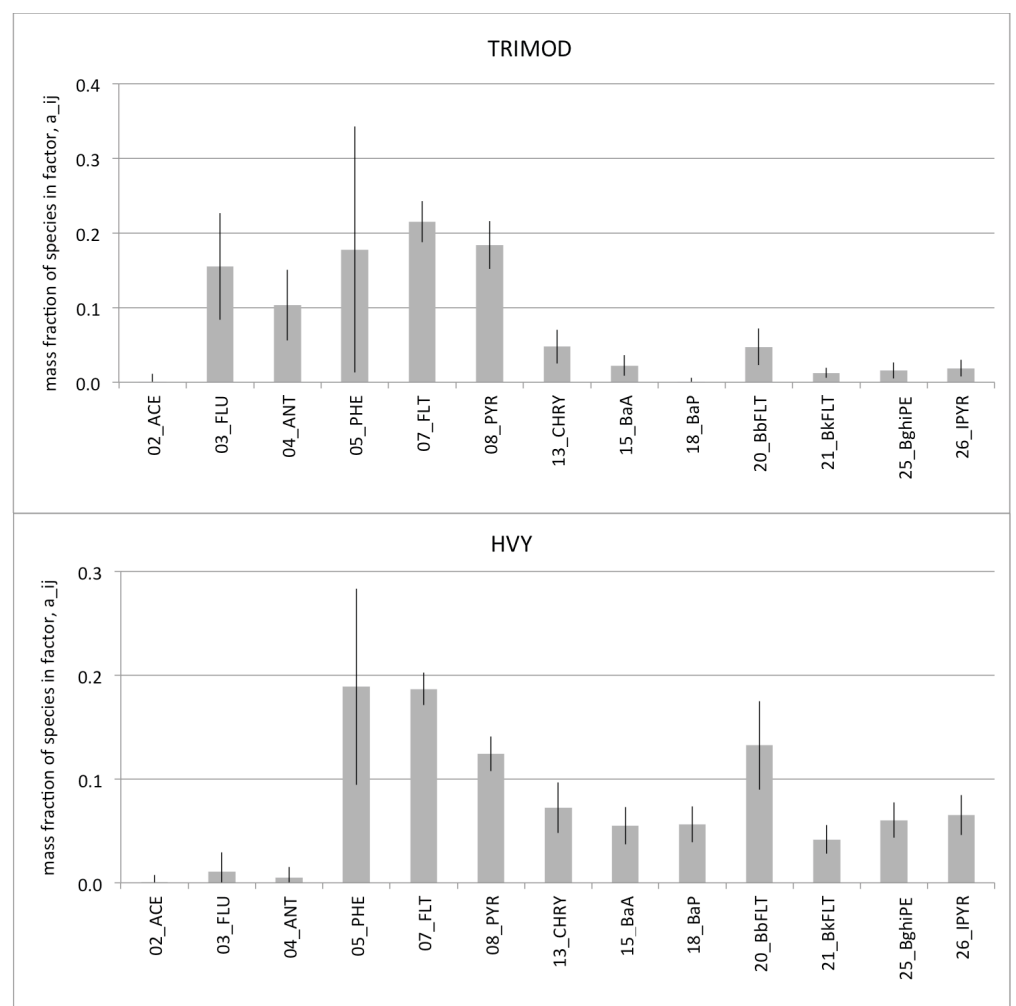

Note

1. Profiles derived by PMF from local central site monitoring data (Hamilton, year-round data, 13 fitting species, 4 factors).

2. Vertical axis scaled to maximum mass fraction. 


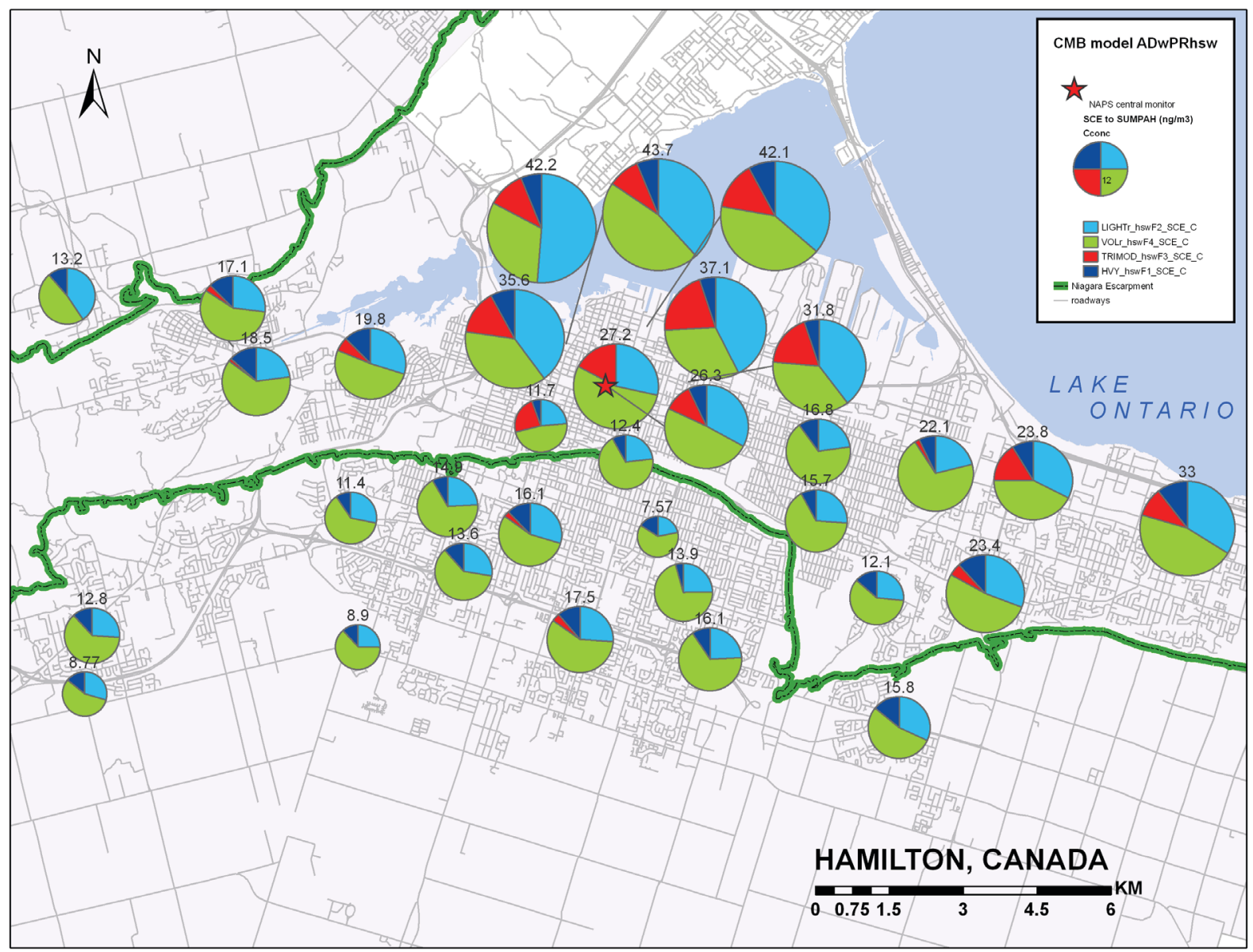

Note: Pie plot size is scaled to sampled SUMPAH $\left(\mathrm{ng} / \mathrm{m}^{3}\right)$, as labeled.

Figure 5-3. Map of fractional source contribution to $\mathrm{\Sigma PAH}$, all modeled factors. 


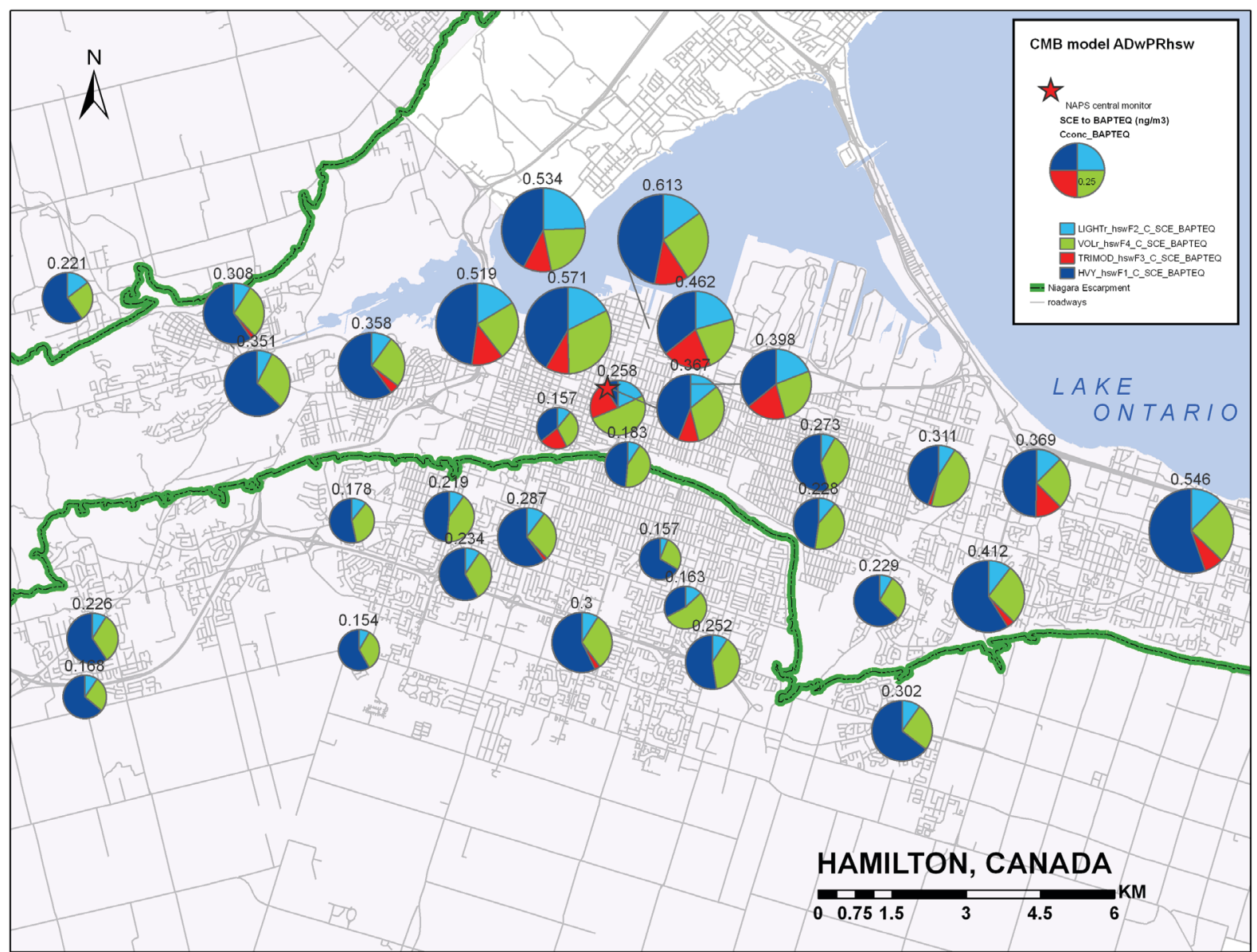

Note: Pie plot size is scaled to sampled BaP-TEQ $\left(\mathrm{ng} / \mathrm{m}^{3}\right)$, as labeled.

Figure 5-4. Map of fractional source contribution to BAPTEQ, all modeled factors. 
(a) $L I G H T r$

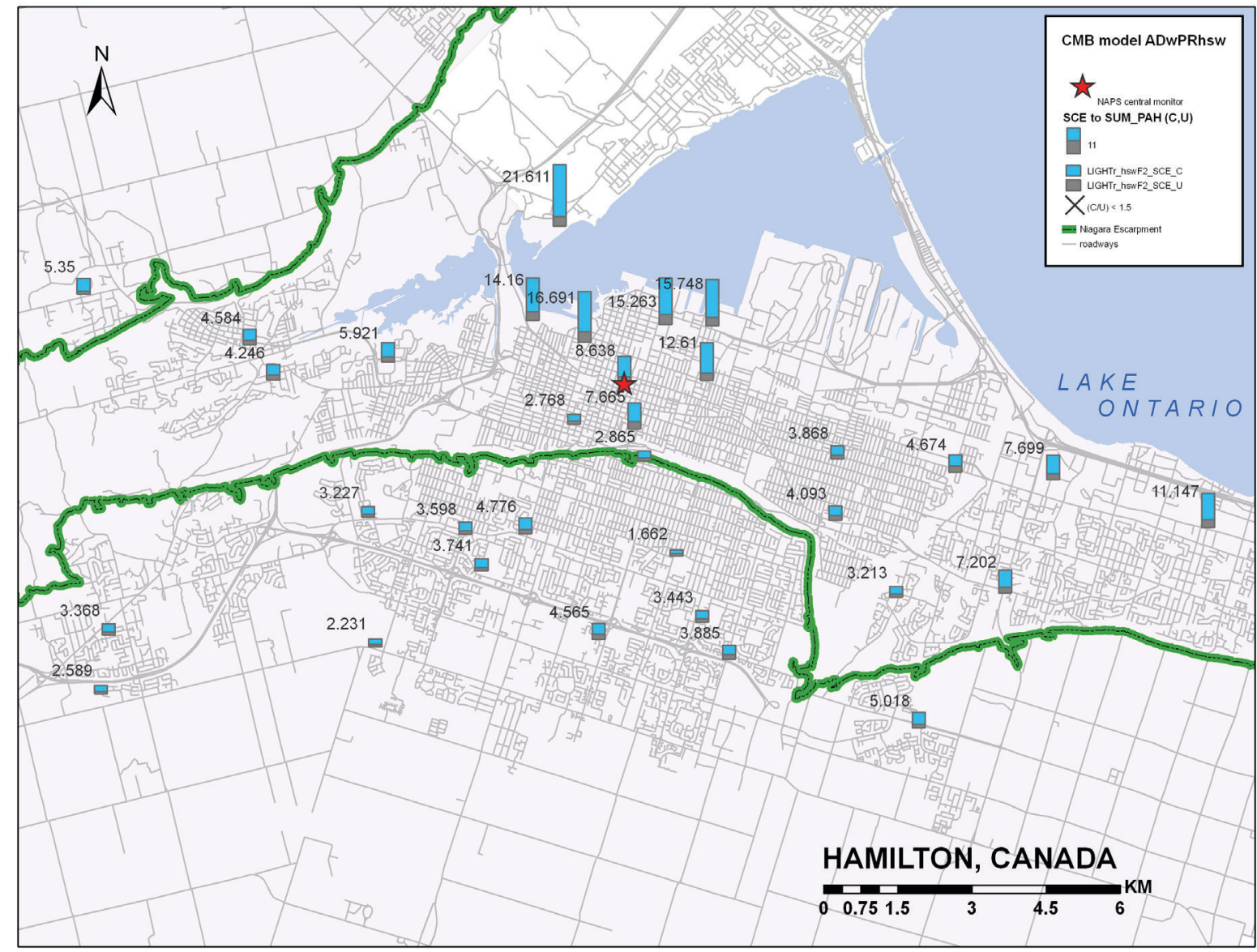

Note: Labels show factor contribution (SCE, $\left.\mathrm{ng} / \mathrm{m}^{3}\right)$

Figure 5-5. Map of source contribution to $\Sigma \mathrm{PAH}$ with uncertainty (sites T-stat $>1.5$ ). 
(b) $\mathrm{VOLr}$

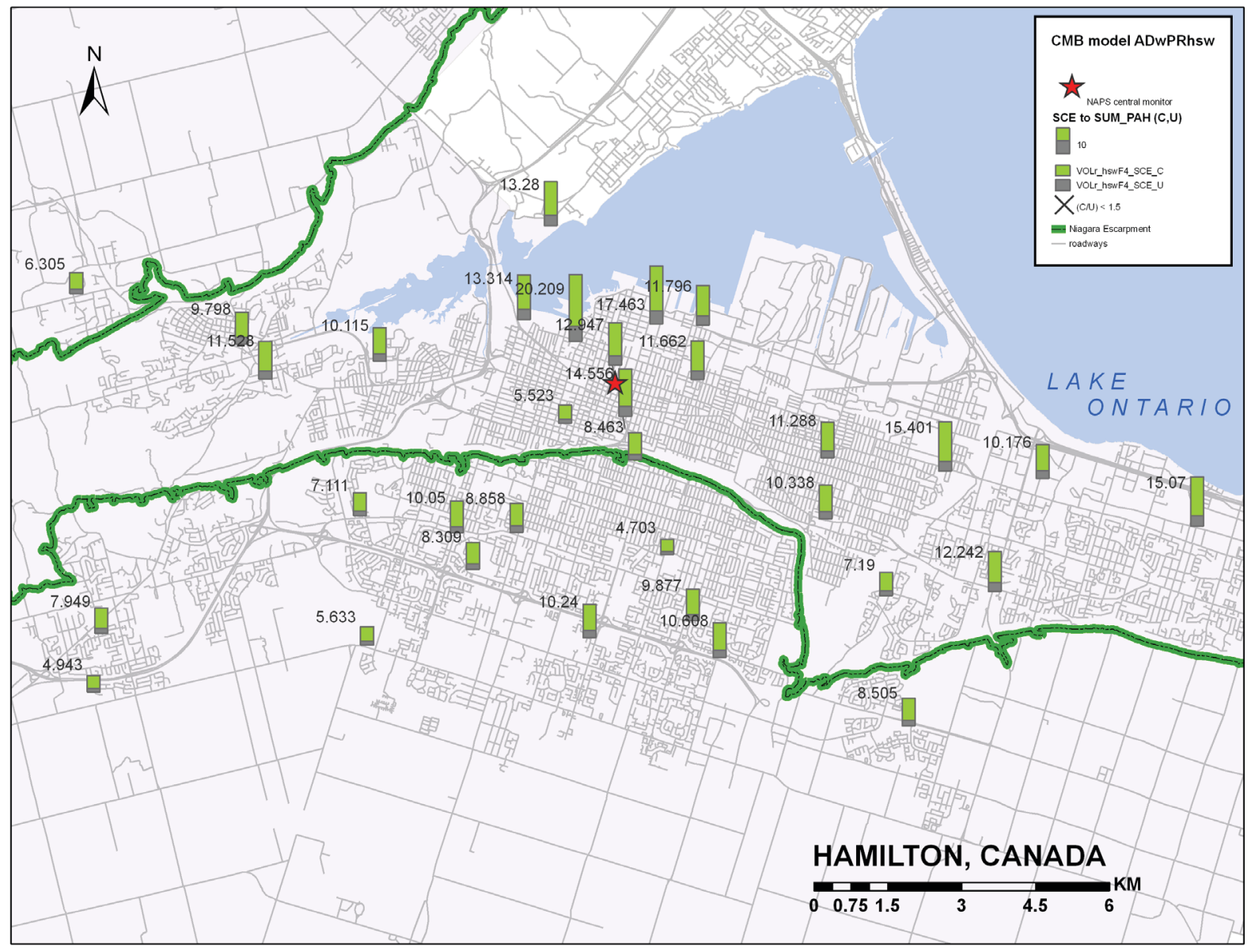

Note: Labels show factor contribution (SCE, $\mathrm{ng} / \mathrm{m}^{3}$ )

Figure 5-5 (continued) 
(c) TRIMODAL

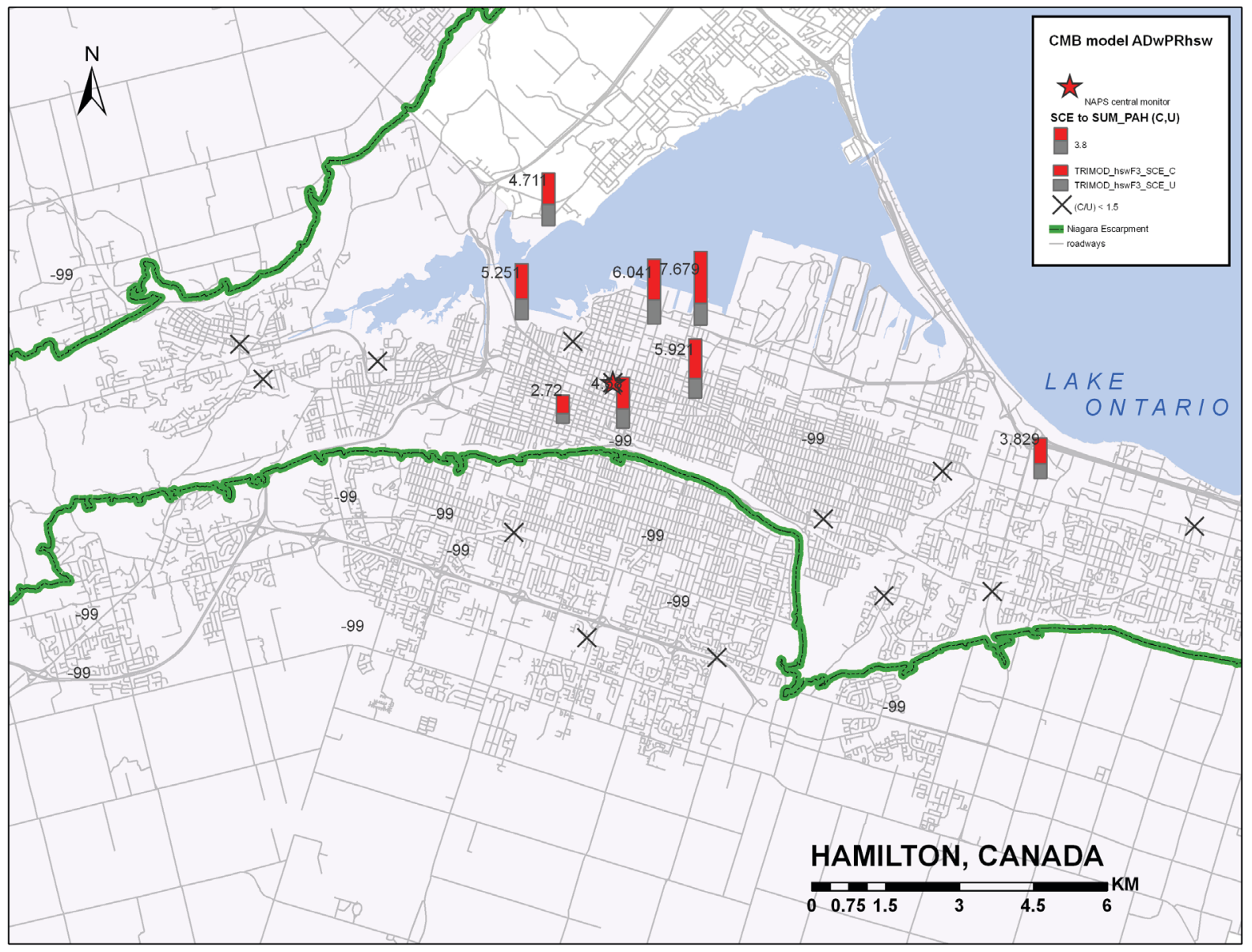

Note: Labels show factor contribution (SCE, $\mathrm{ng} / \mathrm{m}^{3}$ )

Figure 5-5 (continued) 
(d) HEAVY

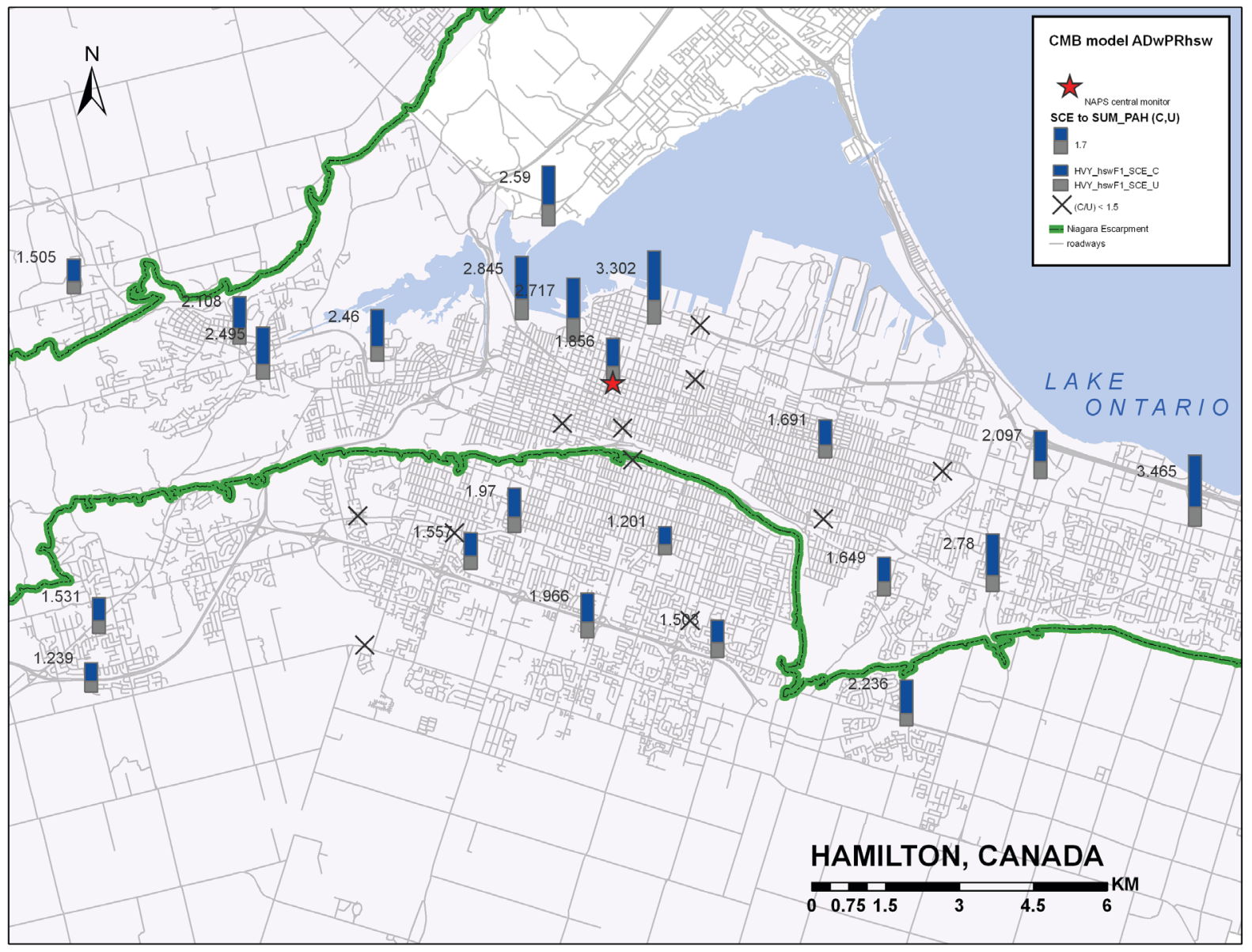

Note: Labels show factor contribution (SCE, $\mathrm{ng} / \mathrm{m}^{3}$ )

Figure 5-5 (continued) 


\section{Chapter: Conclusions and recommendations}

The highlights of the research work undertaken in this dissertation can be summarized in the following points:

- the analysis of long term ambient PAH concentration data from air quality monitoring stations in urban and rural settings with Positive Matrix Factorization (PMF) to identify quantitatively the contributions by factors that can be interpreted in terms of source categories;

- the experimental investigation of spatial and seasonal variability of PAH concentrations at the intraurban scale;

- the analysis of source contributions at intraurban sites by the use of factors identified from long term measurements at one monitoring site with the use of the Chemical Mass Balance (CMB) model; and,

- the extension of PAH concentration source apportionment at intraurban sites to total PAH toxicity (BaP-TEQ) and comparisons of spatial variability of PAH concentrations, PM concentrations, and PAH toxicity. 
The following were the hypotheses:

i. Inter-urban and urban/rural differences in historical ambient PAH levels will be significant and source apportionment will demonstrate these to relate to inter-site differences in dominant source types.

ii. $\quad$ Source apportionment will demonstrate some source types to be 'more toxic' than others (i.e., contributing relatively little to ambient PAH but disproportionately to ambient PAH toxicity).

iii. At the intraurban scale, $P A H$ will show greater variability than $P M_{2.5}$ demonstrating central monitoring as a comparatively poor proxy for estimating PAH exposure.

iv. At the intraurban scale, PAH 'exposure hot spots' (i.e., locations of elevated ambient PAH) will generally be found near concentrated local sources (e.g., traffic, heavy industry); 'exposure toxicity hot spots', however, will not necessarily coincide with 'exposure hot spots', indicating that some source types are contributing comparably more toxicity than others.

v. Pairing two different receptor models (i.e., PMF-CMB) so as to use 'local source profiles' will allow meaningful source apportionment at the intraurban scale.

vi. At the intraurban scale, source contributions to PAH will show spatial variability consistent with interpreted source type and will explain previously identified 'exposure hot spots'; source apportionment of PAH toxicity will distinguish 
sources contributing similarly across an urban centre from those associated with 'exposure toxicity hot spots'.

Key findings are summarized in the respective sections below, followed by the limitations of the experimental and modeling methods, and recommendations for future researchers.

\subsection{Conclusions}

\subsubsection{Urban/rural source apportionment of PAH and PAH toxicity}

The source-based investigation of PAH at the urban/rural scale confirmed the hypotheses and yielded several salient conclusions. First, inter-urban and urban/rural differences in historical ambient PAH were significant and related to inter-site differences in dominant source types: central site monitored levels were highest at Hamilton, the site with a priori known local industry, and lowest at the Egbert (background) site; ambient PAH levels showed the least seasonality at Hamilton, again indicating significant influence of local industry which typically operates year-round.

Second, the factors and interpreted source types resolved by source apportionment of the historical central site ambient PAH dataset were largely consistent across the sites, differing more by relative contribution than by source type. Four source types were found to be contributing to ambient PAH at all study sites: volatilized PAH and long-range transported coal combustion, vehicle traffic exhaust, space heating, biomass combustion. Source apportionment identified an additional local industry source type only at Hamilton, associated with local iron/steel manufacturing and having significant contributions to ambient PAH. Central site ambient PAH at Toronto was attributed to 
significant contributions from vehicle traffic exhaust and space heating; these source types and relative contributions were consistent with the city's large population and with the densely urbanized character and traffic network in the central business district near the sampling site. Coal-related PAH emissions were greatest at Hamilton due to significant local emissions from iron/steel production (i.e., coke ovens) and regional transported coal emissions. Compared with the urban sites, source apportionment at the Egbert site (background) showed less contribution from traffic emissions and greater contributions from space heating and biomass combustion, consistent with its rural location.

Third, source apportionment to PAH toxicity clearly demonstrated that some source types were contributing comparably less to ambient $\mathrm{PAH}$ but disproportionately to $\mathrm{PAH}$ toxicity: vehicle traffic exhaust contributed majority of PAH toxicity at all modeled sites and local industry (e.g., iron/steel manufacturing) contributed significantly to PAH toxicity at Hamilton. As such, the research quantified the degree to which some PMassociated source types can be considered "more toxic" than others (Grahame and Schlesinger, 2007). The specific finding that the traffic and local industry source types were contributing disproportionately to PAH toxicity corroborated previous chronic health effects studies that have identified these sources as possibly contributing to differential exposure toxicity, demonstrating inter-disciplinary consistency between source apportionment modeling (Chapter 3) and previous exposure assessment and epidemiological findings (Schwartz et al, 2005; Gold et al, 2005). 


\subsubsection{Intraurban variation in $\mathrm{PM}_{2.5}, \mathrm{PAH}$ and $\mathrm{PAH}$ toxicity}

Field sampling investigation of $\mathrm{PAH}$ alongside $\mathrm{PM}_{2.5}$ at the intraurban scale further confirmed research hypotheses, with following salient conclusions.

First, PAH showed much greater variability than $\mathrm{PM}_{2.5}$ in both the summer and winter sampling periods and indicated that, due to the more complex intraurban gradients and presence of 'exposure hot spots' found near local sources, central monitoring is a comparatively poor proxy for summarizing ambient PAH exposures. PAH concentrations sampled at the central site monitor were often exceeded at other locations in the city and hence could not be considered conservative estimates of PAH levels for the city as a whole, findings that confirmed the importance of local source contributions to PAH and are expected to apply to many urban centres containing mixed land uses of industrial, commercial, residential.

Second, PAH 'exposure hot spots' were generally found near local sources such as traffic and heavy industry but 'exposure toxicity hot spots' were not always coincident with these. Taken together, these findings indicated the importance of local sources in understanding PAH ambient exposures and indicated that some source types are contributing more toxicity than others.

As a corollary, ambient PAH toxicity (BaP-TEQ) was shown to vary significantly less than ambient PAH concentrations across the urban area, indicating that PAH toxicity remains a concern even where ambient PAH concentrations are comparably low, for example residential areas above the escarpment. Thus, estimates of PAH exposure hazard are very poorly indicated by ambient exposure levels alone underscoring the importance 
of assessing toxicity of pollutants, particularly for: (i) PM-associated air toxics; and, (ii) identifiable classes of toxic compounds.

\subsubsection{Intraurban source apportionment of PAH and PAH toxicity}

Source-based investigation of intraurban ambient PAH also confirmed research hypotheses and yielded salient conclusions.

First, the application of a paired PMF-CMB receptor model so as to model the contributions of 'local source profiles' across an urban area with complex sources and topography yielded meaningful source apportionment results: spatial trends in source contributions were consistent with source, topographical, and prevailing wind characteristics of the urban area.

Second, the source contributions to PAH showed spatial variability consistent with interpreted source types: the PAH volatilization and transported coal combustion + biomass combustion and space heating + biomass combustion source types showed low intraurban variability or above/below escarpment differences; the vehicle traffic exhaust factor showed moderate variability in relative contributions, consistent with variation in road network density and traffic intensity; the local industry factor contributed minimally

at most urban sites yet had significant contributions at sites below the escarpment and near the industrial harbour-front. The spatial variability in source type contributions helped to explain previously identified 'exposure hot spots': areas of high ambient PAH were due to higher contributions of local source types.

6: Synthesis, Conclusions, Recommendations \& Future Research 
Third, source contributions to PAH exposure toxicity (BaP-TEQ) did not follow the same spatial gradients as source contributions to total PAH concentrations, thereby confirming that 'source toxicity' can differ significantly at the local scale: traffic emissions were the predominant source of PAH exposure toxicity despite contributing little to total PAH concentrations and contributed similarly to PAH toxicity throughout the urban area; local industry emissions were found to be contributing significantly to exposure toxicity only near the city's industrial zone; meanwhile, the other modeled source types generally contributed little to PAH exposure toxicity, despite representing the majority of total sampled PAH exposure. The source apportionment of PAH toxicity helped explain previously identified 'exposure toxicity hot spots': these were coincident with high contributions of the local industry source in addition to vehicle traffic contributions. Vehicle traffic exhaust emissions were the predominant source type contributing to PAH toxicity at all field sites, even where ambient PAH concentrations were comparably low, underscoring the importance of this source type in further research or in the design of health interventions.

Last, the intraurban source apportionment results withstood considerations of uncertainty: spatial trends in source type contributions were preserved and accentuated when high-uncertainty sites were excluded from the analysis.

6: Synthesis, Conclusions, Recommendations \& Future Research 


\subsection{Limitations}

\subsubsection{Sampling limitations}

Central site monitoring data was well suited to inter-site and historical trend analysis and also to source apportionment via application of the PMF receptor model. However, the central monitoring site samplers are subject to risk of sample loss for light molecular weight and high volatility PAH species such as acenaphthylene and acenaphthene due to inherent limitations of PUF sorbent cartridges at high flow rates and high temperatures. Sample degradation due to reactive $\mathrm{PAH}$ species is an additional inherent limitation due to potential reactions with ambient oxidants such as ozone, hydroxyl radical, or nitrogen oxides. Since these potentially negative effects were not quantified the field sampled PAH concentrations potentially underrepresent the ambient concentrations, particularly for the summer sampling session.

As with any field sampling study, several inherent practical limitations can be cited. Given constraints of equipment and resource availability, the sampling network implemented in Hamilton achieved a reasonable siting density of $\sim 1$ sampler $/ 5 \mathrm{~km}^{2}$; $1.5 \mathrm{~km}$ average inter-site distance. Finer resolution analysis of spatial variations would require greater densification. While sampling data aligned well with expected seasonal trends, the single two-week sampling campaigns cannot be taken as strictly seasonally representative of summer and winter.

For future field sampling studies, it is desirable to increase sampling air flow rates and decrease sampling period length to obtain field samples with reduced measurement uncertainties and ensure higher sample mass for more species. It is understood that this 
will increase the risk of PUF sorbent breakthrough at high temperatures for few light molecular weight species but such an increase would be largely limited to summer sampling data and could reasonably be considered nominal given central site samplers are routinely able to successfully collect significant mass for light molecular weight species using PUF sorbents despite much higher flow rates.

\subsubsection{Data and modeling limitations}

The central site PAH datasets used a suitably long time series and the intraurban sampling network was denser than has previously been generally available for PAH analysis and source apportionment. Nevertheless, additional PAH speciation, particularly of source marker species, and increased temporal/spatial resolution would enable improved analyses at the urban/rural and intraurban scales.

For the urban and rural site source apportionment, modeling power was limited by data quality in the central site datasets. Quality assurance and quality control procedures (QAQC) limited the modeling to 21 of the 29 species monitored. Additional species with sufficient data quality for inclusion in the receptor model would facilitate factor interpretation in source apportionment; availability of species that could reasonably be applied as source markers could further corroborate factor interpretations.

Two factors resolved by urban/rural PMF modeling were interpreted to represent more than a single source type: $P A H$ volatilization and transported coal combustion; space heating. Sensitivity analysis with multiple model orders and with seasonal model variants showed these factor profiles to be stable; this suggested that more differentiated 'extracted simple' (Shi et al, 2009) source types, interpreted as representing only a single 
source type, would only be possible with a larger PAH species suite that included source marker species or with a modeling suite expanded to include other PM-associated markers (e.g., metals, other organics). Such an expanded modeling suite may also permit better separation of local source components from regionally transported source components.

The exclusion of dibenz(ah)anthracene (DBA) from the source apportionment modeling merits discussion. It was excluded from the source apportionment due to poor data quality at the background (Egbert) site (i.e., missing and/or below detection limit samples) and the desire to maintain a common analysis and modeling suite to facilitate inter-site comparisons. However, DBA had sufficient data quality to permit inclusion in the Hamilton receptor model and, possibly, at Toronto. There is incentive for including DBA in future receptor modeling efforts due to its high relative toxicity. Inclusion of DBA or other highly toxic heavy MW species would further amplify the disproportionate toxicity of vehicle traffic source type or other source types characterized by heavy MW species.

For the intraurban source apportionment, explanatory power of the PMF-CMB modeling was inherently reduced due to species differences in the central site monitoring dataset (21 species modeled) and the comparatively smaller field sampling dataset (i.e., 13 species modeled of the 16 species analyzed for, after QAQC procedures). This would be improved by a larger field sampling suite better matching the central site data. In particularly, inclusion of retene as a useful marker species for biomass combustion would have improved the source apportionment and possibly permitted an increase of model 
order to five factors. Data from the field sampler site co-located with the central site monitor demonstrated generally good comparability between the central site and field datasets (Chapter 4) and CMB source apportionment results were shown to be consistent when applied to either central site data or co-located field sampler data (Chapter 5); nevertheless, dataset compatibility for PMF-CMB modeling and related analyses would be improved by increasing field sampling flow rates and decreasing sampling time.

\subsubsection{Additional methodological limitations}

This research was careful to interpret source types using a preponderance of evidence from receptor model results (e.g., characteristic species, temporal trends, inter-site comparisons) and site-specific environmental context along with reference to other source apportionment literature. At the intraurban scale, the PMF-CMB source apportionment eschewed proxy profiles in favour of 'local source profiles' derived from local time series data. However, since comparison with reference profiles remains a useful interpretive tool, additional availability of source sampling profiles would necessarily improve interpretive power, particularly if available from direct source sampling of local facilities identified in this research as contributing significant PAH emissions to the study area.

Chronic health risk from ambient PAH exposure was quantified using BaP-TEQ for the modeled suite of PAH species, indicating relative trends in ambient PAH toxicity (i.e., allowing comparison of source contributions to toxicity, inter-site and intraurban variation in PAH toxicity). However the BaP-TEQ values must be considered a lower bound on absolute total PAH-associated toxicities due to the small suite of modeled 
species compared with the very large class of parent and reacted PAH found in ambient air. Additionally, given there are several TEF datasets available in the PAH toxicology literature, choice of individual TEF values will increase or decrease estimations of PAH toxicity. Further, potential individual chronic health risk across the urban study area will depend on individual exposure and possible synergistic effects between pollutants and other risk factors.

6: Synthesis, Conclusions, Recommendations \& Future Research 


\subsection{Recommendations and suggested future research}

\subsubsection{Recommendations}

The intraurban source apportionment using PMF-CMB modeling can be considered proof-of-concept and was demonstrated for a particular location (Hamilton, Canada) with specific characteristics (e.g., local land use distribution, local source distribution and emissions patterns, source location relative to prevailing winds, topography) that have necessarily influenced research results. It would be desirable to repeat the PMF-CMB methodological approach for an urban centre comparable to Hamilton in scale but different in contributing local source types and geographic characteristics in order to further support or challenge the observations about the effectiveness of the methodology.

Following from the conclusions, several specific recommendations can be made to other researchers analysing classes of air toxics such as PAH or conducting source apportionment using advanced receptor models.

Source apportionment studies applying advanced receptor models like PMF should make all efforts to reduce subjectivity in the modeling process. This can be done by following best practice guidelines available in the literature (Reff et al, 2007; Watson et al, 2004) as well as the additional methods demonstrated in this dissertation (Chapter 3), emphasizing: (i) prior to modeling, conduct and document investigation of environmental context for source apportionment; (ii) insist on realistic and species-sensitive uncertainty estimates for input data; and, (iii) report uncertainty on model results including the uncertainty associated with factor profiles and with factor contributions.

6: Synthesis, Conclusions, Recommendations \& Future Research 
Comprehensive exposure assessment of PM-associated air toxics such as PAH should be conducted at the intraurban scale using a suitably dense siting network rather than relying on central site monitoring data so as to capture spatial variations in ambient concentrations. The spatial variations in PAH concentrations have been shown to be greater than the spatial variation in fine PM (Chapter 4) in the Hamilton case. PAHassociated ambient toxicity demonstrated significant spatial variation that, if not adequately captured, may lead to exposure risk misclassification and underestimate exposure health risk for some neighbourhoods and sub-populations.

Where a study's primary objective is to quantify total PAH toxicity, analysis of spatial samplers could possibly be restricted to heavy molecular weight species since they are responsible for the majority of total toxicity, based on BaP-Toxicity Equivalency Factors (TEF). This would potentially benefit laboratory analysis resources for dense intraurban studies with a large number of PAH samplers requiring analysis. However, this must be balanced against the advantages of analysing for a larger suite of PAH species that includes light molecular weight species which, despite having lower chronic toxicity, are themselves still a health concern and by their inclusion permit more analyses options (e.g., source apportionment modeling).

Source apportionment studies applying the $\mathrm{CMB}$ receptor model to explain ambient observations in intraurban field sampling data should strive to obtain 'local source profiles' as model input rather than proxy profiles that are unlikely to be sufficiently representative of the area. In lieu of local source sampling, a paired PMF-CMB model can be used, with PMF applied to resolve 'local source profiles' from long time-series 
central site monitoring data and $\mathrm{CMB}$ used to explain ambient observations in intraurban field sampling data in terms of relative source contributions (Chapter 5).

Intraurban exposure assessment (Chapter 4) will better inform interventions (e.g., prioritizing dominant source types in emissions reduction policy or health messaging) when supplemented by source apportionment at the intraurban scale (Chapter 5) since source mix was demonstrated to vary significantly across the urban study area.

Intraurban scale source apportionment maps (Chapter 5) should include uncertainty explicitly, censoring site data for which uncertainty is unacceptably high or, as a sensitivity analysis, ensuring that inferred trends can withstand exclusion of sites with high uncertainty.

Since ambient PAH toxicity varied significantly at the intraurban scale (Chapter 4) and some PAH source types were clearly demonstrated to contribute disproportionately to ambient toxicity (Chapters 3, 5), any investigations of PAH should convert and analyse results on the basis of total benzo(a)pyrene-toxicity equivalent concentration (BaP-TEQ). This is important even for emissions inventories, which can otherwise underestimate importance of source types for sources contributing little pollutant mass but disproportionately to PAH toxicity.

Interventions intended to reduce $\mathrm{PAH}$ source emissions (e.g., emissions control regulation) would best consider contribution to ambient PAH-associated toxicity (BaPTEQ) rather than contributions to ambient PAH concentration ( $\mathrm{PAH})$, so that sources contributing disproportionately to toxicity are adequately addressed. It is understood that 
this requires educating stakeholders designing regulatory tools for air toxics to consider both amount and toxicity of source emissions rather than only amount, despite the latter having worked well in past for single compound criteria pollutants (e.g., $\mathrm{SO}_{\mathrm{x}}$ ).

Analogously, interventions intended to reduce PAH exposures (e.g., central site monitoring, public health messaging) would benefit from using language stressing toxicity and focusing awareness on 'more toxic' source types such as vehicle traffic exhaust or local heavy industry. More nuanced health messaging tools, such as Canada's health-based AQI (AQHI; Stieb et al, 2008), are a step in the right direction and consideration should be given to analogous aggregate indicators for chronic health risk, incorporating PM-associated PAH and other key air toxics.

6: Synthesis, Conclusions, Recommendations \& Future Research 


\subsubsection{Suggested future research}

There are numerous future research opportunities that arise from the research findings and the proof-of-concept PMF-CMB methodology. The use of the PMF-CMB methodology for spatial variation at the intraurban scale for other sites in addition to Hamilton has already been mentioned as the first item under Recommendations. Research related to other methodological issues is presented in brief below.

\section{A. Further development of source apportionment methodology}

Source apportionment analysis of central site data would benefit from an expanded suite to include additional species characterizing PM (e.g., key source marker elements from inorganics data). This would increase model power of analysis and corroborate interpretation of factors as physically meaningful source profiles. The PAH species DBA can also be included in PMF source apportionment at Hamilton, where NAPS data for this species is of sufficient quality; expectation is that the disproportionate toxicity of vehicle traffic source type or other source types characterized by heavy molecular weight species would be further amplified. Inclusion of DBA in the intraurban source apportionment, here precluded due to below detection limit sampled values at all field sampling sites in winter campaign, would not fundamentally alter relative differences in source contribution across sites.

This research avoided the limitations of using particle phase data in receptor modeling by using vapour+particle ambient PAH data. However, many previous studies have used only particle phase, and particle phase only data continues to be field-sampled. This limitation could be explored by applying partitioning estimates to the PAH 
vapour+particle data and comparing results with those in this research. This could enable the re-analysis of previous source apportionment studies conducted using only particle phase PAH data and provide researchers who are limited to particle phase sampling with quantifiable estimates of the effects of omitting the vapour phase.

This research used one set of TEF values, sourced primarily from Nisbet and Lagoy (1992). Given there are several TEF datasets available in the PAH toxicology literature, choice of individual TEF values will increase or decrease estimations of PAH toxicity. Future work can investigate the sensitivity of 'source toxicity' quantifications to TEF values. This can be done by: (i) using TEF datasets with significantly different values (i.e., $>1$ order of magnitude difference from Nisbet and Lagoy, 1992), adapting the sensitivity analysis approach used by Cai et al (2012); or, (ii) by combining all available comparable data and using a median TEF value along with statistical range estimates (first quartile, third quartile) to incorporate an estimate of uncertainty on source type contributions to BaP-TEQ and thus on comparative source toxicity.

Last, opportunity exists to incorporate Conditional Probability Function (CPF) analysis into the PMF-CMB source apportionment methodology. While CPF is most often applied to PMF results for source apportionment of PM, it can also be applied to PAH source apportionment results to further corroborate local source type interpretations. Further, any local source regions indicated by CPF for the PMF-modeled central site data at Hamilton can be compared with the spatial distribution of source contributions indicated by CMB modeling of the intraurban field dataset. To facilitate future work, the CPF methodology is described in detail in Appendix B.3.

6: Synthesis, Conclusions, Recommendations \& Future Research 


\section{B. Meta-analysis and interdisciplinary research}

PAH source apportionment studies typically have not explicitly presented results in terms of toxicity. Meta-analysis of select key studies with PAH data appraised in terms of toxicity (BaP-TEQ) has the potential to highlight trends and conclusions not possible with consideration of only ambient PAH concentrations. Toxicity-based meta-analysis of past studies would contextualize the significance of differences in spatial trends observed in Hamilton between the PAH and BaP-TEQ metrics. Meta-analysis can include sensitivity analysis to choice of TEF values, as mentioned in item 'A' above.

The over-riding purpose of conducting air pollution exposure research is to inform interventions that can reduce exposure health risk, particularly for individuals identified as at-risk for elevated exposure. The insights collected in this research on identification of intraurban zones with elevated ambient PAH and BaP-TEQ and identification of source types contributing disproportionately to PAH toxicity can be used to develop a sourcebased health messaging study. This research would develop suitable aggregate metrics for communicating source-based air pollution health messaging to the public, applying methods from the fields of aggregate indicators / environmental indicators and risk communication science. The research would then determine appropriate media for targeting individuals in neighbourhoods or subpopulations previously identified as at-risk (i.e., such as identified in this dissertation for Hamilton) and deliver meaningful sourcebased air quality information to help reduce personal PAH exposure health risk. A related initiative is to investigate adapting the AQHI (Stieb et al, 2008) to include PAH and other air toxics.

6: Synthesis, Conclusions, Recommendations \& Future Research 


\section{Appendices}

\section{Appendix A Literature review}

This appendix presents material intended to provide additional general background material on PAH to supplement the research methodology (Chapter 2).

\section{A.1 Properties of PAH}

Overview of general properties of $\mathrm{PAH}$, inclusive of: table of chemical properties; chemical structure diagrams; vapour/particle partitioning.

\section{A.2 PAH exposure health effects}

Salient details on health effects from human exposure to air pollution, PM, and

PAH (i.e., emphasis on inhalation exposure pathway). Details of PAH Toxicity Equivalency Factors (TEF).

\section{A.3 Key source apportionment studies}

Summary of salient findings from key studies in which PAH were source apportioned using Positive Matrix Factorization (PMF) or Chemical Mass Balance $(\mathrm{CMB})$ receptor models.

A.4 Approaches to estimating uncertainty on PMF input data

Summary of methods of estimating PMF input uncertainty from key precedents in literature. 


\section{A.1 Properties of PAH}

By general definition, Polycyclic Aromatic Hydrocarbons (PAHs) are compounds containing carbon and hydrogen atoms grouped into a series of aromatic ring structures (Environment Canada, 2007e; US EPA, 2007). PAHs are also sometimes referred to as Polynuclear Aromatic Hydrocarbons.

As PAHs comprise a large class of compounds, their physical properties also vary with their molecular weight (MW) and structure. PAHs are categorized broadly by their MW, with low MW PAHs having fewer than four aromatic rings and high MW PAHs having four or more aromatic rings (CEPA, 1999). The species categorization used throughout this research is adapted from $\mathrm{Li}$ et al (2005), defining "light PAH" as species with $\leq 4$ ring structure and $\mathrm{MW} \leq 202$, "medium PAH" as species with 4-ring structure and MW between 202 and 228, and "heavy PAH" as species with >4-ring structure and MW >228. Additional general physical properties of PAHs include: low solubility in water (exception is naphthalene); low to moderately high vapour pressure; and, relatively high octanol-water partition coefficients $\left(\mathrm{K}_{\mathrm{ow}}\right)$.

As indicated by their relatively high $\mathrm{K}_{\text {ow }}$ (i.e., $\log \mathrm{K}_{\text {ow }}=4-7$ for most species), PAH have high potential to adsorb to suspended particles, either in air or water. The high $\mathrm{K}_{\mathrm{ow}}$ is also indicative of their potential to bio-concentrate in organisms. (CEPA, 1999)

PAHs tend to be environmentally persistent compounds, with larger (i.e., higher ring count) and heavier molecular weight PAHs being more persistent than smaller and lighter molecular weight PAHs. This is consistent with larger and heavier PAHs' increased intermolecular forces and with their decrease in volatilization, as shown by Henry's Law Constant decreasing for larger and heavier PAHs.

Key physical and chemical properties of PAH species sampled by NAPS are shown in Table A.1-1 and chemical structures are depicted in Figure A.1-1.

\section{Vapour/particle partitioning}

PAHs in ambient air are semi-volatile organic compounds (SVOCs) and, as such, are present in vapour phase (i.e., "vapour phase PAH") and also bound to particulate matter (PM; i.e., "particle phase PAH") adsorbing to the particle surface or volatizing into it (Environment Canada, 2007e; CEPA, 1999). Factors influencing gas/particle partitioning of PAHs include the species properties (molecular weight, vapour pressure, $\log \mathrm{K}_{\mathrm{ow}}$ ), ambient temperature, relative humidity, precipitation, ambient species concentration, and amount and type of fine particles present for sorption (Ravindra et al, 2008; Baek et al, 1991a). 
The tendency of lighter MW PAH species to volatilize to vapour phase is indicated by their comparably low vapour pressures (see Table A.1-1 for Henry's Law constant decreasing with increasing PAH MW and thus indicating decreased tendency of heavy species to be found in vapour phase). The tendency of PAH to adsorb to aerosol particles is indicated by their relatively high $\mathrm{K}_{\mathrm{ow}}$ (i.e., $\operatorname{logKow}=4-7$ for most species; $\log \mathrm{K}_{\mathrm{ow}}$ generally increasing with increasing MW as seen in Table A.1-1).

Studies where ambient PAH was sampled in both vapour and particle phase have consistently shown that, generally, lighter MW species with 2 and 3-ring structures (MW $\leq 202$ ) are found largely in vapour phase while heavier MW species with 5-ring and 6ring structures (MW >228) are largely in particle phase. This was shown in early work by Yamasaki et al (1982) and Venkataraman and co-authors (Venkataraman and Friedlander, 1994; Venkataraman et al, 1994) and has been well summarized in key PAH review papers (Baek et al, 1991a; Ravindra et al, 2008).

A more recent rigorous sampling study by Lu et al (2008) corroborated these findings. Lu (2008) sampled ambient PAH (outdoor and indoor) in both vapour (XAD media) and particle phase (Teflon filter; three aerodynamic size fractions: 2.5um, 2.5-10um, >10um), analysed 15 species of $\mathrm{PAH}$, and verified that partitioning to PM phase increased with increasing MW. The "dividing line" for preferential gas/particle partitioning was found to correspond to the sampled species chrysene (MW 228), where vapour/particle partitioning was approximately evenly split; lighter species had greater partitioning to the vapour phase, heavier species had greater partitioning to the particle phase. The preferential vapour/particle phase partitioning for the range of sampled species in Lu et al (2008) is shown in Figure A.1-2.

The PM size fraction distribution of PAH is also of interest. PAH in aerosol phase is associated primarily with smaller size fractions and thus that the clear majority of PMphase ambient PAH mass is represented by the fine size fraction, $\mathrm{PM}_{2.5}$. Several studies have sampled a number of successive PM size fractions and characterized each with respect to elemental and organic composition. Seminal sampling studies quantifying the PM size distribution for PM-phase PAH have been carried out for ambient air (Venkataraman and Friedlander, 1994) and air inside a traffic tunnel to capture mixed fleet vehicle exhaust emissions (Venkataraman et al, 1994). In the tunnel air samples, PAH were shown to exhibit a unimodal size distribution (mode at $0.12 \mathrm{um}$ aerodynamic diameter); in the ambient air samples, PAH were shown to exhibit a bimodal size distribution (emitted mode at $0.05-0.12 \mathrm{um}$, accumulation mode at 0.5 -1.0um aerodynamic diameter). Thus, in both studies, the majority of PM-phase PAH (species ranging from light to heavy $\mathrm{MW}$ ) was found in the ultrafine size fraction and thus well 
within the fine size fraction $\left(\mathrm{PM}_{2.5}\right)$ of concern for health risk due to exposure by inhalation (Lu et al, 2008; ATSDR, 1995).

More recently, Schnelle-Kreis et al (2001), in a sampling study in Munich, Germany found approximately $90 \%$ of the sampled PAH to be on particles smaller than $2 \mu \mathrm{m}$. Other studies have consistently found similar results (Ohura et al, 2004; Guo et al, 2003; Allen et al, 1996; Baek et al, 1991b, 1991c; Liu et al, 2006). A typical size distribution of particle-phase PAH in ambient air is presented from Allen et al (1996) in Figure A.1-3.

An overall summary of preferential gas/particle partitioning and size-fraction abundance of ambient PAH is obtained from Ravindra et al (2008): "Light PAHs with 2-3 benzene rings are mostly found in gas phase while the heavy ones are mainly associated with airborne particles. The majority of PAHs (70-90\%) are adsorbed on suspended particles at ambient temperature. Moreover, PAHs are mostly adsorbed on small inhalable particles with a high concentration on airborne particles of sub-micron diameter."

A related, and important, point is that vapour phase ambient PAH concentrations are much greater than particle phase ambient PAH concentrations; as such, light MW species tend to be found in much greater abundance than heavy MW species (i.e., contribute majority of total vapour plus particle concentrations). This is well summarized in the findings of Lu et al, 2008, reproduced in Figure A.1-4 and shows both gas/particle phase split and, for particle phase $\mathrm{PAH}$, the dominance of the fine size fraction. This stresses the importance of sampling both phases if 'total ambient PAH' (sum of vapour and particle phase) concentrations are desired, important for exposure assessment and thus for source apportionment of PAH toxicity.

\section{Atmospheric transport}

Vapour phase PAH (i.e., light MW species) can be subject to long range transport (Ravindra, 2008). Particle-bound PAHs, however, are considered environmentally persistent; since they are found primarily associated with particles in size range $0.1 \mathrm{um}$ 3um, particle-bound PAHs have relatively long atmospheric residence times (i.e., several days; confirmed by review of species properties tabulated in Mackay et al, 2006) and, since this size range of PM is not effectively removed by rain it may also be subject to long range transport (Baek et al, 1991a).

PAH atmospheric removal is by dry or wet deposition of particles and vapours. Generally, gas phase PAH dissolve within clouds and into raindrops and particle-bound PAHs (predominantly on fine size fraction) is largely by particle washout (i.e., rainfall, snowfall) or dry particle deposition (Ravindra, 2008). 
PAH can also react with sunlight or other atmospheric compounds to form even more persistent or toxic transformation products (e.g., nitro-PAH, hydroxy-PAH, both of which are known carcinogens; Van Noor, 1985; CEPA, 1999), with light MW vapour phase species generally more prone to photochemical reaction than heavy MW and therefore particle-bound species (Ravindra, 2008).

The potential confounding of PAH receptor modeling due to inter-species differences in reactivity was explicitly investigated by a sampling and receptor modeling study conducted by Mari et al (2010) who concluded that atmospheric transformations have little confounding effect. Mari et al (2010) also cite the similar results of the Canadian study by Sanderson et al (2004), which found that PAH source releases age to a stable profile during source-receptor transport and consequently demonstrated relatively long atmospheric lifetimes for PAH.

\section{Seasonal variability}

Some sources may have seasonal variability in their PAH releases and thus contribute to seasonal trends in ambient PAH levels. This is particularly the case for PAHs from biogenic sources such as forest fires, which tend to occur in the summer season and can be significant source contribution to total PAH levels in forested and rural areas. Meanwhile, PAHs from anthropogenic sources such as residential, commercial, and institutional heating will be highest in winter months. Thus, Ontario cities scoped for PAH source apportionment may demonstrate differing degrees and patterns of seasonal variability, depending on their source mix and location.

Typically, higher ambient PAH levels are seen during winter months, particularly in urban centres (Gaga, 2004; Dann, 1997; Waldman et al, 1991; Sisovic et al, 2002, 1996), due to seasonal patterns in source emissions, such as presence of heating sources in fall/winter months, as well as due to meteorology / atmospheric effects, such as reduced mixing height in winter and reduced reaction rates at lower ambient temperatures (Sun et al, 2006; Ravindra et al, 2008). 
Table A.1-1. Physical and chemical properties of PAH species (NAPS central site monitoring suite).

\begin{tabular}{|c|c|c|c|c|c|c|c|c|c|c|}
\hline $\begin{array}{l}\frac{0}{w} \\
\text { 岕 } \\
\text { क }\end{array}$ & 嵓 & 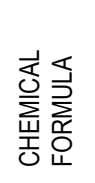 & 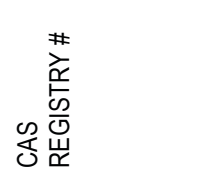 & 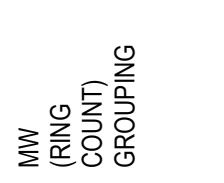 & 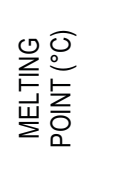 & 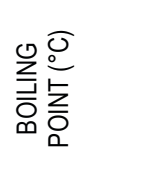 & 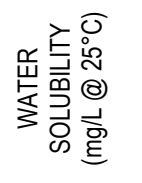 & 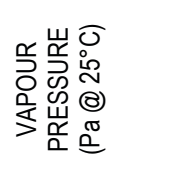 & 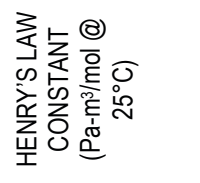 & 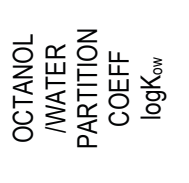 \\
\hline acenaphthylene & $\mathrm{ACY}$ & $\mathrm{C}_{12} \mathrm{H}_{8}$ & $208-96-8$ & $152.192(3)$ light & 92 & 280 & $3.9 \mathrm{E}+00$ & $9.0 \mathrm{E}-01$ & 11.55 & 3.94 \\
\hline acenaphthene & ACE & $\mathrm{C}_{12} \mathrm{H}_{10}$ & $83-32-9$ & 154.207 (3) light & 93 & 279 & $3.9 \mathrm{E}+00$ & $3.8 \mathrm{E}-01$ & 15.70 & 3.92 \\
\hline fluorene & FLU & $\mathrm{C}_{13} \mathrm{H}_{10}$ & 86-73-7 & $166.218(3)$ light & 115 & 295 & $1.9 \mathrm{E}+00$ & $1.6 \mathrm{E}-01$ & 9.75 & 4.18 \\
\hline anthracene & ANT & $\mathrm{C}_{14} \mathrm{H}_{10}$ & $120-12-7$ & $178.229(3)$ light & 216 & 340 & $6.0 \mathrm{E}-02$ & $4.6 \mathrm{E}-03$ & 5.29 & 4.51 \\
\hline phenanthrene & PHE & $\mathrm{C}_{14} \mathrm{H}_{10}$ & $85-01-8$ & $178.229(3)$ light & 99 & 340 & $1.3 \mathrm{E}+00$ & $2.6 \mathrm{E}-02$ & 3.91 & 4.49 \\
\hline 2-methyl-fluorene a & MFLU & $\mathrm{C}_{14} \mathrm{H}_{12}$ & $1430-97-3$ & 180.3 (3) light & 80 & 307 & $5.4 \mathrm{E}-01$ & $5.3 \mathrm{E}-02$ & 17.68 & 4.56 \\
\hline fluoranthene & FLT & $\mathrm{C}_{16} \mathrm{H}_{10}$ & $206-44-0$ & 202.250 (4) light & 110 & 384 & $2.8 \mathrm{E}-01$ & $2.2 \mathrm{E}-03$ & 1.10 & 4.85 \\
\hline pyrene & PYR & $\mathrm{C}_{16} \mathrm{H}_{10}$ & $129-00-0$ & 202.250 (4) light & 151 & 404 & $1.4 \mathrm{E}-01$ & $9.0 \mathrm{E}-04$ & 1.21 & 5.01 \\
\hline 1-methyl-pyrene a & MPYR & $\mathrm{C}_{17} \mathrm{H}_{12}$ & $2381-21-7$ & $216.2(4) \mathrm{med}$ & 132 & 384 & $5.8 \mathrm{E}-02$ & $2.3 \mathrm{E}-04$ & 0.86 & 5.48 \\
\hline benzo(a)fluorene & $\mathrm{BaFLU}$ & $\mathrm{C}_{17} \mathrm{H}_{12}$ & $238-84-6$ & 216.227 (4) med & 190 & 405 & $4.5 \mathrm{E}-02$ & $1.4 \mathrm{E}-03$ & 2.70 & 5.68 \\
\hline benzo(b)fluorene & $\mathrm{BbFLU}$ & $\mathrm{C}_{17} \mathrm{H}_{12}$ & $243-17-4$ & $216.227(4) \mathrm{med}$ & 212 & 401 & $2.0 \mathrm{E}-03$ & $5.4 \mathrm{E}-04$ & $0.39 \mathrm{a}$ & 5.77 \\
\hline benzo $(g, h, i)$ fluoranthene ${ }^{a}$ & BghiFLT & $\mathrm{C}_{18} \mathrm{H}_{10}$ & $203-12-3$ & $226.3(5)$ med & 153 & 415 & $4.8 \mathrm{E}-02$ & $2.3 \mathrm{E}-05$ & 0.11 & 5.52 \\
\hline chrysene & $\mathrm{CHR}$ & $\mathrm{C}_{18} \mathrm{H}_{12}$ & $218-01-9$ & $228.288(4)$ heavy & 256 & 448 & $2.0 \mathrm{E}-03$ & $2.1 \mathrm{E}-06$ & 0.53 & 5.79 \\
\hline triphenylene & TRIP & $\mathrm{C}_{18} \mathrm{H}_{12}$ & $217-59-4$ & $228.288(4)$ heavy & 198 & 425 & $4.1 \mathrm{E}-02$ & $1.2 \mathrm{E}-04$ & $0.02^{a}$ & 5.49 \\
\hline benzo(a)anthracene & BAA & $\mathrm{C}_{18} \mathrm{H}_{12}$ & $56-55-3$ & 228.288 (4) heavy & 161 & 438 & $1.1 \mathrm{E}-02$ & $2.7 \mathrm{E}-05$ & 0.81 & 5.77 \\
\hline retene a & RET & $\mathrm{C}_{18} \mathrm{H}_{18}$ & $483-65-8$ & 234.3 (3) heavy & 101 & 390 & $1.6 \mathrm{E}-02$ & $1.9 \mathrm{E}-03$ & 9.73 & 6.35 \\
\hline 7-methyl-benz(a)anthracene a & MBAA & $\mathrm{C}_{19} \mathrm{H}_{14}$ & $2541-69-7$ & 242.3 (4) heavy & 148 & 411 & $1.3 \mathrm{E}-02$ & 4.1E-05 & 0.74 & 6.07 \\
\hline benzo(a)pyrene & BAP & $\mathrm{C}_{20} \mathrm{H}_{12}$ & $50-32-8$ & 252.309 (5) heavy & 181 & 495 & $2.0 \mathrm{E}-03$ & $6.6 \mathrm{E}-06$ & 0.03 & 6.20 \\
\hline benzo(e)pyrene & BEP & $\mathrm{C}_{20} \mathrm{H}_{12}$ & $192-97-2$ & $252.309(5)$ heavy & 181 & 311 & $6.0 \mathrm{E}-03$ & $1.2 \mathrm{E}-05$ & 0.02 & 6.44 \\
\hline benzo(b)fluoranthene & $\mathrm{BbFLT}$ & $\mathrm{C}_{20} \mathrm{H}_{12}$ & $205-99-2$ & 252.309 (5) heavy & 168 & 481 & $2.0 \mathrm{E}-03$ & $4.4 \mathrm{E}-06$ & 0.05 & 5.78 \\
\hline benzo(k)fluoranthene & BkFLT & $\mathrm{C}_{20} \mathrm{H}_{12}$ & $207-08-9$ & 252.309 (5) heavy & 217 & 480 & $1.0 \mathrm{E}-03$ & $6.6 \mathrm{E}-06$ & 0.04 & 6.35 \\
\hline perylene & PER & $\mathrm{C}_{20} \mathrm{H}_{12}$ & $198-55-0$ & 252.309 (5) heavy & 278 & 503 & $4.0 \mathrm{E}-04$ & $3.6 \mathrm{E}-07$ & 0.44 & 6.25 \\
\hline 3-methyl-cholanthrene & $\mathrm{MCHO}$ & $\mathrm{C}_{21} \mathrm{H}_{16}$ & $56-49-5$ & $268.352(5)$ heavy & 180 & $438^{a}$ & $2.9 \mathrm{E}-03$ & $7.9 \mathrm{E}-07$ & $0.18^{a}$ & 6.69 \\
\hline anthanthrene ${ }^{a}$ & ANTH & $\mathrm{C}_{22} \mathrm{H}_{12}$ & $191-26-4$ & $276.3(6)$ heavy & 162 & 405 & $6.4 \mathrm{E}-04$ & $3.3 \mathrm{E}-05$ & 0.78 & 7.21 \\
\hline benzo(g,h,i)perylene & BghiP & $\mathrm{C}_{22} \mathrm{H}_{12}$ & $191-24-2$ & $276.330(6)$ heavy & 273 & 525 & $5.0 \mathrm{E}-04$ & $1.4 \mathrm{E}-08$ & 0.03 & 6.90 \\
\hline indeno(1,2,3-cd)pyrene & IPYR & $\mathrm{C}_{22} \mathrm{H}_{12}$ & $193-39-5$ & $276.330(6)$ heavy & 162 & 404 a & $4.0 \mathrm{E}-04$ & $1.3 \mathrm{E}-07$ & 0.03 & 7.66 \\
\hline Indeno(1,2,3-cd)fluoranthene a & IFLT & $\mathrm{C}_{22} \mathrm{H}_{12}$ & $193-43-1$ & $276.3(6)$ heavy & 160 & 401 & $1.8 \mathrm{E}-03$ & $1.1 \mathrm{E}-04$ & 24.92 & 7.75 \\
\hline benzo(b)chrysene a & $\mathrm{BbCHR}$ & $\mathrm{C}_{22} \mathrm{H}_{14}$ & $214-17-5$ & $278.4(5)$ heavy & 270 & 524 & $3.3 \mathrm{E}-03$ & $1.3 \mathrm{E}-07$ & 0.0002 & 6.54 \\
\hline dibenzo $(a, c) \&(a, h)$ anthracene & DBA & $\mathrm{C}_{22} \mathrm{H}_{14}$ & $215-58-7 / 53-70-3$ & $278.346(5)$ heavy & 237 & 521 & $1.1 \mathrm{E}-03$ & $8.7 \mathrm{E}-08$ & 0.01 & 6.70 \\
\hline
\end{tabular}

Notes:

1. All data are median of published values from Mackay (2006) except where denoted by superscript "a" sourced from Chemspider, "EPI predicted suite" (2014). Melting point, boiling point data rounded to nearest degree. Dibenzo(a,c)\&(a,h)anthracene data are average of $(a, c)$ and $(a, h)$ isomer values.

2. Synonyms: retene = 1-methyl-7-isopropyl-phenanthrene; anthanthrene = naphtho(7,8,1,2,3-nopqr)tetraphene; benzo(ghi)perylene = 1,12-benzoperylene; benzo(b)chrysene = benzo(c)tetraphene; dibenzo(ac)anthracene = benzo(f)tetraphene; dibenzo(ah)anthracene = benzo(k)tetraphene.

3. Molecular weight grouping adapted from Li et al, 2005 


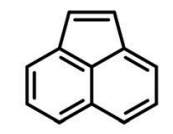

acenaphthylene (ACY)

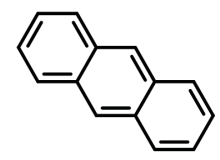

anthracene (ANT)

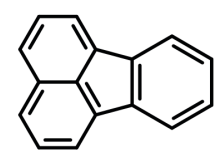

fluoranthene (FLT)

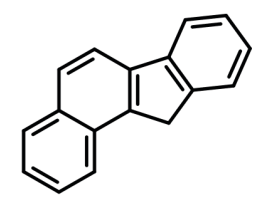

benzo(a)fluorene (BAFLU)

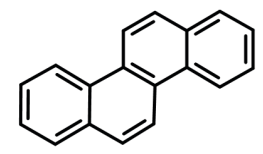

chrysene (CHR)<smiles>Cc1cccc2c1ccc1cc(C(C)C)ccc12</smiles>

retene (RET)

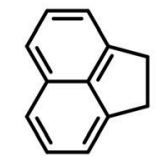

acenaphthene (ACE)

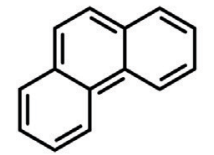

phenanthrene (PHE)

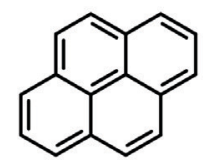

pyrene (PYR)

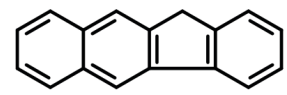

benzo(b)fluorene (BBFLU)

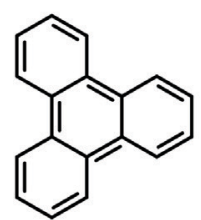

triphenylene (TRIP)<smiles>Cc1c2ccccc2cc2c1ccc1ccccc12</smiles>

7-methyl-benzo(a)anthracene (MBAA)

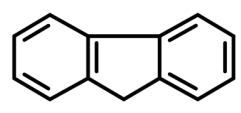

fluorene (FLU)

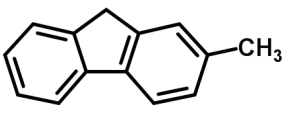

2-methyl-fluorene (MFLU)<smiles>Cc1ccc2ccc3cccc4ccc1c2c34</smiles>

1-methyl-pyrene (MPYR)

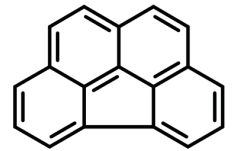

benzo(g,h,i)fluoranthene (BFLT)

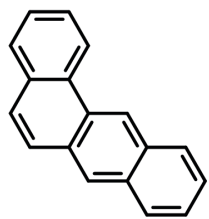

benzo(a)anthracene (BAA)

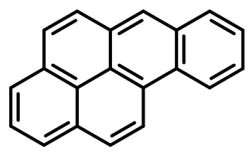

benzo(a)pyrene (BAP)

Figure A.1-1. Chemical structure of PAH species (NAPS central site monitoring suite). 
<smiles></smiles>

benzo(e)pyrene (BEP)<smiles></smiles>

perylene (PER)

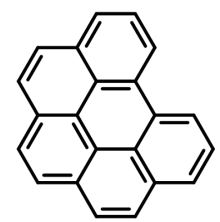

benzo(g,h,i)perylene (BGHIP)<smiles>c1ccc2cc3c(ccc4ccccc43)cc2c1</smiles>

benzo(b)chrysene (BBCHR)

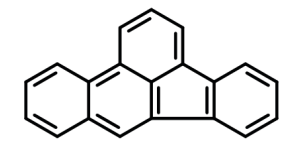

benzo(b)fluoranthene (BBFLT)<smiles>Cc1ccc2cc3c(ccc4ccccc43)c3c2c1CC3</smiles>

3-methyl-cholanthrene ( $\mathrm{MCHO})$<smiles></smiles>

indeno(1,2,3-cd)pyrene (IPYR)<smiles>c1ccc2cc3c4ccccc4c4ccccc4c3cc2c1</smiles>

dibenzo(a,c)anthracene (DBacA)<smiles>c1ccc2cc3c(cc2c1)-c1cccc2cccc-3c12</smiles>

benzo(k)fluoranthene (BKFLT)<smiles></smiles>

anthanthrene (ANTH)

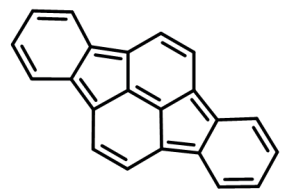

indeno(1,2,3-cd)fluoranthene (IFLT)<smiles>c1ccc2c(c1)ccc1cc3c(ccc4ccccc43)cc12</smiles>

dibenzo(a,h)anthracene (DBahA)

Notes:

1. All structures reproduced from Chemspider (2014).

2. Synonyms: retene $=1$-methyl-7-isopropyl-phenanthrene; anthanthrene $=$ naphtho(7,8,1,2,3-nopqr)tetraphene; benzo(ghi)perylene $=1,12$-benzoperylene;

benzo(b)chrysene $=$ benzo(c)tetraphene; dibenzo(ac)anthracene = benzo(f)tetraphene; dibenzo(ah)anthracene $=$ benzo(k)tetraphene.

Figure A.1-1 (continued) 


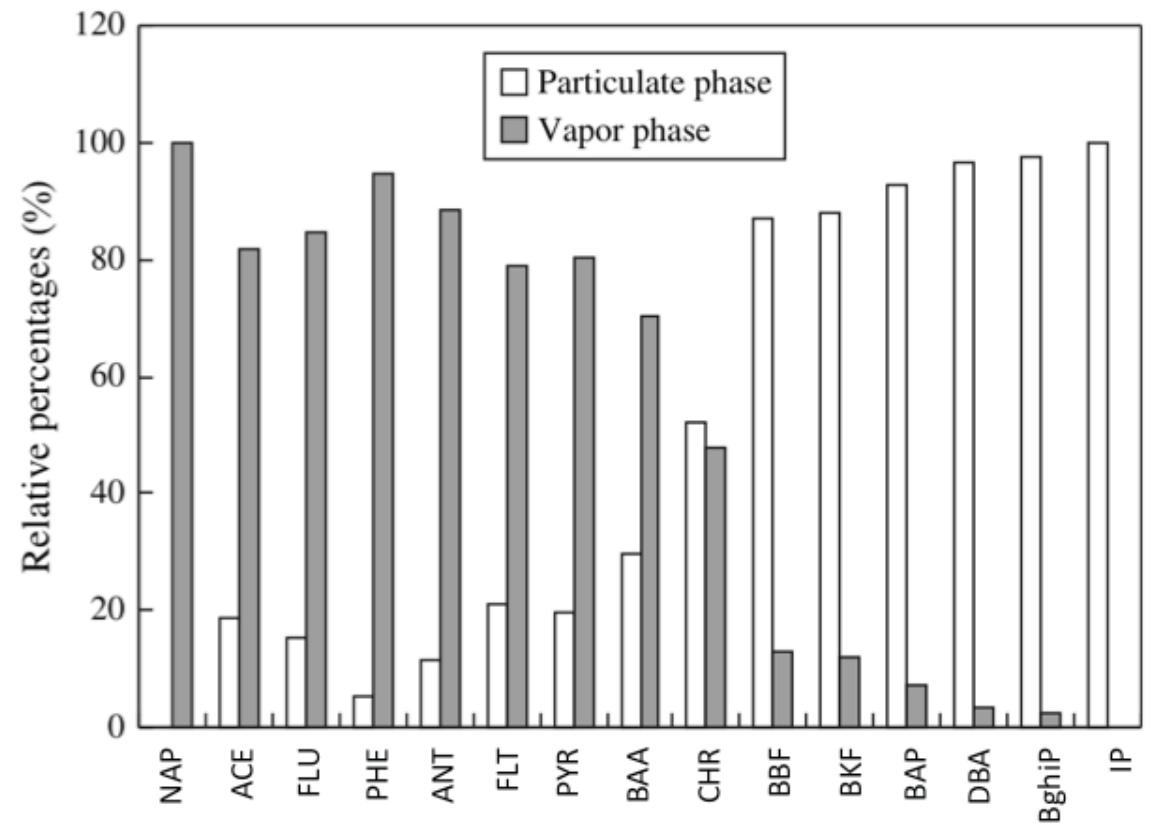

Figure A.1-2. Distribution of sampled ambient PAH in vapour and particulate phase. (reproduced from Lu et al, 2008)

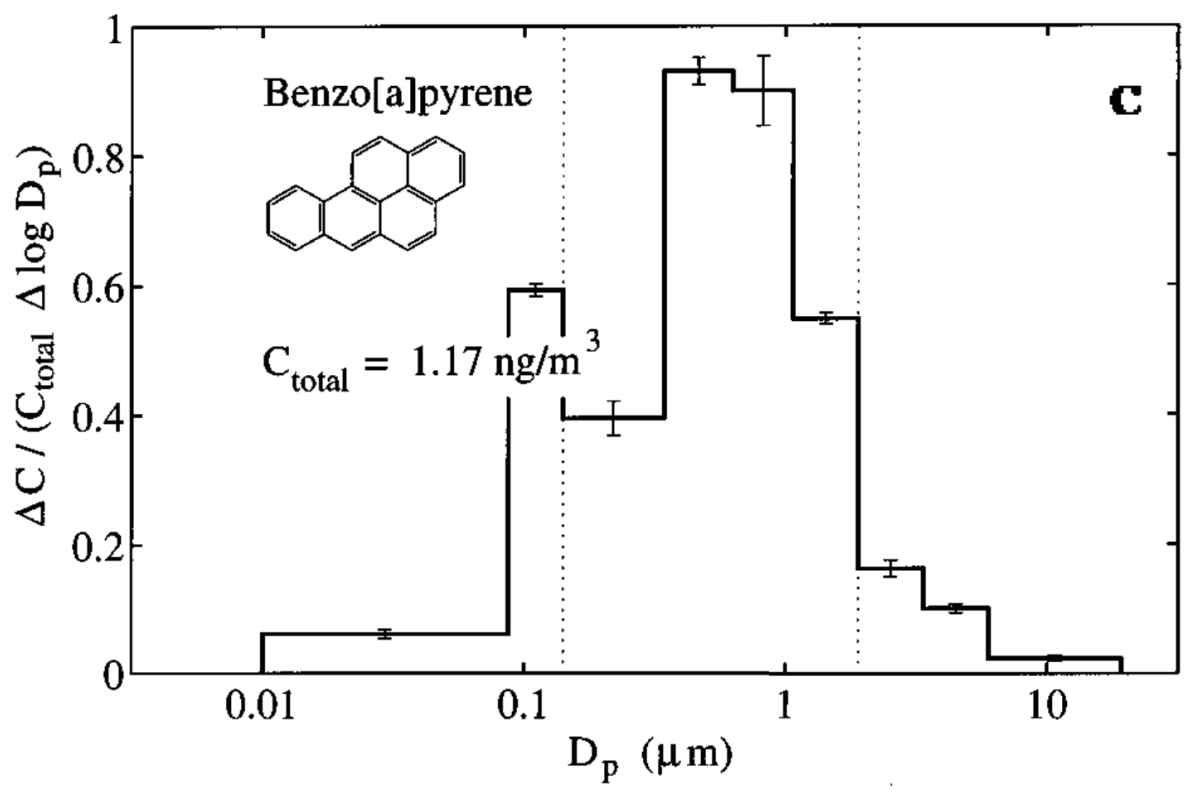

Figure A.1-3. Distribution of sampled BAP with particle size. (reproduced from Allen et al, 1996) 


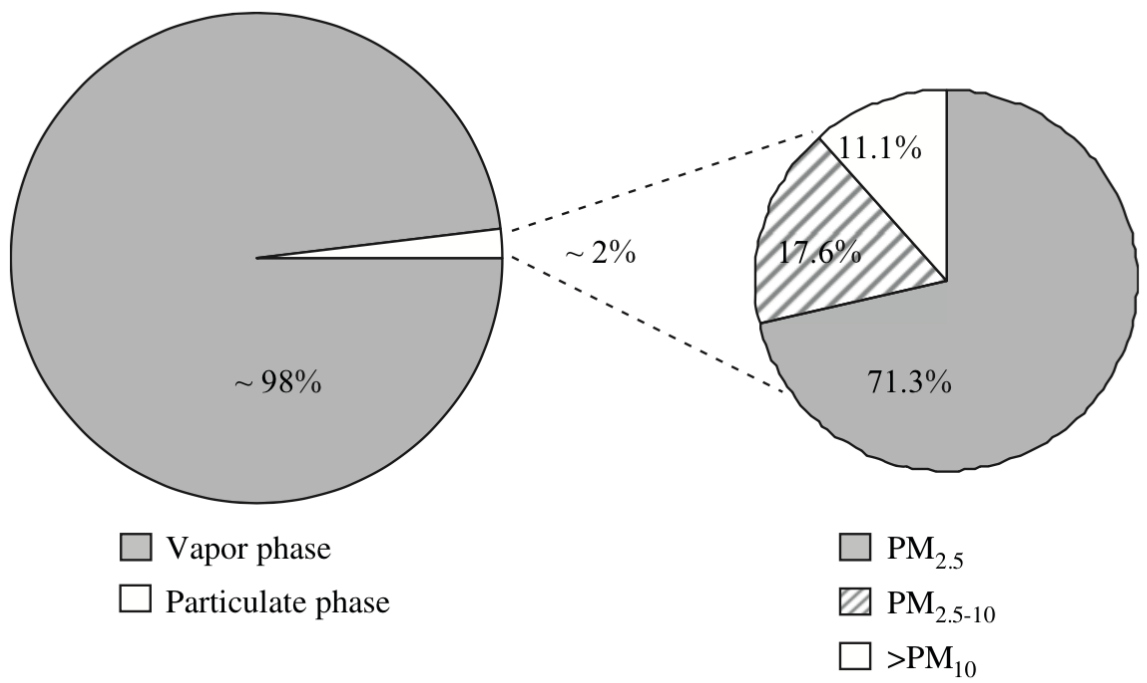

Figure A.1-4. Distribution of sampled PAH by vapour/particulate phase and by particulate size fraction.

(reproduced from Lu et al, 2008) 


\section{A.2 PAH exposure health effects}

\section{Air pollution exposure}

Ecosystems theory and the socio-environmental health model both indicate that an organism's health is linked to the health of its physical macroenvironment and its broader social community (Kindig, 2007; Kjellstrom et al, 2007). For ambient air pollution exposure, individual susceptibility to adverse health effects varies and is dependent on both host factors and environmental factors. Host factors include genetics, gender, age, pharmacokinetic and pharmacodynamic response, nutrition, preexisting disease, and socioeconomic status (SES).

Pertinent to the proposed research, the primary environmental factor is the ambient concentration of air pollutants, as elevated ambient concentrations have long been associated with adverse health effects. (Borm and Donaldson, 2007; Health Canada, 2006a; Peters et al, 1997)

\section{PM exposure}

The primary exposure pathway for particulate matter is inhalation, and ingestion and dermal are co-pathways. Generally, particles larger than $10 \mu \mathrm{m}$ are efficiently filtered by the respiratory system's defenses, such as cilia in the nasal passage or mucus in the mouth and throat. As particle size decreases, however, respiratory penetration increases. The smallest particle sizes (e.g., $0.53-0.65 \mu \mathrm{m}$ ) can penetrate the alveoli and even transfer to the bloodstream (Wilson and Spengler, 1996; Health Canada, 2006a; Borm and Donaldson, 2007).

Accordingly, respirable particulate matter is categorized primarily by size fraction, with particles between 2.5 and $10 \mu \mathrm{m}$ termed "coarse", and less than $2.5 \mu \mathrm{m}$ termed "fine", denoted as $\mathrm{PM}_{2.5}$. As airborne particles are not typically spherical, the diameter used to size-categorize particulate matter is an aerodynamic diameter. (Hinds, 1999)

Due to greater potential penetration of the respiratory system, exposure to fine and ultrafine particulate matter size fractions is considered to be a greater health concern. Indeed, most research investigating particulate matter exposures focuses attention on $\mathrm{PM}_{2.5}$ and is often indicated by the term "respirable particulate matter". (Borm and Donaldson, 2007; Nielsen et al, 1999, 1996; Schnelle-Kreis et al, 2001) 
Acute health endpoints for increased $\mathrm{PM}_{2.5}$ exposure include mortality, pulmonary inflammation, decreased lung function, increased plasma viscosity, heart rate changes or heart rate variability, and exacerbation of existing disease (e.g., asthma, cardiovascular, diabetes). Chronic health endpoints include cardiopulmonary diseases, decreased lung function, and cancers. (Health Canada, 2006a; Borm and Donaldson, 2007; Peters et al, 1997) Several sensitive subpopulations have been identified as experiencing greater effects from exposure to particulate matter. These include individuals with asthma, Chronic Obstructive Pulmonary Disease (COPD), and coronary heart disease, as well as the elderly and infants (National Research Council, 2001).

The relative health risk from exposure to particulate matter has been quantified by mass concentration of the exposure (e.g., $\mu \mathrm{g}_{2.5}$ per $\mathrm{m}^{3}$ of inhaled air). Excess health risk has been estimated as a $5-25 \%$ increase in incidence of bronchitis and $0.4-1.4 \%$ increase in mortality for exposure to an increase of $10 \mu \mathrm{g} / \mathrm{m}^{3}$ in $\mathrm{PM}_{2.5}$ levels. (Borm and Donaldson, 2007; also see Stieb et al, 2008; Dockery et al, 1993; Samet et al, 2000)

The toxicity of respirable particulate matter is characterized further by pollutants that may be adsorbed or otherwise associated with the respirable particles and thus influence exposures. These particle-associated pollutants typically include metals, elemental/organic carbon, soluble ions (e.g., $\mathrm{NO}_{3}, \mathrm{SO}_{4}, \mathrm{NH}_{4}, \mathrm{Na}, \mathrm{Cl}$ ), and PAHs. (Brook et al, 2004; Hinds, 1999) Thus, the toxicity of exposure can vary and is dependent on the source of the aerosol (Grahame and Schlesinger, 2007; Tolbert, 2007; National Research Council, 2001).

\section{PAH exposure}

The primary exposure pathway for PAHs is inhalation. Aside from occupational exposure (e.g., work in industries such as coal tar production, bitumen and asphalt production, aluminum production, mining, oil refining, metalworking, municipal trash incineration, etc.), PAH exposure can occur from inhalation of tobacco smoke, wood smoke, vehicle exhaust, asphalt off-gassing (i.e., volatilization), and ambient air. The exposure mechanism for PAH inhalation most often involves inhaling PM containing adsorbed PAHs that then enter the body through contact with lung tissue. (ATSDR, 1996, 1995; CEPA, 1999)

Acute health effects have been established for inhalation exposure to naphthalene, anthracene, and phenanthrene; short-term exposure at low concentrations is associated with eye and skin irritation while exposure at higher concentrations is associated with headaches, fatigue and nausea. (US EPA, 2000a) Sensitization and allergic responses are also suspected acute health effects. (Environmental Working Group, 2007) 
However, the primary health effects of PAH have been assessed as chronic. PAHs bioaccumulate in fatty tissue, leading to several long-term health effects, primary among them carcinogenicity. Carcinogenicity in humans is associated with several PAH species; the US EPA has determined seven PAH species to be probable human carcinogens (ATSDR, 1995): benz(a)anthracene, benz(a)pyrene, benz(b)fluoranthene, benzo(k)fluoranthene, chrysene, dibenz(a,h)anthracene, indeno(1,2,3-cd)pyrene. The types of cancers seen in animal toxicological studies generally correspond to the site of primary exposure. Thus, inhalation exposure is associated with increased prevalence of lung cancer, ingestion with stomach cancer, and dermal exposure with skin cancers. In humans, PAHs are also suspected as a risk factor for breast cancer. (ATSDR, 1996)

Several studies have examined the health effects of PM-associated PAH. Li N. et al (2002) completed a toxicological study in which macrophages were exposed to PM aerosol from a major roadway and found that their chosen health endpoint, oxidative effects, was positively correlated to the higher OC and PAH content associated with $\mathrm{PM}_{2.5}$. Perera et al (2005) completed an exposure and health effects study with expectant mothers and found that mothers in geographic areas with elevated concentrations of PAHs had elevated adverse health effects in both the mother and fetus (i.e., health endpoint selected was concentration of BaP-DNA adduct selected as proxy for PAH damage to DNA). Additional human cohort studies have shown similar positive correlation between increased aerosol PAH exposure and adverse health endpoints (Miller, 2004; Nadon, 2007; Kinney, 2000).

\section{Toxicity Equivalency Factors (TEF) for PAH exposure}

Toxicity Equivalency Factors (TEF) are used to quantitatively estimate potential cancer risk due to environmental exposure to a mixture of $\mathrm{PAH}$, since ambient exposures are to a number of PAH species, inclusive of the species sampled and analysed in this research.

Toxicity testing on individual PAH species has shown that it can vary significantly by species, as expected given that PAH are a broad class of compounds with varying physico-chemical properties and structures (Appendix A.l). BAP has been used as an appropriate indicator species for PAH toxicity due to its association with chronic health risk, specifically cancer potency, having been long-since characterized for the inhalation pathway (and other exposure routes), by comprehensive health effects evaluation and quantitative risk assessment (i.e., BAP health effects study data is ranked as highest possible data quality as per Collins et al, 1998). Cancer risk for BAP has been established using a slope factor from its dose-response curve from studies that found increased incidence of health risk using various end points such as tumours in laboratory animals, 
mutation frequency in vitro and in vivo bioassays, or adduct formation in DNA. (Equilibrium Environmental Inc., 1996)

The conversion from sampled ambient PAH concentration to 'BaP-toxicity equivalent concentration' (i.e., more conveniently referred to as 'PAH toxicity' in this research) is shown in Equation A.2-1. Individual species concentrations $\left(\mathrm{C}_{\mathrm{j}}\right)$ are converted to BaPtoxicity equivalent species concentrations $\left(\mathrm{BaP}_{-}-\mathrm{q}_{\mathrm{j}}\right)$, multiplying by their respective Toxicity Equivalency Factor $\left(\mathrm{TEF}_{\mathrm{j}}\right)$; summation of individual $\mathrm{BaP}-\mathrm{eq}_{\mathrm{j}}$ yields total sampled or modeled BaP-toxicity equivalent concentration (BaP-TEQ). (Jung et al, 2010)

Equation A.2-1. Calculation of total sampled or modeled BAP-toxicity equivalent concentration.

$$
\begin{gathered}
B A P-T E Q=\sum_{j=1}^{m}\left(B A P-e q_{j}\right)=\sum_{j=1}^{m} C_{j} * T E F_{j} \quad\left[n g / m^{3}\right] \\
B a P-T E Q=\text { Total BAP-toxicity equivalent concentration for } \mathrm{m} \text { summed } \\
\text { species }\left(\mathrm{ng} / \mathrm{m}^{3}\right) \\
\text { BaP-eqj } \mathrm{BAP} \text {-toxicity equivalent concentration of species } \mathrm{j}\left(\mathrm{ng} / \mathrm{m}^{3}\right) \\
C_{j}=\text { concentration of species } j \\
\\
T E F_{j}=\text { Toxicity Equivalency Factor for species } \mathrm{j} \text { (unitless) }
\end{gathered}
$$

This research also explicitly quantified source contributions to PAH toxicity (i.e., BaPtoxicity equivalent total sampled concentrations), converting PMF and CMB source apportionment results by extending the methods of Jung et al (2010); calculation is entirely analogous to Equation A.2-1 but for factor contributions to species concentrations $\left(\mathrm{ng} / \mathrm{m}^{3}\right)$ rather than sampled ambient concentrations. Explicitly, factor contributions to individual species are converted to BaP-eqj, multiplying by their respective TEFj; summation over all species yields the factor contribution to total modeled BaP-toxicity equivalent concentration (BaP-TEQ).

Use of TEF for quantifying relative health risk of PAH species involves several assumptions. First, availability of a compound with well-characterized health risk to serve as reference species or indexing species for all members of the pollutant class; this is chosen as $\mathrm{BaP}$, which has been long-studied and is considered one of the more toxic PAH species. Second, qualitatively similar toxic effects; for PAH this is met since carcinogenicity is the specific toxic effect of concern for majority of risk assessments. Additional assumptions are that TEF should be similar for different toxic endpoints and toxic effects of a PAH mixture are additive, both of which are considered to be reasonably met by the TEF approach. (Nisbet and Lagoy, 1992) 
Nisbet and Lagoy (1992) proposed a reference dataset of TEF for use in risk assessment of exposure to PAHs via the inhalation exposure pathway and their values were used throughout this research, supplemented by additional species reference values as required (Table A.2-1).

The Nisbet and Lagoy TEF dataset addresses a broad range of PAH species (17 species total, from light to heavy MW) and was thus adopted by the US toxicological profile for PAH (ATSDR, 1995). The Nisbet and Lagoy (1992) TEF dataset was derived from comprehensive review of available toxicological literature (13 studies in total). While several health endpoints were used in the toxicology studies reviewed by Nisbet and Lagoy (i.e., lung cancer in laboratory rats, skin cancer of mouse skin, sarcomas at site of subcutaneous injection, PAH-DNA adducts in in vitro studies) the TEF data are considered representative of cancer risk due to inhalation of PAH mixtures.

Nisbet and Lagoy intentionally presented TEF values as order of magnitude data, recognizing the variability in results inherent in survey of multiple toxicology test data and the inherent uncertainty in toxicology tests included in their review. They judge TEFs used from a single toxicology test, particularly where data are presented to many significant figures, as "unreasonably precise" for practical use in risk assessment. They also noted that toxicology studies consulted in preparing their reference dataset showed TEF for most species to generally agree with a maximum difference of a factor of 10 . Uncertainty on the TEFs is stated as "ranging up to a factor of at least 3" and was based on the variation among reviewed toxicology studies. (Nisbet and Lagoy, 1992)

To be clear, the order of magnitude approach to TEF estimates is systematically preferred for purposes of expressing uncertainty in TEF data, due to several sources of uncertainty, including: (i) uncertainty in dose-response curve shape; (ii) limited amount of doseresponse data on carcinogenicity; (iii) wide range of reported TEFs consulted by reference datasets selecting a single value; and, (iv) additional variability introduced by combining results from multiple toxicity tests and reference datasets. (Collins et al, 1998)

By avoiding unreasonable precision through order of magnitude values and encompassing a large suite of PAH of varying MW, use of the Nisbet and Lagoy dataset provides a procedurally rational approach of comparing results in this research with complementary work done by other researchers in estimating PAH health risk (e.g., Jung et al, 2010).

Since the work by Nisbet and Lagoy (1992), other TEF suites have been presented by researchers. Comprehensive sets of TEF are available from California EPA (1993, 2002), Muller (1997; for Ontario MOE), Larsen and Larsen (1998), Malcolm and Dobson (1994), WHO (1998), Collins et al (1998), and Equilibrium Environmental Inc. (2006; for 
Health Canada). Several TEF datasets, inclusive of that of Nisbet and Lagoy (1992) were also summarized in a PAH position paper (Environmental Working Group, 2001; Annex, Table 16). While individual values will of course differ across datasets, recent comparison of TEF data across a number of references (Malcom and Dobson, 1994; Nisbet and Lagoy, 1992, Muller et al, 1995, CalEPA, etc.) found TEF values to generally be within the same order of magnitude for majority of species modeled in this research and, where inter-study differences were greater, to remain within one order of magnitude. (Equilibrium Environmental Inc., 2006; for Health Canada)

Since several species sampled and modeled in this work are not included in the Nisbet and Lagoy (1992) reference dataset, TEF for these was obtained from other TEF datasets, preserving an order of magnitude approach to reflect data uncertainties and inter-dataset variability:

- For MFLU and MPYR assigned same TEF as parent compound.

- For BAF, BBF assigned same TEF as FLU (i.e., toxicity classified equivalent to FLU in report to Health Canada by Equilibrium Environmental Inc., 2006).

- For BghiFLT, assigned TEF value as per Nielsen et al (1996) since grouped with structurally similar B(d)PHEN and recommendation to Health Canada recommends TEF value of B(c)PHEN one order of magnitude lower than TEF for CHR (Equilibrium Environmental Inc., 2006).

- For TRIP, assigned same TEF as CHRY, since Nielsen et al (1996) shows single value for combined CHRY and TRIP.

- Since RET is not as commonly sampled as other PAH species, no toxicity data was found available. Because the compound is an alkylated variant of PHEN, a conservative assumption was made that the compound possesses a degree of toxicity (see also Scott, 2009) and was assigned the same TEF as PHEN (i.e., lowest TEF value in modeled suite).

- For BEP, assigned TEF as per Malcolm and Dobson (1994) and Nielsen et al (1996). 
Given there are several TEF datasets available in the PAH toxicology literature, it is obvious that choice of individual TEF values will increase or decrease estimations of $\mathrm{PAH}$ toxicity. However, there is strong consensus of the following general trends in the TEF data:

- light MW species tend to have low TEF values;

- heavy MW species tend to have high TEF values; and,

- TEF value generally increases with MW weight.

Based on these general species trends in TEF, source types enriched in heavy MW species will necessarily contribute greater PAH toxicity than source types enriched in light MW species. Thus, variability in individual TEF values is expected to have little effect on relative toxicity assessments of source types where these are clearly distinguished with respect to the more toxic heavy MW species.

Future work can investigate the sensitivity of 'source toxicity' quantifications to TEF values. This can be done by:

(i) using TEF datasets with significantly different values (i.e., $>1$ order of magnitude difference from Nisbet and Lagoy, 1992) adapting the sensitivity analysis approach used by Cai et al (2012); and/or,

(ii) combining all available comparable data and using a median TEF value along with statistical range estimates (first quartile, third quartile) to incorporate an estimate of uncertainty on source type contributions to BaP-TEQ and thus on comparative source toxicity. 
Table A.2-1. Benzo(a)Pyrene-equivalent carcinogenic Toxicity Equivalency Factors (BaP-TEF) for inhalation exposure, modeled species.

\begin{tabular}{llr}
\hline Species ID & Species name & BaP-TEF \\
\hline 01_ACY & acenaphthylene & 0.001 \\
02_ACE & acenapthene & 0.001 \\
03_FLU & fluorene & 0.001 \\
04_ANT & anthracene & 0.01 \\
05_PHE & phenanthrene & 0.001 \\
06_MFLU & 2-me-fluorene & $0.001^{\mathrm{a}}$ \\
07_FLT & fluoranthene & 0.001 \\
08_PYR & pyrene & 0.001 \\
09_MPYR & 1-me-pyrene & $0.001^{\mathrm{a}}$ \\
10_BaFLU & benzo[a]fluorene & $0.001^{\mathrm{b}}$ \\
11_BbFLU & benzo[b]fluorene & $0.001^{\mathrm{b}}$ \\
12_BghiFLT & benzo[g,h,i]fluoranthene & $0.001^{\mathrm{c}}$ \\
13_CHRY & chrysene & 0.01 \\
14_TRIPH & triphenylene & $0.01^{\mathrm{a}}$ \\
15_BaA & benzo[a]anthracene & 0.1 \\
16_RET & retene (1-methyl-7- & \\
18_BaP & isopropylphenanthrene) & $0.001^{\mathrm{e}}$ \\
19_BeP & benzo[a]pyrene & 1 \\
20_BbFLT & benzo[e]pyrene & $0.01{ }^{\mathrm{f}}$ \\
21_BkFLT & benzo[b]fluoranthene & 0.1 \\
25_BghiPE & benzo[k]fluoranthene & 0.1 \\
26_IPYR & benzo[g,h,i]perylene & 0.01 \\
\hline & indeno[1,2,3-cd]pyrene & 0.1 \\
\hline
\end{tabular}

Note: Data from Nisbet and Lagoy, 1992 and ATSDR, 1995 except where noted:

(a) Assigned same TEF as parent compound.

(b) Assigned same TEF as FLU (i.e., toxicity classified equivalent to FLU in report to Health Canada, PAH PEF Toxicity Update, 2006).

(c) Assigned TEF value as per Nielsen et al, 1996 (i.e., grouped with structurally similar B[c]PHEN) and recommendation to Health Canada (i.e., recommends TEF value for $\mathrm{B}[\mathrm{C}] \mathrm{PHEN}$ that is one order of magnitude lower than TEF for CHRY; Equilibrium Environmental, 2006).

(d) Assigned same TEF as CHRY (i.e., Nielsen et al, 1996 show single value for CHRY+TRIP).

(e) No toxicity data available since retene less commonly sampled; conservative assumption is that compound has degree of toxicity since an alkylated variant of phenanthrene therefore assigned same TEF as phenanthrene (i.e., lowest toxicity value in modeled species suite; also see Scott, 2009).

(f) Assigned TEF as per Malcolm and Dobson, 1994 and Nielsen et al, 1996. 


\section{A.3 Key source apportionment studies}

\section{Introduction}

Both PMF and CMB techniques have been applied successfully to the source apportionment of PM, most often relying on PM characterization by associated elemental species. However, only a comparably small subset of previous source apportionment studies reference PAH data. This literature review summarizes key studies that have focused on apportioning sources of PAH or have included PAH compounds along with others for source apportionment of atmospheric PM. The summary of key studies below was first presented in part to Environment Canada in the Final Report to the project "Source Apportionment of PAHs at Ontario Sites Using Receptor Modelling Techniques" (Karman and Anastassopoulos, 2009).

Since diagnostic ratio analysis continues to be found in the PAH source apportionment literature, a brief review of diagnostic ratios and recent developments of the method is also provided.

\section{$\underline{\text { PAH source apportionment by PMF }}$}

PAH source apportionment studies at locations around the Great Lakes are of particular interest because of the relative proximity, when considered in the continental scale, of the sampling sites to those in southwestern Ontario being analyzed in the current study. As such, Battelle (2007) modeled data from four sites in the Great Lakes region, representing both urban and rural locations; one of these was Chicago, Illinois, of interest here for its similarity in industry mix to Hamilton, Ontario, inclusive of local steel production, harbour-front activity, and additional regional coal combustion emissions. Battelle used historical time-series data from multiple US sampling networks, the Integrated Atmospheric Deposition Network (IADN) and Speciation Trends Network (STN), and applied the PMF model to a list of species that included PAH, metals, PCBs, and pesticides. The $14 \mathrm{PAH}$ species included in their receptor modeling are all measured by NAPS at Ontario PAH monitoring sites. Resolved PAH sources included diesel emissions, gasoline emissions, wood / vegetative burning, and domestic heating. Validation of PMF model results used wind and pollution rose analysis for local sources and back trajectory analysis (i.e., NOAA's HYSPLIT model) for transported sources.

A comparative study of three receptor models to source apportion atmospheric PAHs in Baltimore, MD, USA was completed by Larsen and Baker (2003). The three source apportionment methods were Principal Component Analysis with multiple linear regression (PCA-MLR), and the US EPA Positive Matrix Factorization (PMF) and UNMIX. The measured vapour- and particulate-phase concentrations of 32 individual 
PAHs were combined to avoid problems associated with vapour-particle partitioning (i.e., preferential partitioning differing between species). PMF modeling arrived at six factors identified as coal, oil, wood, gasoline, diesel, and other. The overall source contributions to PAH compared reasonably well across the three receptor model methods when some of the factors were combined (e.g., vehicles contributed 16-23\% depending on model, coal 28-36\%, oil 15-29\%, and wood/other 23-35\%).

Lee at al (2004) present results of PMF source apportionment for gas and particulate phase PAHs in the Hudson River airshed based on measurements earlier reported by Gigliotti et al (2000). A stable eight-factor model solution was identified, including a factor associated with air-surface exchange. Model factors were distinguished by PAH phase prevalence: motor vehicle emissions and oil combustion factors were the predominant contributors to particle-phase $\mathrm{PAH}$; natural gas combustion, air-surface exchange, and evaporative/uncombusted petroleum factors contributed substantially to gas-phase PAH.

The contribution of motor vehicles to ambient $\mathrm{PM}_{2.5}$ and $\mathrm{PAH}$ is of interest as this is a ubiquitous source with quantifiable contributions in nearly all locations. A comprehensive assessment of the contribution of motor vehicle sources to urban air pollution is given by Brook et al (2007a, 2007b), with PMF modeling for $\mathrm{PM}_{2.5}$ completed for Toronto (Lee et al, 2003). Brook et al differentiated between local sources versus transported $\mathrm{PM}_{2.5}$ as well as primary emissions versus secondary formation, and identified gasoline engine vehicles, diesel vehicles, and coal-fired power plants as significant contributors. PAH emissions from motor vehicles were also reviewed by Karman and Shen (2006) in a study aimed at quantifying emissions from on-road vehicles with the MOBILE6 emission factor model by making use of PAH/VOC or PAH/PM ratios. Finally, a survey paper by Gertler et al (2000) reviewed US source apportionment studies of $\mathrm{PM}_{2.5}$, concluding that a significant advance in receptor modeling is the use of PAHs to better identify mobile sources and even distinguish between gasoline-fueled and diesel-fueled emissions.

\section{$\underline{\text { PAH source apportionment by CMB }}$}

A chemical mass balance model based on organic compounds (Schauer et al, 1996) was used to determine source contributions to $\mathrm{PM}_{2.5}$ mass in four different locations in Southern California. Of interest to this proposed research, the study excluded a number of $\mathrm{PAH}$ species from the $\mathrm{CMB}$ input profiles because of apparent atmospheric transformations that violated the conservation principle of the model.

A number of studies have varied the methodology used in Schauer et al (1996): Schauer and Cass (2000) expanded the application to both gas phase and particle phase pollutants 
in Southern California, with PAH species used for the PM source apportionment; Schauer et al (2002a) again applied CMB to find source contributions to both gas and particle phase pollutants during a severe photochemical smog episode in Southern California; Manchester-Neswig, Schauer, and Cass (2003) report on the application of the method to particle-phase organic compound source apportionment at 12 sites during the Southern California Children's Health Study; recently, Zheng et al (2007) included six PAH compounds to apportion $\mathrm{PM}_{2.5}$ in Atlanta.

In the Northern Front Range Air Quality Study (NFRAQS) completed by Watson et al (1998), CMB modeling for the source apportionment of $\mathrm{PM}_{2.5}$ made use of an extended list of chemical species by including PAH compounds in addition to the more typically used metals and ionic species; the source profiles generated in this study included the highest number of PAH compounds considered in CMB source apportionment studies for $\mathrm{PM}_{2.5}$. Source profiles were determined for wood burning, meat cooking and geological sources. Using extensive vehicle testing, emission profiles were also determined for different classes of vehicles (i.e., diesel, gasoline, high-emitter) and operating conditions (i.e., cold start, hot stabilized operation) providing an indication of the variability of PAH from motor vehicle sources.

A subset of CMB studies have focused on the contribution of mobile source emissions and on distinguishing between gasoline and diesel-fueled vehicles. Fraser et al (2003a, 2003b) used a small set of 5 PAH species in their CMB source apportionment using source profiles of organic molecular markers, as in Schauer et al (1996), aimed at distinguishing PM emissions from gasoline and diesel vehicles in Houston. More recently, the U.S. Department of Energy sponsored a Gasoline/Diesel PM Split Study, carried out collaboratively between the University of Wisconsin Madison and Desert Research Institute (Fujita et al 2005; Schauer and Glynis, 2006; Lough and Schauer, 2007; Lough et al, 2007). The Gasoline/Diesel PM Split Study analyzed PM $_{2.5}$ samples from various vehicles under simulated driving conditions and ambient sampling stations in Los Angeles to determine relative contributions via the CMB model. The chemical species analyzed included a list of $36 \mathrm{PAH}$ compounds, in addition to metals, EC/OC, alkanes, polar compounds, steranes and hopanes. The sensitivity of the source apportionment to uncertainty in the motor vehicle emission profiles is discussed by Lough and Schauer (2007).

A recently completed CMB study by Gannon University (Gannon, 2007) is of interest because of the Great Lakes receptor location and source apportionment of PAH. Ambient PAH data was collected over a one-year period at three sites around the city of Erie on Lake Erie. Reference PAH source profile were used based on available literature, including coke oven emissions, wood burning, gasoline vehicles, diesel vehicles, jet 
exhaust and asphalt roofing manufacturing. The CMB modeling showed highly variable contributions depending on the sources used in the model runs. Many of the model runs explained a relatively low percentage of the total mass observed, suggesting that not all PAH sources may have been adequately included in the model. Caution was therefore recommended for a priori selection of sources to include in the model and in subsequent interpretation of modeling results.

Additionally, Lee and Kim (2007) report on fine particulate PAH source apportionment by $\mathrm{CMB}$, using $13 \mathrm{PAH}$ species and 7 source profiles in Seoul, Korea.

$\underline{\text { PAH source apportionment by diagnostic ratios }}$

Diagnostic ratio (DR) analysis for source identification uses the relative concentration of two PAH species as an indication of source type, with the underlying assumption that the species ratio is reasonably preserved between the source and receptor. A number of species pairings and corresponding diagnostic ratio reference values have been calculated from source sampling studies and tabulated for broad source types (i.e., mobile sources, stationary combustion sources) and more specific sources (e.g., gasoline traffic emissions versus diesel traffic emissions). (Ravindra et al, 2008; Yunker et al, 2002) However, DRs have several limitations arising from changes in the relative species concentrations that may occur between the source (i.e., reference value from PAH profile data reported by source sampling study) and the receptor (i.e., study sample PAH data from which contributing sources wish to be identified). Factors challenging application of DRs arise due to inter-species differences in physico-chemical properties (e.g., atmospheric reactivity such as reaction with ozone, $\mathrm{NO}_{x}$; gas-particle partitioning behaviour due to volatility and solubility) (Ravindra et al, 2008; Tsapakis et al, 2005) along with practical issues such as sampling only particle phase PAH (Dvorska et al, 2011).

Galarneau (2008) examined the use of PAH diagnostic ratios for source identification in a comprehensive literature review and meta-analysis and summarized their limitations, particularly with respect to source specificity (i.e., unique source profile for each source type) and the conservation of relative species mass between source and receptor. The study found that the assumption of source specificity for PAH emissions is often violated when species DRs are incorrectly used as these may vary significantly between locations or even among varying source operating conditions (e.g., diesel engine drive cycle or load). Further, Galarneau demonstrated that species ratio conservation may be significantly affected by several transformative processes in the atmosphere, including inter-species differences in partitioning (i.e., between gas and particle phases); interspecies differences in reactivity (i.e., due to oxidation, solar radiation); inter-species 
differences in atmospheric removal (i.e., due to air-water exchange, particle washout/deposition) as well as by inter-species differences in sampling losses.

Despite their limitations, DRs continue to be used as both primary and supplementary means of source identification for atmospheric PAH sample data (Amodio et al, 2013; Dvorska et al, 2011; Kong et al, 2011; Menezes et al, 2012; Shi et al, 2010; Stella et al, 2012; Zhao et al, 2010; Park et al, 2011; Sofowote et al, 2010a, 2010b). More recent PAH studies employing DRs have explicitly acknowledged their limitations and sought to adapt the DR methodology to better address these (Lima et al, 2005; Tsapakis et al, 2005; Menezes et al, 2012; Yunker et al, 2002; Cavalcante et al, 2012; Sofowote et al, 2010a; Dvorska et al, 2011).

Tobiszewski et al (2012) recently reviewed PAH sampling studies applying DRs and made several positive recommendations for 'best practices' in using them for source identification of atmospheric PAH data. Key recommendations are summarized below:

(i) Selecting less reactive species for use in DRs. (Menezes et al, 2012) As a general rule, species with large difference in MW should not be paired as these tend to have greater differences in their physico-chemical properties (e.g., vapour pressure, gas-particle partitioning) and thus behave differently in the atmosphere, potentially resulting in differing conservation between source and receptor. (Lima et al, 2005)

(ii) Selecting DRs composed of species with similar atmospheric reactivity. $(\mathrm{ANT} / \mathrm{ANT}+\mathrm{PHEN})$ and $(\mathrm{BaA} / \mathrm{BaA}+\mathrm{CHRY})$ are cited as species pairings that are generally not ideal for source identification because the reaction half-life (t1/2) of their constituent species can differ significantly (i.e., two orders of magnitude difference). Conversely, (FLA/FLA+PYR) and (IcD/IcD+BghiP) are cited as more conservative diagnostic ratios (i.e., constituent species in each pairing have similar atmospheric residence times, better preserving their relative concentration between source and receptor).

(iii) Calculating DRs from total PAH sample data (i.e., particulate plus vapour phase) to better account for gas-particle partitioning differences between species. (Dvorska et al, 2011; Zhao et al, 2010). Since many previously published DRs were restricted to particle phase PAH data, there may be value in estimating equivalent vapour plus particle phase DRs, albeit with inherent uncertainty.

(iv) Selecting DR reference values from source sampling studies with comparable PM size fraction to the study dataset being analysed; however, the effect of particle size on DR values can generally be considered less significant since majority of PAH emissions have been shown to occur in the fine size fraction. (Venkataraman and Friedlander, 1994) 
(v) Accounting for expected seasonal effects on PAH concentrations using seasonal DRs. Diagnostic ratios from winter sampling data are preferred as factors affecting PAH source-receptor conservation are typically attenuated in winter conditions (i.e., colder temperature, less solar energy). (Yunker et al, 2002)

(vi) Preferring DR values that have been derived from local source sampling data over arbitrary or remote proxy literature values; where local source sampling studies are not available or are excessively out-of-date, reference values can be calculated from source sampling studies at other locations where these are reasonably representative of local source conditions and sampling methods (i.e., reference diagnostic ratio values should not be arbitrarily selected or 'cherry picked' to match preconceived source types).

(vii) Coupling DRs to better diagnose source types (Cavalcante et al, 2012; Yunker et al, 2002). The traditional application of this principle is to use ratio-ratio plots (i.e., see Yunker et al, 2002; Park et al, 2011) but an interesting variation on this principle is the "linear combination" of DRs to a single ratio (Sofowote et al, 2010a). DR results can sometimes also be clarified by incorporating source marker species into the analysis (Tobiszewski et al, 2012).

(viii) Limiting application of DR analysis to local source types (e.g., local industry, traffic) rather than relying on them to reliably identify regional sources or source types at background/rural sites where source impacts are from regional transport over large distances with associated greater transport effects (i.e., inter-species differences in atmospheric dispersion, reactivity etc.) (Dvorska et al, 2011)

(ix) DR analysis can be used to show intraurban variation in impact of suspected source types since values typically differ between sites upwind/downwind of local sources and with increasing distance from local sources. (Tobiszewski et al, 2012; Mantis et al, 2005) The method was demonstrated successfully for strong local sources by Sofowote et al (2010a): total sampled PAH concentrations were used to classify samples as high/low concentration and wind direction analysis was used to classify samples as downwind/upwind; high/low concentration samples were then compared against downwind/upwind samples to confirm the local source-receptor relationship.

Even with the several possible improvements on DR methods, the general recommendation, which this author agrees with, remains to limit their use to, at most, supplementing the more complex multivariate analysis source apportionment models available (e.g., PCA, US EPA PMF) (Park et al, 2011; Tobiszewski et al, 2012). 


\section{A.4 Approaches to estimating uncertainty on PMF input data}

\section{Introduction}

To obtain PMF receptor modeling results that are both procedurally defensible and physically meaningful, it is essential to:

i. estimate uncertainty realistically for each species time series; and,

ii. appropriately weight the use of that species in the model.

As is typical of environmental datasets, the NAPS PAH datasets do not explicitly report the uncertainty of each sampled and reported concentration. Thus, effort was made to apply sample uncertainty $\left(\mathrm{s}_{\mathrm{ij}}\right)$ that would adequately capture, estimate and aggregate the uncertainties associated with laboratory analysis of PAH samples, thereby ensuring appropriate weighting of individual samples in the PMF receptor model.

A comprehensive review of precedents in literature, summarized below, provided context and corroboration for applying the uncertainty equation available via sub-routine within US EPA PMF3.

\section{Precedents in literature}

A comprehensive literature review of the methodology used in PMF receptor modeling was completed by Reff et al (2007). In their review, Reff note that the PMF uncertainty matrix could be populated in one of two ways:

i. direct quantification of $\mathrm{s}_{\mathrm{ij}}$, reported alongside lab concentration data; or,

ii. estimation of $s_{\mathrm{ij}}$ using equations.

Although Reff et al (2007) cite a couple of studies that were able to directly quantify $\mathrm{s}_{\mathrm{ij}}$ (Poirot et al, 2001; Huang et al, 2001), they show that the clear majority of studies have limited laboratory and sample metadata available (i.e., lab analysis uncertainty) and so are required to estimate $s_{i j}$ from equations.

Reff et al (2007) tabulate a variety of formulae used to estimate uncertainty in datasets modeled via PMF. The basic form of the sample uncertainty equations seen in the literature can be expressed as a function of the analytical uncertainty $\left(\mathrm{u}_{\mathrm{ij}}\right)$, which is an aggregate estimate of sample uncertainty associated with ambient pollutant concentration data (i.e., uncertainty in the sample concentration as analysed by the laboratory 
processing the field samples), and a second term incorporating the laboratory detection limit $\left(D_{i j}\right)$. This can be written in the general form: $s_{i j}=f\left(u_{i j}, D L_{i j}\right)$

Analytical uncertainty $\left(u_{i j}\right)$

The analytical uncertainty $\left(\mathrm{u}_{\mathrm{ij}}\right)$ is used to estimate the sum of known uncertainties associated with PAH sample concentrations measured as being greater than the detection limit (DL). The analytical uncertainty $\left(\mathrm{u}_{\mathrm{ij}}\right)$ is typically not known and so is most often estimated relative to sample concentration $\left(\mathrm{x}_{\mathrm{ij}}\right)$ via an uncertainty scaling coefficient $\left(\mathrm{k}_{\mathrm{j}}\right)$ (Lee PK, 2003; Larsen and Baker, 2003; Ito et al, 2004; Brinkman, 2006; Spengler, 2011). There are sound reasons for this approach in estimating analytical uncertainty for the air pollutant data. First, scaling sampling error to input concentration $\left(\mathrm{x}_{\mathrm{ij}}\right)$ via a coefficient $\left(\mathrm{k}_{\mathrm{j}}\right)$ ensures that concentration and associated sampling uncertainty $\left(\mathrm{s}_{\mathrm{ij}}\right)$ will have similar magnitude and the relative scale of inter-pollutant or inter-species differences in concentration will be reflected in the uncertainty estimates (e.g., for PAH, sampling error on PHEN would be a larger order of magnitude than sampling error on $\mathrm{BaP}$ because PHEN is typically present in high concentrations while $\mathrm{BaP}$ is present at very low concentrations). Next, as indicated by Lee PK et al (2003) and Larsen and Baker (2003), larger $\mathrm{s}_{\mathrm{ij}}$ are desired for high concentration $\mathrm{x}_{\mathrm{ij}}$ to avoid high concentration (and potentially outlier) samples exerting undue influence on the factor model solution (i.e., a sample with large $\mathrm{s}_{\mathrm{ij}}$ is effectively down-weighted in the PMF solution). As stated by Larsen and Baker (2003), "PMF requires an uncertainty estimate for each variable. Part of the uncertainty estimate is proportional to the total concentration; therefore, higher concentrations have larger uncertainties. In addition, PMF was run in robust mode, which down-weights outlying variables i.e., those values beyond 4 times the mean concentration. Therefore, PMF purposely underestimates the modeled concentrations of the highest measured values." Lee PK et al (2003) similarly state the need to, "ensure larger errors apply to the larger values, preventing the high concentration data or outliers from unduly influencing the factor solution."

Analytical uncertainty $\left(\mathrm{u}_{\mathrm{ij}}\right)$ is thus defined as an uncertainty scaling coefficient $\left(\mathrm{k}_{\mathrm{j}}\right)$ multiplied by the sample concentration $\left(\mathrm{x}_{\mathrm{ij}}\right)$, namely:

$$
\mathrm{u}_{\mathrm{ij}}=\mathrm{k}_{\mathrm{j}} * \mathrm{x}_{\mathrm{ij}}
$$

Some previous PMF modeling efforts used a constant $\mathrm{k}_{\mathrm{j}}$ across all modeled species (e.g., Ito et al, 2004 estimated $\mathrm{u}_{\mathrm{ij}}$ as $5 \%$ of the input sample concentration so $\mathrm{k}_{\mathrm{j}}=0.05$ ). However, other modeling studies have attempted to select a $\mathrm{k}_{\mathrm{j}}$ that better reflected expected differences in species uncertainties, under the assumption that, even where one pollutant class is sampled (e.g., PAH), individual species could have different sampling 
uncertainty depending on their relative concentrations and species properties that influence their sampling analysis (e.g., molecular weight and vapour/particle prevalence).

Larsen and Baker (2003), modeling PAH data via PMF, had access to replicate laboratory analysis data for PAH. To estimate uncertainty on concentrations greater than the method detection limit, Larsen and Baker (2003) based $k_{j}$ on the variation in recovery efficiency of each modeled species, calculated as the standard deviation in species recovery efficiency. Analytical uncertainty $\left(\mathrm{u}_{\mathrm{ij}}\right)$ was then calculated as $\mathrm{k}_{\mathrm{j}}$ times the species sample concentration.

Battelle (2007), modeling PAH data via PMF, did not have uncertainty data or speciated detection limits for their lab-reported sample concentration dataset. However, wishing to estimate uncertainty so as to reflect "inter-species differences in measurement quality" (e.g., changes in data quality due to concentration magnitude, namely concentrations below DL should have different uncertainty estimate than those above DL), Battelle made use of duplicate sampling data they had available to them (i.e., co- located sampler data). This was done by setting $\mathrm{k}_{\mathrm{j}}$ equal to a coefficient of variation $(\mathrm{CV})$ defined for the co-located pair of samplers: $\mathrm{k}_{\mathrm{j}}=\mathrm{CV}_{\mathrm{j}}=\left(\mathrm{C}_{\text {sampler1 } \mathrm{j}}-\mathrm{C}_{\text {sampler2 } \mathrm{j}}\right) / \operatorname{mean}\left(\mathrm{C}_{\text {sampler1 } \mathrm{j}}, \mathrm{C}_{\text {sampler2 } \mathrm{j}}\right)$. Analytical uncertainty $\left(\mathrm{u}_{\mathrm{ij}}\right)$ was then calculated as $\mathrm{k}_{\mathrm{j}}$ times the species sample concentration or one-half the speciated detection limit (i.e., $\mathrm{DL}_{\mathrm{j}}$ was estimated from a comparable reference dataset), whichever was higher. This analytical uncertainty was used in the standard form sample uncertainty equation (i.e., $s_{i j}=u_{i j}+D_{j} / 3$ ).

\section{Detection limit $\left(D L_{j}\right)$ uncertainty}

A detection limit term is included in the estimation of uncertainty to represent uncertainty in lab- reported species concentration that is associated with the detection limit $\left(\mathrm{DL}_{\mathrm{ij}}\right)$. Most commonly, the $\mathrm{DL}_{\mathrm{ij}}$ is multiplied by a constant scaling factor $\left(\mathrm{a}_{\mathrm{DL}}\right)$ and added to the analytical uncertainty term, regardless of species (e.g., $\mathrm{a}_{\mathrm{DL}}=1 / 3$ ). The $\mathrm{DL}_{\mathrm{ij}}$ term can also be modified using a variable scaling factor although this approach was cited by Reff et al (2007) as occurring far less frequently in the modeling literature (e.g., Brinkman, 2006 with $\mathrm{PM}_{2.5}$ data; Spengler, 2011 with $\mathrm{PAH}$ data). Since the $\mathrm{DL}_{\mathrm{ij}}$ term is typically a relatively minor component of overall $\mathrm{S}_{\mathrm{ij}}$ there is no compelling argument to impute a species-specific scaling factor for the majority of receptor modeling datasets and a constant scaling factor is used $\left(\mathrm{a}_{\mathrm{DL}}\right)$. 


\section{Forms of the sample uncertainty equation}

With analytical uncertainty and Detection Limit uncertainty terms as outlined above, the form of the uncertainty equation most frequently encountered in the PMF literature is (Reff et al, 2007; e.g., Larsen and Baker, 2003; Battelle, 2007):

$$
\begin{aligned}
& s_{i j}=u_{i j}+a_{D L} * D L_{i j} \\
& \text { where } u_{i j}=k_{j} * x_{i j} \\
& a_{D L}=\text { scaling factor for portion of } D_{i j} \text { to be included in total uncertainty }
\end{aligned}
$$

The fractional scaling factor for the detection limit contribution to total uncertainty is most often set to a value of one-third (Battelle, 2007; Viana et al, 2008) or one-half (Larsen and Baker, 2003). Although much of the PMF literature summarized by Reff et al (2007) involved modeling of PM datasets, previous modeling studies of PAH datasets have tended to use similar formulae for estimating modeling uncertainty (Larsen and Baker, 2003; Battelle, 2007).

A slightly different approach was used to estimate total uncertainty in a recent PMF modeling study completed by Spengler et al (2011). Spengler et al (2011) collected and analysed PAH concentrations using equipment and methods very comparable to NAPS sampling (i.e., inclusive of laboratory co- extraction and co-quantitation of the vapour and particle phase samplers). Along with their PAH sampler data, the authors had access to field blank data (i.e., used to calculate Limit of Detection as $\mathrm{LOD}=3 * \mathrm{SD}_{\text {fieldblank }}$ ), colocated samplers (i.e., used to calculate relative precision as the square root of one-half the Root Mean Standard Error), and comprehensive metadata from the laboratory (Instrument Detection Limits, Method Detection Limits). Similar to other PMF studies, the uncertainty estimate equation $\left(\mathrm{s}_{\mathrm{ij}}\right)$ developed by Spengler et al (2011) employed an analytical uncertainty term $\left(\mathrm{u}_{\mathrm{ij}}\right)$ and detection limit term $\left(\mathrm{DL}_{\mathrm{ij}}\right)$; however, the authors added the $\mathrm{u}_{\mathrm{ij}}$ and $\mathrm{DL}_{\mathrm{ij}}$ terms as sum of squares, an approach which allowed incorporating a weighting coefficient for each term that differed by species. This 'sum of squares form' of the uncertainty equation was given by Spengler et al (2011) as:

$$
\begin{aligned}
& s_{i j}=\left[\left(a_{j} * C_{i j}\right)^{2}+\left(b_{j} * D L_{j}\right)^{2}\right]^{(0.5)} \\
& \text { where } a_{j}=\text { species measurement uncertainty }(\%) \\
& \qquad b_{j}=\text { species method detection limit uncertainty }(\%)
\end{aligned}
$$


The scaling coefficients for analytical uncertainty $\left(a_{j}\right)$ and detection limit $\left(b_{j}\right)$ were calculated from the additional laboratory metadata available to Spengler et al (2011). The species measurement uncertainty $\left(a_{j}\right)$ was determined from replicate analysis of field blank concentrations of each species and as such is an indication of the variation in concentration measurements. The species method detection limit uncertainty $\left(b_{j}\right)$ was determined from the standard deviation of sample surrogate recovery values. The authors then applied the 'sum of squares form' of the uncertainty equation to estimate total uncertainty of sample concentrations above the detection limit. Uncertainty on input concentrations below $\mathrm{DL}_{\mathrm{ij}}$ were estimated using only the second term in their equation (i.e., $\mathrm{s}_{\mathrm{ij}}=\mathrm{b}_{\mathrm{j}} * \mathrm{DL}_{\mathrm{j}}$ ) and uncertainty on missing species concentrations were estimated as one-half the species detection limit (i.e., $\mathrm{s}_{\mathrm{ij}}=\mathrm{DL}_{\mathrm{j}} / 2$ ).

Finally, a pair of PAH-via-PMF modeling studies were recently conducted by Sofowote et al (2008, 2011), modeling PAH data from sediment samples (2008) taken from the harbour in Hamilton, Ontario (2008) and the PAH data from arctic air samples (2011). Both studies provide considerable detail on the PMF methodology used, making them strong reference papers for this work. The authors estimated uncertainty on their PAH data using a 'sum of squares form' equation as seen in Spengler et al (2011); however, as in Larsen and Baker (2003) and the majority of other PMF studies, only the sample concentrations were weighted via a species measurement uncertainty coefficient $\left(\mathrm{k}_{\mathrm{j}}\right)$ :

$$
\begin{aligned}
& \mathrm{S}_{\mathrm{ij}}=\left[\left(\mathrm{k}_{\mathrm{j}} * \mathrm{X}_{\mathrm{ij}}\right)^{2}+(\mathrm{DLj})^{2}\right]^{(1 / 2)} \\
& \text { where } \\
& \mathrm{k}_{\mathrm{j}}=\text { species measurement uncertainty }(\%)
\end{aligned}
$$

The scaling coefficient $\left(\mathrm{k}_{\mathrm{j}}\right)$ was determined from replicate analysis on surrogate compounds, calculated as the standard deviation of recovery efficiency of each species (i.e., similar to methodology of Larsen and Baker, 2003). Uncertainty on concentrations less than the detection limit were estimated as two times the species detection limit (i.e., $\mathrm{s}_{\mathrm{ij}}=2 * \mathrm{DL}_{\mathrm{j}}$ ). The method detection limit for each species was determined from replicate analysis of field blank measurements (i.e., $\mathrm{DL}_{\mathrm{j}}=\mathrm{DL}_{\mathrm{ij} \text { mean }}+3 * \mathrm{DL}_{\mathrm{ij} \text { standard deviation }}$ ). 


\section{$\underline{\text { PMF uncertainty equation for NAPS PAH data }}$}

Uncertainties for PMF modeling of NAPS central site data at the urban and background sites were calculated using the model's 'equation-based' subroutine, which aggregates analytical uncertainty and uncertainty associated with species detection limits to the following formula and is applied to all input concentration data that are above input detection limit values:

$$
\begin{gathered}
\left.\mathrm{s}_{\mathrm{ij}}=\left[\left(\mathrm{k}_{\mathrm{j}} * \mathrm{X}_{\mathrm{ij}}\right)\right)^{2}+\left(\mathrm{DL}_{\mathrm{j}}\right)^{2}\right]^{(1 / 2)} \\
\text { where } \mathrm{k}_{\mathrm{j}}=\text { species measurement uncertainty }(\%) \\
\mathrm{X}_{\mathrm{ij}}=\text { sample species concentration }\left(\mathrm{ng} / \mathrm{m}^{3}\right) \\
\mathrm{DL}_{\mathrm{j}}=\text { species detection limit }\left(\mathrm{ng} / \mathrm{m}^{3}\right)
\end{gathered}
$$

It can be clearly seen that the PMF equation is sample- and species-specific and consistent with the general form of the sample uncertainty equation summarized by Reff et al (2007); namely, $\mathrm{s}_{\mathrm{ij}}=f\left(\mathrm{u}_{\mathrm{ij}}, \mathrm{DL}_{\mathrm{ij}}\right)$ with the first term estimating the analytical uncertainty $\left(\mathrm{u}_{\mathrm{ij}}=\mathrm{k}_{\mathrm{j}}{ }^{*} \mathrm{x}_{\mathrm{ij}}\right)$ and the second term incorporating the method detection limit $\left(\mathrm{DL}_{\mathrm{ij}}\right)$. It can also be seen that the PMF equation-based uncertainty formula is precisely consistent with 'sum of squares' formulation used by Sofowote et al $(2008,2011)$ and Larsen and Baker (2003).

\section{Analytical uncertainty $\left(u_{i j}\right)$ and detection limit $\left(D L_{j}\right)$ values for NAPS PAH data}

Since PAH are a class of compounds of varying molecular weight and ring structure, physico-chemical properties that may affect sampling (e.g., vapour pressure) may vary meaningfully, and so it is not realistic for $\mathrm{k}_{\mathrm{j}}$ to be kept constant across all sampled species. A more defensible approach is to select the uncertainty scaling coefficient $\left(\mathrm{k}_{\mathrm{j}}\right)$ to reflect inter-species differences in laboratory analysis uncertainty; in this way, species with greater measurement uncertainty will be assigned a higher $\mathrm{k}_{\mathrm{j}}$, and thus a greater overall sample uncertainty $\left(\mathrm{s}_{\mathrm{ij}}\right)$ in the model, ensuring that these samples are appropriated down-weighted in the solution.

To avoid being overly subjective or 'ad hoc' with the uncertainty scaling coefficient, however, $\mathrm{k}_{\mathrm{j}}$ was related to known and quantified sources of uncertainty in the laboratory analysis, ensuring a procedurally rational approach to estimating uncertainty for the PMF dataset. Analytical uncertainty information was taken from information made available by Environment Canada (2011f) in "Method 3.03/5.1 - Analytical method for the determination of selected PACs in ambient air samples". This document detailed the analytical methodology for quantifying concentrations of PAH in NAPS samples. The 
document (Environment Canada, 2011f) lists the major uncertainty sources as the accumulation of uncertainty in three measurement steps: preparation of the matrix sample (i.e., sample preparation, spike preparation, instrument response, preparation method, matrix interference, purity), standard preparation (i.e., bias), and sample size (i.e., mass, volume). The cumulative measurement uncertainty associated with these cited uncertainty sources are then quantified for each of the 29 PAH species sampled at NAPS sites; this was calculated as two times the relative standard deviation from the mean (i.e., 95\% confidence interval; units of percent) for data from a replicate sample analysis. The percent uncertainty tabulated in the Environment Canada Method (2011f) is thus meant to estimate the uncertainty associated with sample concentrations reported by NAPS. Since NAPS data is not recovery-corrected (see section 8.3 in Environment Canada, 2011f), the corresponding uncertainty estimates (i.e., Environment Canada, 2011f; "Uncertainty estimates by species, not recovery corrected") were used for the species-specific uncertainty scaling coefficient $\left(\mathrm{k}_{\mathrm{j}}\right)$, which varied significantly between species (i.e., $\mathrm{k}_{\mathrm{j}}$ approximately $10 \%-50 \%$ ), highly volatile and more reactive species assigned greater relative uncertainty than more conservative species.

A single species-specific detection limit $\left(\mathrm{DL}_{\mathrm{j}}\right)$ was used in calculation of $\mathrm{s}_{\mathrm{ij}}$ to ensure consistency with input data preparation of the sample concentration. This approach is also consistent with that used in comparable studies modeling PAH data via PMF (Sofowote et al, 2008, 2011). This was referenced from a 2005 QAQC dataset of NAPS data made available by Environment Canada; calculated as: $\mathrm{DL}_{\mathrm{j}}=\left(\mathrm{t}\right.$-statistic) $* \mathrm{SD}\left(\mathrm{x}_{\mathrm{ij}}\right)$ where $\mathrm{SD}_{\mathrm{j}}=$ calculated as standard deviation of 7 replicate samples analysed for respective PAH species (note: due to outlier species sample concentrations, 5 samples used for ACE, FLU, PHEN; 6 samples used for FLT, PYR); t-value = Student-t test statistic at 95\% confidence interval, for 5-7 samples (4-6 degrees of freedom), dependent on species. 


\section{Appendix B Urban/rural source apportionment}

Supplementary Information to Chapter 3 (research component I).

\section{B.1 PAH source review}

Environmental context for source apportionment via: (i) review of PAH source types, Canadian and Ontario contributions; (ii) source types influencing study area; (iii) aerial maps of study sites; (iv) literature review of relevant available PAH emissions profiles.

\section{B.2 PAH trend analysis}

Environmental context for source apportionment via historical trend analyses of ambient PAH data at study sites: (i) descriptive statistics; (ii) annual, seasonal, species abundance trends; (iii) PAH species monitored by NAPS and comparison with NPRI reporting, CEPA toxicity, US regulation.

\section{B.3 Wind pattern analysis}

Environmental context for source apportionment via wind pattern analysis, including: (i) year-round and seasonal wind roses; (ii) pollutant rose at Hamilton. Description of methodology for Conditional Probability Function (CPF) to facilitate future work.

\section{B.4 Principal Component Analysis}

PCA modeling method and results for NAPS central site data: (i) primary PCA (lognormal data transformation); (ii) supplementary PCA (proportional data transformation).

\section{B.5 PMF modeling details}

PMF modeling details: (i) input data treatment and uncertainty; (ii) model parameters, performance criteria; (iii) F profile uncertainty; (iv) model order; (v) model performance.

\section{B.6 PMF sensitivity analyses}

PMF model checks and sensitivity analyses.

\section{B.7 PMF factors \& source interpretation}

PMF model checks and sensitivity analyses.

\section{B.8 Additional PMF results}

Additional PMF results inclusive of: (i) base run factor profiles; (ii) median and range of source contributions to PAH and PAH toxicity; (iii) total aggregate source contributions to PAH toxicity (pie charts). 


\section{B.1 PAH source review}

\section{Canadian and provincial context for PAH source types}

Broad Canadian and provincial context for PAH source types was established by review of National Pollutant Release Inventory data (NPRI; Environment Canada, 2009) and a recent regional emissions inventory including the study area (Galarneau et al, 2007). PAH emissions are low relative to other NPRI-reportable pollutants but require reporting due to toxic classification under the Canadian Environmental Protection Act (CEPA). ${ }^{17}$

Anthropogenic activities comprise approximately half the annual inventoried PAH releases to air in Canada (Environment Canada, 2009; CEPA, 1999). Anthropogenic sources include space heating (i.e., oil, natural gas), industrial source emissions (e.g., iron/steel production, aluminum smelting), mobile source emissions (i.e., gasoline, diesel-fueled vehicles), coal-fired power generation, and biomass combustion (ATSDR, 1995). With BaP taken as a convenient indicator and referencing recent inventory data for anthropogenic releases: more than two-thirds (69\%) were from non-industrial sources, largely due to wood burning for home heating; nearly a third (28\%) were from industrial sources, dominated by the aluminum industry followed by the iron/steel industry (e.g., facilities located in study region); mobile source contributions were small by comparison (2\%). (Environment Canada, 2009; Figure B.1-1)

In Ontario, recent emission inventories (Environment Canada, 2009; Galarneau et al, 2007) showed major anthropogenic PAH source types: motor vehicle exhaust emissions (i.e., gasoline-fueled, diesel-fueled), iron/steel manufacturing, heating emissions (e.g., oil, natural gas), and residential wood combustion. With $\mathrm{BaP}$ as indicator: majority annual contribution (89\%) was from non-industrial source types, specifically residential fuel wood combustion; next largest contribution was from industrial source types, specifically iron/steel industry $(\sim 5 \%)$; these were followed closely by mobile source contributions ( 4\%). (Environment Canada, 2009; Figure B.1-2)

PAH source types comprising a small portion of overall annual anthropogenic Canadian or Ontario releases may be significant sources at a given receptor location due to their exposing a specific population continuously or disproportionately (e.g., those living near the source or exposed occupationally). This fundamental premise drove the research investigation of source apportionment at the urban and background sites and the

\footnotetext{
${ }^{17}$ Reporting of PAH releases is required under an alternate lower threshold of annual total PAH releases $\geq 50 \mathrm{~kg}$ for all large facilities (annual total operations $\geq 20,000$ employee-hours).
} 
comparisons made between sites. An illustrative example for stationary source types is iron/steel manufacturing: although comprising a small portion of total annual anthropogenic Canadian releases it is the largest industry source in Ontario and is a key local source type in Hamilton, Ontario (i.e., two integrated steel works facilities). A second example is the mobile source type: although mobile source emissions have a relatively small contribution relative to stationary sources on a national or provincial basis, they can be a significant source of PAH exposure to residents in urban centres.

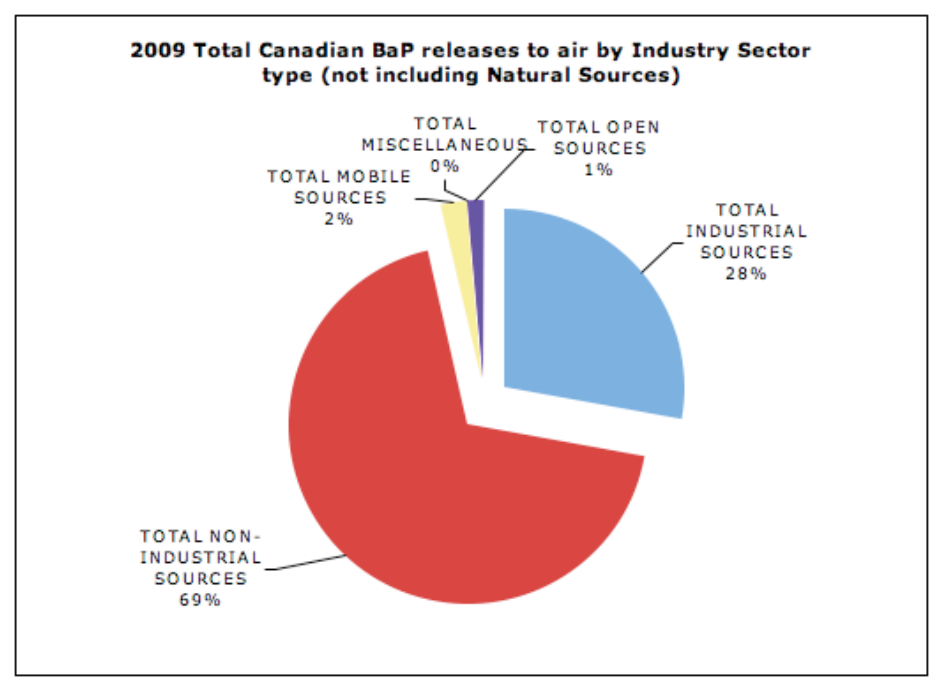

Figure B.1-1. BAP releases to air in Canada by source type (2009 data).

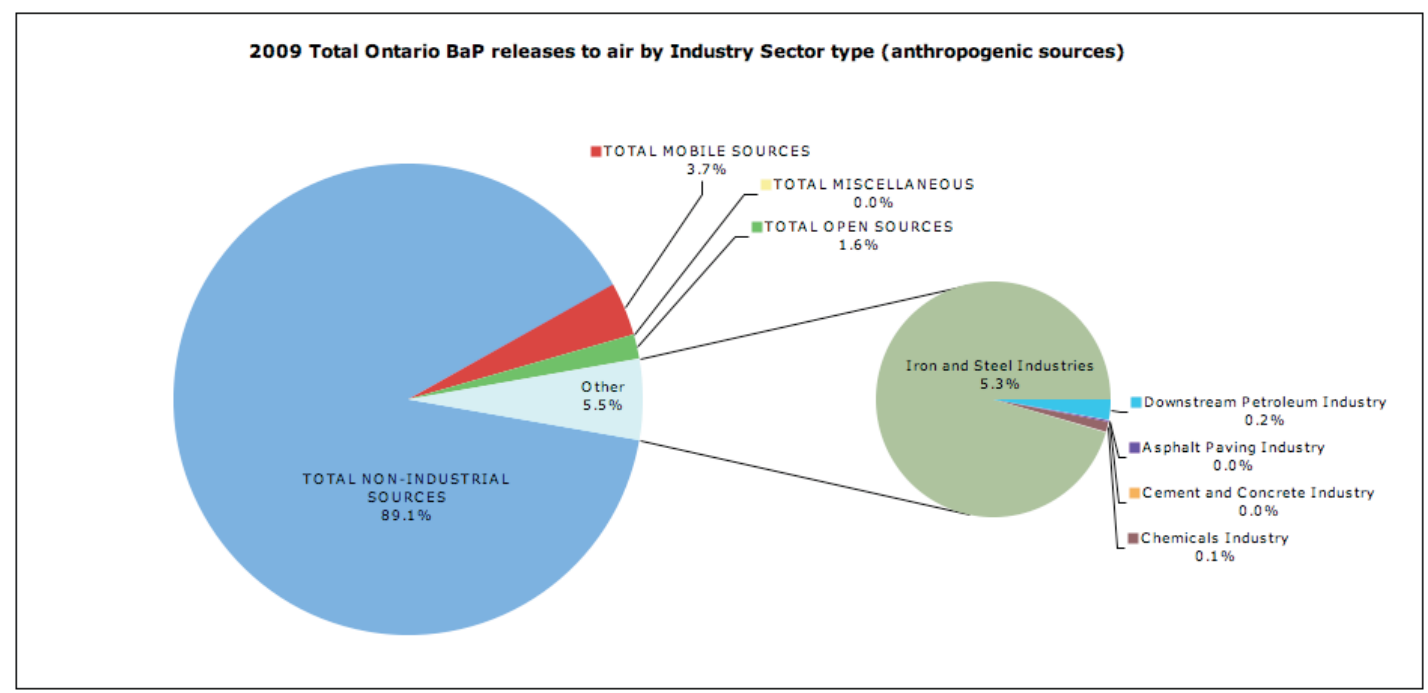

Figure B.1-2. BAP releases to air in Ontario by source type (2009 data). 
$\underline{\text { Source types influencing study area }}$

Industrial stationary sources located near the studies sites and reporting airborne 'Total PAH' or individual species were identified by review of NPRI data for the 2009 year (Environment Canada, 2009), selected to correspond to the related intraurban field sampling completed in 2009 (Anastasopoulos et al, 2012). Additional stationary sources, including institutional and residential, as well as areas and traffic routes with expected concentrated mobile source emissions, were identified by reviewing aerial maps and through local knowledge of the study area.

For the study area, relative contributions by industry type to overall industry-reported PAH releases to air are summarized in Table B.1-1. Reported industrial PAH releases to air were dominated by metals manufacturing (i.e., iron/steel mills and ferro-alloy manufacturing, 45\%; non-ferrous production and processing, 14\%) and chemical manufacturing (39\%); industries reporting relatively minor contributions were petroleum/coal products manufacturing $(1 \%)$, cement/concrete product manufacturing $(1 \%)$, and non-metallic mineral mining/quarrying $(<1 \%)$.

Within the study area, industrial and institutional facilities reporting airborne PAH emissions (Environment Canada, 2009) or otherwise identified as relevant are mapped alongside the scoped sites in Figure B.1-3. Industrial facilities included iron/steel manufacturing and chemical manufacturing in Hamilton and several industry facilities in west Toronto (i.e., steel production, petrochemical refining, cement manufacturing), coalfired power generation (i.e., Ontario Power Generation) and additional iron/steel manufacturing facilities.

Several institutional facilities (i.e., university/college campuses, hospitals) are located near the Hamilton and Toronto central monitoring sites with the most relevant associated source type expected to be space-heating combustion (i.e., natural gas, oil). A military base is also located NW of the study area but was not expected to be influential to the background (Egbert) site due to its location upwind (prevailing winds) of the site and very low PAH levels observed at the site.

Private residences are found in very high density in the greater urban areas around the central monitoring sites (i.e., Toronto 2011 census population of approximately 6M residents in approximately $2 \mathrm{M}$ dwellings; Hamilton 2011 census population of approximately 700,000 residents in approximately 282,000 private dwellings; Statistics Canada, 2012a, 2012b) with the most relevant associated source types expected to be space heating combustion (i.e., natural gas, oil) and biomass combustion (i.e., woodburning fireplaces/supplemental heating). 
In addition to stationary sources (i.e., industrial, institutional, residential), mobile sources are distributed throughout the study region and concentrated in the urban locations; these were aggregated to a single source type termed 'vehicle exhaust', representing exhaust emissions from multiple fuel types (e.g., gasoline, diesel), vehicle classes (e.g., heavy duty vehicles, HDV; light duty vehicles, LDV), and traffic modes (e.g., urban congested, urban free-flowing, highway free-flowing). Vehicle exhaust emissions were expected to be significant at both urban sites, particularly at Toronto due to its larger population base and traffic congestion on several commuting routes (i.e., surface streets in central business district and expressways).

Table B.1-1. Industrial facilities within study region reporting PAH releases to air (2009 NPRI data).

\begin{tabular}{|c|c|c|c|}
\hline NAICS - description & NAICS & Facilities & \begin{tabular}{|c|}
$\%$ reported PAH \\
contribution to study zone \\
(2009 NPRI)
\end{tabular} \\
\hline Iron \& Steel Mills \& Ferro-Alloy Mfg. & 3311 & 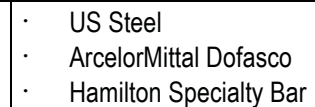 & $45 \%$ \\
\hline Basic Chemical Mfg. & 3251 & $\begin{array}{ll}\text { - } & \text { Ruetgers } \\
\text { - } & \text { Columbian Chemicals }\end{array}$ & $39 \%$ \\
\hline Non-Ferrous (exc. Al) Production \& Processing & 3314 & . $\quad$ Tonolli Canada & $14 \%$ \\
\hline Petroleum \& Coal Products Mfg. & 3241 & $\begin{array}{ll}\text { - } & \text { Imperial Oil } \\
\text { - } & \text { Shell Canada } \\
\text { - Suncor Energy }\end{array}$ & $1 \%$ \\
\hline Cement \& Concrete Product Mfg. & 3273 & - Holcim & $1 \%$ \\
\hline Non-Metallic Mineral Mining \& Quarrying & 2123 & - Walker Aggregates & $<1 \%$ \\
\hline
\end{tabular}




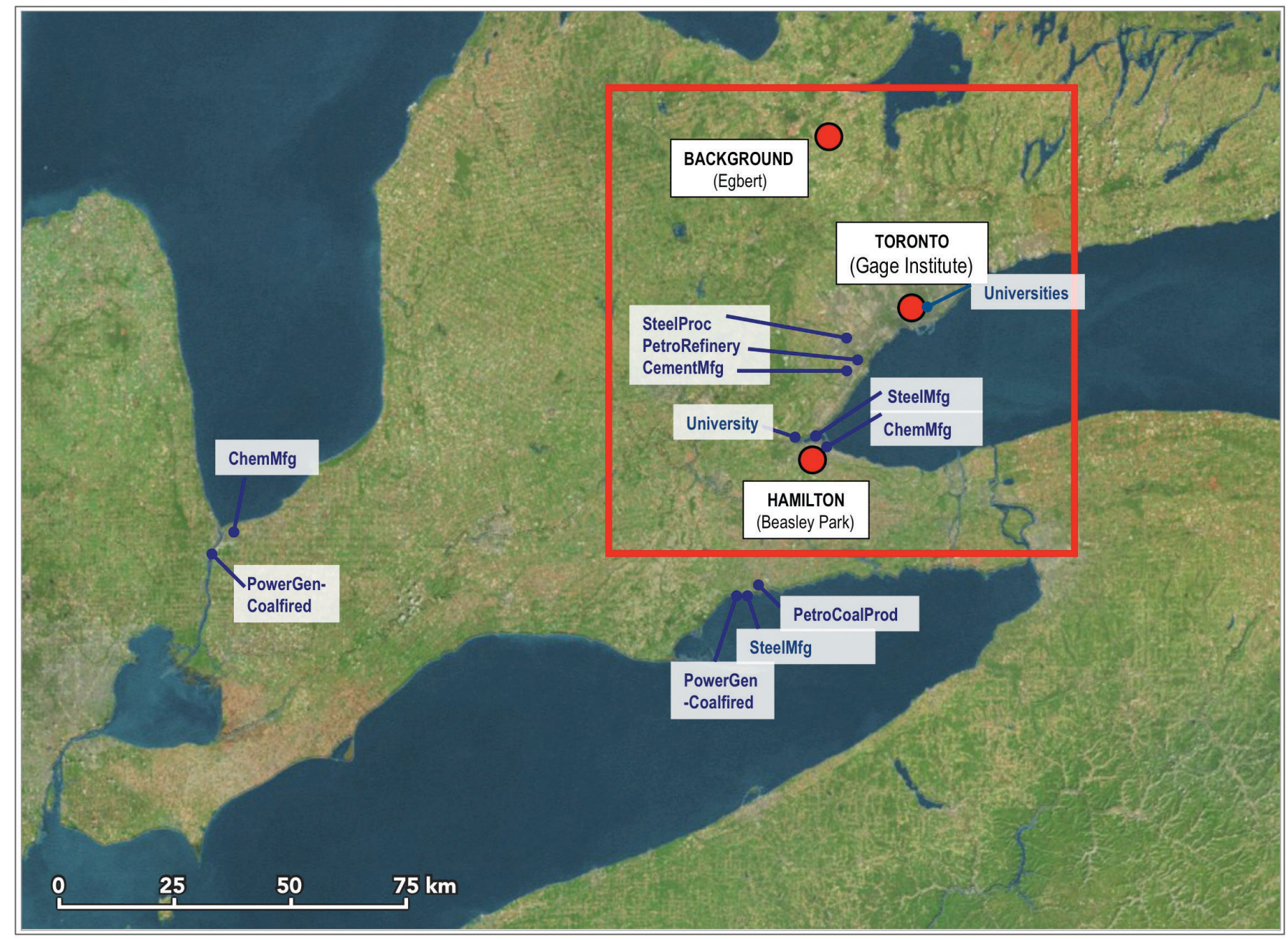

Figure B.1-3. Major stationary PAH sources located in or near study region. 


\section{Aerial Photos Of Receptor Sites}

Aerial 3D maps of the urban (Hamilton, Ontario; Toronto, Ontario) and background (Egbert, Ontario) sites scoped for receptor modeling are shown in Figure B.1-4. Shown at each site is the location of the NAPS central site monitor and immediately surrounding land use (i.e., two scales).

(a) Hamilton central site monitor at Beasley Park $(43.25778 N, 79.86222 \mathrm{~W})$
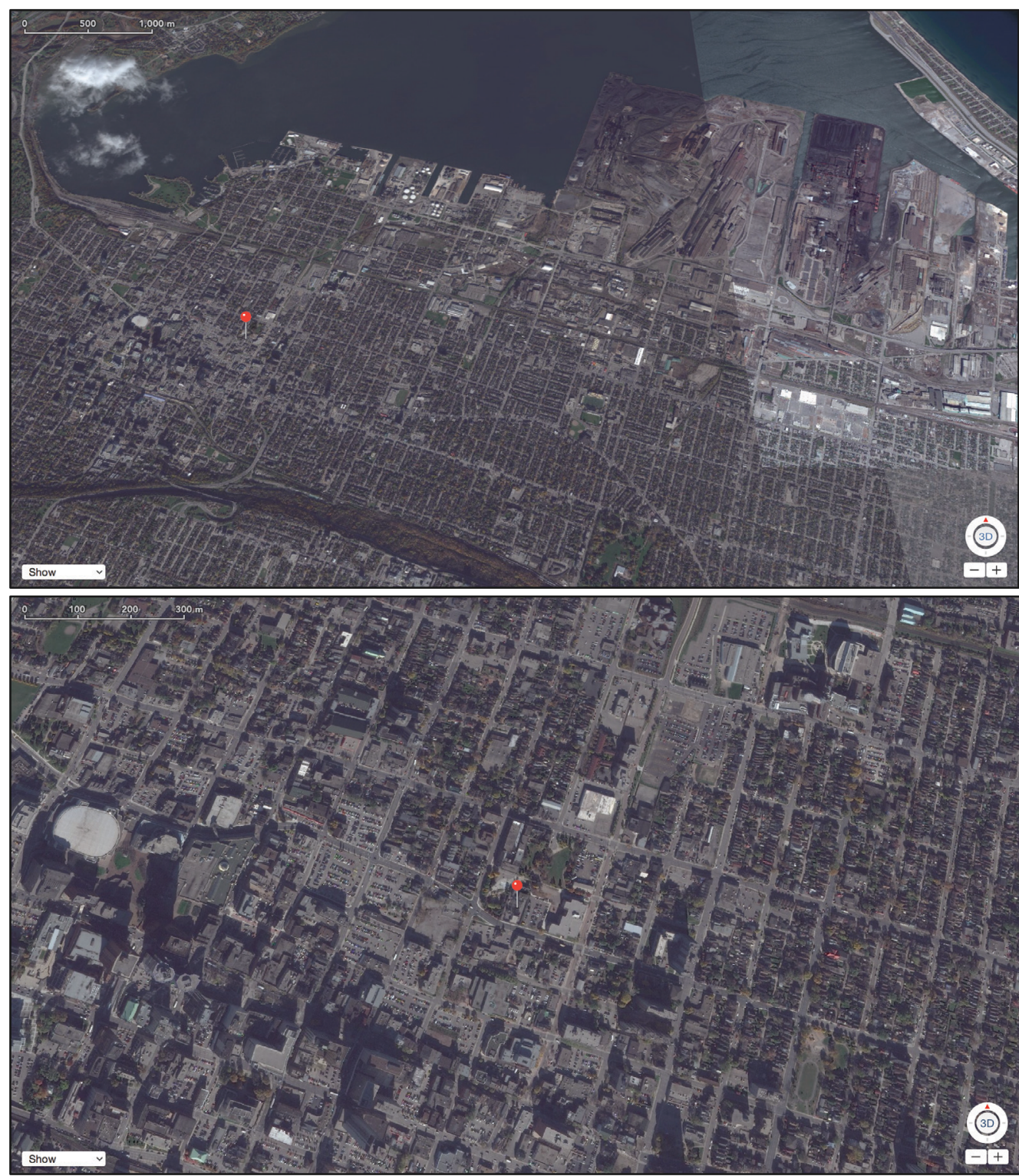

Source: Map generated with Apple Maps v 2.0, Rev. 10/3/2014 (Apple Inc., Cupertino, CA, USA); Canadian map data: MultiNet North America (C) 2006 - 2014 TomTom.

Figure B.1-4. Aerial photos of central site monitor and surrounding land use. 
(b) Toronto central site monitor at Gage Institute (43.65842N, 79.39714W)
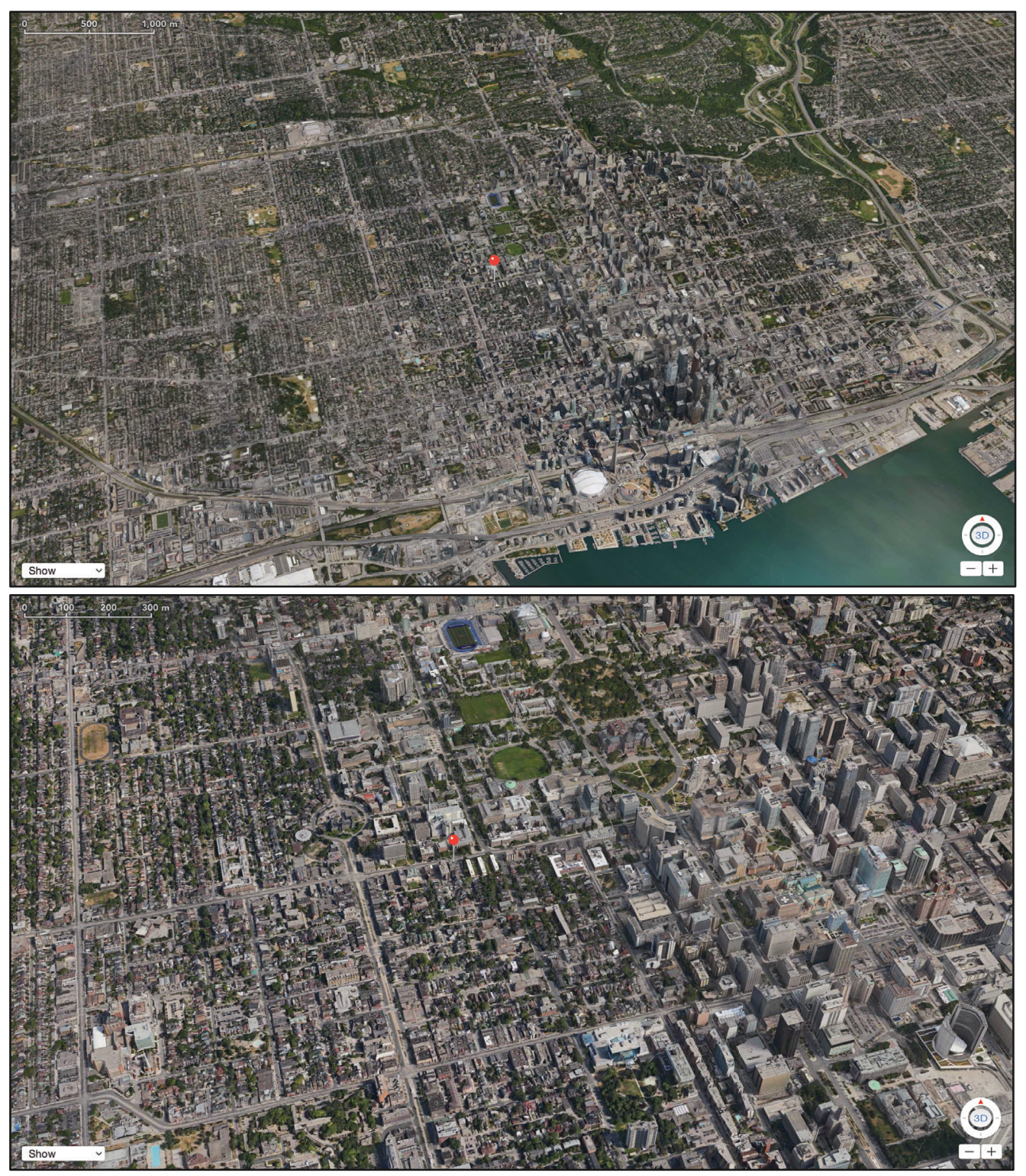

Source: Map generated with Apple Maps v 2.0, Rev. 10/3/2014 (Apple Inc., Cupertino, CA, USA); Canadian map data: MultiNet North America @ 2006 - 2014 TomTom.

Figure B.1-4 (continued) 
(c) Background site monitor at Egbert (44.23250N, 79.78139W)
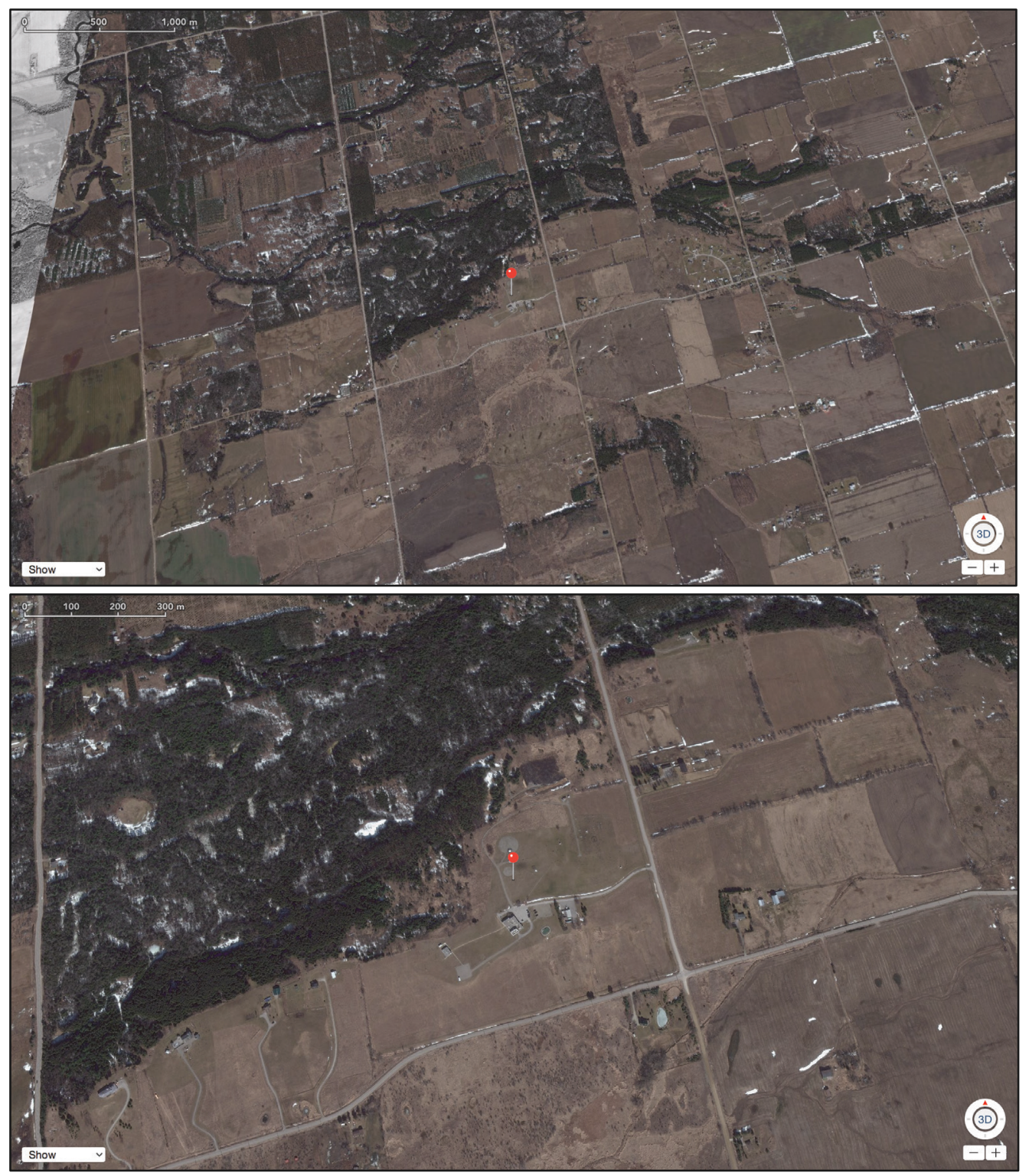

Source: Map generated with Apple Maps v 2.0, Rev. 10/3/2014 (Apple Inc., Cupertino, CA, USA); Canadian map data: MultiNet North America ( 2006 - 2014 TomTom.

Figure B.1-4 (continued) 


\section{LITERATURE REVIEW OF AVAILABLE PAH EMISSIONS PROFILE DATA}

\section{$\underline{\text { Reference emissions profiles }}$}

For major source types identified as influencing the study area, a literature review of emissions databases and source sampling studies was conducted to obtain multi-species PAH emissions profiles and to identify source-characteristic species or source marker species that could inform receptor model factor interpretation. From a review of emissions databases, US EPA SPECIATE yielded emissions profiles for mobile source types (2011a) and US EPA AP-42 / FIRE (2012a, 2012b, 2011b) yielded profiles for stationary source types. ${ }^{18}$ Related source documentation (US EPA, 1998b) was reviewed to identify industry sub-processes contributing most significantly to PAH emission; a highly relevant example in the study area was the coke oven sub-process in iron/steel manufacturing, since integrated iron/steel manufacturing is located at the Hamilton site. Emission profiles reporting combined particle and vapour phase $\mathrm{PAH}$ emissions were preferred to match to NAPS central site dataset analysed and modeled in this research. Where combined phase data were unavailable, particle phase profiles were retained. ${ }^{19}$

Relevant PAH source profiles were also identified by review of source sampling studies, for which in-field traffic or industrial source sampling was preferred over laboratory studies, as well as receptor modeling studies. Source sampling studies were screened for suitability by several criteria, including reporting data for stationary source processes or vehicle fleets reasonably representative of North American conditions, and reporting PAH data that is recent and sampled from particle and vapour phase for a diverse suite of $\mathrm{PAH}$ ranging from light to heavy MW species. For traffic exhaust emissions, profiles derived from tunnel and on-road sampling studies were preferred over chassis dynamometer data (SPECIATE; US EPA, 2011a) since these have been shown to be more representative of emissions from urban vehicle mixed fleets in 'real world' driving modes (Ropkins et al, 2009; Karman and Shen, 2006). Receptor modeling studies

\footnotetext{
${ }^{18}$ Industrial processes in Canada reference the emissions factors in US EPA AP-42 / FIRE when estimating annual PAH emissions for reporting to NPRI.

${ }^{19}$ Profiles reporting only particle phase data may be modified to impute particle plus vapour phase profiles by referencing datasets reporting separate vapour/particle concentration data or percentages, such as found in Wingfors et al (2001), Kristensson et al (2004), Harrison et al (2003) and additional literature cited in Environment Canada (2006). The process is summarized in Bzdusek et al, (2004) with vapour/particle percentage data provided in supplementary material of $\mathrm{Li}$ et al, (2003). Due to inherently large uncertainty with this approach, however, imputed particle plus vapour profiles were not used in this research.
} 
applying diagnostic ratios, PCA, or the PMF model, were screened for reasonably comparable datasets, with preference given to data reporting ambient particle plus vapour phase PAH in North American locations.

Emissions profile data identified via literature review were selected for their relevance to the study region and comparability to NAPS sampling data; as such, the profiles cited in this literature review are not intended to be an exhaustive compilation but are provided for the convenience of other researchers investigating PAH source emissions. Emissions profile data identified by the literature review are tabulated at end of this sub-appendix (Table B.1-2 through Table B.1-4) and are organized by source type with key metadata provided to facilitate comparison to other research datasets (i.e., study type, location, particle/vapour phase sampling, species suite size, etc.).

$\underline{\text { PAH emission profile formats and availability }}$

Emission profile formats: Emissions profiles are the relative species abundance of a suite of pollutants (e.g., PAH species) emitted by a source and are sometimes also termed 'source fingerprints'. The single-species analogs to emissions profiles are 'emission markers', which are pollutant species characteristically associated with a particular process. Typically, however, PAH emissions profiles are characterized by several dominant species (i.e., species with high mass concentration relative to other species and thus contributing a greater portion of the total PAH mass represented by the profile). While profiles can be compared quantitatively (e.g., linearly regressing relative mass concentrations of commonly emitted species in one profile against another), practical comparisons in the literature are most often qualitative and compare presence / absence of dominant species, particularly for source markers or characteristic species.

Emissions profile data is most generally available in either 'source profile' (SP) or 'emissions factor' (EF) format. SPs are compiled from measurements of PAH in the field (e.g., sampling near and downwind of a stationary source; sampling in a tunnel for mobile sources) or in the laboratory (e.g., sampling wood combustion emissions in an environmental chamber). Since ambient PAH is directly measured at close proximity to the emitting process it is presumed that the source profile closely represents the particular source type; other instances or locations of the same source type will have slightly differing profiles due to process variations but dominant PAH species are generally expected to remain characteristic of the profile.

EF data is typically provided as mass of pollutant emitted per unit of activity (e.g., mass $\mathrm{BaP}$ emitted / mass coal combusted). For stationary sources, EFs within a source category are averaged from several industry facilities and so represent long-term averages for any facilities in the source category. (US EPA, 2011) Compared with SPs, the more general 
nature of EFs could be construed as a positive aspect to the receptor modeling research, provided it is understood that any one specific facility (e.g., iron/steel works in Hamilton) will not have identical process as those that went into compiling the associated EF.

Methods for comparing sampling study data with receptor model data: Reference datasets and profiles retained from sampling studies typically report PAH source emissions (e.g., traffic, wood combustion, coal combustion) variously as mass concentration $\left(\mathrm{ng} / \mathrm{m}^{3}\right)$, emission factor (EF; traffic: $\mu \mathrm{g} / \mathrm{km}$; stationary source: $\mu \mathrm{g} / \mathrm{mass}$ fuel combusted), or emission rate (ER; traffic: $\mu \mathrm{g} / \mathrm{km}-\mathrm{hr}$ ). All three forms of reference profile can be compared with each other and with receptor modeling factor profiles, since comparisons are based on the relative concentration of sampled species within a profile. The BeP-normalizing method developed in early PAH sampling studies (Benner et al, 1989) may also facilitate comparison between profile datasets, as summarized by Bzdusek et al (2004) and Li et al (2003) in their source apportionment studies of Chicago sediment PAH data: each species concentration (or EF, or ER) is normalized to BeP, a species selected for its demonstrated atmospheric stability (Venkataraman and Friedlander, 1994) and because it is sampled and analysed in most PAH studies; where $\mathrm{BeP}$ is not available, $\mathrm{BaP}$ is selected as the normalizing species since sampled $\mathrm{BeP} / \mathrm{BaP}$ ratios typically approximate unity in many major source types. The $\mathrm{PAH} / \mathrm{BeP}$ ratio in the resulting BeP-normalized profile will thus be the same regardless of how many species were sampled by a given study, allowing direct comparison across disparate sampling and modeling datasets or even species subsets.

Profiles reporting only particle phase data may also be modified to impute particle plus vapour phase data by referring to datasets reporting separate vapour/particle concentration data or vapour/particle percentages (Wingfors et al, 2001; Kristensson et al, 2004; Harrison et al, 2003; Environment Canada 2006 also includes literature review of several studies reporting vapour/particle phase partitioning percentages). This process is summarized in Bzdusek et al, (2004) with relevant vapour/particle percentage data in Table S1 of the related paper by $\mathrm{Li}$ et al, (2003). Since there is inherently large uncertainty with this approach, imputed particle plus vapour profiles were not used in this research.

A recent trio of receptor modeling papers (Ning et al, 2008; Liacos et al, 2012; Kam et al, 2012) also demonstrate the modification of reference profile data to better represent PAH degradation that may occur between source and receptor, applying a simple first-order decay equation to reference $\mathrm{PAH}$ profile concentration data prior to their use in receptor modeling (i.e., as input profiles for US EPA CMB). This approach may also prove useful for interpreting factors resolved by US EPA PMF from the NAPS dataset, particularly for sources that are at greater distances from the central site monitor and would thus have 
greater potential for degradation by atmospheric processes (e.g., reactivity, particle fallout/washout).

Literature reviewed: SPs for source types relevant to the study region were referenced from source sampling studies in the academic journal literature and in the US EPA database SPECIATE (2009), the central repository of VOC and PM speciation profiles from air pollution sources which also typically provides uncertainty estimates on profiles (US EPA, 2012). EFs for stationary source types relevant to the study region were reviewed in the US EPA database AP-42, Volume I: Stationary point and area emissions, 5th Edition (2012). EF for mobile sources are also available in US EPA databases ${ }^{20}$ but preference was given to SPs from source sampling studies (i.e., tunnel studies, on-road traffic sampling) as these were considered more representative of ambient levels of PAH emissions associated with vehicle traffic.

\section{Source sampling studies}

Search for sampling studies reporting source emissions data: SPs reporting speciated $\mathrm{PAH}$ data were identified by review of air pollution literature published in peer-reviewed journals (e.g., using the "EI Engineering Village" search database). Literature review sought emissions profile data developed from field sampling (e.g., traffic sampling, industrial source sampling) and laboratory-based studies (e.g., combustion of single source and analytical quantification of PAH emission species).

The PAH reference profile data in relevant sampling studies was then screened by the following criteria: (i) recent sampling data (i.e., NAPS dataset is 2000-2010; Hamilton intraurban field dataset is 2009); (ii) sampling data reasonably representative of North American emissions vehicle fleet and driving conditions (e.g., traffic exhaust: vehicle type, vehicle age, fuel type, emission control technology; industry emissions: facility technology, fuel/feed type, air emission control technology); (iii) PAH sampling comparable to that used in NAPS central site monitoring and Hamilton field study (e.g., particle plus vapour phase sampling) or amenable to comparison via estimated vapour/particle phase partitioning (Bzdusek 2004; Li, 2003); (iv) sufficient number of

${ }^{20}$ EF for mobile sources available from US EPA MOBILE6 (2012) and the related US EPA models MOVES (2010) and NMIM (2008) along with reference material in US EPA AP-42, Volume II: Mobile, draft 5th Edition (1989). 
key PAH species to enable interpretation of major source types from receptor model output (i.e., profiles should include light MW through heavy MW species).

Traffic exhaust: On-road sampling studies (i.e., traffic tunnel, roadside, on-road invehicle) have been shown to provide emission data that is more representative of urban vehicle fleets than when composited from single vehicle sampling tests using a chassis dynamometer, such as the mobile source emissions datasets in US EPA SPECIATE (Ropkins et al, 2009; Environment Canada 2006). The primary advantage of on-road sampling studies is their ability to capture emissions from a mixed vehicle fleet operating in various driving modes (e.g., free flow higher speed typically seen in a freeway tunnel; transient lower-speed conditions associated with traffic signal control and congestion typically seen in urban surface streets). For these reasons, PAH reference data from tunnel and roadside sampling studies were given preference over reference datasets derived from chassis dynamometer sampling (e.g., SPECIATE mobile source datasets for gasoline-fueled and diesel-fueled vehicles). Some very recent sampling studies that employed on-road in-vehicle sampling are also cited as these can provide an even more representative sampling of 'real world' driving conditions, particularly in the urban driving mode (Liacos et al, 2012; Kam et al, 2012; Ropkins et al, 2009).

A comprehensive search of studies reporting speciated PAH data collected in tunnel, onroad, or in-vehicle sampling studies was compiled and reviewed. North American sampling studies that best met the screening criteria (i.e., priority given to recent studies with sampling/reporting of particle and vapour phase PAH for large suite of species) were: Khalili et al, 1995; Fraser et al, 1998; Gertler et al, 2002; Environment Canada 2004; Phuleria et al, 2006; Ning et al, 2008. Nearly all of the retained North American studies report profile data for Light Duty Vehicle (LDV) fueled by gasoline separately from Heavy Duty Vehicle (HDV) fueled by diesel (i.e., traffic tunnel and roadside sampling studies used vehicle counts by type to estimate LDV/HDV fleet composition). Further, the sampling studies are consistent in noting a characteristic difference between LDV gasoline and HDV diesel fleet profiles: LDV gasoline emission profiles are dominated by heavy MW species and have comparably less enrichment in light MW species; conversely, HDV diesel emission profiles are dominated by light MW species with comparably less enrichment in heavy MW species.

In addition, three recent European studies are cited as reference date for traffic emissions. First, Harrison et al (2003) study in UK is cited because it reports a large number of species and included vapour phase $\mathrm{PAH}$ in sampling and total particle plus vapour phase concentrations. Second, two studies in Sweden (Wingfors et al, 2001; Kristensson et al, 2004) are cited due to their reporting PM and vapour phase concentrations separately for a large suite of $\mathrm{PAH}$, which may be useful to researchers wishing to estimate 
vapour/particle partitioning of Canada and US datasets that report only particle phase concentrations (note: vapour/particle concentration literature data is also summarized in Karman and Shen, 2006).

Wood combustion and retene as tracer: Using screening criteria similar to those listed earlier for traffic profiles (i.e., priority given to recent and North American-based studies with sampling/reporting of particle and vapour phase PAH for large suite of species), several source sampling studies are cited: Khalili et al, 1995; Rogge et al, 1998; McDonald et al, 2000; Environment Canada, 2000; Schauer et al, 2001. Additional recent North American-based source sampling studies which sampled only particle phase data are cited as secondary references: Hays et al, 2003; Gullett et al, 2003; Oros et al, 2001a, 2001b; Fine et al, 2001, 2002. Source profiles for agricultural burning are found in US EPA SPECIATE but can be supplemented by the following studies reporting multispecies PAH emissions profiles: Jenkins et al, 1996; Oros et al, 2001a, 2001b, 2006.

Retene $\left(\mathrm{C}_{18} \mathrm{H}_{18}\right)$ was first identified as a potentially useful marker species for biomass combustion by Ramdahl (1983). Although widely cited in the receptor modeling literature as a source marker for wood combustion (Jang et al, 2013; Menezes, 2012; Fang, 2006; Larsen and Baker, 2003; Simcik, 1999), it should be considered an imperfect marker (Shen et al, 2012) because it has been identified in sampling studies of other source types (e.g., gasoline-fueled vehicle emissions, certain coal types; Wang, 2009) and because source sampling studies of residential fireplace and woodstoves that compared combustion of various woods (e.g., softwood, hardwood, synthetic logs) showed comparatively more retene is emitted from combustion of softwood such as pine or birch or from synthetic logs than from hardwood such as oak or maple (Schauer et al, 2001; McDonald et al, 2000; Environment Canada, 2000; Rogge et al, 1998; Shen et al, 2012).

Thus, retene can be cited as an imperfect but nevertheless useful organic marker of emissions from residential wood combustion for source type interpretation of receptor PMF model factors if taken in context with other species (i.e., PAH profile also enriched in fluoranthene and pyrene; Rogge et al, 1998; Oros et al, 2001a, 2001b) and with a priori expected seasonal differences (i.e., higher factor contributions in winter where residential wood burning is more common for space heating as well as for aesthetics).

Iron and steel industry: The iron and steel industry is a major stationary source within the study area (i.e., at Hamilton, Ontario). Several manufacturing processes are combined in an integrated iron and steel facility, with significant PAH emissions associated with coke ovens, sintering, cold rolling, and hot rolling; of these, coke oven emissions are cited as contributing the greatest share of an integrated iron and steel facility's airborne PAH emissions (Yang et al, 1998). The source sampling literature was reviewed to obtain 
useful and more recent data for PAH emissions associated with iron and steel manufacturing facility emissions, since the US EPA databases have limited PAH emissions data for the iron and steel industry (i.e., single EF dataset in US EPA AP-42 and no relevant US EPA SPECIATE profiles). Although PAH emissions are dependent on process factors such as coal type, facility type, and emissions control technology (Yang et al, 1998), the limited number of source sampling studies reporting North American data was supplemented with more recent sampling studies from iron and steel mills operating in Europe, Australia, and Asia. With priority given to studies sampling/reporting particle and vapour phase $\mathrm{PAH}$ for a large suite of species, the following studies are cited: Kirton et al, 1991; Khalili et al, 1995; Yang et al, 1998; Yang et al, 2002; Aries et al, 2007, 2009; Ciaparra et al, 2009. Salient points relating to these studies sampling methods and emissions profile data follows.

Kirton et al (1991) sampled coke oven fugitive emissions at an integrated iron/steel plant in Australia; the coke oven fugitive emissions profile was generally enriched in lighter MW species.

Khalili et al (1995) sampled several key source types in Chicago, USA, inclusive of integrated iron/steel industry emissions. The combination of Chicago's large urban population and associated dense traffic emissions, heavy industry, and harbour-front makes this study particularly relevant to the study region since the Greater Toronto Area is a large urban centre and Hamilton has concentrated industry sources along the harbourfront, inclusive of iron/steel manufacturing. For the steel industry sampling, Khalili et al (1995) sampled 100m directly downwind of a coke plant, capturing fugitive emissions and stack emissions, and ensured that no other steel making facilities (e.g., sintering) influenced the sample. The resulting coke oven PAH profile (i.e., sum of particulate and vapour phase) was found to be enriched in light MW species (i.e., ACY, FLU, PHEN) with comparatively little contribution from heavy MW species; useful to receptor modeling, the coke oven profile differed from the traffic emissions and wood combustion profiles that were sampled using comparable methods.

Yang et al (1998) limited sampling to stack emissions from key PAH-emitting processes (i.e., coke oven, blast furnace, basic oxygen furnace, electric arc furnace) at an integrated iron/steel facility in Taiwan, reporting PAH profile data as the sum of particulate and vapour phase. In their 2002 paper, Yang et al applied similar sampling methodology to the stacks of three Taiwanese coal-fueled steel plants but this time reported the particulate and vapour phase data separately as well as summed; their finding was that particulate plus vapour phase differed significantly from the particulate phase profile, a finding explained by the preferential phase partitioning of $\mathrm{PAH}$ species (i.e., light $\mathrm{MW}$ 
species partition primarily to vapour phase, heavy MW species partition primarily to particulate phase).

Aries et al $(2007,2009)$ sampled the coke oven (i.e., stack emissions from under-firing; fugitive emissions from coke oven door and charger hole/lid) and sintering operations (i.e., stack emissions) at a UK integrated iron/steel facility; fugitive coke oven emissions were shown to be the greatest contribution to PAH releases. Also of interest, the authors sampled local air quality at nearby downwind sites and found that, while overall PAH concentrations from the iron/steel operations were attenuated, the PAH profile of the local ambient air sample was largely preserved. Ciaparra et al (2009) carried out a related study on the same UK integrated iron/steel facility as Aries et al $(2007,2009)$ using nearsource ambient sampling sites within the facility's boundaries (i.e., one site near the coke ovens, a second site near the sinter stack); the downwind profiles for coke oven and iron sintering were found to be similar to those reported previously by Aries et al (2007). However, the authors found coke oven and iron sintering PAH profiles to differ in their characteristic species (i.e., coke oven fugitive emissions enriched in low MW species; sintering plant emissions enriched in heavy MW species; results corroborated earlier findings by Khalili et al, 1995).

Two additional studies sampled coke oven PAH emissions, albeit in particle phase (i.e., similar to emission factor data in US EPA AP-42): Oros et al, 2000; Tsai et al, 2007. Oros et al (2000) is a laboratory source sampling study of several coal types of increasing maturity (i.e., lignite, brown, subbituminous, bituminous) to note fuel-based differences in PAH profiles; with sampling restricted to the particulate phase, the PAH profile was enriched in light MW species (e.g., FLT, PYR) alongside some key heavy MW species (i.e., notably PER) and the contribution of heavy MW species in the profile tends to increase with coal maturity (i.e., more heavy MW species contribution in bituminous coal). The PAH profile data in Oros et al (2000) is also useful because it includes retene, a species that has been shown to characterize wood combustion; retene was found to be absent from combustion of brown and bituminous coal, but present in lignite and subbituminous coal. Since coke making in iron/steel manufacturing uses bituminous coal (American Iron and Steel Institute, 2012), retene is thus expected to remain primarily associated with wood combustion.

Tsai et al (2007) sampled PAH from stacks at an integrated iron/steel facility in Taiwan, inclusive of the coke ovens and iron sintering stack. As such, their profile data is comparable to the stack sampling studies by Yang et al $(1998,2002)$, although Tsai report a more limited dataset (i.e., 16 species sampled in particulate phase only and only 8 detected in the stack emissions); coke oven emissions were found to be significantly greater than iron sintering stack emissions, further confirming the dominance of coke 
oven emissions (i.e., fugitive plus stack) in iron/steel manufacturing. The coke oven emissions profile also generally agreed with coke oven profiles reported by others reporting particulate phase PAH (Yang et al, 2002) as well as studies reporting particulate plus vapour phase PAH data with consideration of lighter MW species tending to be greater when the vapour phase is sampled (Khalili et al, 1995; Aries et al, 2007, 2009).

Also cited are reference profiles (i.e., from literature review) reported in a CMB study of sources impacting Chicago, IL, USA (Li et al, 2003; Bzdusek et al, 2004); while this was a receptor modeling study (i.e., US EPA CMB model) using sediment PAH data rather than atmospheric PAH data, it is useful due to similarities between the integrated steel mills and related industry sites in Chicago, USA and those in the study area: US Steel and ArcelorMittal each operate integrated iron/steel facilities in Hamilton, $\mathrm{ON}$ as well as in Chicago, USA (Aim Market Research, 2010). Bzdusek et al (2004) report two variants of the Chicago coke oven PAH profile: (i) primary emitted emissions; and, (ii) weathered emissions.

Coal combustion - power generation: Coal-fired power generation profiles were found in US EPA AP-42 but are usefully supplemented by: Yang et al, 1998; Li et al, 2003 and Bzdusek et al, 2004 (i.e., aforementioned CMB study also reporting coke oven data). As mentioned above, Li et al (2003) and Bzdusek et al (2004) are CMB receptor model studies but are cited as they report reference profiles for coal-fired power generation alongside coke oven steel mill emissions data in Chicago, USA allowing comparison between these two source types (i.e., both reported profiles are for the same PAH species suite but are restricted to the particle phase).

\section{US EPA databases}

US EPA SPECIATE (stationary and mobile sources): SPECIATE provides PAH data for relatively few stationary/area sources relevant to the study area; however, these meaningfully supplement the PAH profiles available in US EPA AP-42 (e.g., SPECIATE provides data for residential fireplace emissions and for agricultural burning). Conversely, SP for mobile source emissions (i.e., vehicle exhaust emissions) are well represented in SPECIATE, with a large number of gasoline-fueled vehicle and dieselfueled vehicle profiles. These are typically single vehicle profiles (i.e., from emissions collected over a Dynamometer Driving Schedule such as the EPA Federal Test Procedure) and can be composited to provide a more representative overall profile of PAH emissions from a mixture of vehicle types, ages, and operating modes. However, while composites of individual vehicle SPs are useful, they do not necessarily represent ambient levels of emissions from mixed traffic; as such, SPs from tunnel study measurements were preferred for purposes of receptor modeling ambient PAH. 
The majority of the SPs identified in SPECIATE (2011) provide emission data for 12 to 20 PAH species common to NAPS PAH sampling. The majority of SPECIATE profiles report PM-associated PAH (i.e., particle phase PAH sampling) and most often for the fine size fraction $\left(\mathrm{PM}_{2.5}\right)$; more recent mobile source data reports $\mathrm{PAH}$ concentrations from co-extracted vapour and particle phase samples (e.g., NREL gas/diesel split study; Kansas city study; Table B.1-3, note $4 \mathrm{~b}, 4 \mathrm{c}, 5 \mathrm{~b})$.

US EPA AP-42 / FIRE (stationary sources): The central database of EF for stationary sources is US EPA AP-42 (2011) and are available in the searchable online database Factor Information Retrieval System (FIRE; 2012a). Since source types and source processes tend to be similar between the USA and Canada, Environment Canada also references US EPA AP-42 for EF needed by facilities reporting to the National Pollutant Release Inventory (NPRI). Environment Canada's "NPRI Toolbox" (Environment Canada, 2012) online material is organized by industry (i.e., North American Industry Classification System, NAICS) and also compiles relevant process types (i.e., Source Classification Code, SCC) within each industry (Environment Canada, 2010). Thus, Environment Canada's NPRI documentation $(2012,2010)$ was used to help identify key process types within each industry relevant to the study area and retrieve these from US EPA AP-42. Additionally, the US EPA document "Locating and estimating air emissions from sources of polycyclic organic matter" (1998), which provides a detailed process description of PAH-emitting sources (i.e., stationary and mobile), was reviewed to help identify the sub-processes within a facility contributing most significantly to PAH emissions (e.g., coke ovens in iron/steel manufacturing).

To extract multi-species PAH EF profiles from FIRE all online records were downloaded in CSV format (US EPA, 2012b), screened for EF containing a sufficient number of PAH species (i.e., typically greater than 10 species), and the resulting candidate EFs were screened for major source activities relevant to the study area.

Tables of available PAH profile data

PAH profile data available in the literature are summarized in tables below for source types relevant to the study area (i.e., source types identified via review of NPRI emissions inventory and maps of the study area; Chapter 3). PAH profile data available in reviewed source sampling literature is summarized in Table B.1-2 (SPs; studies crossreferenced in US EPA SPECIATE are not listed to maintain clarity of presentation) and profile data available in US EPA databases is summarized in Table B.1-3 (SPs) and Table B.1-4 (EFs). 


\section{Table B.1-2. Studies reporting PAH source profile data (SP) for stationary and mobile sources relevant to study area.}

\begin{tabular}{|c|c|c|c|c|c|}
\hline & Study Type & Location & Phase & No.Species & Notes \\
\hline \multicolumn{6}{|l|}{ TRAFFIC (exhaust) } \\
\hline - Khalili et al, 1995 & freeway - tunnel & Chicago, IL, USA & PM+vapour & 20 & $\begin{array}{l}\text { - mixed traffic tunnel, gasoline, diesel } \\
\text { - } \text { also wood burning, coke oven }\end{array}$ \\
\hline - Fraser et al, 1998 & freeway - tunnel & $\begin{array}{l}\text { Los Angeles, CA, } \\
\text { USA }\end{array}$ & PM+vapour & 20 & $\begin{array}{l}\text { - } \text { mixed traffic tunnel but nearly all LDV-gas } \\
\text { - } \quad \text { vapour and particulate reported separately } \\
\text { - fall sampling (late Sep) }\end{array}$ \\
\hline - Gertler et al, 2002 & freeway - tunnel & PA, USA & PM+vapour & 20 & $\begin{array}{l}\text { - } \quad \text { Gertler Mountain Tunnel } \\
\text { - } \quad \text { separate LDV, HDV profiles, uncertainty estimate } \\
\text { - } \quad \text { spring sampling (late May) }\end{array}$ \\
\hline $\begin{array}{l}\text { - Environment Canada, } \\
2004\end{array}$ & freeway - tunnel & $\begin{array}{l}\text { Vancouver, BC, } \\
\quad \text { Canada }\end{array}$ & $\mathrm{PM}$ & 29 & $\begin{array}{l}\text { - } \text { CASSIAR tunnel } \\
\text { - } \text { mixed traffic (HDV in fleet }=2 \%-9.5 \%) \\
\text { - } \text { summer sampling (Aug) }\end{array}$ \\
\hline - Phuleria et al, 2006, 2007 & $\begin{array}{l}\text { freeway - tunnel, } \\
\text { roadside }\end{array}$ & $\begin{array}{l}\text { San Francisco \& } \\
\text { Los Angeles, CA, } \\
\text { USA }\end{array}$ & $\mathrm{PM}$ & 15 & $\begin{array}{l}\text { tunnel, San Francisco (2006) - separate LDV, HDV profiles; } \\
\text { summer sampling (Aug - Sep) } \\
\text { freeway roadside, Los Angeles (2007) - two freeway profiles } \\
\text { (CA-110 is 0\% HDDV; I-710 is 20\% HDDV), congested, } \\
\text { winter sampling (Feb - Mar) }\end{array}$ \\
\hline - Ning et al, 2008 & $\begin{array}{l}\text { freeway - } \\
\text { roadside }\end{array}$ & $\begin{array}{l}\text { Los Angeles, CA, } \\
\text { USA }\end{array}$ & $\mathrm{PM}$ & 12 & $\begin{array}{l}\text { - freeway roadside, comparable to Phuleria } 2007 \\
\text { - CA-110 is 0\% HDDV, summer and winter sampling; I-710 is } \\
20 \% \text { HDDV, winter sampling }\end{array}$ \\
\hline $\begin{array}{l}\text { - Liacos et al, } 2012 \\
\text { \& Kam et al, } 2012\end{array}$ & $\begin{array}{l}\text { freeway \& urban } \\
\text { streets - in- } \\
\text { vehicle }\end{array}$ & $\begin{array}{l}\text { Los Angeles, CA, } \\
\text { USA }\end{array}$ & $\mathrm{PM}$ & 10 & $\begin{array}{l}\text { - freeway in-vehicle, comparable to Phuleria } 2007 \text { / Ning 2008, } \\
\text { CA-110 is 4\% HDDV (low), summer and winter sampling; I- } \\
710 \text { is } 11 \% \text { HDDV (high), spring sampling (Mar - Apr) }\end{array}$ \\
\hline & & & & & $\begin{array}{l}\text { - urban streets in-vehicle, LDV fleet, typical urban driving } \\
\text { mode (with congestion), spring season sampling (Mar - Apr) }\end{array}$ \\
\hline - Harrison et al, 2003 & $\begin{array}{l}\text { freeway - } \\
\text { roadside }\end{array}$ & Birmingham, UK & PM+vapour & 20 & $\begin{array}{l}\text { - } \quad \text { A-class" (A-83), mixed traffic, frequent congestion } \\
\text { - winter season sampling (late Oct - late Jan) }\end{array}$ \\
\hline - Wingfors et al,2001 & freeway - tunnel & $\begin{array}{l}\text { Gothenburg, } \\
\text { Sweden }\end{array}$ & PM+vapour & 29 & $\begin{array}{l}\text { - } \quad \text { mixed traffic }(\mathrm{HDDV} \text { in fleet }=17 \%-24 \%) \\
\text { - } \quad \text { vapour and particulate reported separately } \\
\text { spring season sampling }(\mathrm{Apr})\end{array}$ \\
\hline - Kristensson et al, 2004 & freeway - tunnel & $\begin{array}{l}\text { Stockholm, } \\
\text { Sweden }\end{array}$ & PM+vapour & 29 & $\begin{array}{l}\text { - } \quad \text { separate LDV, HDV profiles, profile data by road speed } \\
\text { - } \quad \text { vapour and particulate reported separately } \\
\text { - } \quad \text { winter season sampling (Dec, Feb) }\end{array}$ \\
\hline
\end{tabular}

Appendix B: Urban/rural source apportionment 
Table B.1-2 (continued)

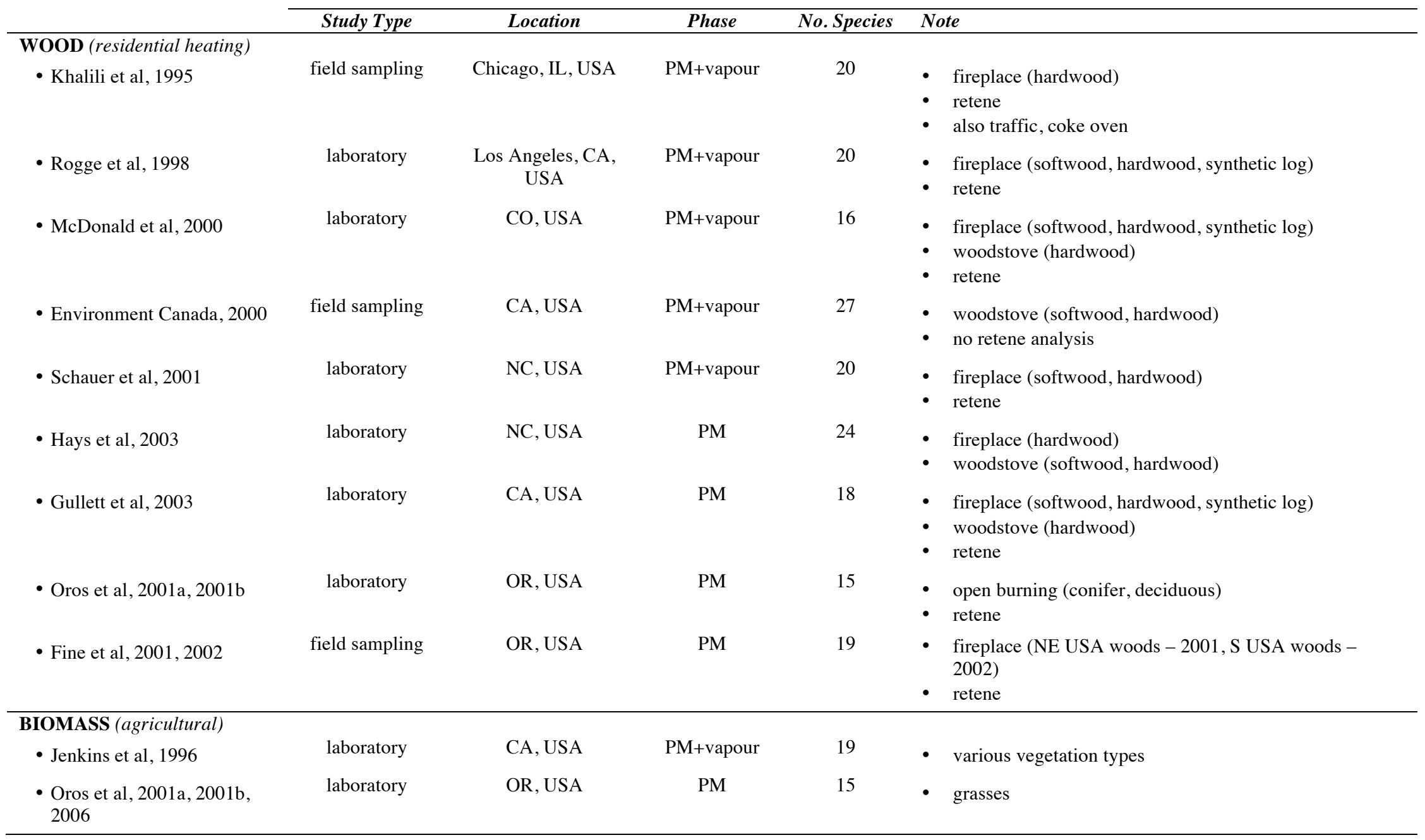


Table B.1-2 (continued)

\begin{tabular}{|c|c|c|c|c|c|}
\hline & Study Type & Location & Phase & No.Species & Note \\
\hline \multicolumn{6}{|l|}{ IRON/STEEL } \\
\hline - Kirton et al, 1991 & field sampling & Australia & PM+vapour & 20 & - coke oven - fugitive \\
\hline - Khalili et al, 1995 & field sampling & Chicago, IL, USA & PM+vapour & 19 & $\begin{array}{ll}\text { - } & \text { coke oven - fugitive, stack } \\
\text { - } & \text { retene } \\
\text { - } & \text { also traffic, wood burning }\end{array}$ \\
\hline - Yang et al, 1998, 2002 & field sampling & Taiwan & PM+vapour & 21 & $\begin{array}{l}\text { - } \quad \text { coke oven, sintering, etc. - stack } \\
\text { - report PM and vapour phase separately in Yang (2002) }\end{array}$ \\
\hline - Aries et al, 2007, 2009 & field sampling & $\mathrm{UK}$ & PM+vapour & 20 & - coke oven, sintering - stack, fugitive \\
\hline - Ciaparra et al, 2009 & field sampling & UK & PM+vapour & 16 & - coke oven, sintering - ambient \\
\hline - Oros et al, 2000 & laboratory & OR, USA & $\mathrm{PM}$ & 16 & - coke oven, various coals \\
\hline - Tsai et al, 2007 & field sampling & Taiwan & $\mathrm{PM}$ & 16 & - coke oven, sintering - stack \\
\hline $\begin{array}{l}\text { - Li et al, } 2003 \\
\text { \& Bzdusek et al, } 2004\end{array}$ & $\begin{array}{l}\text { receptor model } \\
\quad(\mathrm{CMB})\end{array}$ & Chicago, IL, USA & $\mathrm{PM}$ & 16 & $\begin{array}{l}\text { similar to Toronto / Hamilton (dense urban, industry, } \\
\text { harbour) }\end{array}$ \\
\hline \multicolumn{6}{|l|}{ OIL (heating, industry) } \\
\hline - Rogge et al, 1997b & laboratory & CA, USA & & & \\
\hline - Olmez et al, 1988 & laboratory & MA, USA & & & \\
\hline \multicolumn{6}{|l|}{$\begin{array}{l}\text { NATURAL GAS } \\
\text { (heating, appliances) }\end{array}$} \\
\hline - Rogge et al, 1993b & laboratory & CA, USA & & & \\
\hline - Daisey et al, 1979, 1986 & lit survey & - & & & \\
\hline \multicolumn{6}{|l|}{ ASPHALT (roofing, roadwork) } \\
\hline - Rogge et al, 1997a & laboratory & CA, USA & & & \\
\hline
\end{tabular}


Table B.1-3. Source Profiles (SP) available for stationary/area and mobile sources relevant to study area, from US EPA SPECIATE 4.3 (2011).

\begin{tabular}{|c|c|c|c|c|c|c|c|c|c|}
\hline & PROFILE NAME & Profile_ID & $\begin{array}{l}\begin{array}{l}\text { \# species } \\
\text { common to } \\
\text { NAPS }\end{array} \\
\end{array}$ & $\begin{array}{l}\text { PM size } \\
\text { fraction }\end{array}$ & $\begin{array}{c}\text { \% WEIGHT } \\
\text { DATA }\end{array}$ & $\begin{array}{c}\% \\
\text { UNCERTAIN } \\
\text { TY DATA } \\
\end{array}$ & $\begin{array}{c}\text { SPECIATE } \\
\text { version }\end{array}$ & Test Year & NOTE \\
\hline \multirow{4}{*}{ 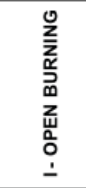 } & $\begin{array}{l}\text { forest fire (lobloly pine, western hemlock, ponderosa pine, mixed hardwood, } \\
\text { palmetto, wiregrass and longleaf pine) }\end{array}$ & $4463-4468$ & $\sim 12$ & $0-2.5 \mathrm{um}$ & YES & YES & 4.0 & 2002 & - episodic, unlikely to be resolved in receptor model. \\
\hline & $\begin{array}{l}\text { Field Burning - Annual Rye Grass } \\
\text { Field Burning - Perenial Rye Grass } \\
\text { Field Burning - Fescye }\end{array}$ & $\begin{array}{l}423222.5 \\
423232.5\end{array}$ & $\begin{array}{l}7 \\
7 \\
7\end{array}$ & $\begin{array}{l}0-2.5 \mathrm{um} \\
0-2.5 \mathrm{~mm}\end{array}$ & $\begin{array}{l}\text { YES } \\
\text { YES } \\
\text { YYS }\end{array}$ & $\begin{array}{l}\text { YES } \\
\text { YES }\end{array}$ & $\begin{array}{l}3.2 \\
3.2 \\
3.2\end{array}$ & $?$ & - few species. \\
\hline & $\begin{array}{l}\text { Field Burning - Fescue } \\
\text { agricultural burning - rice straw }\end{array}$ & $\begin{array}{l}423242.5 \\
4840\end{array}$ & $\frac{7}{>20}$ & $\begin{array}{ll}0-2.20 \mathrm{um} \\
0.2 .5 \mathrm{um}\end{array}$ & $\begin{array}{l}\text { YES } \\
\text { YES }\end{array}$ & 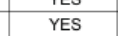 & 4.2 & 2001 & . can composite biomass types. \\
\hline & agricultural burning - wheat straw & 4841 & $>20$ & $0.2 .5 \mathrm{um}$ & YES & YES & 4.2 & 2001 & \\
\hline \multirow{5}{*}{ 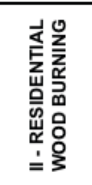 } & FIREPLACE - soft wood & 3920 & 15 & $0-2.5 \mathrm{um}$ & YES & YES & 4.0 & 1997 & - NFRAQS dataset. \\
\hline & FIREPLACE - hard wood & 3925 & 15 & $0-2.5 \mathrm{um}$ & YES & YES & 4.0 & 1997 & \\
\hline & FIREPLACE - hard wood, oak & 4644 & 13 & 0-1.8um & YES & No & 4.0 & 1997 & - Schauer et al dataset. No uncertainty. \\
\hline & FIREPLACE - soft wood, pine & 4645 & 13 & $0-1.8 \mathrm{um}$ & YES & No & 4.0 & 1997 & \\
\hline & $\begin{array}{l}\text { WOODSTOVE - hard wood } \\
\text { WOODSTOVE- composite of several woods }\end{array}$ & 3931 & 15 & $\begin{array}{l}0.2 .5 \mathrm{um} \\
0.25 \mathrm{~m}\end{array}$ & $\begin{array}{l}\text { YES } \\
\text { YES }\end{array}$ & $\begin{array}{l}\text { YES } \\
\text { YES }\end{array}$ & $\begin{array}{l}4.0 \\
32\end{array}$ & 1997 & - NFRAQS dataset. \\
\hline \multirow{2}{*}{ 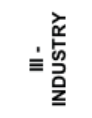 } & natural gas combustion - composite (oil refinery) & 4403,4398 & 16 & $0-2.5 \mathrm{um}$ & YES & YES & 4.0 & 2001 & - natural gas boiler (industry application) \\
\hline & hogged fuel boiler / plywood mfg (running waste wood) & 127062.5 & 12 & $0-2.5 \mathrm{um}$ & YES & YES & 3.2 & ? & $\begin{array}{l}\text { - composite profile, but narrow industry type/fuel may not apply to } \\
\text { study area. }\end{array}$ \\
\hline \multirow{4}{*}{ 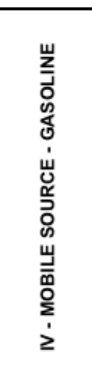 } & $\begin{array}{l}\text { LDV - on road gasoline exhaust - composite } \\
\text { LDV - Gasoline Exhaust - Winter, low emitter } \\
\text { LDV - Gasoline Exhaust - Winter high memitter } \\
\text { LDV - Gasoline Exhaust - Winter, smoker } \\
\text { LDV - Gasoline Exhaust - Summer, low-emitter } \\
\text { LDV - Gasoline Exhaust - Summer, medium-emitter } \\
\text { LDV - Gasoline Exhaust - Summer, high-emitter } \\
\text { LDV - Gasoline Exhaust - Summer, smoker }\end{array}$ & $\begin{array}{r}91122 \\
3884 \\
3892 \\
3904 \\
3947 \\
3951 \\
3955 \\
3959 \\
\end{array}$ & $\begin{array}{l}16 \\
16 \\
16 \\
16 \\
16 \\
16 \\
16 \\
16 \\
16\end{array}$ & $\begin{array}{l}0.2 .5 u m \\
0-2.5 \mathrm{~m} \\
0-2.5 \mathrm{um} \\
0-2.5 \mathrm{um} \\
0-2.5 \mathrm{um} \\
0-2.5 \mathrm{um} \\
0-2.5 \mathrm{um} \\
0-2.5 \mathrm{um}\end{array}$ & $\begin{array}{l}\text { YES } \\
\text { YES } \\
\text { YES } \\
\text { YES } \\
\text { YES } \\
\text { YES } \\
\text { YES } \\
\text { YES }\end{array}$ & $\begin{array}{l}\text { YES } \\
\text { YES } \\
\text { YES } \\
\text { YES } \\
\text { YES } \\
\text { YES } \\
\text { YES } \\
\text { YES }\end{array}$ & $\begin{array}{l}4.0 \\
4.0 \\
4.0 \\
4.0 \\
4.0 \\
4.0 \\
4.0 \\
4.0\end{array}$ & $\begin{array}{l}1996 / 1997 \\
1996 / 1997 \\
1996 / 1997 \\
1996 / 1997 \\
1996 / 1997 \\
1996 / 1997 \\
1996 / 11997 \\
1996 / 1997 \\
\end{array}$ & $\begin{array}{l}\text { NFRAQS dataset, composite profile } \\
\text { - LDV, tested using FTP. } \\
\text { - seasonal profiles. } \\
\text { - can composite by season or overall. }\end{array}$ \\
\hline & LDV exhaust - gasoline (summer) & 5566-5591 & $\sim 16$ & $0-2.5 \mathrm{um}$ & YES & YES & 4.3 & 2004 & Kansas city dataset, composite profiles \\
\hline & LDV exhaust - gasoline (winter) & 5592 - 5617 & $\sim 16$ & $0-2.5 \mathrm{um}$ & YES & YES & 4.3 & 2005 & - PAH data is vapour + particle phase (as in NAPS). \\
\hline & LDV exhaust - gasoline - Califomia, various vehicle types/age/mode & \begin{tabular}{|l|}
$4897-4899 ; 4909-4932 ;$ \\
$4900-4908 ; 4895-4896$
\end{tabular} & $\sim 16$ & $0-2.5 \mathrm{um}$ & YES & YES & 4.2 & 2001 & $\begin{array}{l}\text { NREL gas/diesel split Study dataset, composite profiles } \\
\text { - Multiple vehicle typess/age/mode. } \\
\text { - PAH data is vapour + particle phase (as in NAPS). } \\
\text { - Calfornia market. }\end{array}$ \\
\hline \multirow[b]{2}{*}{ 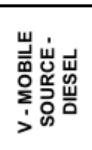 } & HDDV exhaust (winter) & 3914 & $\sim 14$ & $0-2.5 \mathrm{~mm}$ & $\begin{array}{ll}\text { YES } \\
\text { YSES }\end{array}$ & YES & 4.0 & 1997 & NFRAQS dataset, composite profile \\
\hline & $\begin{array}{l}\text { LDDV exhaust combustion - composite } \\
\text { HDDV exhaust - California, various truck type/age/mode }\end{array}$ & $\begin{array}{l}\text { 3912, 3963, 4675 } \\
4842 ; 4845-4858 ; 4861-4868 ; \\
\text { 4879-4884; 4843-4844; } \\
\text { 4875-4878; 4885-4886; } \\
\text { 4869-4874; 4887-4891; } 4894\end{array}$ & $\frac{14 \cdot 21}{12}$ & $\frac{0-2.5 \mathrm{um}}{0-2.5 \mathrm{um}}$ & $\begin{array}{l}\text { YES } \\
\text { YES }\end{array}$ & $\begin{array}{l}\text { YES } \\
\text { YES }\end{array}$ & $\begin{array}{l}4.0 \\
4.2\end{array}$ & $\begin{array}{l}1997 \\
2001\end{array}$ & $\begin{array}{l}\text { - can compositit vehicle types for overall diesel. } \\
\text { NREL gas/dilesel Split study dataset (composite profilies) } \\
\text { - multiple vehicle type/age/mode. } \\
\text { - PAH data is vapour + particle phase (as in NAPS). } \\
\text { - California market. }\end{array}$ \\
\hline
\end{tabular}


1. OPEN BURNING.

(a) Forest fire, various vegetation types. 42323, 42324 are older profiles (SPECIATE 3.2) and also limited number of PAH species so not recommended as reference. Newer and more species are 4463-4468. The composite 91xxx profile for field burning (91109) uses these plus 42321-2.5; however, 42321-2.5 has less PAH species so not recommended for composite PAH profiles. Thus, for field burning of forest vegetation, can retain: 4463 forest fire - lobloly pine; 4464 forest fire western hemlock; 4465 forest fire - ponderosa pine; 4466 forest fire - mixed hardwood; 4467 forest fire - Florida palmetto and slash pine; 4468 forest fire wiregrass and longleaf pine.

(b) Agricultural burning, various vegetation types. Three related datasets available but are older (SPECIATE 3.2) and limited to 7 PAH species: 423222.5, 423232.5, 423242.5. Preferred are newer SPECIATE 4.3 profiles reporting more species, even if these are only for one specific vegetation type: 4840,4841 .

\section{RESIDENTIAL BURNING.}

Combined Fireplace, Woodstove. Composite profile 91032 does not have PAH data. Constituent profiles with PAH data are: 3920 Fireplace - soft wood; 4644 Fireplace hard wood, oak (no uncertainty data); 4645 Fireplace - soft wood, pine (no uncertainty data); 3931 woodstove - hard wood; 423122.5 woodstove - composite of several woods. Can be composited by "fireplace" and "woodstove" types or as single composite "residential wood combustion (fireplace/woodstove)". Two fireplace profiles have no uncertainty data and are for a slightly different $0-1.8$ um size fraction so can supplement with: 3925 Fireplace - hard wood (i.e., hard wood analog to 3920 so suitable for compositing). Additional composite is 91105 , same constituent profiles as for 91032 composite.

\section{INDUSTRY}

(a) Natural gas combustion (industry). Composite profile 91112 does not have PAH data, but constituent profiles do: 4403 and 4398 natural-gas fired process heater (oil refinery facility data). Alternate is "process gas combustion" (typically natural gas) with composite profile 91136 that also does not have PAH data; constituent profiles are $4398,4407,4415$ (all oil refinery facility data).

(b) Hogged fuel boiler (industry with access to waste wood). No new composite profile 91xxx, however, two legacy composite profiles have PAH data: 12706 hogged fuel boiler / plywood mfg (12 PAH species); 12709 hogged fuel boiler / stoker boiler (very few PAH species).

(c) Coal combustion. Composite profile for lignite is 91125 but does not report PAH data. Constituent profiles also do not report PAH data.

\section{MOBILE SOURCE, GASOLINE EXHAUST.}

(a) NFRAQS data. From sampling of light duty vehicle (LDV), gasoline-fueled. Composite profile is 91122 with PAH data in listed constituent profiles. For completeness of reference, three summer and three winter profiles are listed, representing major states of engine emissions control efficiency (low emitter, high emitter, smoker).

(b) Kansas city data. From sampling of light duty vehicles (LDV), gasoline-fueled; Kansas city region, summer and winter season. SVOC including PAH were analyzed together from particle and vapour phase samplers.

(c) NREL Gas/Diesel Split Study data. From sampling of light duty vehicles (LDV), gasoline-fueled. SVOC including PAH were analyzed together from particle and vapour phase samplers. Fifty one vehicles tested of different vehicle type (truck, sedan, etc.), ages, operating mode; California region, summer season.

\section{MOBILE SOURCE, DIESEL EXHAUST.}

(a) NFRAQS data. From sampling of heavy duty diesel-fueled vehicle (HDDV) Composite profile for HDDV is 91106 , composite profile for "mixed diesel" is 91162; PAH data in listed constituent profiles.

(b) NREL Gas/Diesel Split Study data. From sampling of light duty diesel-fueled vehicles (LDDV). SVOC including PAH were analyzed together from particle and vapour phase samplers. Fifty one vehicles tested of different vehicle type (truck, sedan, etc.), ages, operating mode; California region, summer season. 
Table B.1-4. Emission Factors (EF) available for stationary and area sources relevant to study area, from US EPA AP-42 (2012b, 2010).

\begin{tabular}{|c|c|c|c|c|c|c|c|c|c|}
\hline & Facility type & Source type & emission activity & fuel & process/type & scc & $\begin{array}{c}\text { \# species } \\
\text { common to } \\
\text { NAPS }\end{array}$ & Note & Year \\
\hline \multirow{3}{*}{ 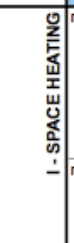 } & \multirow{2}{*}{ residential } & \multirow[t]{2}{*}{$\begin{array}{l}\text { heating } \\
\text { combustion }\end{array}$} & \multirow[t]{2}{*}{ woodstove } & \multirow[t]{2}{*}{ wood } & $\begin{array}{l}\text { catalytic (general) } \\
\text { noncatalytic (EPA } \\
\text { cerififed) }\end{array}$ & $\begin{array}{l}21040080-30 \\
21040080-50\end{array}$ & 17 & $\begin{array}{l}\cdot \text { - no retene in profile. } \\
\cdot \text { no retene in proflile. }\end{array}$ & $\begin{array}{l}1995 \\
1995\end{array}$ \\
\hline & & & & & $\begin{array}{l}\text { noncatalytic (non-EPA } \\
\text { certified) }\end{array}$ & $21040080-51$ & 14 & $\cdot$ no retene in proflile. & 1995 \\
\hline & residential & $\begin{array}{l}\text { heating } \\
\text { combustion }\end{array}$ & residential furnace & $\begin{array}{l}\text { natural gas } \\
\text { coal }\end{array}$ & $\begin{array}{l}\text { residential furnace } \\
\text { residential furnace }\end{array}$ & $\begin{array}{l}21040060-10 \\
21040010-00\end{array}$ & $\begin{array}{l}16 \\
17\end{array}$ & & $\begin{array}{l}1998 \\
1996\end{array}$ \\
\hline 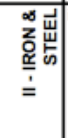 & industrial & metallurgical & iron and steel mfg & coal & $\begin{array}{l}\text { charging \& oven leaks } \\
\text { coke pushing } \\
\text { combustion stack }\end{array}$ & $\begin{array}{r}303003-02,-08,-14 \\
303003-03 \\
303003-17,-18\end{array}$ & $\begin{array}{l}18 \\
17 \\
17\end{array}$ & $\begin{array}{l}\text {-Separate EF for (i) uncontrolled; (ii) pre-NESHAP controls; (ii) post- } \\
\text { NESHAP controls. } \\
\text { - no indication of control. } \\
\text { - separate EF for. (i) controlled, (ii) uncontrolled. }\end{array}$ & \\
\hline \multirow{4}{*}{ 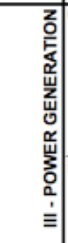 } & \multirow{2}{*}{ utility / industrial } & \multirow[t]{2}{*}{ power generation } & \multirow[t]{2}{*}{$\begin{array}{l}\text { external combustion } \\
\text { boiler }\end{array}$} & & $\begin{array}{l}\begin{array}{c}\text { cyclone feed, pulverized } \\
\text { coal feed }\end{array} \\
\end{array}$ & 101002-03, etc. & 13 & $\begin{array}{l}\text { EF is same for } \mathrm{SCC}=10100203,10100202,10100212,10100223, \\
10100222,1010226,10100303,10100301,10100302 .\end{array}$ & 1998 \\
\hline & & & & $\begin{array}{l}\text { fuel oil } \\
\text { natural gas }\end{array}$ & $\begin{array}{l}\text { Grade } 6 \text { oil, normal or } \\
\text { tangential firing } \\
\text { all except tangential-fired }\end{array}$ & $\begin{array}{l}101004-01,-04 \\
101006-01,-02\end{array}$ & $\begin{array}{l}13 \\
16\end{array}$ & - EF is same for SCC $=10100401,10100404$. & $\begin{array}{l}1998 \\
1998\end{array}$ \\
\hline & \multirow{2}{*}{ industrial } & \multirow[t]{2}{*}{ power generation } & \multirow[t]{2}{*}{$\begin{array}{l}\begin{array}{l}\text { external combustion } \\
\text { boiler }\end{array} \\
\end{array}$} & \multirow[t]{2}{*}{ wood/bark waste } & $\begin{array}{l}\text { wood-fred, bark-fired, dry } \\
\text { wood, wet wood }\end{array}$ & & & - EF same for SCC $=10100901,10100902,10100903,10100908$ & 2003 \\
\hline & & & & & steam co-generation & $102009-05,-07$ & 15 & - EF same for SCC = 10200905, 10200907. & 1999 \\
\hline \multirow{3}{*}{ 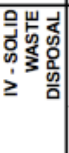 } & \multirow{2}{*}{ industrial } & \multirow[t]{2}{*}{ waste disposal } & \multirow[t]{2}{*}{ open burning } & & used, unused plastic & $503002-02$ & 13 & $\begin{array}{l}\text { average of four EF for open burning of plastic (e.9., waste disposal } \\
\text { operations). }\end{array}$ & 1995 \\
\hline & & & & auto tires & shredded, chunked tires & $503002-03$ & 15 & - open burning (e.g., waste disposal operation for auto parts - rubber tires). & 1995 \\
\hline & \begin{tabular}{|l} 
industrial I \\
institutional
\end{tabular} & waste disposal & incinerator & propane & crematory stack & 315021-01 & 15 & - open burning (e.g., crematory, hospital) & 1992 \\
\hline \multirow{4}{*}{ 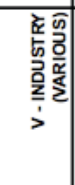 } & \multirow{4}{*}{ industrial } & \multirow[t]{4}{*}{$\begin{array}{l}\text { industrial } \\
\text { processs power }\end{array}$} & \multirow[t]{4}{*}{ IC engine } & natural gas & $\begin{array}{l}\begin{array}{l}\text { reciprocating, 2-cycle } \\
\text { lean burn }\end{array} \\
\end{array}$ & $202002-52$ & 16 & \begin{tabular}{|l|l}
- stationary industrial engine. \\
\end{tabular} & 2000 \\
\hline & & & & natural gas & $\begin{array}{l}\text { reciprocating, 4-cycle } \\
\text { lean burn }\end{array}$ & $202002-54$ & 10 & - stationary industrial engine. & 2000 \\
\hline & & & & distillate oil (diesel) & reciprocating & 203001-01, -02 & 15 & - stationary industrial engine. & 1996 \\
\hline & & & & diesel & large bore & 202004-01 & 15 & - stationary industrial engine. & 1996 \\
\hline \multirow{3}{*}{ 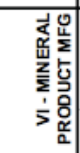 } & \multirow{2}{*}{ industrial } & $\begin{array}{l}\text { asphalt I } \\
\text { concrete mfg }\end{array}$ & hot mix asphalt (HMA) & $\begin{array}{l}\begin{array}{l}\text { not specified (typically } \\
\text { natural gas / oil) }\end{array} \\
\end{array}$ & batch mix & $305002-45$ & 15 & - Batch Mix Plant Hot Elevators, Screens, Bins, Mixer \& NG Rot Dryer. & 2000 \\
\hline & & & & natural gas / oil & $\begin{array}{l}\text { drum mix (natural gas, } \\
\text { oil) }\end{array}$ & $305002-55,-58$ & 16 & - average of natural gas-fired EF and oil-fired EF operations. & 2000 \\
\hline & industrial & cement mfg & kiln combustion & coal / natural gas & diry process, wet process & 305006-06, etc. & 13 & - EF is same for $\mathrm{SCC}=30500606,30500622,30500623,30500706$. & 1995 \\
\hline 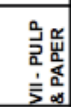 & industrial & pulp/paper & \begin{tabular}{l|}
$\begin{array}{l}\text { Wood pressure treating } \\
\text { via creosotete }\end{array}$ \\
\end{tabular} & creosote & empty cell process & $307005-30,-40$ & 12 & - EF is same for $\mathrm{SCC}=30700530,30700540$. & 1999 \\
\hline
\end{tabular}


1. SPACE HEATING.

(a) EF for residential woodstoves have no retene data (species not typically reported for AP-42).

(b) EF from various residential woodstove types may be averaged to represent emissions from multiple residences (i.e., area source emissions profile).

\section{IRON \& STEEL.}

(a) EF in FIRE is incomplete (very few species); multi-species EF obtained from AP-42 documentation Chapter 12 (US EPA, 2008).

(b) NESHAP controls are referenced in Chapter 12 of AP-42 documentation (US EPA, 2008). The reference provided is another EPA document dated 1987. Thus, one approach is to assume that iron and steel facilities in study area (Hamilton) are "post-NESHAP"; however, recall discussion with City of Hamilton staff regarding exemptions being sought by steel producers because only recently upgrading oven door for less leak emissions. Thus, conservative approach is to reference "preNESHAP" EF data (median EF between "uncontrolled" and "post-NESHAP"). Shape of EF (i.e., relative species concentrations) is similar for all three so minimal error is incurred if controls changed during 2000-2010 period.

\section{POWER GENERATION.}

(a) Power generation does not show significant PAH emissions as reported to NPRI in study area. However, coal-fired power generation EF useful to distinguish from iron and steel emissions.

\section{SOLID WASTE DISPOSAL.}

(a) Open burning expected to be episodic (compared to municipal solid waste incinerators) so distinct PMF factor not expected.

(b) Crematorium and related hospital incineration of biomedical waste also episodic in nature so distinct PMF factor not expected.

\section{INDUSTRY (VARIOUS)}

(a) Diesel industrial engines may be associated with process equipment in industrial facility (pump, etc.).

(b) Large bore diesel stationary engines used nearly exclusively in oil and gas industry, so not expected in study area.

\section{MINERAL PRODUCT MANUFACTURING.}

(a) Hot Mix Asphalt (HMA) industry relevant to study region.

(b) Portland cement kiln emissions relevant to study region.

\section{PULP \& PAPER.}

Industry not relevant to study area but EF provided as reference to others

\section{ADDITIONAL.}

(a) No multi-species PAH EF were found in US EPA AP-42 (i.e., FIRE or related documentation) for several stationary sources relevant to study area (e.g., residential wood fireplace, biomass open burning) but are available in US EPA SPECIATE and source sampling literature.

(b) Process information on petroleum refining (US EPA, 2010) cites severa processes that are known to emit PAH:

- coke ovens: "iron \& steel manufacturing" EF is reasonable proxy.

- process heaters will be found in facility and expected to burn various possible fuels (refinery gas, natural gas, residual fuel oil, etc.) and generate PAH emissions: "power generation" external combustion boiler EF is reasonable proxy.

- compressor engine (natural gas-fueled) are only found in older plants

- asphalt blowing process can generate significant airborne PAH emissions.

(c) No multi-species PAH EF were found in US EPA AP-42 (i.e., FIRE) for mobile sources but are available in SPECIATE and source sampling literature. 


\section{B.2 PAH trend analysis}

Method

Descriptive statistics were calculated for individual PAH and species groupings (i.e., total sampled PAH, $\left.\Sigma_{29} \mathrm{PAH} ; \Sigma_{\mathrm{LIGHT}} \mathrm{PAH} ; \Sigma_{\mathrm{HEAVY}} \mathrm{PAH}\right)$. Statistics were reviewed for data density and sample-to-sample variability to confirm amenability to receptor modeling. Annual and seasonal trends, with seasons defined simply as 'winter' (October - April) and 'summer' (May - September), were reviewed across sites and reconciled with differences in site characteristics and expected general source types. Tests of statistical difference used the Kruskal-Wallis test of medians at a 5\% significance level. Species abundances were calculated by normalizing species concentration to the total of all sampled species $\left(\Sigma_{29} \mathrm{PAH}\right)$ and ranked to determine dominant species and confirm general consistency in source mix over the modeled time period (Kuntasal, 2005; Gaga, 2004).

Descriptive statistics and time series variability

Descriptive statistics (i.e., 2000-2010 time series) for individual PAH and species groupings (i.e., total sampled PAH, $\Sigma_{29} \mathrm{PAH} ; \Sigma_{\mathrm{LIGHT}} \mathrm{PAH} ; \Sigma_{\mathrm{HEAVY}} \mathrm{PAH}$ ) are presented in Table B.2-1 alongside percent of species data below detection limit and missing.

Time series plots for $\Sigma_{29} \mathrm{PAH}$ are shown in Figure B.2-1. $\Sigma_{29} \mathrm{PAH}$ was found to be most variable at Hamilton (i.e., coefficient of variation, $\mathrm{CV}=1.1,2000-2010$; Table B.2-1), next-highest at the background site $(\mathrm{CV}=0.7)$, and least variable at Toronto $(\mathrm{CV}=0.5)$, while confirming each dataset's amenability to receptor modeling (i.e., $\mathrm{CV}>0.3$ suitable for PMF; Sonoma, 2008). Inter-site differences in CV were consistent with the nature of each site: Hamilton has significant local industry sources and an escarpment topography so high variability in ambient PAH was expected; the Toronto site is located in a central business district expected to be subject to consistent patterns of emission from adjacent traffic and commercial/residential land use so less variability was expected; the background site necessitates source-receptor transport over larger distances and greater dependence on meteorology and so moderate variability was reasonably expected. 
Table B.2-1. Summary statistics for PAH at urban and background sites (2000-2010).

(a) Hamilton

\begin{tabular}{|c|c|c|c|c|c|c|c|c|c|c|c|c|c|}
\hline \multirow[b]{2}{*}{ SPECIES } & \multirow[b]{2}{*}{$\begin{array}{r}\mathrm{DL} j \\
(\mathrm{ng} / \mathrm{m} 3)\end{array}$} & \multirow[b]{2}{*}{$\begin{array}{l}\% \mathrm{BDL}+ \\
\text { missing }\end{array}$} & \multirow[b]{2}{*}{$\mathrm{n}$} & \multicolumn{8}{|c|}{$\mathrm{PAH}[\mathrm{ng} / \mathrm{m} 3]$} & \multicolumn{2}{|c|}{ abundance [\%] } \\
\hline & & & & Min & Max & Q1 & median & Q3 & SD & mean & CV & median & $\mathrm{CV}$ \\
\hline $\begin{array}{l}01 \text { ACY } \\
02 \text { ACE }\end{array}$ & $\begin{array}{l}0.001 \\
0.002\end{array}$ & $\begin{array}{l}0 \% \\
0 \%\end{array}$ & $\begin{array}{l}228 \\
228\end{array}$ & $\begin{array}{l}0.007 \\
0.021\end{array}$ & $\begin{array}{l}59.217 \\
69.415\end{array}$ & $\begin{array}{l}0.160 \\
0.357\end{array}$ & $\begin{array}{l}0.553 \\
0.742\end{array}$ & $\begin{array}{l}2.603 \\
1.828\end{array}$ & $\begin{array}{l}5.366 \\
5.214\end{array}$ & $\begin{array}{l}2.388 \\
2.052\end{array}$ & $\begin{array}{l}2.2 \\
2.5\end{array}$ & $\begin{array}{l}2.8 \% \\
3.4 \%\end{array}$ & $\begin{array}{l}1.1 \\
0.9\end{array}$ \\
\hline $03 \mathrm{FLU}$ & 0.003 & $0 \%$ & 228 & 0.219 & 40.322 & 1.405 & 2.323 & 5.077 & 5.830 & 4.575 & 1.3 & $11.4 \%$ & 0.4 \\
\hline 04_ANT & 0.002 & $0 \%$ & 228 & 0.054 & 13.241 & 0.327 & 0.610 & 1.795 & 2.106 & 1.516 & 1.4 & $2.6 \%$ & 0.4 \\
\hline $05 \mathrm{PHE}$ & 0.004 & $0 \%$ & 228 & 0.903 & 107.876 & 4.944 & 8.840 & 18.990 & 16.371 & 15.527 & 1.1 & $34.9 \%$ & 0.3 \\
\hline $06^{-} \mathrm{MFLU}$ & 0.004 & $0 \%$ & 228 & 0.085 & 7.427 & 0.469 & 0.754 & 1.220 & 1.049 & 1.103 & 1.0 & $2.8 \%$ & 0.4 \\
\hline 07_FLT & 0.002 & $0 \%$ & 228 & 0.457 & 41.787 & 1.605 & 3.068 & 6.775 & 6.848 & 5.853 & 1.2 & $12.6 \%$ & 0.2 \\
\hline 08 PYR & 0.001 & $0 \%$ & 228 & 0.317 & 27.062 & 1.132 & 1.962 & 4.357 & 4.428 & 3.768 & 1.2 & $8.2 \%$ & 0.2 \\
\hline 09_MPYR & 0.001 & $0 \%$ & 228 & 0.011 & 1.078 & 0.044 & 0.074 & 0.164 & 0.141 & 0.128 & 1.1 & $0.3 \%$ & 0.4 \\
\hline $10 \mathrm{BaFLU}$ & 0.002 & $0 \%$ & 228 & 0.019 & 4.364 & 0.077 & 0.162 & 0.468 & 0.528 & 0.382 & 1.4 & $0.7 \%$ & 0.4 \\
\hline 11_BbFLU & 0.002 & $0 \%$ & 228 & 0.009 & 2.951 & 0.039 & 0.090 & 0.246 & 0.328 & 0.220 & 1.5 & $0.4 \%$ & 0.4 \\
\hline $12 \mathrm{BghiFL}$ & 0.002 & $0 \%$ & 228 & 0.001 & 2.314 & 0.093 & 0.174 & 0.340 & 0.300 & 0.278 & 1.1 & $0.6 \%$ & 0.4 \\
\hline $13^{-} \mathrm{CHRY}$ & 0.001 & $0 \%$ & 228 & 0.039 & 9.412 & 0.200 & 0.416 & 1.032 & 1.221 & 0.883 & 1.4 & $1.8 \%$ & 0.4 \\
\hline $14^{-} \mathrm{TRIPH}$ & 0.002 & $0 \%$ & 228 & 0.019 & 1.803 & 0.070 & 0.123 & 0.287 & 0.299 & 0.243 & 1.2 & $0.5 \%$ & 0.3 \\
\hline $15 \mathrm{BaA}$ & 0.004 & $0 \%$ & 228 & 0.029 & 6.744 & 0.106 & 0.245 & 0.704 & 0.831 & 0.561 & 1.5 & $1.0 \%$ & 0.5 \\
\hline 16_RET & 0.005 & $12 \%$ & 200 & 0.027 & 2.324 & 0.152 & 0.223 & 0.323 & 0.278 & 0.296 & 0.9 & $0.7 \%$ & 1.1 \\
\hline $17 \mathrm{MBaA}$ & 0.003 & $92 \%$ & 228 & 0.001 & 0.013 & 0.001 & 0.001 & 0.001 & 0.001 & 0.002 & 0.8 & $0.0 \%$ & 0.8 \\
\hline 18_BaP & 0.003 & $1 \%$ & 228 & 0.002 & 4.021 & 0.094 & 0.190 & 0.442 & 0.545 & 0.398 & 1.4 & $0.8 \%$ & 0.6 \\
\hline $19 \mathrm{BeP}$ & 0.002 & $0 \%$ & 228 & 0.033 & 7.035 & 0.124 & 0.268 & 0.636 & 0.836 & 0.578 & 1.4 & $1.2 \%$ & 0.4 \\
\hline 20_BbFLT & 0.003 & $0 \%$ & 228 & 0.052 & 16.371 & 0.256 & 0.545 & 1.372 & 1.842 & 1.254 & 1.5 & $2.5 \%$ & 0.4 \\
\hline 21_BkFLT & 0.003 & $0 \%$ & 228 & 0.019 & 4.790 & 0.082 & 0.163 & 0.430 & 0.548 & 0.373 & 1.5 & $0.7 \%$ & 0.5 \\
\hline 22_PER & 0.002 & $23 \%$ & 228 & 0.001 & 1.135 & 0.012 & 0.035 & 0.102 & 0.136 & 0.087 & 1.6 & $0.2 \%$ & 0.9 \\
\hline $23 \mathrm{MCHO}$ & 0.003 & $100 \%$ & 228 & 0.001 & 0.001 & 0.001 & 0.001 & 0.001 & 0.000 & 0.001 & 0.0 & $0.0 \%$ & 0.8 \\
\hline 24 ANTH & 0.002 & $29 \%$ & 228 & 0.001 & 0.700 & 0.001 & 0.027 & 0.083 & 0.105 & 0.067 & 1.6 & $0.1 \%$ & 0.9 \\
\hline 25_BghiPE & 0.004 & $0 \%$ & 228 & 0.040 & 5.607 & 0.138 & 0.291 & 0.600 & 0.767 & 0.557 & 1.4 & $1.2 \%$ & 0.4 \\
\hline 26 IPYR & 0.003 & $0 \%$ & 228 & 0.001 & 6.096 & 0.131 & 0.261 & 0.647 & 0.800 & 0.572 & 1.4 & $1.2 \%$ & 0.4 \\
\hline 27 IFLT & 0.01 & $58 \%$ & 148 & 0.005 & 0.354 & 0.005 & 0.016 & 0.033 & 0.039 & 0.028 & 1.4 & $0.0 \%$ & 1.0 \\
\hline $28 \mathrm{BbCHRY}$ & 0.002 & $36 \%$ & 228 & 0.001 & 0.819 & 0.001 & 0.014 & 0.047 & 0.080 & 0.042 & 1.9 & $0.1 \%$ & 0.9 \\
\hline 29 dBANT & 0.002 & $14 \%$ & 228 & 0.001 & 3.779 & 0.016 & 0.038 & 0.099 & 0.284 & 0.103 & 2.7 & $0.2 \%$ & 1.5 \\
\hline 30 SUMALL & $\mathrm{n} / \mathrm{a}$ & $\mathrm{n} / \mathrm{a}$ & 228 & 3.388 & 355.283 & 14.231 & 24.224 & 51.597 & 50.210 & 43.790 & 1.1 & $100.0 \%$ & 0.0 \\
\hline 31_SUMLIGHT & $\mathrm{n} / \mathrm{a}$ & $\mathrm{n} / \mathrm{a}$ & 228 & 2.498 & 277.185 & 12.453 & 19.691 & 43.376 & 41.360 & 36.783 & 1.1 & $85.3 \%$ & 0.1 \\
\hline 32 SUMHVY & $\mathrm{n} / \mathrm{a}$ & $\mathrm{n} / \mathrm{a}$ & 228 & 0.593 & 78.099 & 1.710 & 3.476 & 7.979 & 9.313 & 7.008 & 1.3 & $14.7 \%$ & 0.3 \\
\hline
\end{tabular}


(b) Toronto

\begin{tabular}{|c|c|c|c|c|c|c|c|c|c|c|c|c|c|}
\hline \multirow[b]{2}{*}{ SPECIES } & \multirow[b]{2}{*}{$\begin{array}{r}\mathrm{DLj} \\
(\mathrm{ng} / \mathrm{m} 3) \\
\end{array}$} & \multirow[b]{2}{*}{$\begin{array}{l}\text { \%BDL+ } \\
\text { missing }\end{array}$} & \multirow[b]{2}{*}{$n$} & \multicolumn{8}{|c|}{$\mathrm{PAH}[\mathrm{ng} / \mathrm{m} 3]$} & \multicolumn{2}{|c|}{ abundance [\%] } \\
\hline & & & & Min & Max & Q1 & median & Q3 & SD & mean & CV & median & $\mathrm{CV}$ \\
\hline 01_ACY & 0.001 & $0 \%$ & 392 & 0.005 & 6.460 & 0.160 & 0.445 & 1.193 & 1.042 & 0.869 & 1.2 & $2.9 \%$ & 1.0 \\
\hline 02_ACE & 0.002 & $0 \%$ & 392 & 0.031 & 3.187 & 0.437 & 0.689 & 0.992 & 0.477 & 0.783 & 0.6 & $4.1 \%$ & 0.6 \\
\hline 03_FLU & 0.003 & $0 \%$ & 392 & 0.025 & 6.693 & 1.457 & 1.984 & 2.745 & 1.095 & 2.211 & 0.5 & $12.9 \%$ & 0.4 \\
\hline 04_ANT & 0.002 & $0 \%$ & 392 & 0.001 & 5.486 & 0.262 & 0.414 & 0.579 & 0.476 & 0.497 & 1.0 & $2.3 \%$ & 0.7 \\
\hline 05_PHE & 0.004 & $0 \%$ & 392 & 0.061 & 38.299 & 5.051 & 7.654 & 11.489 & 5.609 & 9.145 & 0.6 & $43.2 \%$ & 0.2 \\
\hline 06_MFLU & 0.004 & $0 \%$ & 392 & 0.007 & 3.093 & 0.481 & 0.686 & 0.964 & 0.445 & 0.776 & 0.6 & $3.8 \%$ & 0.3 \\
\hline 07_FLT & 0.002 & $0 \%$ & 392 & 0.080 & 11.479 & 1.163 & 1.627 & 2.243 & 1.185 & 1.898 & 0.6 & $9.1 \%$ & 0.2 \\
\hline 08_PYR & 0.001 & $0 \%$ & 392 & 0.050 & 11.131 & 0.834 & 1.195 & 1.600 & 0.917 & 1.363 & 0.7 & $6.5 \%$ & 0.2 \\
\hline 09_MPYR & 0.001 & $0 \%$ & 392 & 0.001 & 0.562 & 0.035 & 0.050 & 0.070 & 0.052 & 0.061 & 0.8 & $0.3 \%$ & 0.4 \\
\hline 10_BaFLU & 0.002 & $0 \%$ & 392 & 0.004 & 0.617 & 0.046 & 0.068 & 0.097 & 0.067 & 0.084 & 0.8 & $0.4 \%$ & 0.4 \\
\hline 11_BbFLU & 0.002 & $1 \%$ & 392 & 0.001 & 0.396 & 0.022 & 0.032 & 0.047 & 0.036 & 0.041 & 0.9 & $0.2 \%$ & 0.5 \\
\hline 12_BghiFL & 0.002 & $0 \%$ & 392 & 0.008 & 1.150 & 0.074 & 0.107 & 0.166 & 0.104 & 0.136 & 0.8 & $0.7 \%$ & 0.4 \\
\hline 13_CHRY & 0.001 & $1 \%$ & 391 & 0.000 & 2.388 & 0.116 & 0.173 & 0.260 & 0.217 & 0.227 & 1.0 & $1.1 \%$ & 0.5 \\
\hline 14_TRIPH & 0.002 & $1 \%$ & 391 & 0.001 & 0.548 & 0.045 & 0.063 & 0.087 & 0.054 & 0.074 & 0.7 & $0.4 \%$ & 0.5 \\
\hline 15_BaA & 0.004 & $0 \%$ & 392 & 0.002 & 2.737 & 0.065 & 0.099 & 0.165 & 0.201 & 0.149 & 1.3 & $0.6 \%$ & 0.7 \\
\hline 16_RET & 0.005 & $12 \%$ & 345 & 0.031 & 1.403 & 0.115 & 0.164 & 0.237 & 0.148 & 0.204 & 0.7 & $0.8 \%$ & 0.9 \\
\hline 17_MBaA & 0.003 & $97 \%$ & 392 & 0.001 & 0.024 & 0.001 & 0.001 & 0.001 & 0.002 & 0.002 & 1.1 & $0.0 \%$ & 1.3 \\
\hline 18_BaP & 0.003 & $2 \%$ & 392 & 0.002 & 2.429 & 0.054 & 0.084 & 0.143 & 0.167 & 0.124 & 1.3 & $0.5 \%$ & 0.8 \\
\hline 19_BeP & 0.002 & $1 \%$ & 392 & 0.001 & 1.564 & 0.084 & 0.128 & 0.194 & 0.134 & 0.160 & 0.8 & $0.8 \%$ & 0.5 \\
\hline 20_BbFLT & 0.003 & $0 \%$ & 392 & 0.014 & 3.168 & 0.160 & 0.242 & 0.374 & 0.274 & 0.311 & 0.9 & $1.5 \%$ & 0.6 \\
\hline 21_BkFLT & 0.003 & $1 \%$ & 392 & 0.001 & 1.120 & 0.050 & 0.075 & 0.117 & 0.090 & 0.097 & 0.9 & $0.5 \%$ & 0.7 \\
\hline 22_PER & 0.002 & $32 \%$ & 392 & 0.001 & 0.568 & 0.001 & 0.015 & 0.026 & 0.038 & 0.022 & 1.7 & $0.1 \%$ & 1.1 \\
\hline 23_MCHO & 0.003 & $100 \%$ & 392 & 0.001 & 0.001 & 0.001 & 0.001 & 0.001 & 0.000 & 0.001 & 0.0 & $0.0 \%$ & 1.3 \\
\hline 24_ANTH & 0.002 & $53 \%$ & 392 & 0.001 & 0.285 & 0.001 & 0.001 & 0.021 & 0.027 & 0.015 & 1.8 & $0.0 \%$ & 1.2 \\
\hline 25_BghiPE & 0.004 & $0 \%$ & 392 & 0.010 & 1.393 & 0.111 & 0.161 & 0.263 & 0.158 & 0.209 & 0.8 & $1.0 \%$ & 0.5 \\
\hline 26_IPYR & 0.003 & $1 \%$ & 392 & 0.001 & 1.693 & 0.086 & 0.132 & 0.204 & 0.151 & 0.171 & 0.9 & $0.8 \%$ & 0.6 \\
\hline 27_IFLT & 0.01 & $86 \%$ & 227 & 0.005 & 0.078 & 0.005 & 0.005 & 0.005 & 0.010 & 0.009 & 1.1 & $0.0 \%$ & 1.8 \\
\hline 28_BbCHRY & 0.002 & $69 \%$ & 392 & 0.001 & 0.314 & 0.001 & 0.001 & 0.009 & 0.021 & 0.008 & 2.7 & $0.0 \%$ & 1.6 \\
\hline 29_dBANT & 0.002 & $44 \%$ & 392 & 0.001 & 0.355 & 0.001 & 0.012 & 0.022 & 0.027 & 0.016 & 1.7 & $0.1 \%$ & 1.1 \\
\hline 30_SUMALL & $\mathrm{n} / \mathrm{a}$ & $\mathrm{n} / \mathrm{a}$ & 392 & 0.593 & 83.789 & 13.093 & 17.776 & 23.436 & 9.744 & 19.634 & 0.5 & $100.0 \%$ & 0.0 \\
\hline 31_SUMLIGHT & $\mathrm{n} / \mathrm{a}$ & $\mathrm{n} / \mathrm{a}$ & 392 & 0.271 & 73.339 & 11.653 & 15.953 & 21.161 & 8.690 & 17.543 & 0.5 & $89.6 \%$ & 0.1 \\
\hline 32_SUMHVY & $\mathrm{n} / \mathrm{a}$ & $\mathrm{n} / \mathrm{a}$ & 392 & 0.209 & 20.457 & 1.115 & 1.681 & 2.452 & 1.724 & 2.092 & 0.8 & $10.4 \%$ & 0.5 \\
\hline
\end{tabular}


(c) background (Egbert)

\begin{tabular}{|c|c|c|c|c|c|c|c|c|c|c|c|c|c|}
\hline \multirow[b]{2}{*}{ SPECIES } & \multirow[b]{2}{*}{$\begin{array}{r}\mathrm{DLj} \\
(\mathrm{ng} / \mathrm{m} 3)\end{array}$} & \multirow[b]{2}{*}{$\begin{array}{l}\% \text { BDL+ } \\
\text { missing }\end{array}$} & \multirow[b]{2}{*}{$\mathrm{n}$} & \multicolumn{8}{|c|}{$\mathrm{PAH}[\mathrm{ng} / \mathrm{m} 3]$} & \multicolumn{2}{|c|}{ abundance [\%] } \\
\hline & & & & Min & Max & Q1 & median & Q3 & SD & mean & CV & median & $\mathrm{CV}$ \\
\hline 01_ACY & 0.001 & $2 \%$ & 251 & 0.001 & 1.744 & 0.020 & 0.059 & 0.156 & 0.229 & 0.139 & 1.6 & $2.3 \%$ & 1.0 \\
\hline 02_ACE & 0.002 & $1 \%$ & 251 & 0.001 & 0.960 & 0.076 & 0.123 & 0.201 & 0.143 & 0.166 & 0.9 & $4.5 \%$ & 0.6 \\
\hline 03_FLU & 0.003 & $0 \%$ & 251 & 0.002 & 3.538 & 0.288 & 0.535 & 0.894 & 0.559 & 0.680 & 0.8 & $18.8 \%$ & 0.3 \\
\hline 04_ANT & 0.002 & $2 \%$ & 251 & 0.001 & 1.409 & 0.019 & 0.032 & 0.051 & 0.097 & 0.049 & 2.0 & $1.2 \%$ & 0.9 \\
\hline 05_PHE & 0.004 & $0 \%$ & 251 & 0.153 & 4.591 & 0.754 & 1.137 & 1.605 & 0.773 & 1.294 & 0.6 & $38.4 \%$ & 0.2 \\
\hline 06_MFLU & 0.004 & $2 \%$ & 251 & 0.002 & 1.530 & 0.074 & 0.122 & 0.191 & 0.153 & 0.159 & 1.0 & $4.3 \%$ & 0.4 \\
\hline 07_FLT & 0.002 & $0 \%$ & 251 & 0.036 & 1.208 & 0.181 & 0.279 & 0.402 & 0.219 & 0.331 & 0.7 & $9.6 \%$ & 0.2 \\
\hline 08_PYR & 0.001 & $0 \%$ & 251 & 0.019 & 0.702 & 0.123 & 0.177 & 0.258 & 0.127 & 0.211 & 0.6 & $6.1 \%$ & 0.3 \\
\hline 09_MPYR & 0.001 & $6 \%$ & 251 & 0.001 & 0.057 & 0.004 & 0.007 & 0.011 & 0.007 & 0.009 & 0.8 & $0.2 \%$ & 0.6 \\
\hline 10_BaFLU & 0.002 & $6 \%$ & 251 & 0.001 & 0.080 & 0.006 & 0.011 & 0.018 & 0.013 & 0.015 & 0.9 & $0.4 \%$ & 0.5 \\
\hline 11_BbFLU & 0.002 & $14 \%$ & 251 & 0.001 & 0.040 & 0.003 & 0.006 & 0.009 & 0.007 & 0.007 & 0.9 & $0.2 \%$ & 0.7 \\
\hline 12_BghiFL & 0.002 & $4 \%$ & 251 & 0.001 & 0.133 & 0.010 & 0.017 & 0.031 & 0.024 & 0.025 & 1.0 & $0.6 \%$ & 0.5 \\
\hline 13_CHRY & 0.001 & $2 \%$ & 251 & 0.000 & 0.273 & 0.015 & 0.029 & 0.055 & 0.045 & 0.044 & 1.0 & $1.0 \%$ & 0.6 \\
\hline 14_TRIPH & 0.002 & $8 \%$ & 251 & 0.001 & 0.107 & 0.008 & 0.014 & 0.026 & 0.018 & 0.019 & 0.9 & $0.5 \%$ & 0.5 \\
\hline 15_BaA & 0.004 & $23 \%$ & 251 & 0.002 & 0.135 & 0.005 & 0.010 & 0.017 & 0.017 & 0.015 & 1.2 & $0.3 \%$ & 0.7 \\
\hline 16_RET & 0.005 & $10 \%$ & 228 & 0.003 & 1.243 & 0.048 & 0.082 & 0.132 & 0.132 & 0.120 & 1.1 & $2.5 \%$ & 1.0 \\
\hline 17_MBaA & 0.003 & $100 \%$ & 251 & 0.001 & 0.003 & 0.001 & 0.001 & 0.001 & 0.000 & 0.001 & 0.1 & $0.0 \%$ & 0.7 \\
\hline 18_BaP & 0.003 & $54 \%$ & 251 & 0.002 & 0.086 & 0.002 & 0.002 & 0.016 & 0.016 & 0.011 & 1.5 & $0.2 \%$ & 1.2 \\
\hline 19_BeP & 0.002 & $18 \%$ & 251 & 0.001 & 0.203 & 0.008 & 0.017 & 0.032 & 0.032 & 0.027 & 1.2 & $0.6 \%$ & 0.8 \\
\hline 20_BbFLT & 0.003 & $7 \%$ & 251 & 0.002 & 0.463 & 0.019 & 0.040 & 0.079 & 0.075 & 0.065 & 1.2 & $1.5 \%$ & 0.7 \\
\hline 21_BkFLT & 0.003 & $40 \%$ & 251 & 0.001 & 0.140 & 0.001 & 0.008 & 0.020 & 0.020 & 0.015 & 1.3 & $0.3 \%$ & 0.9 \\
\hline 22_PER & 0.002 & $94 \%$ & 251 & 0.001 & 0.015 & 0.001 & 0.001 & 0.001 & 0.002 & 0.001 & 1.5 & $0.0 \%$ & 0.8 \\
\hline 23_MCHO & 0.003 & $100 \%$ & 251 & 0.001 & 0.001 & 0.001 & 0.001 & 0.001 & 0.000 & 0.001 & 0.0 & $0.0 \%$ & 0.7 \\
\hline 24_ANTH & 0.002 & $96 \%$ & 251 & 0.001 & 0.013 & 0.001 & 0.001 & 0.001 & 0.002 & 0.001 & 1.3 & $0.0 \%$ & 0.8 \\
\hline 25_BghiPE & 0.004 & $33 \%$ & 251 & 0.002 & 0.214 & 0.002 & 0.013 & 0.032 & 0.032 & 0.024 & 1.3 & $0.5 \%$ & 0.9 \\
\hline 26_IPYR & 0.003 & $37 \%$ & 251 & 0.001 & 0.234 & 0.001 & 0.014 & 0.036 & 0.035 & 0.026 & 1.3 & $0.5 \%$ & 1.0 \\
\hline 27_IFLT & 0.01 & $94 \%$ & 175 & 0.005 & 0.023 & 0.005 & 0.005 & 0.005 & 0.003 & 0.006 & 0.5 & $0.1 \%$ & 0.9 \\
\hline 28_BbCHRY & 0.002 & $99 \%$ & 251 & 0.001 & 0.009 & 0.001 & 0.001 & 0.001 & 0.001 & 0.001 & 0.5 & $0.0 \%$ & 0.7 \\
\hline 29_dBANT & 0.002 & $88 \%$ & 251 & 0.001 & 0.021 & 0.001 & 0.001 & 0.001 & 0.003 & 0.002 & 1.5 & $0.0 \%$ & 0.8 \\
\hline 30_SUMALL & $\mathrm{n} / \mathrm{a}$ & $\mathrm{n} / \mathrm{a}$ & 251 & 0.364 & 15.061 & 1.885 & 2.915 & 4.326 & 2.318 & 3.451 & 0.7 & $100.0 \%$ & 0.0 \\
\hline 31_SUMLIGHT & $\mathrm{n} / \mathrm{a}$ & $\mathrm{n} / \mathrm{a}$ & 251 & 0.331 & 13.621 & 1.597 & 2.534 & 3.797 & 2.034 & 3.029 & 0.7 & $89.0 \%$ & 0.1 \\
\hline 32_SUMHVY & $\mathrm{n} / \mathrm{a}$ & $\mathrm{n} / \mathrm{a}$ & 251 & 0.033 & 2.131 & 0.180 & 0.311 & 0.550 & 0.370 & 0.423 & 0.9 & $11.0 \%$ & 0.5 \\
\hline
\end{tabular}


(a) Hamilton

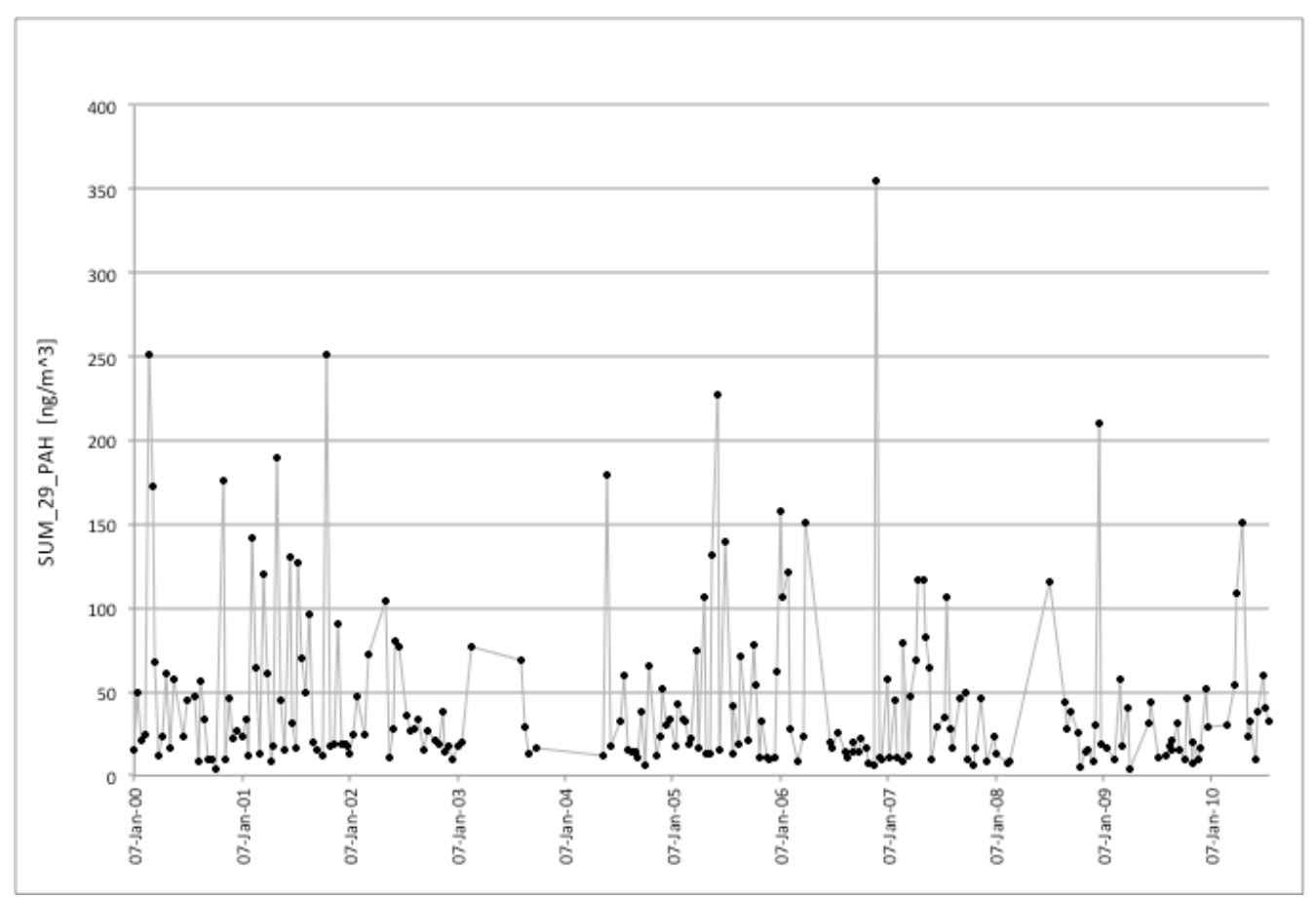

(b) Toronto

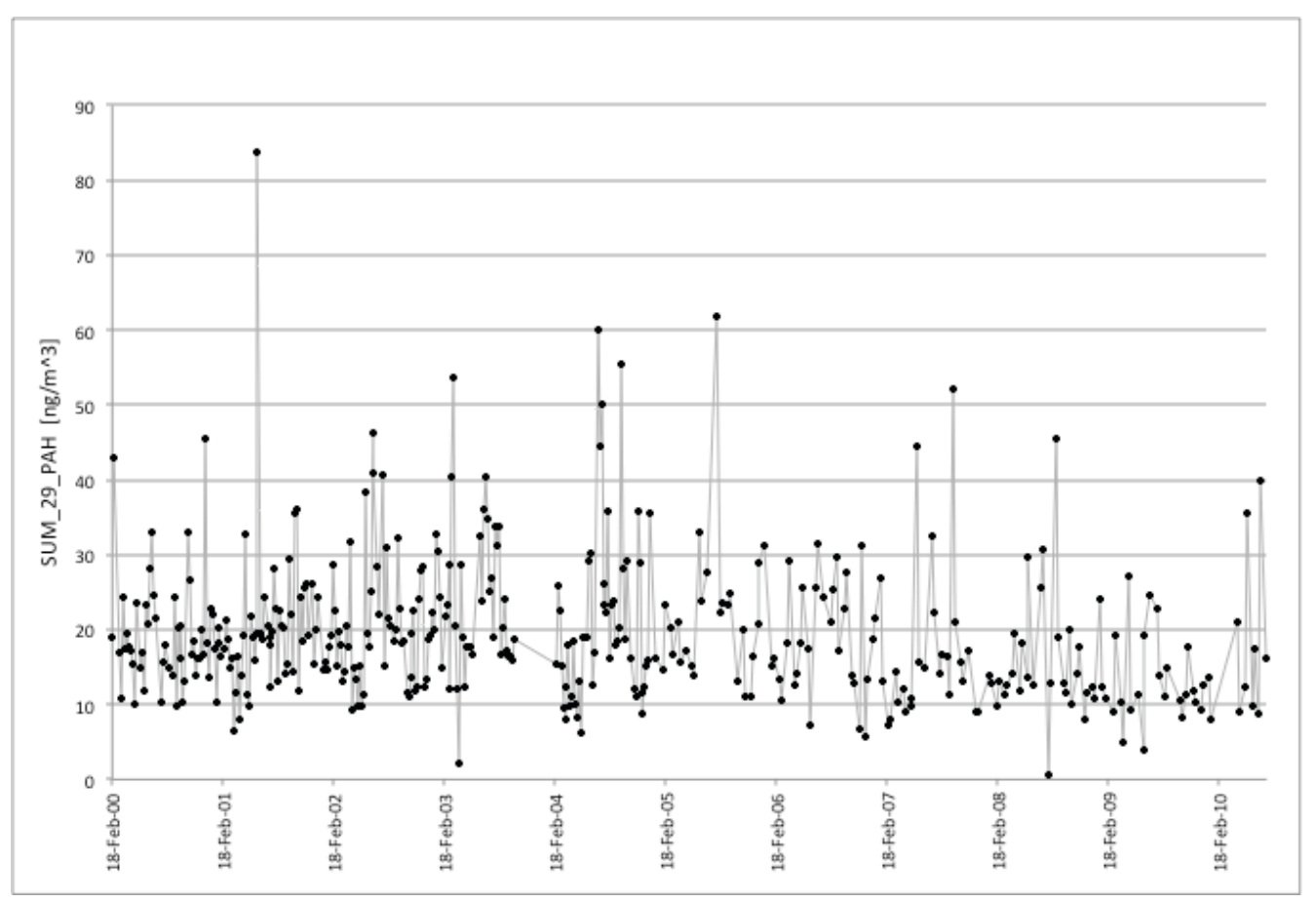

Figure B.2-1. Time series of $\Sigma_{29} \mathrm{PAH}$ at urban and background sites. 
(c) background (Egbert)

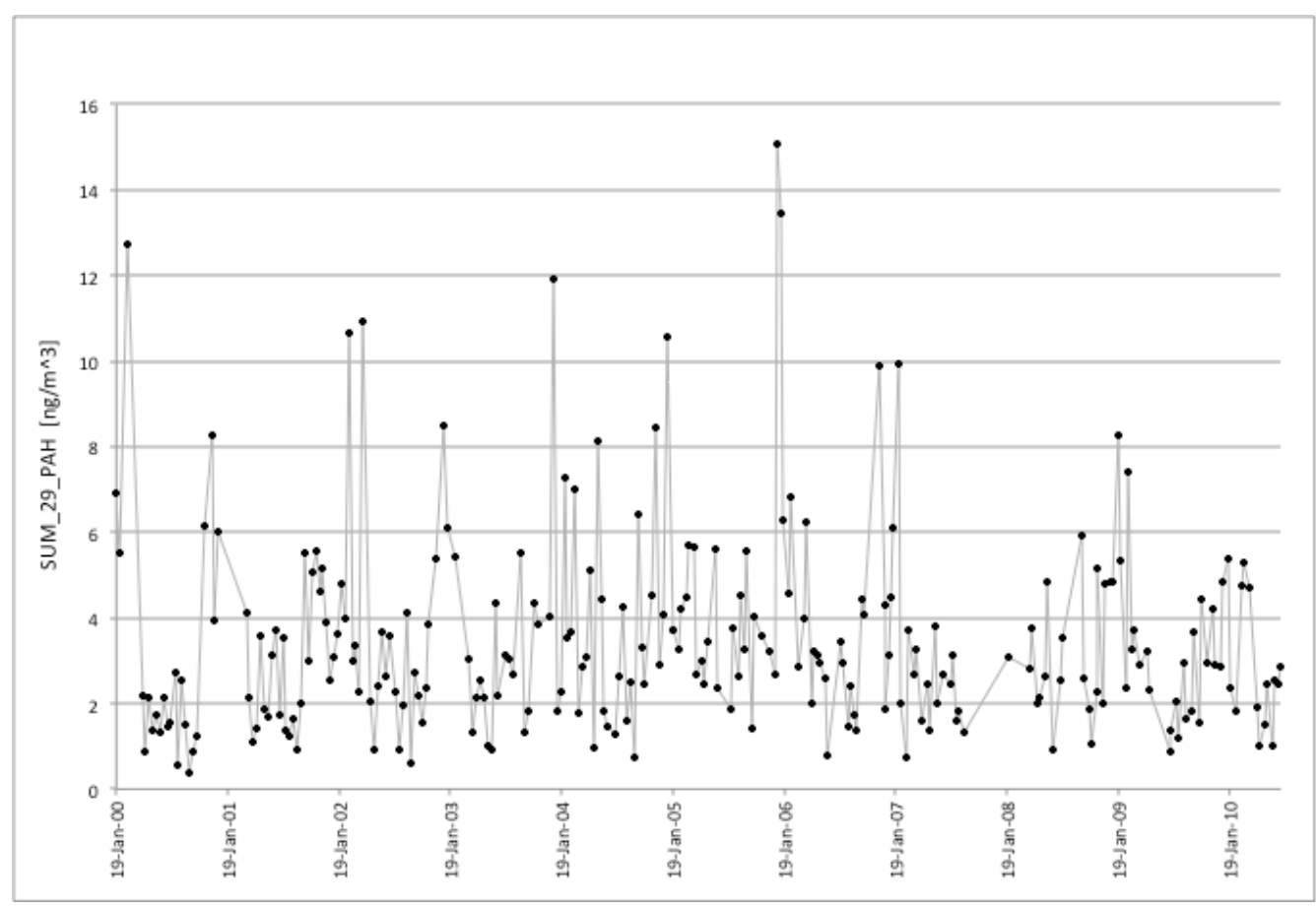

Figure B.2-1 (continued) 
Annual trends

Over a long historical period (1989-2010), $\Sigma_{29}$ PAH levels were observed to generally decrease at the urban sites (Hamilton, Toronto), with levels prior to 2000 being notably higher (i.e., similar trend for BAP, albeit with smaller changes). The longer-term historical decrease in levels is consistent with prior trend analysis at Canadian sites (Dann, 1997) and recommended scoping to a shorter and more recent period (i.e., overly long historical time span and large changes in ambient levels may indicate significant discontinuities in source mix or occurrence of technological changes such as emissions control technologies or regulations). $\Sigma_{29} \mathrm{PAH}$ and BAP levels at the background site followed a generally horizontal trend even over a long historical time period (19892010), confirming its selection as a background site. After scoping to the 2000-2010 period, ambient PAH levels were relatively consistent at all sites and thus amenable to receptor modeling while preserving sufficient data density (i.e., Toronto densest 20002010 dataset, 392 samples; Hamilton, $\mathrm{n}=228$; background, $\mathrm{n}=251$ ).

As expected, the urban sites had monitored ambient levels of $\Sigma_{29} \mathrm{PAH}$ several times higher than the background site (i.e., annual mean, annual median; Figure B.2-2). Highest PAH levels were seen at Hamilton, a trend consistent with the station's long-term monitoring records (Dann, 1997). Comparison of the background site data to an even more remote monitoring site (i.e., Pt. Petre; 43.856611N, 77.150000W) showed similar levels of total named PAH and BAP, confirming it as a suitable background site for the study area. There was no correlation in $\Sigma_{29} \mathrm{PAH}$ between sites (i.e., low Pearson correlation coefficient) and the inter-site differences in median PAH levels (i.e., $\Sigma_{29}$ PAH, $\Sigma_{\text {LIGHT }} \mathrm{PAH}, \Sigma_{\text {HEAVY }} \mathrm{PAH}$ ) were confirmed as statistically significant (i.e., Kruskal-Wallis, common sampling dates, 2000-2010). Annual trends in median PAH concentrations for the five most abundant species at each site were relatively consistent over the scoped time series, similar to $\Sigma_{29} \mathrm{PAH}$, and thus amenable to receptor modeling (Figure B.2-3). 
(a) annual mean

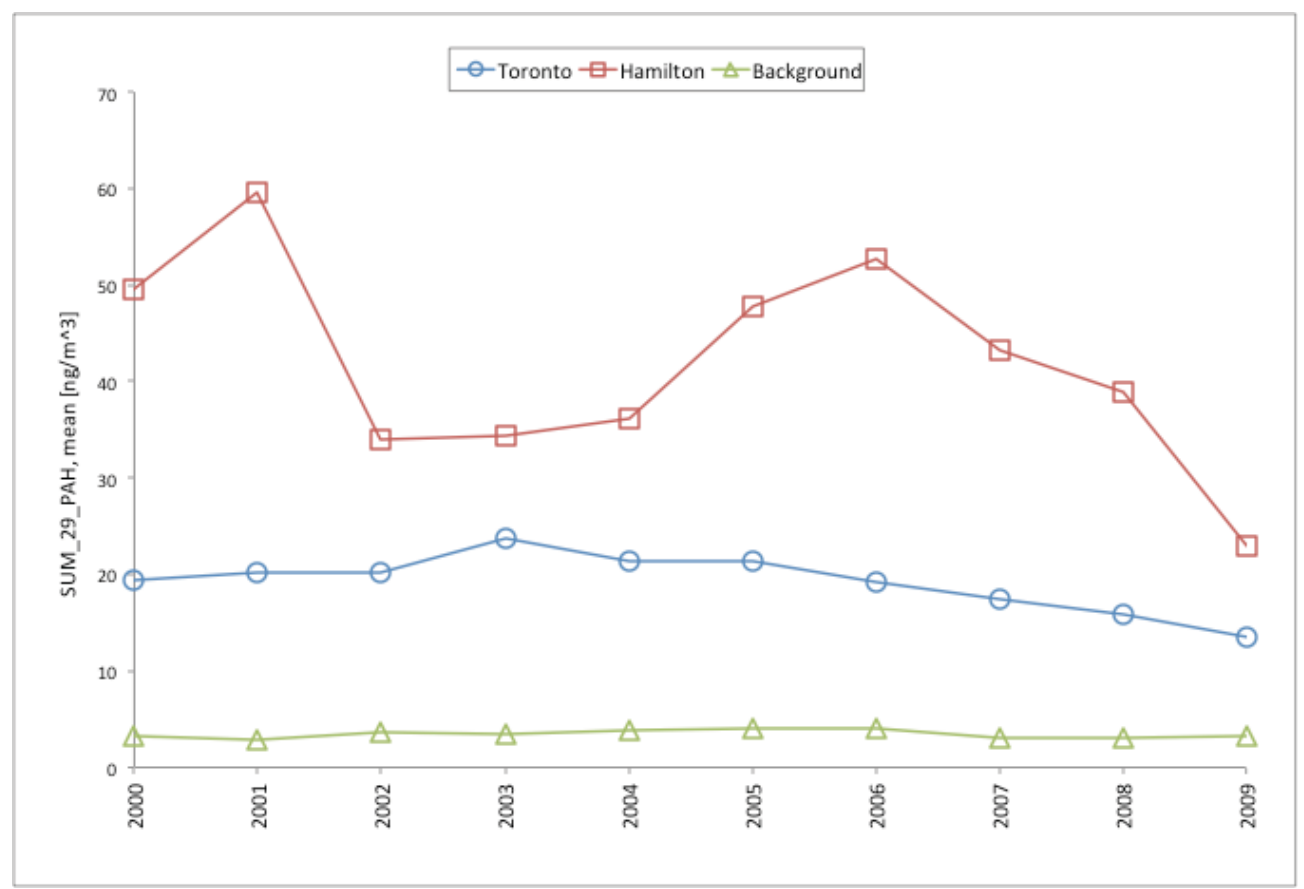

(b) annual median

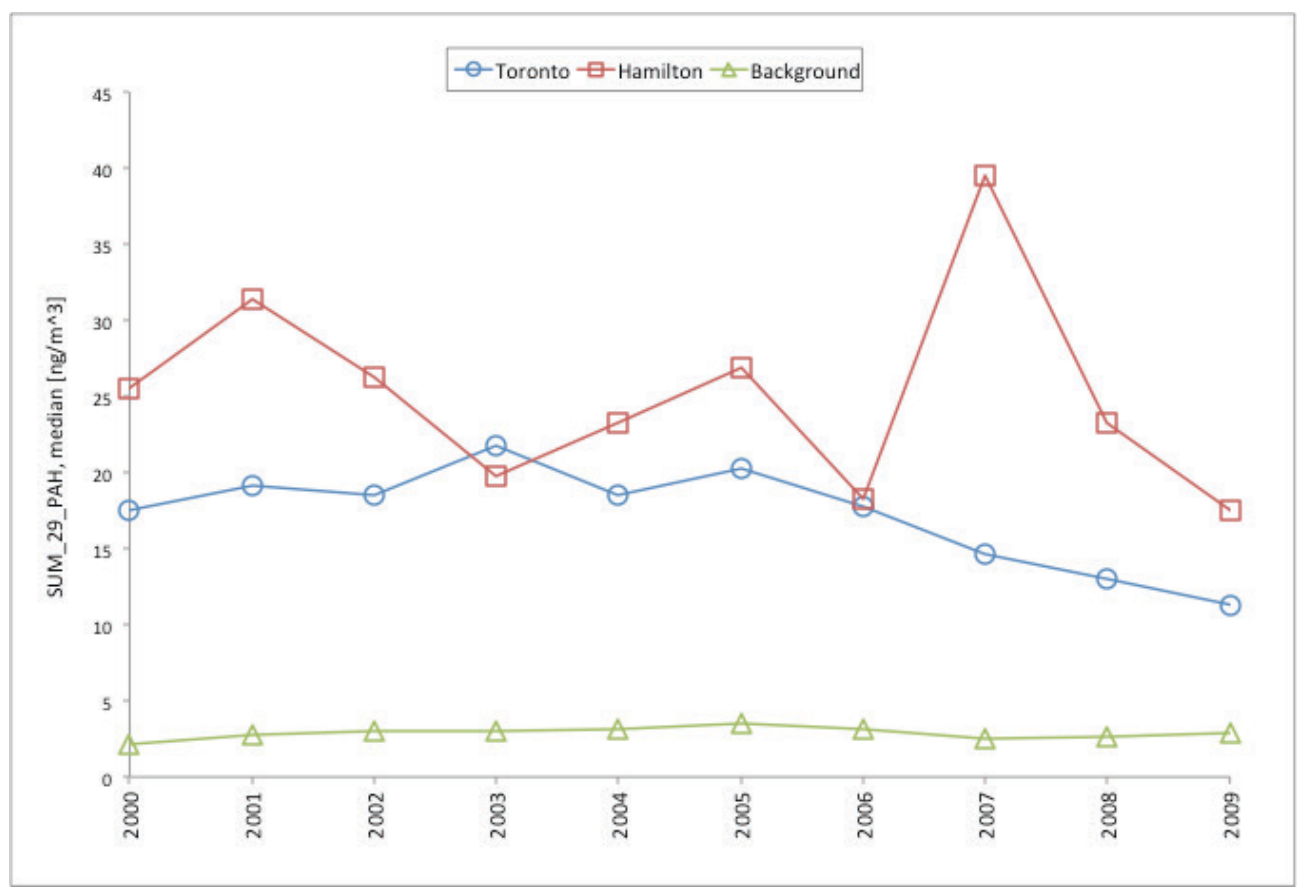

Figure B.2-2. Trend in annual mean and median $\Sigma$ 29PAH at urban and background sites. 


\section{(a) Hamilton}

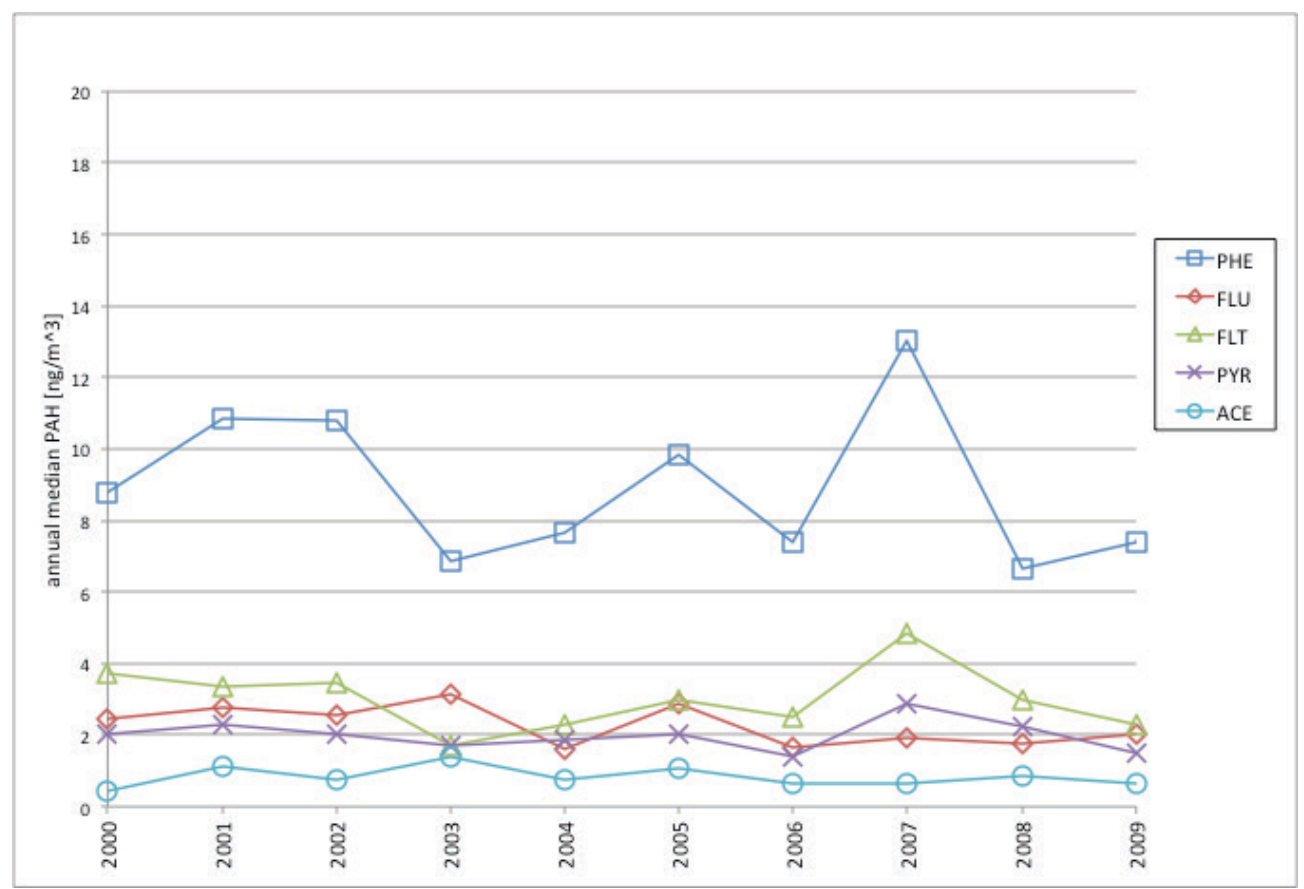

(b) Toronto

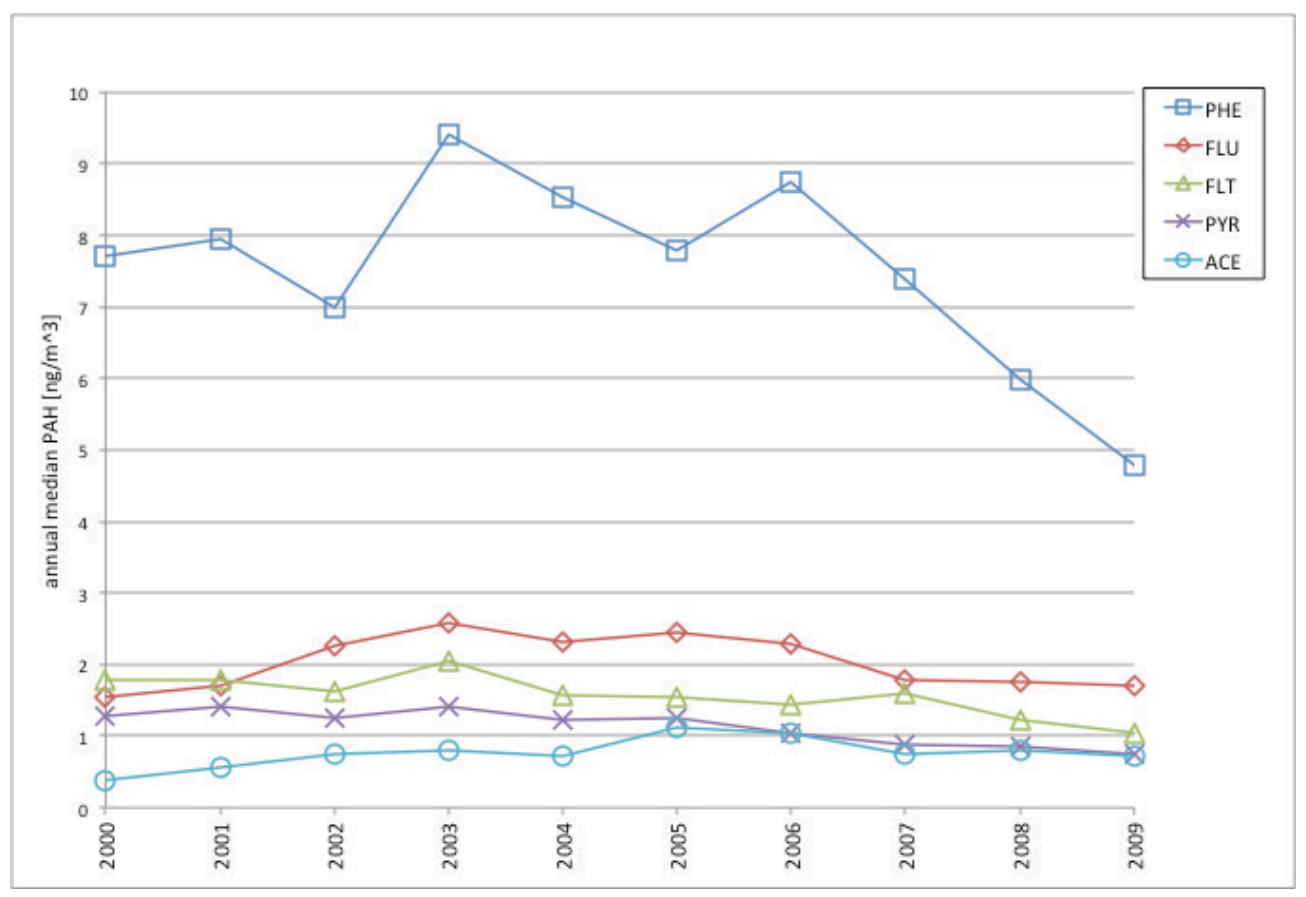

Figure B.2-3. Trend in annual median species concentration (five most abundant species). 
(c) background (Egbert)

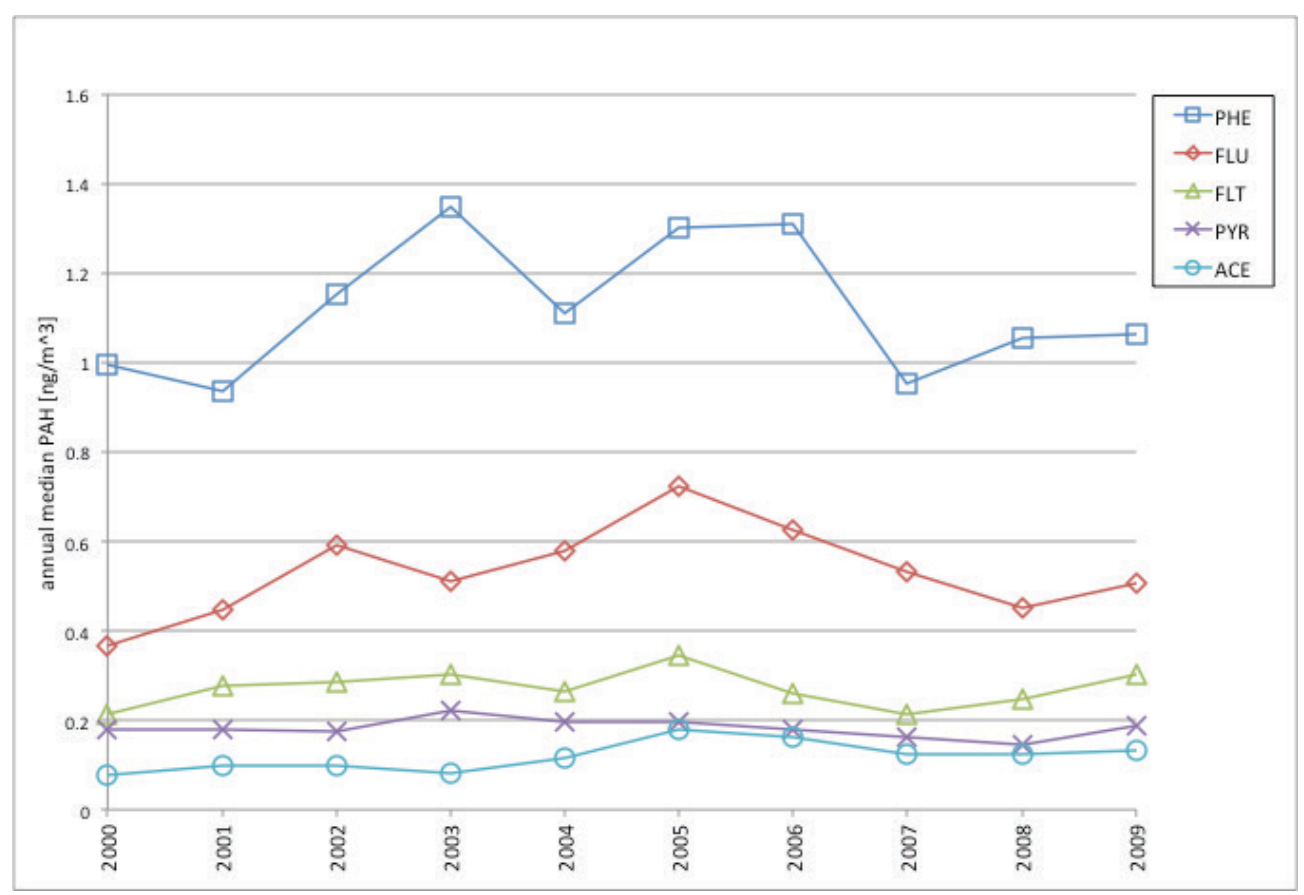

Figure B.2-3 (continued) 


\section{$\underline{\text { Seasonal trends }}$}

Higher ambient levels of particle-phase PAH are typically sampled during winter months, particularly in urban centres (Sun et al, 2006; Gaga, 2004; Dann, 1997; Waldman et al, 1991; Sisovic et al, 2002, 1996), due to seasonal patterns in source emissions (e.g., space heating in winter months) as well as meteorology/atmospheric effects (e.g., reduced mixing height in winter increasing ambient PM concentration, reduced $\mathrm{PAH}$ reaction rates at lower temperatures). For total ambient PAH (i.e., particle and vapour phase), however, summer levels can be higher due to PAH volatilization of light MW species, particularly in urban locations (Dimashki et al, 2001). Median ambient PAH levels $\left(\Sigma_{29} \mathrm{PAH}, \Sigma_{\text {LIGHT }} \mathrm{PAH}, \Sigma_{\text {HEAVY }} \mathrm{PAH}\right)$ were confirmed as statistically different between seasons (i.e., Kruskal-Wallis, median, common sampling dates, 2000-2010) at all three sites. The seasonal trends in annual 'winter' (i.e., October - April) and 'summer' (i.e., May - September) $\Sigma_{29} \mathrm{PAH}$ and $\Sigma_{\mathrm{HEAVY}} \mathrm{PAH}$ levels are shown in Figure B.2-4.

As the most highly urbanized site, Toronto followed expected seasonal trends over majority of the scoped time series (i.e., consistent with comparatively low sample-tosample variability indicated by $\mathrm{CV}$ for $\Sigma_{29} \mathrm{PAH}$ and individual species). Median sampled $\Sigma_{\text {HEAVY }}$ PAH levels (i.e., predominantly particle phase; Venkataraman and Friedlander, 1994) were generally higher in winter and so were typical of particle phase ambient PAH in urban centres (Sun et al, 2006); median $\Sigma_{29}$ PAH levels (i.e., including lighter MW species found predominantly in vapour phase; Venkataraman and Friedlander, 1994) were higher in summer. This was consistent with factors characterizing the densely urbanized central business district location around the monitoring site, including: PAH volatilization from uncombusted liquid fossil fuels (e.g., fuel storage and handling) and re-volatilization from built surfaces higher in summer months due to high temperatures (Dimashki et al, 2001); addition of PAH emissions from summer road maintenance and related construction (e.g., asphalt paving; Dann, 1997); and, high levels of particle phase $\mathrm{PAH}$ expected during smog episodes where PM in the fine size fraction is elevated, which most frequently occur in summer weather conditions (Environment Canada, 2004).

Hamilton urban site data showed less consistent seasonality for $\Sigma_{29} \mathrm{PAH}$ and $\Sigma_{\mathrm{HEAVY}} \mathrm{PAH}$ (i.e., some years higher seasonal median in winter, others in summer). This suggested influence from local sources tending to attenuate seasonal differences (i.e., industry emissions, traffic emissions) and a more variable source mix (i.e., CV indicated highest sample-to-sample variability for $\Sigma_{29} \mathrm{PAH}$ and most individual species). At the background site, median sampled concentrations of $\Sigma_{29} \mathrm{PAH}$ and $\Sigma_{\mathrm{HEAVY}} \mathrm{PAH}$ were consistently higher in winter, suggesting that ambient PAH was primarily due to transported regional source emissions rather than from appreciable local sources (i.e., source-receptor transport over longer distances occurs primarily with transport of fine PM). 
(a) Hamilton
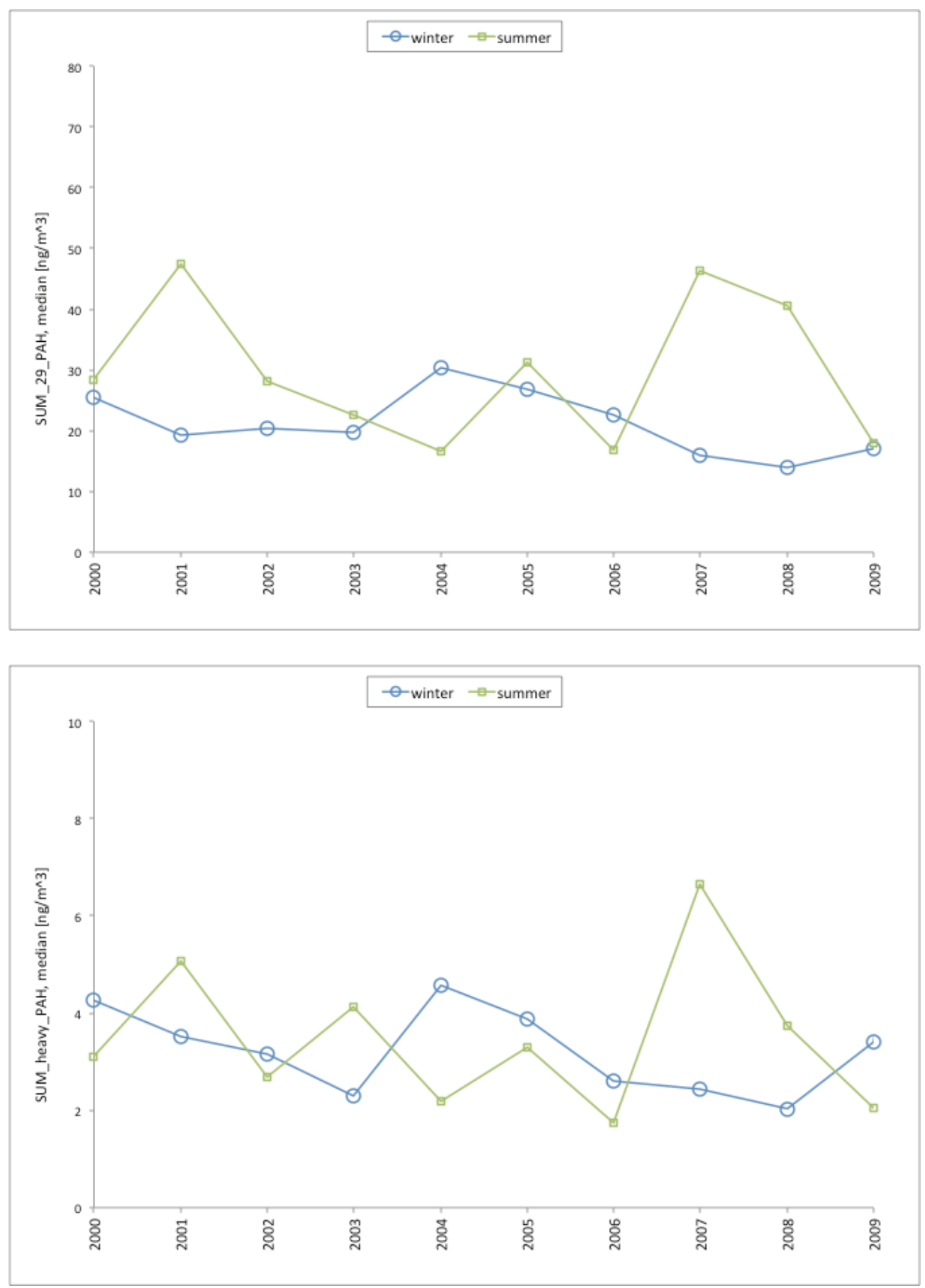

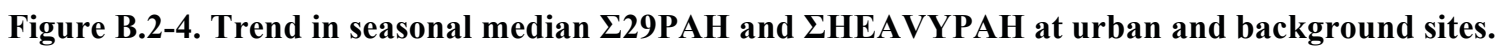


(b) Toronto
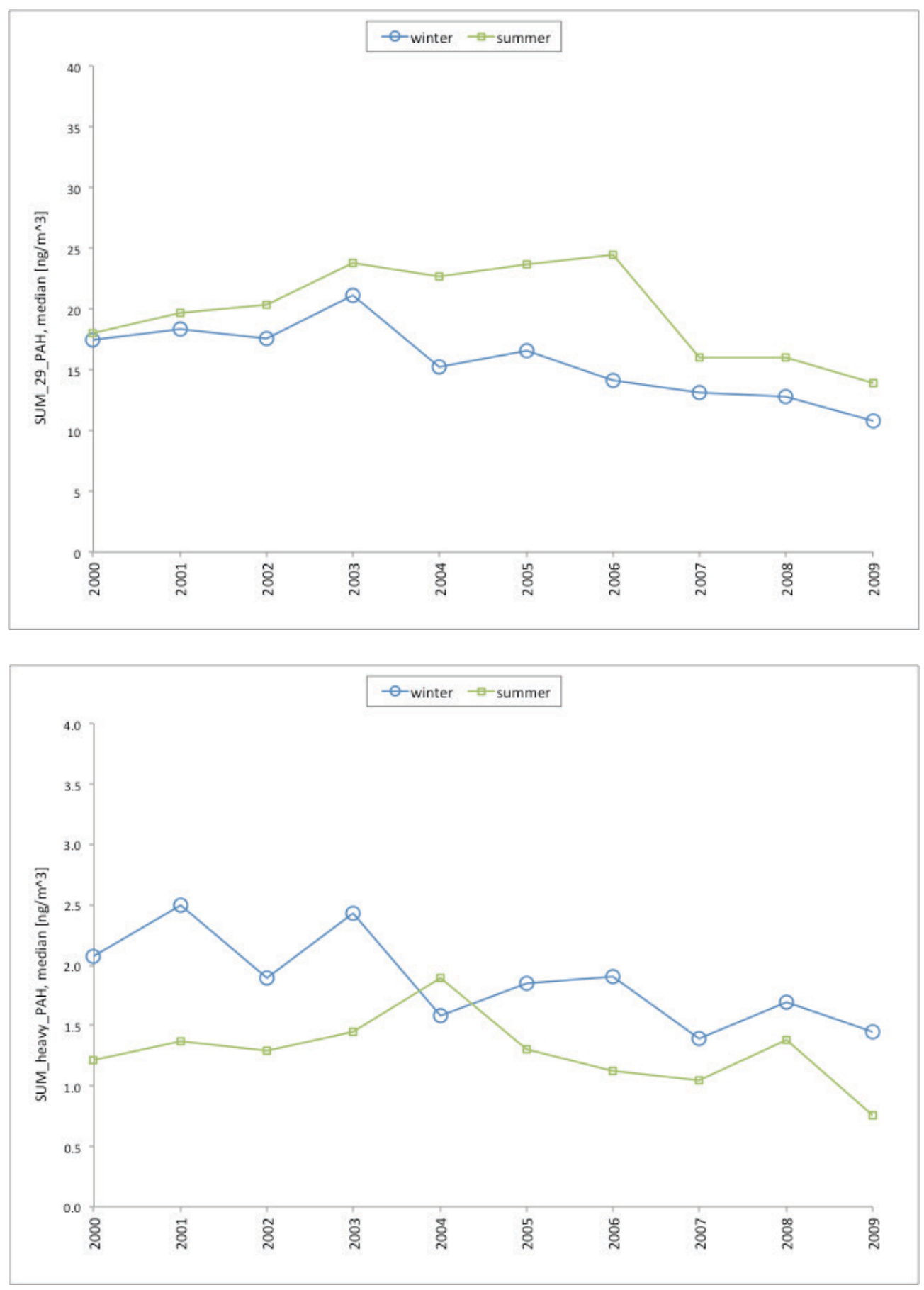

Figure B.2-4 (continued) 
(c) background (Egbert)
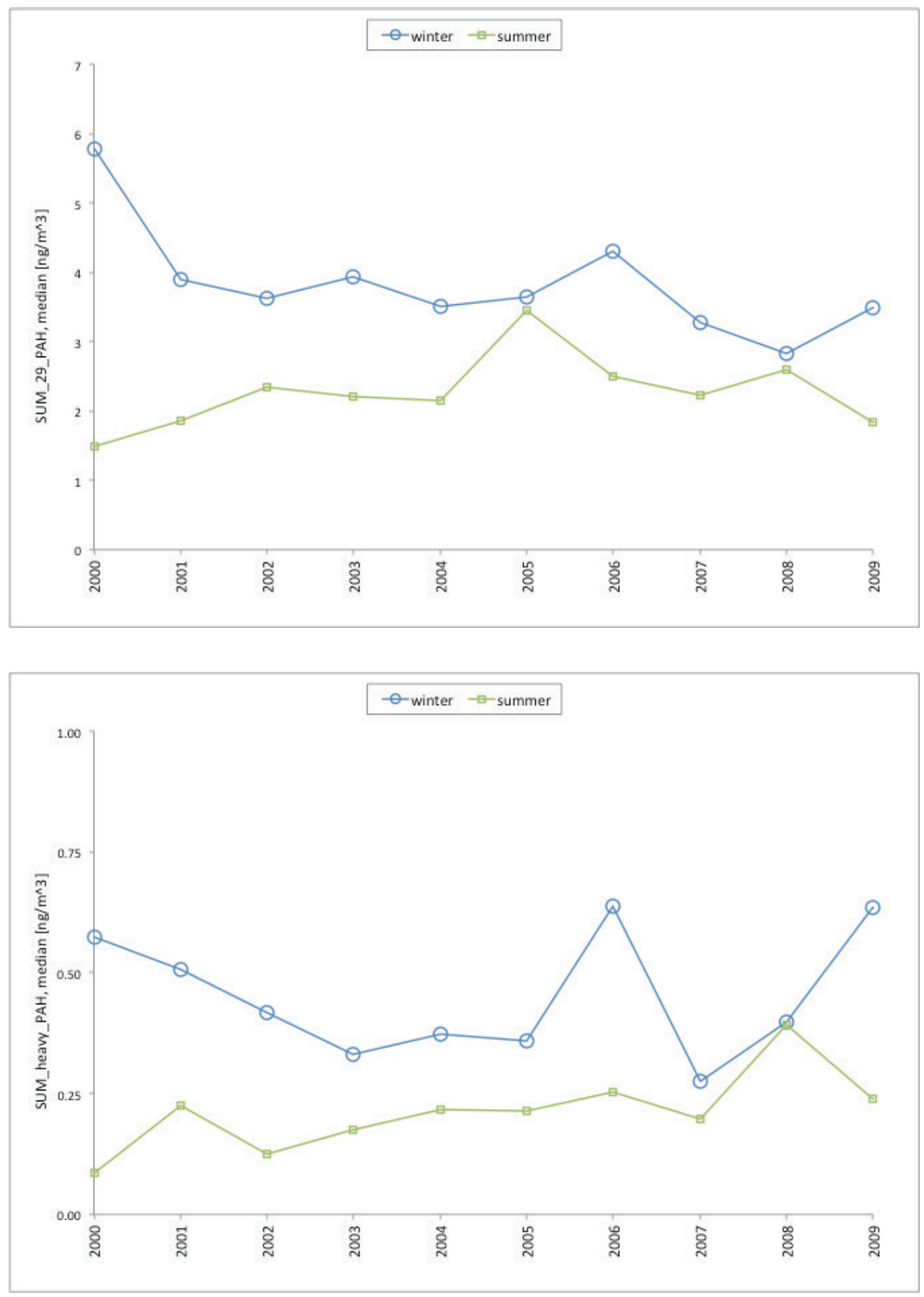

Figure B.2-4 (continued) 


\section{Species abundance}

By broad species grouping, light MW species contributed between approximately $85 \%$ (Hamilton) and 90\% (Toronto) of sample mass at the urban sites and $89 \%$ at the background site (Table B.2-2, median species abundance, 2000-2010). This is typical of total ambient PAH data; vapour phase concentrations are much higher than particle phase concentrations and so species predominantly found apportioned to vapour phase will dominate sample mass (Venkataraman and Friedlander, 1994; Lu et al., 2008). Results are also consistent with recent PAH field sampling studies with vapour and particle phase sampled together (Anastasopoulos et al, 2012; Miller et al, 2010).

The majority of the contribution to $\Sigma_{29} \mathrm{PAH}$ was concentrated among relatively few dominant species. By median species abundance, seven species contributed $>75 \%$ of sampled PAH mass at all sites (i.e., 76\% at Hamilton, $83 \%$ at Toronto, $84 \%$ at background). In generally decreasing order (i.e., rank position varied slightly between sites, Table B.2-2), these were: PHEN, FLU, FLT, PYR, ACE, MFLU, ACY. The most abundant species held relatively constant trends in annual median abundance, suggesting no significant change in source mix over the monitoring period and corroborating time series suitability for receptor modeling. Broad comparison between sites showed statistically significant inter-site differences in median abundance and in $\Sigma_{\mathrm{HEAVY}} \mathrm{PAH}$, however, suggesting inter-site differences in source mix: at the urban sites, Hamilton had a notably higher contribution of heavy MW species, lowest abundance of PHEN, and higher abundance of FLT, PYR, ANT; Toronto was most dominated by PHEN; the rural site showed some interesting differences from both urban sites with RET abundance approximately twice higher and notably lower abundance of the heavier sampled species BGHIP and IPYR.

Since the two urban sites showed a sizable difference in the overall contribution of heavy MW species (i.e., approximately $15 \%$ at Hamilton; $10 \%$ at Toronto; Table B.2-2), the abundance data (i.e., $\Sigma_{\text {HEAVY }} \mathrm{PAH} / \Sigma \mathrm{PAH}$ ) was tested and found to be significantly different (i.e., Kruskal-Wallis, common sampling dates, 2000-2010). The inter-urban difference in species abundances for top-ranked species and for the $\Sigma_{\text {HEAVY }}$ PAH group were useful coarse indicators that predicted inter-urban differences in relative source contributions consistent with differences in local sources known a priori (i.e., significant heavy industry at Hamilton, significant traffic at Toronto). 
Table B.2-2. Species abundance (median, 2000-2010) for urban and background sites.

\begin{tabular}{|c|c|c|c|c|c|c|}
\hline \multirow[b]{2}{*}{ SPECIES } & \multicolumn{6}{|c|}{ median abundance [\%] } \\
\hline & Hamilton & rank & Toronto & rank & $\begin{array}{r}\text { Egbert } \\
\text { (background) }\end{array}$ & rank \\
\hline 01_ACY & $2.8 \%$ & 7 & $2.9 \%$ & 7 & $2.3 \%$ & 8 \\
\hline 02_ACE & $3.4 \%$ & 5 & $4.1 \%$ & 5 & $4.5 \%$ & 5 \\
\hline 03_FLU & $11.4 \%$ & 3 & $12.9 \%$ & 2 & $18.8 \%$ & 2 \\
\hline 04_ANT & $2.6 \%$ & 8 & $2.3 \%$ & 8 & $1.2 \%$ & 10 \\
\hline 05_PHE & $34.9 \%$ & 1 & $43.2 \%$ & 1 & $38.4 \%$ & 1 \\
\hline 06_MFLU & $2.8 \%$ & 6 & $3.8 \%$ & 6 & $4.3 \%$ & 6 \\
\hline 07_FLT & $12.6 \%$ & 2 & $9.1 \%$ & 3 & $9.6 \%$ & 3 \\
\hline 08_PYR & $8.2 \%$ & 4 & $6.5 \%$ & 4 & $6.1 \%$ & 4 \\
\hline 09_MPYR & $0.3 \%$ & 22 & $0.3 \%$ & 21 & $0.2 \%$ & 20 \\
\hline 10_BaFLU & $0.7 \%$ & 18 & $0.4 \%$ & 19 & $0.4 \%$ & 17 \\
\hline 11_BbFLU & $0.4 \%$ & 21 & $0.2 \%$ & 22 & $0.2 \%$ & 21 \\
\hline 12_BghiFL & $0.6 \%$ & 19 & $0.7 \%$ & 15 & $0.6 \%$ & 12 \\
\hline 13_CHRY & $1.8 \%$ & 10 & $1.1 \%$ & 10 & $1.0 \%$ & 11 \\
\hline 14_TRIPH & $0.5 \%$ & 20 & $0.4 \%$ & 20 & $0.5 \%$ & 16 \\
\hline 15_BaA & $1.0 \%$ & 14 & $0.6 \%$ & 16 & $0.3 \%$ & 18 \\
\hline 16_RET & $0.7 \%$ & 17 & $0.8 \%$ & 13 & $2.5 \%$ & 7 \\
\hline 17_MBaA & $0.0 \%$ & 28 & $0.0 \%$ & 28 & $0.0 \%$ & 25 \\
\hline 18_BaP & $0.8 \%$ & 15 & $0.5 \%$ & 17 & $0.2 \%$ & 22 \\
\hline 19_BeP & $1.2 \%$ & 13 & $0.8 \%$ & 14 & $0.6 \%$ & 13 \\
\hline 20_BbFLT & $2.5 \%$ & 9 & $1.5 \%$ & 9 & $1.5 \%$ & 9 \\
\hline 21_BkFLT & $0.7 \%$ & 16 & $0.5 \%$ & 18 & $0.3 \%$ & 19 \\
\hline 22_PER & $0.2 \%$ & 24 & $0.1 \%$ & 23 & $0.0 \%$ & 29 \\
\hline 23_MCHO & $0.0 \%$ & 29 & $0.0 \%$ & 29 & $0.0 \%$ & 24 \\
\hline 24_ANTH & $0.1 \%$ & 25 & $0.0 \%$ & 26 & $0.0 \%$ & 28 \\
\hline 25_BghiPE & $1.2 \%$ & 11 & $1.0 \%$ & 11 & $0.5 \%$ & 15 \\
\hline 26_IPYR & $1.2 \%$ & 12 & $0.8 \%$ & 12 & $0.5 \%$ & 14 \\
\hline 27_IFLT & $0.0 \%$ & 27 & $0.0 \%$ & 25 & $0.1 \%$ & 23 \\
\hline 28_BbCHRY & $0.1 \%$ & 26 & $0.0 \%$ & 27 & $0.0 \%$ & 27 \\
\hline 29_dBANT & $0.2 \%$ & 23 & $0.1 \%$ & 24 & $0.0 \%$ & 26 \\
\hline 30_SUMALL & $100.0 \%$ & $n / a$ & $100.0 \%$ & $n / a$ & $100.0 \%$ & $n / a$ \\
\hline 31_SUMLIGHT & $85.3 \%$ & $n / a$ & $89.6 \%$ & $n / a$ & $89.0 \%$ & $n / a$ \\
\hline 32_SUMHVY & $14.7 \%$ & $n / a$ & $10.4 \%$ & $n / a$ & $11.0 \%$ & $n / a$ \\
\hline
\end{tabular}


$\underline{\text { PAH Species Monitoring and Regulation }}$

Species monitored by Environment Canada at NAPS central sites are compared with species subject to NPRI reporting, CEPA toxicity, and US regulation in Table B.2-3.

Table B.2-3. PAH species comparison (NAPS, NPRI, CEPA-toxicity, US regulation).

\begin{tabular}{|c|c|c|c|c|c|}
\hline Count & PAH & $\begin{array}{c}\text { NAPS } \\
\text { Monitoring }\end{array}$ & $\begin{array}{c}\text { NPRI } \\
\text { Reporting }\end{array}$ & $\begin{array}{c}\text { CEPA-toxic } \\
\text { Status }\end{array}$ & $\begin{array}{l}\text { US ATSDR } \\
\text { Regulation }\end{array}$ \\
\hline 1 & PAHs (total) & $\cdot$ & $\cdot$ & $\mathrm{N} / \mathrm{A}$ & $\mathrm{N} / \mathrm{A}$ \\
\hline 2 & acenaphthylene & • & $\cdot$ & & \\
\hline 3 & acenapthene & $\cdot$ & • & & \\
\hline 4 & anthanthrene & • & & & \\
\hline 5 & anthracene & $\cdot$ & $\cdot$ & & \\
\hline 6 & benzo(a)anthracene & • & $\cdot$ & $\cdot$ & $\cdot$ \\
\hline 7 & benzo(a)phenanthrene & & $\cdot$ & $\cdot$ & \\
\hline 8 & benzo(a)pyrene & • & $\cdot$ & $\cdot$ & $\cdot$ \\
\hline 9 & benzo(b)chrysene & • & & & \\
\hline 10 & benzo(b)fluoranthene & - & • & • & • \\
\hline 11 & benzo(b)fluorene & • & & & \\
\hline 12 & benzo(e)pyrene & • & $\cdot$ & $\cdot$ & \\
\hline 13 & benzo(g,h,i)fluoranthene & $\cdot$ & & & \\
\hline 14 & benzo(g,h,i)perylene & • & $\cdot$ & $\cdot$ & $\cdot$ \\
\hline 15 & benzo(j)fluoranthene & & $\bullet$ & $\cdot$ & \\
\hline 16 & benzo(k)fluoranthene & • & $\cdot$ & $\cdot$ & $\cdot$ \\
\hline 17 & chrysene & • & & & $\cdot$ \\
\hline 18 & coronene & & & & $\cdot$ \\
\hline 19 & dibenz(a,j)acridine & & $\cdot$ & $\cdot$ & \\
\hline 20 & dibenzo(a,h)anthracene & • & $\cdot$ & $\cdot$ & \\
\hline 21 & dibenzo(a,h)perylene & & & & $\bullet$ \\
\hline 22 & dibenzo(a,i)pyrene & & $\cdot$ & $\cdot$ & \\
\hline 23 & fluoranthene & $\cdot$ & $\cdot$ & $\cdot$ & $\cdot$ \\
\hline 24 & fluorene & • & • & & • \\
\hline 25 & $\begin{array}{l}\text { indeno(1,2,3- } \\
\text { c,d)fluoranthene }\end{array}$ & - & & & \\
\hline 26 & indeno(1,2,3-c,d)pyrene & • & • & • & • \\
\hline 27 & napthalene & & $\bullet$ & & $\cdot$ \\
\hline 28 & perylene & $\cdot$ & $\cdot$ & $\cdot$ & \\
\hline 29 & phenanthrene & $\bullet$ & $\bullet$ & $\cdot$ & $\cdot$ \\
\hline 30 & pyrene & $\cdot$ & $\bullet$ & $\bullet$ & $\cdot$ \\
\hline 31 & retene & $\bullet$ & & & \\
\hline 32 & triphenylene & $\bullet$ & & & \\
\hline 33 & 1-Me-pyrene & • & & & \\
\hline 34 & 2-Me-fluorene & $\cdot$ & & & \\
\hline 35 & 3-me-cholanthrene & $\cdot$ & & & \\
\hline 36 & 7H-Dibenzo(c,g)carbazole & & $\cdot$ & $\cdot$ & \\
\hline 37 & 7-Me-benz(a)anthracene & $\cdot$ & & & \\
\hline
\end{tabular}

Notes:

1. NAPS-monitored species shown in bold typeface.

2. Species ordered alphabetically.

3. Data sourced from: NAPS monitoring suite (Environment Canada, 2007a); NPRI reporting (Environment Canada, 2007c); CEPA toxicity (2007d); US regulation (ATSDR, 1995). 


\section{B.3 Wind pattern analysis}

\section{$\underline{\text { Wind roses }}$}

Wind roses were prepared for the year-round dataset (2000-2010) and for winter and summer months (2000-2010) to determine seasonal differences in wind patterns. Historical hourly wind data were downloaded from Environment Canada's online searchable database (2011e) for the weather station nearest each central site monitor (i.e., Toronto airport 43.68 N, 79.63 W; Hamilton airport 43.17 N, 79.93 W; background colocated with NAPS sampler). Wind roses were constructed using WRPlot software (Lakes Environmental Inc.) and predominant wind directions compared across sites and assessed against the location of local PAH source areas (e.g., stationary sources, mobile sources); 24-hr and 48-hr wind roses were also constructed for days with the highest and lowest sampled PAH concentrations to better identify the probable influence of local source areas. Since Hamilton was to be analyzed more closely via intraurban field sampling (Anastasopoulos et al, 2012) and receptor modeled at the intraurban scale (Chapter 5), wind data were combined with central site PAH sampling data to construct a pollutant rose for $\Sigma_{29} \mathrm{PAH}$ (i.e., by concentration, by quartiles; WR Plot software, Lakes Environmental Inc.); the pollutant rose more directly indicated local source areas associated with high ambient PAH (i.e., upwind of central monitor during periods of high ambient PAH).

Westerly (i.e., NW/W/SW) winds were predominant at all sites (Figure B.3-1) and speeds were typically higher at the urban sites. Inter-site differences were relevant to source interpretation of receptor model results: at Hamilton, winds were predominantly from the $\mathrm{W}$ and SW but also NE (i.e., resultant vector $273^{\circ}$ ); at Toronto, winds were largely from the $\mathrm{W}$ or $\mathrm{N}$ (i.e., resultant vector $299^{\circ}$ ); at the background site the wind rose was more rounded than at the urban sites, with frequent NW and S winds (i.e., resultant vector $241^{\circ}$ ). Seasonal wind roses (Figure B.3-2) showed generally similar predominant wind direction as for the year-round data and similar wind direction between seasons, though wind speeds were more frequently higher in winter. 
$\underline{\text { Potential source areas for highest and lowest sampled PAH concentrations }}$

To identify potential source areas (i.e., located upwind of site), predominant wind directions were reviewed alongside land use surrounding the monitors. At Hamilton, predominant westerly winds indicated the site as most often downwind of mobile sources and moderately dense residential / commercial land use and often upwind of the city's industrial harbour-front (i.e., location of heavy and medium industry facilities inclusive of two iron/steel manufacturing facilities). At Toronto, predominant westerly winds indicated the site as most often downwind of mobile sources and dense residential / commercial land use. At the background site, local sources were few and NW winds indicated the site as downwind of a sparsely populated and forested region while $\mathrm{S}$ winds indicated lightly trafficked roadways as potential sources.

To better assess the probable influence of local source areas, a review was made of wind directions and speeds for days with the highest and lowest sampled PAH concentrations (i.e., statistically extreme outlier days; wind rose constructed for sampling day and prior day). At Hamilton, this confirmed that high PAH days were typically characterized by winds from the NE (i.e., industrial harbour-front and downtown source region rendered upwind) and by low wind speeds (i.e., reduced dispersion of pollutants); conversely, low PAH days were typically characterized by westerly winds (i.e., industrial harbour-front and downtown source regions downwind) despite low wind speeds (i.e., reduced dispersion of pollutants). At Toronto, some high PAH days (i.e., since Toronto had less data variance, reviewed statistically mild and statistically extreme outlier days) coincided with predominant wind directions (i.e., westerly), indicating mobile sources and sources associated with residential/commercial land use (e.g., space heating); however, several high PAH days also coincided with moderate-speed SW and S winds, which indicated influence of emissions from nearby industry (i.e., industry facilities reporting to NPRI and airport) and more distant industrialized regions across the narrow span of the lake (e.g., Hamilton iron/steel manufacturing). Conversely, low PAH days typically coincided with N/NE/E wind directions (i.e., more suburban land use to north and northeast, long span of the lake towards the east) and higher wind speeds (i.e., greater dispersion). At the background site, high PAH days (i.e., statistical extreme and mild outliers) coincided with low-speed southerly winds $(\mathrm{SE} / \mathrm{S} / \mathrm{SW})$ indicating nearby roadways and highway as the source region; conversely extreme low PAH days coincided with NW winds indicating the sparsely populated and forested area as local source region. 


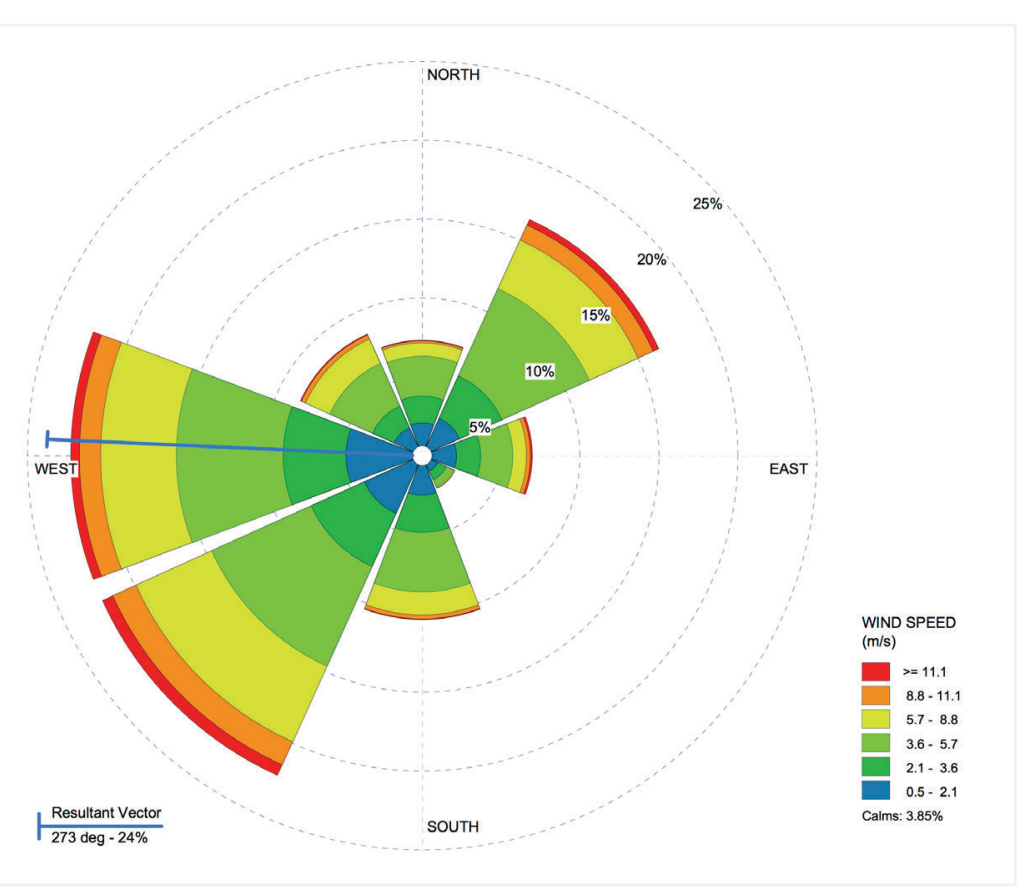

(b) Toronto (1999-2010)

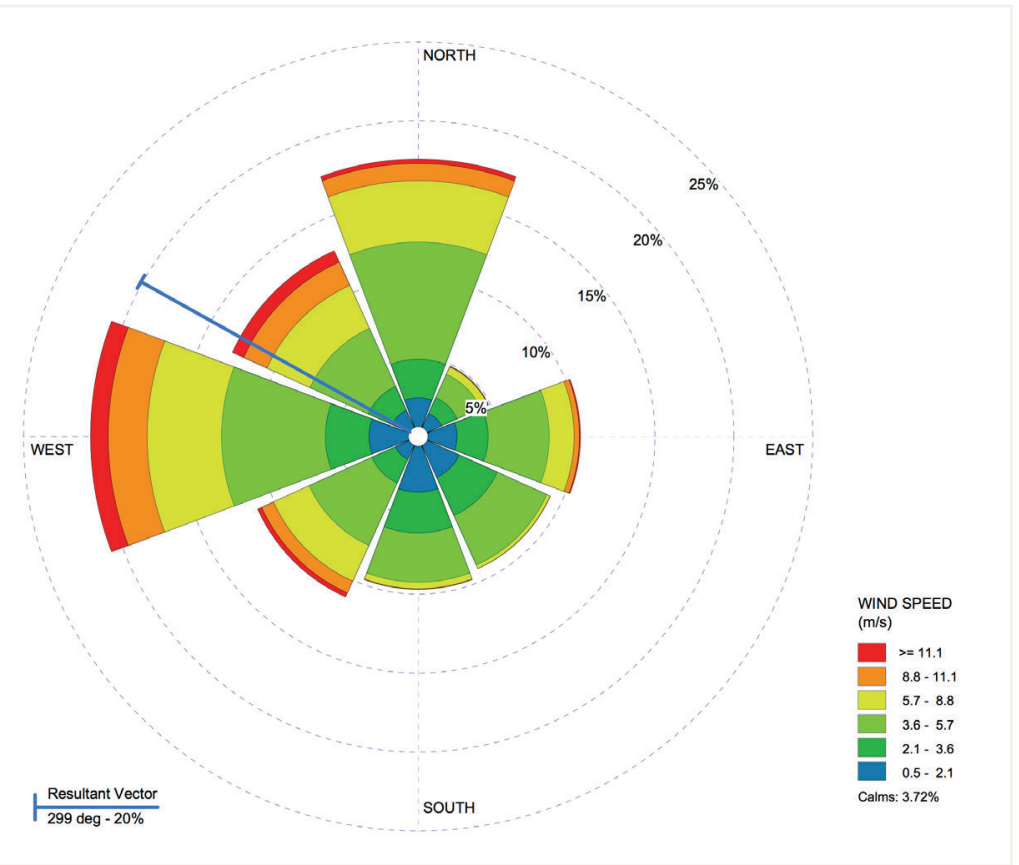

Figure B.3-1. Wind rose at urban and background sites. 
(c) background (Egbert; 2000-2010)

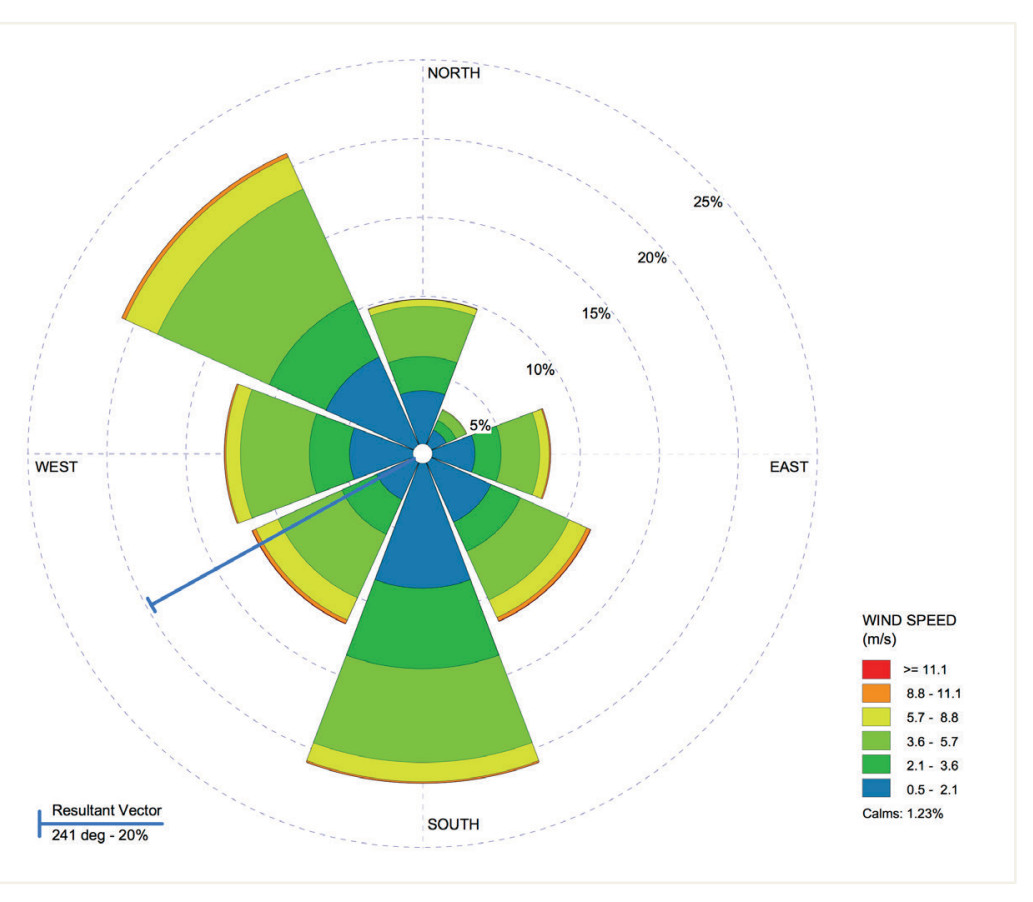

Figure B.3-1 (continued) 

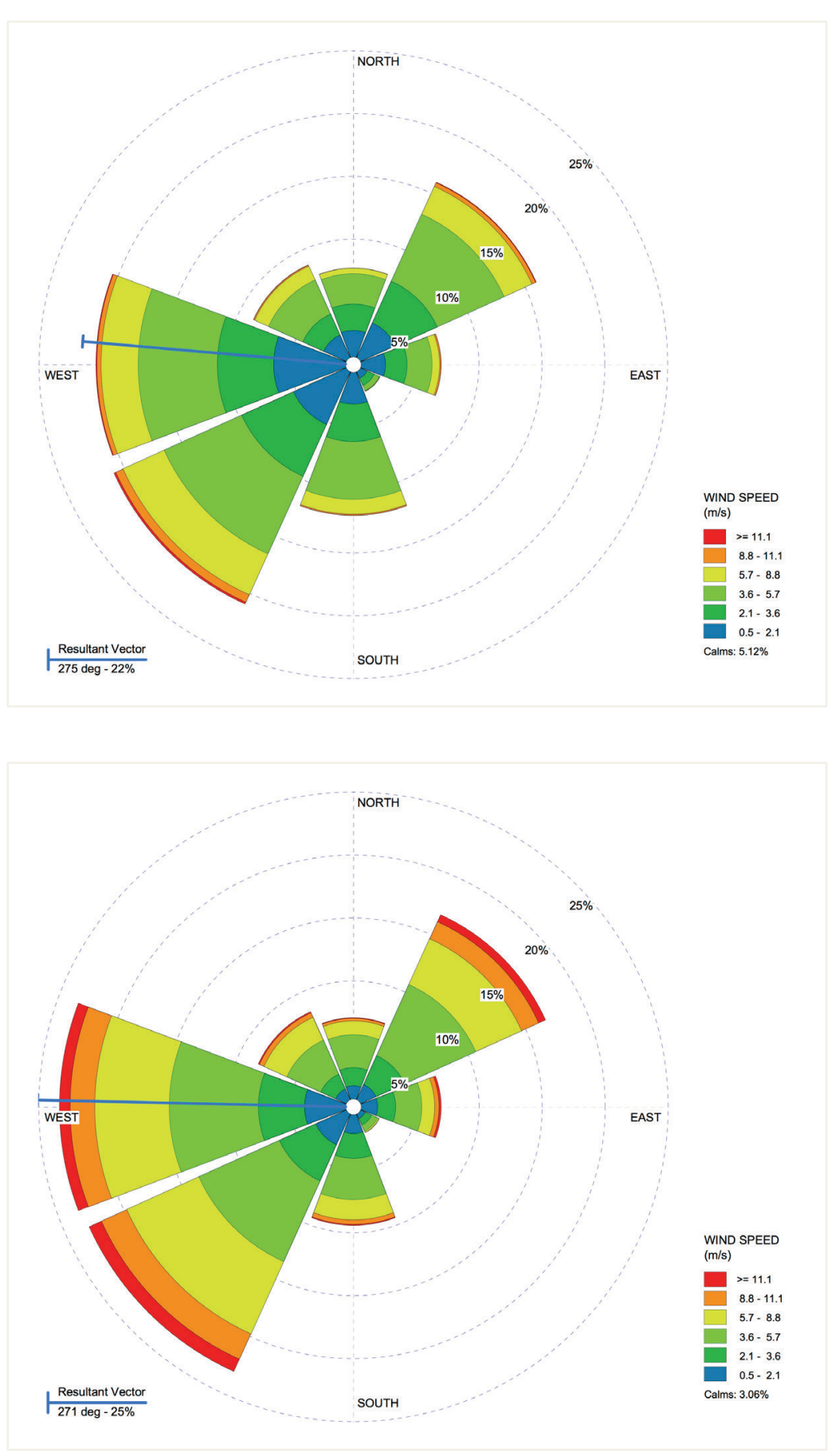

Figure B.3-2. Seasonal wind rose at urban and background sites (summer, winter). 
(b) Toronto (1999-2010)
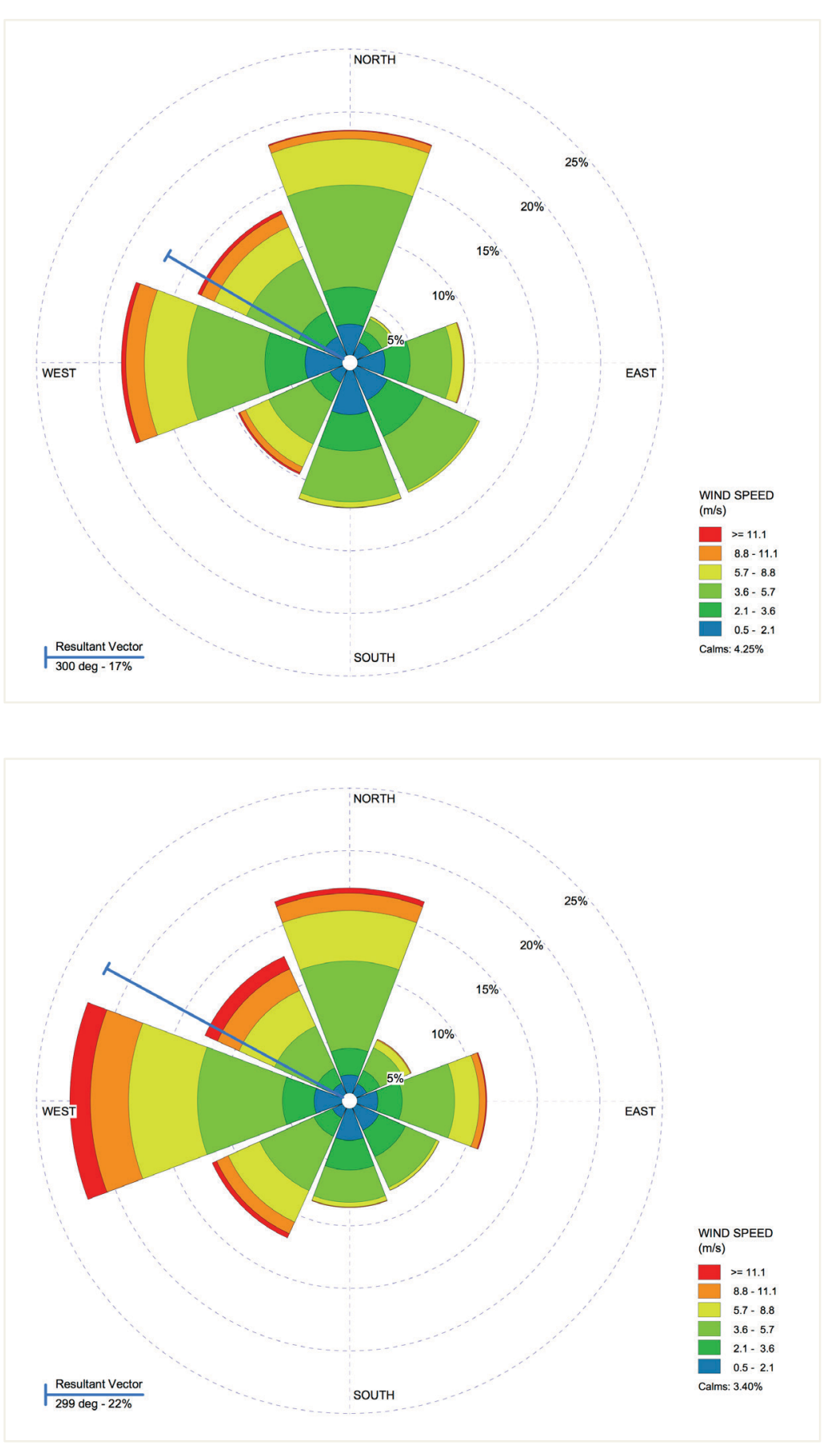

Figure B.3-2 (continued) 
(c) background (Egbert; 2000-2010)

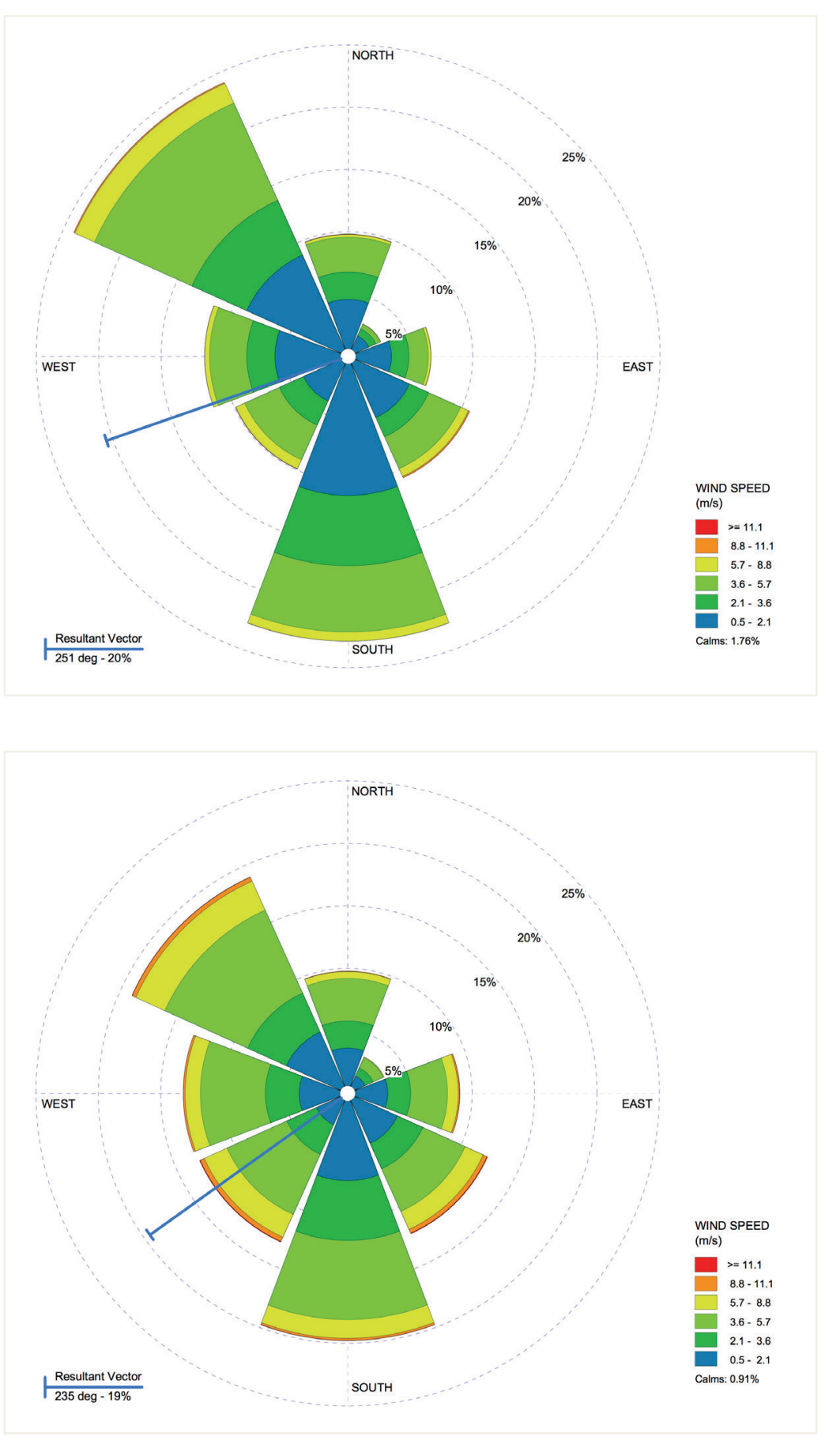

Figure B.3-2 (continued) 


\section{Pollutant rose analysis at Hamilton}

Since related research was to investigate ambient PAH in Hamilton more closely (i.e., intraurban field sampling: Anastasopoulos et al, 2012; intraurban source apportionment: Chapter 5), wind data were combined with the central site PAH data to construct a pollutant rose for $\Sigma_{29} \mathrm{PAH}$ (Figure B.3-3, $\mathrm{ng} / \mathrm{m}^{3}$ and quartiles). The pollutant rose proved a key observation as it confirmed the highest ambient PAH levels (third-quartile and greater concentrations) occurred when winds were from the NE direction, aligned with the city's industrial harbour-front. This occurred in summer and winter periods, consistent with industrial source emissions being significant year-round and thus influencing the central monitoring site when winds were conducive to source-receptor transport (i.e., industrial harbour-front upwind of central site). High PAH levels also occurred frequently for winds from the $\mathrm{N}$ and $\mathrm{E}$ suggesting that, in addition to upwind sources, the escarpment had an effect on increasing ambient concentrations by impeding further dispersion (i.e., central site monitor is located below the escarpment; N/NW/E classified as 'towards the escarpment'). In contrast, westerly winds (i.e., predominant wind direction, Figure B.3-1, Figure B-3-2) were sometimes coincident with thirdquartile and greater PAH concentrations but were more frequently coincident with low ambient PAH (i.e., first and second-quartile concentrations), suggesting less local PAH

sources in that direction and generally less contribution from sources above the escarpment. 
(a) $\Sigma$ PAH concentration $\left(\mathrm{ng} / \mathrm{m}^{3}\right)$

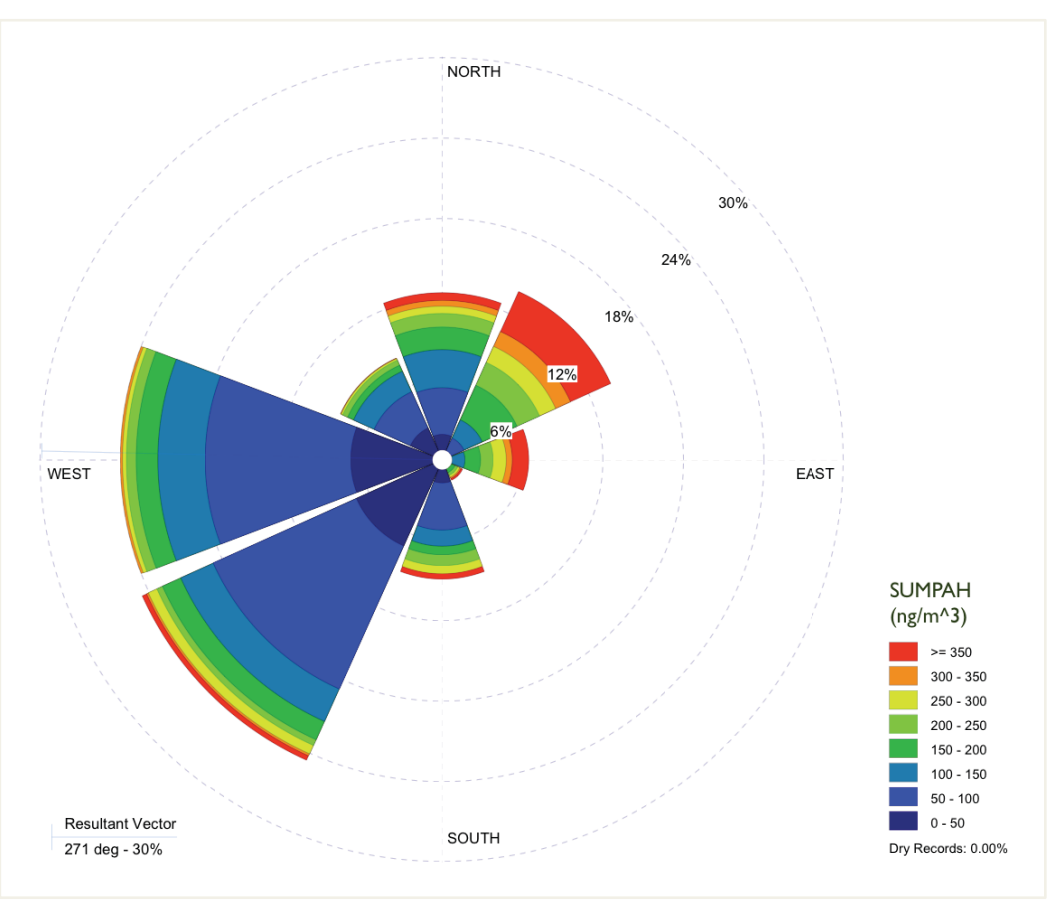

(b) $\Sigma P A H$ concentration $\left(\mathrm{ng} / \mathrm{m}^{3}\right.$, by quartile)

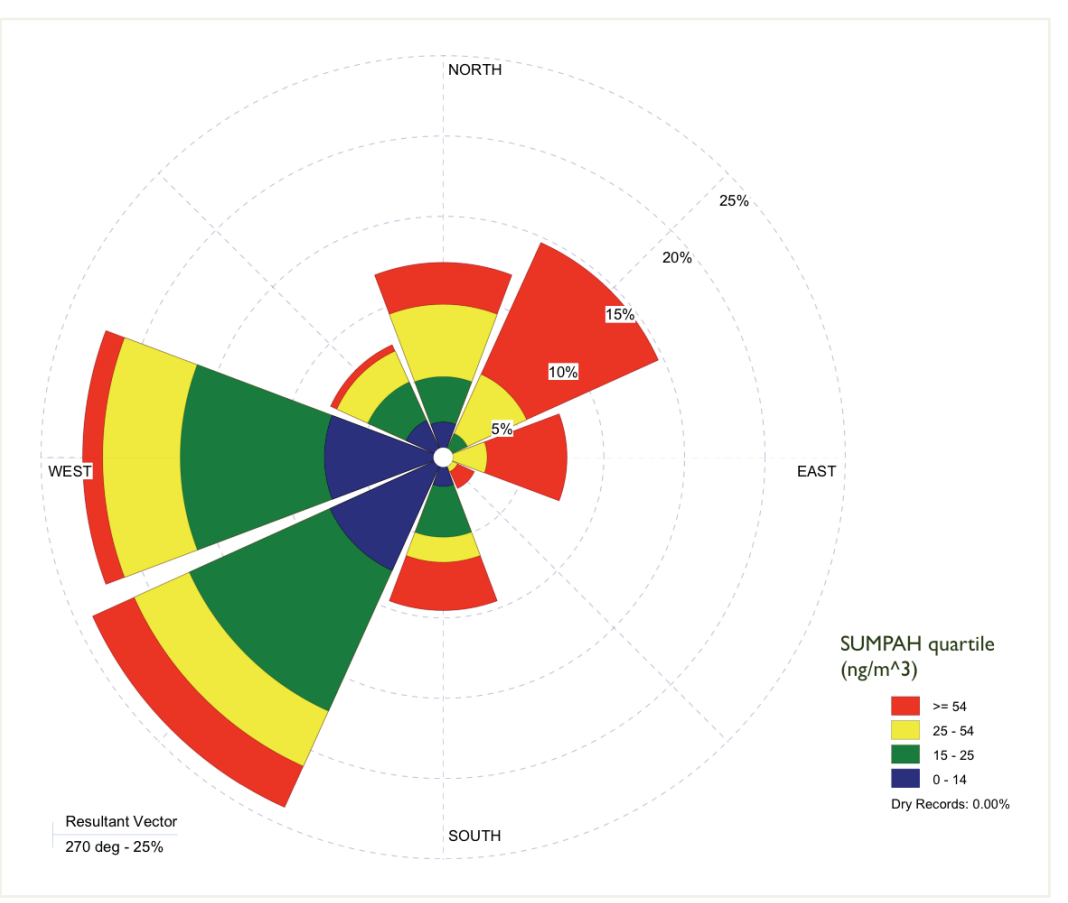

Figure B.3-3. Pollutant rose for $\Sigma$ PAH at urban site (Hamilton). 


\section{Conditional Probability Function (CPF)}

Probable source regions influencing the receptor sites, as indicated by wind pattern analysis and wind pollutant rose analysis, were found to be generally consistent with known local source regions (e.g., ambient PAH concentrations frequently higher in Hamilton for winds from the NE, aligned with location of harbour-front industry inclusive of iron/steel manufacturing).

Conditional Probability Function ( $\mathrm{CPF}$ ) analysis is an additional means of indicating local source regions that are likely influencing the receptor. CPF combines wind direction data with PMF model output (i.e., $F^{*} \mathrm{G}$ ) over the modeled time series, separately for each factor; as such, CPF is generally analogous to a pollutant rose but extended to PMF source contribution estimates. Wind directions that frequently align with high factor contributions can indicate a local source region, which can be checked for consistency with known local point or area sources. (Sonoma, 2008)

The frequency with which wind directions coincide with high factor contributions, and thus indicate the direction of likely local sources, is quantified by the factor's CPF, shown in Equation B.3-1.

As a probability, CPF can range from 0 (factor contributions were never high for a given wind direction) to 1 (factor contributions were always 'high' for a given wind direction).

'High factor contribution' sampling dates are typically defined as sampling dates where PMF source contribution estimate for a given factor are in the top-most portion of the range of source contribution estimates (i.e., above a set 'threshold value'). A typical and convenient threshold is the top $25 \%$ of source contribution estimates, since this corresponds to values greater than the third quartile $\left(\mathrm{Q}_{3}\right)$. However, depending on the range of a particular dataset, several different threshold criteria can be used, the goal being to indicate source directionality most clearly (Hopke, 2006).

Wind direction data is grouped (i.e., binned) into 'sectors' $(\Delta \Theta)$, with larger datasets (greater than 500 sampling dates in time series) permitting finer scale sectors $(\Delta \Theta=10$ $15^{\circ}$ ). Smaller PMF datasets, comparable to those modeled in this research, are typically analysed using a wind direction sector size of 20 to 30 degrees $\left(\Delta \Theta=20-30^{\circ}\right)$. 


\section{Equation B.3-1. Calculation of Conditional Probability Function (CPF).}

$$
\left.C P F_{\Delta \Theta}=\frac{m_{\Delta \Theta}}{n_{\Delta \Theta}} \quad \quad \text { [unitless }\right]
$$

$C P F_{\Delta \Theta_{k}}=\mathrm{CPF}$ value for sector $\Delta \Theta$, for source factor $\mathrm{k}$

$m_{\Delta \Theta}=$ \# of times factor contributions are 'high' while wind direction from sector $\Delta \Theta$

$n_{\Delta \Theta}=\#$ of times wind direction is from sector $\Delta \Theta$

For each factor $(\mathrm{k}), \mathrm{CPF}$ values (probabilities) corresponding to each wind direction sector $(\Delta \Theta)$ can then be plotted using a polar plot, visually identifying wind direction sectors where $\mathrm{CPF}$ was high for the factor. If a wind direction sector shows a CPF close to 1.0 , the method has indicated a high probability that a local source is found in that direction; the study area can then be reviewed for presence of local sources aligned with the wind direction indicated by the CPF plot to further corroborate the factor's source type interpretation.

CPF plots where no wind direction sectors are notably higher than others (e.g., a 'round' $\mathrm{CPF}$ plot) can also be useful, indicating that a particular source type does not have a single local source region (e.g., this may occur for area sources surrounding the receptor, such as space heating or PAH volatilization, depending on sampling site location, prevailing winds, and local topography) or that the receptor is influenced predominantly by sources outside the local area (i.e., regional transported sources and thus better located via regional-scale wind pattern analysis using backward trajectory model output such as from HYSPLIT coupled with PMF source contribution output using Potential Source Contribution Function analysis, PSCF; Heo et al, 2009). ${ }^{21}$

For clarity, an example of a CPF plot is shown in Figure B.3-4. The example plot shows that high factor contributions were most often aligned with easterly winds, indicating a possible source region $\mathrm{E} / \mathrm{SE}$ of the sampling site. (Kim et al, 2003)

${ }^{21} \mathrm{CPF}$ plots associated with known local sources (e.g., industry) that do not indicate a clear predominant wind direction may also be indicative of complex meteorological conditions (see Ogulei et al, 2005). 
Figure B.3-4. Example of plotted Conditional Probability Function (CPF); reproduced from Kim et al (2003).

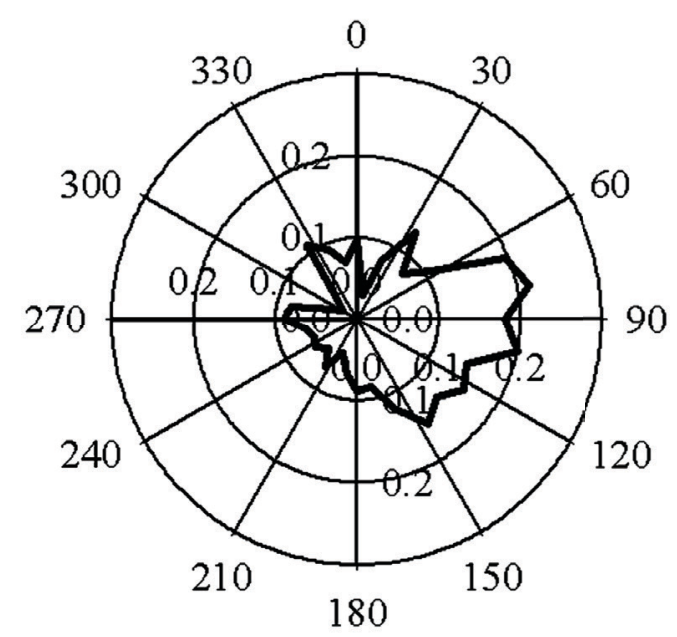

Additional practical notes on the CPF methodology (Kim et al, 2003; Hopke, 2006):

(i) exclude calm winds $(<1 \mathrm{~m} / \mathrm{s})$ as source directions are not well indicated in such conditions;

(ii) match temporal resolution of PMF output (single source contribution estimate for the sampling day, since based on 24-hr sampling) to wind direction data (hourly) by assigning the sampling date's source contribution estimate to each hour of the sampling date;

(iii) use fractional source contributions (i.e., dividing factor $\mathrm{k}$ contribution by sum of source contributions from all factors), rather than absolute source contributions so as to minimize the effect of atmospheric dilution (i.e., normalize for ambient $\Sigma \mathrm{PAH}$ level); and,

(iv) be mindful of anomalously high CPF values caused by wind directions with relatively few corresponding samples.

Additional details of the CPF methodology can be found in Kim et al (2003) and Hopke (2006). CPF can be calculated and plotted using Microsoft Excel or similar spreadsheet programs, with method described in Hopke (2006). 


\section{B.4 Principal Component Analysis (PCA)}

\section{METHODS}

PCA used as input data the same PAH dataset and species suite prepared for PMF modeling and revealed data 'structure' in the urban and background PAH datasets, principal components (PC) indicating patterns of co-varying PAH species. PCA was also used to indicate the number of factors that could be reasonably extracted from the dataset, providing a lower bound on the number of physically meaningful factors expected from application of the PMF receptor model, and to explore data sensitivity to seasonal stratification.

Majority of species datasets were found to log-normally distributed and were logtransformed (Equation B.4-1) prior to PCA modeling to reduce skew, reduce sensitivity to outliers, and provide more equal inter-species weighting in the model (Cao, 1999; Praveena, 2012; Reyment and Joreskog, 1993). While more complex PCA data transformations are available, such as conversion to proportional data using an indexing species (Reyment and Joreskog, 1993; Mudge, 2007) these were not advantageous to the analysis due to requiring exclusion of additional species (i.e., indexing species or most abundant species), abstracting the intended comparison to PMF, and particularly since source type interpretations were restricted to the more advanced PMF receptor model.

Equation B.4-1. Logarithmic data transformation for PCA receptor modeling.

$$
y_{i j}=\log _{10}\left(x_{i j}+1\right)
$$

$y_{i j}=\log$-transformed concentration of species $j$ in receptor sample $i$ (unitless)

$x_{i j}=$ concentration of species $j$ in receptor sample $i\left(\mathrm{ng} / \mathrm{m}^{3}\right)$

(concentrations increased by unity prior to calculation of base 10 logarithm to avoid zero or negative data values in PCA correlation matrix)

A Z-standardization (Equation B.4-2) was performed within the PCA software to equalize the large differences in concentration ranges typical of total ambient PAH data, since light and heavy MW species concentrations can differ by orders of magnitude, and to equalize species influence in the model (Joliffe, 2002; Reyment and Joreskog, 1993; Kavouras, 2001; Berg, 1994).

The PCA was run using SYSTAT 13 (Systat Software Inc., Chicago, IL, USA) with a correlation matrix and Varimax rotation. Extreme outliers in species datasets were defined as values greater than or less than three times the IQR and identified using Microsoft Excel and MINITAB 12 and, since the PCA showed moderate sensitivity to 
outliers, sample dates with extreme outlier concentrations in any species aside from retene ${ }^{22}$ were generally omitted. This was done to ensure greater consistency with PMF modeling protocol, in which extreme outliers are generally excluded (US EPA, 2008; Reff et al, 2007). Extreme outliers were also excluded to improve factor separation, observed as greater factor loading strength for characteristic species and better distinction between factors as per Thurstone's criteria, which are summarized as low loading in each row of the rotated factor loading matrix, a number of low species loadings in each factor equal or greater than the model order, and all factor pairings showing some species with high loadings on one factor and near-zero loadings on the other as well as several species with low loadings on both factors (Reyment and Joreskog, 1993).

\section{Equation B.4-2. Z-transformation applied to PCA data.}

$$
\begin{gathered}
S N_{j}=\sqrt{\frac{\sum_{i=1}^{n}\left(x_{i j}-s_{i j}\right)^{2}}{\sum_{i=1}^{n} s_{i j}{ }^{2}}} \\
S N_{j}=\text { signal-to-noise ratio for species } j \\
x_{i j}=\text { concentration of species } j \text { in receptor sample } i\left(\mathrm{ng} / \mathrm{m}^{3}\right) \\
S_{i j}=\text { uncertainty of species } j \text { in receptor sample } i\left(\mathrm{ng} / \mathrm{m}^{3}\right)
\end{gathered}
$$

Models with three to eight factors were run and the optimal model order retained for analysis and comparison across sites was selected by examining several criteria simultaneously, including slope changes in the scree plot, Thurstone's criteria for factor separation mentioned above, and retaining only factors explaining $>5 \%$ of total variance. (Reyment and Joreskog, 1993) All factors with eigenvalues $>0.25$ were examined, a lower bound set less than Kaiser's rule of 1.0 to ensure that factors are not underextracted by the PCA (Hopke, 1982). Absolute rotated factor loadings values greater than 0.6 were classified as 'high' so as to be consistent with the correlation strength typically accepted as 'mapped' in bootstrap analysis of PMF results; absolute factor loading values between 0.4 and 0.6 were classified as 'moderate'.

\footnotetext{
${ }^{22}$ Excluding dates with statistically extreme retene concentration did not improve factor loading or factor separation at any of the modeled sites, for either year-round or seasonally-stratified datasets, and generally only served to reduce variance in the retene dataset thereby hindering model's ability to clearly extract a separate retene factor. This was expected a priori, since retene was not found to be well correlated with other species and is generally considered a useful, albeit imperfect, marker species (Jang et al, 2013; Shen et al, 2012; Ramdahl et al, 1983).
} 


\section{RESULTS}

\section{Factor loading patterns}

PCA results at the three sites are shown in Figure B.4-1 (i.e., rotated factor loadings, eigenvalue, $\%$ variance explained, cumulative $\%$ variance). Site data showed an optimal model order of four (i.e., supported by elbow in scree plot, good separation of species groupings, retene as separate factor, eigenvalues greater than or near 1.0 for all factors, each factor explaining approximately $\geq 5 \%$ variance). ${ }^{23}$ Cumulatively, four factors explained approximately 94\% (Hamilton), 88\% (Toronto) and 85\% (Egbert) of total data variance. The PCA model explained the most data variance at Hamilton, expected to show greatest number of influencing local sources, and least at the rural background site Egbert.

PCA factor loading patterns were generally expected: (i) they are consistent with PAH vapour/particle partitioning behaviour (i.e., volatile/light MW species found primarily in vapour phase, medium MW species found in vapour and particle phases, heavy MW species found in particle phase; Venkataraman and Friedlander, 1994); (ii) retene is uncorrelated with other sampled species and considered a useful, albeit imperfect, marker for wood combustion (Jang et al, 2013; Shen et al, 2012; Ramdahl et al, 1983); and, (iii) PCA model results were less well resolved (i.e., less distinct differences in loadings for some species across factors, lower explained variance) at the background site where data quality was lowest (i.e., less samples in time series, sampled concentrations much nearer method detection limits) and source-receptor distances were greatest. Thus, while the results confirmed the general correlations between sampled species and expected urbanrural differences, source interpretation was left to the more advanced PMF receptor model. ${ }^{24}$

PCA factor loading patterns were very similar between Toronto and Hamilton and differed more for the background site. This was consistent with the urban sites having a number of local sources contributing to their variance and the remote location of the

\footnotetext{
${ }^{23}$ At the rural background site retene appeared as a sole-species factor at a higher order fivefactor solution; however, the four-factor solution was retained as 'optimal' for comparison with the urban sites since retene was still clearly discernable as a separate factor, appearing alongside medium MW species.

${ }^{24}$ A complementary PCA using a proportional data transformation is presented in Appendix 0. This showed results similar to the PCA on log-transformed data, inclusive of seasonal sensitivity analysis, but with enhanced species separation in factor loading patterns.
} 
background site meaning inherently poorer source-receptor conservation as well as reduced species data variance and poorer data quality.

The first factor (i.e., explained $40 \%$ - $45 \%$ of data variance) was characterized by high loadings ( $>0.6)$ on all heavy MW species (CHRY through IPYR, exception of RET) and was labeled "heavy", summarized as: primary factor at all sites; factor loading pattern generally similar across sites suggesting a corresponding source type impacting all locations; FLT and PYR loading less clearly separated to a single factor at Hamilton, indicating the PCA modeled Hamilton data variance less well while the more advanced PMF model could be reasonably expected to resolve a site-unique source type at a higher order solution. ${ }^{25}$ The second factor $(21 \%-27 \%$ explained variance) was most highly loaded in the light species PHEN alongside high loadings in FLT and PYR, labeled "light": similar at the urban sites (Hamilton, Toronto); minor difference at the background site (i.e., moderately loaded in the lighter species ACE, FLU alongside otherwise similar high loadings in PHEN, FLT, PYR). The third factor (8\% - 16\% explained variance) was highly loaded in some or all of the lightest and most volatile species (ACY, ACE, FLU), labeled "volatile": most similar at the urban sites; differed more at background site (i.e., high loading of ACY and moderate loading of ACE, low loading of FLU). Last, a variant of a "retene" factor (i.e., characterized by high loading in the wood combustion marker species RET) was resolved at all sites (5\% to $16 \%$ explained variance): RET the sole highly loaded species and explained similar amount of data variance at the urban sites (5\% at Hamilton, $8 \%$ at Toronto); RET co-extracted alongside reacted species MPYR at background site, suggesting wood combustion source type with greater transport (i.e., me-PAH indicating aging of parent $\mathrm{PAH}$ ), consistent with the low and sparsely distributed nearby rural population.

${ }^{25}$ Higher order PCA models at Hamilton did not resolve additional factors with high loading $(>0.6)$ in any species, limiting PCA model to four factors.

Appendix B: Urban/rural source apportionment 
(a) by site

Toronto (urban) factors.

\begin{tabular}{|l|rrrr|}
\hline TOR_0010 & F1 & F2 & F3 & F4 \\
\hline -01_ACY & 0.21 & 0.70 & -0.16 & 0.51 \\
_02_ACE & 0.07 & 0.91 & 0.04 & 0.12 \\
_03_FLU & 0.17 & 0.85 & 0.36 & 0.08 \\
_04_ANT & 0.37 & 0.16 & 0.75 & 0.25 \\
_05_PHE & 0.13 & 0.03 & 0.93 & -0.13 \\
_06_MFLU & 0.19 & 0.49 & 0.71 & 0.08 \\
_07_FLT & 0.42 & -0.03 & 0.85 & -0.02 \\
_08_PYR & 0.55 & 0.08 & 0.79 & 0.16 \\
_09_MPYR & 0.60 & 0.05 & 0.56 & 0.35 \\
_10_BAFLU & 0.76 & 0.07 & 0.49 & 0.31 \\
-11_BBFLU & 0.68 & 0.09 & 0.46 & 0.41 \\
-12_BGHIFL & 0.66 & 0.18 & 0.39 & 0.50 \\
_13_CHRY & 0.89 & 0.08 & 0.35 & 0.19 \\
_14_TRIPH & 0.81 & 0.05 & 0.46 & 0.14 \\
_15_BAA & 0.91 & 0.02 & 0.28 & 0.08 \\
_16_RET & 0.12 & 0.26 & 0.04 & 0.76 \\
_18_BAP & 0.93 & 0.07 & 0.16 & -0.07 \\
_19_BEP & 0.94 & 0.16 & 0.22 & 0.12 \\
_20_BBFLT & 0.94 & 0.18 & 0.20 & 0.12 \\
_21_BKFLT & 0.96 & 0.10 & 0.16 & 0.01 \\
_25_BGHIP & 0.84 & 0.24 & 0.23 & 0.26 \\
_26_IPYR & 0.93 & 0.18 & 0.21 & 0.13 \\
\hline eigenvalue & 13.9 & 2.4 & 2.2 & 0.9 \\
\% variance & 45 & 12 & 23 & 8 \\
\% cum var & 45 & 57 & 80 & 88 \\
\hline
\end{tabular}

Hamilton (urban) factors.

\begin{tabular}{|c|c|c|c|c|}
\hline HAM_0010 & F1 & $\mathrm{F} 2$ & F3 & $\mathrm{F} 4$ \\
\hline 01_ACY & 0.28 & 0.91 & 0.08 & 0.09 \\
\hline 02 ACE & 0.30 & 0.83 & 0.06 & 0.25 \\
\hline 03_FLU & 0.41 & 0.64 & 0.03 & 0.59 \\
\hline 04_ANT & 0.55 & 0.33 & 0.10 & 0.71 \\
\hline 05_PHE & 0.46 & 0.13 & 0.11 & 0.83 \\
\hline _06_MFLU & 0.38 & 0.39 & 0.11 & 0.72 \\
\hline _07_FLT & 0.61 & 0.16 & 0.07 & 0.74 \\
\hline _08_PYR & 0.63 & 0.23 & 0.09 & 0.72 \\
\hline _09_MPYR & 0.72 & 0.15 & 0.17 & 0.60 \\
\hline _10_BAFLU & 0.73 & 0.23 & 0.06 & 0.59 \\
\hline _11_BBFLU & 0.73 & 0.25 & 0.03 & 0.56 \\
\hline _12_BGHIFL & 0.71 & 0.38 & 0.17 & 0.47 \\
\hline _13_CHRY & 0.79 & 0.31 & 0.08 & 0.50 \\
\hline _14_TRIPH & 0.77 & 0.28 & 0.06 & 0.52 \\
\hline _15_BAA & 0.83 & 0.23 & 0.12 & 0.45 \\
\hline _16_RET & 0.10 & 0.09 & 0.98 & 0.12 \\
\hline _18_BAP & 0.88 & 0.24 & 0.08 & 0.32 \\
\hline _19_BEP & 0.81 & 0.35 & 0.05 & 0.43 \\
\hline _20_BBFLT & 0.81 & 0.39 & 0.05 & 0.43 \\
\hline 21_BKFLT & 0.84 & 0.38 & 0.06 & 0.37 \\
\hline _25_BGHIP & 0.82 & 0.41 & 0.07 & 0.33 \\
\hline 26_IPYR & 0.82 & 0.39 & 0.06 & 0.39 \\
\hline eigenvalue & 17.6 & 1.3 & 1.0 & 0.7 \\
\hline$\%$ variance & 45 & 16 & 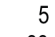 & 27 \\
\hline$\%$ cum var & 45 & 61 & 66 & 94 \\
\hline
\end{tabular}

Egbert (rural/background) factors.

\begin{tabular}{|l|rrrr|}
\hline EGB_0010 & F1 & F2 & F3 & F4 \\
\hline -01_ACY & 0.27 & 0.27 & 0.21 & 0.82 \\
_02_ACE & 0.22 & -0.03 & 0.60 & 0.53 \\
_03_FLU & 0.36 & 0.08 & 0.85 & 0.18 \\
_04_ANT & 0.05 & 0.53 & 0.37 & 0.52 \\
_05_PHE & 0.25 & 0.32 & 0.87 & 0.14 \\
_06_MFLU & 0.24 & 0.17 & 0.87 & 0.08 \\
_07_FLT & 0.52 & 0.33 & 0.71 & 0.15 \\
_08_PYR & 0.35 & 0.52 & 0.64 & 0.11 \\
_09_MPYR & 0.33 & 0.82 & 0.22 & 0.19 \\
_10_BAFLU & 0.47 & 0.71 & 0.38 & 0.24 \\
_11_BBFLU & 0.40 & 0.71 & 0.31 & 0.19 \\
-12_BGHIFL & 0.79 & 0.37 & 0.31 & 0.21 \\
_13_CHRY & 0.86 & 0.26 & 0.28 & 0.21 \\
-14_TRIPH & 0.79 & 0.15 & 0.39 & 0.25 \\
_15_BAA & 0.80 & 0.35 & 0.19 & -0.07 \\
_16_RET & -0.09 & 0.80 & 0.01 & -0.03 \\
-18_BAP & 0.86 & 0.05 & 0.21 & -0.24 \\
_19_BEP & 0.92 & 0.10 & 0.23 & 0.18 \\
_20_BBFLT & 0.91 & 0.12 & 0.24 & 0.26 \\
_21_BKFLT & 0.92 & 0.09 & 0.21 & 0.18 \\
_25_BGHIP & 0.90 & 0.10 & 0.26 & 0.11 \\
_26_IPYR & 0.90 & 0.09 & 0.18 & 0.21 \\
\hline eigenvalue & 13.4 & 2.8 & 1.6 & 1.0 \\
\% variance & 40 & 16 & 21 & 8 \\
\% cum var & 40 & 56 & 77 & 85 \\
\hline & & & &
\end{tabular}

(b) species patterns

HEAVY factor.

\begin{tabular}{|c|c|c|c|}
\hline & TOR & HAM & EGB \\
\hline species & $\overline{F 1}$ & F1 & $\overline{F 1}$ \\
\hline 01_ACY & 0.21 & 0.28 & 0.2 \\
\hline 02_ACE & 0.07 & 0.30 & 0.2 \\
\hline _03_FLU & 0.17 & 0.41 & 0.36 \\
\hline _04_ANT & 0.37 & 0.55 & 0.05 \\
\hline 05_PHE & 0.13 & 0.46 & \\
\hline 06_MFLU & 0.19 & 0.38 & 0.2 \\
\hline _07_FLT & 0.42 & 0.61 & 0.5 \\
\hline 08_PYR & 0.55 & 0.63 & 0.35 \\
\hline 09_MPYR & 0.60 & 0.72 & 0.3 \\
\hline _10_BAFLU & 0.76 & 0.73 & 0.4 \\
\hline _11_BBFLU & 0.68 & 0.73 & 0.40 \\
\hline 12_BGHIFL & 0.66 & 0.71 & 0.7 \\
\hline 13_CHRY & 0.89 & 0.79 & 0.8 \\
\hline -14_TRIPH & 0.81 & 0.77 & 0.79 \\
\hline _15_BAA & 0.91 & 0.83 & $0 . \varepsilon$ \\
\hline 16_RET & 0.12 & 0.10 & -0.0 \\
\hline _18_BAP & 0.93 & 0.88 & 0.1 \\
\hline _19_BEP & 0.94 & 0.81 & 0.92 \\
\hline 20_BBFLT & 0.94 & 0.81 & 0.6 \\
\hline 21_BKFLT & 0.96 & 0.84 & 0. \\
\hline 25_BGHIP & 0.84 & 0.82 & $0.9 c$ \\
\hline 26_IPYR & 0.93 & 0.82 & 0.5 \\
\hline eigenvalue & 13.9 & 17.6 & \\
\hline$\%$ & 45 & 45 & \\
\hline
\end{tabular}

LIGHT factor.

TOR HAM EGB

\begin{tabular}{|c|c|c|c|}
\hline species & $\overline{F 3}$ & $\overline{F 4}$ & $\overline{F 3}$ \\
\hline 01_ACY & -0.16 & 0.09 & 0.21 \\
\hline 02_ACE & 0.04 & 0.25 & $0 . t$ \\
\hline 03_FLU & 0.36 & 0.59 & 0.8 \\
\hline _04_ANT & 0.75 & 0.71 & 0.3 \\
\hline _05_PHE & 0.93 & 0.83 & 0.87 \\
\hline 06_MFLU & 0.71 & 0.72 & 0.8 \\
\hline 07_FLT & 0.85 & 0.74 & 0.7 \\
\hline _08_PYR & 0.79 & 0.72 & 0.64 \\
\hline 09_MPYR & 0.56 & 0.60 & 0.22 \\
\hline 10_BAFLU & 0.49 & 0.59 & 0.38 \\
\hline 11_BBFLU & 0.46 & 0.56 & 0.3 \\
\hline 12_BGHIF & 0.39 & 0.47 & 0.31 \\
\hline _13_CHRY & 0.35 & 0.50 & 0.28 \\
\hline 14_TRIPH & 0.46 & 0.52 & 0.3 \\
\hline _15_BAA & 0.28 & 0.45 & 0.1 \\
\hline _16_RET & 0.04 & 0.12 & 0.01 \\
\hline 18_BAP & 0.16 & 0.32 & 0.2 \\
\hline _19_BEP & 0.22 & 0.43 & 0.2 \\
\hline 20_BBFLT & 0.20 & 0.43 & 0.24 \\
\hline 21_BKFLT & 0.16 & 0.37 & 0.2 \\
\hline 25_BGHIP & 0.23 & 0.33 & 0.2 \\
\hline 26_IPYR & 0.21 & 0.39 & 0.18 \\
\hline eigenvalue & 2.2 & 0.7 & 1.6 \\
\hline$\%$ variance & 23 & 27 & \\
\hline
\end{tabular}

VOLATILE factor.

\begin{tabular}{|c|c|c|c|}
\hline species & $\bar{F} 2$ & $\overline{\mathrm{F} 2}$ & $\overline{F 4}$ \\
\hline _01_ACY & 0.70 & 0.91 & 0.8 \\
\hline _02_ACE & 0.91 & 0.83 & 0.53 \\
\hline _03_FLU & 0.85 & 0.64 & 0.1 \\
\hline _04_ANT & 0.16 & 0.33 & \\
\hline _05_PHE & 0.03 & 0.13 & 0.1 \\
\hline _06_MFLU & 0.49 & 0.39 & 0.08 \\
\hline _07_FLT & -0.03 & 0.16 & 0.1 \\
\hline _08_PYR & 0.08 & 0.23 & \\
\hline 09_MPYR & 0.05 & 0.15 & 0.1 \\
\hline _10_BAFLU & 0.07 & 0.23 & 0.24 \\
\hline _11_BBFLU & 0.09 & 0.25 & 0.1 \\
\hline 12 B BGHIF & 0.18 & 0.38 & 0.2 \\
\hline _13_CHRY & 0.08 & 0.31 & 0.2 \\
\hline _14_TRIPH & 0.05 & 0.28 & 0.2 \\
\hline 15 & 0.02 & 0.23 & -0.0 \\
\hline _16_RET & 0.26 & 0.09 & -0.0 \\
\hline _18_BAP & 0.07 & 0.24 & -0.2 \\
\hline _19_BEP & 0.16 & 0.35 & 0.1 \\
\hline 20_BBFLT & 0.18 & 0.39 & 0.2 \\
\hline 21_BKFLT & 0.10 & 0.38 & 0.1 \\
\hline 25_BGHIP & 0.24 & 0.41 & 0. \\
\hline $26 \_$IPYR & 0.18 & 0.39 & 0.2 \\
\hline eigenvalue & 2.4 & 1.3 & 1. \\
\hline$\%$ variance & 12 & 16 & \\
\hline
\end{tabular}

RETENE factor.

\begin{tabular}{|l|r|r|r|}
\multicolumn{1}{c}{ TOR } & HAM & EGB \\
\hline species & F4 & F 3 & F2 \\
\hline -01_ACY & 0.51 & 0.08 & 0.27 \\
-02_ACE & 0.12 & 0.06 & -0.03 \\
-03_FLU & 0.08 & 0.03 & 0.08 \\
-04_ANT & 0.25 & 0.10 & 0.53 \\
-05_PHE & -0.13 & 0.11 & 0.32 \\
-06_MFLU & 0.08 & 0.11 & 0.17 \\
-07_FLT & -0.02 & 0.07 & 0.33 \\
-08_PYR & 0.16 & 0.09 & 0.52 \\
-09_MPYR & 0.35 & 0.17 & 0.82 \\
-10_BAFLU & 0.31 & 0.06 & 0.71 \\
-11_BBFLU & 0.41 & 0.03 & 0.71 \\
-12_BGHIFL & 0.50 & 0.17 & 0.37 \\
-13_CHRY & 0.19 & 0.08 & 0.26 \\
-14_TRIPH & 0.14 & 0.06 & 0.15 \\
-15_BAA & 0.08 & 0.12 & 0.35 \\
-16_RET & 0.76 & 0.98 & 0.80 \\
-18_BAP & -0.07 & 0.08 & 0.05 \\
-19_BEP & 0.12 & 0.05 & 0.10 \\
-20_BBFLT & 0.12 & 0.05 & 0.12 \\
21_BKFLT & 0.01 & 0.06 & 0.09 \\
25_BGHIP & 0.26 & 0.07 & 0.10 \\
-26_IPYR & 0.13 & 0.06 & 0.09 \\
\hline eigenvalue & 0.9 & 1.0 & 2.8 \\
\% variance & 8 & 5 & 16 \\
\hline
\end{tabular}

Figure B.4-1. PCA rotated factor loadings at urban and rural sites (log-transformed data). 
To explore the model's sensitivity to seasonal stratification of the input data, year-round and seasonally stratified (i.e., 'summer', 'winter') PCA model factors were compared (Figure B.4-2-a). Urban PCA results showed little seasonal sensitivity (i.e., model order and factor loading patterns similar between year-round, winter, and summer models) while the background site showed moderate sensitivity. Generally, year-round PCA factors more closely resembled winter factors than summer factors; this was expected: winter dates comprised a greater portion of the year-round dataset at all sites; local source changes in winter (i.e., space heating sources, volatilization/re-volatilization source emissions reduced); and, transport-related effects on source-receptor conservation greatest in summer due to elevated temperatures and reduced atmospheric stability.

By species (Figure B.4-2-b), heavy MW PAH showed the least sensitivity to seasonal split (i.e., factors extracted for the summer and winter data subsets had similar factor loading patterns as those extracted from the entire dataset); light MW species, inclusive of more volatile species (ACE, FLU) also showed fairly small sensitivity to seasonal split. The arrangement of heavy and light species in factor patterns that were broadly similar between year-round and seasonal model results is consistent with phase partitioning of these species groups being relatively independent of season (i.e., light species largely in vapour phase, heavy species largely in particle phase); medium MW species proved moderately sensitive to seasonal split at all sites and this was unsurprising as their vapour/particle phase partitioning is more variable (Venkataraman and Friedlander, 1994). Greater sensitivity to seasonal stratification was also seen for the most highly volatile species ACY but attributing this result solely to seasonality (i.e., high temperatures) was confounded by a small summer sample size at two of the three sites (i.e., Hamilton, background). Even so, results implied an advantage for the more advanced PMF receptor model: species with larger seasonal sensitivity, if included in the model, could be assigned greater uncertainty.

Overall, while winter sampling data were considered more amenable to receptor modeling a priori (i.e., greater source-receptor conservation due to lower temperatures, greater atmospheric stability, and additional and/or more intense source emissions), PCA results showed little seasonal sensitivity. Since other pollutant datasets may differ in this regard, a sensitivity analysis to seasonal stratification remains recommended as part of receptor modeling best practice, as demonstrated in this research by comparison of yearround and seasonally stratified model factors. 
(a) by site

TORONTO (urban)

\begin{tabular}{|c|c|c|c|c|c|c|c|c|c|c|c|c|c|}
\hline $\log (C)$ & SW & \multicolumn{3}{|c|}{$n=365$} & \multicolumn{2}{|c|}{ SUMMER } & \multicolumn{3}{|c|}{$n=150$} & \multicolumn{2}{|c|}{ WINTER } & \multicolumn{2}{|l|}{$n=205$} \\
\hline \begin{tabular}{l|l} 
TOR_0010 \\
\end{tabular} & $\overline{F 1}$ & $\overline{F 2}$ & F3 & F4 & F1 & $\overline{F 2}$ & $\overline{F 3}$ & $\overline{F 4}$ & F5 & F1 & $\overline{F 2}$ & F3 & F4 \\
\hline 01_ACY & 0.36 & -0.17 & 0.65 & 0.50 & 0.00 & 0.01 & 0.48 & -0.81 & 0.01 & 0.38 & 0.15 & 0.73 & 0.33 \\
\hline 02_ACE & 0.17 & 0.04 & 0.88 & 0.14 & 0.10 & 0.06 & 0.91 & -0.15 & 0.16 & 0.11 & 0.28 & 0.90 & 0.03 \\
\hline 03_FLU & 0.27 & 0.34 & 0.83 & 0.06 & 0.11 & 0.31 & 0.88 & -0.12 & -0.10 & 0.26 & 0.80 & 0.42 & 0.02 \\
\hline 04_ANT & 0.26 & 0.82 & 0.24 & 0.23 & 0.21 & 0.84 & 0.16 & -0.22 & 0.14 & 0.43 & 0.75 & 0.28 & 0.20 \\
\hline 05_PHE & 0.06 & 0.95 & 0.00 & -0.11 & 0.19 & 0.81 & 0.37 & 0.27 & 0.19 & 0.29 & 0.91 & 0.03 & 0.05 \\
\hline _06_MFLU & 0.21 & 0.69 & 0.51 & 0.07 & 0.13 & 0.67 & 0.45 & -0.33 & -0.15 & 0.28 & 0.84 & 0.23 & 0.02 \\
\hline _07_FLT & 0.32 & 0.89 & -0.05 & -0.06 & 0.35 & 0.79 & 0.22 & 0.34 & 0.20 & 0.60 & 0.74 & 0.03 & 0.06 \\
\hline _08_PYR & 0.47 & 0.84 & 0.09 & 0.13 & 0.43 & 0.83 & 0.15 & 0.17 & 0.16 & 0.64 & 0.70 & 0.13 & 0.14 \\
\hline _09_MPYR & 0.55 & 0.65 & 0.12 & 0.38 & 0.55 & 0.74 & -0.02 & -0.14 & 0.19 & 0.62 & 0.59 & 0.13 & 0.32 \\
\hline _10_BAFLU & 0.72 & 0.49 & 0.20 & 0.30 & 0.68 & 0.65 & -0.05 & -0.07 & 0.09 & 0.73 & 0.53 & 0.23 & 0.19 \\
\hline _11_BBFLU & 0.70 & 0.39 & 0.25 & 0.37 & 0.66 & 0.61 & -0.06 & -0.10 & 0.11 & 0.69 & 0.48 & 0.26 & 0.23 \\
\hline _12_BGHIFL & 0.71 & 0.27 & 0.27 & 0.48 & 0.60 & 0.69 & -0.06 & -0.14 & 0.19 & 0.77 & 0.35 & 0.22 & 0.31 \\
\hline _13_CHRY & 0.90 & 0.29 & 0.18 & 0.17 & 0.89 & 0.39 & 0.06 & 0.08 & 0.07 & 0.89 & 0.33 & 0.21 & 0.11 \\
\hline _14_TRIPH & 0.80 & 0.46 & 0.13 & 0.08 & 0.75 & 0.53 & 0.09 & 0.15 & 0.11 & 0.82 & 0.40 & 0.14 & 0.02 \\
\hline 15_BAA & 0.88 & 0.28 & 0.11 & 0.12 & 0.92 & 0.24 & 0.01 & 0.00 & -0.02 & 0.85 & 0.28 & 0.22 & 0.10 \\
\hline _16_RET & 0.19 & 0.05 & 0.17 & 0.83 & 0.16 & 0.41 & 0.06 & 0.02 & 0.87 & 0.10 & 0.09 & 0.16 & 0.92 \\
\hline _18_BAP & 0.91 & 0.24 & 0.07 & 0.02 & 0.95 & 0.16 & 0.07 & 0.05 & 0.01 & 0.89 & 0.25 & 0.11 & 0.02 \\
\hline _19_BEP & 0.93 & 0.18 & 0.19 & 0.13 & 0.94 & 0.22 & 0.13 & 0.01 & 0.12 & 0.91 & 0.32 & 0.07 & 0.07 \\
\hline 20_BBFLT & 0.93 & 0.14 & 0.22 & 0.12 & 0.96 & 0.20 & 0.13 & 0.03 & 0.08 & 0.91 & 0.28 & 0.15 & 0.07 \\
\hline 21_BKFLT & 0.95 & 0.13 & 0.15 & 0.04 & 0.96 & 0.16 & 0.11 & 0.05 & 0.05 & 0.93 & 0.26 & 0.09 & -0.02 \\
\hline 25_BGHIP & 0.82 & 0.21 & 0.25 & 0.32 & 0.83 & 0.34 & 0.08 & -0.18 & 0.12 & 0.85 & 0.33 & 0.13 & 0.19 \\
\hline 26_IPYR & 0.92 & 0.17 & 0.23 & 0.18 & 0.94 & 0.24 & 0.09 & -0.06 & 0.06 & 0.91 & 0.29 & 0.18 & 0.08 \\
\hline eigenvalue & 13.9 & 2.8 & 1.9 & 0.9 & 13.5 & 3.0 & 2.0 & 0.9 & 0.7 & 15.4 & 1.9 & 1.3 & 0.8 \\
\hline$\%$ variance & 44 & 23 & 12 & 0 & 43 & 27 & 11 & 5 & 5 & 47 & 26 & 9 & 6 \\
\hline$\%$ cum var & 44 & 68 & 80 & 89 & 43 & 70 & 81 & 86 & 91 & 47 & 73 & 82 & 88 \\
\hline
\end{tabular}

HAMILTON (urban)

\begin{tabular}{|c|c|c|c|c|c|c|c|c|c|c|c|c|}
\hline $\log (C)$ & SW & \multicolumn{3}{|c|}{$n=216$} & \multicolumn{2}{|c|}{ SUMMER } & \multicolumn{2}{|l|}{$n=84$} & \multicolumn{2}{|c|}{ WINTER } & \multicolumn{2}{|c|}{$n=124$} \\
\hline HAM_0010 & F1 & F2 & F3 & $\mathrm{F} 4$ & F1 & $\overline{F 2}$ & F3 & $\overline{F 4}$ & F1 & F2 & F3 & $\mathrm{F} 4$ \\
\hline _01_ACY & 0.27 & 0.91 & 0.11 & 0.06 & 0.27 & 0.88 & -0.13 & -0.09 & 0.25 & 0.85 & 0.15 & 0.29 \\
\hline _02_ACE & 0.28 & 0.84 & 0.03 & 0.24 & 0.05 & 0.89 & 0.02 & 0.19 & 0.39 & 0.84 & 0.04 & 0.17 \\
\hline _03_FLU & 0.44 & 0.68 & 0.04 & 0.52 & 0.32 & 0.81 & 0.08 & 0.39 & 0.59 & 0.50 & 0.07 & 0.59 \\
\hline _04_ANT & 0.58 & 0.33 & 0.06 & 0.69 & 0.62 & 0.39 & 0.22 & 0.55 & 0.68 & 0.34 & 0.05 & 0.60 \\
\hline 05_PHE & 0.47 & 0.13 & 0.06 & 0.84 & 0.50 & 0.25 & 0.26 & 0.75 & 0.66 & 0.35 & 0.07 & 0.62 \\
\hline _06_MFLU & 0.37 & 0.41 & 0.13 & 0.71 & 0.35 & 0.56 & 0.34 & 0.40 & 0.48 & 0.33 & 0.15 & 0.72 \\
\hline _07_FLT & 0.62 & 0.15 & 0.02 & 0.74 & 0.65 & 0.16 & 0.08 & 0.71 & 0.75 & 0.34 & 0.03 & 0.53 \\
\hline _08_PYR & 0.64 & 0.23 & 0.05 & 0.71 & 0.71 & 0.21 & 0.11 & 0.64 & 0.72 & 0.36 & 0.07 & 0.56 \\
\hline _09_MPYR & 0.70 & 0.14 & 0.15 & 0.62 & 0.80 & 0.16 & 0.23 & 0.45 & 0.72 & 0.16 & 0.19 & 0.58 \\
\hline _10_BAFLU & 0.75 & 0.24 & 0.02 & 0.58 & 0.80 & 0.25 & 0.04 & 0.50 & 0.82 & 0.24 & 0.03 & 0.47 \\
\hline _11_BBFLU & 0.74 & 0.27 & 0.00 & 0.55 & 0.79 & 0.27 & -0.05 & 0.47 & 0.80 & 0.22 & 0.03 & 0.48 \\
\hline _12_BGHIFL & 0.72 & 0.39 & 0.16 & 0.46 & 0.82 & 0.26 & 0.02 & 0.45 & 0.68 & 0.40 & 0.24 & 0.44 \\
\hline _13_CHRY & 0.80 & 0.30 & 0.05 & 0.50 & 0.87 & 0.19 & 0.03 & 0.45 & 0.85 & 0.37 & 0.08 & 0.37 \\
\hline _14_TRIPH & 0.78 & 0.29 & 0.03 & 0.51 & 0.83 & 0.21 & 0.01 & 0.45 & 0.84 & 0.36 & 0.08 & 0.37 \\
\hline _15_BAA & 0.83 & 0.20 & 0.09 & 0.47 & 0.90 & 0.20 & 0.10 & 0.33 & 0.87 & 0.20 & 0.13 & 0.35 \\
\hline _16_RET & 0.06 & 0.10 & 0.99 & 0.08 & 0.07 & -0.02 & 0.95 & 0.12 & 0.09 & 0.11 & 0.98 & 0.08 \\
\hline _18_BAP & 0.88 & 0.21 & 0.06 & 0.31 & 0.94 & 0.12 & 0.15 & 0.10 & 0.92 & 0.21 & 0.11 & 0.22 \\
\hline _19_BEP & 0.84 & 0.36 & 0.03 & 0.38 & 0.94 & 0.19 & 0.07 & 0.25 & 0.86 & 0.38 & 0.05 & 0.33 \\
\hline 20_BBFLT & 0.83 & 0.38 & 0.03 & 0.40 & 0.93 & 0.22 & 0.06 & 0.27 & 0.84 & 0.40 & 0.06 & 0.34 \\
\hline _21_BKFLT & 0.84 & 0.36 & 0.03 & 0.37 & 0.93 & 0.22 & 0.08 & 0.25 & 0.87 & 0.37 & 0.05 & 0.29 \\
\hline _25_BGHIP & 0.83 & 0.39 & 0.08 & 0.34 & 0.94 & 0.21 & 0.04 & 0.21 & 0.82 & 0.38 & 0.13 & 0.35 \\
\hline _26_IPYR & 0.84 & 0.38 & 0.05 & 0.37 & 0.94 & 0.25 & 0.06 & 0.19 & 0.85 & 0.36 & 0.10 & 0.34 \\
\hline eigent & 17.6 & $\overline{1.4}$ & 1.0 & 0.7 & 16.5 & 2.0 & 1.3 & 0.7 & 18.3 & 1.1 & 0.9 & 0.5 \\
\hline$\%$ varia & 46 & 16 & 5 & 26 & 54 & 16 & 6 & 17 & 53 & 17 & 5 & 20 \\
\hline$\%$ cum var & 46 & 63 & 68 & 94 & 54 & 70 & 76 & 93 & 53 & 70 & 75 & 95 \\
\hline
\end{tabular}

Figure B.4-2. Sensitivity analysis to seasonal stratification (log-transformed data). 
EGBERT (background)

\begin{tabular}{|c|c|c|c|c|c|c|c|c|c|c|c|c|c|}
\hline $\log (C)$ & SW & \multicolumn{3}{|c|}{$n=227$} & \multicolumn{2}{|c|}{ SUMMER } & \multicolumn{3}{|c|}{$n=81$} & \multicolumn{4}{|c|}{ WINTER $\quad \mathrm{n}=126$} \\
\hline EGB_0010 & F1 & F2 & F3 & F4 & $\overline{F 1}$ & $\bar{F} 2$ & F3 & $\mathrm{F} 4$ & F5 & $\mathrm{F} 1$ & $\mathrm{~F} 2$ & F3 & F4 \\
\hline 01_ACY & 0.25 & 0.19 & 0.22 & 0.85 & 0.17 & 0.32 & -0.02 & $\overline{0.81}$ & 0.01 & 0.23 & 0.40 & 0.22 & $\overline{0.76}$ \\
\hline _02_ACE & 0.27 & -0.05 & 0.59 & 0.51 & 0.19 & 0.17 & 0.71 & 0.40 & -0.15 & 0.23 & 0.73 & -0.18 & 0.22 \\
\hline _03_FLU & 0.39 & 0.06 & 0.82 & 0.22 & 0.19 & 0.16 & 0.90 & 0.02 & 0.09 & 0.29 & 0.90 & 0.16 & 0.02 \\
\hline 04_ANT & 0.10 & 0.48 & 0.45 & 0.41 & 0.35 & 0.17 & 0.43 & 0.61 & 0.19 & 0.11 & 0.60 & 0.39 & 0.34 \\
\hline _05_PHE & 0.24 & 0.31 & 0.88 & 0.12 & 0.61 & 0.03 & 0.72 & 0.03 & 0.13 & 0.32 & 0.87 & 0.23 & 0.15 \\
\hline _06_MFLU & 0.26 & 0.17 & 0.85 & 0.11 & 0.25 & 0.06 & 0.90 & 0.00 & 0.24 & 0.23 & 0.87 & 0.28 & -0.02 \\
\hline _07_FLT & 0.49 & 0.34 & 0.73 & 0.09 & 0.85 & 0.15 & 0.41 & 0.02 & 0.05 & 0.52 & 0.74 & 0.27 & 0.09 \\
\hline _08_PYR & 0.29 & 0.51 & 0.68 & 0.00 & 0.86 & 0.10 & 0.34 & -0.01 & 0.06 & 0.45 & 0.69 & 0.46 & 0.12 \\
\hline _09_MPYR & 0.24 & 0.85 & 0.24 & 0.11 & 0.69 & 0.14 & 0.08 & 0.53 & 0.29 & 0.36 & 0.37 & 0.75 & 0.01 \\
\hline _10_BAFLU & 0.44 & 0.72 & 0.42 & 0.16 & 0.87 & 0.14 & 0.10 & 0.38 & 0.15 & 0.45 & 0.56 & 0.65 & 0.05 \\
\hline _11_BBFLU & 0.35 & 0.71 & 0.36 & 0.10 & 0.81 & 0.17 & 0.04 & 0.47 & 0.17 & 0.34 & 0.49 & 0.70 & -0.03 \\
\hline _12_BGHIFL & 0.73 & 0.41 & 0.34 & 0.28 & 0.80 & 0.25 & 0.21 & 0.34 & 0.10 & 0.79 & 0.32 & 0.36 & 0.18 \\
\hline _13_CHRY & 0.82 & 0.30 & 0.31 & 0.22 & 0.88 & 0.26 & 0.19 & 0.07 & 0.14 & 0.89 & 0.30 & 0.18 & 0.16 \\
\hline _14_TRIPH & 0.77 & 0.21 & 0.39 & 0.26 & 0.80 & 0.27 & 0.23 & 0.06 & 0.10 & 0.83 & 0.39 & 0.05 & 0.19 \\
\hline _15_BAA & 0.78 & 0.34 & 0.24 & -0.08 & 0.42 & 0.39 & 0.25 & 0.47 & 0.32 & 0.82 & 0.24 & 0.23 & -0.25 \\
\hline _16_RET & -0.09 & 0.77 & -0.04 & 0.00 & 0.33 & 0.19 & 0.10 & 0.35 & 0.77 & 0.03 & -0.03 & 0.77 & 0.14 \\
\hline _18_BAP & 0.83 & 0.02 & 0.25 & -0.25 & 0.12 & 0.31 & 0.14 & -0.04 & 0.85 & 0.83 & 0.22 & 0.08 & -0.33 \\
\hline _19_BEP & 0.92 & 0.10 & 0.24 & 0.18 & 0.33 & 0.81 & 0.20 & 0.12 & 0.11 & 0.94 & 0.20 & 0.16 & 0.15 \\
\hline _20_BBFLT & 0.90 & 0.12 & 0.24 & 0.27 & 0.54 & 0.73 & 0.16 & 0.23 & 0.09 & 0.92 & 0.22 & 0.10 & 0.24 \\
\hline _21_BKFLT & 0.92 & 0.07 & 0.22 & 0.15 & 0.34 & 0.77 & 0.00 & -0.06 & 0.10 & 0.90 & 0.24 & 0.19 & 0.12 \\
\hline _25_BGHIP & 0.90 & 0.09 & 0.24 & 0.12 & -0.02 & 0.71 & 0.14 & 0.33 & 0.20 & 0.88 & 0.29 & 0.22 & 0.02 \\
\hline _26_IPYR & 0.90 & 0.09 & 0.15 & 0.22 & 0.03 & 0.75 & 0.05 & 0.42 & 0.24 & 0.90 & 0.21 & 0.15 & 0.13 \\
\hline eigenvalue & 13.1 & 2.7 & 1.6 & 1.0 & 11.6 & 2.6 & 1.9 & 1.3 & 1.1 & 13.8 & 2.8 & 1.4 & 0.9 \\
\hline$\%$ variance & 38 & 16 & 22 & 8 & 31 & 16 & 16 & 12 & 9 & 40 & 26 & 14 & 5 \\
\hline$\%$ cum var & 38 & 54 & 76 & 84 & 31 & 48 & 63 & 75 & 84 & 40 & 67 & 81 & 86 \\
\hline
\end{tabular}

Figure B.4-2 (continued) 
HEAVY factor.

\begin{tabular}{|c|}
\hline \begin{tabular}{|l} 
SITE \\
DATASET \\
outliers \\
sample size, $n$ \\
model order, $p$ \\
FACTOR\# \\
\end{tabular} \\
\hline 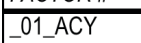 \\
\hline 02_ACE \\
\hline _03_FLU \\
\hline _04_ANT \\
\hline 05_PHE \\
\hline _06_MFLU \\
\hline _07_FLT \\
\hline 08_PYR \\
\hline _09_MPYR \\
\hline 10_BAFLU \\
\hline _11_BBFLU \\
\hline 12_BGHIFL \\
\hline _13_CHRY \\
\hline _14_TRIPH \\
\hline _15_BAA \\
\hline _16_RET \\
\hline _18_BAP \\
\hline _19_BEP \\
\hline 20_BBFLT \\
\hline 21_BKFLT \\
\hline 25_BGHIP \\
\hline 26_IPYR \\
\hline $\begin{array}{l}\text { eigenvalue } \\
\% \text { variance }\end{array}$ \\
\hline
\end{tabular}

\begin{tabular}{|rrr|}
\hline TOR & TOR & TOR \\
SW & S & W \\
out1 & out1 & out1 \\
365 & 150 & 205 \\
4 & 5 & 4 \\
$\mathrm{~F} 1$ & $\mathrm{~F} 1$ & $\mathrm{~F} 1$ \\
\hline 0.36 & 0.00 & 0.38 \\
0.17 & 0.10 & 0.11 \\
0.27 & 0.11 & 0.26 \\
0.26 & 0.21 & 0.43 \\
0.06 & 0.19 & 0.29 \\
0.21 & 0.13 & 0.28 \\
0.32 & 0.35 & 0.60 \\
0.47 & 0.43 & 0.64 \\
0.55 & 0.55 & 0.62 \\
0.72 & 0.68 & 0.73 \\
0.70 & 0.66 & 0.69 \\
0.71 & 0.60 & 0.77 \\
0.90 & 0.89 & 0.89 \\
0.80 & 0.75 & 0.82 \\
0.88 & 0.92 & 0.85 \\
0.19 & 0.16 & 0.10 \\
0.91 & 0.95 & 0.89 \\
0.93 & 0.94 & 0.91 \\
0.93 & 0.96 & 0.91 \\
0.95 & 0.96 & 0.93 \\
0.82 & 0.83 & 0.85 \\
0.92 & 0.94 & 0.91 \\
\hline 13.9 & 13.5 & 15.4 \\
44 & 43 & 47 \\
\hline
\end{tabular}
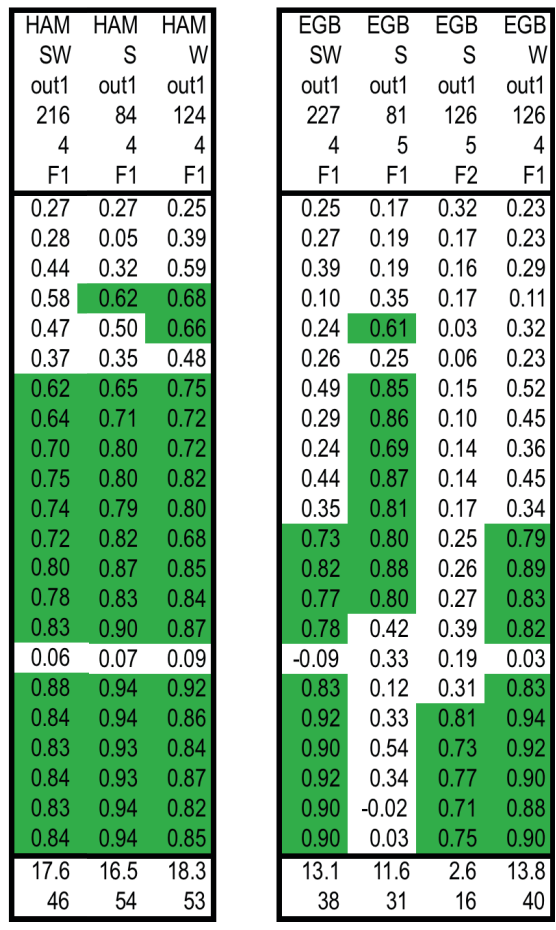

LIGHT factor.

\begin{tabular}{|l|}
\hline SITE \\
DATASET \\
outliers \\
sample size, $n$ \\
model order, $p$ \\
FACTOR\# \\
\hline -01_ACY \\
-02_ACE \\
-03_FLU \\
-04_ANT \\
-05_PHE \\
-06_MFLU \\
-07_FLT \\
-08_PYR \\
-09_MPYR \\
-10_BAFLU \\
-11_BBFLU \\
-12_BGHIFL \\
-13_CHRY \\
-14_TRIPH \\
-15_BAA \\
-16_RET \\
-18_BAP \\
-19_BEP \\
-20_BBFLT \\
21_BKFLT \\
-25_BGHIP \\
26_IPYR \\
\hline eigenvalue \\
\% variance \\
\hline
\end{tabular}

\begin{tabular}{|rrr|}
\hline TOR & TOR & TOR \\
SW & S & W \\
out1 & out1 & out1 \\
365 & 150 & 205 \\
4 & 5 & 4 \\
F2 & F2 & F2 \\
\hline-0.17 & 0.01 & 0.15 \\
0.04 & 0.06 & 0.28 \\
0.34 & 0.31 & 0.80 \\
0.82 & 0.84 & 0.75 \\
0.95 & 0.81 & 0.91 \\
0.69 & 0.67 & 0.84 \\
0.89 & 0.79 & 0.74 \\
0.84 & 0.83 & 0.70 \\
0.65 & 0.74 & 0.59 \\
0.49 & 0.65 & 0.53 \\
0.39 & 0.61 & 0.48 \\
0.27 & 0.69 & 0.35 \\
0.29 & 0.39 & 0.33 \\
0.46 & 0.53 & 0.40 \\
0.28 & 0.24 & 0.28 \\
0.05 & 0.41 & 0.09 \\
0.24 & 0.16 & 0.25 \\
0.18 & 0.22 & 0.32 \\
0.14 & 0.20 & 0.28 \\
0.13 & 0.16 & 0.26 \\
0.21 & 0.34 & 0.33 \\
0.17 & 0.24 & 0.29 \\
\hline 2.8 & 3.0 & 1.9 \\
23 & 27 & 26 \\
\hline
\end{tabular}

\begin{tabular}{|rrr|}
\hline HAM & HAM & HAM \\
SW & S & W \\
out1 & out1 & out1 \\
216 & 84 & 124 \\
4 & 4 & 4 \\
F4 & F4 & F4 \\
\hline 0.06 & -0.09 & 0.29 \\
0.24 & 0.19 & 0.17 \\
0.52 & 0.39 & 0.59 \\
0.69 & 0.55 & 0.60 \\
0.84 & 0.75 & 0.62 \\
0.71 & 0.40 & 0.72 \\
0.74 & 0.71 & 0.53 \\
0.71 & 0.64 & 0.56 \\
0.62 & 0.45 & 0.58 \\
0.58 & 0.50 & 0.47 \\
0.55 & 0.47 & 0.48 \\
0.46 & 0.45 & 0.44 \\
0.50 & 0.45 & 0.37 \\
0.51 & 0.45 & 0.37 \\
0.47 & 0.33 & 0.35 \\
0.08 & 0.12 & 0.08 \\
0.31 & 0.10 & 0.22 \\
0.38 & 0.25 & 0.33 \\
0.40 & 0.27 & 0.34 \\
0.37 & 0.25 & 0.29 \\
0.34 & 0.21 & 0.35 \\
0.37 & 0.19 & 0.34 \\
\hline 0.7 & 0.7 & 0.5 \\
26 & 17 & 20 \\
\hline & & \\
\hline
\end{tabular}

\begin{tabular}{|rrr|}
\hline EGB & EGB & EGB \\
SW & S & W \\
out1 & out1 & out1 \\
227 & 81 & 126 \\
4 & 5 & 4 \\
F3 & F3 & F2 \\
\hline 0.22 & -0.02 & 0.40 \\
0.59 & 0.71 & 0.73 \\
0.82 & 0.90 & 0.90 \\
0.45 & 0.43 & 0.60 \\
0.88 & 0.72 & 0.87 \\
0.85 & 0.90 & 0.87 \\
0.73 & 0.41 & 0.74 \\
0.68 & 0.34 & 0.69 \\
0.24 & 0.08 & 0.37 \\
0.42 & 0.10 & 0.56 \\
0.36 & 0.04 & 0.49 \\
0.34 & 0.21 & 0.32 \\
0.31 & 0.19 & 0.30 \\
0.39 & 0.23 & 0.39 \\
0.24 & 0.25 & 0.24 \\
-0.04 & 0.10 & -0.03 \\
0.25 & 0.14 & 0.22 \\
0.24 & 0.20 & 0.20 \\
0.24 & 0.16 & 0.22 \\
0.22 & 0.00 & 0.24 \\
0.24 & 0.14 & 0.29 \\
0.15 & 0.05 & 0.21 \\
\hline 1.6 & 1.9 & 2.8 \\
22 & 16 & 26 \\
\hline
\end{tabular}

Figure B.4-2 (continued) 
VOLATILE factor.

\begin{tabular}{|c|}
\hline \begin{tabular}{|l} 
SITE \\
DATASET \\
outliers \\
sample size, $n$ \\
model order, $p$ \\
FACTOR \#
\end{tabular} \\
\hline _01_ACY \\
\hline 02_ACE \\
\hline 03_FLU \\
\hline _04_ANT \\
\hline 05_PHE \\
\hline _06_MFLU \\
\hline 07_FLT \\
\hline _08_PYR \\
\hline 09_MPYR \\
\hline _10_BAFLU \\
\hline _11_BBFLU \\
\hline _12_BGHIFL \\
\hline _13_CHRY \\
\hline _14_TRIPH \\
\hline _15_BAA \\
\hline _16_RET \\
\hline _18_BAP \\
\hline _19_BEP \\
\hline 20_BBFLT \\
\hline _21_BKFLT \\
\hline 25_BGHIP \\
\hline 26_IPYR \\
\hline $\begin{array}{l}\text { eigenvalue } \\
\% \text { variance }\end{array}$ \\
\hline
\end{tabular}

\begin{tabular}{|rrrr|}
\hline TOR & TOR & TOR & TOR \\
SW & S & S & W \\
out1 & out1 & out1 & out1 \\
365 & 150 & 150 & 205 \\
4 & 5 & 5 & 4 \\
F3 & F3 & F4 & F3 \\
\hline 0.65 & 0.48 & -0.81 & 0.73 \\
0.88 & 0.91 & -0.15 & 0.90 \\
0.83 & 0.88 & -0.12 & 0.42 \\
0.24 & 0.16 & -0.22 & 0.28 \\
0.00 & 0.37 & 0.27 & 0.03 \\
0.51 & 0.45 & -0.33 & 0.23 \\
-0.05 & 0.22 & 0.34 & 0.03 \\
0.09 & 0.15 & 0.17 & 0.13 \\
0.12 & -0.02 & -0.14 & 0.13 \\
0.20 & -0.05 & -0.07 & 0.23 \\
0.25 & -0.06 & -0.10 & 0.26 \\
0.27 & -0.06 & -0.14 & 0.22 \\
0.18 & 0.06 & 0.08 & 0.21 \\
0.13 & 0.09 & 0.15 & 0.14 \\
0.11 & 0.01 & 0.00 & 0.22 \\
0.17 & 0.06 & 0.02 & 0.16 \\
0.07 & 0.07 & 0.05 & 0.11 \\
0.19 & 0.13 & 0.01 & 0.07 \\
0.22 & 0.13 & 0.03 & 0.15 \\
0.15 & 0.11 & 0.05 & 0.09 \\
0.25 & 0.08 & -0.18 & 0.13 \\
0.23 & 0.09 & -0.06 & 0.18 \\
\hline 1.9 & 2.0 & 0.9 & 1.3 \\
12 & 11 & 5 & 9 \\
\hline & & &
\end{tabular}

\begin{tabular}{|rrr|}
\hline HAM & HAM & HAM \\
SW & S & W \\
out1 & out1 & out1 \\
216 & 84 & 124 \\
4 & 4 & 4 \\
F2 & F2 & F2 \\
\hline 0.91 & 0.88 & 0.85 \\
0.84 & 0.89 & 0.84 \\
0.68 & 0.81 & 0.50 \\
0.33 & 0.39 & 0.34 \\
0.13 & 0.25 & 0.35 \\
0.41 & 0.56 & 0.33 \\
0.15 & 0.16 & 0.34 \\
0.23 & 0.21 & 0.36 \\
0.14 & 0.16 & 0.16 \\
0.24 & 0.25 & 0.24 \\
0.27 & 0.27 & 0.22 \\
0.39 & 0.26 & 0.40 \\
0.30 & 0.19 & 0.37 \\
0.29 & 0.21 & 0.36 \\
0.20 & 0.20 & 0.20 \\
0.10 & -0.02 & 0.11 \\
0.21 & 0.12 & 0.21 \\
0.36 & 0.19 & 0.38 \\
0.38 & 0.22 & 0.40 \\
0.36 & 0.22 & 0.37 \\
0.39 & 0.21 & 0.38 \\
0.38 & 0.25 & 0.36 \\
\hline 1.4 & 2.0 & 1.1 \\
16 & 16 & 17 \\
\hline
\end{tabular}

\begin{tabular}{|rrr|}
\hline EGB & EGB & EGB \\
SW & S & W \\
out1 & out1 & out1 \\
227 & 81 & 126 \\
4 & 5 & 4 \\
F4 & F4 & F4 \\
\hline 0.85 & 0.81 & 0.76 \\
0.51 & 0.40 & 0.22 \\
0.22 & 0.02 & 0.02 \\
0.41 & 0.61 & 0.34 \\
0.12 & 0.03 & 0.15 \\
0.11 & 0.00 & -0.02 \\
0.09 & 0.02 & 0.09 \\
0.00 & -0.01 & 0.12 \\
0.11 & 0.53 & 0.01 \\
0.16 & 0.38 & 0.05 \\
0.10 & 0.47 & -0.03 \\
0.28 & 0.34 & 0.18 \\
0.22 & 0.07 & 0.16 \\
0.26 & 0.06 & 0.19 \\
-0.08 & 0.47 & -0.25 \\
0.00 & 0.35 & 0.14 \\
-0.25 & -0.04 & -0.33 \\
0.18 & 0.12 & 0.15 \\
0.27 & 0.23 & 0.24 \\
0.15 & -0.06 & 0.12 \\
0.12 & 0.33 & 0.02 \\
0.22 & 0.42 & 0.13 \\
\hline 1.0 & 1.3 & 0.9 \\
8 & 12 & 5 \\
\hline
\end{tabular}

RETENE factor

\begin{tabular}{|c|}
\hline \begin{tabular}{|l} 
SITE \\
DATASET \\
outliers \\
sample size, $n$ \\
model order, $p$ \\
FACTOR\# \\
\end{tabular} \\
\hline _01_ACY \\
\hline 02_ACE \\
\hline _03_FLU \\
\hline _04_ANT \\
\hline -05_PHE \\
\hline _06_MFLU \\
\hline _07_FLT \\
\hline _08_PYR \\
\hline _09_MPYR \\
\hline 10_BAFLU \\
\hline _11_BBFLU \\
\hline 12_BGHIFL \\
\hline 13_CHRY \\
\hline _14_TRIPH \\
\hline $15 \_\mathrm{BAA}$ \\
\hline _16_RET \\
\hline _18_BAP \\
\hline _19_BEP \\
\hline 20_BBFLT \\
\hline 21_BKFLT \\
\hline 25_BGHIP \\
\hline _26_IPYR \\
\hline $\begin{array}{l}\text { eigenvalue } \\
\% \text { variance }\end{array}$ \\
\hline
\end{tabular}

\begin{tabular}{|rrr|}
\hline TOR & TOR & TOR \\
SW & S & W \\
out1 & out1 & out1 \\
365 & 150 & 205 \\
4 & 5 & 4 \\
F4 & F5 & F4 \\
\hline 0.50 & 0.01 & 0.33 \\
0.14 & 0.16 & 0.03 \\
0.06 & -0.10 & 0.02 \\
0.23 & 0.14 & 0.20 \\
-0.11 & 0.19 & 0.05 \\
0.07 & -0.15 & 0.02 \\
-0.06 & 0.20 & 0.06 \\
0.13 & 0.16 & 0.14 \\
0.38 & 0.19 & 0.32 \\
0.30 & 0.09 & 0.19 \\
0.37 & 0.11 & 0.23 \\
0.48 & 0.19 & 0.31 \\
0.17 & 0.07 & 0.11 \\
0.08 & 0.11 & 0.02 \\
0.12 & -0.02 & 0.10 \\
0.83 & 0.87 & 0.92 \\
0.02 & 0.01 & 0.02 \\
0.13 & 0.12 & 0.07 \\
0.12 & 0.08 & 0.07 \\
0.04 & 0.05 & -0.02 \\
0.32 & 0.12 & 0.19 \\
0.18 & 0.06 & 0.08 \\
\hline 0.9 & 0.7 & 0.8 \\
9 & 5 & 6 \\
\hline
\end{tabular}

\begin{tabular}{|rrr|}
\hline HAM & HAM & HAM \\
SW & S & W \\
out1 & out1 & out1 \\
216 & 84 & 124 \\
4 & 4 & 4 \\
F3 & F3 & F3 \\
\hline 0.11 & -0.13 & 0.15 \\
0.03 & 0.02 & 0.04 \\
0.04 & 0.08 & 0.07 \\
0.06 & 0.22 & 0.05 \\
0.06 & 0.26 & 0.07 \\
0.13 & 0.34 & 0.15 \\
0.02 & 0.08 & 0.03 \\
0.05 & 0.11 & 0.07 \\
0.15 & 0.23 & 0.19 \\
0.02 & 0.04 & 0.03 \\
0.00 & -0.05 & 0.03 \\
0.16 & 0.02 & 0.24 \\
0.05 & 0.03 & 0.08 \\
0.03 & 0.01 & 0.08 \\
0.09 & 0.10 & 0.13 \\
0.99 & 0.95 & 0.98 \\
0.06 & 0.15 & 0.11 \\
0.03 & 0.07 & 0.05 \\
0.03 & 0.06 & 0.06 \\
0.03 & 0.08 & 0.05 \\
0.08 & 0.04 & 0.13 \\
0.05 & 0.06 & 0.10 \\
\hline 1.0 & 1.3 & 0.9 \\
5 & 6 & 5 \\
\hline
\end{tabular}

\begin{tabular}{|rrr|}
\hline EGB & EGB & EGB \\
SW & S & W \\
out1 & out1 & out1 \\
227 & 81 & 126 \\
4 & 5 & 4 \\
F2 & F5 & F3 \\
\hline 0.19 & 0.01 & 0.22 \\
-0.05 & -0.15 & -0.18 \\
0.06 & 0.09 & 0.16 \\
0.48 & 0.19 & 0.39 \\
0.31 & 0.13 & 0.23 \\
0.17 & 0.24 & 0.28 \\
0.34 & 0.05 & 0.27 \\
0.51 & 0.06 & 0.46 \\
0.85 & 0.29 & 0.75 \\
0.72 & 0.15 & 0.65 \\
0.71 & 0.17 & 0.70 \\
0.41 & 0.10 & 0.36 \\
0.30 & 0.14 & 0.18 \\
0.21 & 0.10 & 0.05 \\
0.34 & 0.32 & 0.23 \\
0.77 & 0.77 & 0.77 \\
0.02 & 0.85 & 0.08 \\
0.10 & 0.11 & 0.16 \\
0.12 & 0.09 & 0.10 \\
0.07 & 0.10 & 0.19 \\
0.09 & 0.20 & 0.22 \\
0.09 & 0.24 & 0.15 \\
\hline 2.7 & 1.1 & 1.4 \\
16 & 9 & 14 \\
\hline
\end{tabular}

Figure B.4-2 (continued) 
Overall, receptor modeling via PCA revealed strong inter-site similarities in factors (i.e., rotated factor loading patterns), consistent with the major source types expected a priori (i.e., from review of emissions inventories and related literature) tending to occur at all sites as either local or proximal transported sources (e.g., vehicle traffic exhaust emissions, residential heating). Thus, PMF modeling was quite reasonably expected to demonstrate that PAH source types influencing the sites would differ more by degree (i.e., relative contribution) than by kind (i.e., source identification); e.g., 'traffic emissions' source type is expected at all sites with relative contribution greatest at Toronto and least at background. At Hamilton, however, the more advanced PMF model was expected to enable a higher model order than seen with PCA, since distinctions between factor loadings were less sharp than seen at Toronto for several abundant species (FLU, MFLU, FLT, PYR) and PMF was thus reasonably expected to resolve a local source type unique to this highly industrialized urban center.

PCA analysis of sensitivity to seasonal stratification corroborated the decision to model the NAPS central site data in PMF without seasonal stratification, particularly since restricting PMF modeling to a winter only time series would severely reduce modeling power and impede corroborating factor interpretations by seasonality in contribution. The particularly strong similarity between year-round PCA factors and winter factors indicated that PMF source profiles derived from a non-seasonally stratified dataset would be representative of winter conditions where $\mathrm{PAH}$ levels are typically higher (Anastasopoulos et al, 2012) and could be legitimately applied to combined receptor modeling (e.g., PMF-CMB) of a winter season field sampling dataset (Chapter 5). 


\title{
Complementary PCA with proportional data
}

\author{
Document history and overview
}

Preliminary PCA modeling using log transformation and proportional data transformation was completed in summer 2013 and summarized via internal report to Dr. Deniz Karman, Carleton University dated August $12^{\text {th }} 2013$ (i.e., report: PCA-results-FINAL-aug13.pdf; results: PCA-ALLSITES-jul13.xlsx; PCA-seasonal-comparisons.xlsx).

Log-transformed PCA results were presented and discussed in Chapter 3. The proportional data transformation was not presented alongside the log-transformed results for several reasons including:

- clarity of presentation;

- primary benefit of proportional data (i.e., factor loading patterns potentially more easily interpretable as source types) not needed since interpretation of factors reserved for PMF analysis;

- proportional data transform resulted in exclusion of phenanthrene from the PCA because it dominated the normalizing value $(\Sigma \mathrm{PAH})$ for essentially all samples, which complicated comparison to PMF results since phenanthrene is a key species contributing majority of sample mass in some factors; and,

- desire to minimally transform sample data for receptor modeling rather than analyse more abstracted data.

However, the transformation to proportional data was useful as a complementary analysis. The general factor loading patterns resolved by the PCA on proportional data did not contra-indicate factor loading patterns resolved by the PCA on log-transformed data although greater separation of PAH species was seen (e.g., heavy MW species more clearly separated from lighter MW species at all sites, revealing clearer inter-site factor differences). Further, the PCA seasonal sensitivity analysis completed on proportional data found identical results as the PCA seasonal sensitivity analysis completed using logtransformed data. 


\section{METHODS}

\section{Proportional data transformation}

Several recent PCA studies of PAH have employed a transformation to proportional data (i.e., also termed "compositional data" in the literature), most often normalizing sample species concentration data on the basis of $\mathrm{PPAH}$ (Dickhut, 2000; Shi, 2009a; Wang, 2010; Guo, 2004) and have demonstrated more physically meaningful (i.e., interpretable) factors from compositional data (Wingfors et al, 2001; normalized to mass of $\mathrm{PM}_{2.5}$ ). The proportional data transformation (Equation B.4-3) was used to allow PCA to resolve factors representing sample-to-sample variation in species composition rather than sample-to-sample variation in absolute concentration (Praveena, 2012; Reid and Spencer, 2009; Baxter, 2005, 2006, 2008).

Consideration was given to normalizing sample species concentrations to the respective sample's concentration of BeP (i.e., 'BeP-indexing'), chosen as a highly stable species (Simcik, 1999; Ciaparra, 2009). However, creating proportional data via normalization by $\mathrm{BeP}$ requires that $\mathrm{BeP}$ be omitted from the $\mathrm{PCA}$ as an independent variable, resulting in several undesirable modeling effects: reduced modeling power; loss of BeP for use in factor interpretation as source type; and, loss of $\mathrm{BeP}$ for indication of atmospheric stability (Park, 2011, 2002). Statistical analysis of trial BeP-normalized datasets in MINITAB also showed kurtosis and skew to be worsened by the transformation.

Rather, the data were normalized on the basis of $\Sigma \mathrm{PAH}$ (i.e., linear sum of species concentrations in each sample). In addition to avoiding the issues associated with using any single species, normalization by $\Sigma \mathrm{PAH}$ offered a significant interpretive advantage, since the resulting compositional species fractions are intuitive and physically meaningful when expressed on the basis of their contribution to sampled PAH mass. Normalization by a variant of $\Sigma \mathrm{PAH}$ also generally reduced skew in species distributions (i.e., confirmed via kurtosis and skewness statistics and histogram plots in MINITAB 12). Independence between modeled proportional species concentrations was ensured by normalizing by a $\Sigma$ PAH comprising a larger number of variables than those retained in the model (Dickhut, 2000): $\Sigma_{28}$ PAH (i.e., all NAPS-reported species less phenanthrene). ${ }^{26}$

\footnotetext{
${ }^{26}$ A theoretical closure restraint for compositional data in PCA (i.e., sum of the species fractions across the sample $=1$; Reyment $\&$ Joreskog, 1993) has demonstrated issues where the unity closure restraint was preserved strictly (i.e., variables across each row sum to unity; Joliffe, 2002; Dickhut, 2000) as would occur with normalization by the sum of all PCA-modeled species (e.g., $\Sigma_{22} \mathrm{PAH}$ for 22 modeled species).
} 
Phenanthrene was selected for omission from the normalizing sum and the PCA variable set because its concentration nearly always dominated the NAPS central site samples (i.e., typically by at least an order of magnitude, even relative to other abundant light MW species) with following undesired effects: inclusion of phenanthrene in the normalizing constant rendered its proportion close to unity with minimal sample-tosample variance; correlation between phenanthrene and the normalizing sum was exceedingly strong; inclusion of phenanthrene tended to 'mask' sample-to-sample differences in other species proportions. Trial PCA runs with phenanthrene included in the normalizing constant (i.e., $\Sigma_{29} \mathrm{PAH}$ ) and model also showed greater sensitivity to outliers and generally less well-resolved factors than by using $\Sigma_{28} \mathrm{PAH}$ and omitting phenanthrene (i.e., modeling 21 species).

Equation B.4-3. Proportional data transformation for PCA receptor modeling.

$$
\begin{aligned}
& \qquad Y_{i j}=\frac{X_{i j}}{\sum_{j=1}^{28} P A H_{j}} \\
& \text { where } \quad Y_{i j}=\text { species proportion in sample [unitless] } \\
& X_{i j}=\text { PMF input species concentration }\left[\mathrm{ng} / \mathrm{m}^{3}\right] \\
& \sum_{j=1}^{28} P A H_{j}=\text { sample sum all species concentrations less phenanthrene }\left[\mathrm{ng} / \mathrm{m}^{3}\right. \text { ] }
\end{aligned}
$$

\section{Model parameters}

Model parameters for the PCA using proportional data were identical to those used for the PCA using log-transformed data.

The PCA was run using SYSTAT 13 (ww.systat.com) with a correlation matrix (R-mode; Z-standardization transformation: mean-centre each sample within variable and normalize by variable's standard deviation) and Varimax rotation. PCA used as input data the PAH concentration dataset prepared for PMF modeling (i.e., values below detection limit replaced with one-half the species detection limit; missing values replaced with the species median; $22 \mathrm{PAH}$ species screened for model inclusion via PMF screening criteria such as excessive missing or BDL samples in a species time series). Factors were retained for eigenvalues as low as 0.25 (i.e., chosen less than Kaiser's rule of 1.0 to ensure do not under-extract factors; Hopke, 1982).

Models with three to eight factors were run and the optimal model order retained for analysis and comparison across sites was selected by examining several criteria simultaneously including: (i) scree plot (elbow where slope changes from steep to not 
steep); (ii) Kaiser's rule (eigenvalues greater than 1); (iii) Thurstone's criteria for well resolved factors (low loading in each row of the rotated factor loading matrix; a number of low species loadings in each factor equal or greater than the model order; all pairs of factors to have some species with high loadings on one factor and near-zero loadings on the other as well as several species with low loadings on both factors); and, (iv) retaining only factors that explain more than $5 \%$ of a species time series variance. (Reyment and Joreskog, 1993)

Absolute rotated factor loadings values greater than 0.6 were classified as "high" so as to be consistent with the correlation strength typically accepted as "mapped" in bootstrap analysis of PMF results; absolute factor loading values between 0.4 and 0.6 were classified as "moderate".

\section{$\underline{\text { RESULTS }}$}

\section{PCA sensitivity to outliers (proportional data)}

Extreme outliers (i.e., values greater than or less than three times interquartile range) in individual species datasets were identified for potential exclusion from the PCA using Microsoft Excel and MINITAB 12 (i.e., via boxplots); this was done for original untransformed concentration data and the transformed proportional datasets. As a rule, far fewer outlier dates were observed in the proportional data than in the original data, confirming the utility of the proportional data transform to reduce skew due to its emphasis of sample-to-sample variance in species compositions rather than concentrations.

The PCA (i.e., proportion data transform) showed moderate sensitivity to outliers and thus dates with extreme outlier concentrations in any species aside from retene ${ }^{27}$ were generally omitted as this improved the separation and interpretation of factors (i.e., greater factor loading strength for characteristic species, better distinction between factors as per Thurstone's criteria; Reyment and Joreskog, 1993).

\footnotetext{
${ }^{27}$ Excluding dates with statistically extreme retene concentration did not improve factor loading or factor separation (i.e., either data transform, any modeled site, year-round or seasonally stratified dataset variants) and generally only served to reduce variance in the retene dataset thereby hindering the PCA model's ability to clearly extract a separate retene factor. The insensitivity of the PCA to retene outliers was expected a priori, since retene was not found to be well correlated with other species and is considered a tracer species.
} 
PCA results for the proportional-transformed PAH data at the three sites is shown in Figure B.4-3 (i.e., rotated factor loadings, eigenvalue, \% variance explained, cumulative $\%$ variance). At all three sites, a four-factor PCA model was retained as 'optimal' model order (i.e., supported by elbow in scree plot, good separation of species groupings, retene as separate factor, eigenvalues greater than or near 1.0 for all factors, each factor explaining greater than approximately $5 \%$ variance), facilitating inter-site comparisons. ${ }^{28}$ Cumulatively, four factors explained approximately 77\% (Toronto, Hamilton) and 69\% (Egbert) of the data variance.

Rotated factor loadings were grouped by similarity or uniqueness across sites in Figure B.4-3-b. The first factor (by $\%$ variance explained) common across sites was characterized by high loadings $(>0.6)$ on all heavy molecular weight species (CHRY through IPYR, exception of RET). This factor was labeled as "heavy" and was the primary factor at all sites, explaining 33\% - 38\% of the data variance. The "heavy" factor loading pattern was highly similar across all sites, suggesting that the corresponding source type was impacting all sites. The second factor $(10 \%-21 \%$ data variance) common across sites was characterized by high loadings of the light species FLT and PYR alongside high/moderate loadings in some volatile species (ACY, ACY, FLU). This factor was labeled as "volatile + light" and was similar at the two urban sites (Toronto, Hamilton); the background site showed minor differences (i.e., minimal loading of ACY alongside the otherwise similar high loading in FLT and PYR). A variant of a "retene" factor was also resolved at all sites (i.e., characterized by high loading in the wood combustion marker species retene), explaining between $8 \%$ and $17 \%$ of data variance. The "retene" factor was least distinctly resolved at the background site, consistent with its remote location; the co-extraction of RET alongside the aged species MPYR (i.e., MePAH indicating aging of parent PAH in source-receptor transport) suggested that a wood combustion source type is being transported from more distant and variable sources, consistent with the low and sparsely distributed rural population living near the rural site.

In addition to the three common factors, the PCA on proportional data resolved a siteunique factor at each of the sites. The site-unique factor at Toronto was characterized by

\footnotetext{
${ }^{28}$ At Hamilton, the retene factor was identifiable in the four-factor model (i.e., retene co-extracted alongside BghiFL and ANT) so was retained for comparison with the other sites. A five-factor model failed to extract retene as highly loaded in any factors; a six-factor model extracted retene as a sole-species factor but resulted in an additional factor that was not useful (i.e., BghiFL extracted alone).
} 
the medium MW species alongside FLU (i.e., labeled "medium + volatile", Figure B.4$3)$. This factor loading pattern was observed at the other sites only at much higher model orders, suggesting that the highly urbanized Toronto central site monitor was strongly influenced by few but intense local PAH source types; this is consistent with the variance in the Toronto data supporting only a low model order (i.e., scree plot showed sharp elbow at $\mathrm{p}=4$ for all PCA transforms and variants modeled). The site-unique factor at Hamilton was loaded in fluorene and its methylated compound (i.e., labeled "FLU + MFLU", Figure B.4-3-b). Since these species were consistently extracted together, insensitive to model order, and because this factor explains a sizable $11 \%$ of the Hamilton dataset variance, the PCA results indicated significant influence from a fluorenecharacterized source; the unique source type was indicated as local to Hamilton by the presence of both parent and methylated fluorene in similar proportion (i.e., receiving emissions in both "fresh" and "aged" form together). Finally, a factor characterized by high loading in the most volatile species ACY was extracted only at the background site (i.e., at the urban sites ACY was extracted alongside several other volatile and light molecular weight species); the factor was also highly loaded in the aged species MFLU, consistent with the site being impacted by PAH emissions transported greater distances (i.e., 'aged' species). 
(a) by site

Toronto (urban) factors.

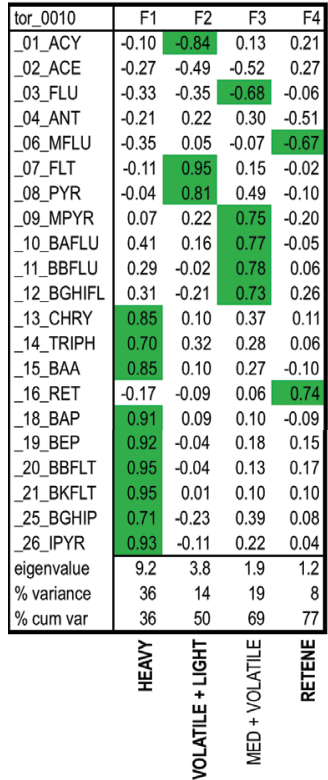

Hamilton (urban) factors.

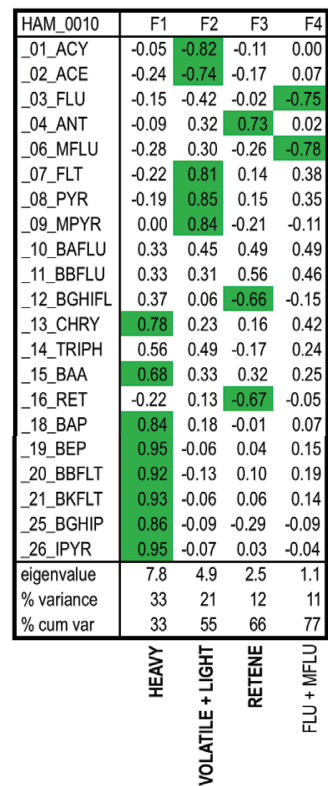

Egbert (rural/background) factors.

\begin{tabular}{|c|c|c|c|c|}
\hline EGB_0010 & $\overline{F 1}$ & $\overline{F 2}$ & F3 & $\mathrm{F} 4$ \\
\hline 01_ACY & 0.10 & 0.15 & 0.50 & 0.63 \\
\hline 02_ACE & -0.27 & -0.31 & 0.25 & 0.43 \\
\hline 03_FLU & -0.12 & -0.61 & 0.39 & -0.43 \\
\hline 04_ANT & -0.29 & 0.44 & 0.16 & 0.10 \\
\hline _06_MFLU & -0.36 & -0.16 & 0.06 & -0.74 \\
\hline 07_FLT & 0.08 & -0.02 & -0.92 & -0.04 \\
\hline 08_PYR & -0.27 & 0.27 & -0.80 & -0.09 \\
\hline 09_MPYR & -0.04 & 0.83 & -0.04 & -0.01 \\
\hline 10 BAFLU & 0.23 & 0.83 & -0.15 & 0.02 \\
\hline _11_BBFLU & 0.20 & 0.84 & -0.01 & -0.06 \\
\hline 12_BGHIFL & 0.67 & 0.34 & -0.26 & 0.25 \\
\hline _13_CHRY & 0.84 & 0.11 & -0.14 & 0.16 \\
\hline _14_TRIPH & 0.72 & 0.03 & -0.20 & 0.25 \\
\hline _15_BAA & 0.69 & 0.27 & 0.14 & -0.20 \\
\hline _16_RET & -0.31 & 0.63 & -0.04 & 0.09 \\
\hline _18_BAP & 0.73 & -0.07 & 0.04 & -0.39 \\
\hline - 19 BEP & 0.90 & -0.09 & 0.02 & 0.12 \\
\hline 20_BBFLT & 0.90 & -0.10 & 0.02 & 0.23 \\
\hline 21__BKFLT & 0.88 & -0.13 & 0.02 & 0.05 \\
\hline 25_BGHIP & 0.87 & 0.01 & 0.17 & -0.10 \\
\hline _26_IPYR & 0.85 & 0.01 & 0.21 & 0.05 \\
\hline eigenvalue & 7.2 & 3.7 & 2.1 & 1.5 \\
\hline$\%$ variance & 34 & 17 & 10 & 8 \\
\hline$\%$ cum var & 34 & 51 & 61 & 69 \\
\hline & 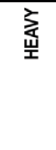 & 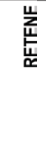 & 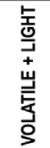 & $\begin{array}{l}\text { ¿े } \\
\text { 岀 } \\
\text { 夏 } \\
\text { g }\end{array}$ \\
\hline
\end{tabular}

(b) by similar / site-unique species pattern

HEAVY factor.

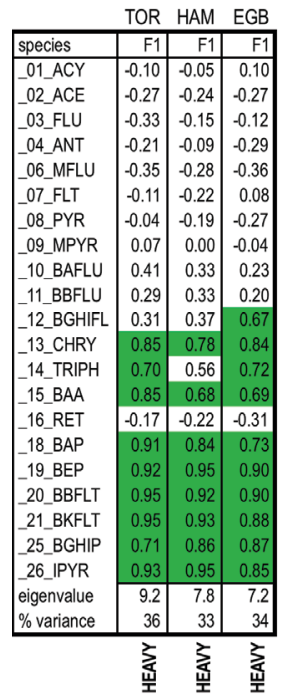

VOLATILE + LIGHT factor.

TOR HAM EGB

\begin{tabular}{|c|c|c|c|}
\hline species & F2 & F2 & $F_{3}$ \\
\hline 01_ACY & -0.84 & -0.82 & 0.50 \\
\hline 02_ACE & -0.49 & -0.74 & 0.25 \\
\hline 03_FLU & -0.35 & -0.42 & 0.39 \\
\hline 04_ANT & 0.22 & 0.32 & 0.16 \\
\hline 06_MFLU & 0.05 & 0.30 & 0.06 \\
\hline 07_FLT & 0.95 & 0.81 & -0.92 \\
\hline _08_PYR & 0.81 & 0.85 & -0.80 \\
\hline 09_MPYR & 0.22 & 0.84 & -0.04 \\
\hline 10_BAFLU & 0.16 & 0.45 & -0.15 \\
\hline 11_BBFLU & -0.02 & 0.31 & -0.01 \\
\hline _12_BGHIFL & -0.21 & 0.06 & -0.26 \\
\hline 13_CHRY & 0.10 & 0.23 & -0.14 \\
\hline 14_TRIPH & 0.32 & 0.49 & -0.20 \\
\hline _15_BAA & 0.10 & 0.33 & 0.14 \\
\hline _16_RET & -0.09 & 0.13 & -0.04 \\
\hline 18_BAP & 0.09 & 0.18 & 0.04 \\
\hline _19_BEP & -0.04 & -0.06 & 0.02 \\
\hline _20_BBFLT & -0.04 & -0.13 & 0.02 \\
\hline _21_BKFLT & 0.01 & -0.06 & 0.02 \\
\hline 25_BGHIP & -0.23 & -0.09 & 0.17 \\
\hline _26_IPYR & -0.11 & -0.07 & 0.21 \\
\hline eigenvalue & 3.8 & 4.9 & 2.1 \\
\hline$\%$ variance & 14 & 21 & 10 \\
\hline & 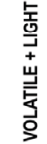 & 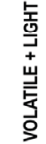 & 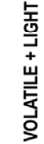 \\
\hline
\end{tabular}

RETENE factor.

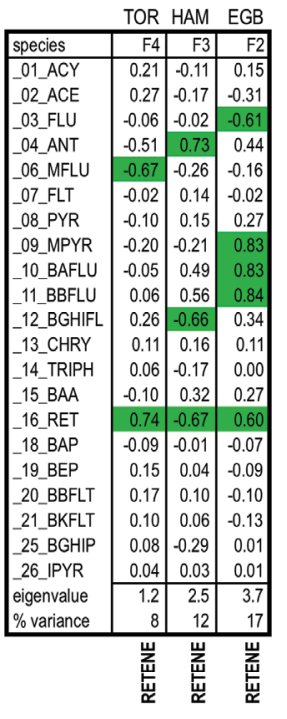

Site-unique factors.

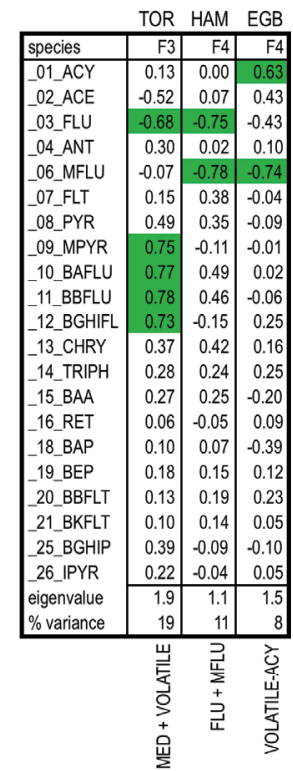

Figure B.4-3. PCA rotated factor loadings at urban and rural sites (proportional data). 
To explore the receptor model's sensitivity to seasonal stratification, year-round and seasonally stratified (i.e., "summer", "winter") PCA model factors were compared (Figure B.4-4, Figure B.4-5). PCA seasonal sensitivity analysis using proportional data found identical results to the seasonal sensitivity analysis using log-transformed data.

By species group: (i) heavy MW species showed the least sensitivity to seasonal split (i.e., factors extracted for the summer and winter data subsets had similar factor loading patterns as those extracted from the entire dataset); (ii) light MW species, inclusive of more volatile species (ACE, FLU) showed fairly small sensitivity to seasonal split; and, (iii) medium MW species proved more sensitive to seasonal split at all sites. The arrangement of heavy and light species in factor patterns that were broadly similar between no-split, summer, and winter model results is consistent with the phase partitioning of these species groups being relatively independent of season (i.e., light species largely in vapour phase, heavy species largely in particle phase). The greater seasonal sensitivity of medium molecular weight species was unsurprising as their vapour/particle phase partitioning is more variable and poses greater challenge to sourcereceptor mass conservation.

By species, greater sensitivity to seasonal stratification was seen for the most highly volatile species ACY and, to a lesser extent, ANT. This recommended that greater model uncertainty be assigned to these species in the advanced PMF receptor model.

By site type, the urban results showed little sensitivity to seasonal split (i.e., model order and factor loading patterns similar between no-split, winter, and summer models); the rural site showed moderate sensitivity to seasonal split as expected due to its distant location from sources.

By season, year-round PCA factors more closely resembled winter factors than summer factors. This was expected since winter dates comprised a greater portion of the yearround dataset for all three sites but is also partially due to greater local source changes in summer (i.e., space heating sources typically absent) and transport-related effects on source-receptor conservation being greatest in summer due to elevated temperatures and reduced atmospheric stability. 


\section{(a) Toronto}

\begin{tabular}{|c|c|c|c|c|c|c|c|c|c|c|c|c|}
\hline SITE & TOR & TOR & TOR & TOR & TOR & TOR & TOR & TOR & TOR & TOR & TOR & TOR \\
\hline DATASET & SW & SW & sw & sw & s & S & $S$ & s & w & W & W & \\
\hline outliers & out1 & out1 & out1 & out1 & out1 & out1 & out1 & out1 & out1 & out1 & out1 & out1 \\
\hline sample size, $n$ & 367 & 367 & 367 & 367 & 151 & 151 & 151 & 151 & 205 & 205 & 205 & 205 \\
\hline model order, $p$ & 4 & 4 & 4 & 4 & 4 & 4 & 4 & 4 & 4 & 4 & 4 & \\
\hline FACTOR\# & F1 & F2 & F3 & F4 & F1 & F2 & F3 & $\mathrm{F} 4$ & $\mathrm{~F} 1$ & F2 & F3 & $\mathrm{F} 4$ \\
\hline -01_ACY & 0.02 & 0.04 & 0.83 & 0.28 & -0.04 & -0.10 & -0.79 & -0.13 & -0.08 & 0.09 & -0.87 & 0.29 \\
\hline -02_ACE & -0.20 & -0.63 & 0.42 & 0.21 & -0.20 & -0.67 & -0.44 & -0.23 & -0.23 & -0.72 & -0.28 & 0.10 \\
\hline _03_FLU & -0.26 & -0.69 & 0.29 & -0.23 & -0.28 & -0.69 & -0.49 & 0.08 & -0.45 & -0.47 & 0.42 & -0.43 \\
\hline _04_ANT & -0.30 & 0.59 & -0.30 & -0.42 & -0.30 & 0.73 & 0.00 & 0.19 & -0.23 & 0.61 & -0.09 & -0.55 \\
\hline _06_MFLU & -0.33 & 0.09 & -0.01 & -0.76 & -0.36 & 0.21 & -0.57 & 0.34 & -0.29 & 0.18 & 0.21 & -0.68 \\
\hline -07_FLT & -0.14 & 0.24 & -0.94 & 0.01 & $-0,14$ & 0.25 & 0.92 & -0.04 & 0.16 & 0.20 & 0.88 & 0.11 \\
\hline _08_PYR & -0.04 & 0.55 & -0.78 & -0.03 & -0.01 & 0.68 & 0.67 & 0.00 & 0.19 & 0.59 & 0.59 & 0.08 \\
\hline _09_MPYR & 0.06 & 0.82 & -0.19 & -0.15 & 0.22 & 0.81 & 0.04 & -0.26 & 0.06 & 0.83 & 0.01 & -0.13 \\
\hline _10_BAFLU & 0.48 & 0.10 & -0.06 & 0.03 & 0.51 & 0.72 & 0.17 & 0.05 & 0.40 & 0.64 & 0.09 & -0.02 \\
\hline _11_BBFLU & 0.42 & 0.65 & 0.13 & 0.12 & 0.44 & 0.68 & 0.11 & 0.04 & 0.29 & 0.61 & 0.00 & 0.03 \\
\hline _12_BGHIFL & 0.45 & 0.59 & 0.31 & 0.35 & 0.36 & 0.79 & 0.01 & -0.13 & 0.47 & 0.54 & -0.10 & 0.50 \\
\hline -13_CHRY & 0.87 & 0.28 & -0.07 & 0.17 & 0.84 & 0.31 & 0.22 & 0.12 & 0.86 & 0.22 & 0.09 & 0.20 \\
\hline _14_TRIPH & 0.69 & 0.22 & -0.39 & 0.03 & 0.64 & 0.35 & 0.41 & 0.09 & 0.73 & 0.10 & 0.32 & -0.07 \\
\hline _15_BAA & 0.81 & 0.28 & -0.02 & -0.14 & 0.86 & 0.23 & 0.06 & 0.14 & 0.77 & 0.31 & -0.23 & -0.24 \\
\hline _16_RET & -0.16 & 0.05 & 0.17 & 0.68 & -0.20 & 0.07 & -0.02 & -0.89 & -0.18 & -0.01 & 0.03 & 0.62 \\
\hline _18_BAP & 0.86 & 0.11 & -0.05 & -0.15 & 0.91 & 0.06 & 0.09 & 0.09 & 0.83 & 0.22 & -0.10 & -0.24 \\
\hline _19_BEP & 0.91 & 0.09 & 0.09 & 0.16 & 0.94 & 0.11 & 0.04 & -0.06 & 0.85 & 0.19 & 0.26 & 0.23 \\
\hline _20_BBFLT & 0.94 & 0.03 & 0.09 & 0.19 & 0.97 & 0.07 & 0.05 & 0.03 & 0.89 & 0.05 & 0.21 & 0.24 \\
\hline _21_BKFLT & 0.94 & 0.01 & 0.04 & 0.08 & 0.95 & 0.04 & 0.07 & 0.01 & 0.90 & 0.09 & 0.16 & 0.09 \\
\hline 25_BGHIP & 0.69 & 0.34 & 0.34 & 0.10 & 0.76 & 0.34 & -0.19 & -0.12 & 0.64 & 0.50 & -0.06 & 0.22 \\
\hline _26_PYR & 0.91 & 0.18 & 0.20 & 0.03 & 0.92 & 0.20 & -0.08 & 0.05 & 0.88 & 0.28 & -0.05 & 0.04 \\
\hline eigenvalue & 8.6 & 4.4 & 1.8 & 1.3 & 9.7 & 4.0 & 1.9 & 1.2 & 8.7 & 2.9 & 2.4 & 1.5 \\
\hline$\%$ variance & 36 & 19 & 14 & 8 & 37 & 23 & 14 & 6 & 33 & 18 & 12 & 10 \\
\hline$\%$ cum var & 36 & 54 & 69 & 77 & 37 & 60 & 74 & 80 & 33 & 52 & 64 & 74 \\
\hline & 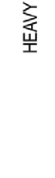 & 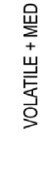 & 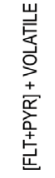 & 㞻 & 离 & 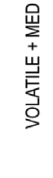 & 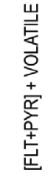 & 勉 & 充 & 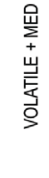 & 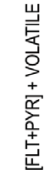 & 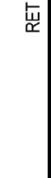 \\
\hline
\end{tabular}

Figure B.4-4. Sensitivity to seasonal stratification, by site. 


\section{(b) Hamilton}

\begin{tabular}{|c|c|c|c|c|c|c|c|c|c|c|c|c|}
\hline SITE & IAM & HAM & HAM & HAM & HAM & HAM & HAM & HAM & HAM & HAM & HAM & HAM \\
\hline ATASET & SW & SW & SW & sW & S & S & S & s & $v$ & $w$ & v & \\
\hline tliers & out1 & out1 & out1 & out1 & out1 & out1 & out1 & out1 & out1 & out1 & out1 & out1 \\
\hline sample size, $n$ & 217 & 217 & 217 & 217 & 84 & 84 & 84 & 84 & 128 & 128 & 128 & 128 \\
\hline model or & 4 & 4 & 4 & 4 & 4 & 4 & 4 & 4 & 4 & 4 & 4 & 4 \\
\hline FACTOR\# & $\mathrm{F} 1$ & F2 & F3 & $\mathrm{F} 4$ & F1 & F2 & F3 & $\mathrm{F} 4$ & $\mathrm{~F} 1$ & F2 & F3 & F4 \\
\hline _01_ACY & -0.08 & -0.83 & -0.16 & 0.14 & -0.04 & -0.76 & 0.10 & 0.04 & -0.39 & -0.70 & 0.04 & 0.35 \\
\hline _02_ACE & -0.25 & -0.74 & -0.10 & 0.04 & -0.29 & -0.72 & -0.14 & -0.29 & -0.25 & -0.69 & -0.15 & 0.17 \\
\hline _03_FLU & -0.20 & -0.51 & 0.03 & -0.70 & -0.44 & -0.78 & -0.26 & -0.10 & -0.32 & -0.09 & -0.15 & -0.82 \\
\hline _04_ANT & -0.11 & 0.32 & 0.69 & 0.05 & -0.11 & 0.02 & 0.14 & 0.88 & -0.13 & 0.48 & -0.55 & 0.26 \\
\hline _06_MFLU & -0.28 & 0.17 & -0.21 & -0.85 & -0.36 & -0.28 & -0.70 & 0.11 & -0.20 & 0.11 & 0.28 & -0.82 \\
\hline _07_FLT & -0.21 & 0.84 & 0.13 & 0.29 & -0.16 & 0.89 & 0.26 & -0.22 & 0.13 & 0.82 & -0.11 & -0.02 \\
\hline _08_PYR & -0.15 & 0.89 & 0.12 & 0.28 & -0.04 & 0.90 & $0.2^{7}$ & 0.09 & 0.01 & 0.87 & 0.11 & 0.10 \\
\hline _09_MPYR & 0.11 & 0.80 & -0.28 & -0.08 & 0.35 & 0.63 & -0.50 & 0.20 & 0.13 & 0.65 & 0.52 & -0.14 \\
\hline _10_BAFLU & 0.35 & 0.52 & 0.47 & 0.45 & 0.40 & 0.42 & 0.64 & 0.34 & 0.29 & 0.74 & -0.31 & 0.37 \\
\hline _11_BBFLU & 0.35 & 0.40 & 0.54 & 0.43 & 0.42 & 0.30 & 0.68 & 0.30 & 0.20 & 0.71 & -0.41 & 0.39 \\
\hline _12_BGHIFL & 0.38 & 0.09 & -0.69 & 0.01 & 0.56 & 0.44 & -0.12 & -0.04 & 0.21 & 0.01 & 0.82 & -0.07 \\
\hline _13_CHRY & 0.76 & 0.32 & 0.15 & 0.41 & 0.69 & 0.42 & 0.49 & 0.13 & 0.86 & 0.27 & -0.09 & 0.23 \\
\hline _14_TRIPH & 0.56 & 0.53 & -0.17 & 0.21 & 0.63 & 0.49 & 0.09 & -0.26 & 0.76 & 0.29 & 0.16 & -0.12 \\
\hline _15_BAA & 0.69 & 0.36 & 0.34 & 0.19 & 0.74 & 0.24 & 0.36 & 0.33 & 0.70 & 0.35 & -0.23 & 0.19 \\
\hline _16_RET & -0.18 & 0.11 & -0.67 & -0.11 & -0.18 & 0.08 & -0.75 & -0.10 & -0.16 & 0.01 & 0.71 & 0.0 \\
\hline-18 & 0.85 & 0.20 & 0.06 & -0.01 & 0.90 & 0.04 & 0.11 & -0.04 & 0.86 & 0.19 & -0.02 & 0.01 \\
\hline _19_BEP & 0.94 & 0.02 & -0.04 & 0.20 & 0.93 & 0.13 & 0.24 & 0.03 & 0.92 & 0.10 & 0.05 & 0.20 \\
\hline _20_BE & 0.93 & -0.05 & 0.07 & 0.23 & 0.90 & 0.05 & 0.36 & 0.05 & 0.93 & 0.05 & -0.07 & 0.21 \\
\hline _21_BKFLT & 0.94 & -0.01 & 0.01 & 0.16 & 0.89 & 0.11 & 0.33 & 0.06 & 0.93 & 0.08 & 0.03 & 0.17 \\
\hline 25_BGHIP & 0.85 & -0.06 & -0.37 & -0.01 & 0.93 & 0.08 & -0.01 & -0.10 & 0.77 & 0.02 & 0.49 & 0.05 \\
\hline _26_IPYR & 0.95 & -0.05 & -0.03 & 0.01 & 0.92 & -0.03 & 0.23 & 0.01 & 0.93 & 0.07 & 0.07 & 0.03 \\
\hline eigenvalue & 8.3 & 4.6 & 2.4 & 1.1 & 10.3 & 3.5 & 2. & 1.1 & 8.6 & 3.5 & 2.8 & 1.3 \\
\hline$\%$ variance & 34 & 23 & 12 & 10 & 36 & 23 & 15 & 7 & 34 & 21 & 12 & 10 \\
\hline$\%$ cum var & 34 & 56 & 68 & 78 & 36 & 59 & 74 & 81 & 34 & 55 & 67 & 77 \\
\hline & 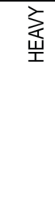 & 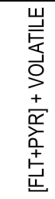 & 卢 & 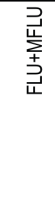 & 岌 & 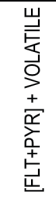 & 苫 & 䘨 & 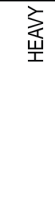 & 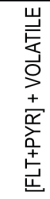 & 点 & 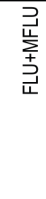 \\
\hline
\end{tabular}

Figure B.4-4 (continued) 
(c) background site

\begin{tabular}{|c|c|c|c|c|c|c|c|c|c|c|c|c|c|c|c|}
\hline SITE & EGB & EGB & EGB & EGB & EGB & $E G B$ & EGB & EGB & EGB & EGB & EGB & EGB & EGB & $E G B$ & EGB \\
\hline DATASET & SW & SW & SW & SW & SW & $S$ & $S$ & $S$ & $S$ & $s$ & W & W & W & W & W \\
\hline outliers & out1 & out1 & out1 & out1 & out1 & out1 & out1 & out1 & out1 & out1 & all & all & all & all & all \\
\hline sample size, $n$ & 232 & 232 & 232 & 232 & 232 & 83 & 83 & 83 & 83 & 83 & 138 & 138 & 138 & 138 & 138 \\
\hline model order, $p$ & 5 & 5 & 5 & 5 & 5 & 5 & 5 & 5 & 5 & 5 & 5 & 5 & 5 & 5 & 5 \\
\hline FACTOR\# & F1 & F2 & F3 & F4 & F5 & $\mathrm{F} 1$ & F2 & F3 & F4 & F5 & $\mathrm{F} 1$ & $\mathrm{~F} 2$ & F3 & F4 & F5 \\
\hline _01_ACY & 0.17 & 0.03 & 0.43 & 0.77 & -0.01 & 0.06 & 0.06 & -0.20 & 0.24 & 0.78 & 0.03 & -0.11 & 0.11 & -0.46 & -0.79 \\
\hline 02_ACE & -0.18 & -0.10 & 0.17 & 0.07 & -0.76 & -0.26 & 0.01 & -0.19 & -0.33 & 0.52 & -0.27 & -0.30 & 0.52 & -0.46 & 0.25 \\
\hline _03_FLU & -0.31 & -0.64 & 0.39 & -0.37 & 0.25 & -0.45 & -0.05 & -0.58 & -0.51 & -0.22 & -0.60 & -0.36 & -0.47 & -0.10 & 0.42 \\
\hline -04_ANT & -0.28 & 0.25 & 0.00 & 0.40 & 0.06 & 0.30 & -0.34 & -0.10 & -0.01 & 0.53 & -0.21 & 0.15 & 0.01 & 0.20 & -0.65 \\
\hline _06_MFLU & -0.54 & -0.12 & 0.12 & -0.54 & 0.29 & -0.30 & -0.28 & -0.39 & -0.45 & -0.37 & -0.35 & 0.03 & -0.80 & -0.14 & 0.17 \\
\hline _07_FLT & 0.08 & -0.05 & -0.91 & -0.09 & 0.15 & 0.22 & -0.01 & 0.84 & -0.07 & -0.32 & 0.11 & -0.04 & 0.05 & 0.91 & 0.12 \\
\hline _08_PYR & -0.23 & 0.27 & -0.81 & -0.09 & 0.07 & 0.14 & -0.10 & 0.91 & 0.04 & -0.20 & 0.06 & 0.48 & 0.09 & 0.73 & -0.13 \\
\hline _09_MPYR & 0.00 & 0.86 & 0.01 & -0.03 & 0.04 & 0.69 & -0.13 & 0.03 & 0.42 & 0.12 & 0.28 & 0.76 & -0.12 & 0.05 & -0.04 \\
\hline _10_BAFLU & 0.20 & 0.73 & -0.10 & 0.15 & 0.47 & 0.87 & -0.13 & 0.16 & 0.29 & 0.05 & 0.32 & 0.72 & -0.28 & 0.20 & -0.05 \\
\hline _11_BBFLU & 0.18 & 0.77 & -0.01 & 0.07 & 0.44 & 0.80 & -0.10 & 0.01 & 0.38 & 0.06 & 0.00 & 0.75 & 0.02 & 0.03 & 0.00 \\
\hline _12_BGHIFL & 0.74 & 0.26 & -0.16 & 0.20 & 0.20 & 0.75 & 0.07 & 0.38 & 0.17 & -0.10 & 0.77 & 0.23 & 0.10 & 0.20 & -0.12 \\
\hline _13_CHRY & 0.87 & 0.02 & -0.10 & 0.16 & 0.16 & 0.79 & 0.29 & 0.26 & -0.11 & -0.04 & 0.91 & 0.00 & 0.10 & 0.17 & -0.09 \\
\hline -14_TRIPH & 0.77 & -0.05 & -0.20 & 0.20 & 0.00 & 0.65 & 0.36 & 0.28 & -0.01 & -0.04 & 0.75 & -0.18 & 0.24 & 0.33 & 0.02 \\
\hline _15_BAA & 0.72 & 0.30 & 0.07 & -0.28 & -0.08 & 0.57 & 0.39 & -0.17 & 0.04 & 0.20 & 0.77 & 0.26 & -0.02 & 0.05 & 0.17 \\
\hline _16_RET & -0.28 & 0.68 & -0.07 & 0.05 & -0.24 & 0.27 & -0.04 & -0.05 & 0.89 & 0.00 & -0.24 & 0.64 & 0.40 & 0.03 & -0.02 \\
\hline _18_BAP & 0.74 & 0.03 & 0.06 & -0.44 & 0.07 & -0.16 & 0.71 & 0.09 & -0.02 & -0.29 & 0.76 & 0.16 & -0.13 & -0.06 & 0.30 \\
\hline _19_BEP & 0.91 & -0.06 & 0.06 & 0.03 & 0.07 & -0.07 & 0.75 & 0.01 & 0.01 & 0.14 & 0.93 & 0.08 & 0.10 & 0.10 & 0.02 \\
\hline 20_BBFLT & 0.92 & -0.11 & 0.05 & 0.17 & 0.08 & 0.34 & 0.66 & 0.16 & 0.01 & 0.06 & 0.93 & -0.07 & 0.19 & 0.14 & -0.06 \\
\hline _21_BKFLT & 0.89 & -0.09 & 0.03 & 0.00 & 0.09 & 0.03 & 0.74 & 0.02 & -0.16 & -0.19 & 0.91 & 0.03 & 0.00 & 0.07 & -0.01 \\
\hline 25_BGHIP & 0.83 & 0.05 & 0.23 & -0.20 & 0.13 & 0.15 & 0.78 & -0.11 & 0.02 & -0.02 & 0.87 & 0.16 & -0.22 & -0.14 & 0.10 \\
\hline _26_IPYR & 0.87 & 0.06 & 0.20 & 0.00 & 0.02 & 0.11 & 0.81 & -0.14 & 0.11 & 0.12 & 0.88 & 0.04 & -0.01 & -0.13 & 0.06 \\
\hline eigenvalue & 7.8 & 3.5 & 2.0 & 1.5 & 1.0 & 6.2 & 3.8 & 2.6 & 1.1 & 1.0 & 8.6 & 2.9 & 1.9 & 1.7 & 1.0 \\
\hline$\%$ variance & 36 & 15 & 10 & 8 & 6 & 22 & 19 & 12 & 9 & 8 & 39 & 13 & 8 & 10 & 7 \\
\hline \multirow[t]{2}{*}{$\%$ cum var } & 36 & 51 & 61 & 69 & 75 & 22 & 40 & 52 & 62 & 70 & 39 & 52 & 59 & 70 & 77 \\
\hline & 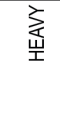 & 㟧 & 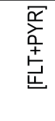 & 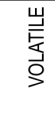 & 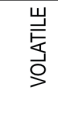 & 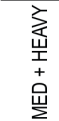 & 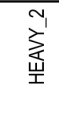 & 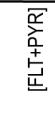 & 岀 & 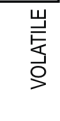 & 岌 & 卢 & $\begin{array}{l}\vec{\partial} \\
\frac{1}{\Sigma}\end{array}$ & 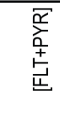 & 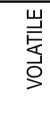 \\
\hline
\end{tabular}

Figure B.4-4 (continued) 
HEAVY factors (SW, S, W).

\begin{tabular}{|c|c|c|c|c|c|c|c|c|c|c|}
\hline SITE & TOR & TOR & TOR & $\overline{\text { HAM }}$ & HAM & HAM & EGB & EGB & EGB & EGB \\
\hline DATASET & SW & $S$ & W & SW & S & W & SW & $S$ & $S$ & W \\
\hline outliers & out1 & out1 & out1 & out1 & out1 & out1 & out1 & out1 & out1 & all \\
\hline sample size, $n$ & 367 & 151 & 205 & 217 & 84 & 128 & 232 & 83 & 83 & 138 \\
\hline model order, $p$ & 4 & 4 & 4 & 4 & 4 & 4 & 5 & 5 & 5 & 5 \\
\hline FACTOR \# & $\mathrm{F} 1$ & F1 & $\mathrm{F} 1$ & $\mathrm{~F} 1$ & F1 & $\mathrm{F} 1$ & F1 & F1 & $\mathrm{F} 2$ & $\mathrm{~F} 1$ \\
\hline _01_ACY & 0.02 & -0.04 & -0.08 & -0.08 & -0.04 & -0.39 & 0.17 & 0.06 & 0.06 & $\overline{0.03}$ \\
\hline $02 \mathrm{ACE}$ & -0.20 & -0.20 & -0.23 & -0.25 & -0.29 & -0.25 & -0.18 & -0.26 & 0.01 & -0.27 \\
\hline O3_FLU & -0.26 & -0.28 & -0.45 & -0.20 & -0.44 & -0.32 & -0.31 & -0.45 & -0.05 & -0.60 \\
\hline _04_ANT & -0.30 & -0.30 & -0.23 & -0.11 & -0.11 & -0.13 & -0.28 & 0.30 & -0.34 & -0.21 \\
\hline _06_MFLU & -0.33 & -0.36 & -0.29 & -0.28 & -0.36 & -0.20 & -0.54 & -0.30 & -0.28 & -0.35 \\
\hline _07_FLT & -0.14 & -0.14 & 0.16 & -0.21 & -0.16 & 0.13 & 0.08 & 0.22 & -0.01 & 0.11 \\
\hline 08_PYR & -0.04 & -0.01 & 0.19 & -0.15 & -0.04 & 0.01 & -0.23 & 0.14 & -0.10 & 0.06 \\
\hline _09_MPYR & 0.06 & 0.22 & 0.06 & 0.11 & 0.35 & 0.13 & 0.00 & 0.69 & -0.13 & 0.28 \\
\hline _10_BAFLU & 0.48 & 0.51 & 0.40 & 0.35 & 0.40 & 0.29 & 0.20 & 0.87 & -0.13 & 0.32 \\
\hline _11_BBFLU & 0.42 & 0.44 & 0.29 & 0.35 & 0.42 & 0.20 & 0.18 & 0.80 & -0.10 & 0.00 \\
\hline 12_BGHIFL & 0.45 & 0.36 & 0.47 & 0.38 & 0.56 & 0.21 & 0.74 & 0.75 & 0.07 & 0.77 \\
\hline 13_CHRY & 0.87 & 0.84 & 0.86 & 0.76 & 0.69 & 0.86 & 0.87 & 0.79 & 0.29 & 0.91 \\
\hline _14_TRIPH & 0.69 & 0.64 & 0.73 & 0.56 & 0.63 & 0.76 & 0.77 & 0.65 & 0.36 & 0.75 \\
\hline 15_BAA & 0.81 & 0.86 & 0.77 & 0.69 & 0.74 & 0.70 & 0.72 & 0.57 & 0.39 & 0.77 \\
\hline _16_RET & -0.16 & -0.20 & -0.18 & -0.18 & -0.18 & -0.16 & -0.28 & 0.27 & -0.04 & -0.24 \\
\hline 18_BAP & 0.86 & 0.91 & 0.83 & 0.85 & 0.90 & 0.86 & 0.74 & -0.16 & 0.71 & 0.76 \\
\hline _19_BEP & 0.91 & 0.94 & 0.85 & 0.94 & 0.93 & 0.92 & 0.91 & -0.07 & 0.75 & 0.93 \\
\hline 20 BBFLT & 0.94 & 0.97 & 0.89 & 0.93 & 0.90 & 0.93 & 0.92 & 0.34 & 0.66 & 0.93 \\
\hline 21_BKFLT & 0.94 & 0.95 & 0.90 & 0.94 & 0.89 & 0.93 & 0.89 & 0.03 & 0.74 & 0.91 \\
\hline 25_BGHIP & 0.69 & 0.76 & 0.64 & 0.85 & 0.93 & 0.77 & 0.83 & 0.15 & 0.78 & 0.87 \\
\hline 26_IPYR & 0.91 & 0.92 & 0.88 & 0.95 & 0.92 & 0.93 & 0.87 & 0.11 & 0.81 & 0.88 \\
\hline eigenvalue & 8.6 & 9.7 & 8.7 & 8.3 & 10.3 & 8.6 & 7.8 & 6.2 & 3.8 & 8.6 \\
\hline$\%$ variance & 35.7 & 37.4 & 33.4 & 33.5 & 36.5 & 34.2 & 36.3 & 21.8 & 18.7 & 38.6 \\
\hline
\end{tabular}

$(F L T+P Y R)+$ VOLATILE factors (SW, S, W).

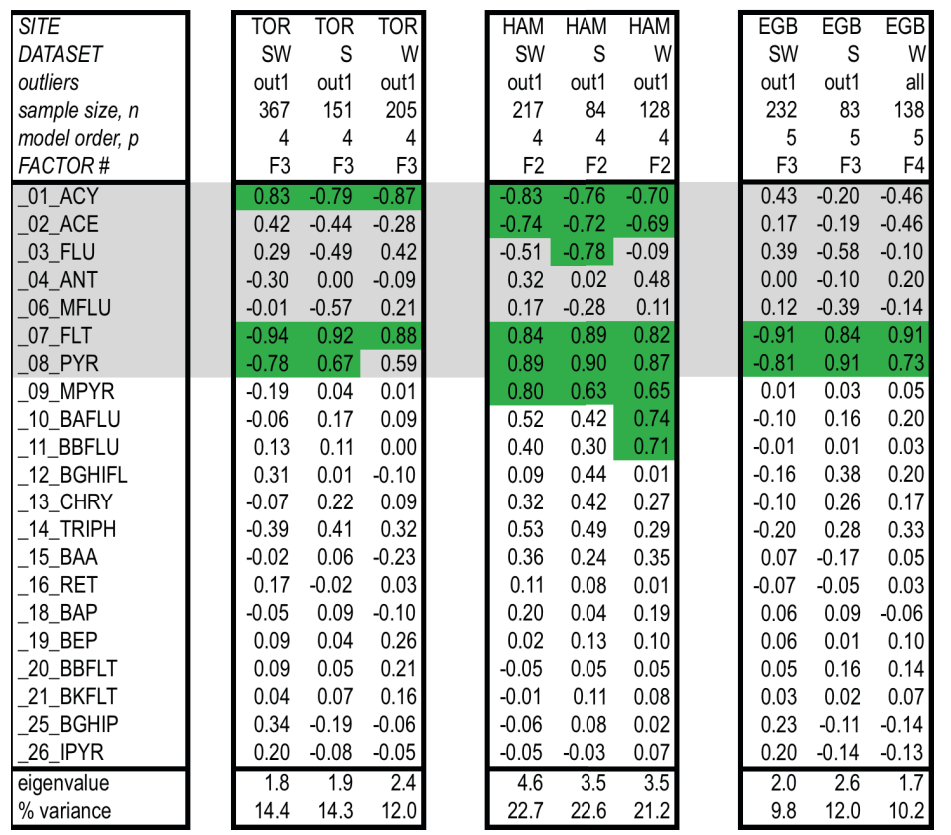

Figure B.4-5. Sensitivity to seasonal stratification, by similar / site-unique species pattern. 
RETENE factors (SW, S, W)

\begin{tabular}{|l|}
\hline SITE \\
DATASET \\
outliers \\
sample size, $n$ \\
model order, $p$ \\
FACTOR\# \\
\hline -01_ACY \\
_02_ACE \\
_03_FLU \\
_04_ANT \\
_06_MFLU \\
_07_FLT \\
-08_PYR \\
_09_MPYR \\
-10_BAFLU \\
-11_BBFLU \\
-12_BGHIFL \\
-13_CHRY \\
-14_TRIPH \\
_15_BAA \\
-16_RET \\
-18_BAP \\
-19_BEP \\
20_BBFLT \\
_1_BKFLT \\
25_BGHIP \\
_6_IPYR \\
\hline eigenvalue \\
\% variance \\
\hline
\end{tabular}

\begin{tabular}{|rrr|}
\hline TOR & TOR & TOR \\
SW & S & W \\
out1 & out1 & out1 \\
367 & 151 & 205 \\
4 & 4 & 4 \\
F4 & F4 & F4 \\
\hline 0.28 & -0.13 & 0.29 \\
0.21 & -0.23 & 0.10 \\
-0.23 & 0.08 & -0.43 \\
-0.42 & 0.19 & -0.55 \\
-0.76 & 0.34 & -0.68 \\
0.01 & -0.04 & 0.11 \\
-0.03 & 0.00 & 0.08 \\
-0.15 & -0.26 & -0.13 \\
0.03 & 0.05 & -0.02 \\
0.12 & 0.04 & 0.03 \\
0.35 & -0.13 & 0.50 \\
0.17 & 0.12 & 0.20 \\
0.03 & 0.09 & -0.07 \\
-0.14 & 0.14 & -0.24 \\
0.68 & -0.89 & 0.62 \\
-0.15 & 0.09 & -0.24 \\
0.16 & -0.06 & 0.23 \\
0.19 & 0.03 & 0.24 \\
0.08 & 0.01 & 0.09 \\
0.10 & -0.12 & 0.22 \\
0.03 & 0.05 & 0.04 \\
\hline 1.3 & 1.2 & 1.5 \\
8.1 & 5.6 & 9.7 \\
\hline & &
\end{tabular}

\begin{tabular}{|rrr|}
\hline HAM & HAM & HAM \\
SW & S & W \\
out1 & out1 & out1 \\
217 & 84 & 128 \\
4 & 4 & 4 \\
F3 & F3 & F3 \\
\hline-0.16 & 0.10 & 0.04 \\
-0.10 & -0.14 & -0.15 \\
0.03 & -0.26 & -0.15 \\
0.69 & 0.14 & -0.55 \\
-0.21 & -0.70 & 0.28 \\
0.13 & 0.26 & -0.11 \\
0.12 & 0.27 & 0.11 \\
-0.28 & -0.50 & 0.52 \\
0.47 & 0.64 & -0.31 \\
0.54 & 0.68 & -0.41 \\
-0.69 & -0.12 & 0.82 \\
0.15 & 0.49 & -0.09 \\
-0.17 & 0.09 & 0.16 \\
0.34 & 0.36 & -0.23 \\
-0.67 & -0.75 & 0.71 \\
0.06 & 0.11 & -0.02 \\
-0.04 & 0.24 & 0.05 \\
0.07 & 0.36 & -0.07 \\
0.01 & 0.33 & 0.03 \\
-0.37 & -0.01 & 0.49 \\
-0.03 & 0.23 & 0.07 \\
\hline 2.4 & 2.1 & 2.8 \\
11.5 & 15.1 & 11.9 \\
\hline
\end{tabular}

\begin{tabular}{|rrr|}
\hline EGB & EGB & EGB \\
SW & S & W \\
out1 & out1 & all \\
232 & 83 & 138 \\
5 & 5 & 5 \\
F2 & F4 & F2 \\
\hline 0.03 & 0.24 & -0.11 \\
-0.10 & -0.33 & -0.30 \\
-0.64 & -0.51 & -0.36 \\
0.25 & -0.01 & 0.15 \\
-0.12 & -0.45 & 0.03 \\
-0.05 & -0.07 & -0.04 \\
0.27 & 0.04 & 0.48 \\
\hline 0.86 & 0.42 & 0.76 \\
0.73 & 0.29 & 0.72 \\
0.77 & 0.38 & 0.75 \\
0.26 & 0.17 & 0.23 \\
0.02 & -0.11 & 0.00 \\
-0.05 & -0.01 & -0.18 \\
0.30 & 0.04 & 0.26 \\
0.68 & 0.89 & 0.64 \\
0.03 & -0.02 & 0.16 \\
-0.06 & 0.01 & 0.08 \\
-0.11 & 0.01 & -0.07 \\
-0.09 & -0.16 & 0.03 \\
0.05 & 0.02 & 0.16 \\
0.06 & 0.11 & 0.04 \\
\hline 3.5 & 1.1 & 2.9 \\
14.7 & 9.1 & 13.1 \\
\hline & & \\
\hline
\end{tabular}

VOLATILE + MED factors (SW, S, W)

Site-unique factor, Toronto (SW, S, W).

\begin{tabular}{|l|}
\hline SITE \\
DATASET \\
outliers \\
sample size, $n$ \\
model order, $p$ \\
FACTOR\# \\
\hline 01_ACY \\
_02_ACE \\
-03_FLU \\
-04_ANT \\
-06_MFLU \\
_07_FLT \\
08_PYR \\
-09_MPYR \\
10_BAFLU \\
11_BBFLU \\
12_BGHIFL \\
13_CHRY \\
14_TRIPH \\
-15_BAA \\
16_RET \\
18_BAP \\
19_BEP \\
20_BBFLT \\
21_BKFLT \\
25_BGHIP \\
26_IPYR \\
\hline eigenvalue \\
$\%$ variance \\
\hline
\end{tabular}

\begin{tabular}{|rrr|}
\hline TOR & TOR & TOR \\
SW & S & W \\
out1 & out1 & out1 \\
367 & 151 & 205 \\
4 & 4 & 4 \\
F2 & F2 & F2 \\
\hline 0.04 & -0.10 & 0.09 \\
-0.63 & -0.67 & -0.72 \\
-0.69 & -0.69 & -0.47 \\
0.59 & 0.73 & 0.61 \\
0.09 & 0.21 & 0.18 \\
0.24 & 0.25 & 0.20 \\
0.55 & 0.68 & 0.59 \\
0.82 & 0.81 & 0.83 \\
0.70 & 0.72 & 0.64 \\
0.65 & 0.68 & 0.61 \\
0.59 & 0.79 & 0.54 \\
0.28 & 0.31 & 0.22 \\
0.22 & 0.35 & 0.10 \\
0.28 & 0.23 & 0.31 \\
0.05 & 0.07 & -0.01 \\
0.11 & 0.06 & 0.22 \\
0.09 & 0.11 & 0.19 \\
0.03 & 0.07 & 0.05 \\
0.01 & 0.04 & 0.09 \\
0.34 & 0.34 & 0.50 \\
0.18 & 0.20 & 0.28 \\
\hline 4.4 & 4.0 & 2.9 \\
18.6 & 22.6 & 18.5 \\
\hline & &
\end{tabular}

Figure B.4-5 (continued) 
Site-unique factors (SW, S, W).

Site-unique factor, Hamilton (SW, S, W).

\begin{tabular}{|c|c|c|c|}
\hline $\begin{array}{l}\text { SITE } \\
\text { DATASET } \\
\text { outliers } \\
\text { sample size, } n \\
\text { model order, } p \\
\text { FACTOR\# }\end{array}$ & $\begin{array}{r}\text { HAM } \\
\text { SW } \\
\text { out1 } \\
217 \\
4 \\
\text { F4 }\end{array}$ & $\begin{array}{r}\text { HAM } \\
W \\
\text { out1 } \\
128 \\
4 \\
\text { F4 }\end{array}$ & $\begin{array}{r}\text { HAM } \\
\text { S } \\
\text { out1 } \\
84 \\
4 \\
\text { F4 }\end{array}$ \\
\hline _01_ACY & 0.14 & 0.35 & 0.04 \\
\hline _02_ACE & 0.04 & 0.17 & -0.29 \\
\hline _03_FLU & -0.70 & -0.82 & -0.10 \\
\hline _04_ANT & 0.05 & 0.26 & 0.88 \\
\hline _06_MFLU & -0.85 & -0.82 & 0.11 \\
\hline _07_FLT & 0.29 & -0.02 & -0.22 \\
\hline _08_PYR & 0.28 & 0.10 & 0.09 \\
\hline 09_MPYR & -0.08 & -0.14 & 0.20 \\
\hline _10_BAFLU & 0.45 & 0.37 & 0.34 \\
\hline _11_BBFLU & 0.43 & 0.39 & 0.30 \\
\hline _12_BGHIFL & 0.01 & -0.07 & -0.04 \\
\hline -13_CHRY & 0.41 & 0.23 & 0.13 \\
\hline _14_TRIPH & 0.21 & -0.12 & -0.26 \\
\hline 15_BAA & 0.19 & 0.19 & 0.33 \\
\hline _16_RET & -0.11 & 0.05 & -0.10 \\
\hline _18_BAP & -0.01 & 0.01 & -0.04 \\
\hline _19_BEP & 0.20 & 0.20 & 0.03 \\
\hline 20_BBFLT & 0.23 & 0.21 & 0.05 \\
\hline 21_BKFLT & 0.16 & 0.17 & 0.06 \\
\hline 25_BGHIP & -0.01 & 0.05 & -0.10 \\
\hline _26_IPYR & 0.01 & 0.03 & 0.01 \\
\hline \multirow{3}{*}{$\begin{array}{l}\text { eigenvalue } \\
\% \text { variance }\end{array}$} & 1.1 & & 1.1 \\
\hline & 10 & 10 & 7 \\
\hline & 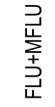 & 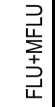 & $\overline{5}$ \\
\hline
\end{tabular}

VOLATILE factor (SW, S, W).

Site-unique factor, Egbert (SW, S, W).

\begin{tabular}{|l|}
\hline SITE \\
DATASET \\
outliers \\
sample size, $n$ \\
model order, $p$ \\
FACTOR\# \\
\hline 01_ACY \\
02_ACE \\
03_FLU \\
04_ANT \\
06_MFLU \\
07_FLT \\
08_PYR \\
09_MPYR \\
\hline 10 10_BAFLU \\
11_BBFLU \\
12_BGHIFL \\
13_CHRY \\
14_TRIPH \\
15_BAA \\
16_RET \\
18_BAP \\
19_BEP \\
20_BBFLT \\
21_BKFLT \\
25_BGHIP \\
26_IPYR \\
\hline eigenvalue \\
$\%$ variance \\
\hline
\end{tabular}

\begin{tabular}{|rr|r|r|}
\hline EGB & EGB & EGB & EGB \\
SW & SW & S & W \\
out1 & out1 & out1 & all \\
232 & 232 & 83 & 138 \\
5 & 5 & 5 & 5 \\
F4 & F5 & F5 & F5 \\
\hline 0.77 & -0.01 & 0.78 & -0.79 \\
0.07 & -0.76 & 0.52 & 0.25 \\
-0.37 & 0.25 & -0.22 & 0.42 \\
0.40 & 0.06 & 0.53 & -0.65 \\
-0.54 & 0.29 & -0.37 & 0.17 \\
-0.09 & 0.15 & -0.32 & 0.12 \\
-0.09 & 0.07 & -0.20 & -0.13 \\
-0.03 & 0.04 & 0.12 & -0.04 \\
0.15 & 0.47 & 0.05 & -0.05 \\
0.07 & 0.44 & 0.06 & 0.00 \\
0.20 & 0.20 & -0.10 & -0.12 \\
0.16 & 0.16 & -0.04 & -0.09 \\
0.20 & 0.00 & -0.04 & 0.02 \\
-0.28 & -0.08 & 0.20 & 0.17 \\
0.05 & -0.24 & 0.00 & -0.02 \\
-0.44 & 0.07 & -0.29 & 0.30 \\
0.03 & 0.07 & 0.14 & 0.02 \\
0.17 & 0.08 & 0.06 & -0.06 \\
0.00 & 0.09 & -0.19 & -0.01 \\
-0.20 & 0.13 & -0.02 & 0.10 \\
0.00 & 0.02 & 0.12 & 0.06 \\
\hline 1.5 & 1.0 & 1.0 & 1.0 \\
8.0 & 6.4 & 8.2 & 7.2 \\
\hline
\end{tabular}

Figure B.4-5 (continued) 


\section{B.5 PMF modeling details}

\section{Input data treatment}

The PMF data treatment protocol for input concentrations (xij) and uncertainties (sij) reflected best practice guidelines (Reff et al, 2007; US EPA, 2000). NAPS data showed three classes of lab-reported species concentrations: (i) species concentration above $\mathrm{DL}_{\mathrm{j}}$; (ii) species concentration below $\mathrm{DL}_{\mathrm{j}}$; and, (iii) species concentration missing. Species concentration data were treated for PMF input consistent with these three classes: (i) for concentrations above $\mathrm{DL}_{\mathrm{j}}, \mathrm{x}_{\mathrm{ij}}$ set equal to the lab-reported species sample concentration; (ii) for concentrations below $\mathrm{DL}_{\mathrm{j}}, \mathrm{x}_{\mathrm{ij}}$ replaced with one-half $\mathrm{DL}_{\mathrm{j}}$; and, (iii) for missing data, $x_{i j}$ replaced within PMF by the species concentration median. Species $D_{\mathrm{j}}$ were obtained from a laboratory QAQC dataset (Environment Canada, 2005).

\section{Input uncertainty}

Effort was made to apply sample uncertainty $\left(\mathrm{s}_{\mathrm{ij}}\right)$ that would adequately capture, estimate and aggregate the uncertainties associated with laboratory analysis of PAH samples, thereby ensuring appropriate weighting of individual samples in the PMF receptor model. Species uncertainty data were calculated using the 'equation-based' subroutine available in PMF3 and were consistent with species concentration data treatment: (i) for concentrations above $\mathrm{DL}_{\mathrm{j}}$, $\mathrm{s}_{\mathrm{ij}}$ equal to sum of squares of the concentration $\left(\mathrm{x}_{\mathrm{ij}}\right)$ multiplied by the species error fraction $\left(\mathrm{k}_{\mathrm{j}}\right)$ plus the $\mathrm{DL}_{\mathrm{j}}$; (ii) for concentrations below $\mathrm{DL}_{\mathrm{j}}, \mathrm{s}_{\mathrm{ij}}$ equal to $\mathrm{DL}_{\mathrm{j}}$ multiplied by (5/6); and, (iii) for missing data, $\mathrm{s}_{\mathrm{ij}}$ equal to four times the species concentration median. In this fashion, species concentrations below $\mathrm{DL}_{\mathrm{j}}$ or missing were assigned greater modeling uncertainty than species concentrations above $\mathrm{DL}_{\mathrm{j}}$, for which uncertainties were effectively scaled to concentration by the species error fraction. Species error fraction data were obtained from the appropriate laboratory methods document (Environment Canada, 2011f) and more conservative species were assigned less uncertainty than highly volatile and more reactive species, varying from approximately $10 \%$ to $50 \%$. 


\section{Model parameters}

Since PMF down-weights outliers (Hopke, 2003a), and because efforts were made to retain model power and adequate sample-to-sample variability, a conservative protocol was applied for excluding outliers: samples were excluded only if they contained statistically extreme outlier species concentrations (i.e., higher or lower than three times IQR) and outlier status was corroborated by poor model performance for that sample date and exclusion would not meaningfully alter source profiles or trends in contribution.

Additional uncertainty can be assigned to individual species by categorizing a species as 'strong', 'weak', or 'bad' within PMF. The species categorization instructs the model to apply uncertainty to species samples, $\mathrm{s}_{\mathrm{ij}}$, as input for species categorized as 'strong', triple the input file $\mathrm{s}_{\mathrm{ij}}$ value for species categorized as 'weak', and exclude a species from the model for species categorized as 'bad'. A convenient metric for deciding if a species warrants additional uncertainty is the species signal-to-noise ratio ( $\mathrm{SN}$ ), available within the model software, which summarily compares species concentration data with its associated uncertainty (Equation B.5-1). Guidelines for SN are to categorize species as 'strong' for $\mathrm{SN}>2$, 'weak' for $\mathrm{SN}$ between 0.2 and 2, and 'bad' for $\mathrm{SN}<0.2$ (Paatero and Hopke, 2003b). However, since input data uncertainty estimates were judged sufficiently conservative and the species suite was relatively small, it was generally preferred to include species as 'strong', down-weighting species further only if warranted by consistently poor species fit.

Last, additional modeling uncertainty can be added to all species within PMF using the model's C3 parameter. Since the uncertainty estimates on the ambient PAH data were conservative and species-specific (Equation B.5-2), additional modeling uncertainty was explored in sensitivity analysis but otherwise not applied.

Equation B.5-1. Species signal-to-noise ratio in PMF input data.

$$
\begin{aligned}
& S N_{j}=\sqrt{\frac{\sum_{i=1}^{n}\left(x_{i j}-s_{i j}\right)^{2}}{\sum_{i=1}^{n} s_{i j}^{2}}} \\
& S N_{j}=\text { signal-to-noise ratio for species } j \\
& x_{i j}=\text { concentration of species } j \text { in receptor sample } i\left(\mathrm{ng} / \mathrm{m}^{3}\right) \\
& S_{i j}=\text { uncertainty of species } j \text { in receptor sample } i\left(\mathrm{ng} / \mathrm{m}^{3}\right)
\end{aligned}
$$




\section{Model performance criteria}

PMF modeling followed best practice guidelines (US EPA, 2008; Sonoma, 2008) and recommendations in the comprehensive literature review of PMF studies completed by Reff et al (2007). Briefly: explored 3 to 7 factor solutions and selected optimal model order with respect to model performance and to ensure physical meaning of solution; verified solution unique and stable (i.e., converged to local minimum with narrow range in Q; further validated using random seed); verified solution converged to theoretical global minimum (i.e., compared $\mathrm{Q}_{\text {robust }}$ to $\mathrm{Q}_{\text {theory }}$ ) and not subject to outlier influence (i.e., compared $\mathrm{Q}_{\text {robust }}$ to $\mathrm{Q}_{\text {true }}$ ); verified all fitted species modeled well (e.g., high $\mathrm{r}^{2}$, low species residuals, normally distributed species residuals); and, verified solution was reasonably free from rotational ambiguity (i.e., scatter in inter-factor contribution plots, 'G-space'; exploration of F-peak solution rotation). Most critically, model solutions were verified for robustness via bootstrapping analysis, the PMF3 subroutine that repeatedly models differing subsets of the original concentration dataset and statistically summarizes their solutions (US EPA, 2008). This ensured that interpreted solutions were statistically representative results and that factors were sufficiently distinct from each other to merit interpreting as a distinct source type, determined by bootstrap factors being mapped consistently to the same base factor.

\section{Uncertainty on factor profiles}

Bootstrapping analysis, available via subroutine within PMF3, ensured a statistically representative PMF solution and provided a procedurally rational method of quantifying uncertainty on the PMF solution (Reff et al, 2007; Watson et al, 2008) as well as for application of profiles in related investigations at the intraurban scale (Chapter 5). PMF factor profiles retained for interpretation as source types referenced median bootstrap values (i.e., median species concentration, median species explained variance) thereby precluding interpretation of 'singular solutions', such as solutions with atypical values in the concentration profile that may yield a 'better' / lower Q value but may not be statistically representative.

Uncertainty on profiles was estimated by the bootstrap IQR statistics (i.e., narrower IQR quantifying less uncertainty) following an uncertainty formula (Equation B.5-2) devised to distinguish 'characteristic' from 'non-characteristic' profile species, with the latter defined by a first quartile explained variance value less than $5 \%$. Effectively, the majority of profile species were assigned uncertainty based on the IQR (i.e., $Q_{3}-Q_{1}$ ) of the bootstrap runs and relatively few 'non-characteristic' species were assigned uncertainty based on the top-half of the IQR (i.e. $Q_{3}-Q_{2}$; species with very low EV can be assigned near-zero factor profile concentrations in some bootstrap runs, possibly skewing $Q_{1}$ concentration unrealistically below $\mathrm{DL}_{\mathrm{j}}$ ). 
Equation B.5-2. Uncertainty for 'characteristic' and 'non-characteristic' species in PMF factor profiles.

$$
\begin{aligned}
& u_{j}=\frac{\left(Q_{3_{j}}-Q_{1_{j}}\right)}{2} \text { where } Q_{1_{j}} \geq 5 \% \\
& u_{j}=\frac{\left(Q_{3_{j}}-Q_{2_{j}}\right)}{2} \text { where } Q_{1_{j}}<5 \%
\end{aligned}
$$

$u_{j}=$ concentration uncertainty for species $\mathrm{j}$ in bootstrap concentration profile $\left(\mathrm{ng} / \mathrm{m}^{3}\right)$

$Q_{1 j}, Q_{2 j}, Q_{3 j}=$ first, second, third quartile for species $\mathrm{j}$ in bootstrap explained variance profile (\%)

\section{Model order}

The decision process for selecting a PMF model order for interpretation was complex and balanced good model performance with need for model factors interpretable as source types. (Reff et al, 2007) Optimal model order for PMF considered PCA results along with several PMF-specific quantitative diagnostics: overall fit (i.e., proximity of $\mathrm{Q}_{\mathrm{robust}}$ to $\mathrm{Q}_{\text {theory }}$; slope inflection in $\mathrm{p}-\mathrm{Q}$ plot; overall regression of predicted concentrations versus observed concentrations); individual species fit (i.e., shape of residuals histograms; abrupt over-fitting of poorly fitting species; inspection of residuals matrix for measure of least fit and least precise fit versus model order to avoid over-fitment due to excess factors); robustness (i.e., minimal cross-mapping of bootstrapping factors to base run factors). (US EPA, 2008; Sonoma, 2008; Reff et al, 2007; Zhao, 2004; Hopke, 2000; Sofowote, 2011) Quantitative analyses for optimal model order were then subject to the primary practical requirement that all model factors resolved be reasonably and defensibly interpretable as source types (Reff et al, 2007).

On the balance of these analyses, a five-factor PMF solution proved optimal at the urban sites and a four-factor solution proved optimal at the rural site. For Hamilton, the fivefactor model was preferred due to: greater inter-factor distinction; greater profile similarity to Toronto for source types expected a priori at both urban sites (i.e., volatilization factor, traffic factor); expected weekday/weekend trend in the traffic factor; reasonable agreement of source type contributions to emissions inventory and other a priori estimates; added separation between wood and oil combustion source types. For Toronto, the five-factor model was preferred due to: greater inter-factor distinction (i.e., blending of factors apparent at lower model order due to under-extraction); resolving two variants of traffic source type; better model performance (i.e., robustness via bootstrapping results). For the background site, data quality (i.e., low concentrations relative to detection limits; more missing data) and the inherently greater uncertainty associated with its background location (i.e., minimal local sources, greater sourcereceptor distances) permitted a four-factor solution with acceptable model performance and distinct, interpretable profiles. 


\section{Model performance}

The PMF receptor model predicted the original sampled ambient PAH well at all sites (Figure B.5-1), explaining the original ambient dataset best at Hamilton $\left(\mathrm{r}^{2}=0.995\right)$, next-best at Toronto $\left(\mathrm{r}^{2}=0.985\right)$, and slightly less well at the background site $\left(\mathrm{r}^{2}=\right.$ 0.952).
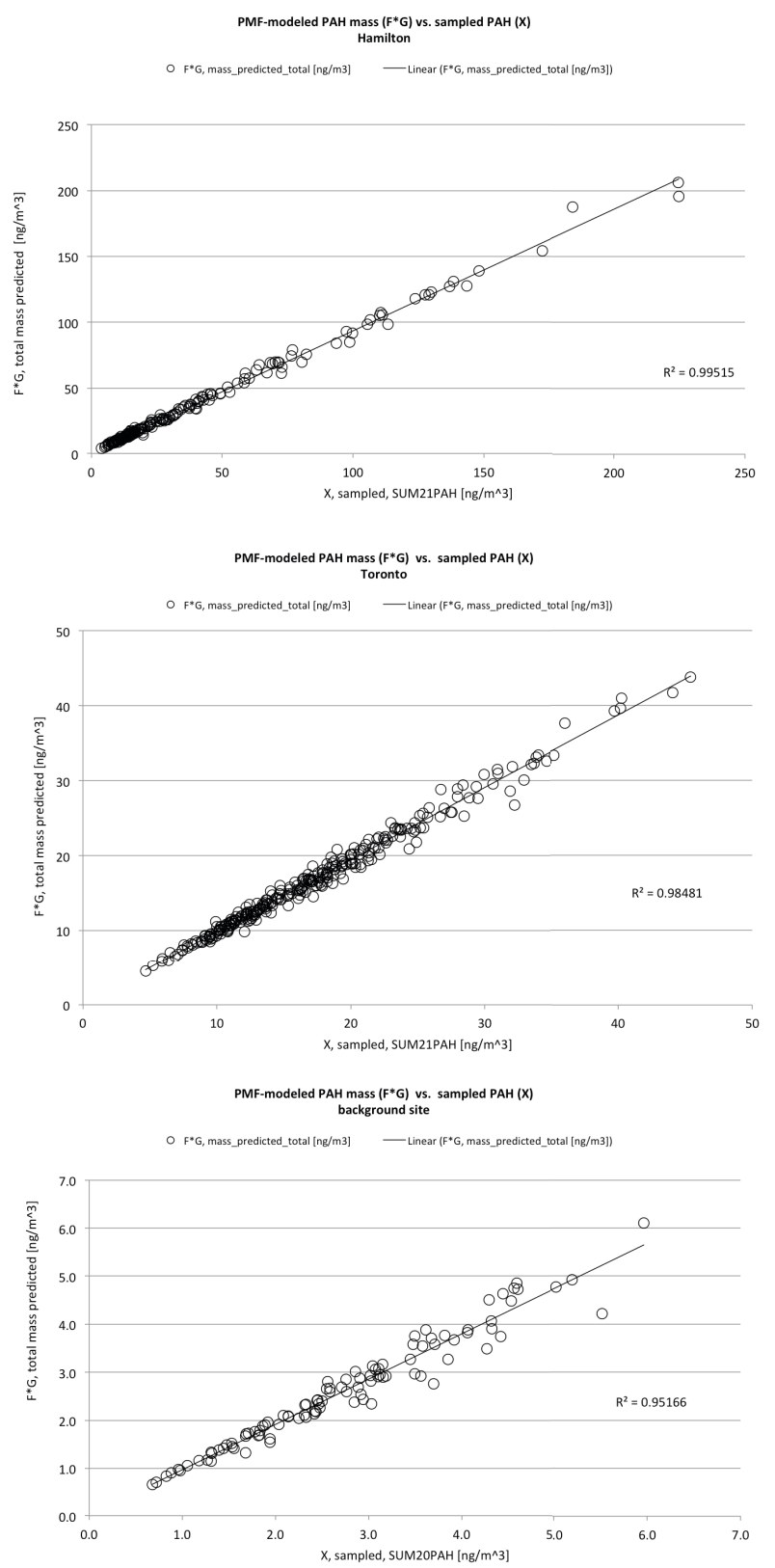

Figure B.5-1. Correlation of PMF-predicted versus measured sampled mass, year-round model; modeled species (urban, $\Sigma_{21} \mathrm{PAH}$; background, $\Sigma_{20} \mathrm{PAH}$ ). 
Model results were also regressed (Figure B.5-2) against the total of all 29 sampled species $\left(\Sigma_{29} \mathrm{PAH}\right)$ to confirm that the larger species suite was adequately being predicted by the subset of fitting species (i.e., 21 species at the urban sites, 20 species at background site) and good correlation was confirmed at all sites (i.e., Hamilton, $\mathrm{r}^{2}=$ 0.989; Toronto, $\mathrm{r}^{2}=0.976$; background, $\mathrm{r}^{2}=0.956$ ).
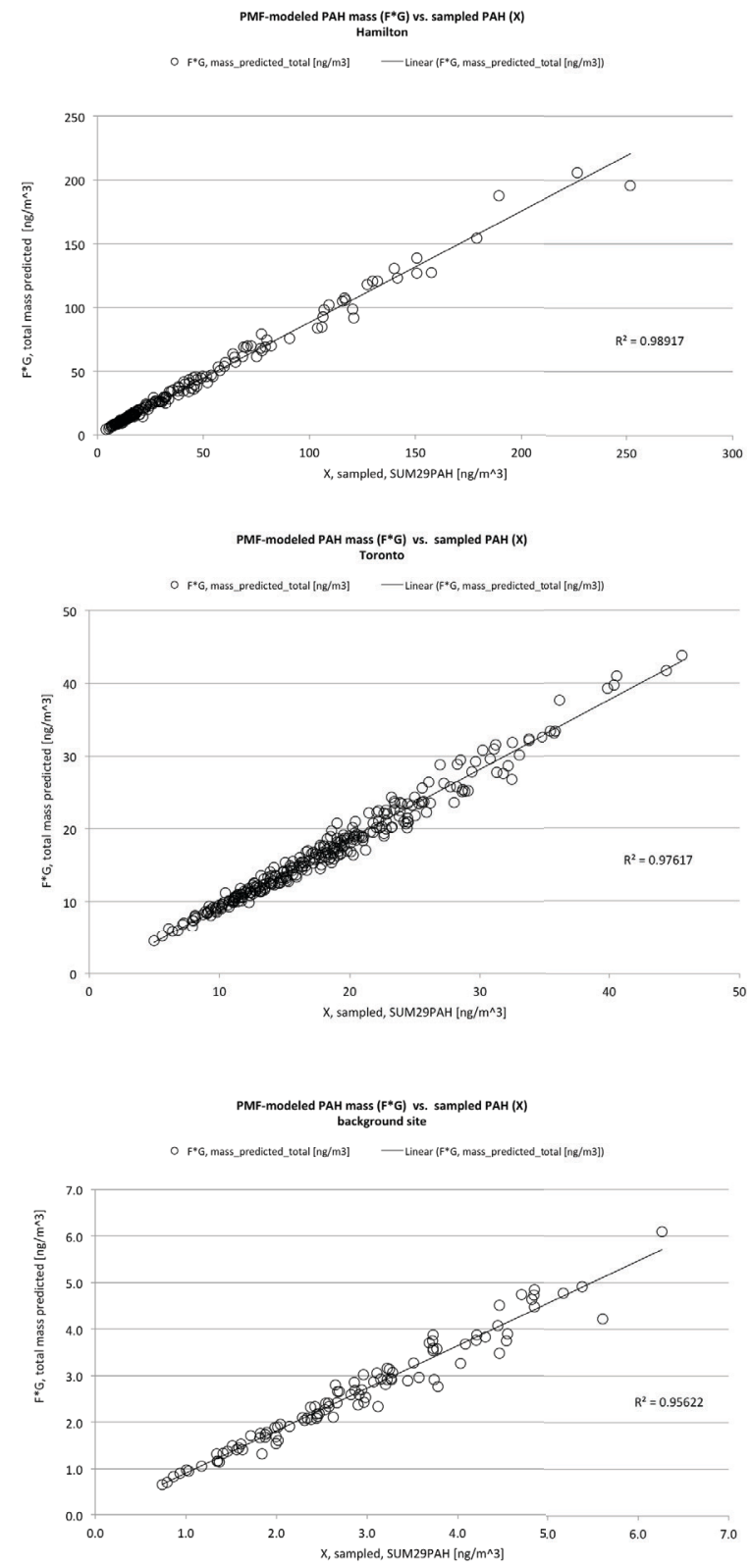

Figure B.5-2. Correlation of PMF-predicted versus measured sampled mass, year-round model; all sampled species $\left(\Sigma_{29} \mathrm{PAH}\right)$. 
As an additional confirmation of overall PMF model performance, a plot of PMFpredicted versus sampled average species concentration (Figure B.5-3) showed excellent overall correlation at all sites (i.e., Hamilton, $\mathrm{r}^{2}=0.998$; Toronto, $\mathrm{r}^{2}=0.999$; background, $\left.r^{2}=0.997\right)$.
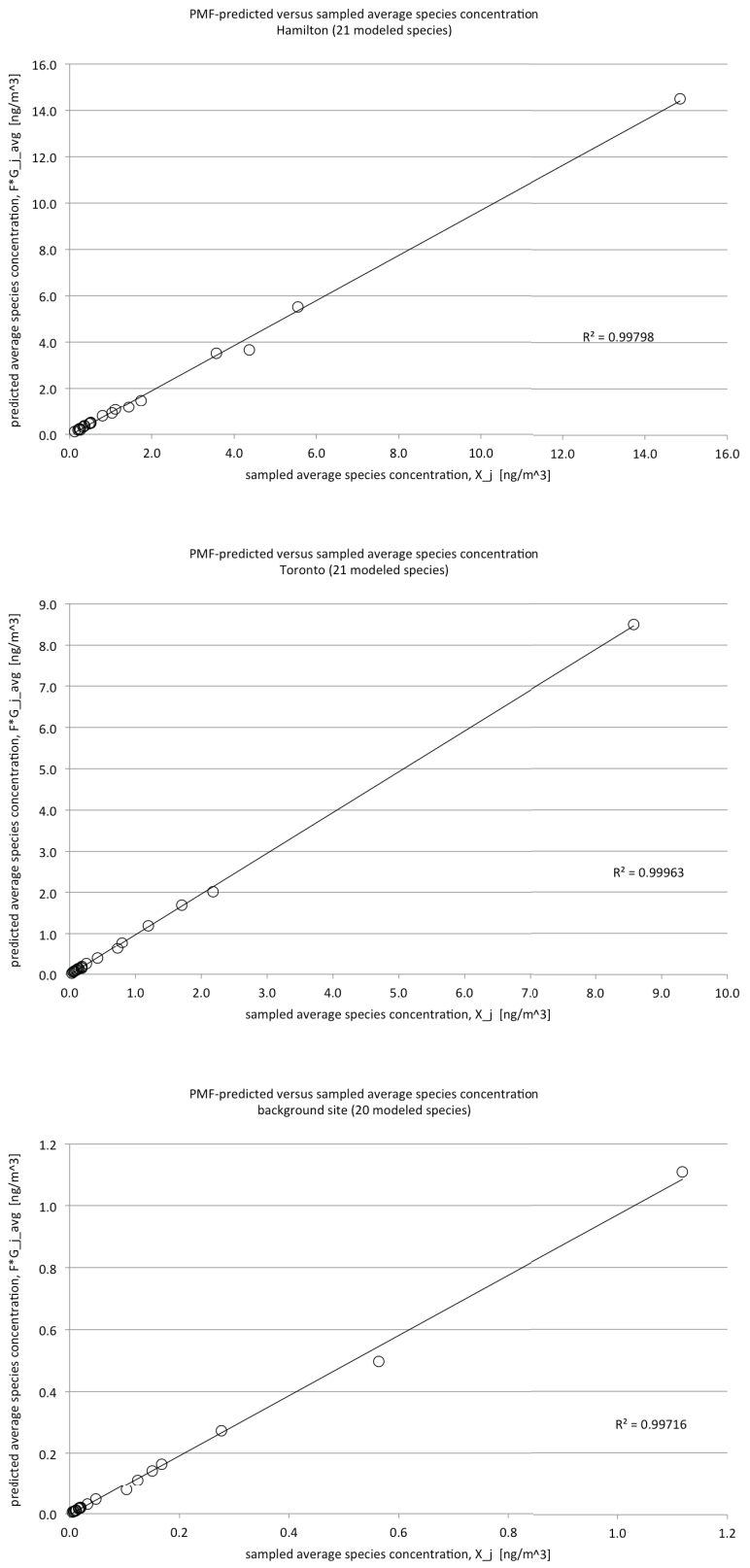

Figure B.5-3. Correlation of PMF-predicted versus measured sampled mass, year-round model; average species concentration. 
The model solutions selected as optimal met PMF best practice requirements (i.e., stable, good fit, robust, rotationally unique) and, most important, resolved distinct factor profiles that could be reasonably interpreted as PAH source types. Model performance metrics for the solutions at the urban and background sites are summarized in Table B.5-1.

As expected, there were some differences in model performance between the three site datasets. The Hamilton data permitted very good model performance with minimal exclusion of outliers or down-weighting of species; only $5 \%$ of outlier samples required exclusion and all fitted species could be modeled as 'strong' to yield a well-fit, stable, unique, and robust solution. Briefly: the $\mathrm{Q}_{\text {robust }}$ value was within $12 \%$ of theoretical $\mathrm{Q}$; the solution was stable (range of $\mathrm{Q}_{\text {robust }}=0.06, \Sigma \mathrm{d}^{2}<004$ ); outliers had little effect on the solution $\left(\mathrm{Q}_{\text {robust }} \sim \mathrm{Q}_{\text {true }}\right)$; there were few sample dates with high residuals; majority of modeled species were well fit $\left(\mathrm{r}^{2}>0.9\right)$; factors were rotationally unique (good fill/scatter in all factor pairs). Hamilton model results were also highly robust with minimal crossmapping between factors at a correlation level of 0.6; unmapped factors generally resembled the "retene $+m e P A H$ " factor profile pattern, expected given the higher input uncertainty and reduced fit of these species.

The Toronto data also showed very good model performance but greater sensitivity to outliers (15\% exclusion), expected and less critical given the higher density of the Toronto dataset (293 sampling dates retained). Similar to Hamilton, all modeled species could be modeled as 'strong' to yield: $\mathrm{Q}_{\text {robust }}$ value within $25 \%$ of theoretical Q; stable solution (range of $\mathrm{Q}_{\text {robust }}=0.13, \Sigma \mathrm{d}^{2}<0.16$ ); very few sample dates with high residuals, as expected from greater outlier exclusion; majority of modeled species were well fit $\left(\mathrm{r}^{2}>\right.$ 0.8 ); factors were rotationally unique (good fill/scatter in all pairs). The Toronto model results were highly robust with minimal cross-mapping or unmapped factors (correlation level of 0.6); the minimal cross-mapping seen between the two variants of the "heavy" factor (i.e., "heavy_BAABAP", "heavy_2") confirmed them as distinct variants of a common source type rather than artifacts of over-extraction (i.e., mass splitting can occur at too-high model order; Reff et al, 2007).

Last, the background site data permitted good model performance at a lower model order four-factor solution but the comparably poorer data quality and lower sample size challenged the fit of some species (i.e., ANT showed very poor fit and contributed to poor robustness so was excluded from the model; BAA and BAP could be included but required down-weighting to 'weak'). However, with appropriate input species weightings the model yielded performance metrics similar to the urban sites: $\mathrm{Q}_{\text {robust }}$ value within $2 \%$ of $\mathrm{Q}_{\text {theory }}$; outliers had little effect on the solution $\left(\mathrm{Q}_{\text {robust }} \sim \mathrm{Q}_{\text {true }}\right)$; stable solution (range of $\mathrm{Q}_{\text {robust }}=0.15, \Sigma \mathrm{d}^{2}<0.10$ ); very few sample dates with high residuals (least of all sites); majority of modeled species were well fit $\left(\mathrm{r}^{2}>0.8\right)$; factors rotationally unique (good 
fill/scatter in all pairs). Perhaps most important, the background model results were robust with little cross-mapping and minimal unmapped factors (correlation level of 0.6).

RET fit comparably poorly at all sites but was retained (i.e., assigned high uncertainty) to aid in interpreting the biomass combustion source type; however, fit was notably better at Hamilton $\left(\mathrm{r}^{2}=0.35\right)$ and background $\left(\mathrm{r}^{2}=0.35\right)$ than at Toronto $\left(\mathrm{r}^{2}=0.26\right)$. Consequently, a clear majority of RET was apportioned to a single well-characterized factor at Hamilton and at the background site (i.e., $80 \%$ at Hamilton, $72 \%$ at background) but apportioned across several factors at Toronto in the otherwise optimal solution, suggesting the corresponding source type was less clearly resolved to a single factor at the most highly urbanized site. 
Table B.5-1. PMF performance summary.

\begin{tabular}{|c|c|}
\hline INPUT \& CFG & HAMILTON, year-round data (h0110sw5p) \\
\hline Filename & SvarEC-ham0110sw-oa-0c3-5pF.cfg \\
\hline $\mathrm{n}$, outliers & $\begin{array}{l}\mathrm{n} \_0110 \mathrm{~s} w=200, \mathrm{n} \_ \text {out }=10(5 \% \text { exclusion }) \\
\left.\mathrm{n} \_ \text {model }=190\right)\end{array}$ \\
\hline $\mathrm{S}$ & SvarEC \\
\hline C3 & $0 \%$ \\
\hline species - bad & $\mathrm{ACY}$ \\
\hline m (fitting species) & 21 \\
\hline species - weak & none \\
\hline \multicolumn{2}{|l|}{ STABILITY } \\
\hline$Q_{\text {_t theory }}$ & $3990\left(n^{\star} m\right), 3040$ (Sonoma) \\
\hline Q_rob (range) & $3392(0.06) \rightarrow$ Q_theory $+12 \%$ \\
\hline Q_true (range) & $3401(0.03) \rightarrow \sim Q \_$rob \\
\hline$\Sigma \_d^{2}$ & $<0.04$ \\
\hline \multicolumn{2}{|l|}{ FIT } \\
\hline$r^{2}$ & $\begin{array}{ll} & \text { majority }>0.9 \\
- & \mathrm{RET}=0.35, \mathrm{ACE}=0.79\end{array}$ \\
\hline \# res $>|3|$ & 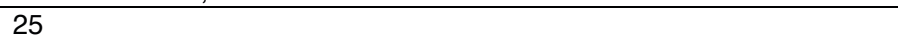 \\
\hline high res species $\left(\#\right.$ res, $\left.r^{2}\right)$ & BAA (9 samples, $\left.r^{2}=0.95\right) ;$ TRIP $\left(4, r^{2}=0.96\right) ; \operatorname{MPYR}\left(4, r^{2}=0.95\right)$ \\
\hline \multicolumn{2}{|l|}{ G-space } \\
\hline edge? & $\begin{array}{l}\text { good fill/scatter in all F pairs } \\
\text { (i.e., slight edge in } 4 \mathrm{~F} \text { pairs expected since did not screen all } 0123 \\
\text { outliers) }\end{array}$ \\
\hline \multicolumn{2}{|l|}{ ROBUST } \\
\hline bootstrapping $(R \geq 0.6)$ & $97 / 94 / 100 / 97 / 99 / 7 U$ \\
\hline shape unmapped? & - $\quad$ largely RET \\
\hline notes & - $\quad$ excellent bootstrap (mapping, narrow IQR, base run $\sim$ IQR median) \\
\hline \multicolumn{2}{|l|}{ FACTORS } \\
\hline label (i.e., based on F_EV) & $\begin{array}{ll}\text { - } & \text { VOL }(16 \% \text { G) } \\
\text { - } & \text { RET+MePAH (15\%, 80\% retene) } \\
\text { - } & \text { HVY }(13 \%) \\
\text { - } & \text { LIGHT }(41 \%, 15 \% \text { retene }) \\
\end{array}$ \\
\hline year trend? & - $\quad$ none \\
\hline season trend? & $\begin{array}{ll} & \text { > summer: LIGHT (moderate) } \\
\text { - } & \text { > winter: VOL (mild) } \\
\text { - } & \text { no seasonal trend: HVY, TRIMOD, RET }\end{array}$ \\
\hline weekday/weekend trend? & $\begin{array}{ll} & \text { no day trends (i.e., small weekend sample size) }\end{array}$ \\
\hline
\end{tabular}

(a) Hamilton 
Table B.5-1 (continued)

\begin{tabular}{|c|c|}
\hline INPUT \& CFG & TORONTO, year-round data (t0110sw5p) \\
\hline Filename & t0110sw-5-p-o123.cfg \\
\hline $\mathrm{n}$, outliers & $\begin{array}{l}\text { n_0110sw }=345, \mathrm{n} \_ \text {out }=52(15 \% \text { exclusion }) \\
\text { n_model }=293\end{array}$ \\
\hline $\mathrm{S}$ & SvarEC \\
\hline $\mathrm{C} 3$ & $0 \%$ \\
\hline species - bad & $\mathrm{ACY}$ \\
\hline m (fitting species) & 21 \\
\hline species - weak & none \\
\hline \multicolumn{2}{|l|}{ STABILITY } \\
\hline Q_theory & $6153\left(n^{*} m\right), 4688$ (Sonoma) \\
\hline Q_rob (range) & $3515(0.13) \rightarrow$ Q_theory $-25 \%$ \\
\hline Q_true (range) & $3517(0.13) \rightarrow \sim Q \_$rob \\
\hline$\Sigma \_d^{2}$ & $<0.16$ \\
\hline \multicolumn{2}{|l|}{ FIT } \\
\hline$r^{2}$ & $\begin{array}{ll}\cdot & \text { majority }>0.8 \\
\text { - } & \mathrm{RET}=0.26, \mathrm{MFLU}=0.58, \mathrm{FLU}=0.73 \\
\end{array}$ \\
\hline \# res > |3| & 7 \\
\hline high res species $\left(\#\right.$ res, $\left.r^{2}\right)$ & TRIP (4 samples, $r^{2}=0.88$ ) \\
\hline \multicolumn{2}{|l|}{ G-space } \\
\hline edge? & good scatter/fill in all pairs \\
\hline \multicolumn{2}{|l|}{ ROBUST } \\
\hline bootstrapping $(R \geq 0.6)$ & $100 / 97 / 100 / 98 / 100 / 2 U$ \\
\hline shape unmapped? & - $\quad$ none \\
\hline notes & $\begin{array}{ll}- & \text { excellent bootstrap (mapping, narrow IQR, base run } \sim \text { IQR median) } \\
\text { three runs of HVY_2 cross-mapped to HVY_BAABAP F } \\
\text { (reasonable for closely related F and source types) }\end{array}$ \\
\hline \multicolumn{2}{|l|}{ FACTORS } \\
\hline label (i.e., based on F_EV) & $\begin{array}{ll}- & \text { HVY_BAABAP }(13 \% \mathrm{G}) \\
\cdot & \text { HVY_2 }(7 \%, 31 \% \text { retene }) \\
\text { - } & \text { LIGHT }(39 \%) \\
\text { - } & \text { RET+med }(8 \%, 25 \% \text { retene }) \\
\text { - } & \text { VOL+RET }(33 \%, 31 \% \text { retene }) \\
\end{array}$ \\
\hline year trend? & • none \\
\hline season trend? & $\begin{array}{ll} & \text { > summer: LIGHT (strong trend) } \\
\text { - } & \text { > winter: VOL+RET (moderate), HVY_2 (strong) } \\
\text { - } & \text { no seasonal trend: HVY_BAABAP, RET+med }\end{array}$ \\
\hline weekday/weekend trend? & $\begin{array}{ll}\text { - } & \text { > weekday: HVY_BAABAP (moderate), RET+med (moderate) } \\
\text { - } & \text { > weekend: HVY_2 (moderate) } \\
\text { - } & \text { no day trend: LIGHT, VOL+RET } \\
\end{array}$ \\
\hline
\end{tabular}

(b) Toronto 
Table B.5-1 (continued)

\begin{tabular}{|c|c|}
\hline INPUT \& CFG & BACKGROUND, year-round data (e0110sw5p) \\
\hline Filename & egb-0510-sw-o-0c3-SvarEC.cfg \\
\hline $\mathrm{n}$, outliers & $\begin{array}{l}\text { n_0510sw }=127, \mathrm{n} \_ \text {out }=26 \\
\text { n_model }=101\end{array}$ \\
\hline $\mathrm{S}$ & SvarEC \\
\hline C3 & $0 \%$ \\
\hline species - bad & ACY, ANT (note: ANT very poor fit and robustness at background site) \\
\hline m (fitting species) & 20 \\
\hline species - weak & $\mathrm{BAA}, \mathrm{BAP}$ \\
\hline \multicolumn{2}{|l|}{ STABILITY } \\
\hline Q_theory & $2020\left(n^{*} m\right), 1481$ (Sonoma) \\
\hline Q_rob (range) & $1447(0.15) \rightarrow$ Q_theory $-2 \%$ \\
\hline Q_true (range) & $1455(0.14) \rightarrow \sim Q \_$rob (within $\left.1 \%\right)$ \\
\hline$\Sigma \_d^{2}$ & $<0.10$ \\
\hline \multicolumn{2}{|l|}{ FIT } \\
\hline$r^{2}$ & $\begin{array}{ll}\text { - } & \text { majority }>0.8 \\
\text { - } & R E T=0.35, \mathrm{BAP}=0.61, \mathrm{FLU}=0.62, \mathrm{BAA}=0.65, \mathrm{MFLU}=0.67 \\
\end{array}$ \\
\hline$\#$ res $>|3|$ & 4 \\
\hline high res species (\# res, $r^{2}$ ) & $\begin{array}{l}\left.\text { PYR (1 sample, } r^{2}=0.88\right) \text {, BBFLU }(1,0.80) \text {, TRIP }(1,0.91), \text { BkFLT } \\
(1,0.92)\end{array}$ \\
\hline \multicolumn{2}{|l|}{ G-space } \\
\hline edge? & good scatter/fill, all F pairs \\
\hline \multicolumn{2}{|l|}{ ROBUST } \\
\hline bootstrapping $(R \geq 0.6)$ & $94 / 10099 / 98 / 2 U$ \\
\hline shape unmapped? & - $\quad \sim$ RET factor \\
\hline notes & $\begin{array}{ll} & \text { good bootstrap (mapping, narrow IQR, base run } \sim \text { IQR median) } \\
- & \text { F2-HVY has base run overestimated versus median of bootstraps } \\
\end{array}$ \\
\hline \multicolumn{2}{|l|}{ FACTORS } \\
\hline label (base on F_EV) & $\begin{array}{ll} & \text { LIGHT }(29 \% G) \\
\text { - } & \text { HVY }(11 \%) \\
\text { - } & \text { RET+med }(25 \%, 72 \% \text { retene }) \\
\text { - } & \text { VOL }(35 \%) \\
\end{array}$ \\
\hline year trend? & - $\quad$ none (some variability only for RET factor) \\
\hline season trend? & $\begin{array}{l}\text { - } \quad \text { > summer: LIGHT (very mild, much attenuated versus urban) } \\
\text { > winter/fall: HVY (strong), VOL (winter, strong), RET+med (fall, } \\
\text { moderate) }\end{array}$ \\
\hline weekday/weekend trend? & $\begin{array}{ll} & >\text { weekend: HVY (strong), VOL (strong), RET+med (moderate) } \\
\text { - } & \text { no day trend: LIGHT }\end{array}$ \\
\hline
\end{tabular}

(c) background 


\section{B.6 PMF Sensitivity Analyses}

\section{Method}

PMF modeling remains a source apportionment technique with several significant subjective elements and these are typically not discussed in any detail in published work. In the interests of transparency (Reff et al, 2007; Watson et al, 2008), replication ${ }^{29}$ (Ioannidis et al, 2005; Moonesinghe et al, 2007) and advancing modeling practice for other practitioners, modeling applied a comprehensive suite of model checks (e.g., effect of time-series length; degree of solution stability, robustness, rotational uniqueness; factor mapping of excluded species) and detailed sensitivity analyses (i.e., sensitivity to input uncertainty estimates, outlier exclusion, base run choice and robustness, seasonal stratification).

While many of these are 'best practice' and have been previously described in general terms (US EPA, 2008; Reff et al, 2007), several have not been explicitly presented (e.g., sensitivity to input uncertainty, mapping of excluded species to corroborate factor interpretations; sensitivity of robustness to base run choice). The intent is to emphasize the nuances involved in PMF receptor modeling and, by example, refine modeling practice so that reasonably experienced practitioners with reasonable familiarity of a given dataset would arrive at generally similar model factors, thereby limiting the more subjective aspects of PMF modeling to factor interpretation.

\section{Model checks}

PMF model sensitivity to time series was assessed at the two urban sites ${ }^{30}$ by comparing with a model variant that used approximately half the dataset (i.e., 2005-2010 samples) and confirmed consistency in model results (i.e., comparable factors and contribution trends); a related check successfully compared model results from the earlier 2001-2004 period with the later 2005-2010 period, confirming significant source types were relevant and consistent in their factor profile over the entire scoped sampling period. Model stability of the interpreted solution was confirmed over a suitably large number of base runs (e.g., 100, 1000) with fixed seed (i.e., for replication of results) and with random

\footnotetext{
${ }^{29}$ In the broader scientific community there has been increasing awareness of "truth" of research findings (Ioannidis, 2005) and replication has been shown as one effective way to confirm research findings or, minimally, corroborate conclusions drawn from research findings; "true replication" is cited as only possible where data and methods are sufficiently well documented (Moonesinghe et al, 2007).

${ }^{30}$ Background site was scoped to 2005-2010 time period due to data quality issues.
} 
seed, verifying a global minimum solution by base run convergence (i.e., narrow range of $\mathrm{Q}_{\text {robust }}, \mathrm{Q}_{\text {true }}$, low $\Sigma \mathrm{d}^{2}$ ). Model robustness of the interpreted solution was confirmed at a suitably large number of bootstrap runs (i.e., 1000 runs, correlation $=0.6$ ) with random seed and with higher correlation coefficients: at all three sites, bootstrap mapping results and bootstrap factor profiles remained robust for all factors at correlations as high as 0.8 and for several factors as high as 0.9 .

The model solutions were considered sufficiently rotationally unique (i.e., good scatter in paired factor contribution plots). However, to investigate the possibility of further optimization, additional runs were made assessing sensitivity to F-peak rotation (i.e., tested values from -0.5 to +0.5 to ensure dQ within tens of units so did not deviate excessively from original solution; US EPA, 2008). While this showed the expected changes in the factor profiles and contributions (i.e., positive rotations rendered factor profiles more distinct which could aid interpretation but simultaneously decreased contributions thereby worsening G-space scatter; negative rotations 'smeared' profiles which rendered them less distinct although generally improved G-space scatter) application of F-peak was judged to provide no net advantage to the model solution with respect to performance parameters or to factor interpretation.

Two additional model checks were made to investigate model exclusion of fitting species. First, since PHEN species abundance was significantly higher than other species (i.e., $35 \%-44 \%$ median across sites) a check was made to ensure that it would not drive collinearity between factors (i.e., a condition for use of PMF-derived input profiles in CMB receptor models; Chapter 5). A model variant excluding PHEN found model results well preserved (i.e., performance statistics maintained; factor profile patterns F_C and F_EV largely maintained with only minor mass reallocation for other modeled species), confirming that model results were not being driven by the most abundant species. Also, while the most volatile species ACY was excluded from the PMF model due to known sampling issues (US EPA, 1999; confirmed for the NAPS dataset by very high species uncertainty estimate), a model variant including the species was found to generally apportion it alongside ACE, thereby corroborating the "volatile" factor profile pattern and its corresponding source type interpretation. 
$\underline{\text { Sensitivity Analyses }}$

Input uncertainty

While this research was able to source representative estimates of uncertainty for the PAH concentration data that reflected inter-species differences, appropriate uncertainty estimates are expected to remain a challenge to obtain for many ambient PAH datasets. To explore the model's sensitivity to input uncertainty, alternate model variants set a fixed uncertainty estimate (i.e., error fraction) for all modeled species, a limitation often seen in the literature. To establish boundaries on the sensitivity analysis, the error fraction was first fixed at two levels (i.e., 10\%, 25\%; all species modeled as 'strong'): the lower fixed error fraction of $10 \%$ led to overly high fitting function values $\left(\mathrm{Q}_{\text {robust }}>\mathrm{Q}_{\text {theory }}\right)$, and very high outlier influence $\left(\mathrm{Q}_{\text {true }}>>\mathrm{Q}_{\text {robus }}\right)$, and a very large number of species-dates with high residuals, clearly confirming insufficient uncertainty had been assigned to majority of fitting species; conversely, the higher fixed error fraction of $25 \%$ reduced outlier influence but led to an unstable solution with overly low $\mathrm{Q}_{\text {robust }}\left(\mathrm{Q}_{\text {robust }}<<\mathrm{Q}_{\text {theory }}\right)$ and poor robustness, confirming excessive uncertainty had been assigned to many fitting species. Informed by these boundaries, a fixed error fraction was set to the approximate median of the known species uncertainty estimates $(\sim 15 \%)$ : this returned improved model performance only when some species (e.g., RET) were categorized as 'weak' to further increase their uncertainty estimate, confirming the limitations of using a fixed uncertainty estimate.

Last, additional runs were made with $\mathrm{C} 3$ used to add additional 'in-model' uncertainty (e.g., 10\%) to the species error fractions used in the optimal models. ${ }^{31}$ While model fit was further improved (i.e., residuals decreased) and factor profile patterns (F_C, F_EV) and trends in factor contributions remained largely insensitive, $\mathrm{Q}_{\text {robust }}$ rapidly decreased from $\mathrm{Q}_{\text {theory }}$, run-to-run stability decreased, and robustness deteriorated. This demonstrated that, where input uncertainties are estimated appropriately, significant 'inmodel' adjustment via species down-weighting or C3 should not be needed and may significantly decrease some aspects of model performance; rather than being viewed as a panacea, excessive 'in-model' uncertainty is a strong indication that initial uncertainty estimates require review and better estimates should be sourced (e.g., laboratory analysis Quality Assurance/Quality Control data, field sampling uncertainty estimates). The species-specific error fractions sourced for the ambient PAH data were thus shown to be appropriate for receptor modeling, yielding well-scaled model results $\left(\mathrm{Q}_{\text {robust }} \sim \mathrm{Q}_{\text {true }}\right)$ with

${ }^{31}$ General PMF model recommendation is that, since it is unlikely that all sources of uncertainty in field sampling data would be identified and estimated a priori, a typical lower value of $\mathrm{C} 3=$ $5 \%$ or higher can be used (US EPA, 2008). 
strong model performance (e.g., solution stability, uniqueness, robustness) without excessive 'in-model' uncertainty adjustments.

\section{Outlier exclusion}

Model variants were run with increasing degree of outlier exclusion (i.e., all samples included, exclusion of samples with extreme outlier $\Sigma_{29} \mathrm{PAH}$ concentrations, exclusion of samples with extreme outliers in $\Sigma_{29} \mathrm{PAH}$ and/or individual species concentrations, etc.). The basic factor profile patterns (F_EV, F_C) resolved by PMF and their contributions proved largely insensitive to outlier exclusion (i.e., factor patterns preserved, contributions consistent), confirming that model results were interpreted from a stable set of factor profiles and associated factor contributions. Model fit $\left(\mathrm{r}^{2}\right)$ generally improved as outlier samples were excluded from the model, as expected; however, run-to-run stability, solution uniqueness and robustness could be affected negatively by excessive sample exclusion. Additional consequences of outlier exclusion are reduced modeling power (i.e., reduced sample size may result in lower model order than would otherwise be ideal to discriminate between source types) and loss of explanatory power (e.g., muting of seasonal trends for sources with otherwise strong seasonality).

Since excluding outliers decreased dataset variability, a check of CV confirmed that species time series would retain sufficient variability for modeling by PMF even with all statistically extreme outliers excluded (i.e., best practice guideline of $\mathrm{CV}>0.3$ ): modeled species retained CV between 0.6 and 1.1 at Hamilton, 0.4 to 0.7 at Toronto, and 0.4 and 1.0 at the background site; RET retained a CV of 0.5 at the two urban sites and 0.7 at the background site. The degree to which excluding all outliers reduced species CV was found to provide useful indication of whether outlier exclusion would benefit or disadvantage PMF model performance. The Hamilton dataset (i.e., no outliers excluded) had the greatest time series variability (i.e., high species CV) and excluding all statistical outliers resulted in a large decrease in $\mathrm{CV}$ for most species (i.e., approximate $25 \%$ decrease); thus, outlier exclusion tended to decrease PMF model stability without greatly improving model fit. Conversely, Toronto had the lowest initial dataset variability and outlier exclusion caused only a small further decrease in CV for most species; thus, outlier exclusion improved PMF model fit while retaining model performance and actually improving robustness. The results at the background site lay between the two cases demonstrated by the urban sites.

The sensitivity analysis thus validated the nuanced and conservative outlier exclusion protocol applied in the PMF modeling, retaining good model power and reducing procedural subjectivity by ensuring that decisions on outlier exclusion were procedurally rational (i.e., samples excluded only if statistical outliers and outlier status corroborated 
by poor model performance for that sample date and exclusion would not meaningfully alter source profiles or trends in contribution).

\section{Base run choice}

Typically, the base run yielding the lowest fitting function value (minimum $\mathrm{Q}_{\text {robust }}$ ) is judged 'best' and forms the basis for bootstrapping analysis (i.e., it is the default selection for bootstrapping within PMF software). A final sensitivity analysis at the Hamilton urban site investigated whether PMF bootstrap results were sensitive to choice of base run and, if so, if this could affect the interpreted solution (i.e., F_EV, F_C factor profile patterns constructed from median of bootstrap runs) or its uncertainty estimate (i.e., quantified from IQR of F_EV, F_C bootstrap runs). This sensitivity analysis was not found discussed or explicitly presented in prior receptor modeling literature.

The sensitivity analysis first reviewed 20 base runs for differences in goodness of fit parameters, factor profile patterns (F_EV, F_C), and factor apportionment of characteristic species; this merely confirmed that best practice modeling had been rigorously followed, since exploration of run-to-run consistency is recommended in the PMF practical literature (US EPA, 2008; Reff et al, 2007). Briefly, close review of the base runs showed: model fit was consistent across base runs (i.e., $\mathrm{r}^{2}$, residuals) as expected for a stable solution (i.e., $\mathrm{Q}_{\text {robust }}$ narrow range, low maximum $\Sigma \mathrm{d}^{2}$ ); factor profile patterns showed some degree of difference across base runs, although differences were typically seen for species with low/near-zero EV (i.e., non-characteristic species); trends in median contributions showed inter-run variation insufficient to reverse a characteristic trend but with some attenuation/exaggeration and corresponding small differences in Gspace scatter; and, moderate differences in species apportionment for some base runs (i.e., facilitated by review of species apportionment pie charts within PMF software). Thus, small differences in model results do occur even for a highly stable solution, reinforcing the need to base factor interpretation and uncertainty estimates on factor profiles derived from bootstrap analysis rather than on any single base run.

For a reduced suite of base runs showing moderate inter-run differences, a bootstrap analysis was performed for each base run (i.e., fixed seed $=1$; correlation $=0.6$ ) and robustness was found to differ slightly across base runs (i.e., one run of 20 less robust, remainder comparably robust) but not meaningfully: for F_EV, the most robust base run solution (i.e., narrow IQR, base run species EV near bootstrapping median) was not always the base run with the lowest $Q_{\text {robust }}$ but source type interpretation proved insensitive to base run choice since median bootstrap F_EV profile patterns were similar for all base runs; for F_C, the most robust base run solution did typically correspond to the base run with lowest $\mathrm{Q}_{\text {robust }}$; median bootstrap F_EV and F_C profile patterns were 
similar for all base runs and bootstrap IQR were similar across base runs for majority of species (i.e., narrow IQR for characteristic species in each factor).

The sensitivity analysis thus confirmed that, for a stable set of PMF base run solutions (i.e., global minimum found as indicated by narrow $\mathrm{Q}_{\text {robust }}$ range, low $\Sigma \mathrm{d}^{2}$ ) the base run solution with the minimum $\mathrm{Q}_{\text {robust }}$ can be retained as 'best' and carried forward for bootstrap analysis with assurance that the resulting factor profile patterns (i.e., median and IQR in F_EV, F_C) will be representative of alternate base run bootstrap analyses. However, the recommendation remains to verify consistency in factor interpretation and reporting by comparing bootstrap results across alternate base run solutions (e.g., maximum $\mathrm{Q}_{\text {robust }}$, randomly selected alternate base run), even for stable solutions.

\section{Seasonal stratification}

Although few PAH receptor modeling studies have seasonally stratified time-series data, fall/winter source profiles held some initial interest primarily due to fall/winter sampling data being generally considered more amenable to receptor modeling (i.e., greater sourcereceptor conservation due to lower temperatures, greater atmospheric stability, and additional and/or more intense source emissions). This was balanced with consideration of several advantages afforded by modeling year-round data (i.e., improved source type interpretation via seasonal trends in factor contributions, significantly increased modeling power via increased sample size, ensuring all contributing sources are accounted for, etc.).

To explore model sensitivity to seasonal stratification of the ambient PAH dataset, a variant of the two urban site models was run with only fall/winter ambient samples in the time series and solutions compared with respect to model performance, factor characteristics and factor contributions (Table B.6-1). A number of disadvantages were seen to accrue for the fall/winter PMF model at both sites: generally reduced PMF model performance and more sensitive to outlier exclusion; robustness proved more challenging to attain and bootstrapping results were less robust than for the non-seasonally stratified model; and, the fall/winter model inherently could not resolve seasonal trends (i.e., since spring/summer samples not modeled) thereby precluding seasonal corroboration of interpreted source type. ${ }^{32}$

32 This limitation may be partially mitigated by 'mapping' seasonally stratified model factors to their closest factor analog in the year-round PMF solution under coarse assumption that temporal trends noted for the year-round factor would apply to the seasonally stratified factor variant. 
Factor profile patterns were generally consistent between year-round and fall/winter variants, confirming that source emission profiles observed at the receptor could be well represented by profiles derived from year-round data. Related observations that corroborated the decision to model year-round data were: PCA modeling also showed similar results between year-round and seasonally stratified models (Appendix B.4); seasonal wind rose analysis showed generally similar predominant wind directions between seasons (Appendix B.3); and, many species with larger seasonal sensitivity were assigned suitably greater input uncertainty in the PMF model (e.g., highly volatile or reactive species).

It is understood that other pollutant datasets may show greater differences in seasonal factors without associated PMF model performance issues and thus may merit seasonal stratification; recommendation is that practitioners include a sensitivity analysis to seasonal stratification as part of receptor modeling best practice. 
Table B.6-1. PMF performance comparison, year-round and fall/winter models (NAPS data).

\begin{tabular}{|c|c|c|}
\hline INPUT \& CFG & $\begin{array}{l}\text { HAMILTON, year-round } \\
\text { (h0110sw5p) }\end{array}$ & HAMILTON, winter (h0110w5p) \\
\hline Filename & $\begin{array}{l}\text { SvarEC-ham0110sw-oa-0c3- } \\
\text { 5pF.cfg }\end{array}$ & h0110w-SvarEC-0123-5p5.cfg \\
\hline $\mathrm{n}$, outliers & $\begin{array}{l}\mathrm{n} \_0110 \mathrm{sw}=200, \mathrm{n} \_ \text {out }=10(5 \% \\
\text { exclusion }), \mathrm{n} \_ \text {model }=190\end{array}$ & $\begin{array}{l}\text { n_0110w }=115, \mathrm{n} \_ \text {out }=19(17 \% \text { exclusion }) \\
\text { n_model }=96 \text { (i.e., all of o123) }\end{array}$ \\
\hline $\mathrm{S}$ & SvarEC & SvarEC \\
\hline C3 & $0 \%$ & $0 \%$ \\
\hline species - bad & ACY & ACY \\
\hline m (fitting species) & 21 & 21 \\
\hline species - weak & none & $\begin{array}{l}\text { ANT, PYR, medium MW (MPYR, BAFLU, } \\
\text { BBFLU, BGFLT), TRIP }\end{array}$ \\
\hline \multicolumn{3}{|l|}{ STABILITY } \\
\hline Q_theory & $3990\left(n^{\star} \mathrm{m}\right), 3040$ (Sonoma) & $2016\left(n^{\star} m\right), 1088$ (Sonoma) \\
\hline Q_rob (range) & $3392(0.06) \rightarrow$ Q_theory $+12 \%$ & $816(0.08) \rightarrow$ Q_theory $-25 \%$ \\
\hline Q_true (range) & $3401(0.03) \rightarrow \sim Q \_$rob & $816(0.08) \rightarrow \sim Q \_$rob \\
\hline$\Sigma \_d^{\wedge} 2$ & $<0.04$ & $<0.11$ \\
\hline \multicolumn{3}{|l|}{ FIT } \\
\hline$r^{2}$ & $\begin{array}{l}\cdot \quad \text { majority }>0.9 \\
\text { RET }=0.35, A C E=0.79\end{array}$ & $\begin{array}{l}\cdot \text { majority }>0.9 \\
\cdot \text { RET, } r^{2}=0.53, \text { ANT } r^{2}=0.79\end{array}$ \\
\hline$\#$ res $>|3|$ & 25 & 1 (BAA) \\
\hline high res species (\# res, $r^{2}$ ) & $\begin{array}{l}\left.\text { BAA (9 samples, } r^{2}=0.95\right) ; \operatorname{TRIP}(4, \\
\left.r^{2}=0.96\right) ; \operatorname{MPYR}\left(4, r^{2}=0.95\right)\end{array}$ & BAA (1 sample, $\left.r^{2}=0.94\right)$ \\
\hline \multicolumn{3}{|l|}{ G-space } \\
\hline edge? & good scatter/fill in all pairs & $\begin{array}{l}\text { good fill/scatter in all F pairs } \\
\text { (i.e., moderate edge in some pairs) }\end{array}$ \\
\hline \multicolumn{3}{|l|}{ ROBUST } \\
\hline bootstrapping $(R \geq 0.6)$ & 97 / 94 / 100 / 97 / 99 / $7 \mathrm{U}$ & $95 / 96 / 81 / 100 / 99 / 3 U$ \\
\hline shape unmapped? & - $\quad$ largely RET & - $\quad$ none \\
\hline notes & $\begin{array}{l}\text { excellent bootstrap (mapping, } \\
\text { narrow IQR, base run } \sim \text { IQR } \\
\text { median) }\end{array}$ & $\begin{array}{l}\text { reasonable bootstrap (mapping, narrow } \\
\text { IQR, base run IQR median all except } \\
\text { HVY) but less robust than "sw" } \\
\text { HVY cross-mapping; most often to } \\
\text { HVY_BAABAP ("w" shows "split" of } \\
\text { traffic factor to different traffic modes) }\end{array}$ \\
\hline \multicolumn{3}{|l|}{ FACTORS } \\
\hline label (i.e., based on F_EV) & $\begin{array}{ll}\text { - } & \text { VOL }(16 \% \text { G) } \\
\text { - } & \text { RET+MePAH (15\%, 80\% } \\
& \text { retene) } \\
\text { - } & \text { HVY }(13 \%) \\
\text { - } & \text { LIGHT }(41 \%, 15 \% \text { retene }) \\
\text { - } & \text { TRIMODAL (16\%) } \\
\end{array}$ & 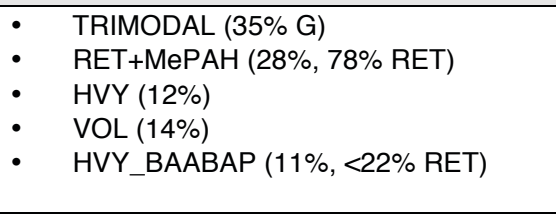 \\
\hline year trend? & - $\quad$ none & - $\quad$ none \\
\hline season trend? & $\begin{array}{ll}- & \text { > summer: LIGHT (moderate) } \\
\text { - } & \text { > winter: VOL (mild) } \\
\text { - } & \text { no seasonal trend: HVY, } \\
& \text { TRIMOD, RET }\end{array}$ & - $\quad$ n/a (no spring/summer samples) \\
\hline weekday/weekend trend? & $\begin{array}{ll} & \text { no day trends (i.e., small } \\
& \text { weekend sample size) }\end{array}$ & - $\quad$ n/a (low weekend sample size) \\
\hline
\end{tabular}

(a) Hamilton 
Table B.6-1 (continued)

\begin{tabular}{|c|c|c|}
\hline INPUT \& CFG & TORONTO, year-round (t0110sw5p) & TORONTO, winter (t0110w5p) \\
\hline Filename & t0110sw-5-p-o123.cfg & t0110w-oa-svarec-5p.cfg \\
\hline $\mathrm{n}$, outliers & $\begin{array}{l}\text { n_0110sw }=345, n \_ \text {out }=52(15 \% \\
\text { exclusion }), n \_ \text {model }=293\end{array}$ & $\begin{array}{l}\mathrm{n} \_0110 \mathrm{w}=190, \mathrm{n} \_ \text {out }=3(2 \% \\
\text { exclusion }), \mathrm{n} \_ \text {model }=187\end{array}$ \\
\hline $\mathrm{S}$ & SvarEC & SvarEC \\
\hline $\mathrm{C3}$ & $0 \%$ & $0 \%$ \\
\hline species - bad & ACY & ACY \\
\hline m (fitting species) & 21 & 21 \\
\hline species - weak & none & TRIP, BAA \\
\hline \multicolumn{3}{|l|}{ STABILITY } \\
\hline Q_theory & $6153\left(n^{\star} m\right), 4688$ (Sonoma) & $3927\left(n^{*} m\right), 2743$ (Sonoma) \\
\hline Q_rob (range) & $3515(0.13) \rightarrow$ Q_theory $-25 \%$ & 1924 ( ) $\rightarrow$ Q_theory $-30 \%$ \\
\hline Q_true (range) & $3517(0.13) \rightarrow \sim Q \_r o b$ & 1924 ( ) $\rightarrow \sim Q \_$rob \\
\hline$\Sigma \Sigma_{-} d^{2}$ & $<0.16$ & $<0.12$ \\
\hline \multicolumn{3}{|l|}{ FIT } \\
\hline$r^{2}$ & $\begin{array}{ll} & \text { majority }>0.8 \\
R E T=0.26, M F L U=0.58, F L U=0.73\end{array}$ & $\begin{array}{ll}\text { - } & \text { majority }>0.8 \\
\text { - } & \mathrm{RET}=0.18, \mathrm{ANT}=0.72, \mathrm{MFLU}=0.74 \\
\end{array}$ \\
\hline$\#$ res $>|3|$ & 7 & 4 \\
\hline $\begin{array}{l}\text { high res species (\# res, } \\
r^{2} \text { ) }\end{array}$ & TRIP (4 samples, $r^{2}=0.88$ ) & $\begin{array}{l}\text { none with more than one date or } \\
\text { particularly high }\end{array}$ \\
\hline \multicolumn{3}{|l|}{ G-space } \\
\hline edge? & good scatter/fill in all pairs & $\begin{array}{l}\text { good scatter/fill (edge in } 2 \text { pair due } \\
\text { solely to inclusion of outliers) }\end{array}$ \\
\hline \multicolumn{3}{|l|}{ ROBUST } \\
\hline bootstrapping $(\mathrm{R} \geq 0.6)$ & $100 / 97 / 100 / 98 / 100 / 2 U$ & $90 / 91 / 100 / 99 / 94 / 11 U$ \\
\hline shape unmapped? & - $\quad$ none & - $\quad$ largely LIGHT (8U) \\
\hline notes & $\begin{array}{l}\text { excellent bootstrap (mapping, narrow } \\
\text { IQR, base run } \sim \text { IQR median) } \\
\text { three runs of HVY_2 cross-mapped to } \\
\text { HVY_BAABAP F (reasonable for } \\
\text { closely related F and source types) }\end{array}$ & $\begin{array}{l}\text { - } \quad \text { overall, good bootstrap (narrow } \\
\text { IQR, base run IQR median) but } \\
\text { mapping worse than "sw" } \\
\text { - } \quad \text { reduced robustness from "sw" } \\
\text { - } \\
\text { cross-mapping to several F for } \\
\text { HVY_2, TRIMODAL }\end{array}$ \\
\hline \multicolumn{3}{|l|}{ FACTORS } \\
\hline $\begin{array}{l}\text { label (i.e., based on } \\
\text { F_EV) }\end{array}$ & $\begin{array}{ll}\cdot & \text { HVY_BAABAP }(13 \% \mathrm{G}) \\
\text { - } & \text { HVY_2 }(7 \%, 31 \% \text { retene }) \\
\text { - } & \text { LIGHT }(39 \%) \\
\text { - } & \text { RET+med }(8 \%, 25 \% \text { retene }) \\
\text { - } & \text { VOL+RET }(33 \%, 31 \% \text { retene }) \\
\end{array}$ & $\begin{array}{ll}\text { - } & \text { LIGHT }(36 \% \text { G) } \\
\text { - } & \text { HVY_2 (19\%) } \\
\text { - } & \text { HVY_BAABAP (9\%) } \\
\text { - } & \text { VOL+RET }(29 \%, 54 \% \text { RET) } \\
\text { - } & \text { RET+med }(7 \%, 28 \% \text { RET }) \\
\end{array}$ \\
\hline year trend? & - none & - $\quad$ none \\
\hline season trend? & $\begin{array}{ll}- & >\text { summer: LIGHT (strong trend) } \\
\text { - } & \text { > winter: VOL+RET (moderate), HVY_2 } \\
\text { (strong) } \\
\text { no seasonal trend: HVY_BAABAP, } \\
\text { RET+med }\end{array}$ & - $\quad$ n/a (no spring/summer samples) \\
\hline $\begin{array}{l}\text { weekday/weekend } \\
\text { trend? }\end{array}$ & $\begin{array}{ll}\text { - } & \text { > weekday: HVY_BAABAP, RET+med } \\
\text { - } & \text { > weekend: HVY_2 } \\
\text { - } & \text { no day trend: LIGHT, VOL+RET }\end{array}$ & $\begin{array}{ll}\text { - } & >\text { weekday: HVY_BAABAP, } \\
& \text { RET+med } \\
\text { - } & >\text { weekend: HVY_2 } \\
\text { - } & \text { no day trend: LIGHT, VOL+RET }\end{array}$ \\
\hline
\end{tabular}

(b) Toronto 


\section{B.7 PMF Factors and Source Type Interpretation}

\section{PMF factors}

The set of factors resolved by PMF at each of the modeled sites are shown in Figure B.71 (i.e., bootstrap results: concentration profile, F_C; explained variance profile, F_EV). Factor profile patterns were stable with model order, generally remaining consistent at a model order one lower or one higher than retained for interpretation.

Factor profile patterns were labeled according to salient species in the F_EV profile. Variants of five basic profile patterns were resolved across the sites: "light", "heavy", "volatile", "trimodal", and "retene".

Factors resolved by PMF could be grouped into broadly similar profile patterns (Figure B.7-2), as anticipated from PCA results (Appendix B.4) as well as due to the general similarity in ambient PAH profile for many Canadian urban centers (Sanderson et al, 2004) as well as seen in previous PCA studies of multiple sites (Mari et al, 2004). For convenience, factor profile patterns were labeled according to the most salient species in their explained variance profiles (i.e., F_EV, \% species apportioned to factor; e.g., factor profile pattern enriched in heavy species was labeled "heavy"). Variants of five basic profile patterns were resolved across the sites: "light", "heavy", "volatile", "trimodal", and "retene". With exception of the "retene" factor, these factor profile patterns were generally consistent even at a model order one lower or one higher than the optimal solution. The factor profile patterns were also broadly similar to factor loading patterns resolved by the PCA model, indicating that PMF factors legitimately explained data variance.

PMF factors were most similar between the two urban sites, as expected: sites were regionally proximal; generally common source types were expected to be influencing the sites; and, inter-site differences in data were highly controlled (i.e., same sampling methods, analysing lab, time period for common species suite). Given the broad similarities in profile patterns (i.e., particularly for F_EV), remaining inter-site

differences in model results (e.g., factor differences, especially in F_C; trends in factor contributions; relative quantification of factor contributions) were deemed meaningful and helped corroborate interpretation of factor profile patterns as source types. 


\section{(a) Hamilton}
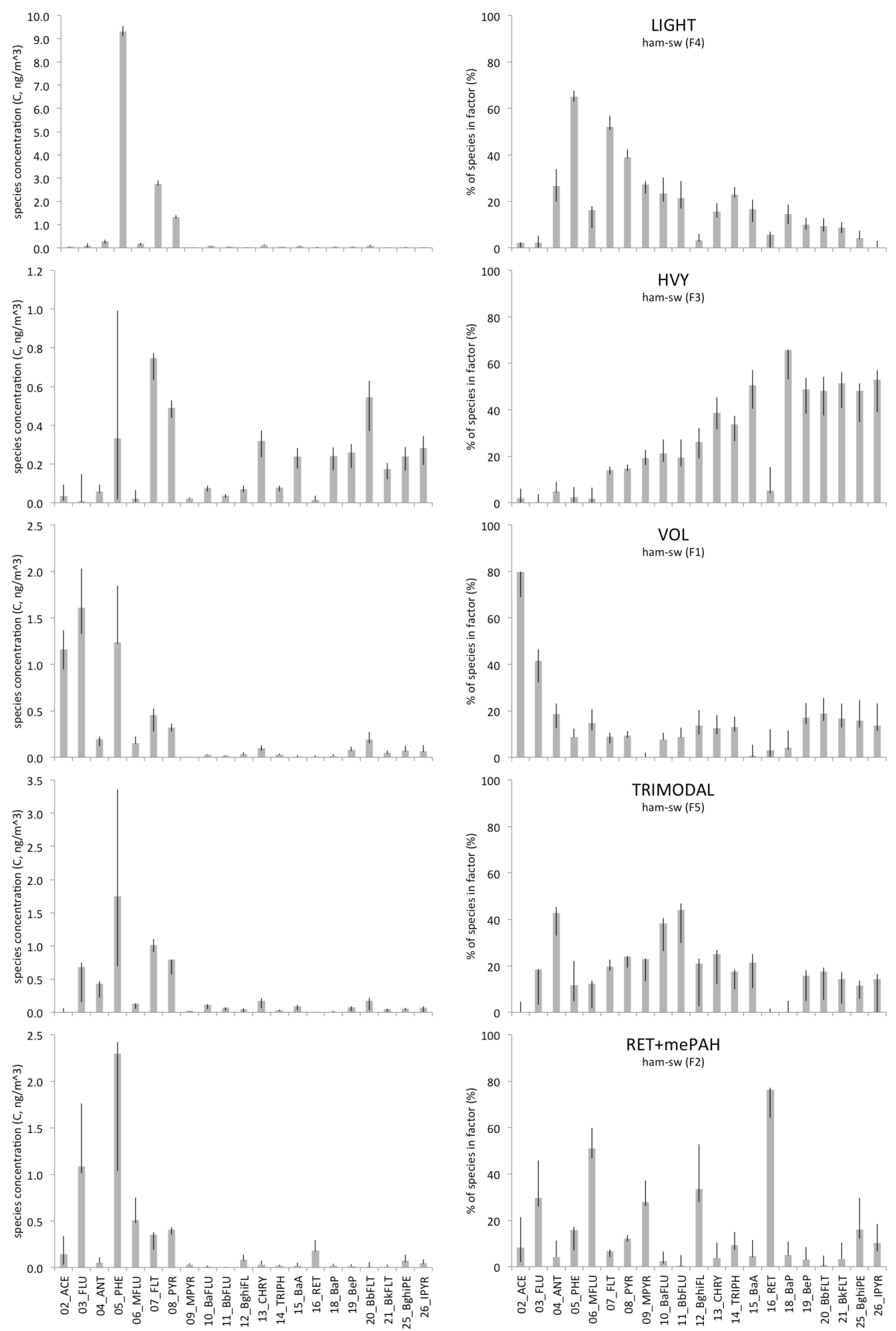

Figure B.7-1. Bootstrap factor profiles (concentration, \% variance). 


\section{(b) Toronto}
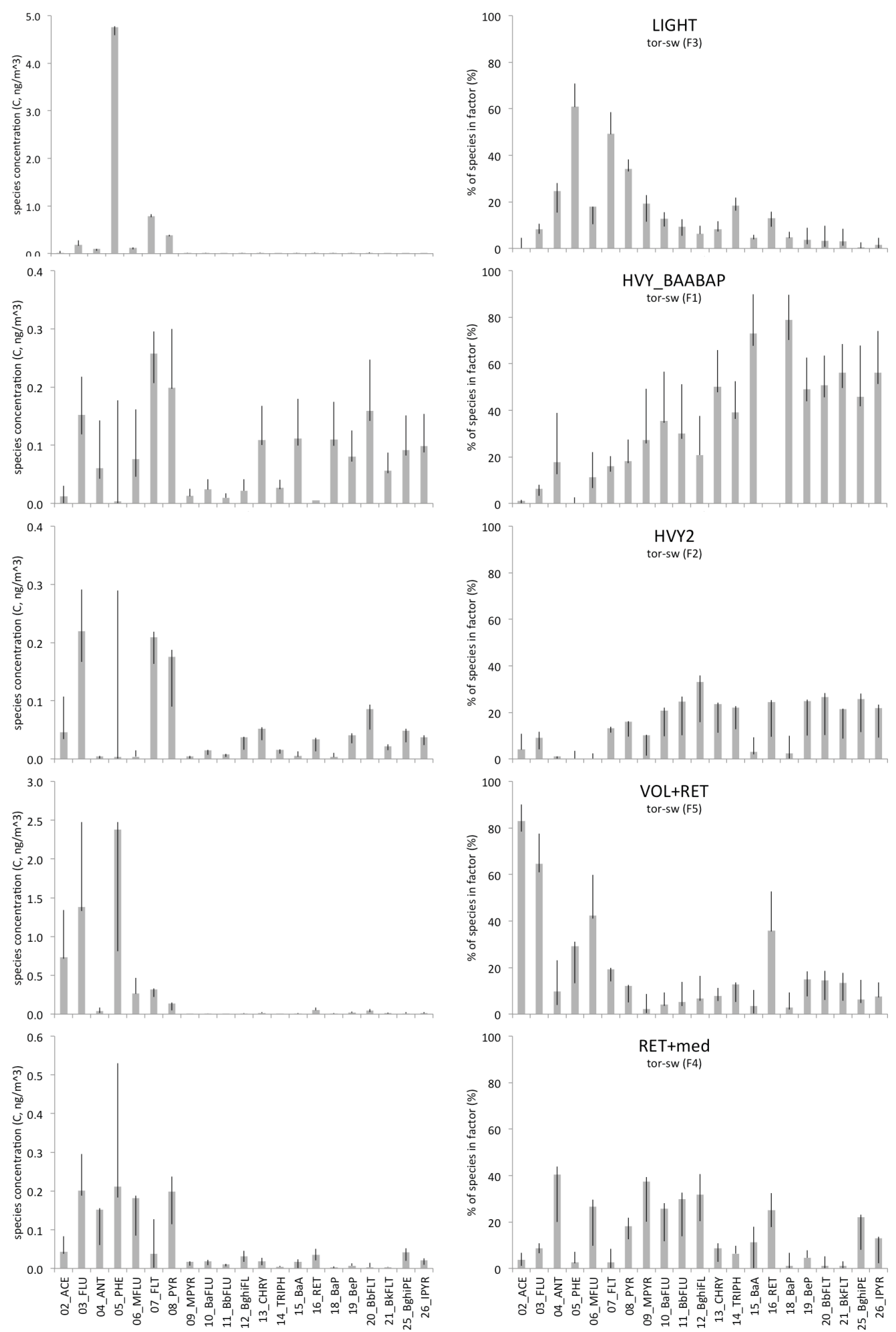

Figure B.7-1 (continued) 
(c) background
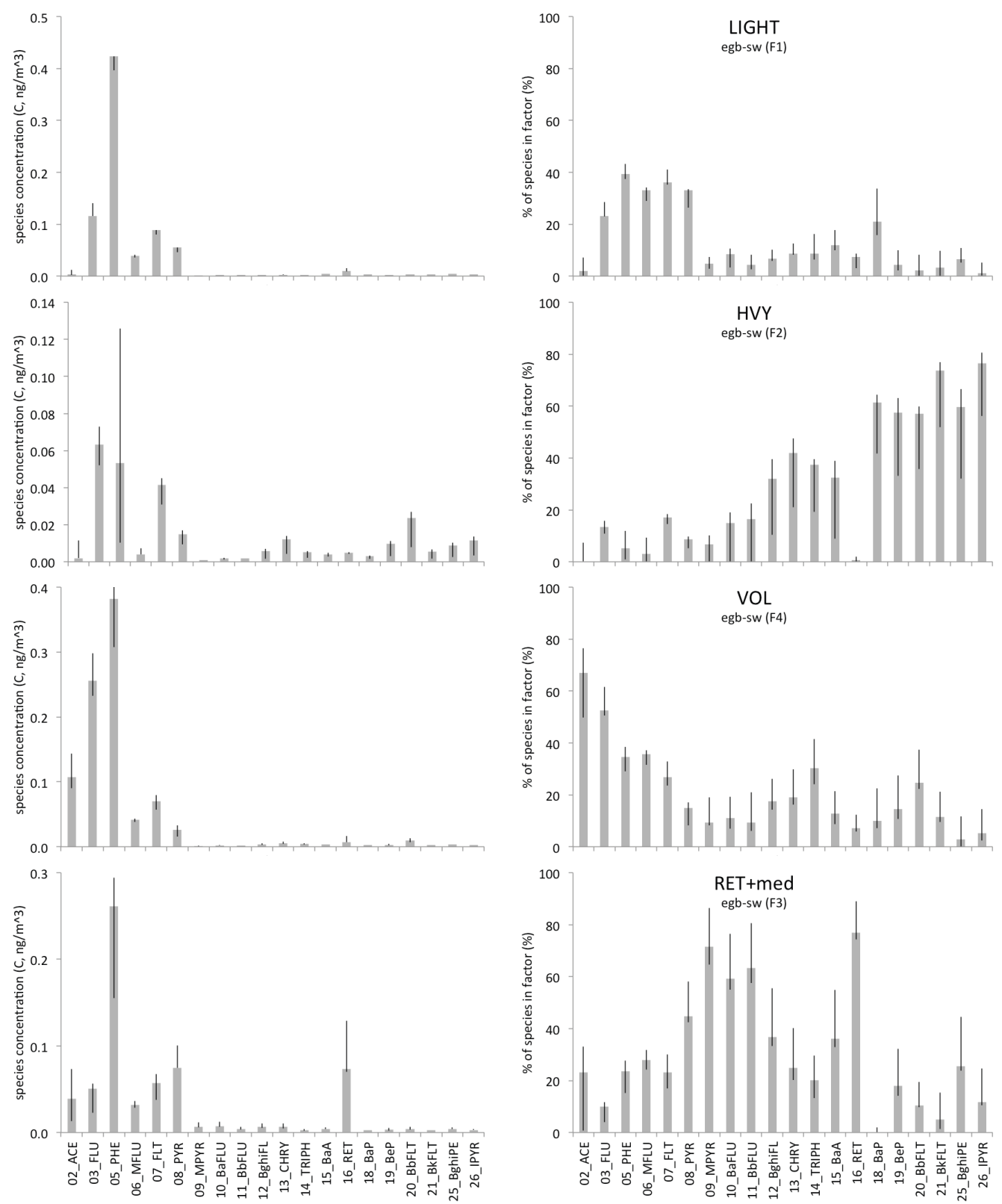

Note: ANT not modeled at background site due to poor data quality.

Figure B.7-1 (continued) 


\section{PAH volatilization + LRTAP}
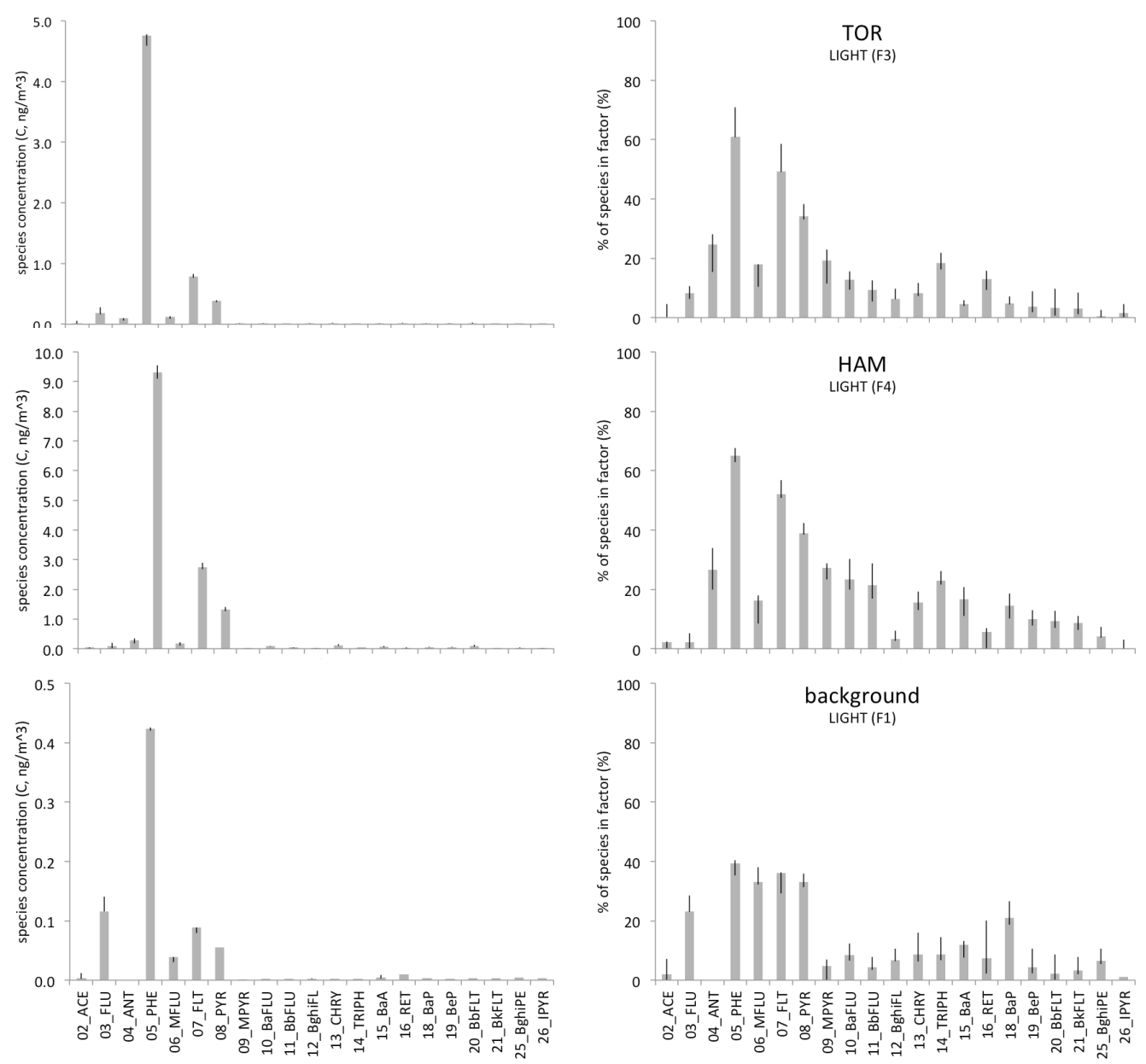

Note: ANT not modeled at background site due to poor data quality.

Figure B.7-2. Bootstrap factor profiles (concentration, \% variance) by source type. 
traffic emissions
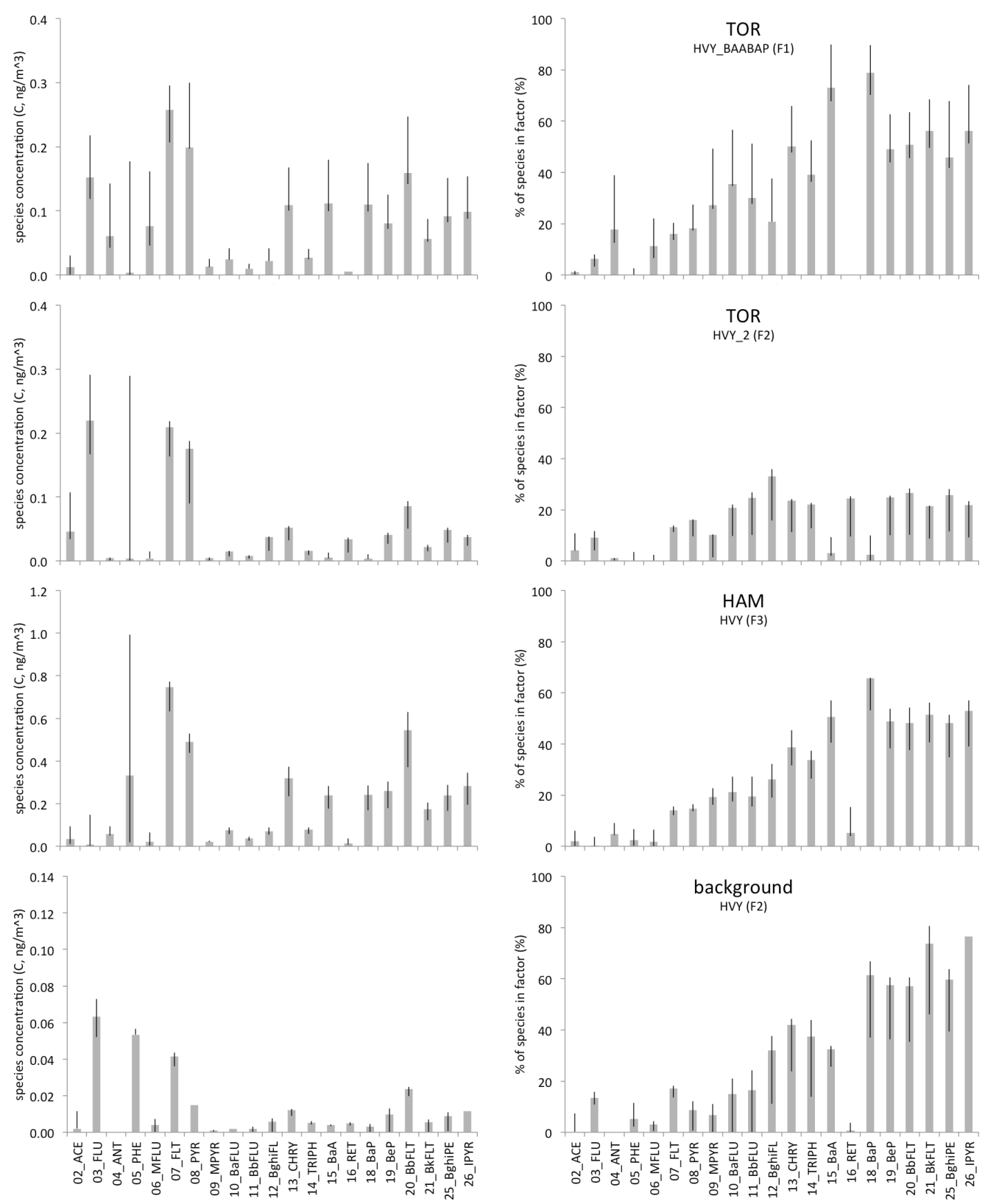

Note: ANT not modeled at background site due to poor data quality.

Figure B.7-2 (continued) 
space heating - oil and natural gas combustion
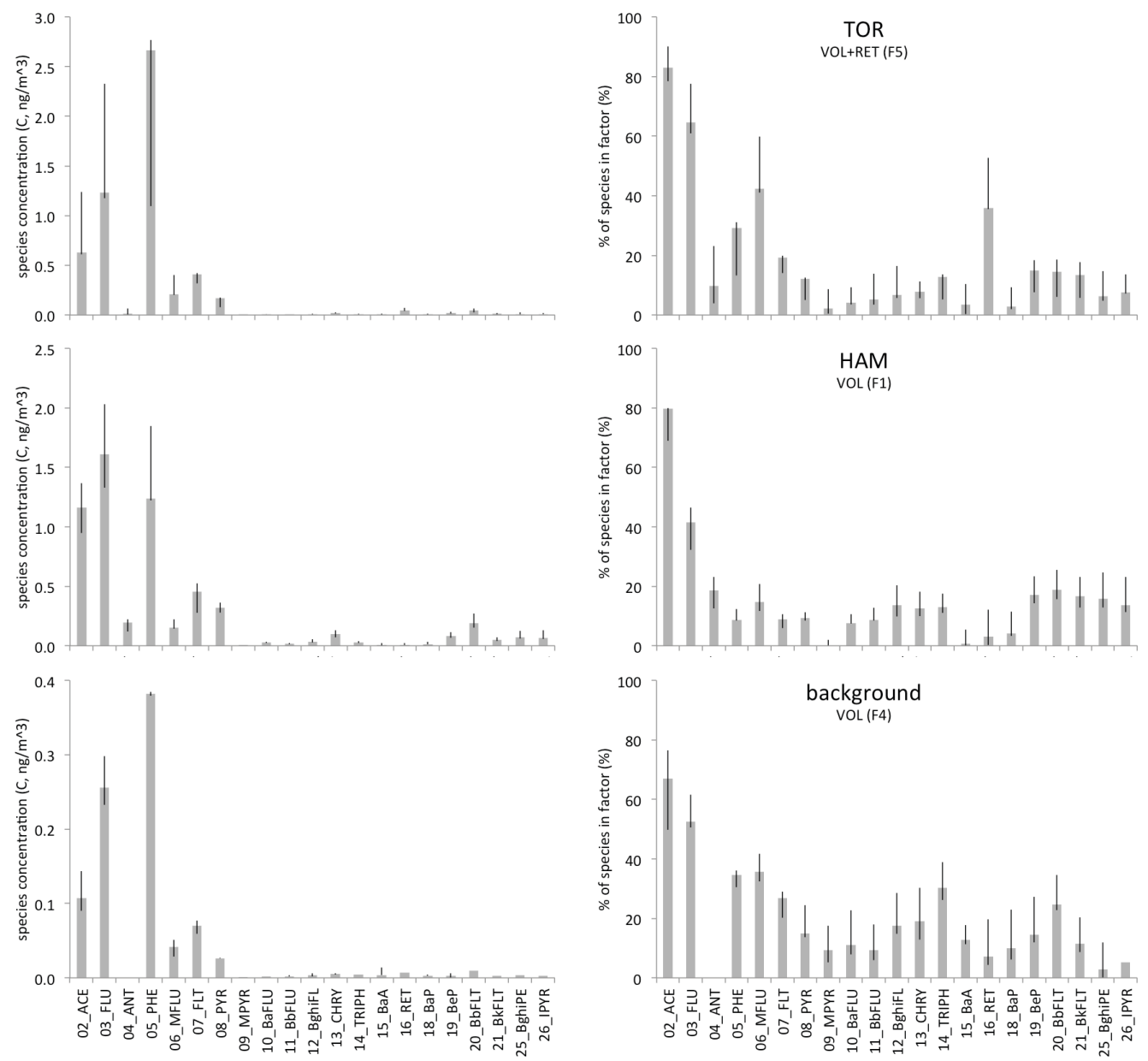

Note: ANT not modeled at background site due to poor data quality.

Figure B.7-2 (continued) 


\section{local industry}
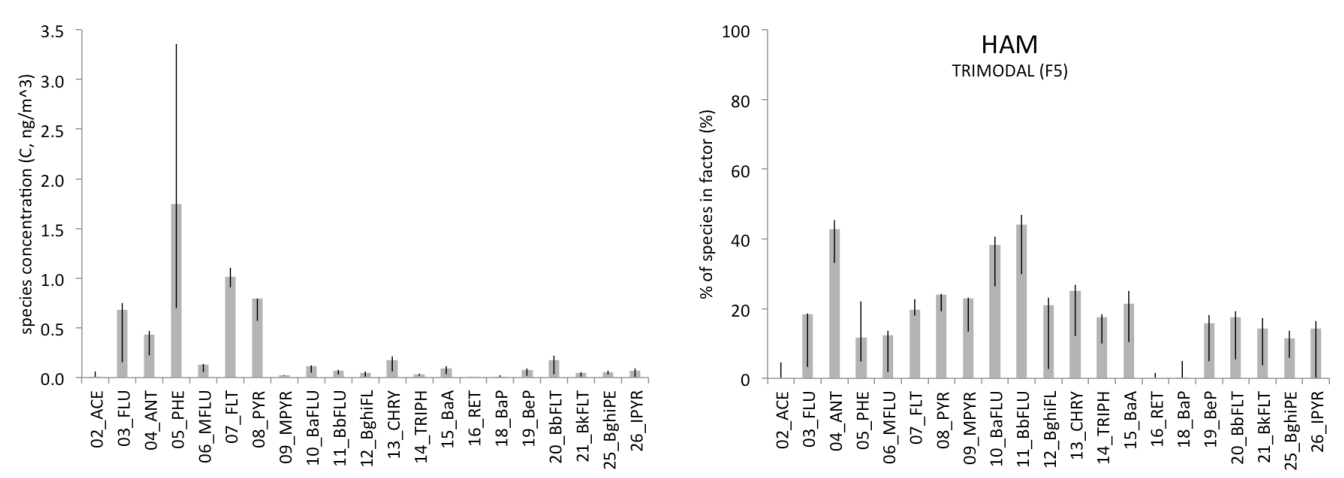

space heating - wood combustion
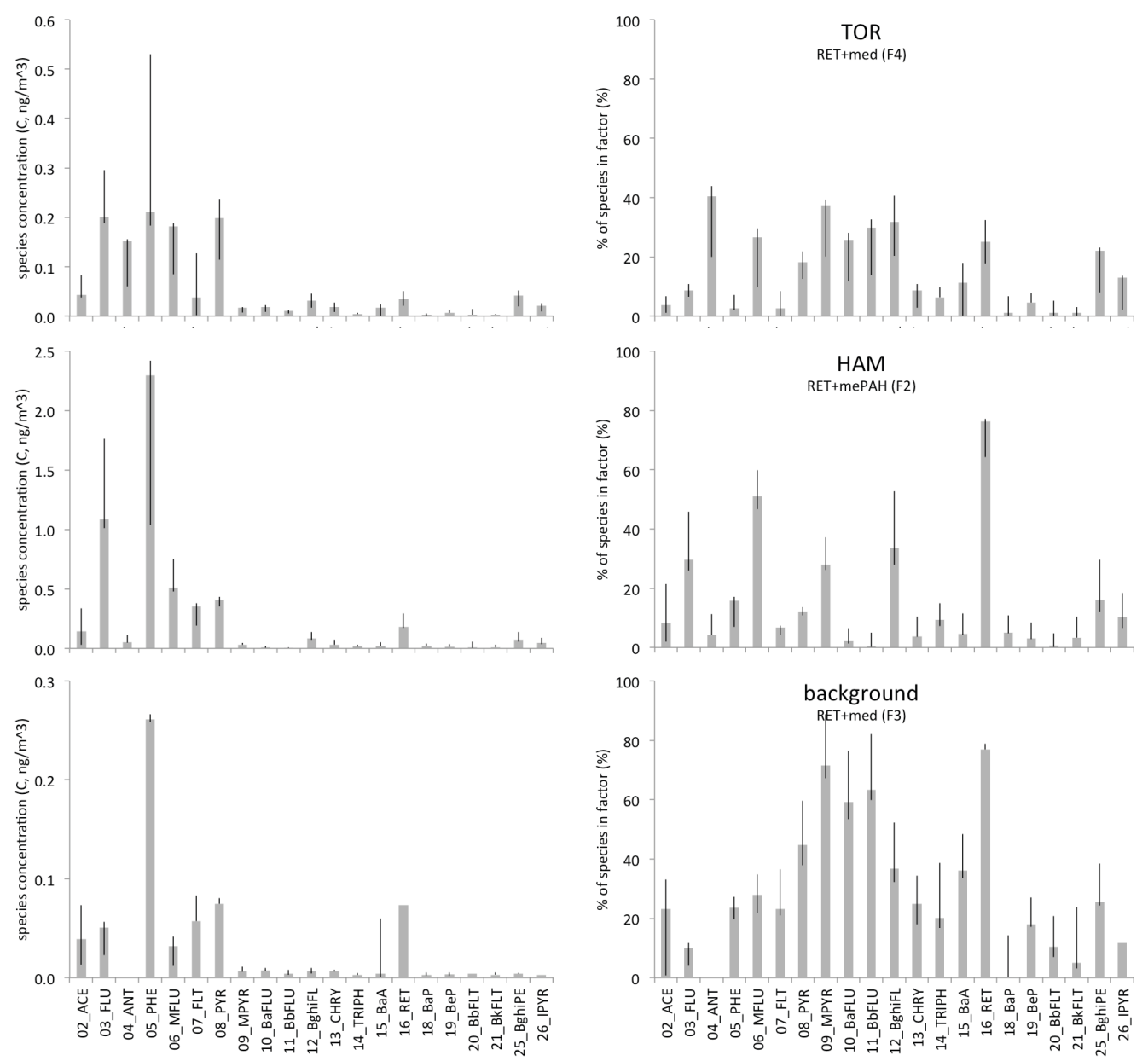

Note: ANT not modeled at background site due to poor data quality.

Figure B.7-2 (continued) 
Source type interpretation for PMF factors

Interpretation of model factors as physically meaningful source types is the primary challenge of PMF and requires knowledgeable application of several layers of evidence, inclusive of: corroboration of profiles with alternate receptor model results (e.g., PCA), factor enrichment in known source marker or source-characteristic species, comparison with reference profiles, evaluation of seasonal or other temporal trends in factor contributions, and comparison of source apportionment estimates with emissions inventories. (Reff et al, 2007; Brook et al, 2004) Inter-site comparison also helped identify similar source types or confirm site-unique local source types. Overall, source types were interpreted from model factors based on a preponderance of contextual evidence (Reff et al, 2007).

\section{PAH volatilization and LRTAP coal combustion}

The "light" factor profile pattern was observed at all sites (Figure B.7-2).

The PMF F_EV profile was enriched in light MW species ANT through PYR along with MPYR, explaining the clear majority of variance in PHEN, FLT, PYR; the corresponding F_C profile was enriched in PHEN, FLT, PYR at all sites and FLU at the rural site.

Strong inter-site similarity in F_EV and F_C profiles indicated a source type influencing all sites (i.e., a transported regional source and/or ubiquitous source type).

The PMF-resolved "light" factor was highly consistent with PCA model results at all sites (i.e., PC labeled "light" with high loading in PHEN, FLT, PYR at all sites; moderate loading in FLU and MPYR at background site; explained $21 \%$ - 27\% of data variance; Appendix B.4: Figure B.4-1).

Factor analysis and receptor modeling studies of PAH consistently cite high variance in these species as characterizing coal related emissions such as in power generation or iron/steel manufacturing (Park, 2011; Shi, 2010; Larsen and Baker, 2003; Simcik, 1999). Review of source sampling studies confirmed these species as enriched in coal combustion emissions (particle+vapour phase: Ciappara et al, 2009; Aries et al, 2007; Khalili et al, 1995; Kirton et al, 1991; particle phase: Li, 2003; Oros et al, 2000). Thus, the factor was interpreted as representing regionally transported PAH emissions from coal combustion (i.e., power generation facility releases transported to the study area via LRTAP).

Appendix B: Urban/rural source apportionment 
In addition to the distinct factor profile pattern, the "light" factor showed a highly distinct seasonal trend indicating an additional source type: median concentrations to modeled $\mathrm{PAH}\left(\Sigma_{22} \mathrm{PAH}\right)$ were notably higher in spring/summer. This distinctive and pronounced seasonality was indicative of the factor also representing $P A H$ releases due to volatilization of uncombusted liquid fossil fuel (e.g., gasoline/diesel/oil storage, handling, dispensation) as well as re-volatilization of PAH from asphalt roadways and otherwise generally adsorbed to surfaces in the built environment. Recent receptor modeling studies that sampled PAH in both vapour and particle phase have found a highly similar factor and have consistently attributed it to PAH volatilization (Jang et al, 2013; Spengler, 2011; Hanedar, 2014). The factor showed no weekday/weekend contribution trend at any site, as expected for regional transported PAH and for volatilized PAH emissions (i.e., salient observation at the background site, since the only factor to show no weekday/weekend trend).

The total aggregate contribution of "light" to $\Sigma_{22} \mathrm{PAH}$ was very similar at the urban sites $(40 \%)$ and lower at the background site $(\sim 30 \%)$, consistent with the factor representing a combined source with a local plus regionally transported component. While relative individual source contributions of LRTAP of coal combustion versus PAH volatilization cannot be quantified, the factor rationally contained a sizable volatilization contribution at the urban sites, consistent with their large number of relevant local source locations (e.g., fueling stations and storage, pavements, built surfaces) and short source-receptor transport distances and thus strong source-receptor conservation. Conversely, source contribution was least at the background site with most attenuated seasonal trend; the inference is that the factor represented regionally transported LRTAP comparatively more at the background site than at the urban sites, consistent with few local volatilization sources (Appendix B.1: Figure B.1-4).

\section{Traffic exhaust}

Variants of the "heavy" factor profile pattern were observed at all sites (Figure B.7-2). A single "heavy" factor was resolved at Hamilton and at background while two variants were resolved at Toronto ("heavy_BAABAP", "heavy_2"). For all variants, the F_EV profile was characteristically enriched in heavy MW species (CHRY through IPYR) with exception of RET; at the urban sites, the factor notably explained the majority of variance in BAA and BAP (i.e., "heavy" factor at Hamilton, "heavy_BAABAP" factor at Toronto). The corresponding F_C profile showed greater inter-site differences but the defining characteristic remained: high concentrations of heavy MW species relative to light MW species and relative absence of medium MW species.

Appendix B: Urban/rural source apportionment 
The similarity in F_EV profiles with inter-site differences in F_C suggested a local source type.

The PMF-resolved "heavy" factor was consistent with PCA model results, which resolved a PC labeled "heavy" at all sites that was characterized by a high loading in heavy MW species, with exception of RET, and moderate loading on FLT and PYR (Appendix B.4: Figure B.4-1).

Factor analysis and receptor modeling studies of PAH consistently cite factors enriched in heavy species as characterizing traffic exhaust emissions (Menezes, 2012; Park, 2011; Shi, 2010; Ravindra, 2008; Guo, 2003; Larsen and Baker, 2003; Marr, 1999; Simcik, 1999); variance in heavy species has also been associated more specifically with dieselfueled traffic emissions (Park, 2011). The F_C profiles were comparatively bimodal, with significant concentrations of both light and heavy species, thereby showing visual similitude with profiles from mixed gasoline and diesel-fueled vehicle traffic or diesel traffic exhaust profiles rather than strictly gasoline traffic exhaust profiles (PM+vapour phase: Kristensson, 2004; Li, 2003; Harrison, 2003; Wingfors, 2001; Fraser, 1998; Khalili, 1995). ${ }^{33}$ Generally, F_EV and F_C profiles were enriched in species characterizing diesel exhaust emissions (BAA, BaP, BbFLT, BkFLT, IPYR; Liacos, 2012 / Kam, 2012; Li, 2003; Simcik, 1999), suggesting the factor included a strong diesel traffic component or represented emissions from mixed traffic (i.e., gasoline- and dieselfueled vehicles) operating in the urban mode, which can be characterized by frequent deceleration/acceleration phases that have been shown to emit heavy MW species disproportionately relative to light MW species (Harrison, 2003; Phuleria, 2007; Shah, 2005). At Hamilton and Egbert, the "heavy" factor was thus interpreted as representing PAH emissions from mixed traffic (gasoline + diesel).

The two related traffic profile variants ("heavy_BAABAP”, “heavy_2”) at Toronto could be interpreted as physically meaningful rather than model order artifacts, consistent with higher traffic loadings influencing the sampler due to its central location characterized by high traffic volumes on surface streets and nearby elevated freeways (Appendix B.1: Figure B.1-4). As implied by the label, "heavy_BAABAP" F_EV explained the majority of the variance in BAA and BAP while the "heavy_2" F_EV was conspicuous by absence

\footnotetext{
${ }^{33}$ Preference was given to fleet-based source sampling studies such as tunnel, roadside, and invehicle sampling as these have been shown to be more representative of real world traffic emissions than composite single vehicle emissions data from chassis dynamometer sampling; Ropkins et al, 2009; Environment Canada 2006.
} 
of these species and F_C was less bimodal. As cited previously, with these species (BAA, BAP) and concentration profile bimodality as useful indicators of diesel traffic and/or mixed-fleet urban mode emissions, the two traffic factors resolved at Toronto could be interpreted as reflecting a difference in traffic mode: (i) the Toronto "heavy_BAABAP" represented PAH emissions from urban mode mixed traffic (gasoline + diesel); and, (ii) the Toronto "heavy_2" represented PAH emissions from highway mode mixed fleet traffic emissions. ${ }^{34}$

Interpretations of the Toronto traffic factor variants were consistent with the other sites: (i) "heavy_BAABAP" profiles (F_EV, F_C) showed high degree of visual similitude to Hamilton "heavy", which was similarly located near the central business district (Appendix B.1: Figure B.1-4) and thus also subject to congestion (i.e., vehicles operating in the urban mode with frequent deceleration/acceleration phases emitting heavy MW species) and to emissions from commercial traffic, inclusive of diesel-fueled heavy duty vehicles; while, (ii) the "heavy_2" profile showed some similarities to "heavy" at background, which was influenced primarily by adjacent highway traffic due to its rural location (Appendix B.1: Figure B.1-4).

Seasonal trends in contribution were generally consistent with interpretation of the traffic factor variants. For the "heavy" factor at Hamilton and the "heavy_BAABAP" factor at Toronto, no seasonality was seen, as expected since urban traffic volumes do not typically show seasonality, particularly where these include significant commercial traffic and, thus, emissions from heavy duty diesel-fueled vehicles. However, the "heavy_2" factor at Toronto and the "heavy" factor at the background site showed winter contributions to increase slightly; likely explained by increased traffic volumes and increased vehicle emissions in cold temperature conditions, along with winter conditions being generally more conducive to buildup of pollutants and greater transport of highway traffic emissions to the sites due to reduced mixing height typical in the winter season and decreased reactivity due to colder temperatures (Sun et al, 2006).

At Toronto, the "heavy_BAABAP" factor contributed more on weekdays as expected for urban mode mixed traffic emissions, since local traffic volumes are typically higher on weekdays due to commuting trips and commercial/business traffic includes more heavy

\footnotetext{
${ }^{34}$ Relatively low receptor model order, lack of additional specific source markers for gas and diesel in the species suite, a priori knowledge of fleet and traffic patterns on the road network near the sites, and conservative forensic reasoning precluded interpreting the factors overly narrowly as emissions from a single fuel type.
}

Appendix B: Urban/rural source apportionment 
duty diesel vehicles on weekdays. Conversely, the "heavy_2" factor contributed more on weekends as expected for a highway mode mixed traffic source, since overall traffic volumes in the central business district are typically reduced on weekends due to absence of commuting traffic and since the diesel-fueled portion of fleet typically decreases on weekends with less commercial vehicles servicing centrally located businesses. At the background site, weekend traffic contributions were higher, consistent with greater highway traffic expected from weekend extra-urban and recreational trips. At Hamilton, limited weekend data precluded inferring a weekday/weekend trend.

Traffic contributions to ambient PAH, aggregated over the entire modeling period, can be readily compared across the two urban sites, since both sites have samplers located in their central business district. The contribution of the traffic source type was greater at Toronto $(\sim 20 \%)$ than at Hamilton $(13 \%)$, consistent with greater population density and higher traffic volumes near the site. As an additional consideration, the urban traffic factors showed annual median contributions remaining essentially level across the sampling period at both urban sites, but peak contributions higher in 2005 and 2007, years with unseasonably hot weather favouring increased buildup of traffic pollutants, affirmed by a very high number of smog advisory days (Ontario Ministry of Environment, 2013). Contributions at the background site (13\%) indicated traffic emissions to be a significant source type even in rural locations.

\section{Space heating combustion}

A variant of the "volatile" factor profile pattern was observed at all sites (Figure B.7-2). The F_EV was highly enriched in the lightest and most volatile species (ACE, FLU) with moderate enrichment in a range of heavy species; corresponding F_C was similarly characterized by high ACE and FLU alongside high PHEN.

The PMF-resolved "volatile" factor was consistent with PCA model results, since a PC labeled "volatile" with high loading in lightest species was resolved by PCA at all three sites and explained $8 \%$ to $16 \%$ of data variance (Appendix B.4: Figure B.4-1). The factor's consistent and stable appearance in PCA results and in PMF results at even lower model orders strongly indicated a source type present at all sites.

Critically, the factor showed a strong seasonal trend with higher median contributions in cold months.

The factor profile and seasonal trend together indicated space heating oil and natural gas fuel combustion (Ravindra, 2008; Larsen and Baker, 2003; Lee, 2004; Rogge et al, 1993; Mari, 2010; Hanedar, 2014; Okuda, 2010), both fuels being used for space heating in 
Ontario (Sanderson et al, 2004). Additional oil combustion emissions from industry (Yang, 2002, 1998; Ravindra, 2008) were deemed comparably minor since these do not typically show strong seasonality, while space heating does. Further distinction between space heating fuel types was not possible at the model order permitted by the dataset.

Only at Toronto, the factor also explained a large percentage of variance in the RET, indicating significant additional wood combustion space heating emissions were apportioned to the factor (Menezes, 2012; Fang, 2006; Larsen and Baker, 2003; Simcik, 1999); the factor variant was labeled "vol+ret" to reflect this difference. (Additional PMF solution variants at Toronto confirmed the presence of RET was not a model artifact; higher RET was found in the factor by a lower order model, a five-factor model with less outliers excluded, and a model with less uncertainty assigned to RET.) Also only at Toronto, the factor's median annual contribution to modeled PAH showed more year-toyear variation than seen for other factors, consistent with the factor representing sizable wood-fueled space heating emissions, which are a supplemental heating source in urban areas (i.e., in-home wood-fired appliances often used for aesthetics) and thus expected to vary more than use of primary heating fuels. Last, the Toronto factor's higher total aggregate contribution to ambient PAH (33\% for "vol+ret"), compared with the analogous factor's contribution at the other urban site (16\% at Hamilton for "vol") was consistent with its representing space heating from several combined fuel types (i.e., oil, natural gas, wood) in the Toronto model solution.

\section{Biomass combustion}

A variant of the "retene" factor profile pattern was observed at all sites (Figure B.7-2) and was characterized by being highly enriched in the wood marker species retene (Jang et al, 2013; Menezes, 2012; Fang, 2006; Larsen and Baker, 2003; Simcik, 1999; Ramdahl, 1983).

The PMF-resolved "retene" factor was consistent with the PCA model results for Hamilton and the background site, since retene appeared as the sole highly loaded species in a separate PC at Hamilton and alongside high loadings of the me-PAH species at the background site (Appendix B.4: Figure B.4-1). Despite the PCA anticipating a separate retene factor at Toronto, however, the wood combustion factor was less clearly resolved by PMF, and retene was also apportioned to the Toronto space heating factor ("vol+ret") as discussed previously.

Different from the other factors, however, the factor showed greater inter-site variability in its profile pattern and relative contribution. This was expected for a factor representing biomass combustion emissions since this inherently comprises a large variability of fuels

Appendix B: Urban/rural source apportionment 
(e.g., hardwood, softwood, wood moisture content), a large number of combustion apparatus such as fireplaces, wood stoves with or without emissions controls, outdoor chimineas, and open burning and agricultural burning activity (Schauer et al, 2001; McDonald et al, 2000; Environment Canada, 2000; Rogge et al, 1998). Greater inter-site variability in profile pattern was also anticipated because retene has been recently shown to be an imperfect marker of biomass combustion, characterizing some wood types more than others and also having been sampled in other combustion source types (Shen et al, 2012).

The "retene" factor variants showed no seasonality at the urban sites, indicating that biomass combustion emissions are common year-round at urban centers. This is consistent with the broad range of common combustion activities in addition to space heating (e.g., residential outdoor cooking, use of residential and commercial wood burning appliances for aesthetics, agricultural burning) and also with the relatively mild climate in southern Ontario. At background, contributions were higher in fall, consistent with seasonal biomass combustion activities expected for rural locations (e.g., agricultural burning at end of growing season).

Local industry (iron/steel manufacturing)

The "trimodal" factor profile pattern was observed only at Hamilton (Figure B.7-2) and remained clearly resolved and unique even with a four-factor PMF model, precluding artifact effects from model order. The F_EV was most enriched in ANT and medium species BAFLU, BBFLU and explained the greatest portion of variance of these species ( $\sim 50 \%$ species mass apportioned to factor); next-most enriched were FLT, PYR, MPYR, along with moderate enrichment of heavy species; no RET was apportioned to this factor. The corresponding F_C was enriched in PHEN, FLT, PYR along with ANT, FLU and had comparably high concentrations of heavy species. The factor profiles showed cursory similarity to the "light" factor but also physically meaningful differences: accentuated enrichment of FLU, ANT, BAFLU, BFLU; attenuated PHEN; conspicuous absence of RET; and, higher contributions of heavy MW species.

A similar factor profile to the PMF-resolved "trimodal" was not indicated by the PCA model results at Hamilton, an expected consequence of the inherent limitations of PCA, since the solution was of lower model order and since PCA is challenged by species with higher known uncertainty such as ANT or medium MW species due to uncertainty not being included in the model. Even so, the PCA results showed several species (FLU, MFLU, FLT, PYR) as not being well-resolved to a single PC (i.e., appeared highly 
loaded in more than one PC; Appendix B.4: Figure B.4-1), suggesting that PMF would better resolve these species to separate and interpretable factors, as indeed occurred.

The similarities of the site-unique "trimodal" profile to the "light" factor at first suggested an association with coal combustion based on previous receptor model studies (Park, 2011; Shi, 2010; Larsen and Baker, 2003; Simcik, 1999) and source sampling studies (Ciappara et al, 2009; Aries et al, 2007; Khalili et al, 1995; Kirton et al, 1991 for PM+vapour phase; Li, 2003; Oros et al, 2000 PM phase). However, unique characteristics of the Hamilton "trimodal" profile characterized it more specifically as emissions associated with iron/steel manufacturing from coke oven and sintering operations based on observations on specific components. High ANT and BAFLU, BBFLU are associated with coke oven emissions rather than coal-fired power generation (Larsen and Baker, 2003; Jang et al, 2013); sintering operations in steel manufacture emit more heavy species relative to coal-fired power generation emissions (Ravindra, 2008; Ciaparro, 2009); PHEN, FLT, PYR are more strongly associated with a coal-fired power generation emissions profile and are comparably attenuated in coke oven emissions (Bzdusek, 2004); FLU, and its methylated compound MFLU, are more pronounced in coke oven emissions than in general coal combustion emissions (Ciaparra, 2009; Khalili et al, 1995) and have been associated with iron/steel emissions in previous receptor modeling (Fang et al, 2006; Simcik et al, 1999); and, RET is associated with combustion of less mature coals such as sub-bituminous (Shen, 2012) and therefore not generally emitted from coking (Simcik, 1999; Khalili, 1995), which requires bituminous coal (Mckay, 2006; World Coal Institute, 2007).

The factor showed no seasonal or day trend in median contributions to modeled PAH, consistent with a heavy industry source that operates year-round and seeks to minimize downtime (i.e., coke oven require significant start up/shut down operations). Median contributions were also relatively unchanged from year-to-year, also consistent with local industry operations: one of the steelworks facilities has maintained production even after international acquisition and the second steelworks facility has consolidated sintering operations with US facilities but maintained coke oven operations (Environment Canada, 2011g). Last, the factor was consistent with the city's dominant local industry, with two steelworks facilities located near the sampling site at the city's industrial harbour-front (Appendix B.1: Figure B.1-4). 


\section{B.8 Additional PMF results}

Table B.8-1. First quartile, median, third quartile source contributions to ambient PAH at urban and background sites (year-round PMF model, NAPS data).

\begin{tabular}{|l|c|c|c|}
\cline { 2 - 4 } \multicolumn{1}{c|}{} & \multicolumn{3}{c|}{\begin{tabular}{c} 
SOURCE APPORTIONMENT \\
\multicolumn{1}{c|}{$(\%$ G to __modeled_PAH; Q1 / median / Q3) }
\end{tabular}} \\
\hline SOURCE TYPE & $\begin{array}{c}\text { Urban - } \\
\text { Hamilton }\end{array}$ & $\begin{array}{c}\text { Urban - } \\
\text { Toronto }\end{array}$ & background \\
\hline volatized PAH + LRTAP (coal) & $\begin{array}{c}38 \% \\
(15 \%-54 \%)\end{array}$ & $\begin{array}{c}29 \% \\
(11 \%-55 \%)\end{array}$ & $\begin{array}{c}26 \% \\
(13 \%-37 \%)\end{array}$ \\
\hline local industry (coal) & $\begin{array}{c}9 \% \\
(3 \%-16 \%)\end{array}$ & -- & - \\
\hline traffic exhaust & $10 \%$ & $19 \%$ & $10 \%$ \\
$(5 \%-16 \%)$ & $(10 \%-29 \%)$ & $(2 \%-15 \%)$ \\
\hline space heating (oil + wood) & $14 \%$ & $36 \%$ & $37 \%$ \\
$(19 \%-53 \%)$ & $(25 \%-46 \%)$ \\
\hline biomass combustion & $\begin{array}{c}(7 \%-25 \%) \\
(11 \%-38 \%)\end{array}$ & $\begin{array}{c}8 \% \\
(5 \%-12 \%)\end{array}$ & $\begin{array}{c}27 \% \\
(13 \%-39 \%)\end{array}$ \\
\hline
\end{tabular}

Note: Source type contribution data (\%) from first quartile, median, and third quartile of sample contributions.

Table B.8-2. First quartile, median, third quartile source contributions to PAH toxicity (BaP-TEQ) at urban and background sites (year-round PMF model, NAPS data).

\begin{tabular}{|c|c|c|c|}
\hline \multirow[b]{2}{*}{ SOURCE TYPE } & \multicolumn{3}{|c|}{$\begin{array}{c}\text { Source apportionment to BaP-TEQ } \\
\text { (\% G to } \Sigma_{\text {modeled }} \text { BAPTEQ; Q1 / median / Q3) }\end{array}$} \\
\hline & $\begin{array}{l}\text { Urban - } \\
\text { Hamilton }\end{array}$ & $\begin{array}{l}\text { Urban - } \\
\text { Toronto }\end{array}$ & background \\
\hline volatized PAH + LRTAP (coal) & $\begin{array}{c}13 \% \\
(5 \%-25 \%) \\
\end{array}$ & $\begin{array}{c}5 \% \\
(2 \%-15 \%) \\
\end{array}$ & $\begin{array}{c}10 \% \\
(5 \%-23 \%) \\
\end{array}$ \\
\hline local industry (coal) & $\begin{array}{c}9 \% \\
(3 \%-15 \%)\end{array}$ & -- & -- \\
\hline traffic exhaust & $\begin{array}{c}53 \% \\
(37 \%-66 \%)\end{array}$ & $\begin{array}{c}64 \% \\
(47 \%-80 \%)\end{array}$ & $\begin{array}{c}60 \% \\
(25 \%-69 \%)\end{array}$ \\
\hline space heating (oil + wood) & $\begin{array}{c}8 \% \\
(3 \%-16 \%)\end{array}$ & $\begin{array}{c}10 \% \\
(6 \%-16 \%)\end{array}$ & $\begin{array}{c}17 \% \\
(10 \%-28 \%)\end{array}$ \\
\hline biomass combustion & $\begin{array}{c}11 \% \\
(4 \%-18 \%)\end{array}$ & $\begin{array}{c}16 \% \\
(9 \%-22 \%)\end{array}$ & $\begin{array}{c}11 \% \\
(7 \%-23 \%)\end{array}$ \\
\hline
\end{tabular}

Note: Source type contribution data (\%) from first quartile, median, and third quartile of sample contributions. 


\section{Hamilton}
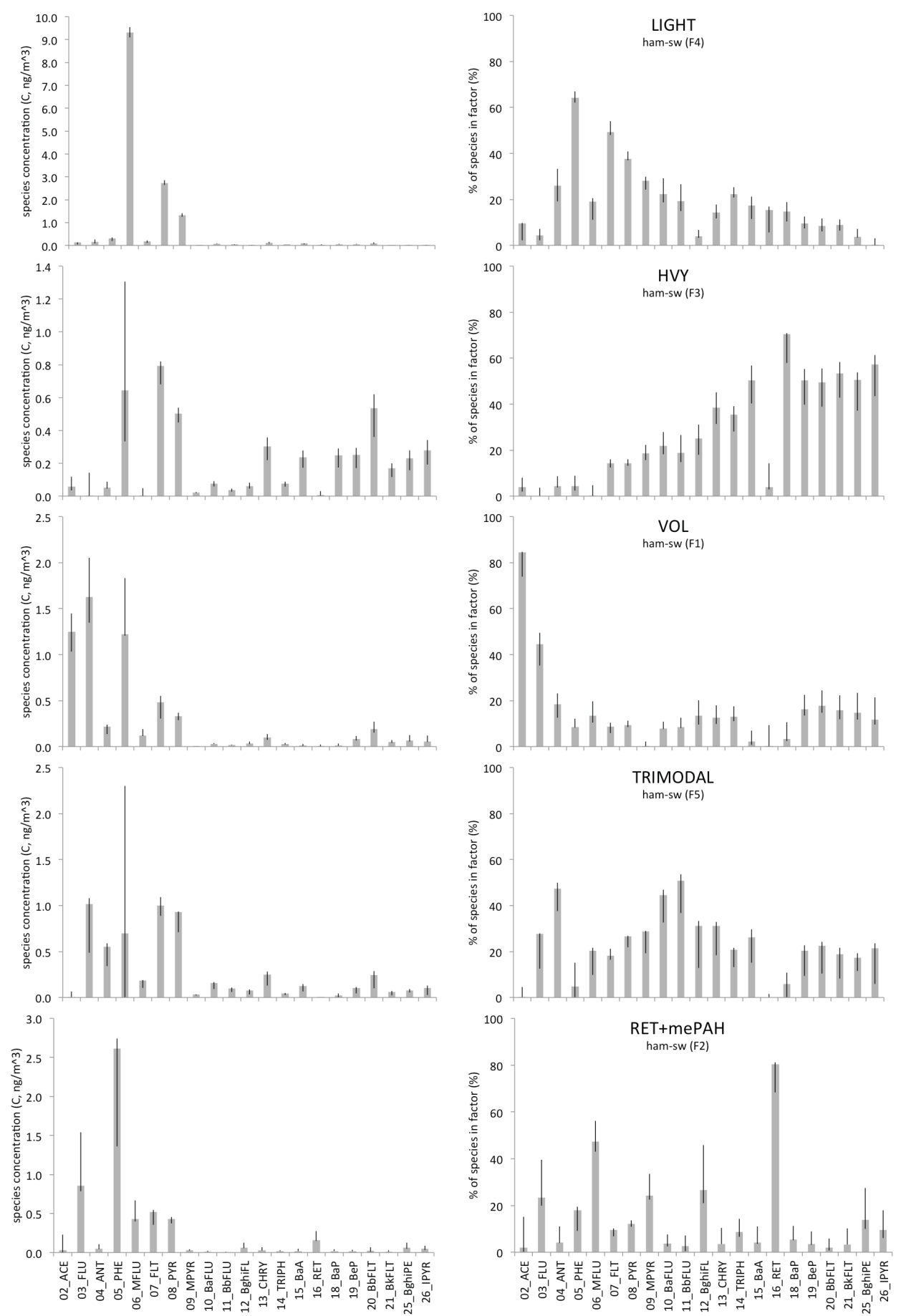

Figure B.8-1. Base factor profiles (concentration, \% variance), year-round PMF model. 
Toronto
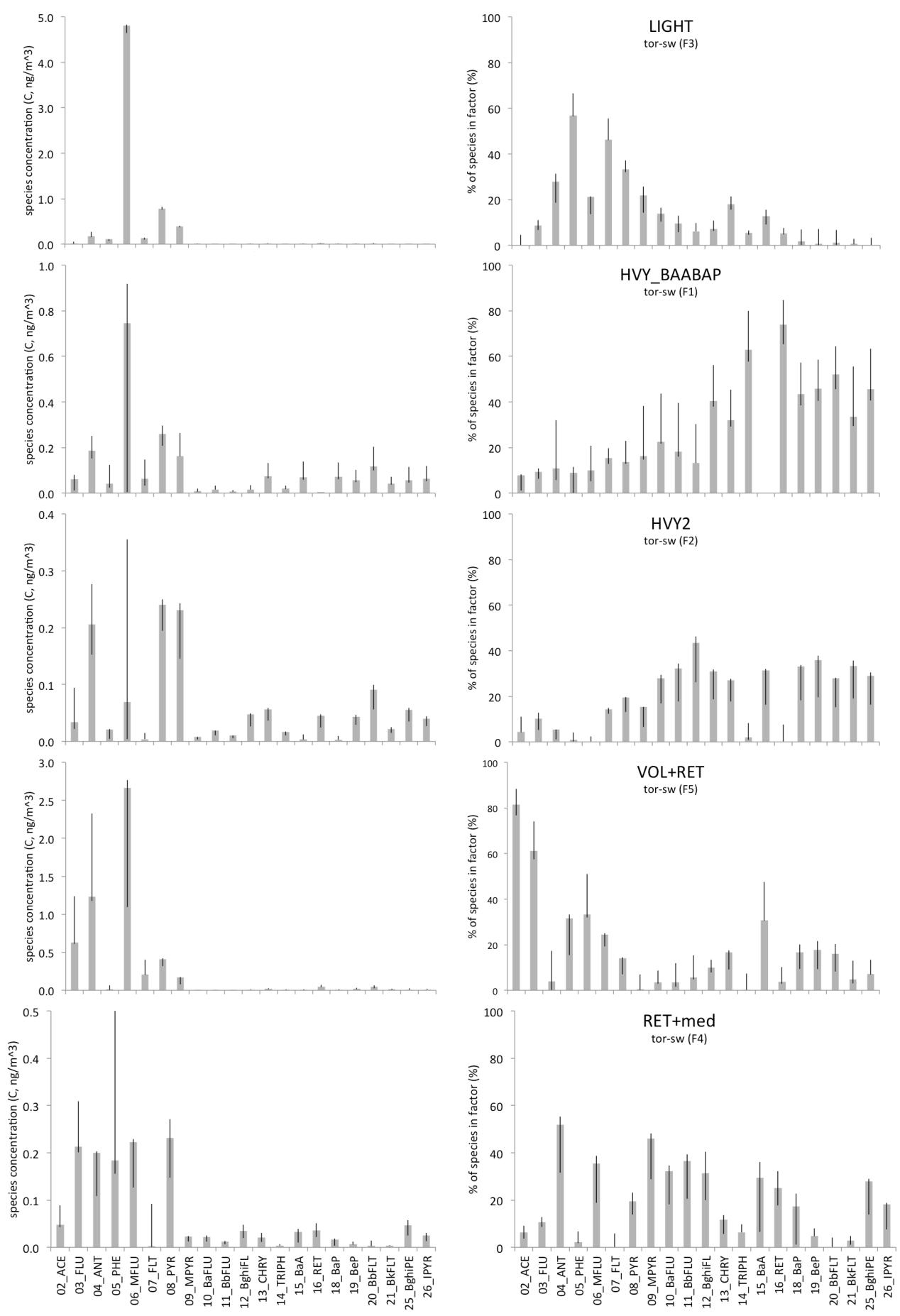

Figure B.8-1 (continued) 
background (Egbert)
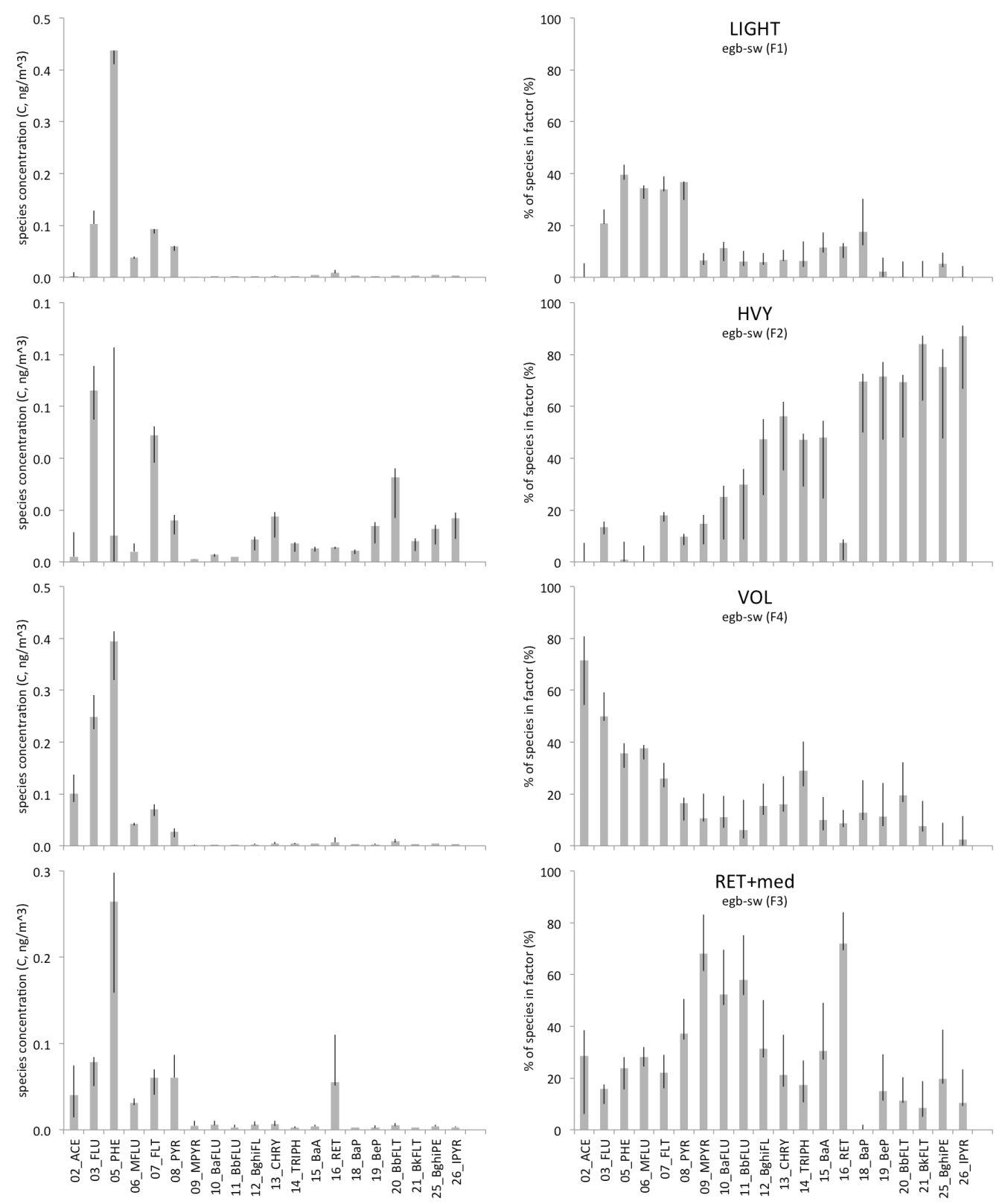

Figure B.8-1 (continued) 


\section{Hamilton}

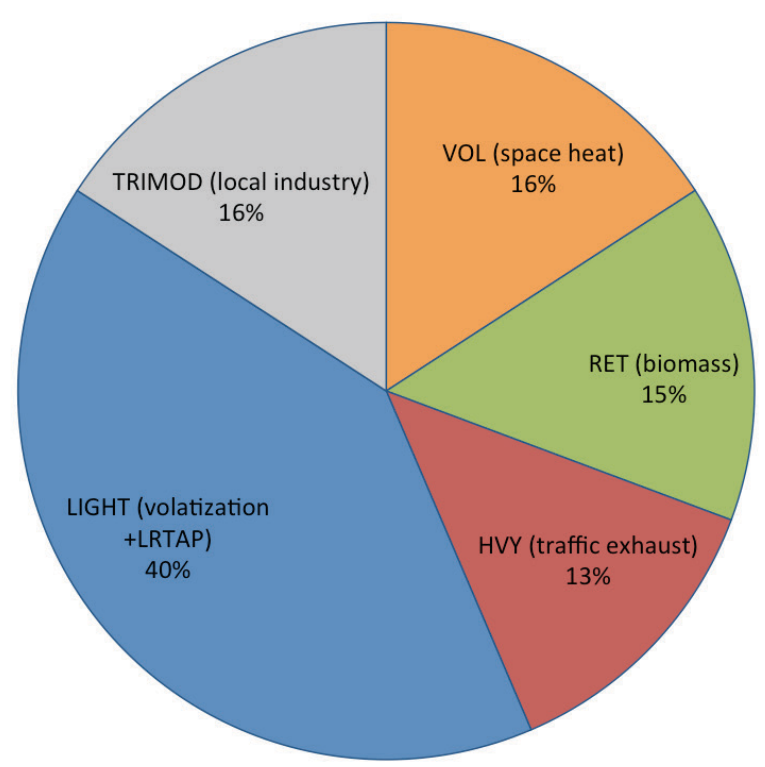

Toronto

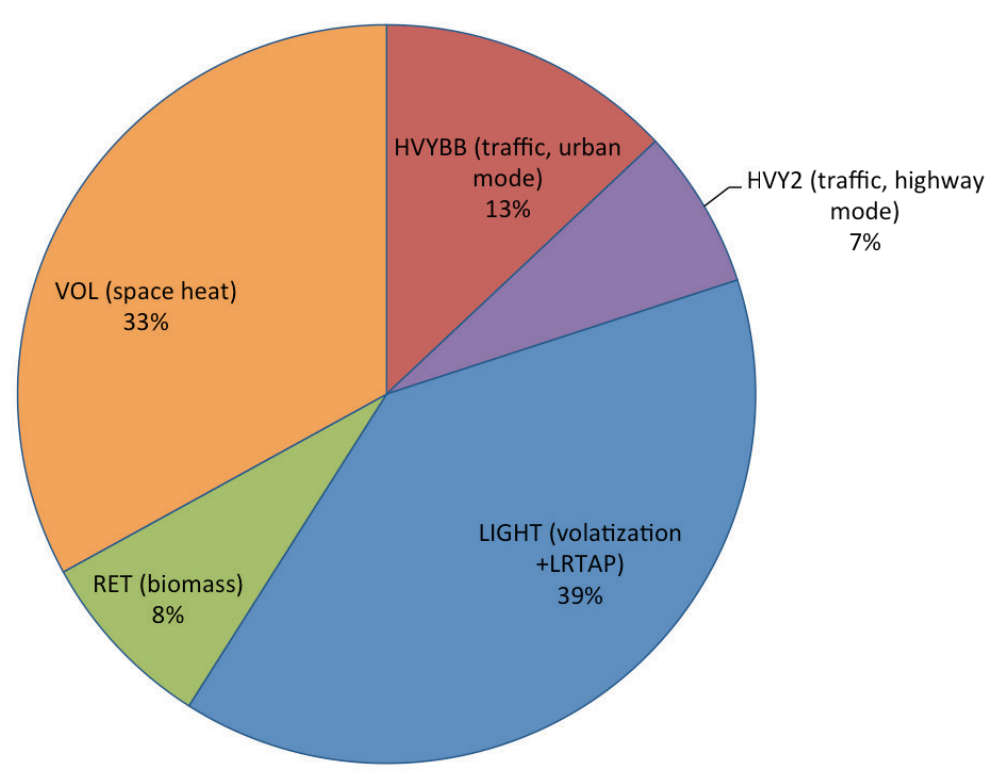

Figure B.8-2. Source apportionment of PAH to PMF factors (all modeled mass). 


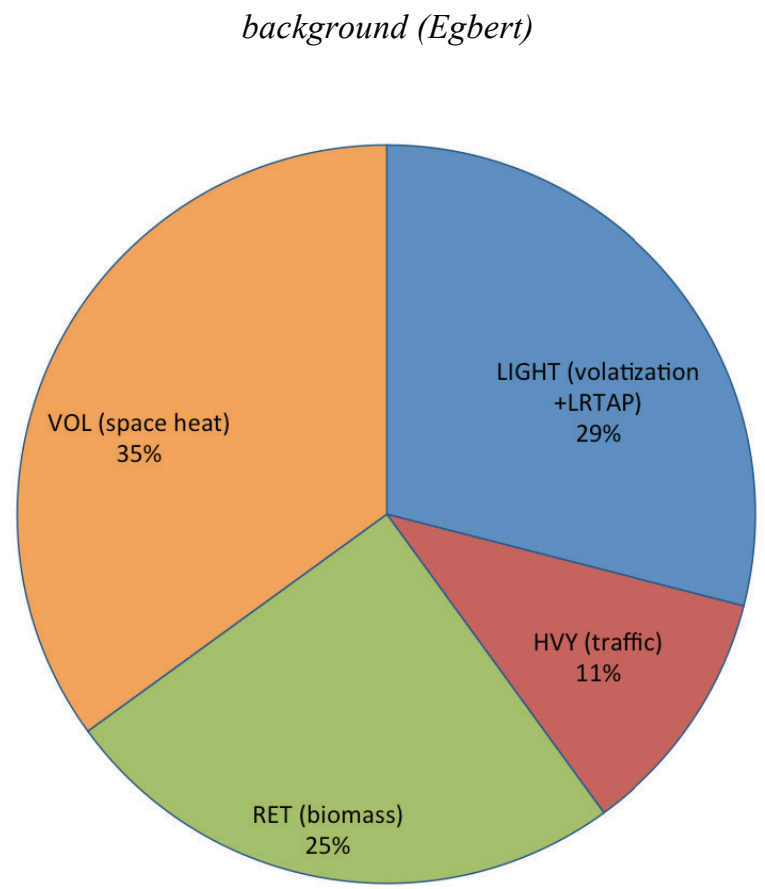

Figure B.8-2 (continued)

Appendix B: Urban/rural source apportionment 


\section{Hamilton}

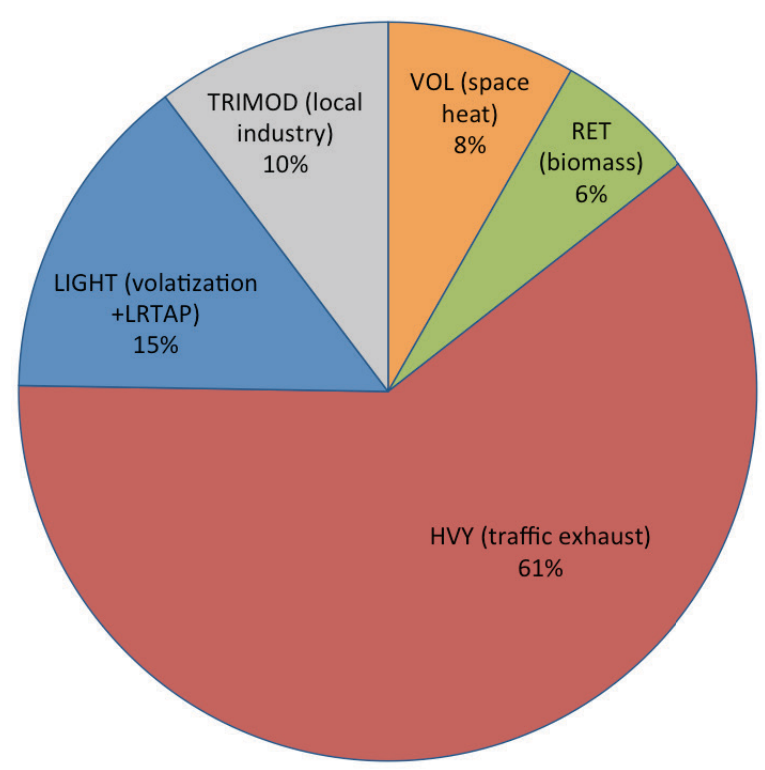

Toronto

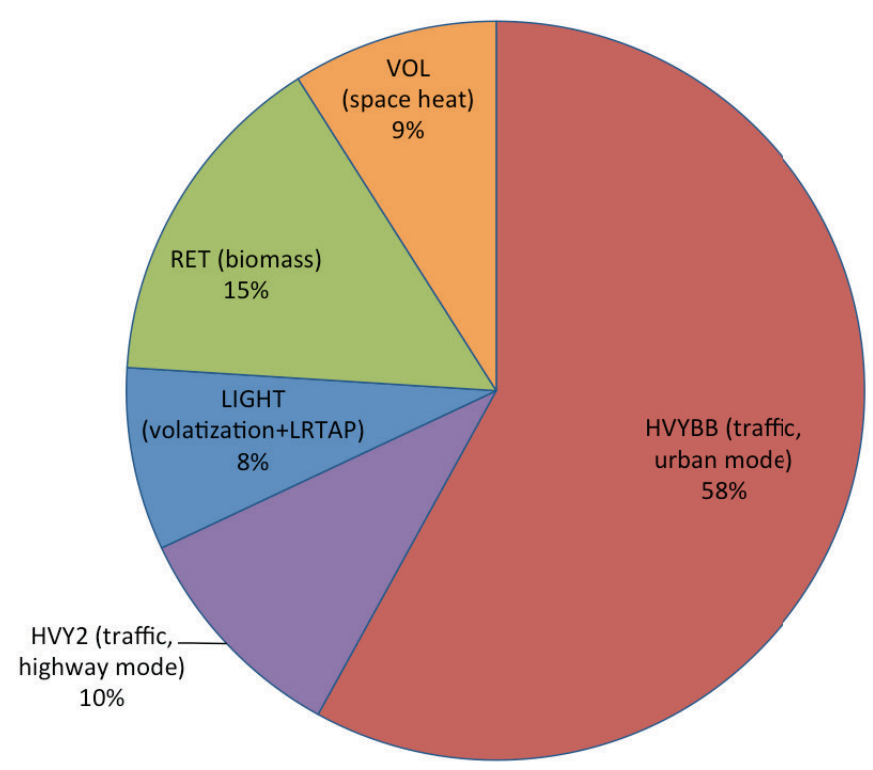

Figure B.8-3. Source apportionment to PAH toxicity (BaP-TEQ) (all modeled mass). 
background (Egbert)

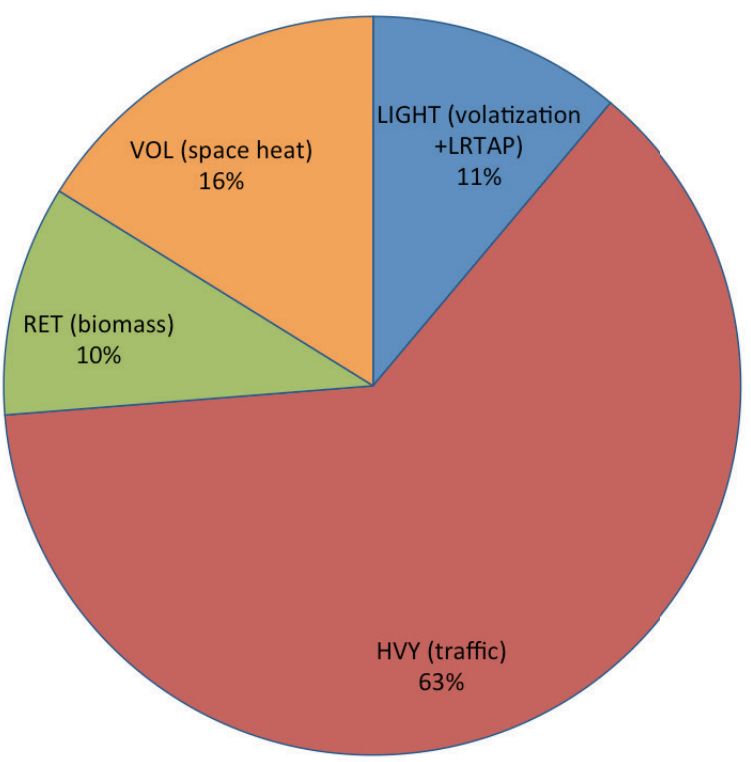

Figure B.8-3 (continued) 


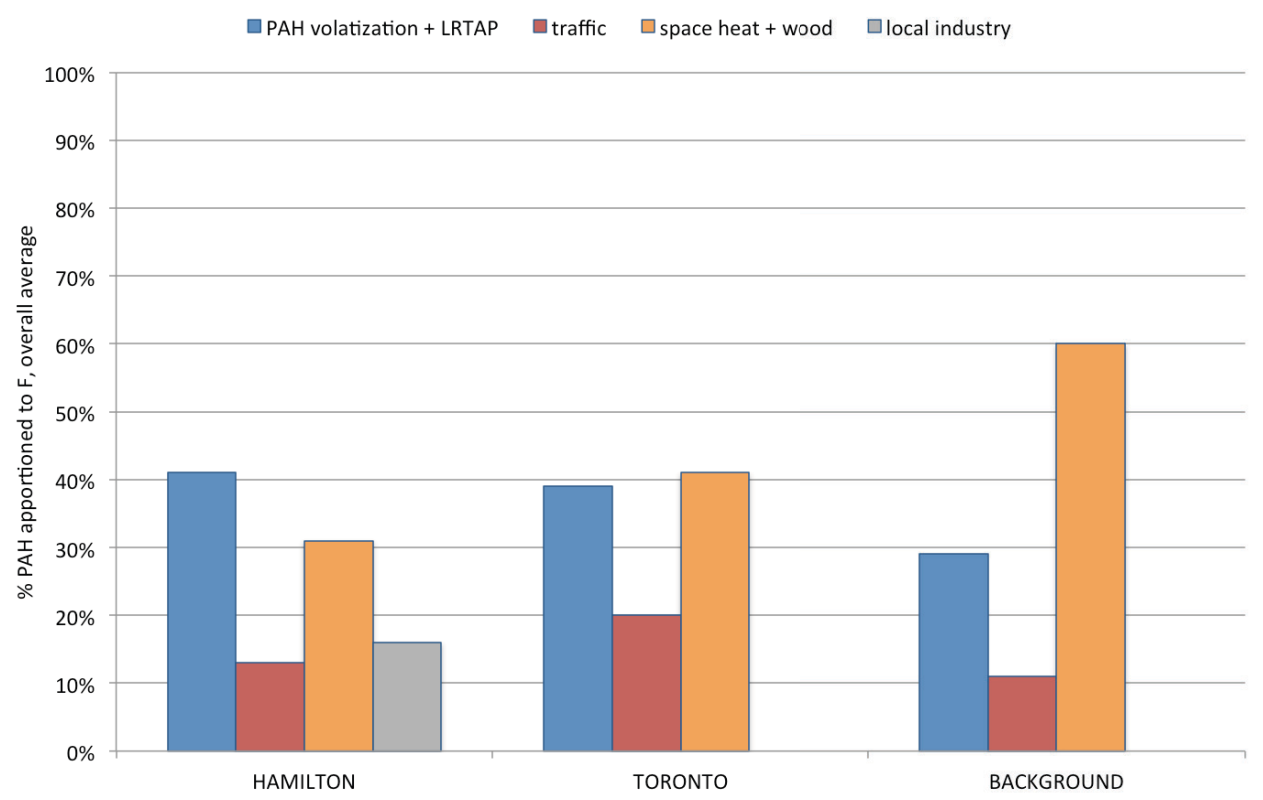

Note: Traffic factors grouped at Toronto; space heating and wood combustion factors grouped at all sites.

Figure B.8-4. Inter-site comparison of PAH source apportionment (all modeled mass).

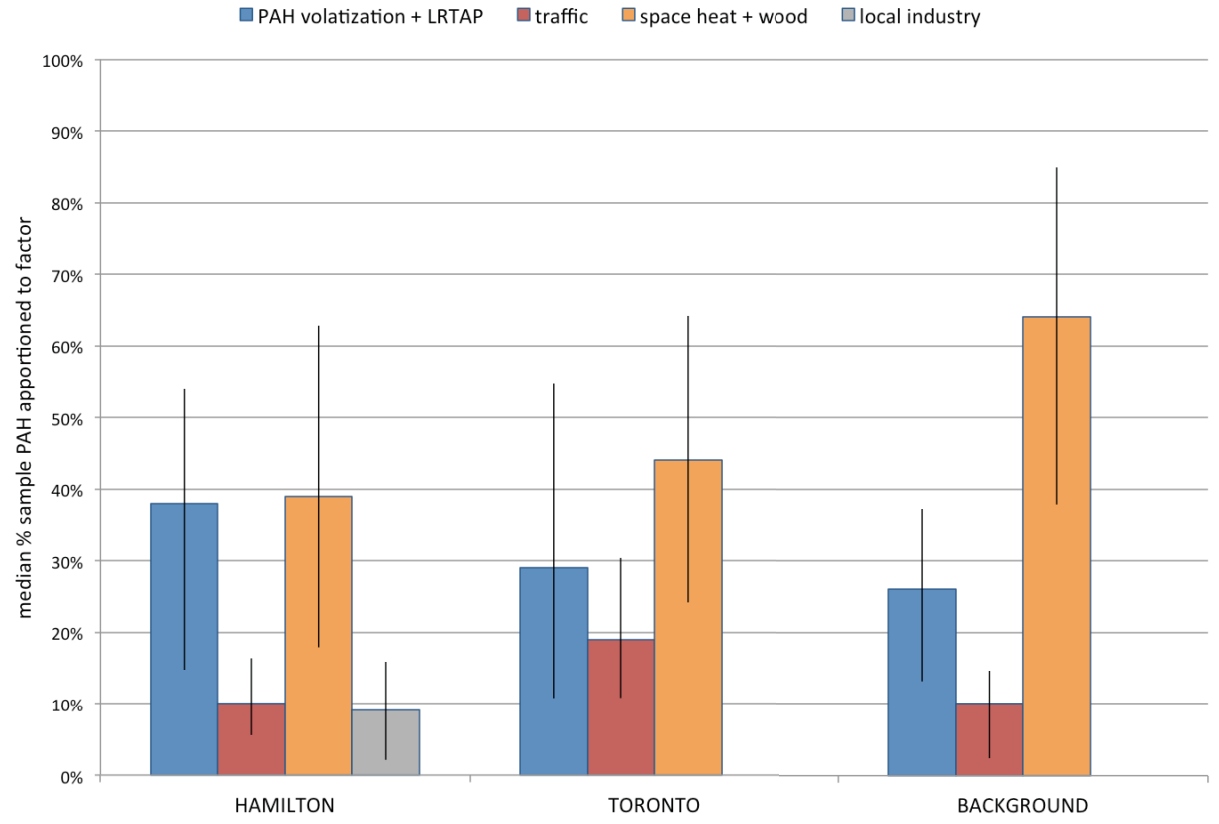

Note: Traffic factors grouped at Toronto; space heating and wood combustion factors grouped at all sites.

Figure B.8-5. Inter-site comparison of PAH source apportionment (Q1, median, Q3 contribution to sample). 


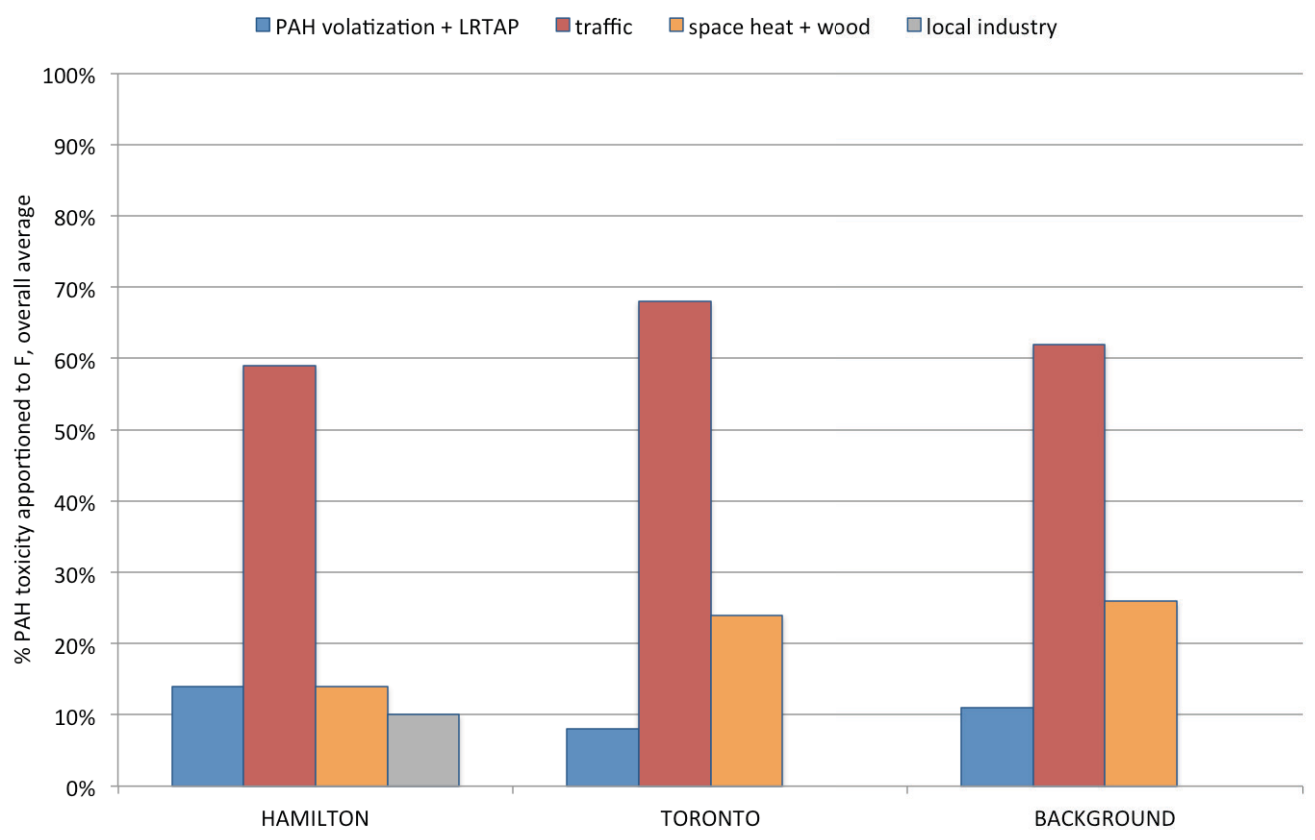

Note: Traffic factors grouped at Toronto; space heating and wood combustion factors grouped at all sites.

Figure B.8-6. Inter-site comparison of BaP-TEQ source apportionment, all modeled mass.

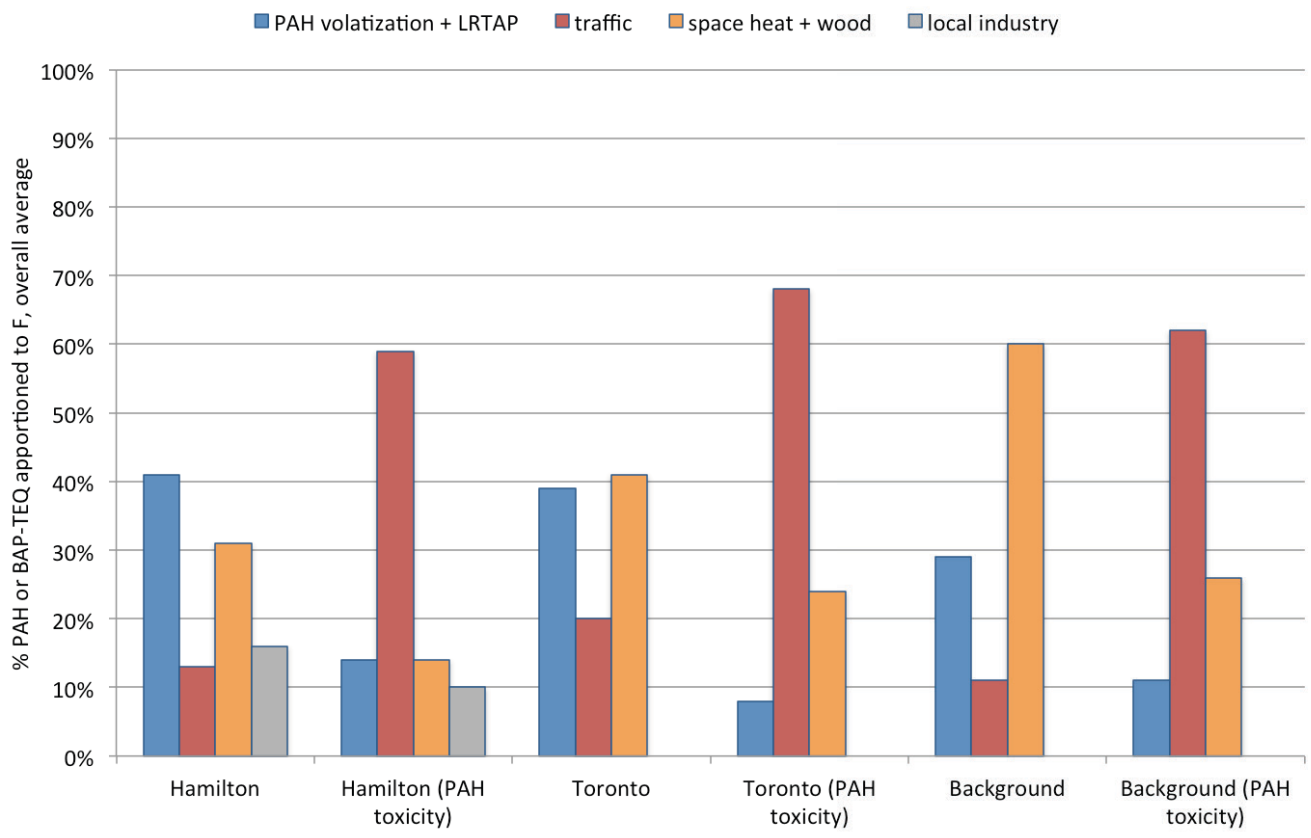

Note: Traffic factors grouped at Toronto; space heating and wood combustion factors grouped at all sites.

Figure B.8-7. Inter-site comparison of source apportionment to PAH versus toxicity (BaP-TEQ), all modeled mass. 

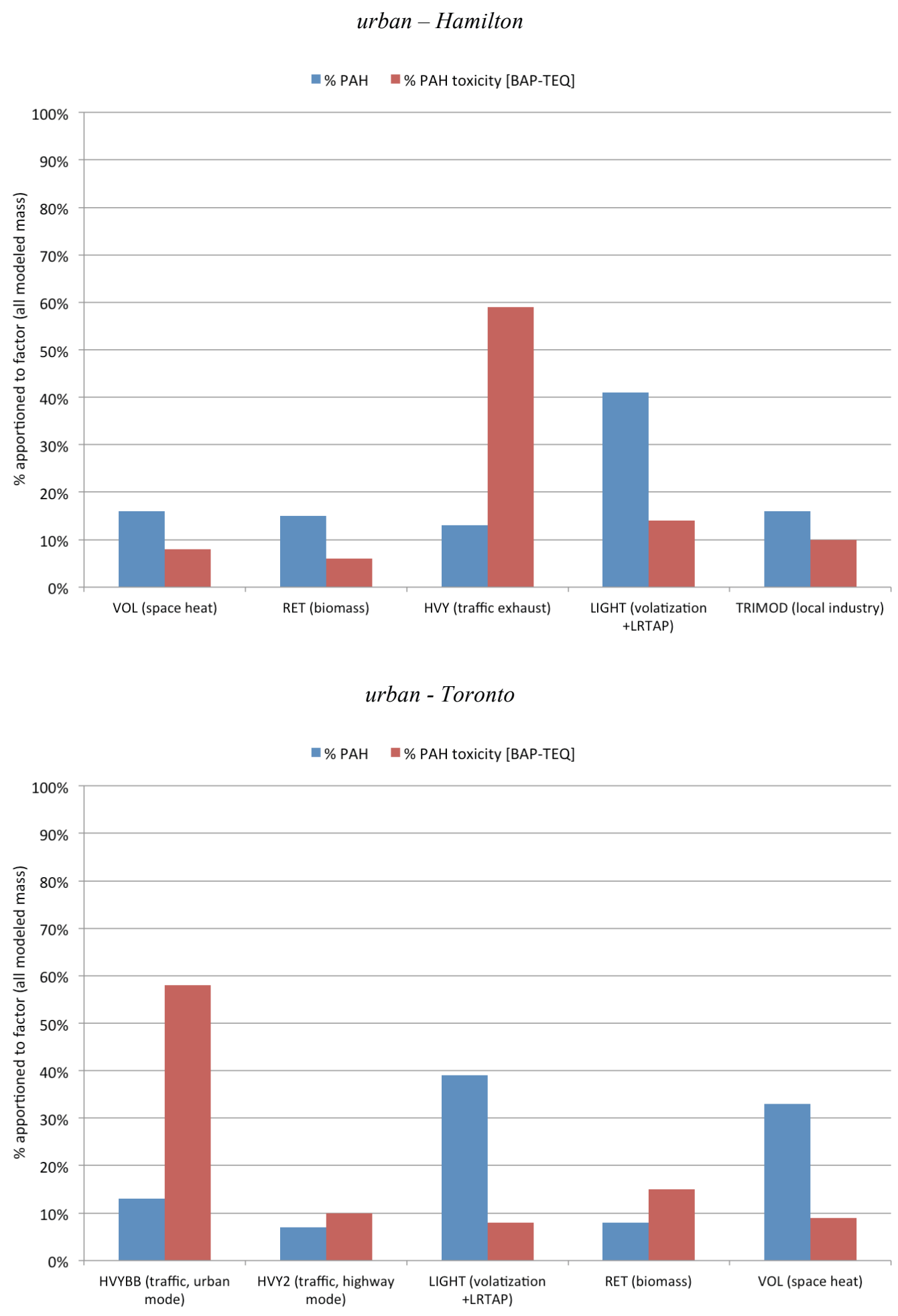

Figure B.8-8. Source apportionment to PAH versus toxicity (BaP-TEQ), all modeled mass. 


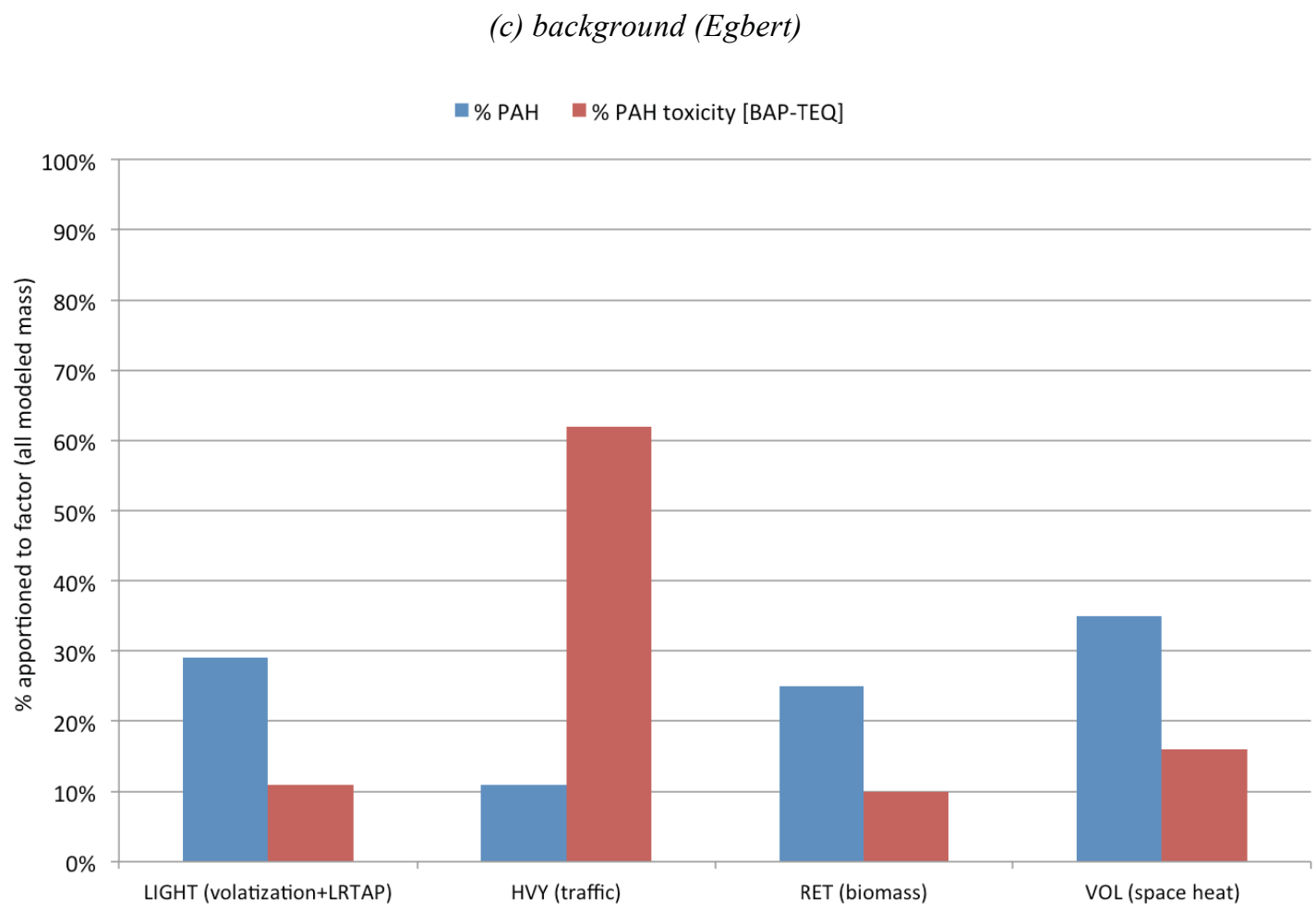

Figure B.8-8 (continued) 


\section{Appendix C}

\section{Detailed intraurban field sampling work}

Supplementary Information to Chapter 4 (research component II).

Describes the scope of work completed by this author in preparing for, coordinating, and completing the two intraurban field sampling campaigns carried out in Hamilton, Canada in 2009 .

Details of intraurban sampling campaign research activities completed are presented in Appendix C.1 for:

- Site selection protocol (i.e., inclusive of maps comparing deployed sampling sites with sites selected for sampling via locate-allocate model).

- Fieldwork instructions.

- Sampling campaign teams, equipment, and documentation (i.e., inclusive of example completed field logsheet).

- Fieldwork summary of sampling issues or site attrition. 


\section{C.1 Intraurban sampling campaign details}

\section{Site selection}

Selection protocol: Sampling locations were selected based on results of a locationallocation model (Jerrett et al 2001, 2005) to ensure representative coverage of land use types and the Niagara escarpment, a key geological feature expected to influence air pollutant concentrations. To provide specific sampling site locations, a partnership was established with the City of Hamilton for use of municipal properties.

Initial contact with the City of Hamilton was made and a working relationship was established with the Senior Project Manager of Environmental Health - Hamilton Public Health Services (Matt Lawson). A list of available municipal property sites, with associated maps, was obtained from the City of Hamilton GIS group.

Municipal property sites with good potential for sampling were identified near each sampling location indicated by the locate-allocate model (i.e., locate-allocate modeling by Michael Jerrett at University of California at Berkeley). Screening criteria included:

- proximity to locate-allocate model location and other nearby sites;

- availability of electrical power;

- sufficient security for the equipment; and,

- possibility of significant on-property sources (e.g., parking lot emissions).

Next, contact was made through Matt Lawson with the City of Hamilton manager for facilities, and individual site supervisors, for facility types identified as potentially suitable for sampling (e.g., libraries, heritage sites, nursing homes, parks/greenhouses, fire stations). Some areas of the city could not be adequately represented with selection limited to municipal properties and so additional non-municipal sites were sought, inclusive of churches and private homes.

Final site selection from screened candidate sites was then made this author traveling to Hamilton and completing extensive on-site visits across the city. Site visits at municipal properties were closely coordinated with Matt Lawson and site supervisors. Site visits at non-municipal sites were coordinated with the appropriate on-site authority (e.g., homeowner at private homes, caretaker at church sites). Siting visits were completed in partnership with Ryan Kulka (Health Canada) and included documenting each site with photographs of the optimal location for sampler set up and location of available electrical 
power and notes on equipment required for each site setup (e.g., ground versus roof setup, length of electrical cord, security or safety concerns, etc.).

Maps (i.e., summer, winter campaign) showing sampling sites overlaid with locateallocate model sites are provided in Figure C.1-1.

Sites selected: For the summer sampling session, 46 sites were identified. Sampling sites provided good coverage of the city and its suburbs, above and below the escarpment. All sites identified for summer sampling were available for repeat sampling in winter. In the winter sampling campaign, one new site was added to densify the sampling network, for a total of 47 sites.

Fieldwork instructions: A field binder was prepared for each sampling team containing a field map, site setup instructions for assigned sites, and sampling logsheets. Fieldwork instructions were based on the siting visits made to Hamilton during the site selection phase of the research and incorporated step-by-step instructions with photographs, providing the following information to the field technicians:

- $\quad$ site name and sampling identification number (i.e., 'site ID');

- address of site;

- contact name and information of site supervisor or other on-site authority that field technicians would need to meet (i.e., for sites were on-site person required for access to electrical power, roof, etc.);

- equipment setup details (e.g., ladder, cord length, etc.) inclusive of recommended electrical cord routing to satisfy safety /security concerns at each site;

- where applicable, specific setup / takedown time.

Due to their visual nature, the fieldwork instructions were prepared using Microsoft PowerPoint software and printed in colour. Field maps were also prepared to show the location and site ID of each sampling site and facilitate efficient route planning.

Sampling setup instructions as shown for a typical site in Figure C.1-2. 


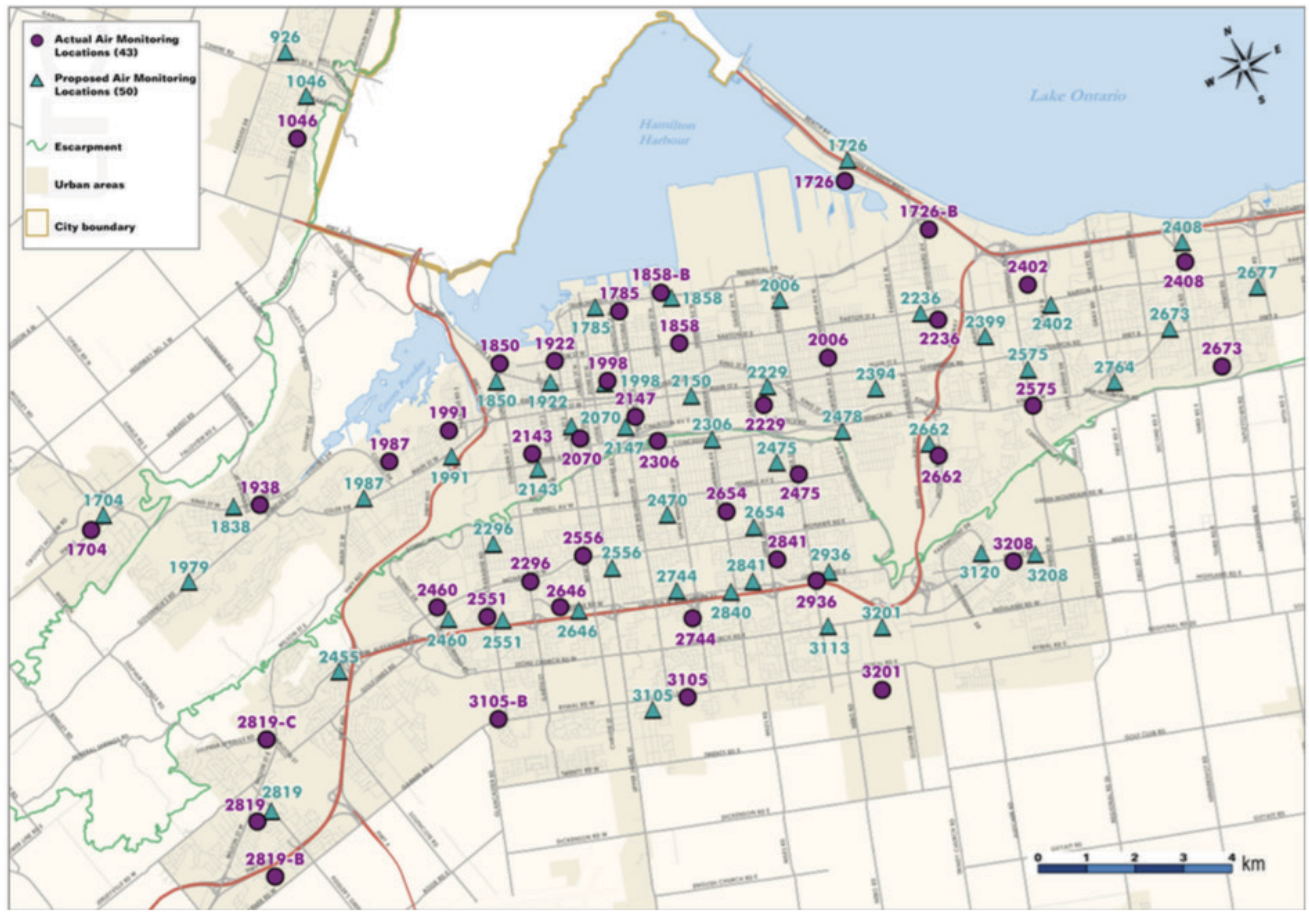

Winter campaign

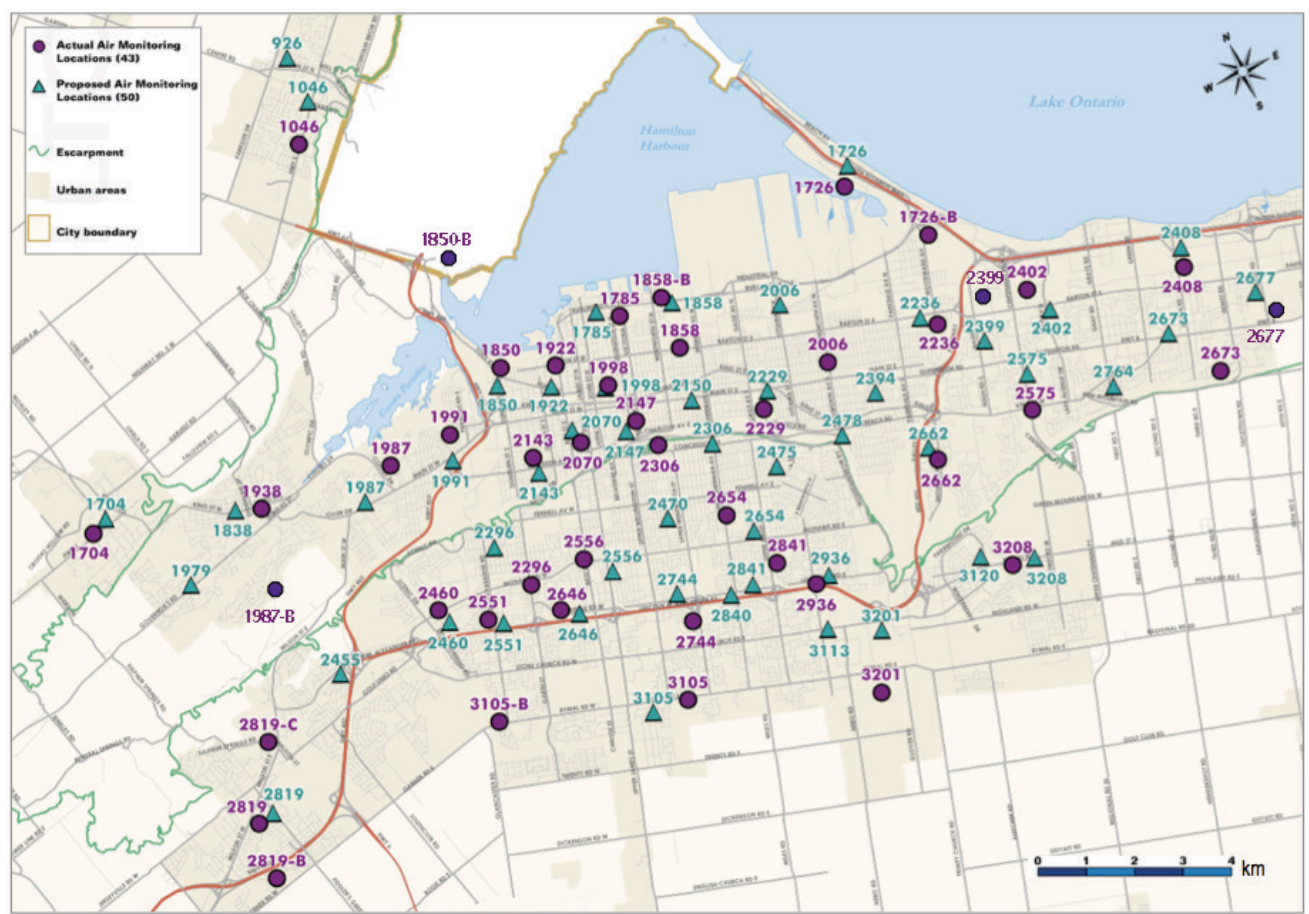

Figure C.1-1. Map of sampling sites (symbol: purple circle) versus locate-allocate model sites (symbol: teal triangle); adapted from City of Hamilton GIS group, Department of Public Health. 


\section{Site ID 1858}

\section{Barton Public Library}

Address: 571 Barton St. East

Equipment:

- 60' extension cord

- long ladder

- lock

Protocol:

- $\quad$ Branch Mgr = Meg Uttangi Matsos

- $\quad$ go to front desk and let know you are setting up

- $\quad$ (site supervisor $=$ Mike Sands)

Setup:

1. city will have provided standard $15 \mathrm{~A}$ electrical outlet off security cam or light box.

2. strap sampler box to "roof fence"

3. secure cord (anti-tripping practice), including along wall (duct tape)

4. Lock case.
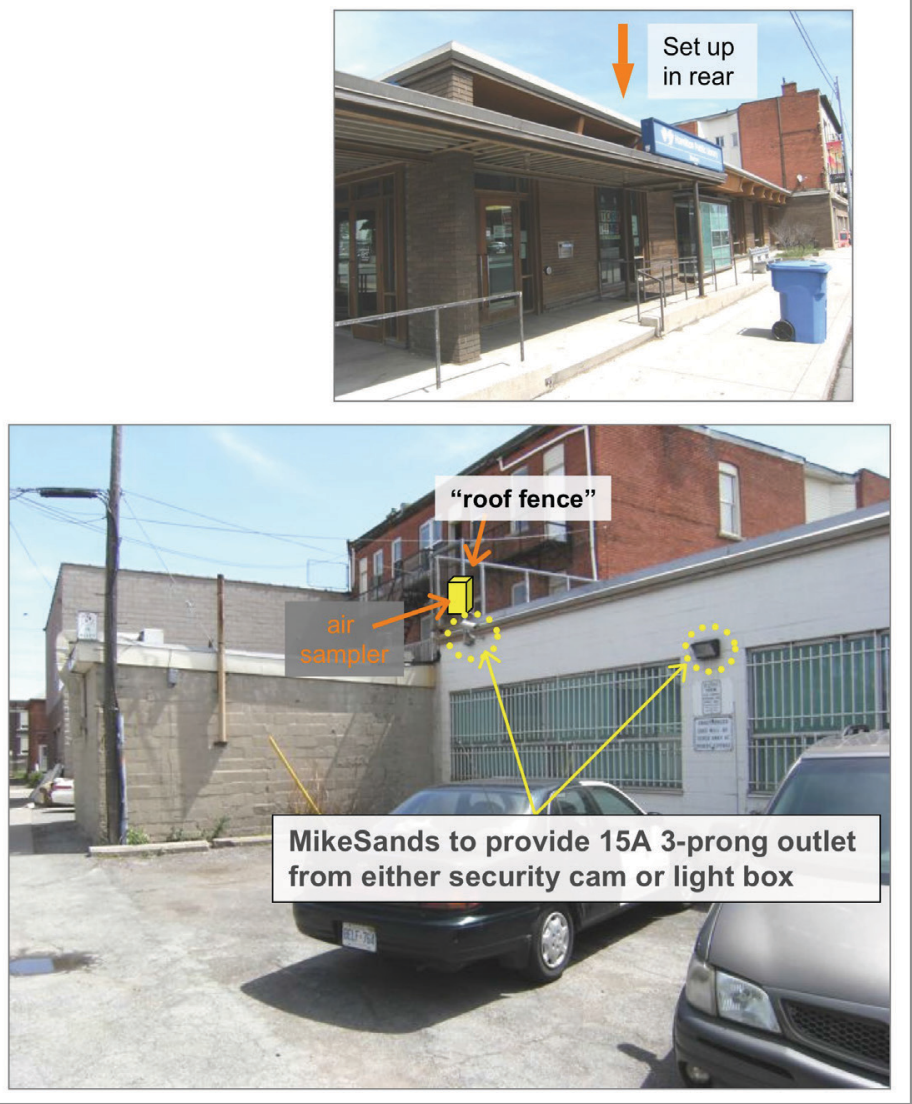

Figure C.1-2. Example of setup instructions for typical sampling site. 


\section{Sampling campaign timing, equipment, teams, documentation}

Seasonal timing: In collaboration with Health Canada, historical trend analysis of National Air Pollution Surveillance (NAPS) Beasley Park data for multiple pollutants was carried out to select seasonally representative sampling periods. This corroborated known seasonal trends for PAHs (i.e., heating and non-heating season). Additional consideration of equipment and personnel availability led to selection of June/July 2009 and December 2009 as the two preferred sampling sessions for intraurban sampling.

Equipment: Sampling equipment was stored in Windsor, Ontario at a Health Canada offsite storage locker. Coordination for both summer and winter seasons required travel to Windsor, Ontario and preparation of equipment for each team in conjunction with Health Canada (Hongyu You). For sampling setup, equipment and samplers were driven from Windsor, Ontario to Hamilton, Ontario in two vans rented for this purpose; equipment was similarly returned to Windsor, Ontario for storage after sampling takedown.

Sampling technician teams: Four sampling teams of two persons each were organized and an approximately equal number of sites assigned to each. A request was made for City of Hamilton individuals to help form the sampling teams, furthering the partnership with the City. In summer sampling, three City of Hamilton staff were assigned to field teams alongside Health Canada personnel (i.e., inclusive of the key City of Hamilton contact, Matt Lawson). In winter sampling, only one City of Hamilton staff member was required and assigned to a field team alongside Health Canada personnel.

In addition to coordination activities for all teams, this author was part of one field sampling team for sampling setup and takedown days (summer, winter campaigns).

Samplers and logsheets: Field samplers were sent by Health Canada (Hongyu You) to contracted labs for analysis. PM samplers were sent to Alberta Research Council (lab contact: Annette Bugnet) and PAH samplers to AirZone (lab contact: Phil Fellin).

Completed field logsheets from the summer and winter sampling sessions were scanned and an electronic copy (i.e., PDF) was emailed to Health Canada personnel for filing. Original hard copy logsheets were returned to Health Canada head office via tracked Canada Post mail.

An example of a sampling logsheet as completed in the field is provided in Figure C.1-3. 
HAMILTON

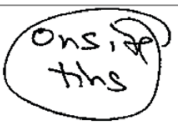

LOG SHEET: SPATIAL MONITORING - June 2009

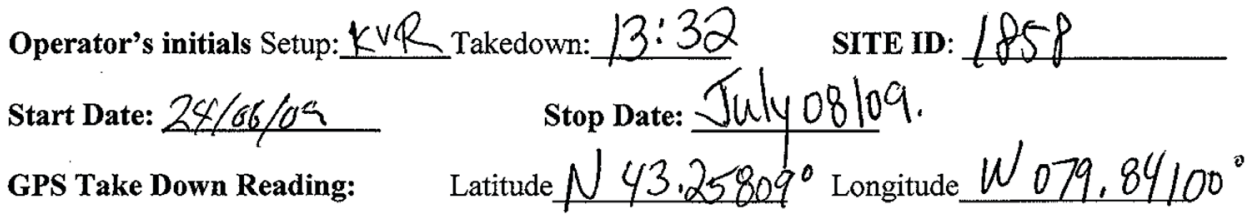

\begin{tabular}{|c|c|c|c|c|c|c|}
\hline $\begin{array}{l}\text { Pump } \\
\text { No. }\end{array}$ & Dlow Meter in & $\begin{array}{l}\text { Start rime } \\
\text { (lihomm) }\end{array}$ & $\begin{array}{l}\text { Rump Displa } \\
\text { Time (lours) }\end{array}$ & Hon Meterin & $\begin{array}{l}\text { Stop } 1 \text { ime } \\
(\text { hibmin) }\end{array}$ & $\begin{array}{l}\text { Pump Display } \\
\text { Time (hours) }\end{array}$ \\
\hline & $B-4$ & $13: 45$ & 0 & Buck & $13: 32$ & 21052 \\
\hline
\end{tabular}

\begin{tabular}{|c|c|c|c|c|c|c|}
\hline Pollutant & Labl ID & Start $S$ & How (1) & pii) & EndSplit & Comments \\
\hline & & Initial & Adju & vited & Flow (pw) & \\
\hline PAH $(2.0 \mathrm{lpm})^{*}$ & H09-PAH 043 & & 210 & $2 / 19$ & 20532075 & \\
\hline Coarse PM & HO9-PMO05 & & 5052 & 5052 & 5461 & \\
\hline & HO9-PU & & & & & \\
\hline
\end{tabular}

$* 1 \mathrm{lpm}=$ ideal flow rate. Actual flow rate can be $\pm 5 \%$

\section{Ogawa Passive Sample}

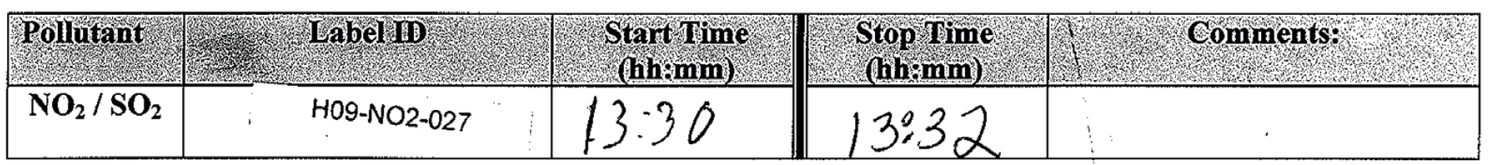

\section{VOC Badge}

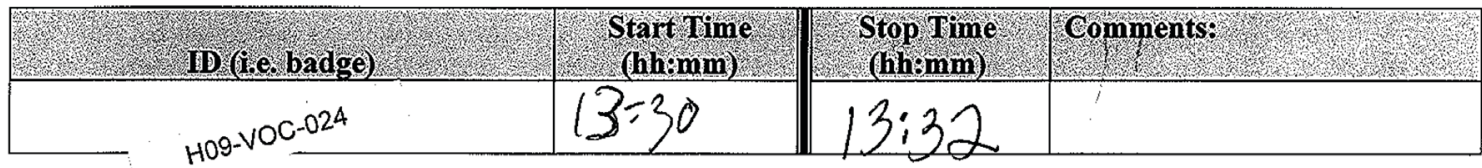

\section{Blank IDs (as applicable)}

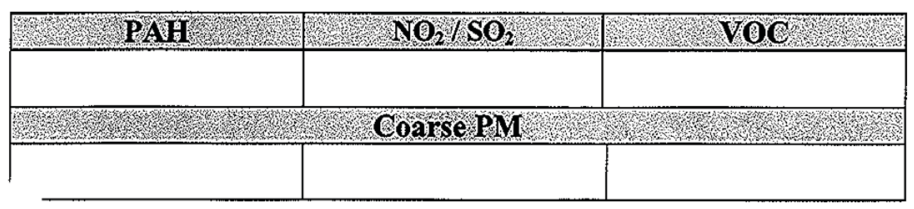

Figure C.1-3. Example of completed sampling logsheet for typical sampling site. 


\section{Fieldwork summary}

Summer season: For the summer season, samplers were installed across the city on Wednesday 24 June 2009 and taken down two weeks later on Wednesday 8 July 2009. Samplers were successfully deployed at 43 of 46 available sites (Figure C.1-4).

At sampling takedown, 40 of the 43 sites had a complete suite of samplers available for analysis (i.e., active and passive samplers). At three sites, the pump failed and was not operating at takedown, but passive sampler data were available. None of the sites experienced vandalism or other security or safety-related concerns.

Winter season: For the winter season, samplers were installed across the city on Wednesday 2 December 2009 and taken down two weeks later on Wednesday 16 December 2009. Samplers were successfully deployed at 46 of 47 available sites (Figure C.1-4).

At sampling takedown, 40 of the 46 sites had a complete suite of samplers available for analysis (i.e., active and passive samplers). At six sites, the pump had failed during sampling (i.e., not found to be operating at takedown), but passive sampler data remained available. None of the sites experienced vandalism or other security or safety-related concerns.

At four rooftop sites, the tripod fell over during the sampling period; however, with the exception of a single PAH sampler at one of these rooftop sites, end flow rates were within range, suggesting that the active samplers were not compromised. Similarly, passive samplers were partially exposed to the elements at these sites due to the rain shelter being displaced from its vertical orientation but samplers were flagged and sent for lab analysis. This sampling failure mode is believed to be partially due to a severe windstorm that occurred in Hamilton during the sampling period. However, it was noted in follow-up conversations with the field technician teams that sandbags or similar weighting was not used to secure the roof tripod as per standard operating procedures; this likely contributed to tripod failure and subsequent potential loss of sampling data at these sites.

City of Hamilton site supervisors were supportive of the sampling at their respective sites and were notified that sampling was completed successfully either directly or through the key City of Hamilton contact. 


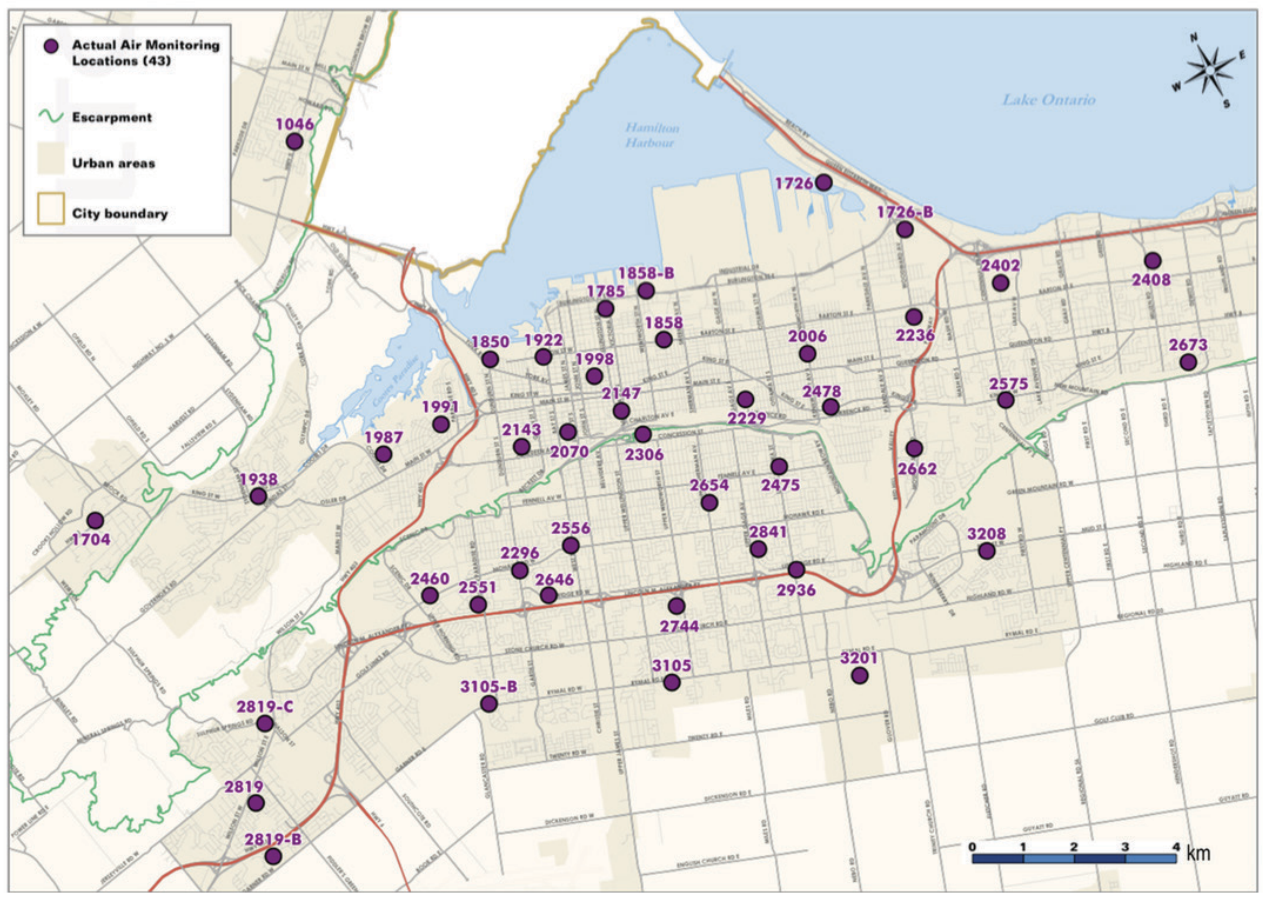

Winter campaign (46 sites)

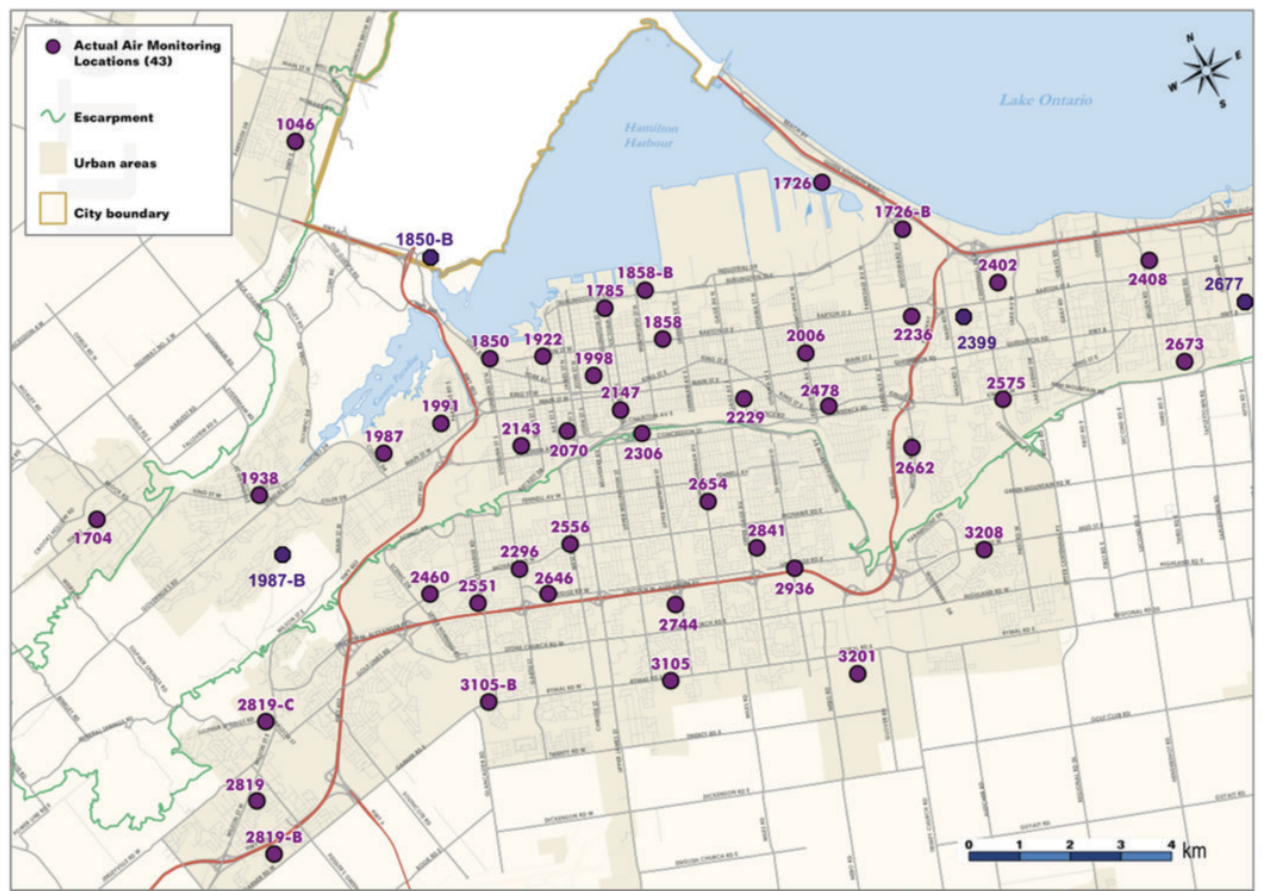

Figure C.1-4. Deployed field sampling sites in summer and winter campaigns; adapted from City of Hamilton GIS group, Department of Public Health. 


\section{Appendix D Intraurban source apportionment}

Supplementary Information for Chapter 5 (research component III).

D.1 PMF-CMB model parameters and performance criteria

Details of modeling parameters and performance criteria for PMF modeling of Hamilton central site data and CMB modeling of Hamilton intraurban field data.

D.2 Preliminary PMF modeling for generating CMB input source profiles

Preliminary PMF modeling iterations to generate CMB input source profiles: (i) Determination of appropriate PMF model order. (ii) Determination of appropriate suite of fitting species. (iii) Investigation of seasonally stratified PMF model.

D.3 PMF-CMB model performance verification

Model performance for PMF-CMB verification: (i) comparison with NAPS data (central monitoring station); (ii) comparison with field data site co-located at central monitoring station.

\section{D.4 Detailed results for intraurban source apportionment via PMF-CMB}

Detailed results for source apportionment of intraurban field data. PMF model solution summary and bootstrap factor profile output used to generate local source profiles input to $\mathrm{CMB}$ model ( $\mathrm{PR}$ file). $\mathrm{CMB}$ model performance metrics are presented as summary statistics and by site.

Fractional and absolute source contribution estimates (SCE) to $\Sigma \mathrm{PAH}$ and BaPTEQ, with associated uncertainty (UNC) and related metadata (T-stat), presented as summary statistics, tabulated by site, and mapped across study area.

D.5 Intraurban source apportionment using seasonal source profiles

Summary of sensitivity analysis for intraurban source apportionment using seasonal variants of CMB input source profiles. 


\section{D.1 PMF-CMB model parameters and performance criteria}

\section{$\underline{\text { PMF modeling }}$}

US EPA PMF 3 modeling retained fitting species based on data quality criteria (e.g., omit species with very high portion of time series missing or below $\mathrm{DL}_{\mathrm{j}}$, omit species with known sampling/sample analysis issues, include marker species even where lower data quality using conservative error estimate; US EPA, 2008; Zhao et al, 2006; Hopke et al, 2003b; Reff et al, 2007) and PMF-calculated signal-to-noise (SN) ratio (US EPA, 2008). Species categorization within PMF referenced commonly used guidelines (Paatero and Hopke, 2003) but since input data uncertainty estimates were judged sufficiently conservative and the species suite was relatively small, it was generally preferred to model species as input, down-weighting further only if warranted by consistently poor species fit. Additional extra modeling uncertainty was not applied (i.e., $\mathrm{C} 3=0 \%$ ) because PAH sample uncertainty estimates were conservative and species-specific, as confirmed by sensitivity analysis.

Outliers are down-weighted within PMF (Hopke, 2003a), so a conservative sample exclusion protocol was applied: samples were excluded only if they contained statistically extreme outlier species concentrations (i.e., higher or lower than three times IQR) and outlier status was corroborated by poor model performance for that sample date and exclusion would not meaningfully alter source profiles or trends in contribution.

PMF modeling followed best practice guidelines (US EPA, 2008; Sonoma, 2008) and recommendations in the comprehensive literature review of PMF studies completed by Reff et al (2007). Briefly: explored 3 to 7 factor solutions and selected optimal model order with respect to model performance and to ensure physical meaning of solution; verified solution unique and stable (i.e., converged to local minimum with narrow range in Q; further validated using random seed); verified solution converged to theoretical global minimum (i.e., $\mathrm{Q}_{\text {robust }}$ compared to $\mathrm{Q}_{\text {theory }}$ ) and not subject to outlier influence (i.e., $\mathrm{Q}_{\text {robust }}$ compared to $\mathrm{Q}_{\text {true }}$ ); verified all fitted species modeled well (e.g., high $\mathrm{r}^{2}$, low species residuals, normally distributed species residuals); and, verified solution was reasonably free from rotational ambiguity (i.e., scatter in inter-factor contribution plots, 'G-space'; exploration of F-peak solution rotation). Most critically, model solutions were verified for robustness via bootstrapping analysis, the PMF subroutine that repeatedly models differing subsets of the original dataset and statistically summarizes their solutions (US EPA, 2008). This ensured that solutions were statistically representative and factors were sufficiently distinct from each other to merit interpreting as a distinct source type, determined by bootstrap factors being mapped consistently to the same base factor. 


\section{CMB modeling}

US EPA CMB 8.2 model parameters were consistent with modeling practice guidelines (i.e., iteration delta $=20$; maximum source uncertainty $=20 \%$; minimum source projection $=0.95$, source elimination option $=$ yes, Britt and Lueck algorithm $=$ no; US EPA, 2004; Watson et al, 2004). Model quality was first characterized by goodness of fit (GOF) metrics (i.e., $\mathrm{r}^{2} \geq 0.8$; chi $^{2}<2$; percent mass $80 \%-120 \%$; US EPA, 2004) and the model's "best fit" function was used as an aggregate metric of model fit (i.e., fitting measure, $\mathrm{FM}=$ weighted sum of squares of $\mathrm{r}^{2}, \mathrm{chi}^{2}$, percent mass, number of estimable sources; equal weighting of 1.0 for FM components; US EPA, 2004).

At each site, apportioned factor source contribution estimates (SCE) were reviewed for quality (i.e., magnitude of SCE to uncertainty) via reported T-stat values, reflecting uncertainty in the ambient data, the source profiles and any collinearities among profiles (i.e., $\mathrm{T}$-stat $=\mathrm{SCE} /$ standard error where standard error $=$ one standard deviation).

Collinearities between CMB fitting factor profiles are generally not desired but manifest only in relation to a specific ambient data set (i.e., could arise at one sampling site but not at another) so cannot be reliably predicted a priori by using tests of factor similitude such as Pearson's $r$ (Watson et al, 2004). Instead, collinearities between non-estimable sources (i.e., SCE value calculated but reported by the model as being outside the estimable space) were reviewed for frequency of occurrence; source profile pairs appearing as a linear combination at many sites indicated the need for more distinct input source profiles. 


\section{D.2 Preliminary PMF modeling for generating CMB input source profiles}

\section{$\underline{\text { PMF model order }}$}

Initial PMF-CMB iterations referenced the Hamilton PMF solution previously identified as optimal for source type interpretation (Chapter 3), a 5-factor model resolving: "light" denoting PAH from volatilization of fossil fuel and re-volatilization from built surfaces plus coal combustion emissions via long range transport of air pollutants (i.e., abbreviated for convenience to PAH volatilization and LRTAP coal combustion); "heavy" denoting vehicle traffic exhaust emissions; "volatile" denoting space heating; "retene" denoting biomass combustion; "trimodal" denoting local industry emissions from iron/steel manufacturing. ${ }^{35}$

Corresponding CMB source profiles (i.e., PR) proved inappropriate due to a difference in species resolution between the PMF and CMB models (i.e., 21 of 29 fitting species retained from NAPS central site data; 13 of 16 species retained from intraurban field sampling data). While a larger number of fitting species boosts PMF modeling power and assists more nuanced interpretation of source types, particularly where marker species are available (Reff et al, 2007), the significant difference in PMF-CMB species resolution revealed a practical constraint on model order: one of the factors resolved at the fifth model order denoted a source type characterized by species that were not field-sampled (i.e., biomass combustion, with characteristic species retene alongside me-PAH); such a factor profile would lose key characteristics upon 'redaction' of these species in the CMB model (i.e., retene and me-PAH cannot be CMB fitting species since they were not sampled for the intraurban field dataset and thus not present in the AD file) and the resulting 'redacted' factor profile would have a high likelihood of collinearity with other factor profiles in the CMB source apportionment. Review of the 5-factor PMF model source profiles redacted to the 13-species field suite available as CMB fitting species confirmed strong similarity among some factors (Figure D.2-1) and CMB model runs using the 5-factor PMF source profiles confirmed collinearity issues (i.e., estimable linear combination of factors found at half the sampled sites: "light + retene" at 11 sites; "volatile + retene" at 6 sites).

\footnotetext{
${ }^{35}$ A 5-factor model restricted to seasonally stratified fall/winter data resolved similar factors but was not retained as a viable solution due to poorer fit, reduced robustness, greater sensitivity to outliers, and poorer separation of the PAH volatilization factor (i.e., "light") due to exclusion of spring/summer samples where contributions are greater driven by high ambient temperatures.
} 
Thus, 4-factor PMF models were run on the Hamilton NAPS central site data to resolve local CMB source profiles (PR) that would be more compatible with the field data species suite $(\mathrm{AD})$.

\section{$\underline{\text { PMF fitting species suite }}$}

Mindful of potential modeling effects of differences in species resolution between the PMF and CMB models, 4-factor PMF solutions were generated using a larger species suite (i.e., 21 species) and then the smaller field suite available as CMB fitting species (i.e., 13 species, all modeled as 'strong' to ensure they were well-represented in the PMF model rather than down-weighted). Visual comparison of PMF results showed preference for the smaller field-sampling suite due to greater distinction between PMF output source profiles, both concentration and explained variance profiles and thus more distinct CMB input source profiles. In $\mathrm{CMB}$, the source profile set derived from a species suite larger than the AD field suite tended to yield poorer $\mathrm{CMB}$ results than the source profile set derived from the smaller field-matched suite (i.e., worse fit, more collinearity between factors at several sites; observed for year-round and fall/winter PR variants). Restricting fitting species to a common PMF-CMB suite ensured that inter-factor differences in the local source profiles (PR) were adequately modeled and apportioned to the field sampling data (AD) for all species.

A precautionary comparison of PMF results using both species suites (i.e., 13 species; 21 species) confirmed that source type interpretations remained consistent, with all four factors maintaining consistent species patterns, temporal trends in contribution, and source contributions to ambient PAH with the reduced 13-species suite. 


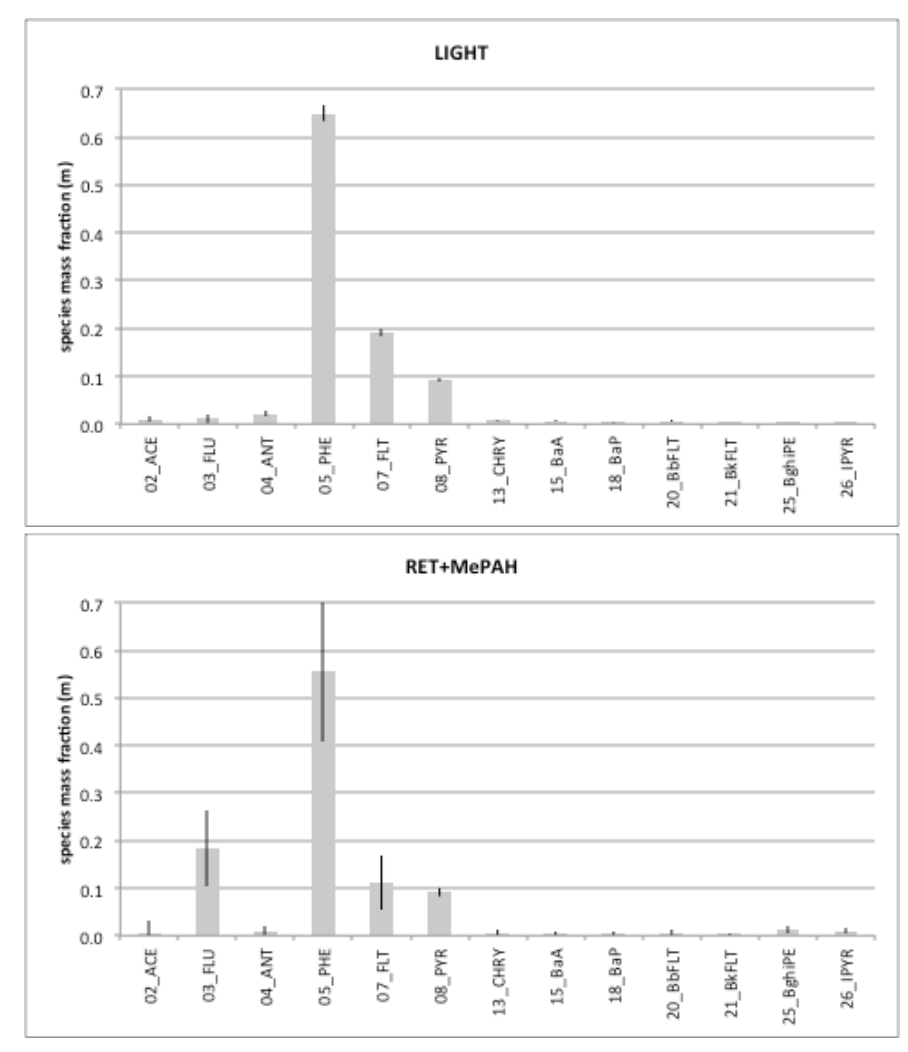

Note: Strong similarity seen between "light" and "retene+mePAH" factor profiles. Confirmed as collinear in CMB model.

Figure D.2-1. CMB source profiles from 5-factor PMF sw model, redacted to AD species suite.
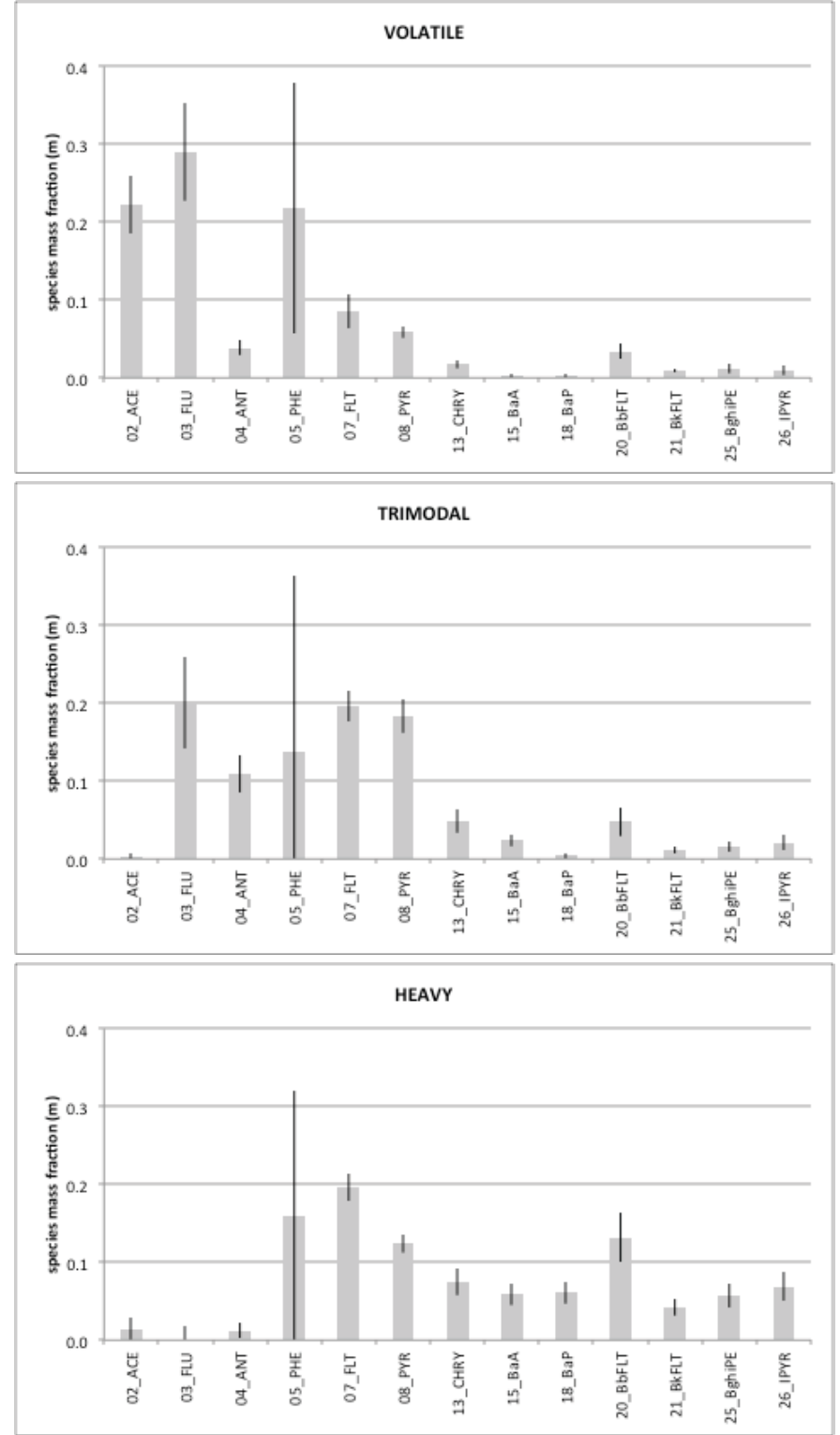


\section{PMF seasonal stratification}

Seasonally stratified source profiles held initial interest as a strictly analogous match to ambient data collected in the same season (e.g., PR from PMF model of fall/winter data applied to $\mathrm{CMB}$ of winter $\mathrm{AD}$ ) but the advantages afforded by modeling year-round data were compelling, including significantly increased modeling power via increased sample size and improved interpretative power for source types via seasonal trends in factor contributions.

Results confirmed preference for a year-round PMF model (Table D.2-1): factors between year-round and fall/winter model variants (i.e., 4-factor model, 13 fitting species as per AD field suite) were generally similar but a number of strong disadvantages accrued for the fall/winter model (i.e., reduced model performance; increased sensitivity to outliers; loss of seasonal trend information; less robust, higher degree of crossmapping indicating less factor distinction and greater risk of factor collinearity in $\mathrm{CMB}$ ). 
Table D.2-1. PMF performance summary comparison between year-round and fall/winter models.

\begin{tabular}{|c|c|c|}
\hline & year-round (h0110sw4p) & fall/winter (h0110w4p) \\
\hline \multicolumn{3}{|l|}{ INPUT \& CFG } \\
\hline Filename & h0110sw-sec-0123-4p-fieldsuite.cfg & h0110w-sec-0123-4p-fieldsuite-TEST.cfg \\
\hline $\mathrm{n}$, outliers & $\begin{array}{l}\text { n_0110sw }=200, n \_o u t=21(11 \% \\
\text { exclusion }), \\
\text { n_model }=179 \\
\text { (i.e., } 0123+2 \text { very poor fit dates) }\end{array}$ & $\begin{array}{l}\mathrm{n} \_0110 \mathrm{w}=115, \mathrm{n} \_ \text {out }=18(16 \% \\
\text { exclusion }) \\
\text { n_model }=97 \\
\text { (i.e., o123) }\end{array}$ \\
\hline $\mathrm{S}$ & SvarEC & SvarEC \\
\hline C3 & $0 \%$ & $0 \%$ \\
\hline species - bad & $\begin{array}{l}\text { ACY, MFLU, medium (MPYR, BAFLU, } \\
\text { BBFLU, BGFLT), TRIP, RET, BEP } \\
\text { (i.e., limited to CMB AD field suite) }\end{array}$ & $\begin{array}{l}\text { ACY, MFLU, medium (MPYR, BAFLU, } \\
\text { BBFLU, BGFLT), TRIP, RET, BEP } \\
\text { (i.e., limited to CMB AD field suite) }\end{array}$ \\
\hline species - weak & none & none \\
\hline \multicolumn{3}{|l|}{ STABILITY } \\
\hline Q_theory & $2327\left(n^{*} m\right), 1611$ (Sonoma) & $1261\left(n^{\star} m\right), 873$ (Sonoma) \\
\hline Q_rob (range) & $1755(0.03)+9 \%$ Qtheory & $1038(0.04)+19 \%$ Qtheory \\
\hline Q_true (range) & $1755(<0.01)$ & $1038(0.02)$ \\
\hline$\Sigma \_d^{\wedge} 2 \_\max$ & $<0.01$ & 0.13 \\
\hline \multicolumn{3}{|l|}{ FIT } \\
\hline$r^{\wedge} 2$ & majority $>0.8 ;$ FLU r2=0.77 & all >0.8; FLU r2=0.80 \\
\hline \# res $>|3|$ & 6 & 6 \\
\hline high res species ( $r^{\wedge} 2, \#$ res) & BAA, $r 2=0.93,3$ hi res & $\mathrm{FLU}, \mathrm{r} 2=0.80,2$ hi res \\
\hline \multicolumn{3}{|l|}{ G-space } \\
\hline edge? & good fill in all $\mathrm{F}$ pairs & moderate edge in $3 \mathrm{~F}$ pairs \\
\hline \multicolumn{3}{|l|}{ ROBUST } \\
\hline bootstrapping & $98 / 100 / 94 / 99 / 1 U$ & $100 / 74 / 79 / 81 / 14 U$ \\
\hline shape unmapped? & none (FLU, PHEN) & $\sim$ LIGHT \\
\hline notes & $\begin{array}{l}\text { excellent bootstrap (mapping, narrow } \\
\text { IQR, base run } \sim \text { IQR median) }\end{array}$ & $\begin{array}{l}\text { poor bootstrap (cross-mapping, } \\
\text { especially for HVY crossmapped to } \\
\text { TRIMOD; many LIGHT unmapped) }\end{array}$ \\
\hline \multicolumn{3}{|l|}{ FACTORS } \\
\hline label & $\begin{array}{l}\text { HEAVY }(\sim 15 \% \mathrm{G}) \\
\text { LIGHT }(\sim 38 \% \mathrm{G}) \\
\text { TRIMODAL }(\sim 18 \% \mathrm{G}) \\
\text { VOLATILE }(\sim 29 \% \mathrm{G})\end{array}$ & $\begin{array}{l}\text { VOLATILE }(\sim 31 \% G) \\
\text { TRIMODAL }(\sim 14 \% \mathrm{G}) \\
\text { HEAVY }(\sim 25 \% \mathrm{G}) \\
\text { LIGHT }(\sim 31 \% \mathrm{G})\end{array}$ \\
\hline year trend? & no trend & no trend \\
\hline season trend? & $\begin{array}{l}\text { LIGHT > summer; VOL > winter } \\
\text { no seasonal trend: HVY, TRIMOD }\end{array}$ & n/a (no spring/summer samples) \\
\hline weekday/weekend trend? & $\begin{array}{l}\text { mild trend but noted difference } \\
\text { between HVY and other factors: } \\
\text { HVY }>\text { weekday } \\
\text { LIGHT, TRIMOD, VOL }>\text { weekend }\end{array}$ & $\begin{array}{l}\text { moderate trend seen only for HVY > } \\
\text { weekend; others, no trend }\end{array}$ \\
\hline
\end{tabular}




\section{D.3 PMF-CMB model performance verification}

\section{Comparison with NAPS data}

Performance and viability of the PMF-CMB model was first verified by comparing CMB results with previous PMF source apportionment results (i.e., Chapter 3; 5-factor model) at the central monitoring site for all 2009 NAPS sampling dates.

Use of a five-factor CMB model was to permit all available species to be modeled for purposes of stricter model verification as well as to enable direct comparison with the previous PMF source apportionment results (i.e., PR: five-factor PMF solution from 2001-2010 time series, year-round data, 24-hr sampling, 21 species, details in Chapter 3; AD: identical 24-hr samples and 21 species suite as PR, 26 sample dates in 2009).

CMB model fit was good for the majority of 2009 dates, with values for the goodness of fit metrics (Table D.3-1) well within CMB guideline targets (US EPA, 2004): $\mathrm{R}^{2}$ values were consistently above 0.9 (median, 0.98 ); most dates explained $90 \%-100 \%$ sample mass (median, 92\%); $\mathrm{CHI}^{2}$ values were suitably low (median, 0.6 ); uncertainty on SCE was low for most dates and factors (T-stat $>1.5$ ).

The CMB model resolved contributions to modeled PAH $\left(\Sigma_{21} \mathrm{PAH}\right)$ from all five input source profiles (Table D.3-2) for most 2009 sampling dates and source contributions were generally consistent with PMF results (i.e., SCE and T-stat varied over the year, expected due to variability in source contributions and prevailing weather conditions).

Further, seasonal trends in CMB source contributions were consistent with trends observed in the PMF model from which the source profiles were first derived with no contraindications of interpreted source types. Summarizing: strong seasonality for "light" factor (i.e., 27\% higher median SCE in summer) consistent with seasonality observed in PMF and its interpretation (i.e., higher summer temperatures driving greater volatilization); mild seasonality for "retene" and "volatile" factors (i.e., 10\% - 15\% higher median SCE in winter) consistent with PMF seasonal trends and with their association with space heating emissions; minimal seasonality for "trimodal" and "heavy" factors (i.e., $<10 \%$ difference in summer and winter median SCE), consistent with absence of seasonality in PMF and with interpreted source types (i.e., "trimodal", industrial emissions generally active year-round; "heavy", traffic patterns generally similar year-round). 
Comparison with field data site co-located at central monitoring station

The next verification examined the viability of PMF-CMB to apply source profiles derived from local NAPS data to source apportionment of the intraurban field campaign dataset (Anastasopoulos et al, 2012), referencing the field site co-located with the NAPS central monitor (i.e., site 1998) and comparing source contribution estimates between datasets (i.e., NAPS data versus field data).

For apportioning source contributions to NAPS data at the central site, CMB input PR and $\mathrm{AD}$ were as described in the first verification of PMF-CMB model performance (i.e., 5th model order, 21-species suite). Meanwhile, for apportioning source contributions to field data at the site co-located with the NAPS central monitor, CMB input PR was the 4factor solution (i.e., 4th model order for species suite compatibility with field data; 20012010 time series, year-round data, 13 species) and input AD was the winter campaign field data (i.e., 14-day sample, 13 species) at field site 1998.

Comparing model outputs, estimated source contributions to modeled PAH were found to be consistent for similar source types, despite the reduced modeling power due to the smaller field sampling suite and the ambient data sampling differences (i.e., SCE fraction to NAPS samples: Table D.3-2, average of 3, 21 December 2009 samples \#22 and \#23 model results to align with field campaign period; SCE fraction to field sample: Appendix D.4: Figure D.4-1, site 1998). The "light"/“light-r" contributions were highly similar (i.e., NAPS, $\Sigma_{21} \mathrm{PAH}, 29 \%$; field, $\Sigma_{13} \mathrm{PAH}, 33 \%$ ). The combined "volatile" and "retene" contributions to NAPS-sampled PAH (39\% combined to $\left.\Sigma_{21} \mathrm{PAH}\right)$ agreed reasonably well with the "volatile-r" contribution to field-sampled PAH $\left(49 \%, \Sigma_{13} \mathrm{PAH}\right)$, the difference being within $\mathrm{CMB}$ source contribution uncertainty estimates and the comparison suggesting the biomass combustion source type is largely represented by the "volatile-r" factor in the intraurban source apportionment results. Contribution differences were largest for the "trimodal" factor (NAPS, $\Sigma_{21} \mathrm{PAH}, 25 \%$; field, $\Sigma_{13} \mathrm{PAH}, 11 \%$ ); this was considered reasonable given the large sample-to-sample variability expected for local industry emissions at the central monitoring site (i.e., source-receptor dispersion strongly subject to weather patterns since monitor is upwind of industrial harbour-front, based on prevailing wind direction; corroborated by greater variability of CMB SCE over 2009 NAPS dates and in PMF contributions over 2001-2010, Chapter 3) and the comparatively larger CMB uncertainty estimates (i.e., lower T-stat) typically found for the "trimodal" factor. Finally, contribution differences were minimal for the "heavy" factor (NAPS, $\Sigma_{21} \mathrm{PAH}, 8 \%$; field, $\Sigma_{13} \mathrm{PAH}, 7 \%$ ). 
Table D.3-1. CMB performance for 2009 central site data (5-factors, 21-species).

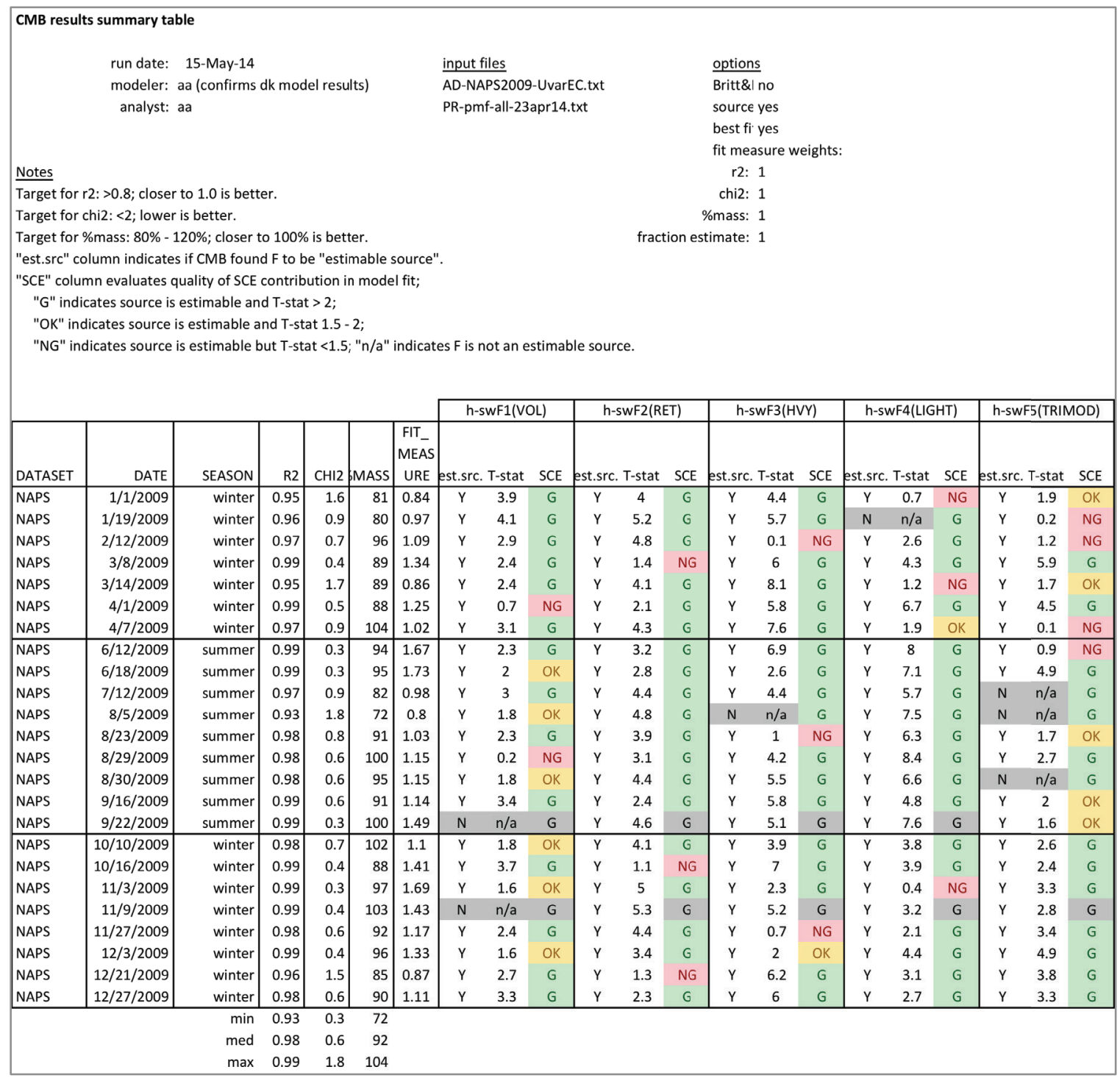


Table D.3-2. CMB source apportionment for 2009 central site data (5-factors, 21-species).

\begin{tabular}{|c|c|c|c|c|c|c|}
\hline \multicolumn{7}{|l|}{$\%$ to species: TM } \\
\hline sample \# season & $\begin{array}{r}\% \text { h-swF1 } \\
\text { (VOL) }\end{array}$ & $\begin{array}{r}\% \text { h-swF2 } \\
\text { (RET) }\end{array}$ & $\begin{array}{r}\text { \%h-swF3 } \\
\text { (HVY) }\end{array}$ & $\begin{array}{r}\text { \%h-swF4 } \\
\text { (LIGHT) }\end{array}$ & $\begin{array}{r}\text { \%h-swF5 } \\
\text { (TRIMOD) }\end{array}$ & $\%$ mass \\
\hline 1 winter & 0.39 & 0.39 & 0.07 & 0.06 & 0.09 & 1.00 \\
\hline 2 winter & 0.44 & 0.45 & 0.10 & 0.00 & 0.01 & 1.00 \\
\hline 3 winter & 0.24 & 0.48 & 0.00 & 0.22 & 0.05 & 1.00 \\
\hline 4 winter & 0.08 & 0.09 & 0.14 & 0.30 & 0.38 & 1.00 \\
\hline 5 winter & 0.19 & 0.44 & 0.17 & 0.11 & 0.09 & 1.00 \\
\hline 6 winter & 0.01 & 0.12 & 0.11 & 0.50 & 0.25 & 1.00 \\
\hline 7 winter & 0.26 & 0.41 & 0.16 & 0.17 & 0.01 & 1.00 \\
\hline 8 summer & 0.08 & 0.20 & 0.10 & 0.58 & 0.04 & 1.00 \\
\hline 9 summer & 0.06 & 0.18 & 0.03 & 0.51 & 0.22 & 1.00 \\
\hline 10 summer & 0.19 & 0.36 & 0.05 & 0.40 & 0.00 & 1.00 \\
\hline 11 summer & 0.08 & 0.41 & 0.00 & 0.50 & 0.00 & 1.00 \\
\hline 12 summer & 0.12 & 0.33 & 0.01 & 0.48 & 0.07 & 1.00 \\
\hline 13 summer & 0.00 & 0.22 & 0.06 & 0.60 & 0.12 & 1.00 \\
\hline 14 summer & 0.08 & 0.41 & 0.06 & 0.44 & 0.00 & 1.00 \\
\hline 15 summer & 0.30 & 0.15 & 0.11 & 0.34 & 0.11 & 1.00 \\
\hline 16 summer & 0.00 & 0.29 & 0.07 & 0.58 & 0.06 & 1.00 \\
\hline 17 winter & 0.08 & 0.40 & 0.06 & 0.33 & 0.13 & 1.00 \\
\hline 18 winter & 0.39 & 0.06 & 0.15 & 0.27 & 0.14 & 1.00 \\
\hline 19 winter & 0.09 & 0.67 & 0.04 & 0.04 & 0.16 & 1.00 \\
\hline 20 winter & 0.00 & 0.49 & 0.09 & 0.29 & 0.13 & 1.00 \\
\hline 21 winter & 0.15 & 0.49 & 0.01 & 0.19 & 0.15 & 1.00 \\
\hline 22 winter & 0.06 & 0.31 & 0.03 & 0.36 & 0.25 & 1.00 \\
\hline 23 winter & 0.32 & 0.09 & 0.13 & 0.22 & 0.24 & 1.00 \\
\hline 24 winter & 0.29 & 0.19 & 0.12 & 0.20 & 0.19 & 1.00 \\
\hline$\% F$ median_sw & $11 \%$ & $34 \%$ & $7 \%$ & $31 \%$ & $11 \%$ & 0.95 \\
\hline$\% \bar{F} \_$median_w & $19 \%$ & $40 \%$ & $10 \%$ & $22 \%$ & $14 \%$ & 1.05 \\
\hline$\% \bar{F}$ median_s & $8 \%$ & $29 \%$ & $6 \%$ & $50 \%$ & $6 \%$ & 0.99 \\
\hline diff, $w-S$ & $11 \%$ & $12 \%$ & $4 \%$ & $29 \%$ & $7 \%$ & \\
\hline $\min$ & $0 \%$ & $6 \%$ & $0 \%$ & $\overline{0 \%}$ & $0 \%$ & \\
\hline $\max$ & $44 \%$ & $67 \%$ & $17 \%$ & $60 \%$ & $38 \%$ & \\
\hline average & $16 \%$ & $32 \%$ & $8 \%$ & $32 \%$ & $12 \%$ & \\
\hline
\end{tabular}




\section{D.4 Detailed results for intraurban source apportionment via PMF-CMB}

As presented in Chapter 5, intraurban source apportionment was completed using a combined PMF-CMB model: CMB input PR was derived from a 4-factor PMF solution of local central site time series data (i.e., 4th model order for species suite compatibility with field data; 2001-2010 time series, year-round data, 13 species) and input AD was the winter campaign intraurban field data (i.e., 14-day sample, 13 species).

Model performance of the PMF model used to generate local source profiles is summarized in Table D.4-1 and PMF output (i.e., bootstrap factor concentration and explained variance profiles; BOOT_C, BOOT_EV) is presented in Figure D.4-1.

CMB model performance metrics are presented as summary statistics and tabulated by site in Table D.4-2 and mapped across the study area in Figure D.4-2. Uncertainty on model source apportionment results is qualified via T-stat metrics and aggregated by site in Table D.4-3.

Fractional source contribution results (SCE fractions) to $\Sigma_{13} \mathrm{PAH}$ and $\Sigma_{13} \mathrm{BaP}-\mathrm{TEQ}$ are tabulated by site in Table D.4-4 and mapped in Figure D.4-3 ( $\left.\Sigma_{13} \mathrm{PAH}\right)$ and Figure D.4-4 $\left(\Sigma_{13} \mathrm{BaP}-\mathrm{TEQ}\right)$.

Source contribution results (SCE) and associated uncertainty (UNC) to $\Sigma_{13} \mathrm{PAH}$ and $\Sigma_{13} \mathrm{BaP}-\mathrm{TEQ}$ are presented in Table D.4-5 (summary statistics, tabulated by site); additional uncertainty metadata on the SCE is presented in Table D.4-6 (summary statistics, tabulated by site).

Finally, source contribution results (SCE) to $\Sigma_{13} \mathrm{PAH}$ and associated uncertainties are mapped in Figure D.4-5 (i.e., stacked bar chart at each site). 
$\underline{\text { PMF model performance and factor profiles }}$

The PMF model used to generate local source profiles for the intraurban source apportionment via CMB met 'best practice' requirements of a good solution (i.e., stable, good fit, robust, rotationally unique; US EPA, 2004; Reff et al, 2007). The PMF solution is summarized in Table D.4-1.

For continuity and clarity of the PMF-CMB method, the PMF model output used to construct the $\mathrm{CMB}$ input source profiles (i.e., bootstrap factor concentration and explained variance profiles; BOOT_C, BOOT_EV) is presented in Figure D.4-1.

Table D.4-1. PMF model performance summary for solution used to generate local source profiles for paired CMB intraurban source apportionment model.

\begin{tabular}{|c|c|}
\hline INPUT \& CFG & h0110sw-4p \\
\hline Filename & h0110sw-sec-o123-4p-fieldsuite.cfg \\
\hline $\mathrm{n}$, outliers & $\begin{array}{l}\text { n_0110sw }=200, \text { n_out }=21 \text { ( } 11 \% \text { exclusion) } \\
\text { n_model }=179 \text { (i.e., o123 }+2 \text { very poor fit dates) }\end{array}$ \\
\hline $\mathrm{S}$ & SvarEC \\
\hline C3 & $0 \%$ \\
\hline species - bad & $\begin{array}{l}\text { ACY, MFLU, medium (MPYR, BAFLU, BBFLU, BGFLT), TRIP, RET, } \\
\text { BEP (i.e., limited to CMB AD field suite; } 13 \text { fitting species) }\end{array}$ \\
\hline species - weak & none \\
\hline \multicolumn{2}{|l|}{ STABILITY } \\
\hline Q_theory & $2327\left(n^{\star} m\right), 1611$ (Sonoma) \\
\hline Q_rob (range) & $1755(0.03)+9 \%$ Qtheory \\
\hline Q_true (range) & $1755(<0.01)$ \\
\hline SUM_d^2_max & $<0.01$ \\
\hline \multicolumn{2}{|l|}{ FIT } \\
\hline$r^{\wedge} 2$ & majority $>0.8 ;$ FLU r2=0.77 \\
\hline$\#$ res $>|3|$ & 6 \\
\hline high res species $\left(r^{\wedge} 2, \#\right.$ res $)$ & $\mathrm{BAA}, \mathrm{r} 2=0.93,3$ hi res \\
\hline \multicolumn{2}{|l|}{ G-space } \\
\hline edge? & good fill in all $F$ pairs \\
\hline \multicolumn{2}{|l|}{ ROBUST } \\
\hline bootstrapping & $98 / 100 / 94 / 99 / 1 U$ \\
\hline shape unmapped? & none (FLU, PHEN) \\
\hline notes & excellent bootstrap (mapping, narrow IQR, base run IQR median) \\
\hline \multicolumn{2}{|l|}{ FACTORS } \\
\hline label & $\begin{array}{c}\text { HEAVY }(\sim 15 \% \mathrm{G}) \\
\text { LIGHT }(\sim 38 \% \mathrm{G}) \\
\text { TRIMODAL }(\sim 18 \% \mathrm{G}) \\
\text { VOLATILE }(\sim 29 \% \mathrm{G})\end{array}$ \\
\hline year trend? & no trend \\
\hline season trend? & $\begin{array}{l}\text { LIGHT > summer; VOL > winter } \\
\text { no seasonal trend: HVY, TRIMOD }\end{array}$ \\
\hline weekday/weekend trend? & $\begin{array}{l}\text { mild trend but noted difference between HVY and other factors: } \\
\text { HVY > weekday } \\
\text { LIGHT, TRIMOD, VOL > weekend }\end{array}$ \\
\hline
\end{tabular}



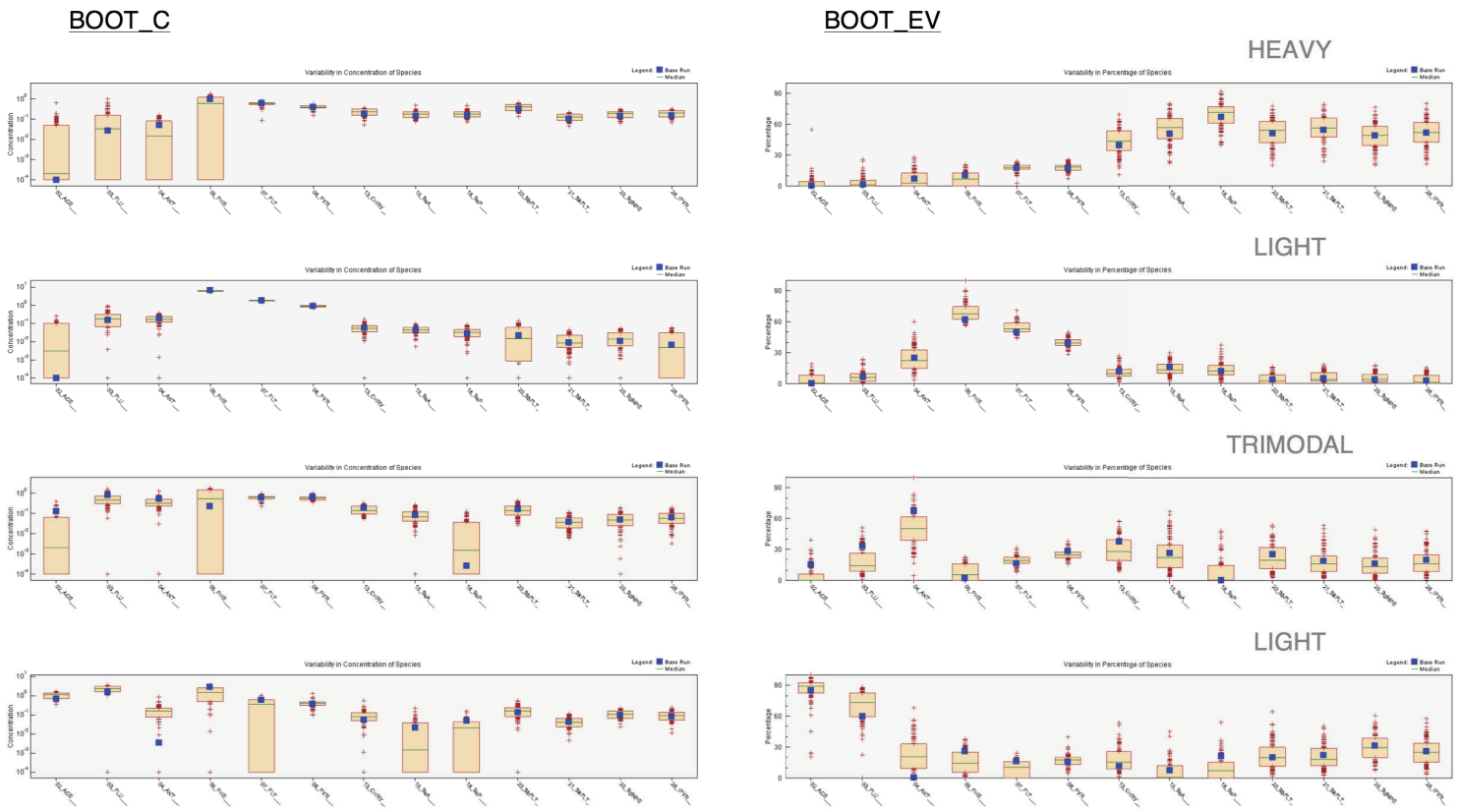

Figure D.4-1. PMF bootstrap factor profiles (concentration, explained variance) used to construct local profiles used in CMB modeling. 
CMB model performance for intraurban field data

CMB model fit (i.e., $\mathrm{r}^{2}, \%$ mass, $\mathrm{chi}^{2}$ ) was good at the majority of sites, both above and below escarpment (i.e., statistical summary and results tabulated by site in Table D.4-2 and mapped in Figure D.4-2). Uncertainty on the SCE for each modeled factor is qualified via T-stat metrics and aggregated by site in Figure D.4-3.

Values for the $\mathrm{r}^{2}$ metric were within CMB guideline targets ( $>0.8$; US EPA, 2004) at all sites, with a median value of 0.90 (all sites) and little intraurban variation (CV $<0.1$ all sites, lower sites, upper sites) although fit was generally better above the escarpment (median, upper $=0.93$; lower $=0.89$ ). Values for the $\%$ mass metric were also within guideline targets (80\% - 120\%; US EPA, 2004) at all sites, and were generally similar above and below the escarpment (median, all sites $=93 \%$; upper $=92 \%$; lower $=93 \%$ ).

Values for the chi ${ }^{2}$ metric were within CMB guideline targets ( $<2$; US EPA, 2004) at most sites (median, all sites, 0.99). However, relative to the more consistent $r^{2}$ and \%mass goodness of fit measures, the $\mathrm{chi}^{2}$ metric showed a higher degree of intraurban variability (CV, 0.6 overall, $\sim 0.4$ lower, upper) and a number of sites showed poorer fit (i.e., 8 sites with chi $\left.^{2}>3\right)$; these occurred where the highest ambient PAH concentrations $\left(\sum_{16} \mathrm{PAH}\right)$ were sampled, which tended to be below the escarpment. Given the dense intraurban sampling network used in the field study, localized high concentrations reasonably indicate local source influence, corroborated by the proximity of these sites to known local sources (Anastasopoulos et al, 2012). At sites where chi ${ }^{2}$ was particularly high (i.e., chi $^{2}>3$ ), review of the individual species concentrations calculated by the model (i.e., RES/UNC by species) showed majority of the fitting species to be modeled reasonably well, with relatively poorer fit for heavy $\mathrm{MW}$ species $\mathrm{BbF}$ and $\mathrm{BkF}$ (i.e., $\mathrm{CMB}$ tended to overestimate and underestimate $\mathrm{BbF}$ and $\mathrm{BkF}$, respectively). Poorer model fit for these species was not anticipated by the PMF results (i.e., both characteristic species of "heavy" factor, similarly enriched in bootstrap profiles, similar model species fit) and so was attributed to field sampling data limitations (i.e., only BkF data permitted quantifying intraurban variability since BbF below DL at all sites); despite the difference in $\mathrm{CMB}$ model fit, however, both congener species were retained to maintain overall model power. 
Table D.4-2. Detailed CMB model performance.

\begin{tabular}{|c|c|c|c|c|c|}
\hline & & 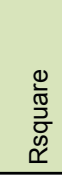 & $\begin{array}{l}\frac{\mathscr{U}}{\mathbb{N}} \\
\vec{D} \\
\frac{\mathscr{O}}{T} \\
\frac{T}{U}\end{array}$ & 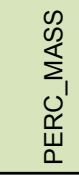 & $\sum_{i}$ \\
\hline \multirow{8}{*}{ 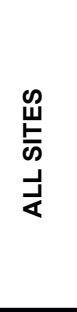 } & $\min$ & 0.85 & 0.50 & 83.9 & 0.73 \\
\hline & mean & 0.90 & 1.97 & 92.9 & 0.90 \\
\hline & $\max$ & 0.96 & 4.39 & 100.8 & 1.22 \\
\hline & Q1 & 0.88 & 1.02 & 91.1 & 0.79 \\
\hline & median & 0.90 & 1.62 & 92.7 & 0.86 \\
\hline & Q3 & 0.92 & 2.68 & 95.3 & 0.97 \\
\hline & SD & 0.03 & 1.19 & 3.7 & 0.13 \\
\hline & $\mathrm{CV}=\mathrm{SD} /$ mean & 0.03 & 0.60 & 0.0 & 0.14 \\
\hline \multirow{8}{*}{ 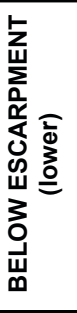 } & $\min$ & 0.85 & 1.00 & 83.9 & 0.73 \\
\hline & mean & 0.89 & 2.66 & 93.4 & 0.82 \\
\hline & $\max$ & 0.94 & 4.39 & 100.8 & 0.97 \\
\hline & Q1 & 0.88 & 1.95 & 91.8 & 0.78 \\
\hline & median & 0.89 & 2.53 & 93.1 & 0.80 \\
\hline & Q3 & 0.90 & 3.59 & 95.6 & 0.84 \\
\hline & SD & 0.02 & 1.08 & 3.8 & 0.06 \\
\hline & $\mathrm{CV}=\mathrm{SD} /$ mean & 0.03 & 0.41 & 0.0 & 0.08 \\
\hline \multirow{8}{*}{ 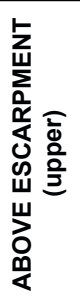 } & $\min$ & 0.89 & 0.50 & 85.4 & 0.82 \\
\hline & mean & 0.93 & 1.03 & 92.2 & 1.00 \\
\hline & $\max$ & 0.96 & 2.05 & 97.6 & 1.22 \\
\hline & Q1 & 0.92 & 0.68 & 91.0 & 0.93 \\
\hline & median & 0.93 & 0.99 & 91.6 & 0.98 \\
\hline & Q3 & 0.94 & 1.14 & 94.9 & 1.07 \\
\hline & SD & 0.02 & 0.45 & 3.7 & 0.12 \\
\hline & $\mathrm{CV}=\mathrm{SD} /$ mean & 0.02 & 0.43 & 0.0 & 0.12 \\
\hline
\end{tabular}

(a) summary statistics 
Table D.4-2 (continued)

\begin{tabular}{|c|c|c|c|c|c|c|c|}
\hline & ADwPR & sw & & & & & \\
\hline 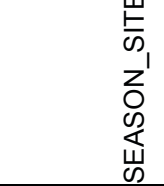 & 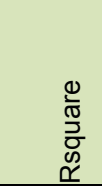 & 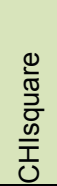 & 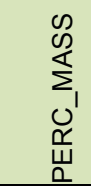 & $\sum_{u}$ & $\begin{array}{l}\frac{\alpha}{3} \\
\frac{\alpha}{\alpha} \\
\frac{\alpha}{\rho}\end{array}$ & 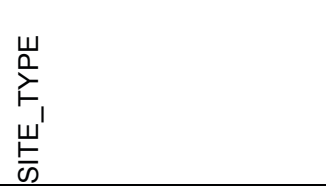 & 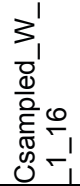 \\
\hline sw_1785 & 0.86 & 4.1 & 92.7 & 0.76 & Iwr & municipal_fire/EMS & 45.4 \\
\hline sw_1838 & 0.89 & 2.0 & 91.9 & 0.83 & Iwr & municipal_library & 18.6 \\
\hline sw_1850_A & 0.88 & 3.7 & 90.0 & 0.76 & Iwr & municipal_heritage & 39.5 \\
\hline w_1850_B & 0.87 & 4.2 & 91.8 & 0.76 & Iwr & municipal_cemetery & 46.0 \\
\hline sw_1858_A & 0.89 & 3.0 & 91.9 & 0.78 & Iwr & municipal_library & 34.6 \\
\hline sw_1858_B & 0.88 & 3.4 & 94.1 & 0.78 & Iwr & private_home & 39.4 \\
\hline sw_1922 & 0.85 & 4.4 & 83.9 & 0.73 & Iwr & municipal_other & 52.1 \\
\hline sw_1987_A & 0.91 & 2.1 & 93.2 & 0.83 & Iwr & institutional_U & 21.2 \\
\hline w_1987_B & 0.89 & 1.9 & 95.7 & 0.84 & Iwr & municipal_nursing home & 19.3 \\
\hline sw_1998 & 0.89 & 2.5 & 93.1 & 0.80 & Iwr & NAPS & 28.3 \\
\hline sw_2006 & 0.89 & 1.5 & 88.8 & 0.86 & Iwr & municipal_library & 19.0 \\
\hline sw_2070 & 0.94 & 1.0 & 95.4 & 0.97 & Iwr & municipal_other & 12.2 \\
\hline sw_2147 & 0.90 & 2.0 & 97.6 & 0.84 & Iwr & municipal_other & 27.8 \\
\hline sw_2478 & 0.91 & 1.3 & 96.7 & 0.91 & Iwr & community_church & 16.3 \\
\hline sw_2236 & 0.85 & 2.4 & 91.0 & 0.80 & Iwr-east & municipal_fire/EMS & 24.3 \\
\hline sw_2402 & 0.88 & 3.1 & 95.3 & 0.79 & Iwr-east & community_church & 25.0 \\
\hline sw_2408 & 0.86 & 3.9 & 98.5 & 0.78 & Iwr-east & municipal_fire/EMS & 33.4 \\
\hline sw_2575 & 0.88 & 2.7 & 92.1 & 0.79 & Iwr-east & municipal_heritage & 25.5 \\
\hline sw_2662 & 0.92 & 1.2 & 100.8 & 0.94 & Iwr-east & community_church & 12.0 \\
\hline sw_1704 & 0.94 & 1.0 & 95.1 & 0.97 & upr & municipal_library & 13.8 \\
\hline sw_2296 & 0.92 & 1.0 & 95.2 & 0.98 & upr & municipal_fire/EMS & 15.6 \\
\hline sw_2306 & 0.93 & 0.8 & 91.8 & 1.02 & upr & private_home & 13.5 \\
\hline sw_2460 & 0.95 & 0.7 & 91.1 & 1.10 & upr & private_home & 12.5 \\
\hline sw_2556 & 0.92 & 1.5 & 97.6 & 0.89 & upr & municipal_library & 16.5 \\
\hline sw_2646 & 0.92 & 1.1 & 93.0 & 0.93 & upr & community_church & 14.6 \\
\hline sw_2654 & 0.96 & 0.5 & 91.0 & 1.22 & upr & municipal_nursing home & 8.3 \\
\hline sw_2744 & 0.89 & 2.1 & 91.4 & 0.82 & upr & private_home & 19.1 \\
\hline sw_2819_A & 0.92 & 1.1 & 90.6 & 0.93 & upr & private_home & 14.2 \\
\hline sw_2819_B & 0.94 & 0.8 & 85.4 & 1.02 & upr & private_home & 10.3 \\
\hline sw_2841 & 0.95 & 0.5 & 91.2 & 1.21 & upr & private_home & 15.3 \\
\hline sw_2936 & 0.92 & 1.1 & 97.4 & 0.95 & upr & municipal_fire/EMS & 16.5 \\
\hline sw_3105_B & 0.94 & 0.7 & 85.5 & 1.08 & upr & private_home & 10.4 \\
\hline sw_3208 & 0.90 & 1.6 & 94.1 & 0.87 & upr & municipal_fire/EMS & 16.7 \\
\hline
\end{tabular}

(a) by site 
Figure D.4-2. Maps of CMB goodness of fit.

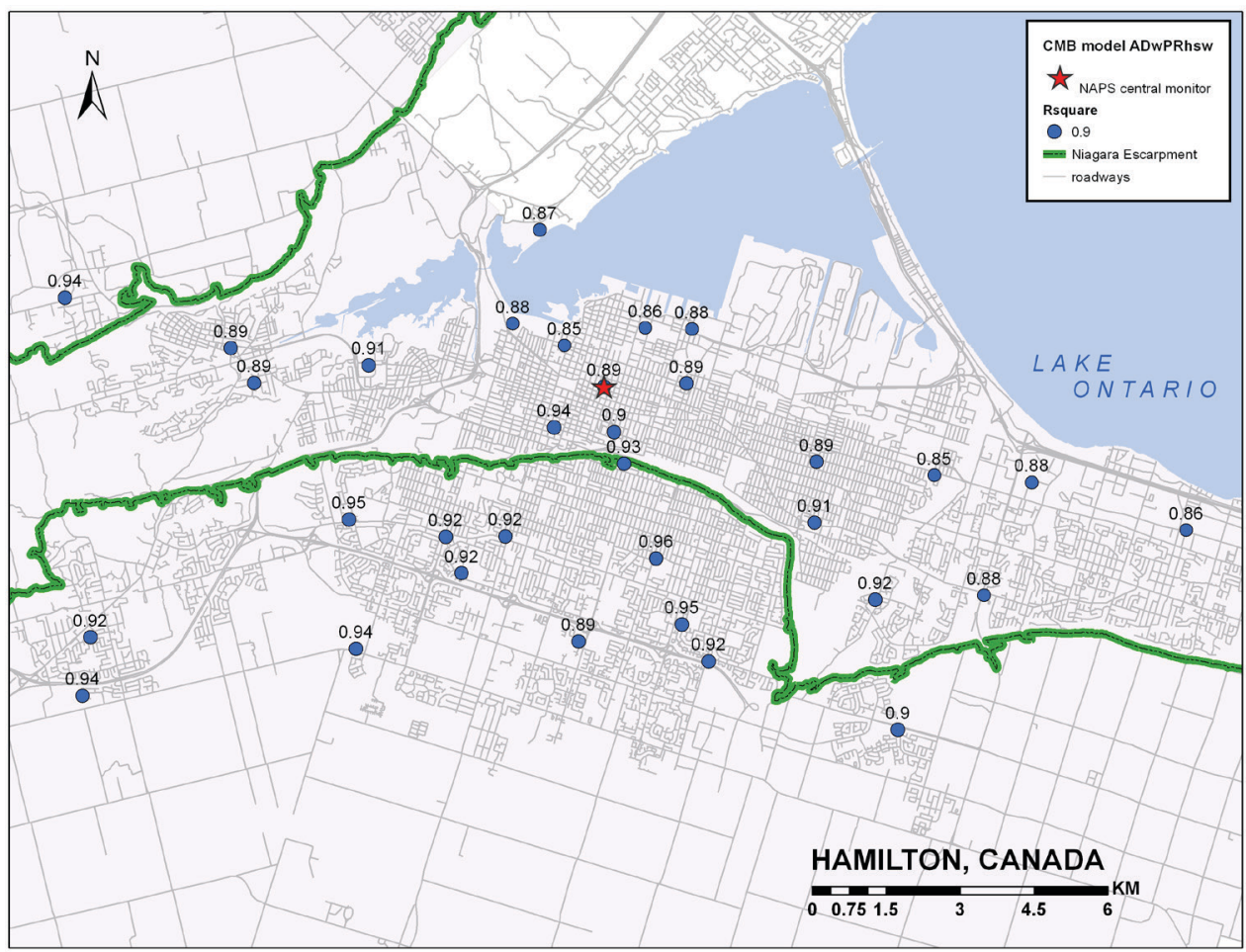

(a) $R^{2}$

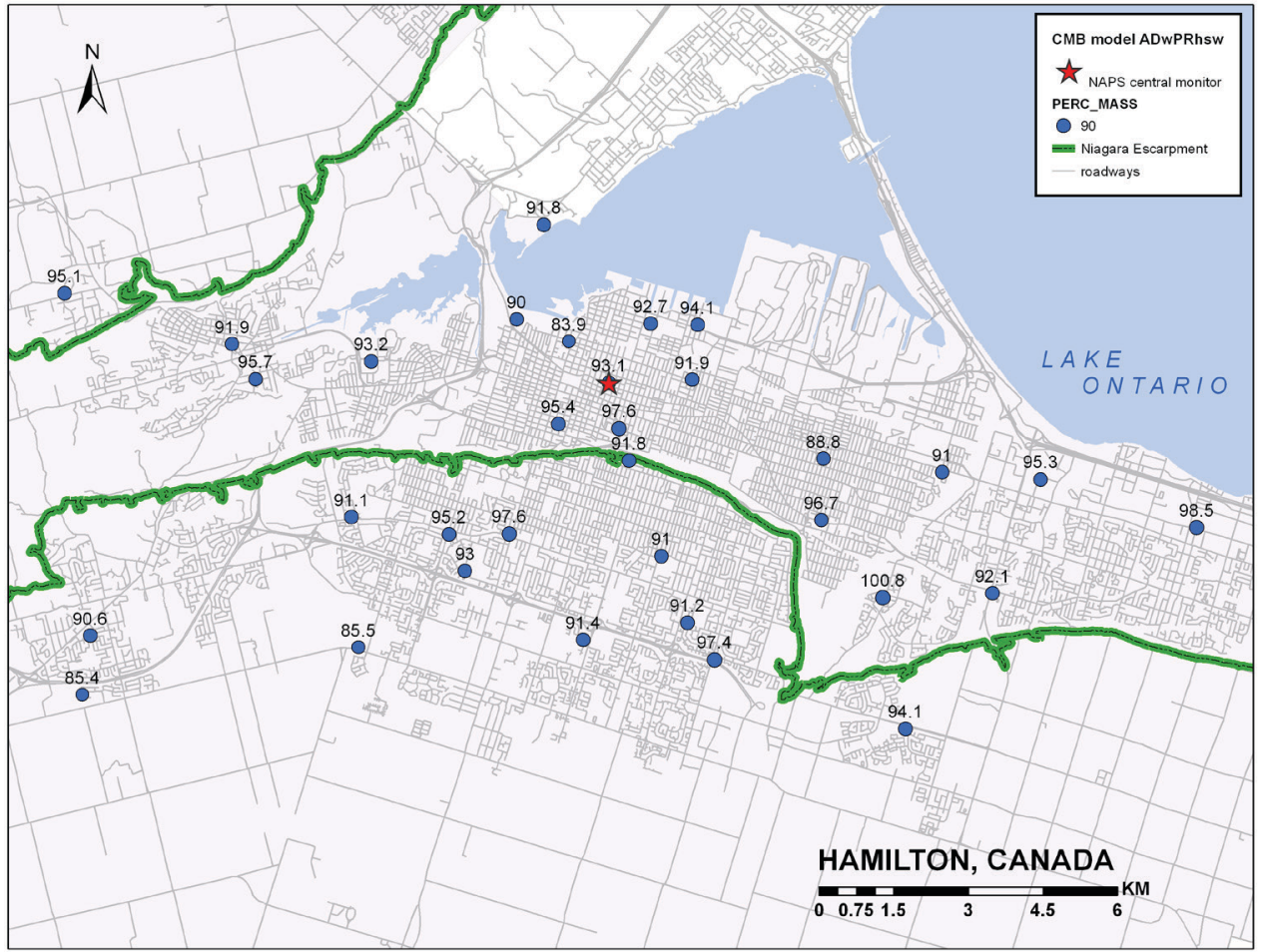

(b) $\%$ mass 


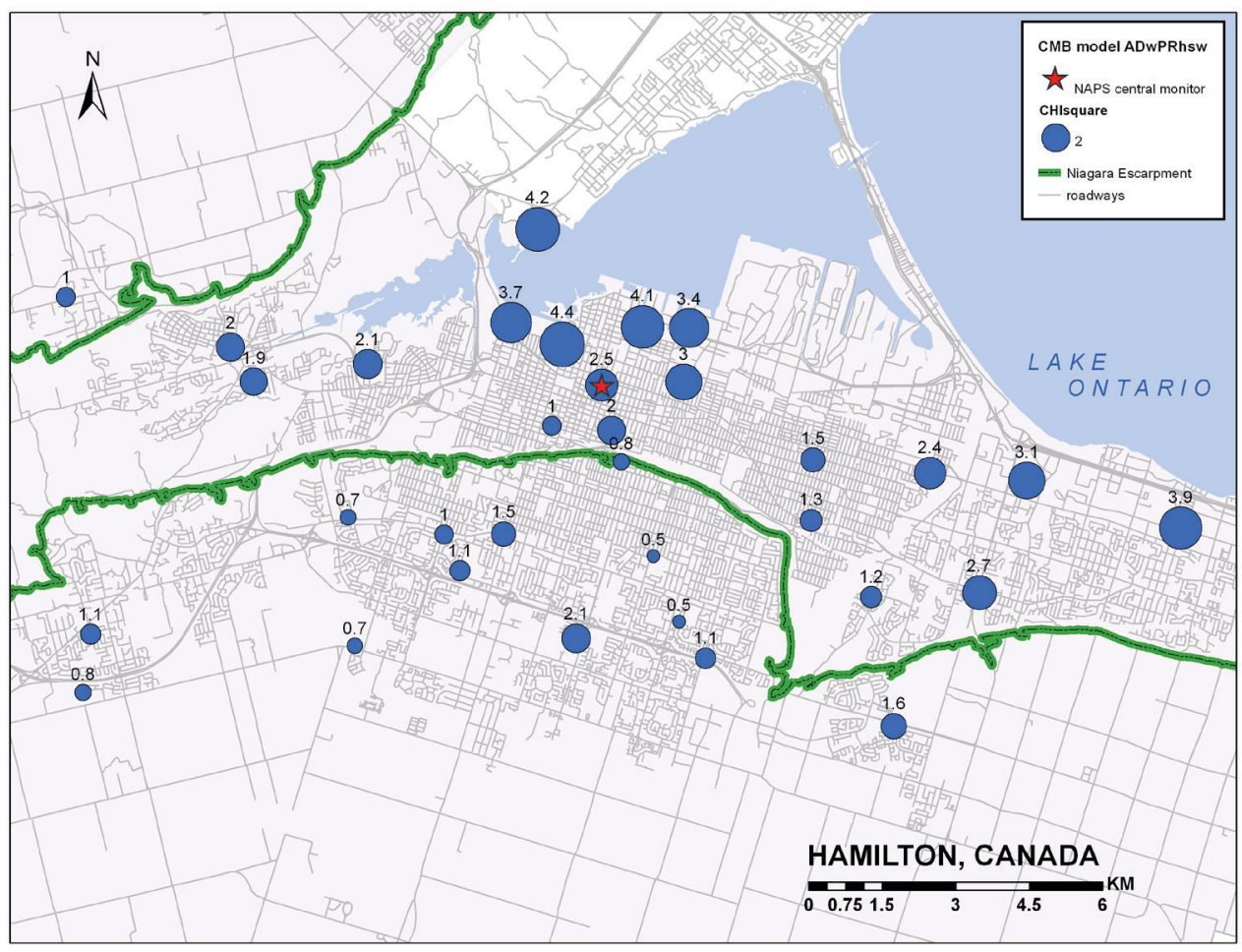

(c) $\mathrm{CHI}^{2}$

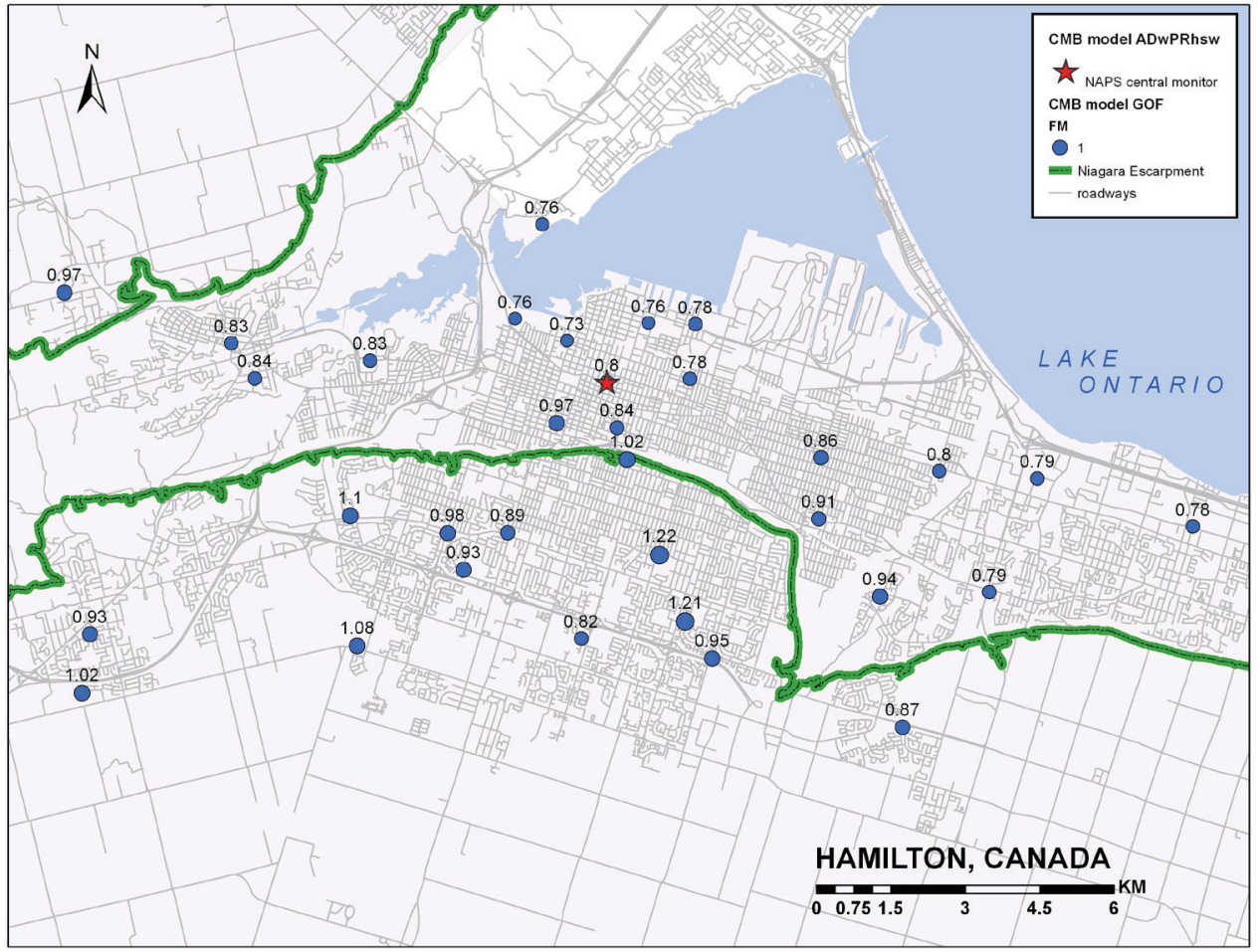

(d) aggregate fit measure (FM) 
Table D.4-3. T-stat classification; by site.

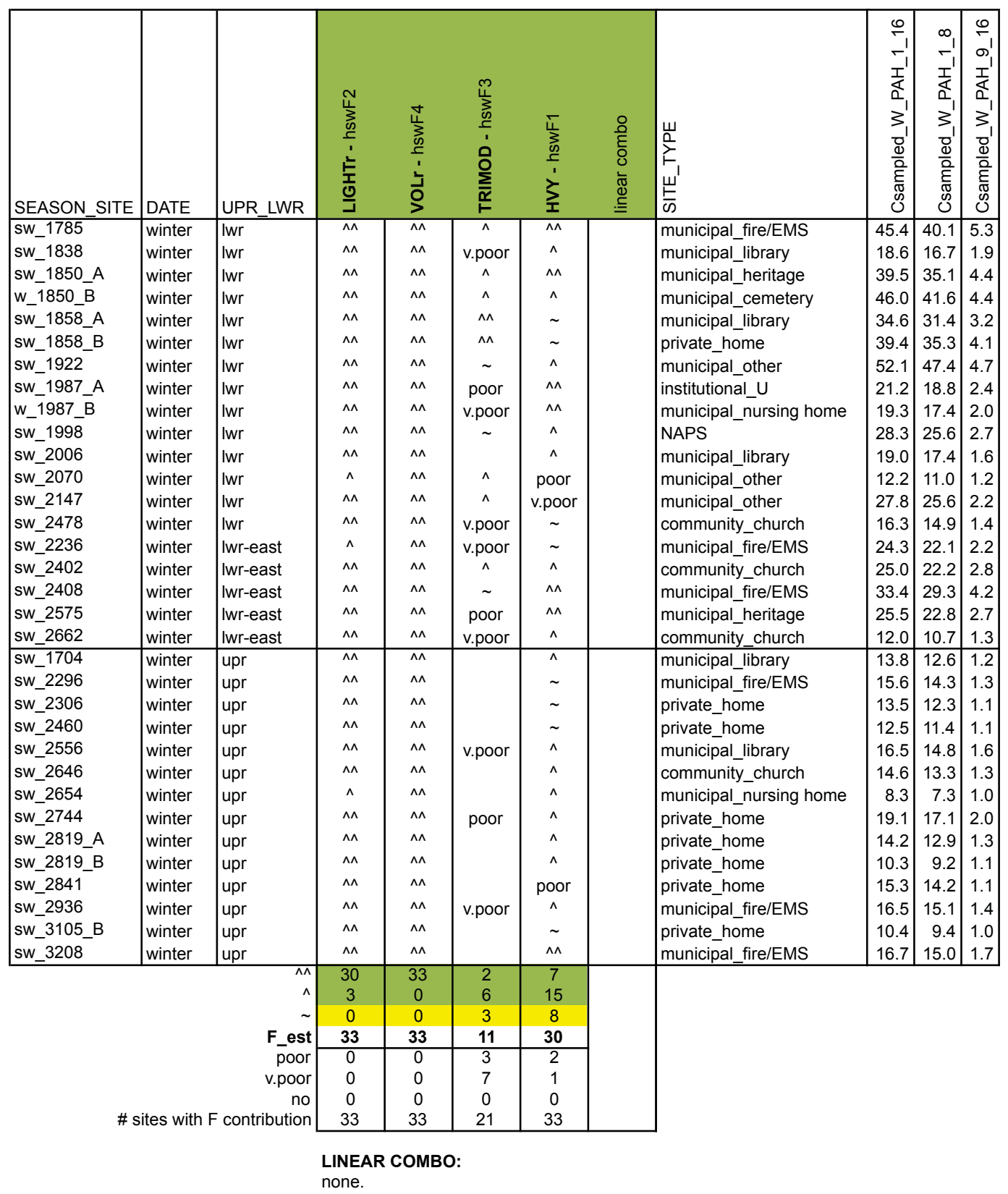

Legend

T-stat $>2.0\left({ }^{\wedge \wedge}\right), 1.5-2.0(\wedge), 1.0-1.5(\sim), 0.5-1.0$ (poor), $<0.5$ (very poor). 
CMB source apportionment results by site

Fractional source contribution results (SCE fractions) to $\Sigma_{13} \mathrm{PAH}$ and $\Sigma_{13} \mathrm{BaP}-\mathrm{TEQ}$ are tabulated by site in Table D.4-4 and mapped in Figure D.4-3 and Figure D.4-4.

SCE and associated uncertainty (UNC) to $\Sigma_{13} \mathrm{PAH}$ and $\Sigma_{13} \mathrm{BaP}$-TEQ are presented in Table D.4-5 (summary statistics, tabulated by site); additional uncertainty metadata on the SCE is presented in Table D.4-6 (summary statistics, tabulated by site). SCE to $\Sigma_{13} \mathrm{PAH}$ and associated uncertainties are mapped in Figure D.4-5.

Table D.4-4. Fractional source contribution (SCE fractions) to $\Sigma$ PAH and BaP-TEQ; by site.

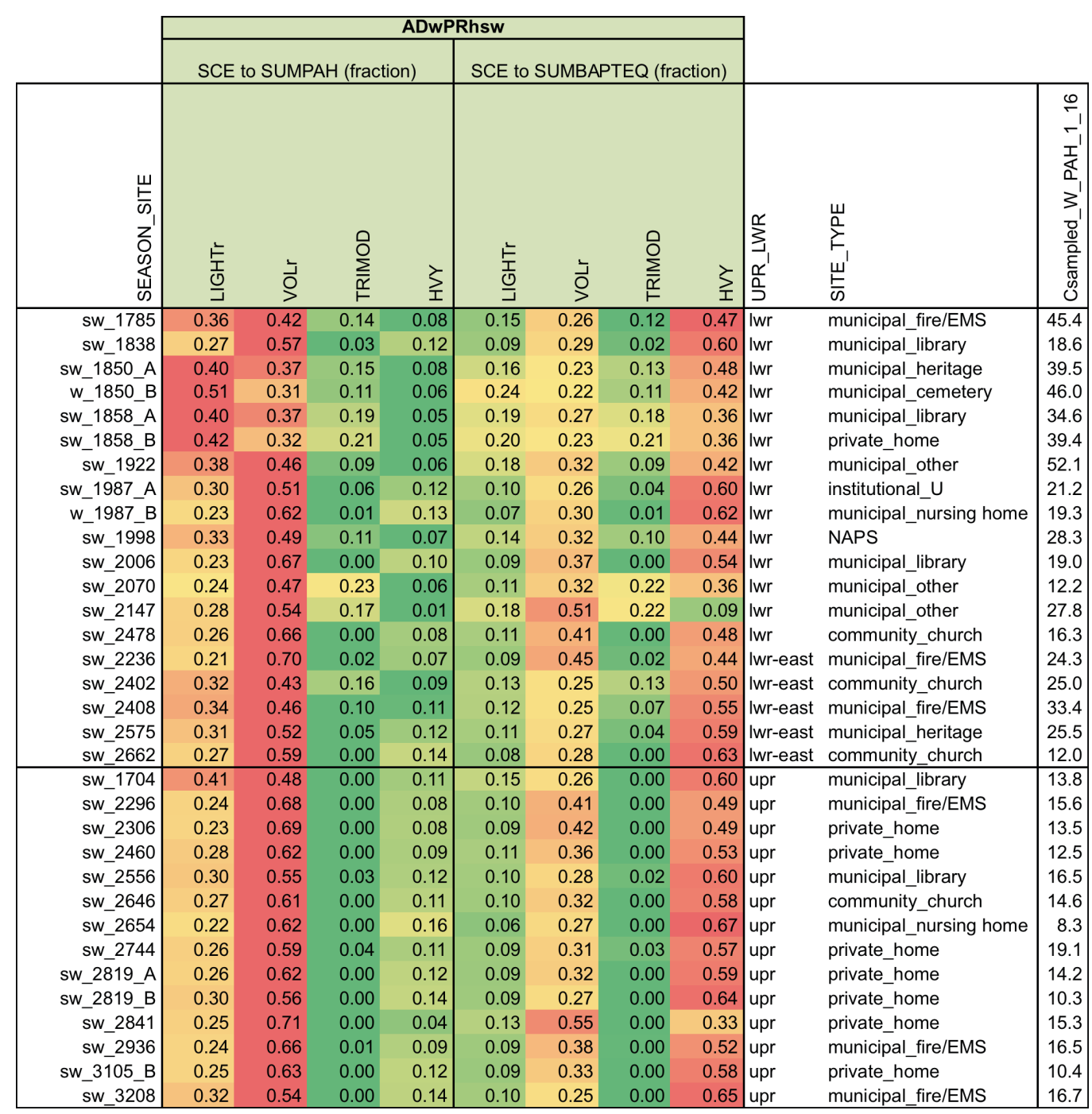

Note: Shading denotes highest SCE fraction (red) to lowest SCE fraction (green). 
(a) $L I G H T r$

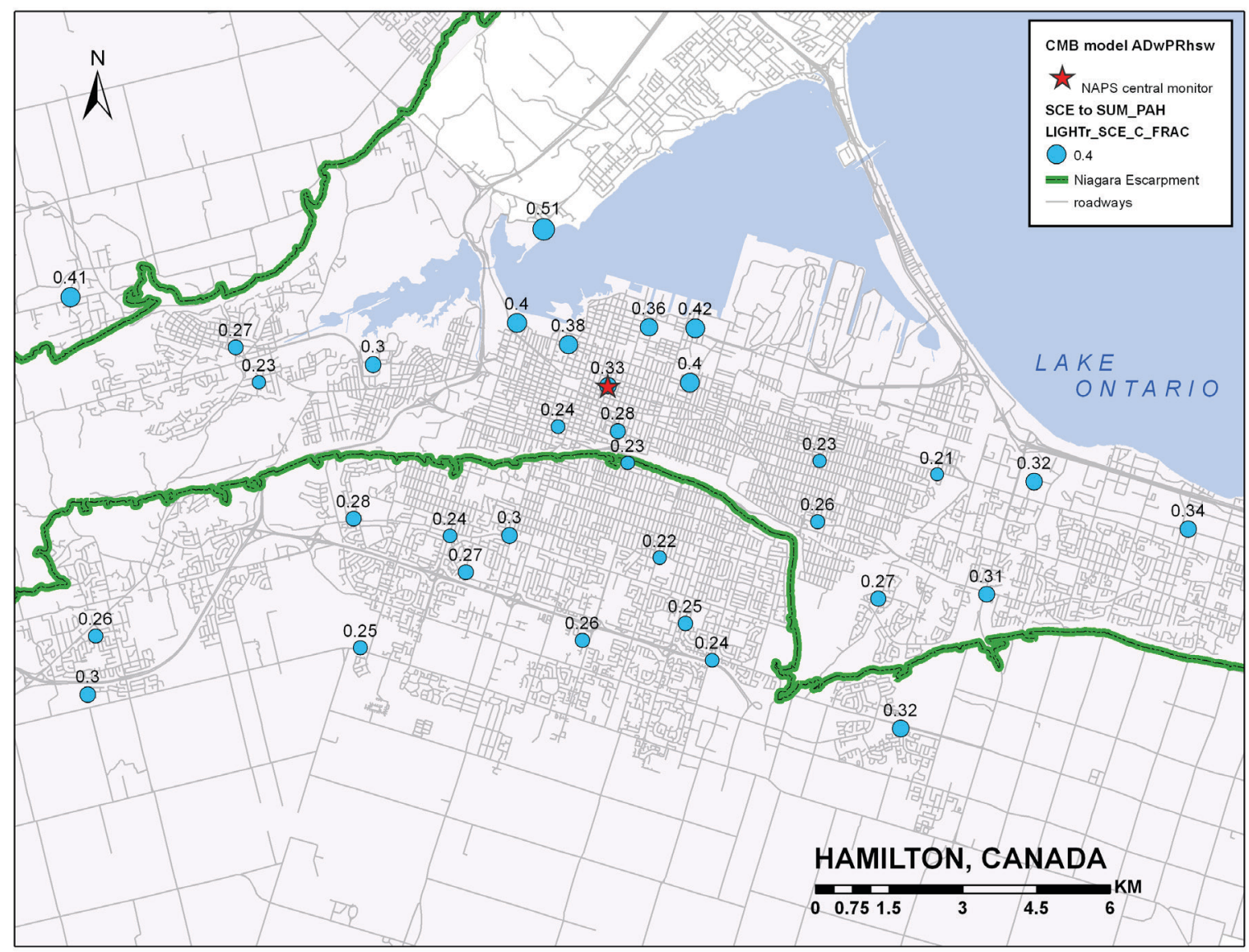

Note: Labels show factor contribution (SCE) as fraction of total modeled mass.

Figure D.4-3. Map of fractional source contributions (SCE fractions) to $\Sigma$ PAH. 
(b) $V O L r$

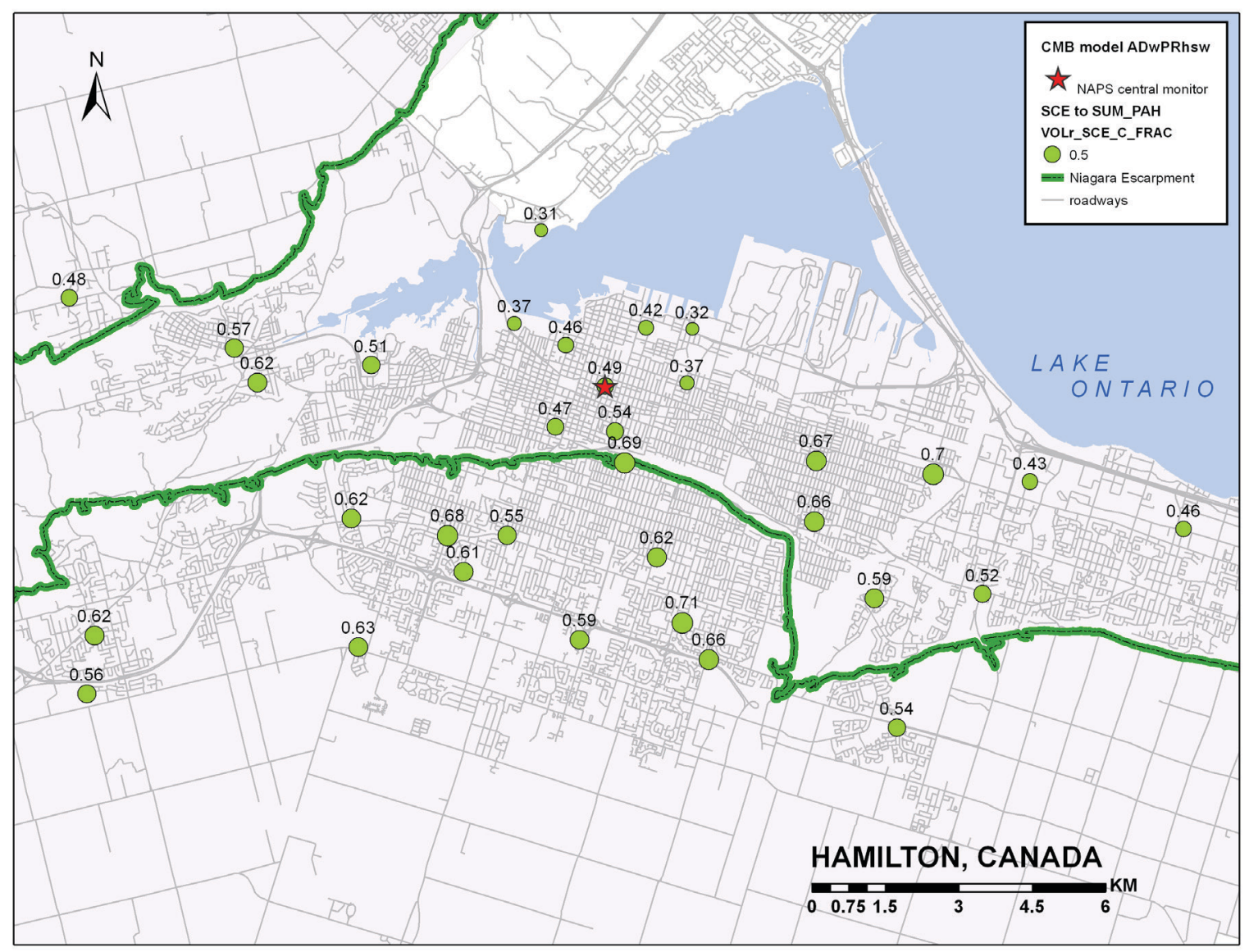

Note: Labels show factor contribution (SCE) as fraction of total modeled mass.

Figure D.4-3 (continued) 
(c) TRIMODAL

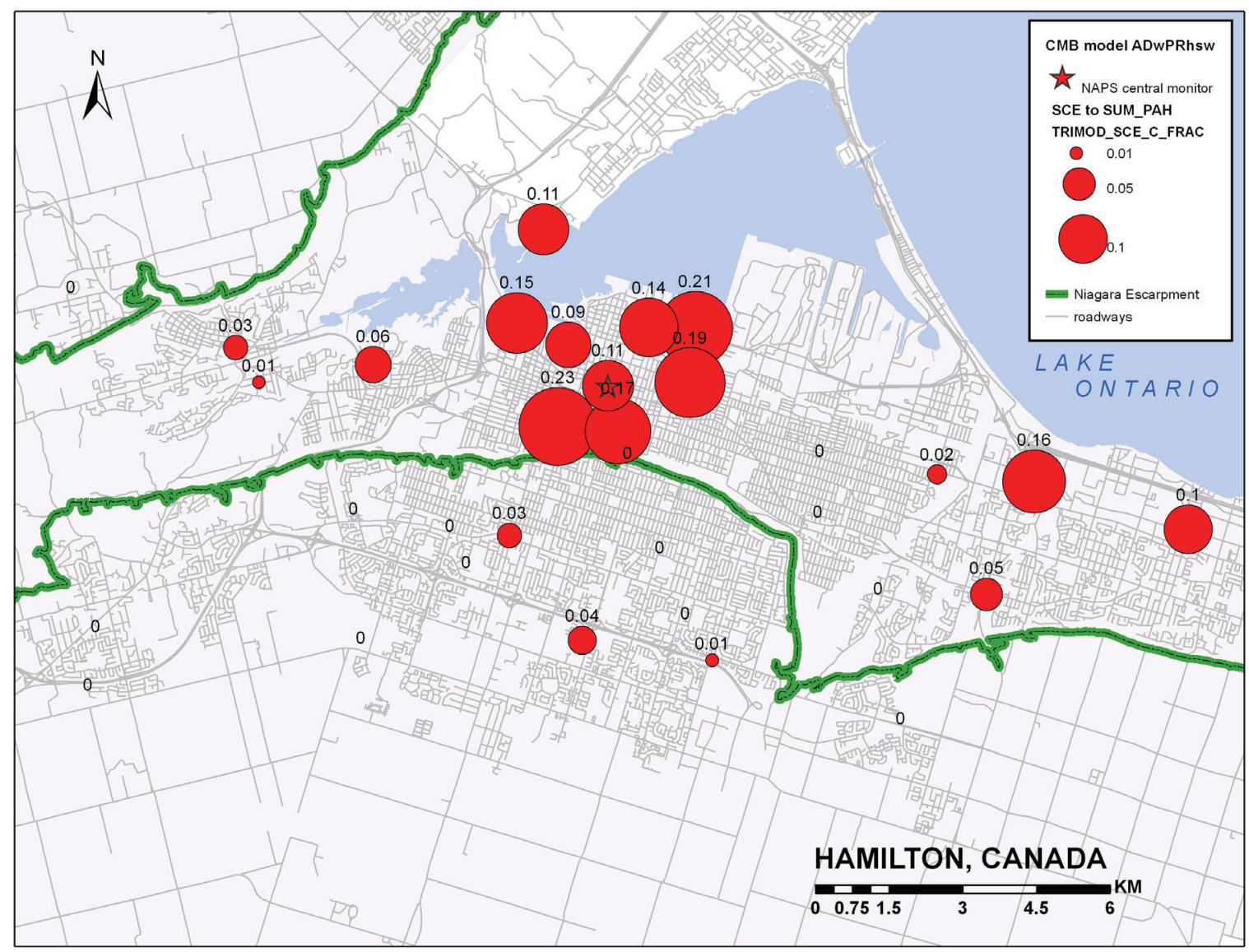

Note: Labels show factor contribution (SCE) as fraction of total modeled mass.

Figure D.4-3 (continued) 
(d) $H V Y$

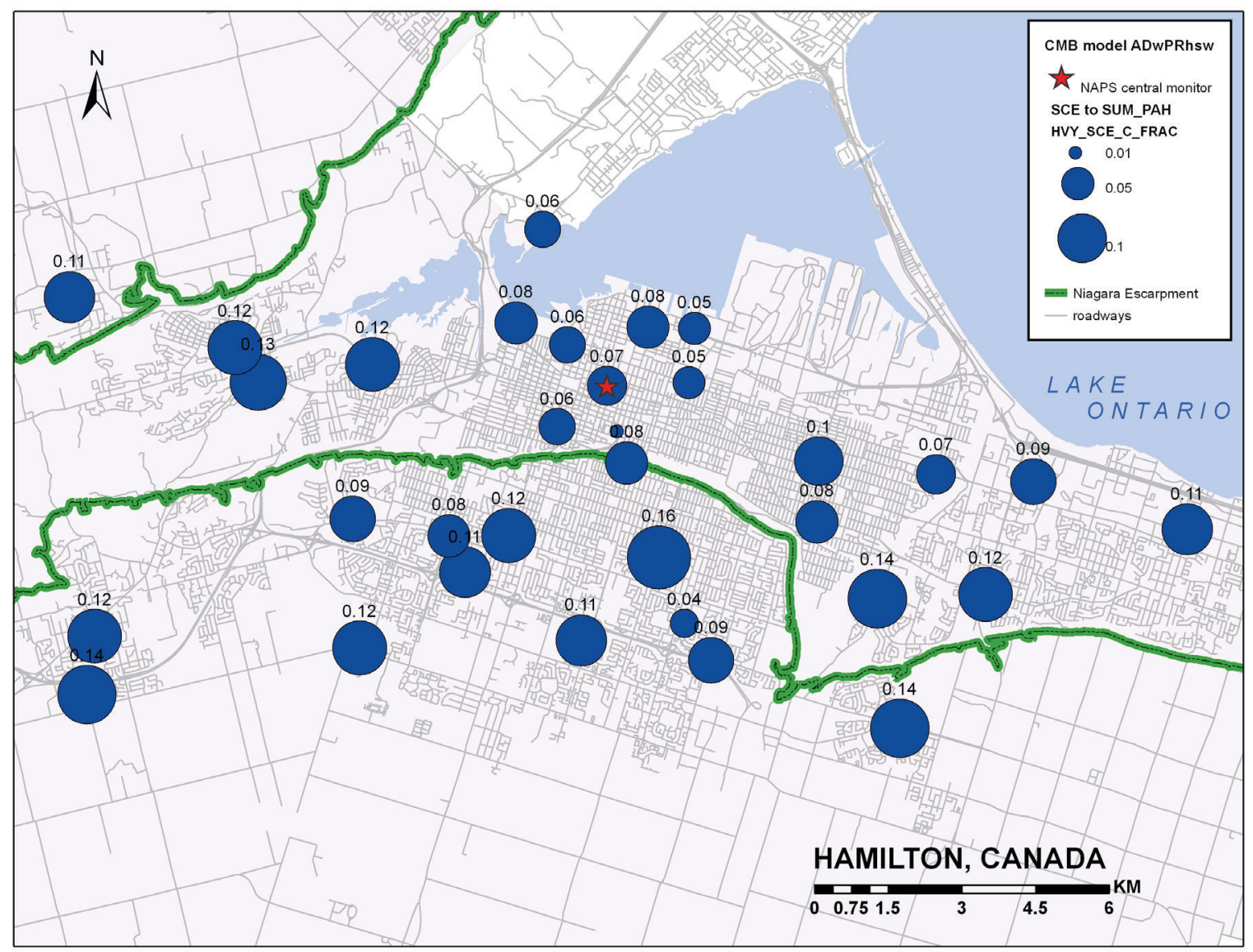

Note: Labels show factor contribution (SCE) as fraction of total modeled mass

Figure D.4-3 (continued) 
(a) $L I G H T r$

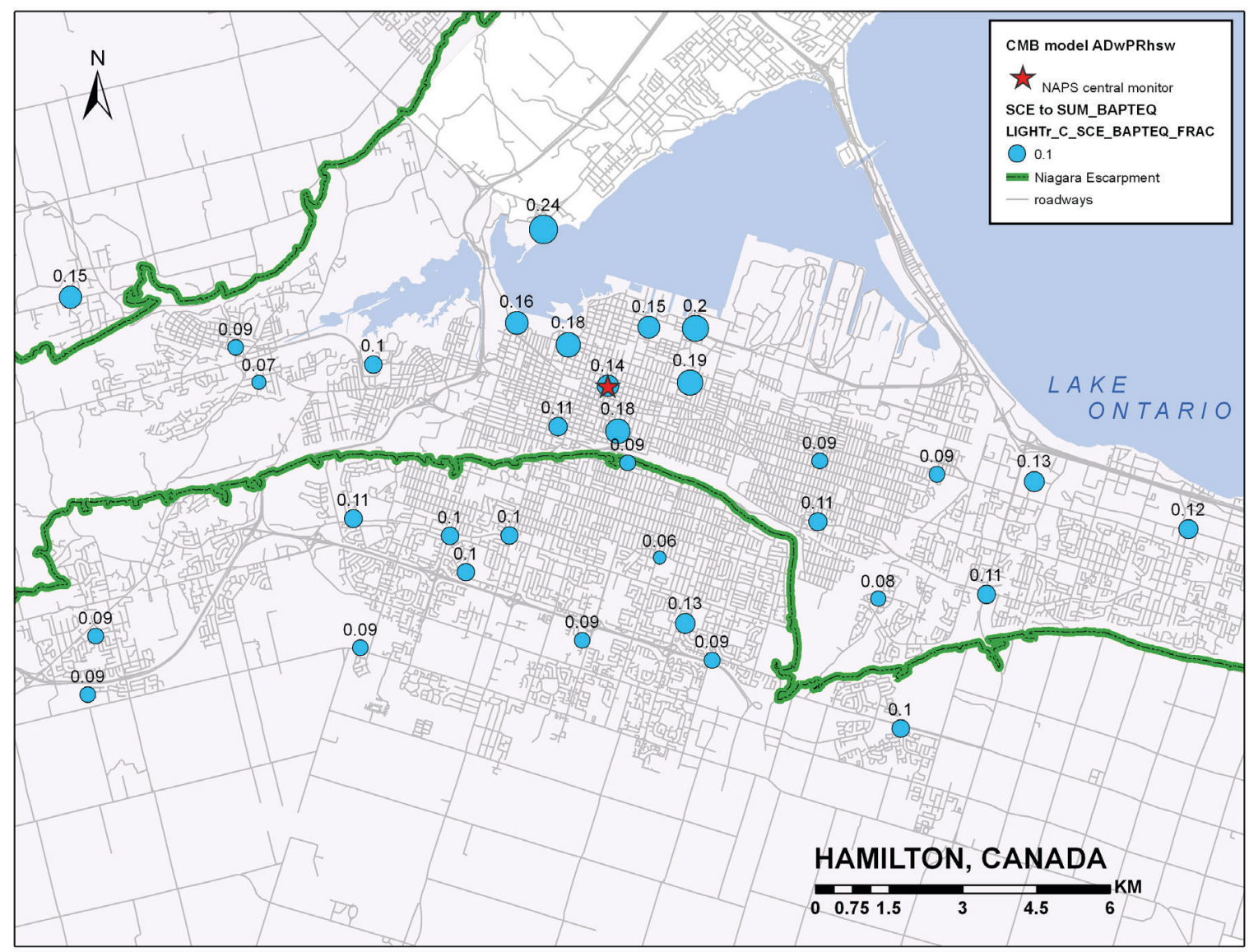

Note: Labels show factor contribution (SCE) as fraction of total modeled mass

Figure D.4-4. Map of fractional source contributions (SCE fractions) to BaP-TEQ. 
(b) $\mathrm{VOLr}$

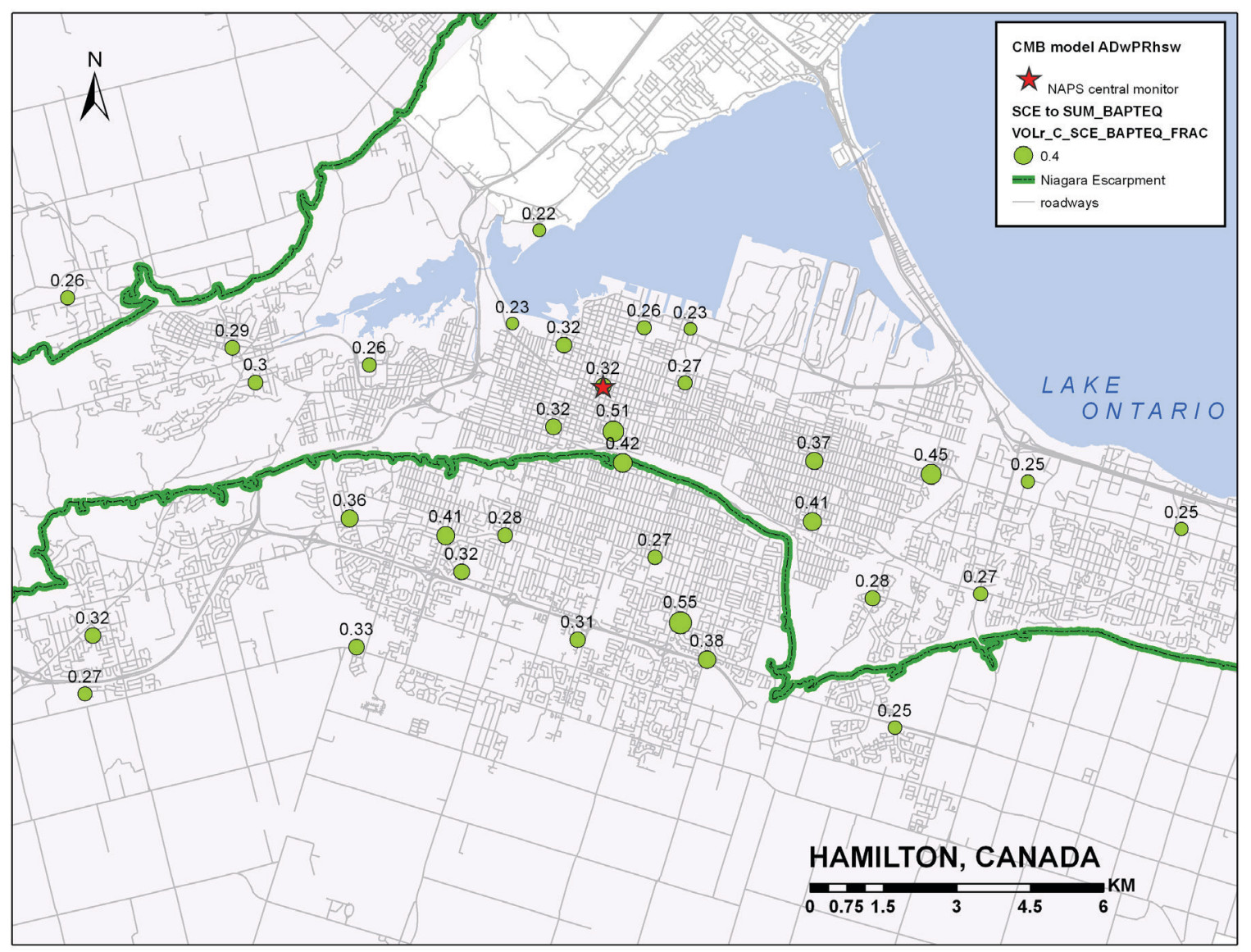

Note: Labels show factor contribution (SCE) as fraction of total modeled mass.

Figure D.4-4 (continued) 
(c) TRIMODAL

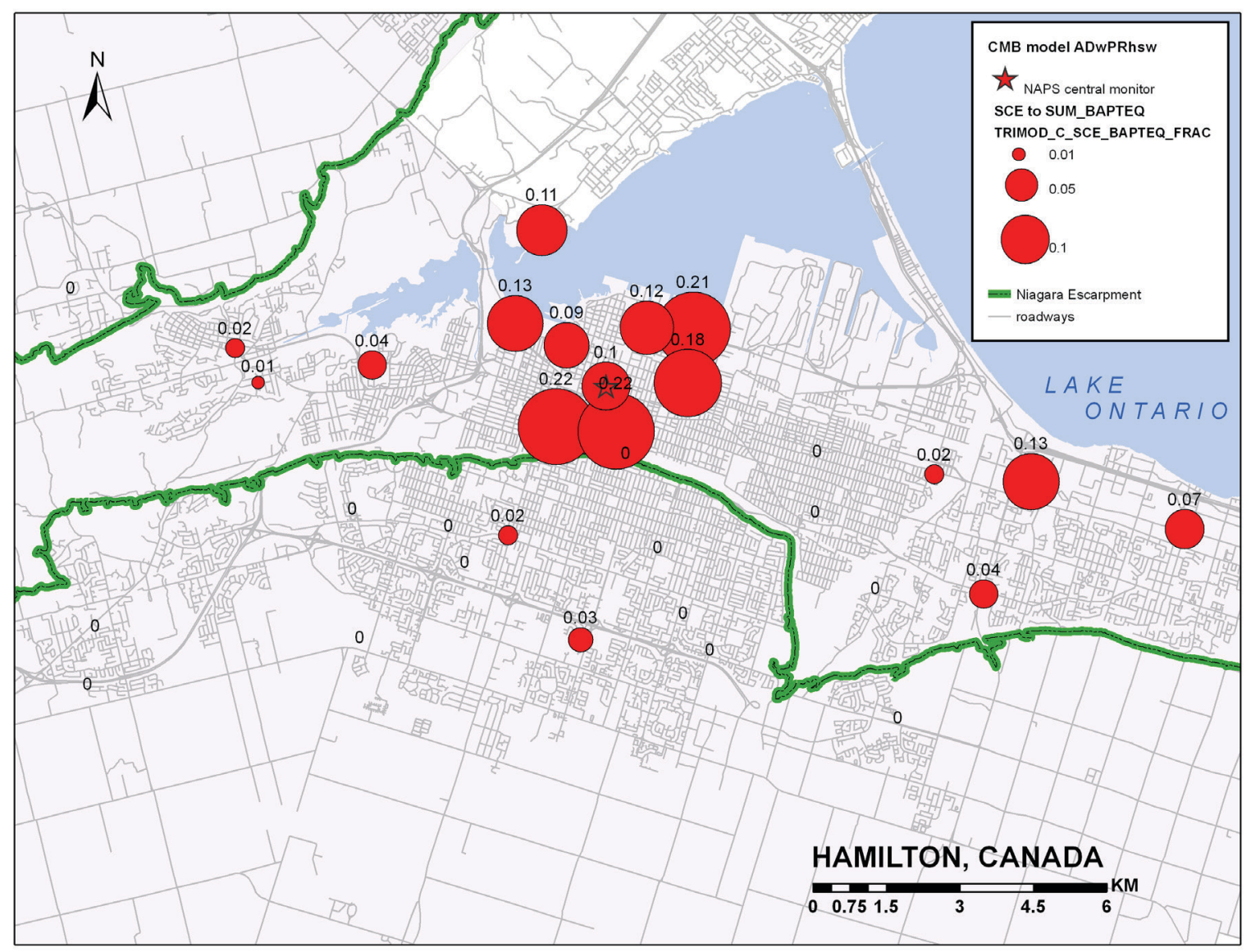

Note: Labels show factor contribution (SCE) as fraction of total modeled mass.

Figure D.4-4 (continued) 
(d) $H V Y$

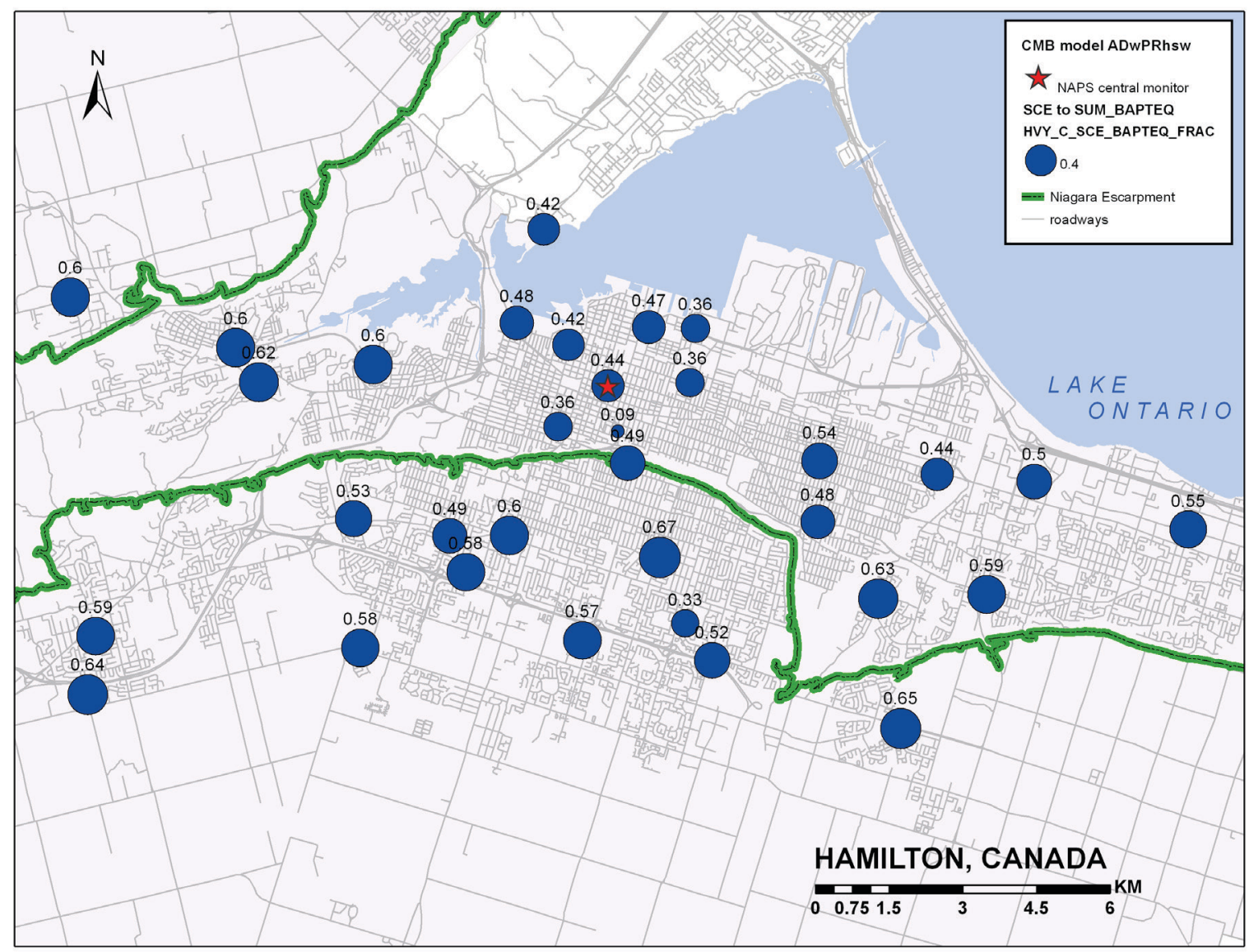

Note: Labels show factor contribution (SCE) as fraction of total modeled mass.

Figure D.4-4 (continued) 
Table D.4-5. Source contributions (SCE) and uncertainty (UNC) to $\Sigma$ PAH and BaP-TEQ.

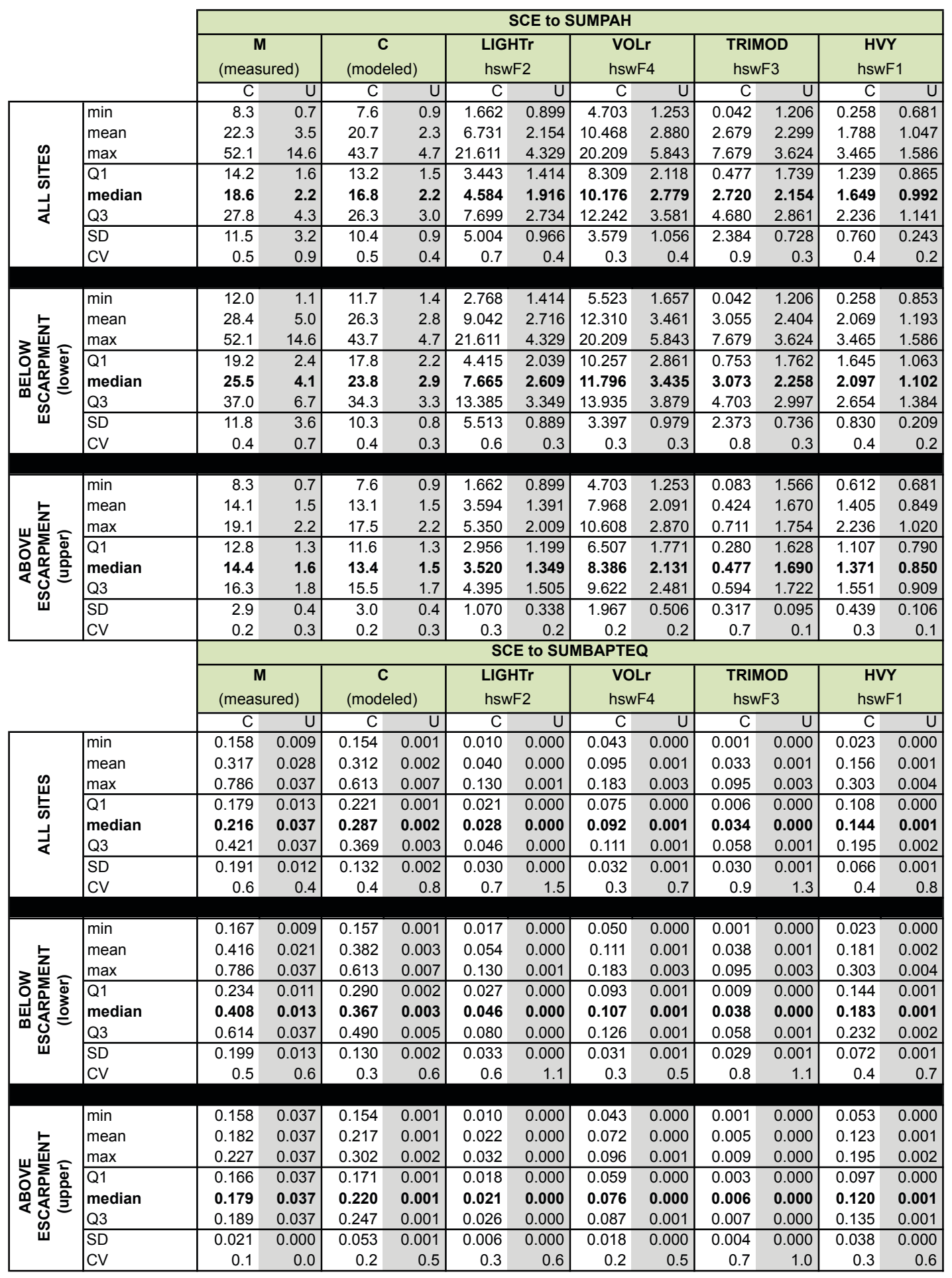

(a) summary statistics. 
Table D.4-5 (continued)

\begin{tabular}{|c|c|c|c|c|c|c|c|c|c|c|c|c|c|c|c|c|c|}
\hline \multirow{3}{*}{ 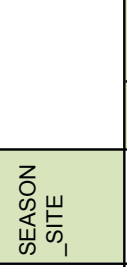 } & \multicolumn{12}{|c|}{ SCE to SUMPAH } & \multirow{3}{*}{$\begin{array}{l}\frac{\alpha}{3} \\
\frac{\alpha}{\alpha} \\
\frac{\alpha}{\partial}\end{array}$} & \multirow[b]{3}{*}{ SITE_TYPE } & \multirow{3}{*}{ 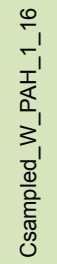 } & \multirow{3}{*}{$\begin{array}{l}\infty_{1} \\
\frac{1}{1} \\
\frac{T}{\sigma} \\
0 \\
3 \\
3 \\
0 \\
\frac{0}{0} \\
\frac{E}{\pi} \\
0 \\
0\end{array}$} & \multirow{3}{*}{ 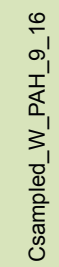 } \\
\hline & \multicolumn{2}{|c|}{$\begin{array}{c}\mathbf{M} \\
\text { (measured) }\end{array}$} & \multicolumn{2}{|c|}{$\begin{array}{c}\mathbf{C} \\
\text { (modeled) }\end{array}$} & \multicolumn{2}{|c|}{$\begin{array}{l}\text { LIGHTr } \\
\text { hswF2 }\end{array}$} & \multicolumn{2}{|c|}{$\begin{array}{l}\text { VOLr } \\
\text { hswF4 }\end{array}$} & \multicolumn{2}{|c|}{$\begin{array}{l}\text { TRIMOD } \\
\text { hswF3 }\end{array}$} & \multicolumn{2}{|c|}{$\begin{array}{c}\text { HVY } \\
\text { hswF1 }\end{array}$} & & & & & \\
\hline & $\mathrm{C}$ & $u$ & C & U & C & U & C & $\mathrm{U}$ & C & $\mathrm{u}$ & C & $u$ & & & & & \\
\hline sw_1785 & 45.4 & 9.9 & 42.1 & 4.2 & 15.263 & 4.140 & 17.463 & 5.108 & 6.041 & 3.585 & 3.302 & 1.586 & Iwr & municipal_fire/EMS & 45.4 & 40.1 & 5.3 \\
\hline sw_1838 & 18.6 & 2.2 & 17.1 & 2.2 & 4.584 & 1.966 & 9.798 & 2.767 & 0.595 & 1.751 & 2.108 & 1.071 & Iwr & municipal_library & 18.6 & 16.7 & 1.9 \\
\hline sw_1850_A & 39.5 & 7.9 & 35.6 & 3.4 & 14.160 & 3.500 & 13.314 & 4.022 & 5.251 & 3.043 & 2.845 & 1.384 & Iwr & municipal_heritage & 39.5 & 35.1 & 4.4 \\
\hline w_1850_B & 46.0 & 10.2 & 42.2 & 3.5 & 21.611 & 3.776 & 13.280 & 3.902 & 4.711 & 3.088 & 2.590 & 1.385 & Iwr & municipal_cemetery & 46.0 & 41.6 & 4.4 \\
\hline sw_1858_A & 34.6 & 6.1 & 31.8 & 3.0 & 12.610 & 3.197 & 11.662 & 3.410 & 5.921 & 2.861 & 1.642 & 1.295 & Iwr & municipal_library & 34.6 & 31.4 & 3.2 \\
\hline sw_1858_B & 39.4 & 7.3 & 37.1 & 3.3 & 15.748 & 3.610 & 11.796 & 3.749 & 7.679 & 3.258 & 1.891 & 1.421 & Iwr & private_home & 39.4 & 35.3 & 4.1 \\
\hline sw_1922 & 52.1 & 14.6 & 43.7 & 4.7 & 16.691 & 4.329 & 20.209 & 5.843 & 4.089 & 3.624 & 2.717 & 1.520 & Iwr & municipal_other & 52.1 & 47.4 & 4.7 \\
\hline sw_1987_A & 21.2 & 2.6 & 19.8 & 2.3 & 5.921 & 2.128 & 10.115 & 2.850 & 1.282 & 1.795 & 2.460 & 0.986 & Iwr & institutionāal_U & 21.2 & 18.8 & 2.4 \\
\hline w_1987_B & 19.3 & 2.4 & 18.5 & 2.4 & 4.246 & 2.112 & 11.528 & 3.181 & 0.252 & 1.739 & 2.495 & 0.983 & Iwr & municipal_nursing home & 19.3 & 17.4 & 2.0 \\
\hline sw_1998 & 28.3 & 4.6 & 26.3 & 2.9 & 8.638 & 2.734 & 12.947 & 3.581 & 2.877 & 2.306 & 1.856 & 1.102 & Iwr & NAPS & 28.3 & 25.6 & 2.7 \\
\hline sw_2006 & 19.0 & 2.5 & 16.8 & 1.9 & 3.868 & 1.665 & 11.288 & 2.676 & & & 1.691 & 0.853 & Iwr & municipal_library & 19.0 & 17.4 & 1.6 \\
\hline sw_2070 & 12.2 & 1.1 & 11.7 & 1.4 & 2.768 & 1.442 & 5.523 & 1.657 & 2.720 & 1.483 & 0.645 & 1.056 & Iwr & municipal_other & 12.2 & 11.0 & 1.2 \\
\hline sw_2147 & 27.8 & 4.3 & 27.2 & 3.1 & 7.665 & 3.039 & 14.556 & 3.802 & 4.680 & 2.819 & 0.258 & 1.390 & Iwr & municipal_other & 27.8 & 25.6 & 2.2 \\
\hline sw_2478 & 16.3 & 1.8 & 15.7 & 2.2 & 4.093 & 1.907 & 10.338 & 2.873 & 0.071 & 1.696 & 1.245 & 1.097 & Iwr & community_church & 16.3 & 14.9 & 1.4 \\
\hline sw_2236 & 24.3 & 4.1 & 22.1 & 3.0 & 4.674 & 2.609 & 15.401 & 3.856 & 0.459 & 2.209 & 1.579 & 1.141 & Iwr-east & municipal_fire/EMS & 24.3 & 22.1 & 2.2 \\
\hline sw_2402 & 25.0 & 3.2 & 23.8 & 2.4 & 7.699 & 2.432 & 10.176 & 2.905 & 3.829 & 2.154 & 2.097 & 1.097 & Iwr-east & community_church & 25.0 & 22.2 & 2.8 \\
\hline sw_2408 & 33.4 & 5.1 & 33.0 & 3.4 & 11.147 & 3.160 & 15.070 & 4.119 & 3.269 & 2.637 & 3.465 & 1.293 & Iwr-east & municipal_fire/EMS & 33.4 & 29.3 & 4.2 \\
\hline sw_2575 & 25.5 & 3.9 & 23.5 & 2.7 & 7.202 & 2.447 & 12.242 & 3.435 & 1.226 & 2.017 & 2.780 & 1.075 & Iwr-east & municipal_heritage & 25.5 & 22.8 & 2.7 \\
\hline sw_2662 & 12.0 & 1.1 & 12.1 & 1.6 & 3.213 & 1.414 & 7.190 & 2.014 & 0.042 & 1.206 & 1.649 & 0.942 & Iwr-east & community_church & 12.0 & 10.7 & 1.3 \\
\hline sw_1704 & 13.8 & 1.4 & 13.2 & 1.3 & 5.350 & 1.241 & 6.305 & 1.751 & & & 1.505 & 0.805 & upr & municipal_library & 13.8 & 12.6 & 1.2 \\
\hline sw_2296 & 15.6 & 1.8 & 14.9 & 1.7 & 3.598 & 1.518 & 10.050 & 2.491 & & & 1.223 & 0.918 & upr & municipal_fire/EMS & 15.6 & 14.3 & 1.3 \\
\hline sw_2306 & 13.5 & 1.5 & 12.4 & 1.5 & 2.865 & 1.317 & 8.463 & 2.144 & & & 1.023 & 0.828 & upr & private_home & 13.5 & 12.3 & 1.1 \\
\hline sw_2460 & 12.5 & 1.3 & 11.4 & 1.3 & 3.227 & 1.185 & 7.111 & 1.830 & & & 1.075 & 0.785 & upr & private_home & 12.5 & 11.4 & 1.1 \\
\hline sw_2556 & 16.5 & 1.7 & 16.1 & 2.0 & 4.776 & 1.795 & 8.858 & 2.513 & 0.477 & 1.566 & 1.970 & 0.992 & upr & municipal_library & 16.5 & 14.8 & 1.6 \\
\hline sw_2646 & 14.6 & 1.6 & 13.6 & 1.5 & 3.741 & 1.371 & 8.309 & 2.118 & & & 1.557 & 0.885 & upr & community_church & 14.6 & 13.3 & 1.3 \\
\hline sw_2654 & 8.3 & 0.7 & 7.6 & 0.9 & 1.662 & 0.899 & 4.703 & 1.253 & & & 1.201 & 0.681 & upr & municipal_nursing home & 8.3 & 7.3 & 1.0 \\
\hline sw_2744 & 19.1 & 2.2 & 17.5 & 2.2 & 4.565 & 2.009 & 10.240 & 2.779 & 0.711 & 1.754 & 1.966 & 1.020 & upr & private_home & 19.1 & 17.1 & 2.0 \\
\hline sw_2819_A & 14.2 & 1.6 & 12.8 & 1.4 & 3.368 & 1.328 & 7.949 & 2.046 & & & 1.531 & 0.867 & upr & private_home & 14.2 & 12.9 & 1.3 \\
\hline sw_2819_B & 10.3 & 1.0 & 8.8 & 1.0 & 2.589 & 0.970 & 4.943 & 1.360 & & & 1.239 & 0.710 & upr & private_home & 10.3 & 9.2 & 1.1 \\
\hline Sw_2841 & 15.3 & 1.8 & 13.9 & 1.7 & 3.443 & 1.451 & 9.877 & 2.448 & & & 0.612 & 0.865 & upr & private_home & 15.3 & 14.2 & 1.1 \\
\hline sw_2936 & 16.5 & 1.9 & 16.1 & 2.2 & 3.885 & 1.916 & 10.608 & 2.870 & 0.083 & 1.690 & 1.503 & 0.981 & upr & municipal_fire/EMS & 16.5 & 15.1 & 1.4 \\
\hline sw_3105_B & 10.4 & 1.0 & 8.9 & 1.0 & 2.231 & 1.005 & 5.633 & 1.499 & & & 1.032 & 0.714 & upr & private_home & 10.4 & 9.4 & 1.0 \\
\hline$\overline{\mathrm{Sw}} \_3208$ & 16.7 & 2.0 & 15.8 & 1.6 & 5.018 & 1.466 & 8.505 & 2.177 & & & 2.236 & 0.834 & upr & municipal_fire/EMS & 16.7 & 15.0 & 1.7 \\
\hline
\end{tabular}

Note: Blanks indicate factor not apportioned at site.

(b) SCE, UNC to $\Sigma P A H$ (by site). 
Table D.4-5 (continued)

\begin{tabular}{|c|c|c|c|c|c|c|c|c|c|c|c|c|c|c|c|c|c|}
\hline \multirow{3}{*}{ 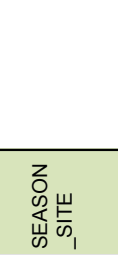 } & \multicolumn{12}{|c|}{ SCE to SUMBAPTEQ } & \multirow{3}{*}{$\begin{array}{l}\frac{\alpha}{3} \\
a^{\prime} \\
\frac{\alpha}{0} \\
\frac{\alpha}{J}\end{array}$} & \multirow[b]{3}{*}{ SITE TYPE } & \multirow{3}{*}{ 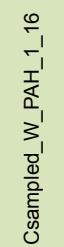 } & \multirow{3}{*}{ 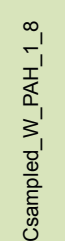 } & \multirow{3}{*}{ 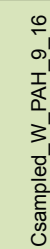 } \\
\hline & \multicolumn{2}{|c|}{$\begin{array}{c}\mathbf{M} \\
\text { (measured) }\end{array}$} & \multicolumn{2}{|c|}{$\begin{array}{c}\mathbf{C} \\
\text { (modeled) }\end{array}$} & \multicolumn{2}{|c|}{$\begin{array}{l}\text { LIGHTr } \\
\text { hswF2 }\end{array}$} & \multicolumn{2}{|c|}{$\begin{array}{c}\text { VOLr } \\
\text { hswF4 } \\
\end{array}$} & \multicolumn{2}{|c|}{$\begin{array}{c}\text { TRIMOD } \\
\text { hswF3 }\end{array}$} & \multicolumn{2}{|c|}{$\begin{array}{c}\text { HVY } \\
\text { hswF1 }\end{array}$} & & & & & \\
\hline & c & $u$ & c & u & c & $u$ & C & $\mathrm{u}$ & c & $\mathrm{u}$ & c & u & & & & & \\
\hline sw_1785 & 0.786 & 0.015 & 0.613 & 0.007 & 0.092 & 0.000 & 0.158 & 0.002 & 0.075 & 0.002 & 0.288 & 0.003 & Iwr & municipal_fire/EMS & 45.4 & 40.1 & 5.3 \\
\hline sw_1838 & 0.227 & 0.037 & 0.308 & 0.002 & 0.028 & 0.000 & 0.089 & 0.001 & 0.007 & 0.000 & 0.184 & 0.001 & Iwr & municipal _library & 18.6 & 16.7 & 1.9 \\
\hline sw_1850_A & 0.643 & 0.013 & 0.519 & 0.005 & 0.085 & 0.000 & 0.120 & 0.001 & 0.065 & 0.001 & 0.248 & 0.003 & Iwr & municipal_heritage & 39.5 & 35.1 & 4.4 \\
\hline W_1850_B & 0.625 & 0.013 & 0.534 & 0.005 & 0.130 & 0.001 & 0.120 & 0.001 & 0.058 & 0.001 & 0.226 & 0.002 & Iwr & municipal_cemetery & 46.0 & 41.6 & 4.4 \\
\hline sw $1858 \mathrm{~A}$ & 0.493 & 0.011 & 0.398 & 0.003 & 0.076 & 0.000 & 0.106 & 0.001 & 0.073 & 0.002 & 0.143 & 0.001 & Iwr & municipal _library & 34.6 & 31.4 & 3.2 \\
\hline sw_1858_B & 0.621 & 0.013 & 0.462 & 0.005 & 0.095 & 0.000 & 0.107 & 0.001 & 0.095 & 0.003 & 0.165 & 0.001 & Iwr & private_home & 39.4 & 35.3 & 4.1 \\
\hline SW_1922 & 0.699 & 0.013 & 0.571 & 0.006 & 0.100 & 0.000 & 0.183 & 0.003 & 0.051 & 0.001 & 0.237 & 0.002 & Iwr & municipal_other & 52.1 & 47.4 & 4.7 \\
\hline sw_1987_A & 0.371 & 0.009 & 0.358 & 0.003 & 0.036 & 0.000 & 0.092 & 0.001 & 0.016 & 0.000 & 0.215 & 0.002 & Iwr & institutional_U & 21.2 & 18.8 & 2.4 \\
\hline w_1987_B & 0.333 & 0.009 & 0.351 & 0.003 & 0.026 & 0.000 & 0.104 & 0.001 & 0.003 & 0.000 & 0.218 & 0.002 & Iwr & municipal_nursing home & 19.3 & 17.4 & 2.0 \\
\hline sw_1998 & 0.408 & 0.010 & 0.367 & 0.003 & 0.052 & 0.000 & 0.117 & 0.001 & 0.036 & 0.000 & 0.162 & 0.001 & iwr & NAPS & 28.3 & 25.6 & 2.7 \\
\hline sw_2006 & 0.205 & 0.037 & 0.273 & 0.002 & 0.023 & 0.000 & 0.102 & 0.001 & & & 0.148 & 0.001 & Iwr & munici & 19.0 & 17.4 & 1.6 \\
\hline sw_2070 & 0.167 & 0.037 & 0.157 & 0.001 & 0.017 & 0.000 & 0.050 & 0.000 & 0.034 & 0.000 & 0.056 & 0.000 & Iwr & municipal_other & 12.2 & 11.0 & 1.2 \\
\hline sw_2147 & 0.241 & 0.037 & 0.258 & 0.002 & 0.046 & 0.000 & 0.132 & 0.001 & 0.058 & 0.001 & 0.023 & 0.000 & Iwr & municipal_other & 27.8 & 25.6 & 2.2 \\
\hline sw_2478 & 0.191 & 0.037 & 0.228 & 0.001 & 0.025 & 0.000 & 0.094 & 0.001 & 0.001 & 0.000 & 0.109 & 0.000 & Iwr & community_church & 16.3 & 14.9 & 1.4 \\
\hline sw_2236 & 0.245 & 0.037 & 0.311 & 0.002 & 0.028 & 0.000 & 0.139 & 0.001 & 0.006 & 0.000 & 0.138 & 0.001 & Iwr-east & municipal_fire/EMS & 24.3 & 22.1 & 2.2 \\
\hline sw_2402 & 0.421 & 0.010 & 0.369 & 0.003 & 0.046 & 0.000 & 0.092 & 0.001 & 0.047 & 0.001 & 0.183 & 0.001 & Iwr-east & community_church & 25.0 & 22.2 & 2.8 \\
\hline sw 2408 & 0.606 & 0.013 & 0.546 & 0.006 & 0.067 & & 0.136 & 0.001 & 0.041 & 0.000 & 0.303 & 0.004 & Iwr-east & municipal fire/EMS & 33.4 & 29.3 & 4.2 \\
\hline sw_2575 & 0.451 & 0.011 & 0.412 & 0.003 & 0.043 & 0.000 & 0.111 & 0.001 & 0.015 & 0.000 & 0.243 & 0.002 & Iwr-east & municipal_heritage & 25.5 & 22.8 & 2.7 \\
\hline sw_2662 & 0.179 & 0.037 & 0.229 & 0.001 & 0.019 & 0.000 & 0.065 & 0.000 & 0.001 & 0.000 & 0.144 & 0.001 & Iwr-east & community_church & 12.0 & 10.7 & 1.3 \\
\hline SW_1704 & 0.178 & 0.037 & 0.221 & 0.001 & 0.032 & 0.000 & 0.057 & 0.000 & & & 0.131 & 0.001 & upr & municipal_library & \begin{tabular}{|l|l|}
13.8 \\
\end{tabular} & 12.6 & 1.2 \\
\hline sw_2296 & 0.183 & 0.037 & 0.219 & 0.001 & 0.022 & 0.000 & 0.091 & 0.001 & & & 0.107 & 0.000 & upr & municipal_fire/EMS & 15.6 & 14.3 & 1.3 \\
\hline sw_2306 & 0.171 & 0.037 & 0.183 & 0.001 & 0.017 & 0.000 & 0.077 & 0.000 & & & 0.089 & 0.000 & upr & private_home & 13.5 & 12.3 & 1.1 \\
\hline sw_2460 & 0.168 & 0.037 & 0.178 & 0.001 & 0.019 & 0.000 & 0.064 & 0.000 & & & 0.094 & 0.000 & upr & private_home & 12.5 & 11.4 & 1.1 \\
\hline sw_2556 & 0.207 & 0.037 & 0.287 & 0.002 & 0.029 & 0.000 & 0.080 & 0.000 & 0.006 & 0.000 & 0.172 & 0.001 & upr & municipal_library & 16.5 & 14.8 & 1.6 \\
\hline sw_2646 & 0.185 & 0.037 & 0.234 & 0.001 & 0.022 & 0.0 & 0.075 & 0.000 & & & 0.136 & 0.001 & upr & community church & 14.6 & 13.3 & 1.3 \\
\hline sw_2654 & 0.158 & 0.037 & 0.157 & 0.001 & 0.010 & 0.000 & 0.043 & 0.000 & & & 0.105 & 0.000 & upr & municipal_nursing home & 8.3 & 7.3 & 1.0 \\
\hline sw_2744 & 0.227 & 0.037 & 0.300 & 0.002 & 0.027 & 0.000 & 0.093 & 0.001 & 0.009 & 0.000 & 0.172 & 0.001 & upr & private_home & 19.1 & 17.1 & 2.0 \\
\hline sw 2819 A & 0.180 & 0.0 & 0.226 & & 0.020 & & 0.072 & 0.0 & & & 0.134 & 0.001 & upr & private home & 14.2 & 12.9 & 1.3 \\
\hline sw_2819_B & 0.164 & 0.037 & 0.168 & 0.001 & 0.016 & 0.000 & 0.045 & 0.000 & & & 0.108 & 0.000 & upr & private_home & 10.3 & 9.2 & 1.1 \\
\hline sw_2841 & 0.165 & 0.037 & 0.163 & 0.001 & 0.021 & 0.000 & 0.089 & 0.001 & & & 0.053 & 0.000 & upr & private_home & 15.3 & 14.2 & 1.1 \\
\hline sw_2936 & 0.190 & 0.03 & 0.252 & 0.0 & 0.023 & 0.0 & 0.096 & 0.001 & 0.001 & 0.000 & 0.131 & 0.001 & upr & municipal_fire/EMS & 16.5 & 15.1 & 1.4 \\
\hline sw_3105_B & 0.162 & 0.037 & 0.154 & 0.001 & 0.013 & 0.000 & 0.051 & 0.000 & & & 0.090 & 0.000 & upr & private_home & 10.4 & 9.4 & 1.0 \\
\hline sw 3208 & 0.216 & 0.037 & 0.302 & 0.002 & 0.030 & 0.000 & 0.077 & 0.000 & & & 0.195 & 0.002 & upr & municipal fire/EMS & 16.7 & 15.0 & 1.7 \\
\hline
\end{tabular}

Note: Blanks indicate factor not apportioned at site.

(c) SCE, UNC to BaP-TEQ (by site). 
Table D.4-6. Source contributions and uncertainty to $\mathrm{\Sigma PAH}$ and BaP-TEQ with uncertainty metadata.

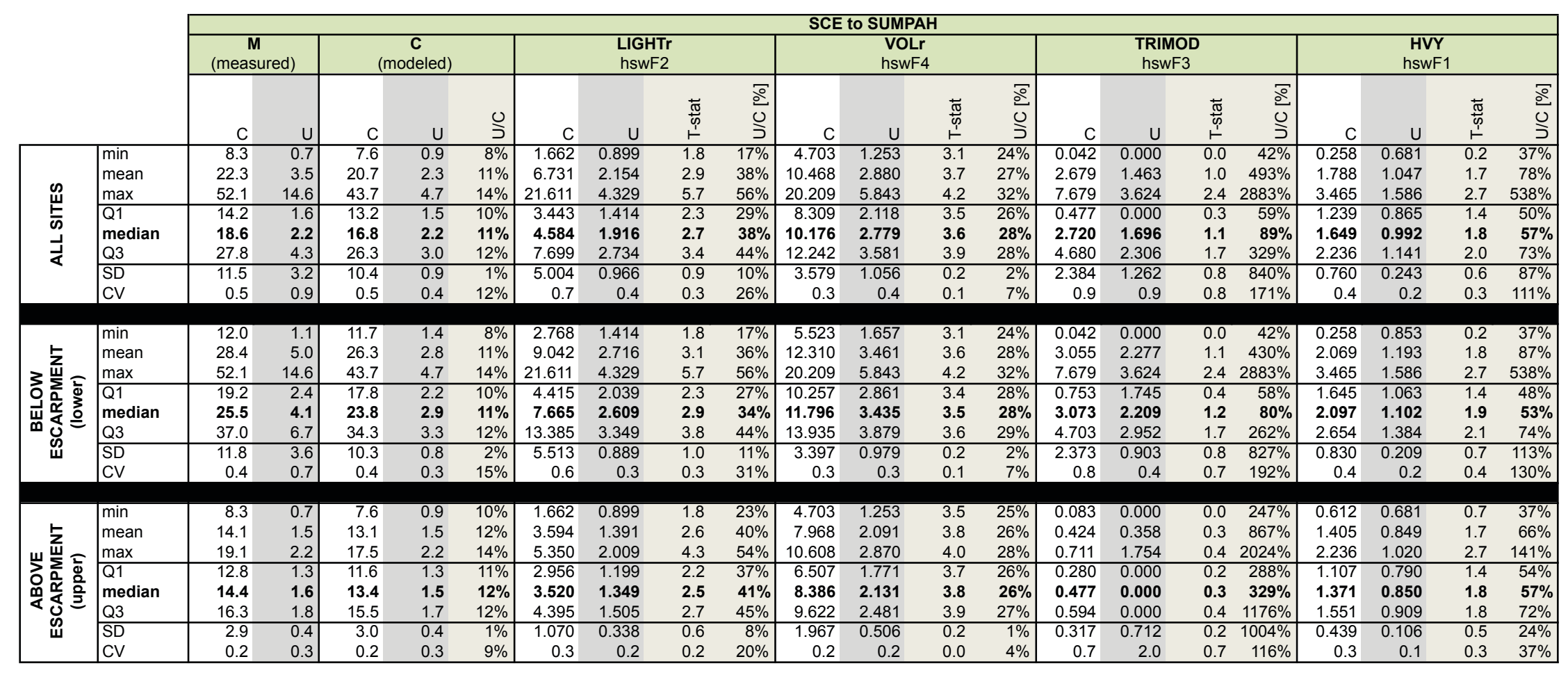

(a) SCE, UNC, metadata to $\mathrm{PPAH}$ (summary statistics). 
Table D.4-6 (continued)

\begin{tabular}{|c|c|c|c|c|c|c|c|c|c|c|c|c|c|c|c|c|c|c|c|c|c|c|c|c|}
\hline \multirow[b]{3}{*}{ 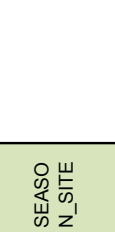 } & \multicolumn{21}{|c|}{ SCE to SUMPAH } & \multirow[b]{3}{*}{$\mid \begin{array}{l}\frac{\alpha}{3} \\
\sum_{1}^{\alpha} \\
\frac{\alpha}{2}\end{array}$} & \multirow[b]{3}{*}{ SITE_TYPE } & $\stackrel{\varphi}{\oplus}$ \\
\hline & \multicolumn{2}{|c|}{$\begin{array}{c}\mathbf{M} \\
\text { (measured) }\end{array}$} & \multicolumn{3}{|c|}{$\begin{array}{c}\mathrm{C} \\
\text { (modeled) }\end{array}$} & \multicolumn{4}{|c|}{$\begin{array}{l}\text { LIGHTr } \\
\text { hswF2 }\end{array}$} & \multicolumn{4}{|c|}{$\begin{array}{c}\text { VOLr } \\
\text { hswF4 }\end{array}$} & \multicolumn{4}{|c|}{$\begin{array}{c}\text { TRIMOD } \\
\text { hswF3 }\end{array}$} & \multicolumn{4}{|c|}{$\begin{array}{c}\text { HVY } \\
\text { hswF1 }\end{array}$} & & & $a_{1}$ \\
\hline & C & $\mathrm{U}$ & C & u & $\mathrm{o}$ & C & u & 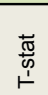 & $\begin{array}{l}\frac{\delta}{0} \\
\frac{0}{3}\end{array}$ & C & $u$ & 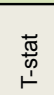 & $\begin{array}{l}\frac{\overline{3}}{\mathrm{o}} \\
\mathrm{u}\end{array}$ & C & $u$ & 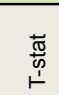 & 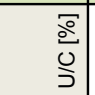 & C & u & 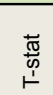 & 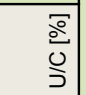 & & & 0 \\
\hline sw_1785 & 45.4 & 9.9 & 42.1 & 4.2 & $10 \%$ & 15.263 & 4.140 & 3.7 & $27 \%$ & 17.463 & 5.108 & 3.4 & $29 \%$ & 6.041 & 3.585 & 1.7 & $59 \%$ & 3.302 & 1.586 & 2.1 & $48 \%$ & Iwr & municipal_fire/EMS & 45.4 \\
\hline sw_1838 & 18.6 & 2.2 & 17.1 & 2.2 & $13 \%$ & 4.584 & 1.966 & 2.3 & $43 \%$ & 9.798 & 2.767 & 3.5 & $28 \%$ & 0.595 & 1.751 & 0.3 & $294 \%$ & 2.108 & 1.071 & 2.0 & $51 \%$ & Iwr & municipal_library & 18.6 \\
\hline sw_1850_A & 39.5 & 7.9 & 35.6 & 3.4 & $9 \%$ & 14.160 & 3.500 & 4.0 & $25 \%$ & 13.314 & 4.022 & 3.3 & $30 \%$ & 5.251 & 3.043 & 1.7 & $58 \%$ & 2.845 & 1.384 & 2.1 & $49 \%$ & Iwr & municipal_heritage & 39.5 \\
\hline W_1850_B & 46.0 & 10.2 & 42.2 & 3.5 & $8 \%$ & 21.611 & 3.776 & 5.7 & $17 \%$ & 13.280 & 3.902 & 3.4 & $29 \%$ & 4.711 & 3.088 & 1.5 & $66 \%$ & 2.590 & 1.385 & 1.9 & $53 \%$ & Iwr & municipal_cemetery & 46.0 \\
\hline sw_1858_A & 34.6 & 6.1 & 31.8 & 3.0 & $9 \%$ & 12.610 & 3.197 & 3.9 & $25 \%$ & 11.662 & 3.410 & 3.4 & $29 \%$ & 5.921 & 2.861 & 2.1 & $48 \%$ & 1.642 & 1.295 & 1.3 & $79 \%$ & Iwr & municipal_library & 34.6 \\
\hline sw_1858_B & 39.4 & 7.3 & 37.1 & 3.3 & $9 \%$ & 15.748 & 3.610 & 4.4 & $23 \%$ & 11.796 & 3.749 & 3.1 & $32 \%$ & 7.679 & 3.258 & 2.4 & $42 \%$ & 1.891 & 1.421 & 1.3 & $75 \%$ & Iwr & private_home & 39.4 \\
\hline Sw_1922 & 52.1 & 14.6 & 43.7 & 4.7 & $11 \%$ & 16.691 & 4.329 & 3.9 & $26 \%$ & 20.209 & 5.843 & 3.5 & $29 \%$ & 4.089 & 3.624 & 1.1 & $89 \%$ & 2.717 & 1.520 & 1.8 & $56 \%$ & Iwr & municipal_other & 52.1 \\
\hline sw_1987_A & 21.2 & 2.6 & 19.8 & 2.3 & $11 \%$ & 5.921 & 2.128 & 2.8 & $36 \%$ & 10.115 & 2.850 & 3.5 & $28 \%$ & 1.282 & 1.795 & 0.7 & $140 \%$ & 2.460 & 0.986 & 2.5 & $40 \%$ & Iwr & institutional_U & 21.2 \\
\hline W_1987_B & 19.3 & 2.4 & 18.5 & 2.4 & $13 \%$ & 4.246 & 2.112 & 2.0 & $50 \%$ & 11.528 & 3.181 & 3.6 & $28 \%$ & 0.252 & 1.739 & 0.1 & $689 \%$ & 2.495 & 0.983 & 2.5 & $39 \%$ & Iwr & municipal_nursing home & 19.3 \\
\hline sw_1998 & 28.3 & 4.6 & 26.3 & 2.9 & $11 \%$ & 8.638 & 2.734 & 3.2 & $32 \%$ & 12.947 & 3.581 & 3.6 & $28 \%$ & 2.877 & 2.306 & 1.2 & $80 \%$ & 1.856 & 1.102 & 1.7 & $59 \%$ & Iwr & NAPS & 28.3 \\
\hline sw_2006 & 19.0 & 2.5 & 16.8 & 1.9 & $11 \%$ & 3.868 & 1.665 & 2.3 & $43 \%$ & 11.288 & 2.676 & 4.2 & $24 \%$ & & & & & 1.691 & 0.853 & 2.0 & $50 \%$ & Iwr & municipal_library & 19.0 \\
\hline sw_2070 & 12.2 & 1.1 & 11.7 & 1.4 & $12 \%$ & 2.768 & 1.442 & 1.9 & $52 \%$ & 5.523 & 1.657 & 3.3 & $30 \%$ & 2.720 & 1.483 & 1.8 & $55 \%$ & 0.645 & 1.056 & 0.6 & $164 \%$ & Iwr & municipal_other & 12.2 \\
\hline sw_2147 & 27.8 & 4.3 & 27.2 & 3.1 & $11 \%$ & 7.665 & 3.039 & 2.5 & $40 \%$ & 14.556 & 3.802 & 3.8 & $26 \%$ & 4.680 & 2.819 & 1.7 & $60 \%$ & 0.258 & 1.390 & 0.2 & $538 \%$ & Iwr & municipal_other & 27.8 \\
\hline sw_2478 & 16.3 & 1.8 & 15.7 & 2.2 & $14 \%$ & 4.093 & 1.907 & 2.1 & $47 \%$ & 10.338 & 2.873 & 3.6 & $28 \%$ & 0.071 & 1.696 & 0.04 & $2399 \%$ & 1.245 & 1.097 & 1.1 & $88 \%$ & Iwr & community_church & 16.3 \\
\hline sw_2236 & 24.3 & 4.1 & 22.1 & 3.0 & $14 \%$ & 4.674 & 2.609 & 1.8 & $56 \%$ & 15.401 & 3.856 & 4.0 & $25 \%$ & 0.459 & 2.209 & 0.2 & $482 \%$ & 1.579 & 1.141 & 1.4 & $72 \%$ & Iwr-east & municipal_fire/EMS & 24.3 \\
\hline sw_2402 & 25.0 & 3.2 & 23.8 & 2.4 & $10 \%$ & 7.699 & 2.432 & 3.2 & $32 \%$ & 10.176 & 2.905 & 3.5 & $29 \%$ & 3.829 & 2.154 & 1.8 & $56 \%$ & 2.097 & 1.097 & 1.9 & $52 \%$ & Iwr-east & community_church & 25.0 \\
\hline sw_2408 & 33.4 & 5.1 & 33.0 & 3.4 & $10 \%$ & 11.147 & 3.160 & 3.5 & $28 \%$ & 15.070 & 4.119 & 3.7 & $27 \%$ & 3.269 & 2.637 & 1.2 & $81 \%$ & 3.465 & 1.293 & 2.7 & $37 \%$ & Iwr-east & municipal_fire/EMS & 33.4 \\
\hline sw_2575 & 25.5 & 3.9 & 23.5 & 2.7 & $11 \%$ & 7.202 & 2.447 & 2.9 & $34 \%$ & 12.242 & 3.435 & 3.6 & $28 \%$ & 1.226 & 2.017 & 0.6 & $165 \%$ & 2.780 & 1.075 & 2.6 & $39 \%$ & Iwr-east & municipal_heritage & 25.5 \\
\hline sw_2662 & 12.0 & 1.1 & 12.1 & 1.6 & $13 \%$ & 3.213 & 1.414 & 2.3 & $44 \%$ & 7.190 & 2.014 & 3.6 & $28 \%$ & 0.042 & 1.206 & 0.03 & $2883 \%$ & 1.649 & 0.942 & 1.8 & $57 \%$ & Iwr-east & community_church & 12.0 \\
\hline sw_1704 & 13.8 & 1.4 & 13.2 & 1.3 & $10 \%$ & 5.350 & 1.241 & 4.3 & $23 \%$ & 6.305 & 1.751 & 3.6 & $28 \%$ & & & & & 1.505 & 0.805 & 1.9 & $54 \%$ & upr & municipal_library & \begin{tabular}{|l|}
13.8 \\
\end{tabular} \\
\hline sw_2296 & 15.6 & 1.8 & 14.9 & 1.7 & $12 \%$ & 3.598 & 1.518 & 2.4 & $42 \%$ & 10.050 & 2.491 & 4.0 & $25 \%$ & & & & & 1.223 & 0.918 & 1.3 & $75 \%$ & upr & municipal_fire/EMS & 15.6 \\
\hline sw_2306 & 13.5 & 1.5 & 12.4 & 1.5 & $12 \%$ & 2.865 & 1.317 & 2.2 & $46 \%$ & 8.463 & 2.144 & 3.9 & $25 \%$ & & & & & 1.023 & 0.828 & 1.2 & $81 \%$ & upr & private_home & 13.5 \\
\hline sw_2460 & 12.5 & 1.3 & 11.4 & 1.3 & $11 \%$ & 3.227 & 1.185 & 2.7 & $37 \%$ & 7.111 & 1.830 & 3.9 & $26 \%$ & & & & & 1.075 & 0.785 & 1.4 & $73 \%$ & upr & private_home & 12.5 \\
\hline sw_2556 & 16.5 & 1.7 & 16.1 & 2.0 & $12 \%$ & 4.776 & 1.795 & 2.7 & $38 \%$ & 8.858 & 2.513 & 3.5 & $28 \%$ & 0.477 & 1.566 & 0.3 & $329 \%$ & 1.970 & 0.992 & 2.0 & $50 \%$ & upr & municipal_library & 16.5 \\
\hline sw_2646 & 14.6 & 1.6 & 13.6 & 1.5 & $11 \%$ & 3.741 & 1.371 & 2.7 & $37 \%$ & 8.309 & 2.118 & 3.9 & $25 \%$ & & & & & 1.557 & 0.885 & 1.8 & $57 \%$ & upr & community_church & 14.6 \\
\hline sw_2654 & 8.3 & 0.7 & 7.6 & 0.9 & $12 \%$ & 1.662 & 0.899 & 1.8 & $54 \%$ & 4.703 & 1.253 & 3.8 & $27 \%$ & & & & & 1.201 & 0.681 & 1.8 & $57 \%$ & upr & municipal_nursing home & 8.3 \\
\hline sw_2744 & 19.1 & 2.2 & 17.5 & 2.2 & $13 \%$ & 4.565 & 2.009 & 2.3 & $44 \%$ & 10.240 & 2.779 & 3.7 & $27 \%$ & 0.711 & 1.754 & 0.4 & $247 \%$ & 1.966 & 1.020 & 1.9 & $52 \%$ & upr & private_home & 19.1 \\
\hline sw_2819_A & 14.2 & 1.6 & 12.8 & 1.4 & $11 \%$ & 3.368 & 1.328 & 2.5 & $39 \%$ & 7.949 & 2.046 & 3.9 & $26 \%$ & & & & & 1.531 & 0.867 & 1.8 & $57 \%$ & upr & private_home & 14.2 \\
\hline sw_2819_B & 10.3 & 1.0 & 8.8 & 1.0 & $11 \%$ & 2.589 & 0.970 & 2.7 & $37 \%$ & 4.943 & 1.360 & 3.6 & $28 \%$ & & & & & 1.239 & 0.710 & 1.7 & $57 \%$ & upr & private_home & 10.3 \\
\hline sw_2841 & 15.3 & 1.8 & 13.9 & 1.7 & $12 \%$ & 3.443 & 1.451 & 2.4 & $42 \%$ & 9.877 & 2.448 & 4.0 & $25 \%$ & & & & & 0.612 & 0.865 & 0.7 & $141 \%$ & upr & private_home & 15.3 \\
\hline sw_2936 & 16.5 & 1.9 & 16.1 & 2.2 & $14 \%$ & 3.885 & 1.916 & 2.0 & $49 \%$ & 10.608 & 2.870 & 3.7 & $27 \%$ & 0.083 & 1.690 & 0.05 & $2024 \%$ & 1.503 & 0.981 & 1.5 & $65 \%$ & upr & municipal_fire/EMS & 16.5 \\
\hline sw_3105_B & 10.4 & 1.0 & 8.9 & 1.0 & $12 \%$ & 2.231 & 1.005 & 2.2 & $45 \%$ & 5.633 & 1.499 & 3.8 & $27 \%$ & & & & & 1.032 & 0.714 & 1.4 & $69 \%$ & upr & private_home & 10.4 \\
\hline sw_3208 & 16.7 & 2.0 & 15.8 & 1.6 & $10 \%$ & 5.018 & 1.466 & 3.4 & $29 \%$ & 8.505 & 2.177 & 3.9 & $26 \%$ & & & & & 2.236 & 0.834 & 2.7 & $37 \%$ & upr & municipal_fire/EMS & 16.7 \\
\hline
\end{tabular}

Note: Blanks indicate factor not apportioned at site.

(b) SCE, UNC, metadata to $\Sigma P A H$ (by site).

Appendix D: Intraurban source apportionment 
Table D.4-6 (continued)

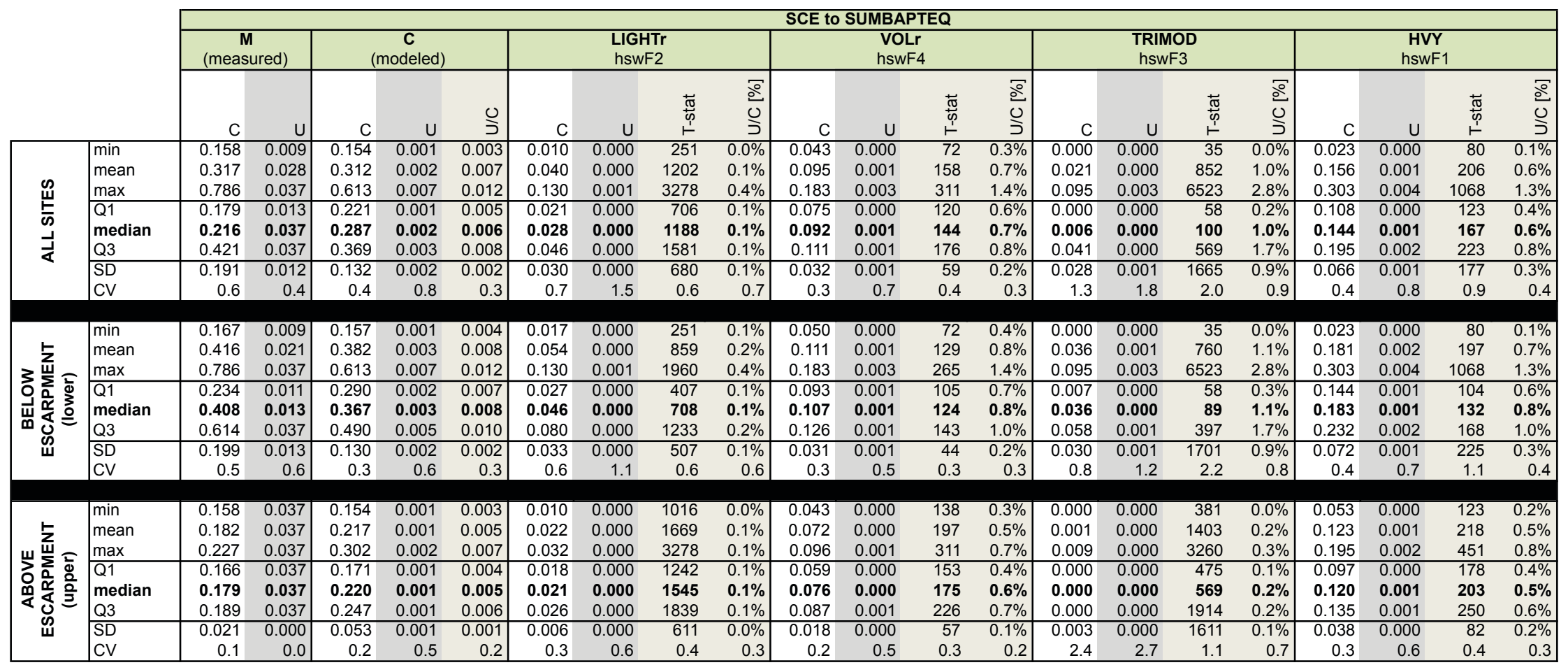

(c) SCE, UNC, metadata to BaP-TEQ (summary statistics). 
Table D.4-6 (continued)

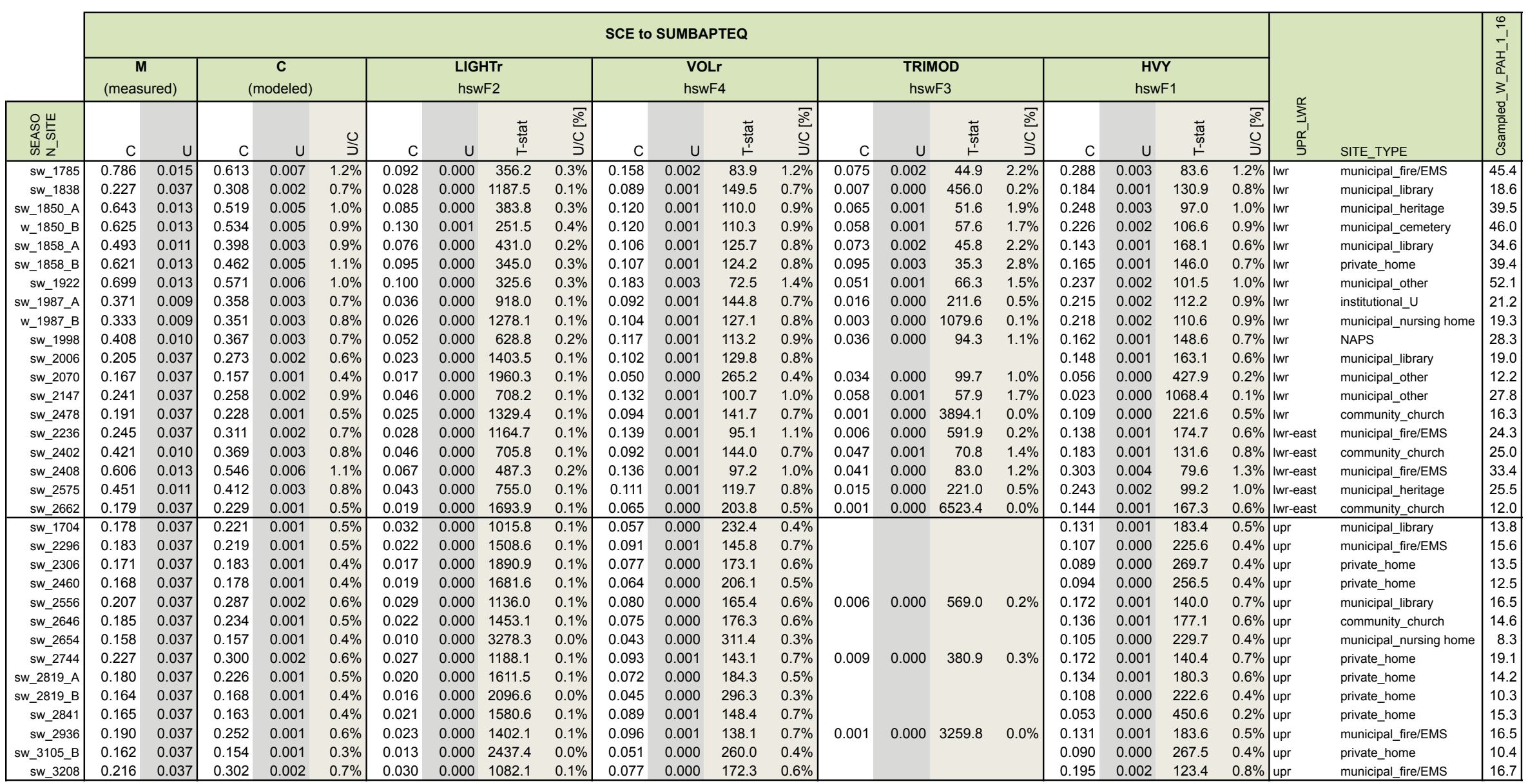

Note: Blanks indicate factor not apportioned at site.

(d) SCE, UNC, metadata to BaP-TEQ, by site. 
(a) LIGHTr

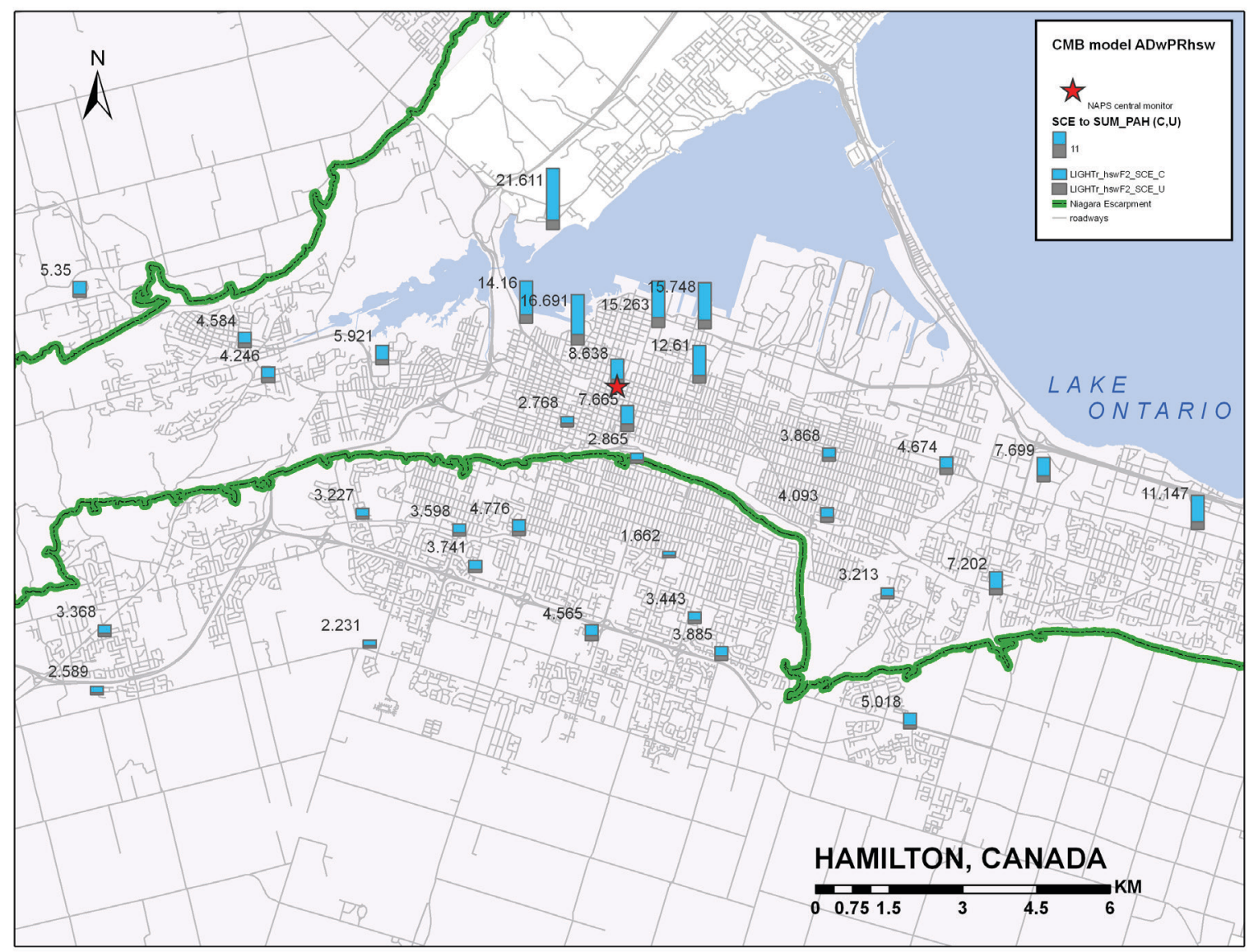

Note: Labels show factor contribution (SCE, $\mathrm{ng} / \mathrm{m}^{3}$ )

Figure D.4-5. Map of source contribution to $\Sigma$ PAH with uncertainty. 
(b) $\mathrm{VOLr}$

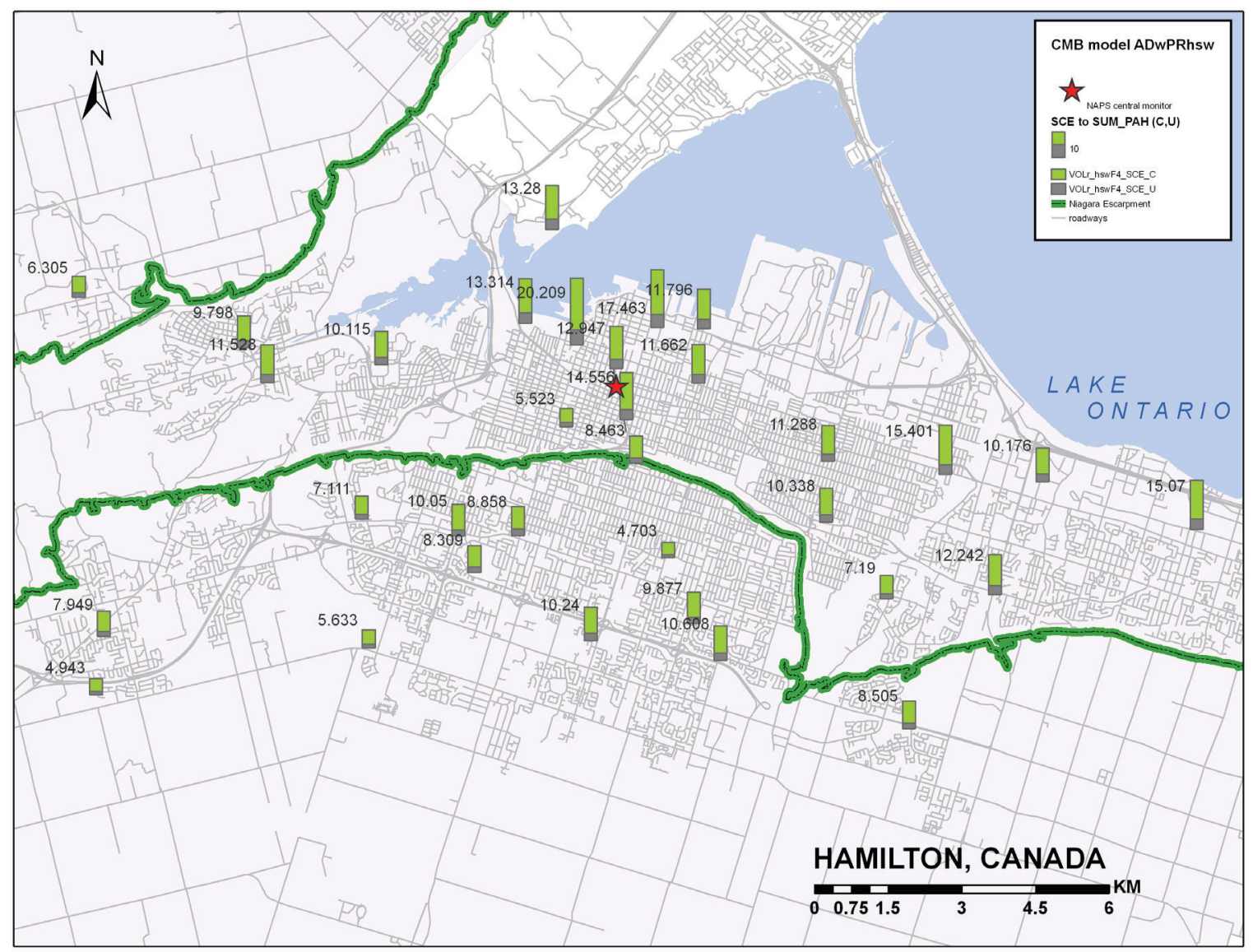

Note: Labels show factor contribution (SCE, $\mathrm{ng} / \mathrm{m}^{3}$ )

Figure D.4-5 (continued) 
(c) TRIMODAL

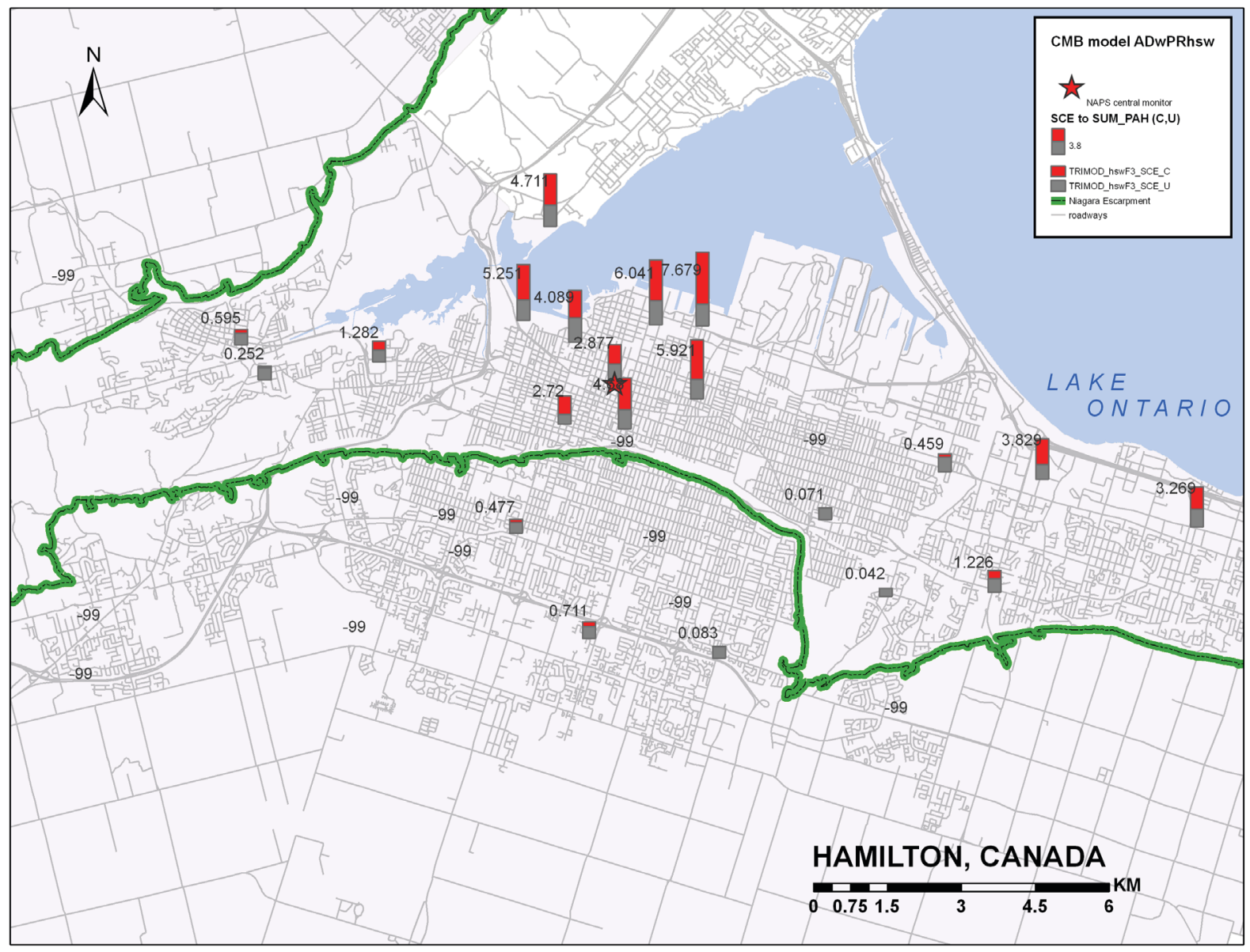

Note: Labels show factor contribution (SCE, $\mathrm{ng} / \mathrm{m}^{3}$ )

Figure D.4-5 (continued) 
(d) $H V Y$

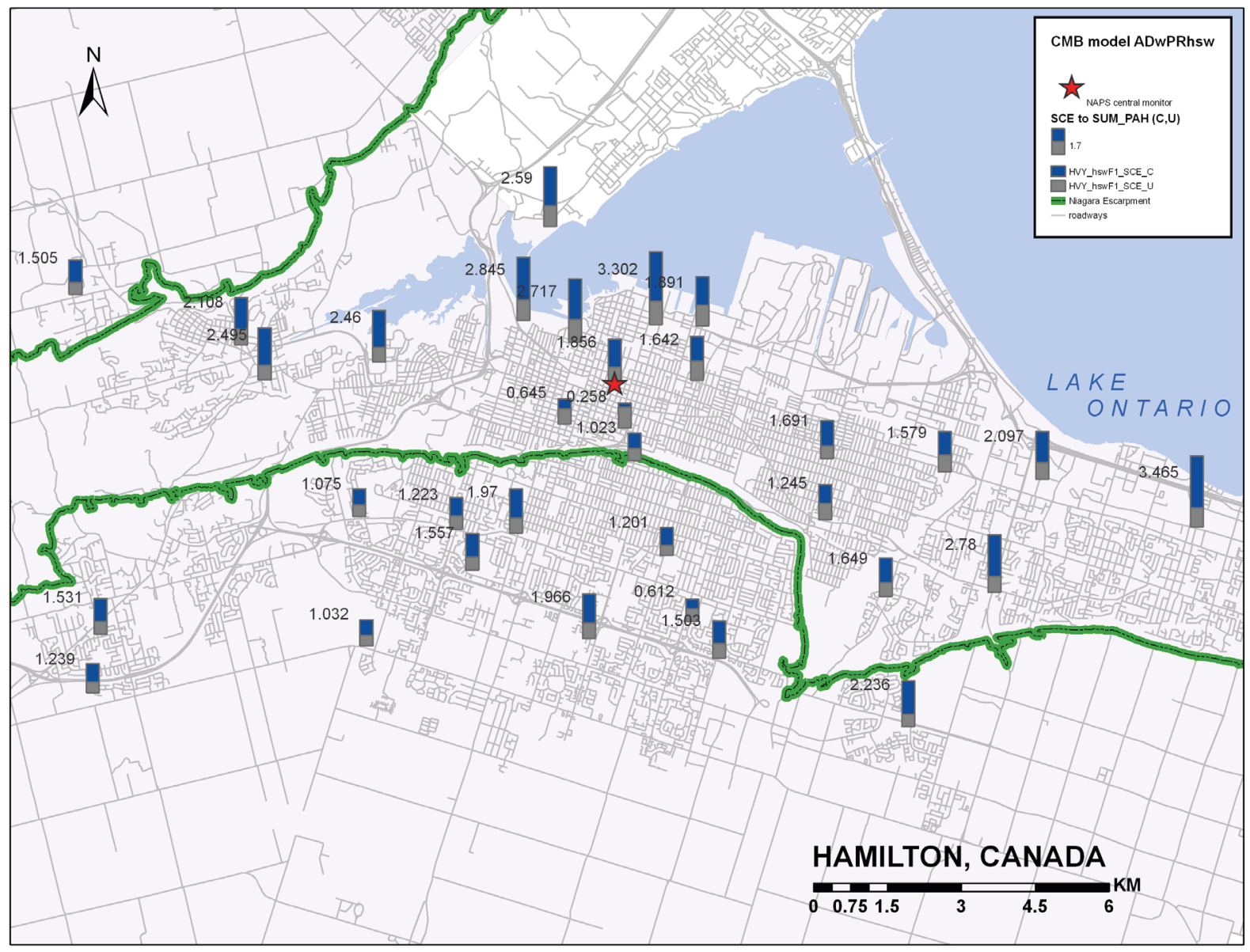

Note: Labels show factor contribution (SCE, $\mathrm{ng} / \mathrm{m}^{3}$ ) 


\section{Uncertainty estimates on source contributions to PAH}

Source contribution estimates to PAH toxicity (BaP-TEQ) were also reviewed against their analogous uncertainty estimates. It was noted that all four modeled factors showed very low uncertainty estimates relative to their contributions to toxicity. This can be understood by recalling that light MW species, in particular PHEN, contributed the clear majority of uncertainty at most sites, as can be seen by review of "contribution by species" model output. These species generally have very low BAP-TEF (e.g., 0.001 for PHEN; Nisbet and Lagoy, 1992) so, while their modeled contribution to $\Sigma \mathrm{PAH}$ is typically high relative to heavy MW species, their contribution to BaP-TEQ is effectively scaled down significantly.

However, a second consideration is the method in which the CMB model estimates uncertainty on the total mass (TM) variable (e.g., $\Sigma$ PAH or BaP-TEQ). Total estimated $\mathrm{PAH}$ concentration and uncertainty are estimated directly by CMB for contributions to $\mathrm{PAH}$ and denoted in CMB output as "TM-C" and "TM-U". However, source apportionment to BaP-TEQ is completed outside the model by converting the factor SCE to equivalent toxicities, as there can be no single BAP-TEF for TM-C and TM-U estimates since TM is an aggregate variable. Thus, uncertainty on contribution to BaPTEQ at each site was estimated by converting individual species uncertainties to BaPTEQ and summing these by sum of squares, applying a formulation frequently recommended for propagation of error (US EPA, 2000b). This method was procedurally rational but may have resulted in non-conservative uncertainty estimates. An alternate method of estimating the uncertainty on contributions to BaP-TEQ was explored, referencing the T-stat value for the source contribution to $\Sigma \mathrm{PAH}$ and 'scaling' the uncertainty for BaP-TEQ to the contribution to BaP-TEQ while assuming the same T-stat (i.e., T-stat = SCE/UNC); obviously, this yielded spatial trends in relative uncertainty on BaP-TEQ to be identical to those previously noted for $\Sigma$ PAH. Scaling BaP-TEQ contribution uncertainties to $\Sigma$ PAH T-stat values can yield more conservative uncertainty values and is thus presented as an alternate approach, while noting its theoretical limitations, namely that different modeled species contribute variously to reported total uncertainty so it may also be reasonable to down-weight reported species uncertainties by their respective BAP-TEF value. 


\section{D.5 Intraurban source apportionment using seasonal source profiles}

The PAH species suite typically sampled in central site monitoring challenges source apportionment that is restricted to just one season, since important information about seasonality is lost that could help interpret source types and, in some cases, even resolve a separate source type that is a strong function of season (i.e., PAH volatilization increased in spring/summer due to higher temperatures). Given this limitation, a seasonally stratified PMF model is not recommended a priori, particularly for the relatively small suite of PAH sampled by NAPS (29 species) and the even smaller suite typically measured in field sampling studies (16 species in "EPA-16"). ${ }^{36}$ However, for purposes of comparison with primary PMF-CMB results (i.e., Chapter 5), the CMB model was run with a seasonally stratified variant of the local source profiles (i.e., 4factor PMF model, fall/winter data, October through April, 13 fitting species).

Year-round and fall/winter model profile variants were generally similar (Figure D.5-1); however, greater inter-factor distinction was seen for year-round profiles (i.e., "light" and "trimodal" noticeably less distinct in winter profile variant). Reviewing CMB model results, non-seasonally stratified local source profiles (PRhsw) yielded better model fit at more sites (Tables D.5-1, D.5-2) and better source contribution estimates relative to uncertainty estimates (Table D.5-3) than the fall/winter variant (PRhw). Metrics were generally similar between source profile variants: $r^{2}$ was very similar (difference in medians of $1 \%$ ); \% mass showed slightly better fit with PRhsw; chi ${ }^{2}$ showed slightly better fit with PRhw (i.e., absolute chi ${ }^{2}$ values slightly improved but sites with high chi ${ }^{2}$ were same for both source profile variants. The aggregate model fit metric showed generally better fit for the non-seasonally stratified model (FM, median, all sites $=0.86$, PRhsw; 0.84, PRhw). Ranking model performance of the non-stratified and stratified source profile models by the aggregate fit measure showed a clear preference: $\mathrm{CMB}$ selected the non-seasonally stratified source profiles as "best fit" for the field-sampled ambient data at 20 of 33 sites.

The difference in aggregate model fit was larger at sites above the escarpment (FM, median $=0.98$, PRhsw; 0.95, PRhw) than below the escarpment (FM, median $=0.80$ for both PRhsw and PRhw). This suggests fall/winter profiles were a poorer representation of source types expected a priori and resolved at nearly all sites above the escarpment (i.e., "light-r", "volatile-r", "heavy", representing volatilized PAH/LRTAP coal combustion

\footnotetext{
${ }^{36}$ A significantly expanded PAH species suite or one augmented with PM inorganics / metals data may prove more amenable to seasonally stratified PMF modeling; however, PAH species may resolve together in only a few factors, reducing resolution of extracted PAH source types.
} 
and biomass combustion, space heating, traffic exhaust emissions, respectively) and offer no advantage to representation of the additional source type resolved below the escarpment (i.e., "trimodal", representing iron/steel industry emissions).

The source contribution estimates (i.e., \% contribution by factor, general spatial trends) for the fall/winter variant (Figure D.5-2 for pie charts across sites; Figure D.5-3 for individual factor contributions across sites) were highly similar to the non-seasonally stratified CMB model results (Chapter 5), as expected from the general similarity of the PRhsw and PRhw factor profiles (Figure D.5-1). However, the non-seasonally stratified model generally resolved factors with less relative uncertainty (i.e., higher T-stat) than the fall/winter variant, observed below and above the escarpment, particularly for "trimodal" and "heavy" factors (i.e., T-stat, median, all sites: ADwPRhsw, 2.7 "light-r", 3.6 "volatile-r", 1.1 "trimod", 1.8 "heavy"; ADwPRhw, 2.5 "light-r", 3.1 "volatile-r", 1.0 "trimodal", 1.4 "heavy"). Uncertainty results were consistent with superior model fit of the non-seasonally stratified local source profiles (i.e., aggregate FM and constituent metrics) and recommended them for intraurban source apportionment of Hamilton field data via PMF-CMB.

The rigorous modeling indicated receptor modeling using year-round PAH central site sampling (i.e., non-seasonally stratified) can yield better results in a PMF model used to generate CMB-input factors (i.e., better PMF model fit, stability, robustness, interpretation of source types; Chapter 3) and then in turn yield more defensible source contribution estimates in a paired CMB model (i.e., better model fit, less uncertainty on source contribution estimates), even with ambient field data sampled in the winter season (i.e., December 2009). Overall, despite any seasonality observed in individual PAH concentrations, the recommendation can be made to resolve 'local source profiles' from PMF on year-round time series data rather than stratifying the dataset, even for purposes of $\mathrm{CMB}$ modeling ambient data which will necessarily be sampled in one season. The superior $\mathrm{CMB}$ model performance and results observed for the non-seasonally stratified source profiles is in addition to other advantages of modeling year-round data in PMF (e.g., increased modeling power due to larger time series; ensure do not miss source types that are seasonal; aid in interpretation of source profiles as source types due to presence / absence of seasonality in factor contributions; increase sample-to-sample variance in time series; etc.). While a time series dataset of sufficient size to permit seasonal stratification (i.e., addressing PMF modeling power and related CMB model fit issues) may be useful as a complementary PMF-CMB solution, results are expected to be in general agreement to modeling of non-seasonally stratified source profiles, as found in this research. 

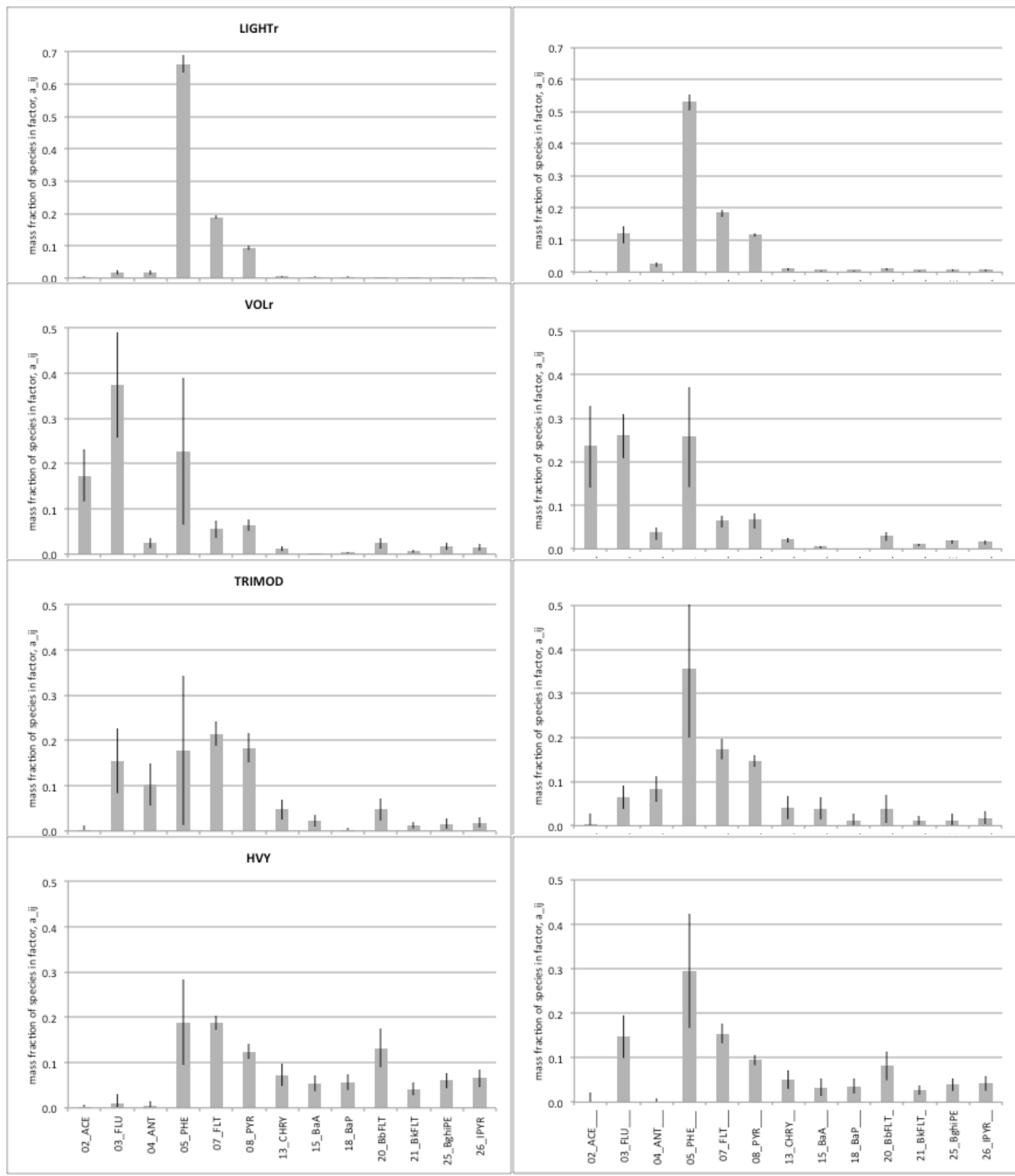

Note: Vertical axis scaled to factor maximum mass fraction and kept equal between year-round and winter profiles to aid visual comparison.

Figure D.5-1. Comparison of year-round (left) and winter (right) source profiles (PR) used in respective CMB models. 
Table D.5-1. CMB model performance (GOF) for PRhsw and PRhw; summary statistics.

\begin{tabular}{|c|c|c|c|c|c|c|c|c|c|}
\hline & & ADwPRhs & 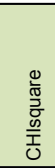 & 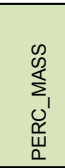 & $\sum_{i}$ & ADwPRhw & 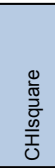 & 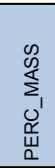 & $\sum_{4}$ \\
\hline \multirow{8}{*}{ 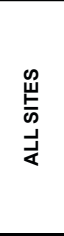 } & $\min$ & 0.85 & 0.50 & 83.9 & 0.73 & 0.86 & 0.58 & 81.8 & 0.74 \\
\hline & mean & 0.90 & 1.97 & 92.9 & 0.90 & 0.91 & 1.92 & 90.5 & 0.88 \\
\hline & $\max$ & 0.96 & 4.39 & 100.8 & 1.22 & 0.95 & 4.21 & 97.6 & 1.15 \\
\hline & Q1 & 0.88 & 1.02 & 91.1 & 0.79 & 0.89 & 1.11 & 88.4 & 0.80 \\
\hline & median & 0.90 & 1.62 & 92.7 & 0.86 & 0.91 & 1.72 & 90.7 & 0.84 \\
\hline & Q3 & 0.92 & 2.68 & 95.3 & 0.97 & 0.93 & 2.62 & 93.5 & 0.94 \\
\hline & SD & 0.03 & 1.19 & 3.7 & 0.13 & 0.02 & 1.02 & 3.7 & 0.10 \\
\hline & $\mathrm{CV}=\mathrm{SD} /$ mean & 0.03 & 0.60 & 0.0 & 0.14 & 0.03 & 0.53 & 0.0 & 0.11 \\
\hline \multirow{8}{*}{ 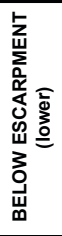 } & $\min$ & 0.85 & 1.00 & 83.9 & 0.73 & 0.86 & 1.11 & 81.8 & 0.74 \\
\hline & mean & 0.89 & 2.66 & 93.4 & 0.82 & 0.90 & 2.52 & 91.3 & 0.82 \\
\hline & $\max$ & 0.94 & 4.39 & 100.8 & 0.97 & 0.94 & 4.21 & 97.6 & 0.95 \\
\hline & Q1 & 0.88 & 1.95 & 91.8 & 0.78 & 0.89 & 1.95 & 89.0 & 0.78 \\
\hline & median & 0.89 & 2.53 & 93.1 & 0.80 & 0.90 & 2.53 & 91.2 & 0.80 \\
\hline & Q3 & 0.90 & 3.59 & 95.6 & 0.84 & 0.91 & 3.19 & 93.9 & 0.83 \\
\hline & SD & 0.02 & 1.08 & 3.8 & 0.06 & 0.02 & 0.92 & 3.9 & 0.06 \\
\hline & $\mathrm{CV}=\mathrm{SD} /$ mean & 0.03 & 0.41 & 0.0 & 0.08 & 0.02 & 0.37 & 0.0 & 0.07 \\
\hline \multirow{8}{*}{ 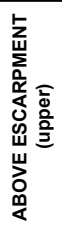 } & $\min$ & 0.89 & 0.50 & 85.4 & 0.82 & 0.90 & 0.58 & 83.5 & 0.83 \\
\hline & mean & 0.93 & 1.03 & 92.2 & 1.00 & 0.93 & 1.12 & 89.6 & 0.96 \\
\hline & $\max$ & 0.96 & 2.05 & 97.6 & 1.22 & 0.95 & 2.03 & 95.7 & 1.15 \\
\hline & Q1 & 0.92 & 0.68 & 91.0 & 0.93 & 0.92 & 0.92 & 88.0 & 0.91 \\
\hline & median & 0.93 & 0.99 & 91.6 & 0.98 & 0.93 & 1.02 & 89.9 & 0.95 \\
\hline & Q3 & 0.94 & 1.14 & 94.9 & 1.07 & 0.94 & 1.22 & 91.2 & 0.97 \\
\hline & SD & 0.02 & 0.45 & 3.7 & 0.12 & 0.01 & 0.39 & 3.4 & 0.09 \\
\hline & $\mathrm{CV}=\mathrm{SD} /$ mean & 0.02 & 0.43 & 0.0 & 0.12 & 0.01 & 0.35 & 0.0 & 0.09 \\
\hline
\end{tabular}


Table D.5-2. CMB model performance (GOF) for PRhsw and PRhw; by site.

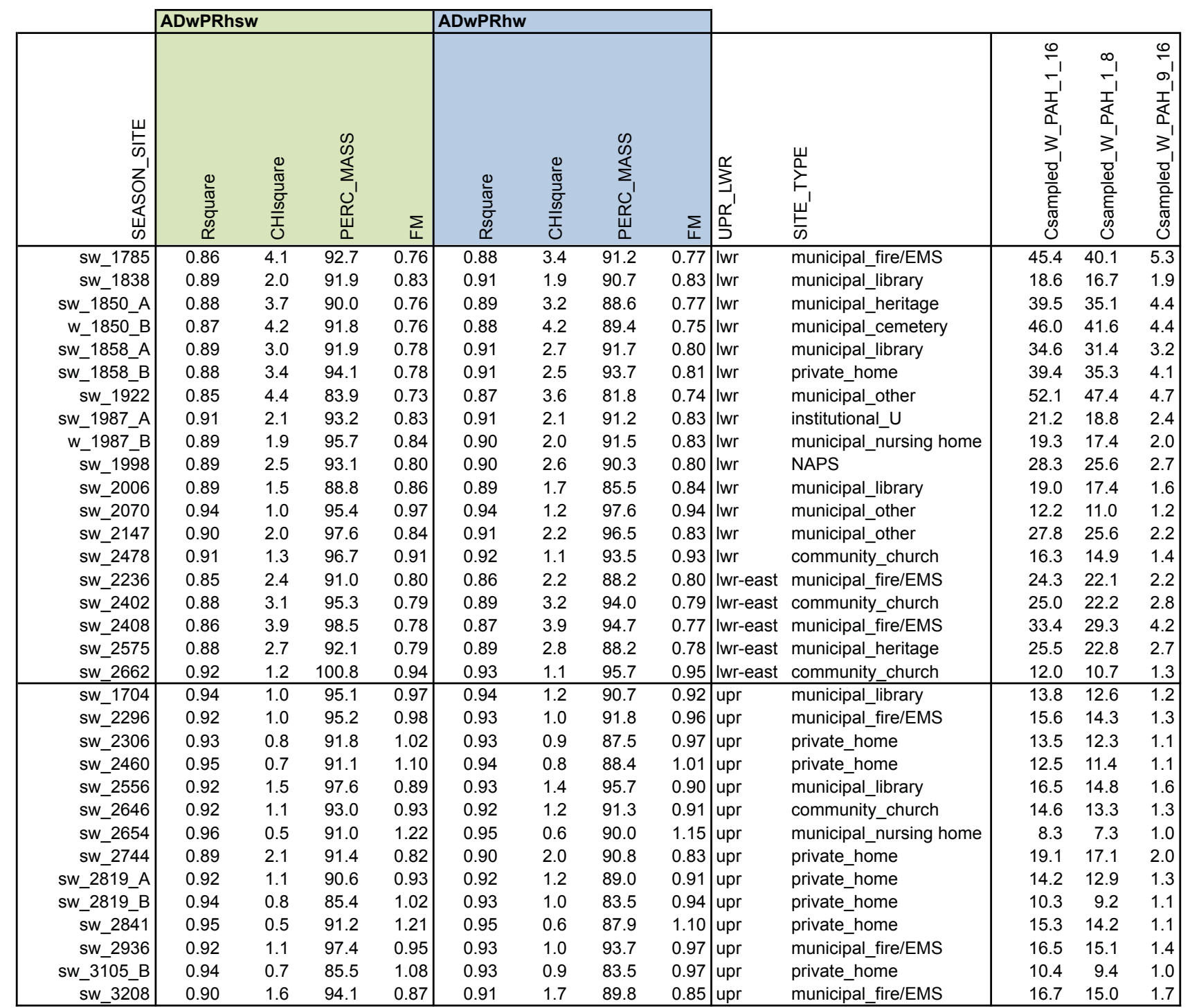

Appendix D: Intraurban source apportionment 
Table D.5-3. T-stat classification for ADwPRhsw and ADwPRhw models; by site.

\begin{tabular}{|c|c|c|c|c|c|c|c|c|c|c|c|c|c|c|c|c|c|}
\hline & & & & & PRhsw & & & & & PRhw & & & & & & & \\
\hline $\begin{array}{l}\text { SEASON_SI } \\
\text { TE }\end{array}$ & DATE & UPR_LWR & 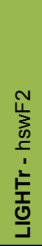 & 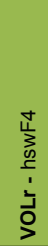 & 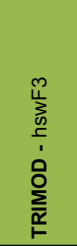 & 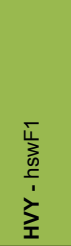 & 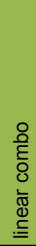 & 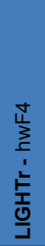 & 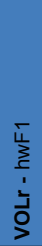 & 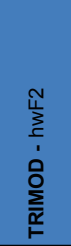 & 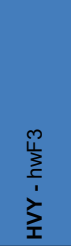 & 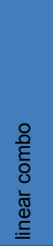 & 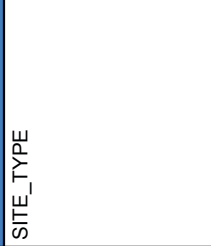 & 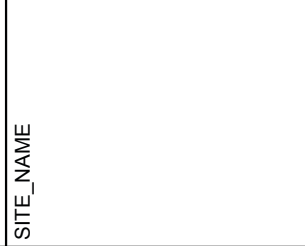 & 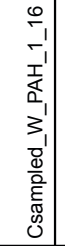 & 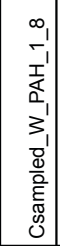 & 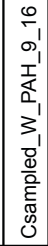 \\
\hline sw_1785 & \begin{tabular}{|l|l|} 
winter \\
\end{tabular} & Iwr & $\wedge^{\wedge \wedge}$ & $\wedge \wedge$ & $\hat{n}$ & $\wedge \wedge$ & & $\wedge^{\wedge}$ & $\wedge^{\wedge}$ & $\hat{n}$ & poor & & municipal_fire/EMS & Ambulance/EMS Stn \#30 & \begin{tabular}{|l|}
45.4 \\
\end{tabular} & \begin{tabular}{|l|}
40.1 \\
\end{tabular} & 5.3 \\
\hline sw_1838 & winter & Iwr & $\wedge \wedge$ & $\wedge \wedge$ & v.poor & $\wedge$ & & $\wedge \wedge$ & $\wedge \wedge$ & v.poor & $\sim$ & & municipal_library & Library_Dundas. & $\mid 18.6$ & 16.7 & 1.9 \\
\hline sw_1850_A & winter & Iwr & $\wedge \wedge$ & $\wedge \wedge$ & $\wedge$ & $\wedge \wedge$ & & $\wedge \wedge$ & $\wedge \wedge$ & $\wedge$ & poor & & municipal_heritage & Dundurn Castle & 39.5 & 35.1 & 4.4 \\
\hline$w_{-}{ }_{1}^{1} 850 \_\bar{B}$ & winter & Iwr & $\wedge \wedge$ & $\wedge \wedge$ & $\wedge$ & $\wedge$ & & $\wedge \wedge$ & $\wedge \wedge$ & $\sim$ & $\sim$ & & municipal_cemetery & Woodland Cemetery & 46.0 & 41.6 & 4.4 \\
\hline Sw_1858_A & winter & Iwr & $\wedge \wedge$ & $\wedge \wedge$ & $\wedge$ & $\sim$ & & $\wedge \wedge$ & $\wedge \wedge$ & $\wedge$ & v.poor & & municipal_library & Barton library & 34.6 & 31.4 & 3.2 \\
\hline sw_1858_B & winter & Iwr & $\wedge \wedge$ & $\wedge \wedge$ & $\wedge$ & $\sim$ & & $\wedge \wedge$ & $\wedge \wedge$ & $\wedge$ & v.poor & & private_home & private home & 39.4 & 35.3 & 4.1 \\
\hline sw_1922 & winter & Iwr & $\wedge \wedge$ & $\wedge \wedge$ & $\sim$ & $\wedge$ & & $\wedge \wedge$ & $\wedge \wedge$ & $\sim$ & v.poor & & municipal_other & West Nile Virus Lab & 52.1 & 47.4 & 4.7 \\
\hline Sw_1987_A & winter & Iwr & $\wedge \wedge$ & $\wedge \wedge$ & poor & $\wedge \wedge$ & & $\wedge \wedge$ & $\wedge \wedge$ & poor & $\wedge$ & & institutional_U U & MacMaster U Meteo Station & 21.2 & 18.8 & 2.4 \\
\hline w_1987_B & winter & Iiwr & $\wedge \wedge$ & $\wedge \wedge$ & v.poor & $\wedge \wedge$ & & $\wedge$ & $\wedge \wedge$ & v.poor & $\wedge$ & & municipal_nursing home & Wentworth Lodge & 19.3 & 17.4 & 2.0 \\
\hline sw_1998 & winter & Iwr & $\wedge \wedge$ & $\wedge \wedge$ & $\sim$ & $\wedge$ & & $\wedge \wedge$ & $\wedge \wedge$ & $\sim$ & $\sim$ & & NAPS & Beasley Park & 28.3 & 25.6 & 2.7 \\
\hline sw_2006 & winter & Iiwr & $\wedge \wedge$ & $\wedge \wedge$ & & $\wedge$ & & $\wedge \wedge$ & $\wedge \wedge$ & $\sim$ & & & municipal_library & Kenilworth Library & $\mid 19.0$ & 17.4 & 1.6 \\
\hline sw_-2070 & winter & Iwr & $\wedge$ & $\wedge \wedge$ & $\wedge$ & poor & & $\wedge \wedge$ & $\wedge \wedge$ & $\sim$ & poor & & municipal_other & Charlton Hall Child \& Family Centre & 12.2 & 11.0 & 1.2 \\
\hline sw_2147 & winter & Iwr & $\wedge \wedge$ & $\wedge \wedge$ & $\wedge$ & v.poor & & $\wedge \wedge$ & $\wedge \wedge$ & poor & v.poor & & municipal_other & Central Memorial Rec Centre & 27.8 & 25.6 & 2.2 \\
\hline sw_2478 & winter & Iwr & $\wedge \wedge$ & $\wedge \wedge$ & v.poor & $\sim$ & & $\wedge \wedge$ & $\wedge \wedge$ & & $\sim$ & & community_church & Church of the Nativity (Anglican) & 16.3 & 14.9 & 1.4 \\
\hline sw_-2236 & winter & Iwr-east & $\wedge$ & $\wedge \wedge$ & v.poor & $\sim$ & & $\wedge \wedge$ & $\wedge \wedge$ & & $\sim$ & & municipal_fire/EMS & Fire Station \#8 & 24.3 & 22.1 & 2.2 \\
\hline sw_2402 & winter & Iwr-east & $\wedge \wedge$ & $\wedge \wedge$ & $\wedge$ & $\wedge$ & & $\wedge \wedge$ & $\wedge \wedge$ & $\sim$ & $\wedge$ & & community_church & Gursikh Temple (sikh) & 25.0 & 22.2 & 2.8 \\
\hline sw_2408 & winter & Iwr-east & $\wedge \wedge$ & $\wedge^{\wedge}$ & $\sim$ & $\wedge \wedge$ & & $\wedge \wedge$ & $\wedge \wedge$ & poor & $\wedge \wedge$ & & municipal_fire/EMS & Fire Station \#15 & 33.4 & 29.3 & 4.2 \\
\hline sw_2575 & winter & Iwr-east & $\wedge \wedge$ & $\wedge \wedge$ & poor & $\wedge \wedge$ & & $\wedge \wedge$ & $\wedge \wedge$ & poor & $\wedge$ & & municipal_heritage & Battlefield Museum_Admin Office & 25.5 & 22.8 & 2.7 \\
\hline sw_2662 & winter & Iwr-east & $\wedge \wedge$ & $\wedge \wedge$ & v.poor & $\wedge$ & & $\wedge \wedge$ & $\wedge \wedge$ & & ^ & & community_church & St. Luke (catholic) & $\mid 12.0$ & $\mid 10.7$ & 1.3 \\
\hline sw_1704 & winter & upr & $\Lambda^{\wedge \Lambda}$ & $\wedge^{\wedge \wedge}$ & & $\Lambda$ & & $\wedge^{\wedge}$ & $\Lambda^{\wedge \wedge}$ & & 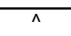 & & municipal_library & Greensville Library & \begin{tabular}{|c|}
13.8 \\
\end{tabular} & \begin{tabular}{|l|}
12.6 \\
\end{tabular} & 1.2 \\
\hline sw_2296 & winter & upr & $\wedge \wedge$ & $\wedge \wedge$ & & $\sim$ & & $\wedge \wedge$ & $\wedge \wedge$ & & $\sim$ & & municipal_fire/EMS & Fire Station \#3 & 15.6 & 14.3 & 1.3 \\
\hline sw_2306 & winter & upr & $\wedge \wedge$ & $\wedge \wedge$ & & $\sim$ & & $\wedge \wedge$ & $\wedge \wedge$ & & $\sim$ & & private_home & private home & 13.5 & 12.3 & 1.1 \\
\hline sw_2460 & winter & upr & $\wedge \wedge$ & $\wedge \wedge$ & & $\sim$ & & $\wedge \wedge$ & $\wedge \wedge$ & & $\sim$ & & private_home & private home & 12.5 & 11.4 & $\mid 1.1$ \\
\hline sw_2556 & winter & upr & $\wedge \wedge$ & $\wedge \wedge$ & v.poor & $\wedge$ & & $\wedge \wedge$ & $\wedge \wedge$ & v.poor & $\wedge$ & & municipal_library & Terry Berry library & 16.5 & 14.8 & 1.6 \\
\hline sw_2646 & winter & upr & $\wedge \wedge$ & $\wedge^{\wedge}$ & & $\wedge$ & & $\wedge \wedge$ & $\wedge \wedge$ & & ^ & & community_church & Annunciation of our Lord (catholic) & $\mid 14.6$ & 13.3 & 1.3 \\
\hline sw_2654 & winter & upr & $\wedge$ & $\wedge \wedge$ & & $\wedge$ & & $\sim$ & $\wedge \wedge$ & & ^ & & municipal_nursing home & Maccassa Lodge & 8.3 & 7.3 & 1.0 \\
\hline sw_2744 & winter & upr & $\wedge \wedge$ & $\wedge \wedge$ & poor & $\wedge$ & & $\wedge \wedge$ & $\wedge \wedge$ & v.poor & $\sim$ & & private_home & private home & 19.1 & 17.1 & 2.0 \\
\hline Sw_2819_A & winter & upr & $\wedge \wedge$ & $\wedge \wedge$ & & $\hat{\imath}$ & & $\wedge \wedge$ & $\wedge \wedge$ & & & & private_home & private home & 14.2 & 12.9 & 1.3 \\
\hline sw_2819_B & winter & upr & $\wedge \wedge$ & $\wedge \wedge$ & & $\wedge$ & & $\wedge \wedge$ & $\wedge \wedge$ & & $\wedge$ & & private_home & private home & 10.3 & 9.2 & 1.1 \\
\hline sw_2841 & winter & upr & $\wedge \wedge$ & $\wedge \wedge$ & & poor & & $\wedge \wedge$ & $\wedge \wedge$ & & v.poor & & private_home & private home & 15.3 & 14.2 & 1.1 \\
\hline sw_2936 & winter & upr & $\wedge \wedge$ & $\wedge \wedge$ & v.poor & $\wedge$ & & $\wedge \wedge$ & $\wedge \wedge$ & & $\sim$ & & municipal_fire/EMS & Fire Station \#5 & $|16.5|$ & 15.1 & $\mid 1.4$ \\
\hline sw_3105_B & winter & upr & $\wedge \wedge$ & $\wedge \wedge$ & & $\sim$ & & $\wedge$ & $\wedge \wedge$ & & $\sim$ & & private_home & private home & $|10.4|$ & \begin{tabular}{|l|}
9.4 \\
\end{tabular} & $\mid 1.0$ \\
\hline sw_3208 & winter & upr & $\wedge \wedge$ & $\wedge \wedge$ & & $\wedge \wedge$ & & $\wedge \wedge$ & $\wedge \wedge$ & & $\wedge \wedge$ & & municipal_fire/EMS & Fire Station \#17 & 16.7 & 15.0 & 1.7 \\
\hline & & & $\begin{array}{c}30 \\
3\end{array}$ & 33 & 2 & $\begin{array}{c}7 \\
15\end{array}$ & & $\begin{array}{c}30 \\
2\end{array}$ & 33 & $\begin{array}{l}0 \\
4\end{array}$ & $\begin{array}{c}2 \\
11\end{array}$ & & & & & & \\
\hline & & & 0 & 0 & $\begin{array}{l}6 \\
3\end{array}$ & 8 & & 1 & 0 & 6 & 11 & & & & & & \\
\hline & & F_es & 33 & 33 & 11 & 30 & & 33 & 33 & 10 & 24 & & & & & & \\
\hline & & poor & 0 & 0 & 3 & 2 & & 0 & 0 & 4 & 3 & & & & & & \\
\hline & & v.poor & 0 & 0 & 7 & 1 & & 0 & 0 & 4 & 5 & & & & & & \\
\hline & & & 0 & 0 & 0 & 0 & & 0 & 0 & 0 & 0 & & & & & & \\
\hline & s wit & ntribution & 33 & 33 & 21 & 33 & & 33 & 33 & 18 & 32 & & & & & & \\
\hline
\end{tabular}

Legend: $T$-stat $>2.0\left({ }^{\wedge \wedge}\right), 1.5-2.0(\wedge), 1.0-1.5(\sim), 0.5-1.0$ (poor), $<0.5$ (very poor). 


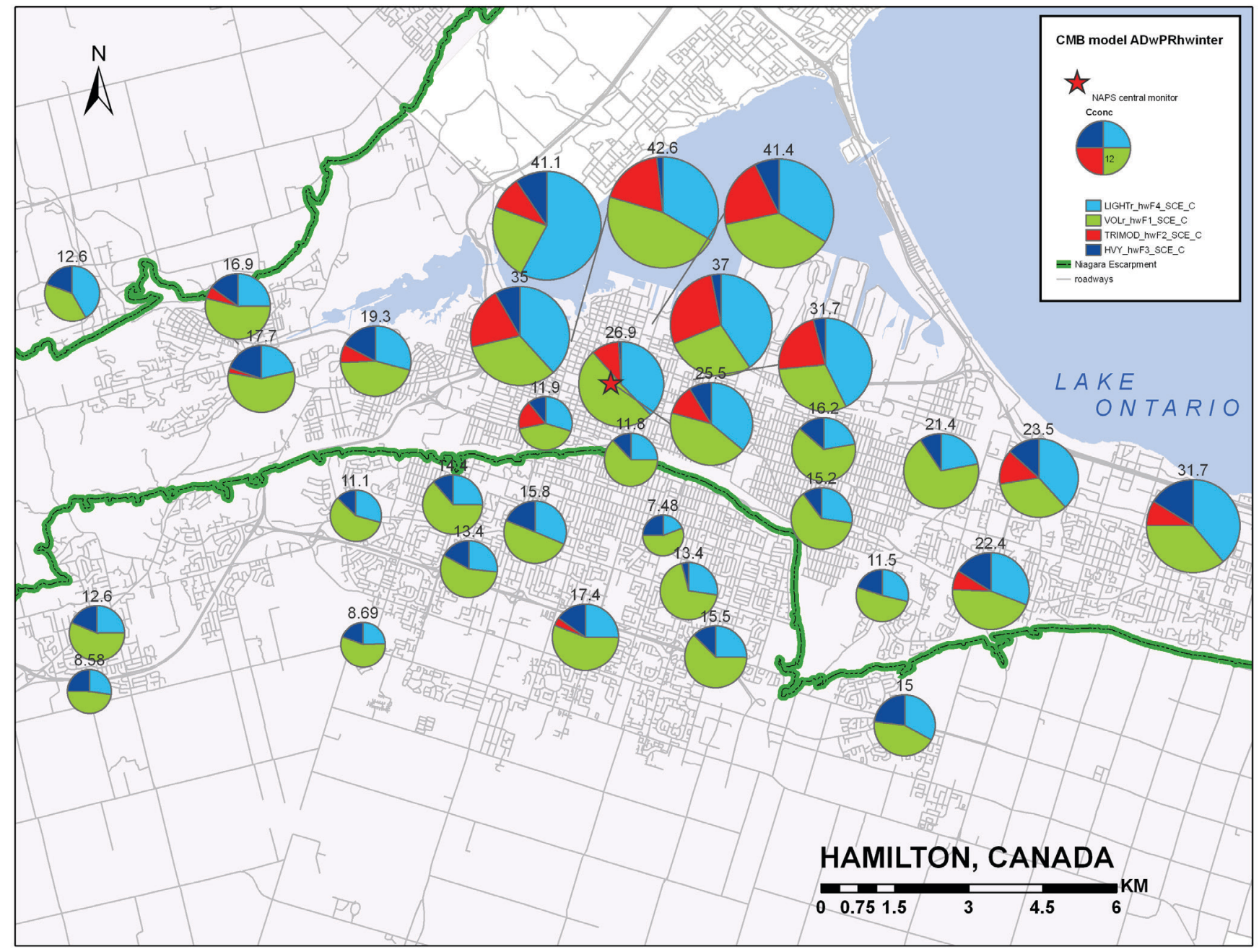

Note: Pie plots are scaled to sampled $\Sigma P A H$.

Figure D.5-2. Map of fractional source contribution to $\Sigma$ PAH, all modeled factors; ADwPRhw. 
(a) LIGHT-r

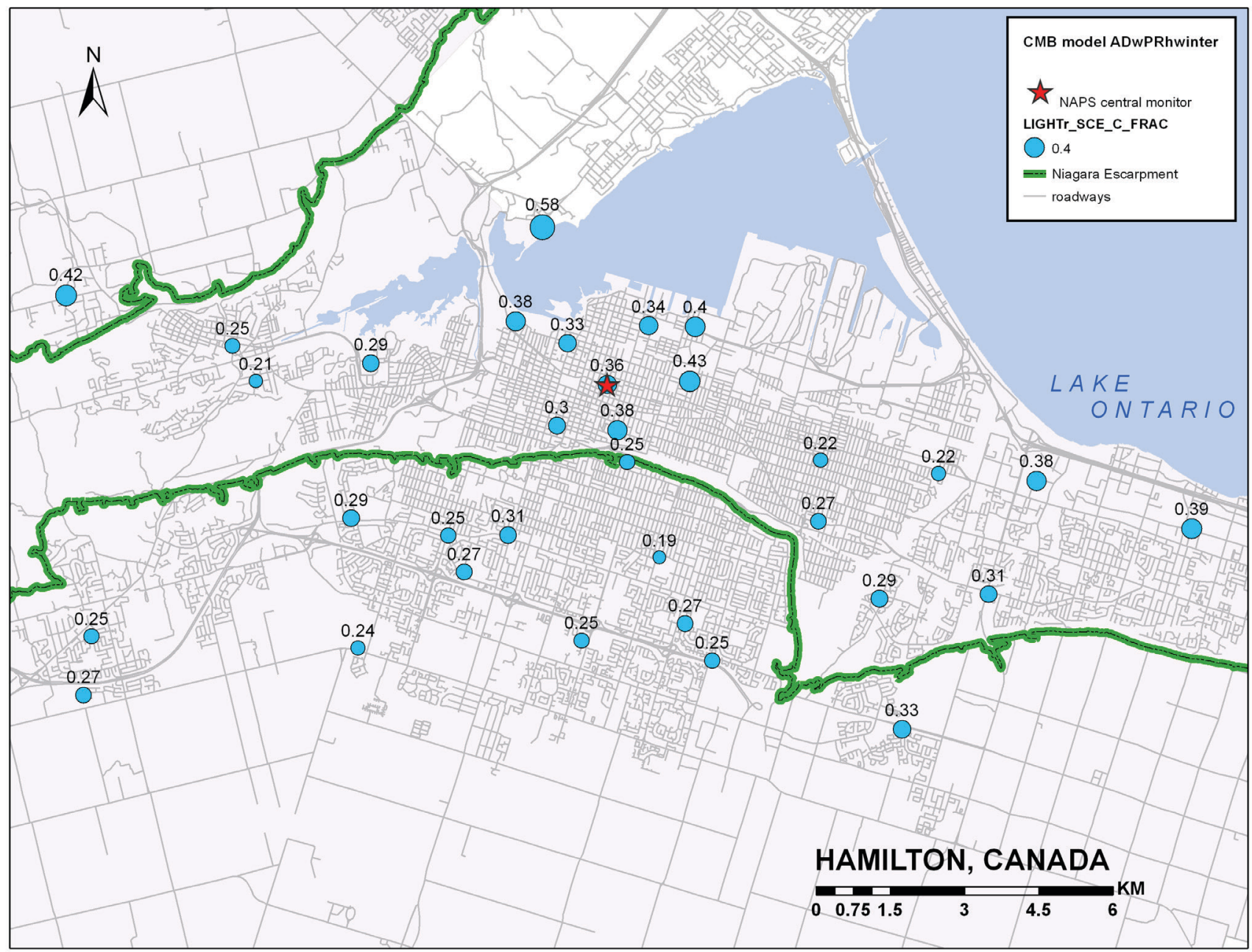

Note: Labels show factor contribution (SCE) as fraction of total modeled mass.

Figure D.5-3. Map of fractional source contribution to $\mathrm{DPAH}$; ADwPRhw. 
(b) VOL-r

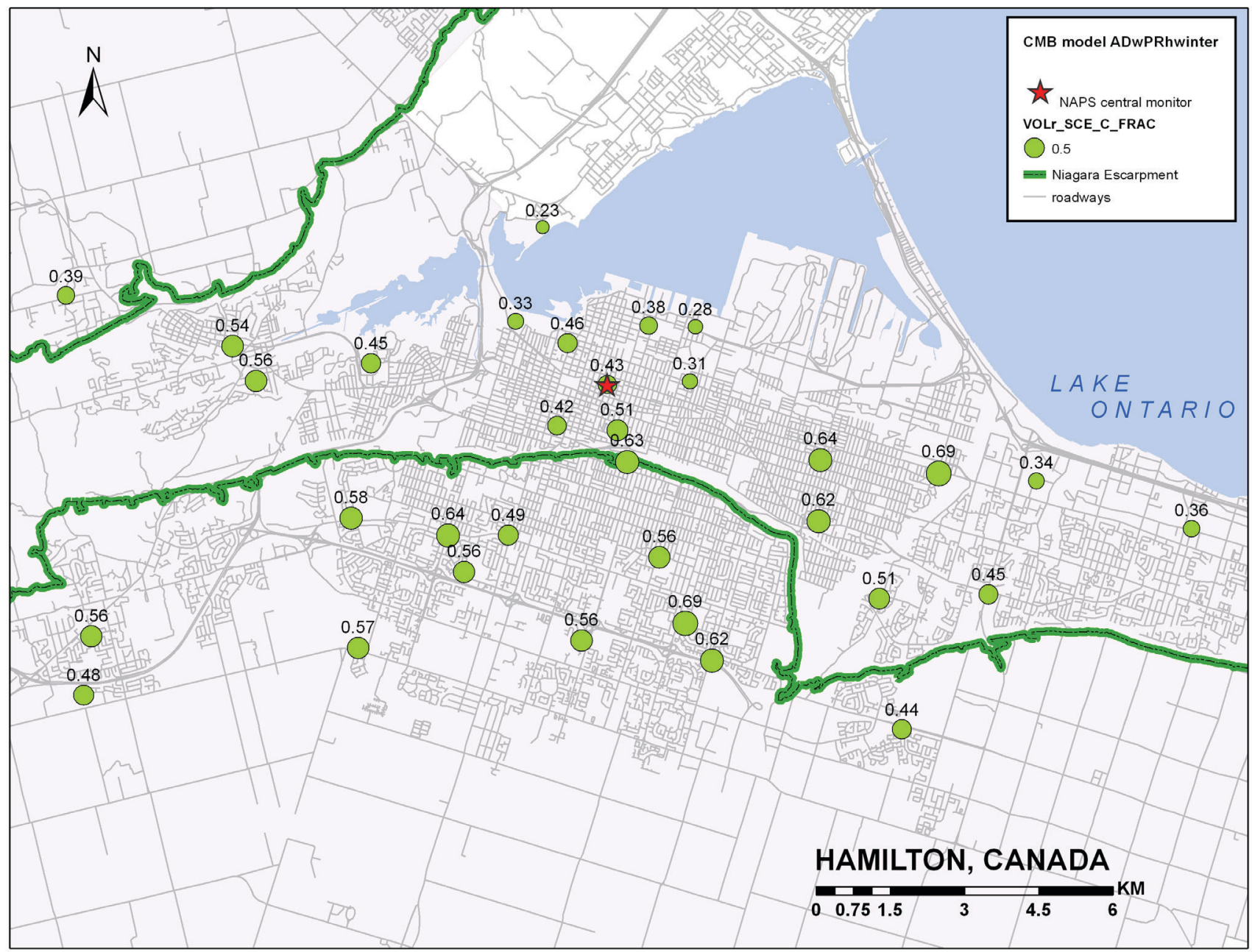

Note: Labels show factor contribution (SCE) as fraction of total modeled mass.

Figure D.5-3 (continued) 
(c) TRIMODAL

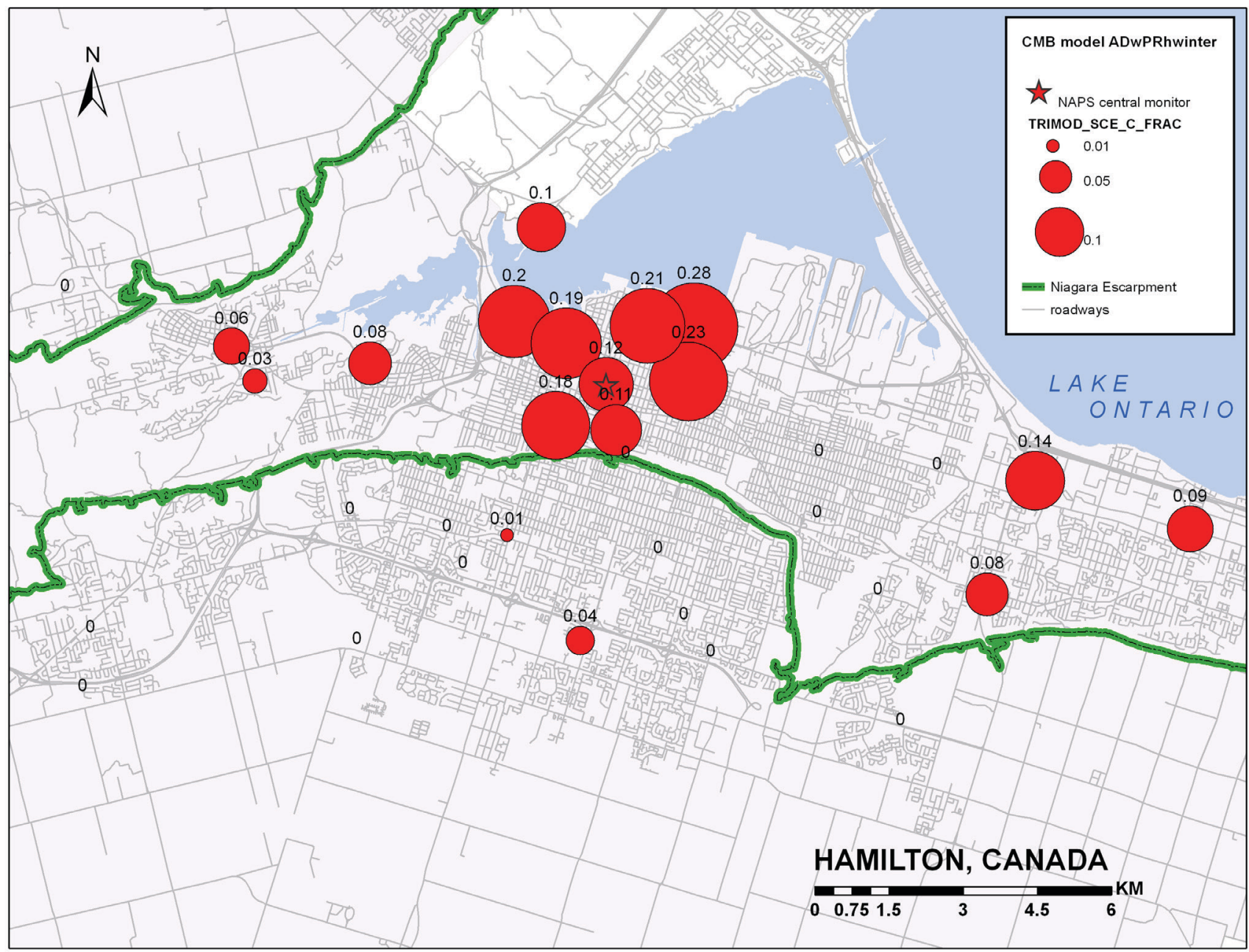

Note: Labels show factor contribution (SCE) as fraction of total modeled mass.

Figure D.5-3 (continued) 
(d) HEAVY

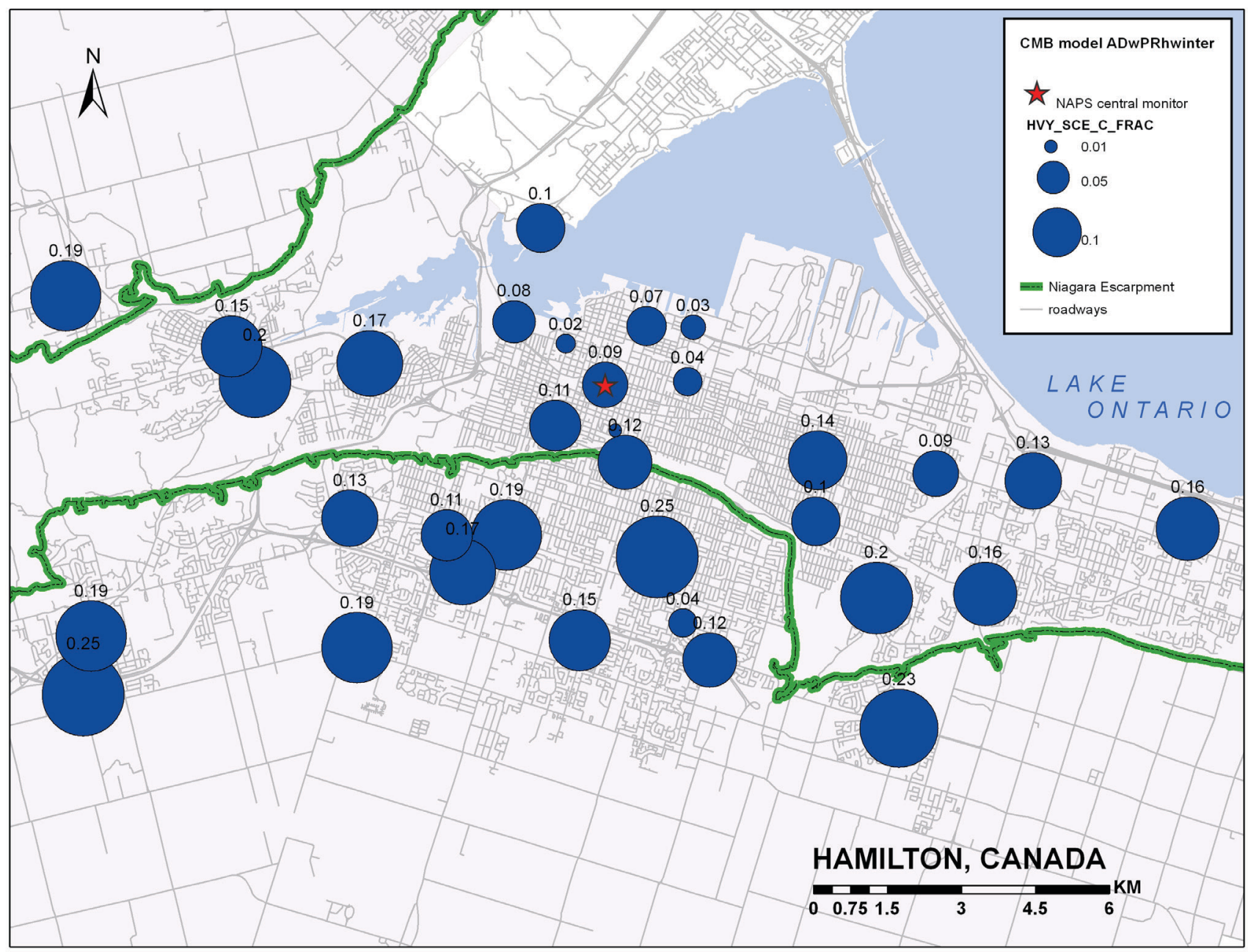

Note: Labels show factor contribution (SCE) as fraction of total modeled mass.

Figure D.5-3 (continued) 


\section{Appendix E Detailed modeling methodology}

Chapter 2 in the body of the thesis has presented an overview of the methodology used for the source apportionment of PAH and PAH toxicity using two distinct PAH datasets. This appendix presents the detailed steps in the methodology for the purposes of verification and the application of the methodology to other datasets of a similar nature.

\section{E.1 PCA protocol}

Details of PCA modeling, inclusive of: strategy, input data treatment, and model parameters.

\section{E.2 PMF protocol}

Details of PMF modeling, inclusive of: strategy, summary of model outputs and sub-functions, input data treatment, model parameters and performance criteria, sensitivity analyses, uncertainty on factor profiles, and interpretation of model factors as physically meaningful source types.

\section{E.3 CMB protocol}

Details of CMB modeling, inclusive of: strategy, input data (ambient data, source profiles), model parameters and performance criteria. 


\section{E.1 PCA protocol}

\section{Modeling strategy}

The urban and background site PAH datasets were assessed first by PCA to reveal data 'structure', since principal components (PC) resolved by the model indicate patterns of co-varying PAH species, as well as to indicate the number of factors that could be reasonably extracted from the dataset, providing a lower bound on the number of physically meaningful factors expected from application of PMF. The PCA was also used to explore data sensitivity to seasonal stratification, permitting ready comparison of results between the model comprised of year-round PAH time-series samples with complementary models restricting the time series to only winter (October - April) or summer (May - September) samples.

\section{Input data treatment}

PCA used as input data the PAH dataset prepared for PMF modeling, in which species concentrations below $\mathrm{DL}_{\mathrm{j}}$ were replaced with one-half $\mathrm{DL}_{\mathrm{j}}$ and missing data were replaced with the respective species median, and was run on the same species suite modeled with PMF. Majority of species datasets were found to log-normally distributed and were log-transformed (Equation E.1-1) prior to PCA modeling to reduce skew, reduce sensitivity to outliers, and provide more equal inter-species weighting in the model (Cao, 1999; Praveena, 2012; Reyment and Joreskog, 1993). While more complex PCA data transformations are available, such as conversion to proportional data using an indexing species (Reyment and Joreskog, 1993; Mudge, 2007), these were not advantageous to the analysis due to requiring exclusion of additional species (i.e., indexing species or most abundant species), abstracting the intended comparison to PMF, and particularly since source type interpretations were restricted to the more advanced PMF receptor model.

Equation E.1-1. Logarithmic data transformation for PCA receptor modeling.

$$
y_{i j}=\log _{10}\left(x_{i j}+1\right)
$$

\footnotetext{
$y_{i j}=\log$-transformed concentration of species $j$ in receptor sample $i$ (unitless)

$x_{i j}=$ concentration of species $j$ in receptor sample $i\left(\mathrm{ng} / \mathrm{m}^{3}\right)$

(concentrations increased by unity prior to calculation of base 10 logarithm to avoid zero or negative data values in PCA correlation matrix)
} 
A Z-standardization (Equation E.1-2) was performed within the PCA software to equalize the large differences in concentration ranges typical of total ambient PAH data, since light and heavy MW species concentrations can differ by orders of magnitude, and to equalize species influence in the model (Joliffe, 2002; Reyment and Joreskog, 1993; Kavouras, 2001; Berg, 1994).

Equation E.1-2. Z-transformation applied to PCA data.

$$
z_{i j}=\frac{x_{i j}-\overline{x_{j}}}{\sigma_{j}}
$$

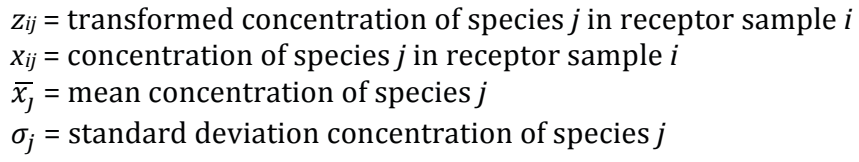

\section{Model parameters}

The PCA was run using SYSTAT 13 (Systat Software Inc., Chicago, IL, USA) with a correlation matrix and Varimax rotation. Extreme outliers in species datasets were defined as values greater than or less than three times the IQR and identified using Microsoft Excel and MINITAB 12 and, since the PCA showed moderate sensitivity to outliers, sample dates with extreme outlier concentrations in any species aside from retene $^{37}$ were generally omitted. This was done to ensure greater consistency with PMF modeling protocol, in which extreme outliers are generally excluded (US EPA, 2008; Reff et al, 2007). Extreme outliers were also excluded to improve factor separation, observed as greater factor loading strength for characteristic species and better distinction between factors as per Thurstone's criteria, which are summarized as low loading in each row of the rotated factor loading matrix, a number of low species loadings in each factor equal or greater than the model order, and all factor pairings showing some species with high loadings on one factor and near-zero loadings on the other as well as several species with low loadings on both factors (Reyment and Joreskog, 1993).

\footnotetext{
${ }^{37}$ Excluding dates with statistically extreme retene concentration did not improve factor loading or factor separation at any of the modeled sites, for either year-round or seasonally-stratified datasets, and generally only served to reduce variance in the retene dataset thereby hindering model's ability to clearly extract a separate retene factor. This was expected a priori, since retene was not found to be well correlated with other species and is generally considered a useful, albeit imperfect, marker species (Jang et al, 2013; Shen et al, 2012; Ramdahl et al, 1983; see Appendix B.1, source sampling studies, "wood combustion and retene as marker species").
} 
Models with three to eight factors were run and the optimal model order retained for analysis and comparison across sites was selected by examining several criteria simultaneously, including slope changes in the scree plot, Thurstone's criteria for factor separation mentioned above, and retaining only factors explaining $>5 \%$ of total variance. (Reyment and Joreskog, 1993) All factors with eigenvalues $>0.25$ were examined, a lower bound set less than Kaiser's rule of 1.0 to ensure that factors are not underextracted by the PCA (Hopke, 1982). Absolute rotated factor loadings values greater than 0.6 were classified as 'high' so as to be consistent with the correlation strength typically accepted as 'mapped' in bootstrap analysis of PMF results; absolute factor loading values between 0.4 and 0.6 were classified as 'moderate'. 


\section{E.2 PMF protocol}

\section{Modeling strategy and key model functions}

The US EPA PMF3 receptor model was applied to the central site monitoring PAH datasets (i.e., NAPS data at two urban sites and one background site) to identify major source types contributing to ambient PAH and quantify source type contributions at each site.

PMF offers several functional advantages over simpler factor analysis models, such as PCA, which assist more complex interpretation of factors as source types (Hopke, 2006). These include incorporating sample uncertainty, which permits data weighting to differ between species and between samples, and estimates of uncertainty on factor profiles, which allows comparisons with reference profiles to be made within uncertainty bounds.

PMF also directly quantifies factor contributions and this enables factor interpretation to extend beyond simplistic comparisons of factor profile: overall relative factor contribution can be compared with a priori expected contributions, such as available from inventory estimates, and seasonal and weekday/weekend trends can be compared with expected source type trends, such as seasonal versus year-round source releases.

Source apportionment by PMF modelling can be considered in two distinct stages: (i) modeling; and, (ii) interpretation.

The modeling stage determines a mathematical solution to the general source-receptor mass balance (Equation E.2-1) as an optimization problem. The interpretation stage requires analysis of this mathematical solution and interpretation of the model output, which are ultimately factors $(\boldsymbol{F})$ and their contributions $(\boldsymbol{G})$, in terms of physically meaningful source types and their relative contributions. 


\section{Equation E.2-1. General source-receptor mass balance.}

\section{matrix form}

$$
\boldsymbol{X}=\boldsymbol{G} \cdot \boldsymbol{F}+\boldsymbol{E}
$$

$\boldsymbol{X}=$ sample concentration matrix

$\boldsymbol{G}=$ factor contribution matrix

$\boldsymbol{F}=$ factor profile matrix

$\boldsymbol{E}=$ model residual matrix

\section{indexed form}

$$
x_{i j}=\sum_{k=1}^{p} g_{i k} f_{k j}+e_{i j}
$$

\footnotetext{
$x_{i j}=$ concentration species $j$ in receptor sample $i\left(\mathrm{ng} / \mathrm{m}^{3}\right)$

$g_{i k}=$ contribution of source factor $k$ to receptor sample $i$

$f_{k j}=$ concentration of species $j$ to source factor $k\left(\mathrm{ng} / \mathrm{m}^{3}\right)$

$e_{i j}=$ model residual species $j$ in receptor sample $i\left(\mathrm{ng} / \mathrm{m}^{3}\right)$
} 
The US EPA PMF3 model software greatly facilitates determination of a mathematical solution to the source-receptor mass balance by providing algorithms for the optimization problem alongside numerous sub-functions and model outputs intended to aid analysis of input data, execute model runs, and review model results. These are presented in flow chart form in Figure E.2-1 and are generally run from left to right.

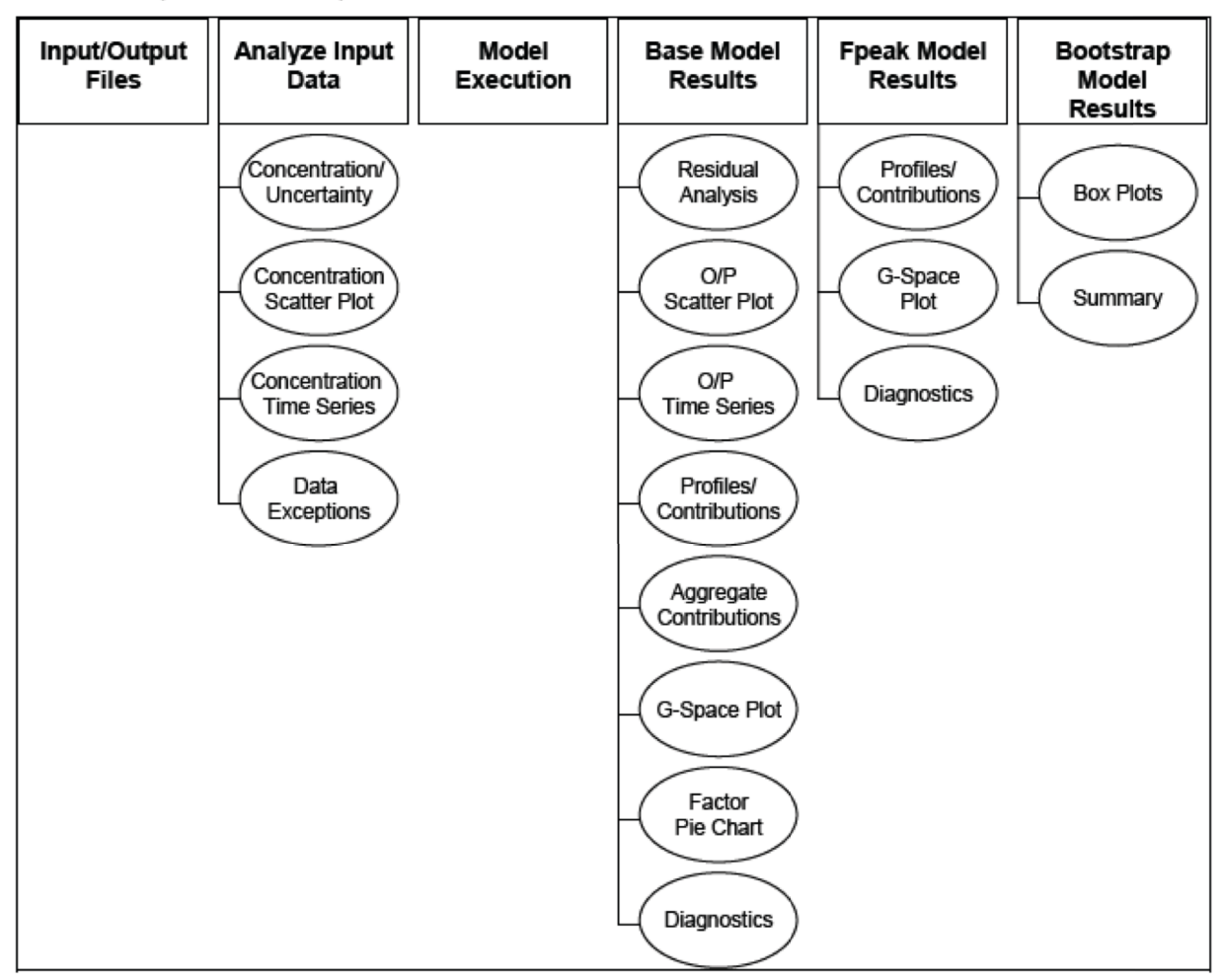

Note: Program sub-functions are generally run in order from left to right.

Figure E.2-1. Overview of US EPA PMF3 tasks and output (reproduced from US EPA, 2008).

Detailed description and user guidance for all sub-functions are left to the US EPA "Fundamentals and User Guide" (2008) and are further supplemented by Sonoma "Multivariate Receptor Modeling Workbook" (2008).

To clarify presentation of modeling protocol and discussion of results in this dissertation, however, key PMF outputs and sub-functions are described in brief below. 
$\underline{\text { PMF outputs and sub-functions }}$

\section{Factor profiles}

Receptor model factor profiles $(\boldsymbol{F})$ are presented as a concentration profile and a factor explained variance profile. The concentration (C) profile shows the relative species abundance in each factor, and can thus be considered a 'true profile' of concentration $\left(\mathrm{ng} / \mathrm{m}^{3}\right)$. The explained variance $(\mathrm{EV})$ profile shows the percentage $(\%)$ of modeled species mass explained by the factor and, as such, can be considered analogous to explained variance profiles obtained by simpler factor analysis models such as PCA.

It is important to note that both forms of the factor profiles are useful for interpretation of model factors as source types. The concentration profile can be compared with reference profiles (e.g., source sampling or other receptor modeling studies) and the explained variance profile will identify species whose variance is largely explained by the factor (i.e., variance presumed to be due to changes in source emissions at the receptor as a fundamental assumption of receptor modeling) and can be compared to information on 'characteristic species' for known source types that are relevant to a study area (e.g., source sampling studies, tracer species, and other receptor modeling studies, inclusive of PCA studies).

Since PMF provides output factor profiles for the base run solution, bootstrap run solution, and F-peak run solutions, the factor profiles are distinguished symbolically in this work by an appropriate prefix: base run concentration profile (F_C), bootstrap run concentration profile (BOOT_C), F-peak run concentration profile (Fpeak_C); analogously, base run explained variance profile (F_EV), bootstrap run explained variance profile (BOOT_EV), F-peak run explained variance profile (Fpeak_EV).

\section{Factor contribution trends}

The contributions $(\boldsymbol{G})$ of each factor to modeled mass are presented as a time-series plot (i.e., contribution of each factor to mass of each sample), trends in contribution, as well as in output data for analysis and reporting outside the model software. Factor contribution trends include annual, seasonal, and weekday/weekend and are presented within the software for direct review as box plots (i.e., median and interquartile range along with whiskers to the $5^{\text {th }}$ percentile and $95^{\text {th }}$ percentile). Each trend in factor contribution provides useful context for meaningful interpretation of the factors as source types. (US EPA, 2008)

Briefly, the annual trend plot should indicate relative similar median trend across the sampled time-series, since one of the fundamental assumptions made in time-series 
scoping is that the number of major source types or their emissions profile did not change significantly over the course of the modeled time series (i.e., no significant discontinuities in source mix or occurrence of technological changes such as emissions control technologies or regulations). Conversely, presence or absence of seasonality in factor contributions can be invaluable in corroborating factor identification (e.g., space heating source type reasonably expected to show increased contributions in fall/winter months versus spring/summer months; industry sources would reasonably be expected to show absence of seasonality where operations are year-round; traffic sources would typically show absence of seasonality or, at most, mild contribution increase in winter months due to increased emissions due to inefficiency and idling in cold weather conditions). Last, presence or absence of weekday/weekend trends can also help corroborate factor identification for source types whose contributions are known a priori to follow such a diurnal pattern (e.g., mobile source emissions from heavy duty vehicles and diesel engines reasonably expected to show increased contributions during the week due to commercial business traffic and commuting). An additional item recommended for use of the factor contribution trends is that a sufficient and comparable number of samples be available within each sample grouping (e.g., similar number of samples within each modeled year).

\section{Base model runs}

The initial set of PMF solutions to the source-receptor mass balance for the input dataset are termed 'base runs' within the PMF software and literature since they form the basis for subsequent model runs by bootstrapping analysis and F-peak analysis. Each 'base run' is an independent solution of the identical input dataset with the same model parameters excepting the 'seed' value that initially populates the $\boldsymbol{F}$ and $\boldsymbol{G}$ matrices.

The seed can be set as either 'fixed' or 'random'. With 'fixed seed' the user-specified value is used by PMF in a pseudo-random number generator to generate the $\boldsymbol{F}$ and $\boldsymbol{G}$ seed values for each run; the matrix seeds will differ from one base run to the next but will be generated in the same order if the model is re-run to permit replication of results or comparative analysis of solutions by the modeler. With 'random seed' the program will randomly select a starting seed for each model run and thus the $F$ and $G$ seeds will differ from one base run to the next and will not necessarily appear in the same model order on subsequent re-runs; random seed is thus recommended to ensure that the model converged to a global minimum rather than a local minimum. (US EPA, 2008) 


\section{Bootstrap model runs}

Any of the base run solutions (i.e., default selection is the 'best' base run, defined by PMF3 as that with minimum $Q$ value) can be further explored by bootstrap runs to determine the robustness and estimate uncertainty of that solution. This is by randomly selecting (i.e., re-sampling) non-overlapping blocks of an equal number of samples (i.e., referred to as 'block size'; details of the algorithm by which PMF3 suggests default block size are in Politis and White, 2004; also see Politis, 2003) from the original data set, and creating a new input data file comprised of the re-sampled blocks that has the same dimensions as the original data set (i.e., same sample size and species count, $n^{*} m$ ).

PMF then solves the source-receptor mass balance for the new 'bootstrapped' data set, resolving a set of 'bootstrap' factors in each bootstrap run. Each of these factors is compared with the factors in the base run and the bootstrap factor is 'mapped' to the base factor with which it correlates with most strongly; the correlation strength required for a bootstrap factor to be considered adequately 'mapped' to a base factor is set by the user (i.e., default value is 0.6). If a bootstrap factor is not sufficiently correlated with any base factor, that bootstrap factor is considered 'unmapped'. This process is repeated for as many bootstrap runs as the user specifies (i.e., 100 bootstrap runs are generally recommended to adequately determine robustness). EPA PMF then statistically summarizes all the bootstrapping runs in a summary table and graphical factor plot (i.e., box plots showing distribution of bootstrap factor profiles around the base run factor profile; for concentration and explained variance factor profiles). (US EPA, 2008)

\section{G-space plots and F-peak model runs}

G-space plots are pair-wise plots of the factor contributions to each sample. Ideally, plots will show scatter, indicating that factors are independent. However, the plots may also reveal well-defined 'edges' within the plot space rather than aligned with the axes.

The presence of any oblique edges indicates inter-dependence between the two factors that suggests rotational ambiguity in the base run solution. When the G-space analysis from the base run suggests rotational ambiguity, the model's F-peak function will rotate the base run solution by a user-input F-peak value to help the modeler assess whether a rotated base run solution would yield greater independence in factors, subject to the practical constraint of the rotated model solution remaining near the global minimum (i.e., recommendation is to limit changes in $\mathrm{Q}$ value from base run, $\mathrm{dQ}$, to tens of units).

Generally, positive F-peak values "sharpen the F matrix and smear the G matrix and negative F-peak values smear the F matrix and sharpen the G matrix”. (US EPA, 2008) 
The results from F-peak runs are presented in similar fashion to base runs and bootstrap runs (i.e., concentration and explained variance factor profiles and factor contributions).

\section{Input data treatment}

The PMF data treatment protocol for input concentrations $\left(\mathrm{x}_{\mathrm{ij}}\right)$ and uncertainties $\left(\mathrm{s}_{\mathrm{ij}}\right)$ reflected best practice guidelines (Reff et al, 2007; US EPA, 2000) and is summarized in Table E.2-1.

NAPS data showed three classes of lab-reported species concentrations: (i) species concentration above $\mathrm{DL}_{\mathrm{j}}$; (ii) species concentration below $\mathrm{DL}_{\mathrm{j}}$; and, (iii) species concentration missing. Species concentration data were treated for PMF input consistent with these three classes: (i) for concentrations above $\mathrm{DL}_{\mathrm{j}}, \mathrm{x}_{\mathrm{ij}}$ set equal to the lab-reported species sample concentration; (ii) for concentrations below $\mathrm{DL}_{\mathrm{j}}, \mathrm{x}_{\mathrm{ij}}$ replaced with one-half $\mathrm{DL}_{\mathrm{j}}$; and, (iii) for missing data, $\mathrm{x}_{\mathrm{ij}}$ replaced within PMF by the species concentration median. Species $\mathrm{DL}_{\mathrm{j}}$ were obtained from a laboratory QAQC dataset (Table E.2-2; Environment Canada, 2005).

As is typical of environmental datasets, the NAPS PAH datasets do not explicitly report the uncertainty of each sampled and reported concentration. Thus, effort was made to apply sample uncertainty $\left(\mathrm{s}_{\mathrm{ij}}\right)$ that would adequately capture, estimate and aggregate the uncertainties associated with laboratory analysis of PAH samples, thereby ensuring appropriate weighting of individual samples in the PMF receptor model. Species uncertainty data were calculated using the model's 'equation-based' subroutine formula available within US EPA PMF3, decided only after considerable literature review, as detailed in Appendix A.4.

Species concentration uncertainties $\left(\mathrm{s}_{\mathrm{ij}}\right)$ were consistent with species concentration data treatment: (i) for concentrations above $\mathrm{DL}_{\mathrm{j}}, \mathrm{s}_{\mathrm{ij}}$ equal to sum of squares of the concentration $\left(\mathrm{x}_{\mathrm{ij}}\right)$ multiplied by the species error fraction $\left(\mathrm{k}_{\mathrm{j}}\right)$ plus the $\mathrm{DL}_{\mathrm{j}}$; (ii) for concentrations below $\mathrm{DL}_{\mathrm{j}}$, $\mathrm{s}_{\mathrm{ij}}$ equal to $\mathrm{DL}_{\mathrm{j}}$ multiplied by (5/6); and, (iii) for missing data, $\mathrm{s}_{\mathrm{ij}}$ equal to four times the species concentration median. In this fashion, species concentrations below $\mathrm{DL}_{\mathrm{j}}$ or missing were assigned greater modeling uncertainty than species concentrations above $\mathrm{DL}_{\mathrm{j}}$, for which uncertainties were effectively scaled to concentration by the species error fraction. Species error fraction data were obtained from the appropriate laboratory methods document (Environment Canada, 2011f) and assigned greater relative uncertainty to highly volatile and more reactive species than to conservative species, varying from approximately $10 \%$ to $50 \%$.

Appendix E: Detailed modeling methodology 
Table E.2-1. Treatment protocols for speciated ambient receptor data used in PMF and CMB.

\begin{tabular}{|c|c|c|}
\hline $\begin{array}{l}\text { Lab-reported sample } \\
\text { concentration }\left(\mathrm{c}_{\mathrm{ij}}\right)\end{array}$ & $\begin{array}{l}\text { Treated sample concentration } \\
\left(\mathrm{PMF}, \mathrm{CMB}, \mathrm{x}_{\mathrm{ij}}\right)\end{array}$ & $\begin{array}{l}\text { Treated sampled uncertainty } \\
\left(\mathrm{PMF}, \mathrm{s}_{\mathrm{ij}} ; \mathrm{CMB}, \mathrm{u}_{\mathrm{ij}}\right)\end{array}$ \\
\hline$c_{i j}>D L_{j}$ & $\begin{array}{l}\frac{P M F}{x_{i j}=C_{i j}} \\
\frac{C M B}{x_{i j}=C_{i j}}\end{array}$ & $\begin{array}{l}\frac{P M F}{S_{i j}=\left[\left(\left(k_{j}\right)\left(x_{i j}\right)\right)^{2}+\left(D L_{j}\right)^{2}\right]^{(1 / 2)}} \\
\frac{\mathrm{CMB}}{\mathrm{u}_{\mathrm{ij}}=\left(\mathrm{k}_{\mathrm{j}}\right)\left(\mathrm{x}_{\mathrm{ij}}\right)+(1 / 3)\left(D L_{\mathrm{j}}\right)}\end{array}$ \\
\hline$c_{i j}<D L_{j}$ & $\begin{array}{l}\frac{P M F}{x_{i j}=(1 / 2) D L_{j}} \\
\frac{C M B}{x_{i j}=(1 / 2) D L_{j}}\end{array}$ & $\begin{array}{l}\frac{P M F}{s_{i j}=(5 / 6) D L_{j}} \\
\frac{C M B}{u_{i j}=D L_{j}}\end{array}$ \\
\hline $\begin{array}{l}\text { missing or invalid data } \\
c_{i j}=-999\end{array}$ & $\begin{array}{l}\frac{P M F}{x_{i j}=\text { median }\left(x_{i j}\right)} \\
\frac{\text { CMB }}{\text { no missing field sampling data }}\end{array}$ & $\begin{array}{l}\frac{P M F}{S_{i j}=(4)} \text { median }\left(x_{i j}\right) \\
\frac{\text { CMB }}{\text { no missing field sampling data }}\end{array}$ \\
\hline
\end{tabular}

Notes:

1. Method Detection Limit (DL).

For PMF, DL data taken from a 2005 QAQC dataset of NAPS data made available by Environment Canada; calculated as: $\mathrm{DL}_{\mathrm{j}}=\left(\mathrm{t}\right.$-statistic) ${ }^{*} \mathrm{SD}\left(\mathrm{x}_{\mathrm{i}}\right)$ where $\mathrm{SD}_{\mathrm{j}}=$ calculated as standard deviation of 7 replicate samples analysed for respective PAH species (due to outlier species sample concentrations, 5 samples used for ACE, FLU, PHEN; 6 samples used for FLT, PYR); t-value = Student-t test statistic at 95\% confidence interval, for 5-7 samples (4-6 degrees of freedom), dependent on species.

For $\mathrm{CMB}, \mathrm{DL}_{\mathrm{j}}$ data as reported by analyzing lab (i.e., 14-day field sampling period at nominal flow rate).

2. Error fraction $\left(\mathrm{k}_{\mathrm{j}}\right)$.

For PMF and CMB, error fraction data from Method 3.03/5.1, Appendix B - "Uncertainty estimates by species, not recovery corrected" (Environment Canada, 2011f). Error fraction data are not recovery corrected, consistent with NAPS and field sampling data. Species error fractions account for major sources of uncertainty in PAH sample preparation and measurement and are determined via: $k_{j}=k{ }^{*} R S D$ where RSD = relative standard deviation = $\mathrm{SD} /$ mean of 30 replicate samples; $\mathrm{k}=\mathrm{t}$-value for $95 \%$ confidence interval on 30 replicate samples $=2.0$ 
Table E.2-2. NAPS-sampled PAH detection limit and error fraction data (PMF modeled suite).

\begin{tabular}{|c|c|c|c|c|}
\hline PAH Species & Label & $\begin{array}{r}\text { MW } \\
\text { Grouping }\end{array}$ & $\begin{array}{r}\text { Method Detection } \\
\text { Limit }(M D L) \\
{\left[\mathrm{ng} / \mathrm{m}^{3}\right]}\end{array}$ & $\begin{array}{r}\text { error fraction }\left(\mathbf{k}_{\mathrm{j}}\right) \\
\text { [fraction] }\end{array}$ \\
\hline acenaphthylene & $\mathrm{ACY}$ & light & 0.001 & 48.7 \\
\hline acenaphthene & ACE & light & 0.002 & 19.8 \\
\hline fluorene & FLU & light & 0.003 & 25.7 \\
\hline anthracene & ANT & light & 0.002 & 27.1 \\
\hline phenanthrene & PHE & light & 0.004 & 10.9 \\
\hline 2-methyl-fluorene & MFLU & light & 0.004 & 27.6 \\
\hline fluoranthene & FLT & light & 0.002 & 9.0 \\
\hline pyrene & PYR & light & 0.001 & 9.2 \\
\hline 1-methyl-pyrene & MPYR & med & 0.001 & 15.2 \\
\hline benzo(a)fluorene & BaFLU & med & 0.002 & 17.1 \\
\hline benzo(b)fluorene & BbFLU & med & 0.002 & 24.0 \\
\hline benzo(g,h,i)fluoranthene & BghiFLT & med & 0.002 & 14.1 \\
\hline chrysene & $\mathrm{CHR}$ & heavy & 0.001 & 10.5 \\
\hline triphenylene & TRIP & heavy & 0.002 & 13.3 \\
\hline benzo(a)anthracene & BAA & heavy & 0.004 & 11.7 \\
\hline retene & RET & heavy & 0.005 & 36.7 \\
\hline benzo(a)pyrene & BAP & heavy & 0.003 & 12.5 \\
\hline benzo(e)pyrene & BEP & heavy & 0.002 & 10.4 \\
\hline benzo(b)fluoranthene & $\mathrm{BbFLT}$ & heavy & 0.003 & 11.3 \\
\hline benzo(k)fluoranthene & BkFLT & heavy & 0.003 & 15.2 \\
\hline benzo(g,h,i)perylene & BghiP & heavy & 0.004 & 14.4 \\
\hline indeno(1,2,3-cd)pyrene & IPYR & heavy & 0.003 & 16.5 \\
\hline
\end{tabular}

Notes:

1. Method Detection Limit (MDL) data taken from a 2005 QAQC dataset of NAPS data made available by Environment Canada; calculated as: $\mathrm{DL}_{\mathrm{j}}=$ (t-statistic) * $\mathrm{SD}\left(\mathrm{x}_{\mathrm{ij}}\right)$ where $\mathrm{SD}_{\mathrm{j}}=$ calculated as standard deviation of 7 replicate samples analysed for respective PAH species (due to outlier species sample concentrations, 5 samples used for ACE, FLU, PHE; 6 samples used for FLT, PYR); t-value = Student-t test statistic at $95 \%$ confidence interval, for 5-7 samples (4-6 degrees of freedom), dependent on species.

2. Error fraction $\left(\mathrm{k}_{\mathrm{j}}\right)$ data from Method 3.03/5.1, Appendix B - "Uncertainty estimates by species, not recovery corrected" (Environment Canada, 2011f). Error fraction data are not recovery corrected, consistent with NAPS and field sampling data. Species error fractions account for major sources of uncertainty in PAH sample preparation and measurement and are determined via: $\mathrm{k}_{\mathrm{j}}=\mathrm{k}^{*} \mathrm{RSD}$ where RSD = relative standard deviation = $\mathrm{SD} /$ mean of 30 replicate samples; $\mathrm{k}=\mathrm{t}$-value for $95 \%$ confidence interval on 30 replicate samples $=2.0$

3. Retene also known as 1-methyl-7-isopropylphenanthrene. 


\section{Model parameters and performance criteria}

Since PMF down-weights outliers (Hopke, 2003a), and because efforts were made to retain model power and adequate sample-to-sample variability, a conservative protocol was applied for excluding samples: samples were excluded only if they contained statistically extreme outlier species concentrations (i.e., higher or lower than three times IQR) and outlier status was corroborated by poor model performance for that sample date and exclusion would not meaningfully alter source profiles or trends in contribution.

Additional uncertainty can be assigned to individual species by categorizing a species as 'strong', 'weak', or 'bad' within PMF. The species categorization instructs the model to apply uncertainty to species samples, $\mathrm{s}_{\mathrm{ij}}$, as input for species categorized as 'strong', triple the input file sij value for species categorized as 'weak', and exclude a species from the model for species categorized as 'bad'. A convenient metric for deciding if a species warrants additional uncertainty is the species signal-to-noise ratio ( $\mathrm{SN}$ ), available within the model software, which summarily compares species concentration data with its associated uncertainty (Equation E.2-2). Guidelines for SN are to categorize species as 'strong' for $\mathrm{SN}>2$, 'weak' for $\mathrm{SN}$ between 0.2 and 2, and 'bad' for $\mathrm{SN}<0.2$ (Paatero and Hopke, 2003). However, since input data uncertainty estimates were judged sufficiently conservative and the species suite was relatively small, it was generally preferred to include species as 'strong', down-weighting species further only if warranted by consistently poor species fit. Last, additional modeling uncertainty can be added to all species within PMF using the model's C3 parameter; since the uncertainty estimates on the ambient PAH data were conservative and species-specific (Table E.2-2), additional modeling uncertainty was explored in sensitivity analysis but otherwise not applied.

Equation E.2-2. Species signal-to-noise ratio in PMF input data.

$$
S N_{j}=\sqrt{\frac{\sum_{i=1}^{n}\left(x_{i j}-s_{i j}\right)^{2}}{\sum_{i=1}^{n} s_{i j}^{2}}}
$$

$S N_{j}=$ signal-to-noise ratio for species $j$

$x_{i j}=$ concentration of species $j$ in receptor sample $i\left(\mathrm{ng} / \mathrm{m}^{3}\right)$

$s_{i j}=$ uncertainty of species $j$ in receptor sample $i\left(\mathrm{ng} / \mathrm{m}^{3}\right)$ 
PMF modeling followed best practice guidelines (US EPA, 2008; Sonoma, 2008) and recommendations in the comprehensive literature review of PMF studies completed by Reff et al (2007), applying a suite of performance criteria as summarized in brief below:

- Modeling explored a range of model orders, from 3 to 7 factors. 'Optimal' model order was chosen because it yielded good model performance but also, and primarily, to ensure the solution could be reasonably interpreted as physically meaningful (i.e., factor profiles and contributions interpretable as source types relevant to the study area).

- The solution was verified as unique and stable. This was determined by a solution with a narrow range in $\mathrm{Q}$ over multiple runs, indicating convergence to a local minimum, even with random starting seed.

- The solution was verified as having converged to a theoretical global minimum. This was determined by comparing $Q_{\text {robust }}$ to $Q_{\text {theory }}$, the value representing an idealized model solution, in which all input data would be perfectly explained and true uncertainty would be fully captured by the input uncertainty estimates. In this research, $Q_{\text {theory }}$ was calculated using the formulation presented in Sonoma (2008), which takes into account the reduced influence that down-weighted 'weak' species have in the PMF model, as can be seen in Equation E.2-3.

- The solution was verified as not being excessively influenced by outlier samples. This was determined by comparing $\mathrm{Q}_{\text {robust }}$ to $\mathrm{Q}_{\text {true }}$ values.

- The solution was verified for good fit of all modeled species. This was determined by examining goodness of fit for each modeled species for high $r^{2}$, low species residuals, and normal distribution of species residuals.

- The solution was verified to be reasonably free from rotational ambiguity. This was determined by good scatter in the inter-factor contribution plots (i.e., 'Gspace') and by exploring F-peak rotations of the solution.

Most critically, model solutions were verified for robustness via bootstrapping analysis (i.e., see summary of PMF3 subroutine above). This ensured that interpreted solutions were statistically representative and that factors were sufficiently distinct from each other to merit interpreting as a distinct source type, determined by bootstrap factors being mapped consistently to the same base factor. 
Equation E.2-3. Calculation of theoretical $Q$ for model validation (Sonoma, 2008).

$$
\begin{aligned}
& Q_{\text {theory }}=\left(n * m_{\text {species strong }}\right)+\frac{1}{3}\left(n * m_{\text {species weak }}\right)-n * p \\
& \mathrm{Q}_{\text {theory }}=\text { theoretical fitting value for the PMF optimization } \\
& \mathrm{n}=\text { number of modeled samples } \\
& \mathrm{m}_{\text {species strong }}=\text { number of fitting species characterized as 'strong' } \\
& \mathrm{n} \text { species weak }=\text { number of fitting species characterized as 'weak' } \\
& \mathrm{p}=\text { number of modeled factors }
\end{aligned}
$$

\section{Sensitivity analyses}

PMF modeling remains a source apportionment technique with several significant subjective elements and these are typically not always detailed in published work. In the interests of transparency (Reff et al, 2007; Watson et al, 2008), replication ${ }^{38}$ (Ioannidis et al, 2005; Moonesinghe et al, 2007) and advancing modeling practice for other practitioners, modeling applied a comprehensive suite of model checks, including:

- Model sensitivity to time series length. This sensitivity analysis was performed by comparing results of a model variant using approximately half the dataset (i.e., 2005-2010 samples) with the primary model that used the entire dataset (i.e., 2000-2010) and confirming consistency in model results (i.e., factor profiles and trends in factor contributions). Model results were also compared between early (i.e., 2001-2004) and later (i.e., 2005-2010) portions of the time series to confirm that significant source types remained relevant and consistent in factor profile and factor contribution trends over the entire scoped sampling period.

- Stability. The stability of the model solution was confirmed over a suitably large number of base runs (e.g., 100, 1000). Stability was checked using a fixed starting seed to populate the $\mathrm{F}$ and $\mathrm{G}$ matrices, run so that presented results could be easily replicated, and also with a random seed, which better verified that convergence was to a global minimum.

\footnotetext{
${ }^{38}$ In the broader scientific community there has been increasing awareness of "truth" of research findings (Ioannidis, 2005) and replication has been shown as one effective way to confirm research findings or, minimally, corroborate conclusions drawn from research findings; "true replication" is cited as only possible where data and methods are sufficiently well documented (Moonesinghe et al, 2007).
} 
- Robustness. As mentioned earlier, model robustness was determined via bootstrapping analysis but robustness results were then checked using a suitably large number of bootstrap runs (i.e., 1000 runs) at correlations that varied from 0.6 to as high as 0.9 . The different correlation coefficients were used to better distinguish more robust factors (i.e., retained good bootstrapping results even at very high correlation coefficients) from less robust factors (i.e., bootstrapping performance reduced at higher correlation coefficients).

- Solution rotations. Model solutions were considered sufficiently rotationally unique when they showed good scatter in paired factor contribution plots. However, additional model runs were made to assess sensitivity to F-peak rotation, which could indicate the possibility of further optimization of the model solution. The model solution was rotated using a range of negative through positive F-peak to ensure the rotated model solutions did not deviate excessively from the un-rotated solution (i.e., quantified by dQ remaining within tens of units; US EPA, 2008).

- Species exclusion. The exclusion of fitting species was also investigated in two model checks. First, a model variant was run excluding the species PHEN and comparing to results of the model that included it as a fitting species; PHEN was selected for investigation as it represented the most abundant species in the ambient data and so risked strongly influencing factor results or driving collinearity between factors, which would preclude using PMF-derived input profiles in a CMB receptor models (Chapter 5) since CMB is sensitive to factor collinearity (US EPA, 2004). Second, model variants were run with and without ACY, selected for investigation due to its very high volatility and related known sampling issues (US EPA, 1999; confirmed for the NAPS dataset by very high species uncertainty estimate, Table E.2-2).

In addition to the above model checks, more detailed sensitivity analyses were also performed, including:

- Sensitivity to input uncertainty estimates. While this research was able to source estimates of uncertainty for the PAH concentration data that reflected interspecies differences (Table E.2-2), appropriate uncertainty estimates are expected to remain a challenge to obtain for many ambient PAH datasets. Thus, the model's sensitivity to input uncertainty was explored by running model variants using a fixed error fraction $\left(\mathrm{k}_{\mathrm{j}}\right)$ for all modeled species, a limitation often seen in the literature. To establish boundaries on this sensitivity analysis, the error fraction was first fixed at two levels (i.e., 10\%, 25\%; all species modeled as 
'strong') and model performance metrics were reviewed to indicate whether uncertainty was too low or too high at these boundaries (i.e., $Q_{\text {robust }}>>Q_{\text {theory }}$, high outlier influence indicated by $\mathrm{Q}_{\text {true }} \gg \mathrm{Q}_{\text {robust }}$, large number of species-dates with high residuals generally indicate insufficient uncertainty; conversely, $\mathrm{Q}_{\text {robust }}<<$ $\mathrm{Q}_{\text {theory }}$, poor stability, poor robustness, indicate excessive uncertainty). A model variant was then run with a fixed error fraction set to the approximate median of species-specific error fractions listed in Table E.2-2 (i.e., 15\%) and results were compared to the model that incorporated the species-specific error fraction estimates available for the NAPS PAH dataset, indicating the degree to which model performance was reduced by the limitation of using a fixed uncertainty estimate. Last, model runs were performed applying additional 'in-model' uncertainty to the input data, via the C3 parameter (US EPA, 2008) and noting changes in model performance as well as stability of factor profiles and factor contribution trends.

- Sensitivity to outlier exclusion. Several variants of the input data were run excluding an increasing number of outliers, from no exclusion, to exclusion of samples with extreme outlier (i.e., greater than or less than $3 *$ IQR) concentrations of $\Sigma_{29} \mathrm{PAH}$, to exclusion of samples with extreme outliers in concentrations of $\Sigma_{29} \mathrm{PAH}$ and/or individual species. Model results with increasing degree of outlier exclusion were compared to note changes in model fit (i.e., indicated by species $\mathrm{r}^{2}$, high residuals, etc.; model fit will generally improve with outlier exclusion but stability, uniqueness and robustness can be adversely affected). In addition to model fit, factor profile patterns and trends in contributions were also examined for sensitivity to outlier exclusion and model results were examined for potential consequences of reduced modeling power (i.e., reduced sample size may result in lower model order than would otherwise be ideal to discriminate between source types) and loss of explanatory power (e.g., muting of seasonal trends for sources with otherwise strong seasonality). As a convenient indicator metric, the $\mathrm{CV}$ was also calculated for each variant of the input data to examine how changes in $\mathrm{CV}$ could relate to the degree to which outlier exclusion would benefit or disadvantage PMF model performance.

- Sensitivity of robustness to base run choice. This sensitivity analysis was not found discussed or explicitly presented in prior receptor modeling literature. Typically, the base run yielding the lowest fitting function value (minimum $\mathrm{Q}_{\text {robust }}$ ) is judged 'best' and forms the basis for bootstrapping analysis, thus it is the default selection for bootstrapping within PMF software. This research investigated whether PMF bootstrap results were sensitive to choice of base run and, if so, if this could affect the interpreted solution (i.e., since the median 
bootstrap factor profile pattern is retained for interpretation and publication) or its uncertainty estimate (i.e., since this is quantified from IQR of bootstrap runs).

- Sensitivity to seasonal stratification. Few source apportionment studies of PAH using PMF have seasonally stratified data as doing so would preclude the inherent advantages of modeling year-round data, such as significantly increased modeling power via increased sample size and improved ability to interpret source types via seasonal trends in factor contributions. In addition, several investigations of seasonality using PAH data and associated meteorology for the 2000-2010 period at Toronto, selected as the site with the greatest number of samples available, inclusive of seasonal differences in photochemical decomposition (Park, 2011; 2002) demonstrated that the data was amenable to PMF modeling without need for seasonal stratification (i.e., see also Appendix B.6). However, since fall/winter PAH data can be generally considered more amenable to receptor modeling (i.e., greater source-receptor conservation due to lower temperatures, greater atmospheric stability, and additional and/or more intense source emissions), a model variant was run with only fall/winter time series samples and the results were compared to the year-round PMF model with respect to model performance, factor characteristics and factor contributions.

While many of the above are 'best practice' and have been previously described in general terms (US EPA, 2008; Reff et al, 2007), several sensitivity analyses conducted and presented in this research have not been explicitly presented or discussed (e.g., sensitivity to input uncertainty, mapping of excluded species to corroborate factor interpretations; sensitivity of robustness to base run choice; sensitivity to seasonal stratification). The intent of including sensitivity analyses alongside source apportionment results was to emphasize the nuances involved in PMF receptor modeling and, by example, refine modeling practice so that reasonably experienced practitioners with reasonable familiarity of a given dataset would arrive at generally similar model factors, thereby limiting the more subjective aspects of PMF modeling to factor interpretation.

Appendix E: Detailed modeling methodology 


\section{Uncertainty on factor profiles}

Bootstrapping analysis (i.e., available via subroutine within PMF3 as summarized above) ensured a statistically representative PMF solution and provided a procedurally rational method of quantifying uncertainty on the PMF solution (Reff et al, 2007; Watson et al, 2008) as well as for application of profiles in related investigations at the intraurban scale (i.e., PMF as input to CMB receptor model, Chapter 5). PMF factor profiles retained for interpretation as source types referenced median bootstrap values (i.e., median species concentration, median species explained variance) thereby precluding interpretation of 'singular solutions', such as solutions with atypical values in the concentration profile that may yield a 'better', or lower, Q value but may not be statistically representative.

Uncertainty on profiles was estimated by the bootstrap IQR statistics (i.e., narrower IQR quantifying less uncertainty) following an uncertainty formula (Equation E.2-4) devised to distinguish 'characteristic' from 'non-characteristic' profile species (i.e., 'noncharacteristic species' defined as first quartile explained variance value less than $5 \% ; Q_{1}$ EV <5\%). Effectively, the majority of profile species were assigned uncertainty based on the IQR of the bootstrap runs and relatively few 'non-characteristic' species were assigned uncertainty based on the top-half of the IQR (i.e. $Q_{3}-Q_{2}$ ). This approach ensured meaningful uncertainty estimates for non-characteristic species (i.e., species with very low EV can be assigned near-zero factor profile concentrations in some bootstrap runs, possibly skewing Q1 concentration unrealistically below $\mathrm{DL}_{\mathrm{j}}$ ).

Equation E.2-4. Species uncertainty for PMF factor profiles.

$$
\begin{gathered}
u_{j}=\frac{\left(Q_{3_{j}}-Q_{1_{j}}\right)}{2} \text { where } E V_{Q 1_{j}} \geq 5 \% \\
u_{j}=\frac{\left(Q_{3_{j}}-Q_{2_{j}}\right)}{2} \text { where } E V_{Q 1_{j}}<5 \%
\end{gathered}
$$

\footnotetext{
$u_{j}=$ uncertainty for species $\mathrm{j}$ in PMF bootstrap concentration profile $\left(\mathrm{ng} / \mathrm{m}^{3}\right)$

$Q_{1 j}, Q_{2 j}, Q_{3 j}=$ first, second, third quartile for species $j$ in PMF bootstrap concentration profile (ng/ $\mathrm{m}^{3}$ )

$E V_{Q 1 j}=$ explained variance first quartile for species $j$ (PMF bootstrap profile; \%)
} 


\section{Interpretation of source types and calculation of source contributions}

Interpretation of model factors as physically meaningful source types is the primary challenge of PMF and requires knowledgeable application of several layers of evidence, inclusive of: corroboration of profiles with alternate receptor model results (e.g., PCA), factor enrichment in known source marker or source-characteristic species, comparison with reference profiles, evaluation of seasonal or other temporal trends in factor contributions, comparison of source apportionment estimates with emissions inventories, etc. (Reff et al, 2007; Brook et al, 2004) Inter-site comparison also helped identify similar source types or confirm site-unique local source types. Overall, source types were interpreted from model factors based on a preponderance of contextual evidence (Reff et al, 2007).

Relative factor contributions (\%) to ambient PAH were first calculated as 'total aggregate contributions', indicating source type contributions to total modeled PAH (i.e., sum of modeled PAH aggregated over all modeled samples; 2001-2010 and $\Sigma_{21} \mathrm{PAH}$ at urban sites; 2005-2010 and $\Sigma_{20} \mathrm{PAH}$ at background site). These provided the overall source apportionment results and were useful for comparison with emissions inventory data (i.e., emissions inventories similarly aggregate total PAH releases over a given time period) or with other receptor modeling results.

Since source contributions can vary significantly from sample-to-sample and some source types may show greater variability in relative contribution (e.g., due to dependence on wind direction), source type contributions to modeled PAH (\%) were also calculated for each sample and statistically summarized by median and IQR (i.e., adapting mean contribution calculation of Larsen and Baker, 2003). Thus, where most source apportionment papers provide a single source apportionment value (i.e., total aggregate contribution), this research also reported median and interquartile range for source contributions. Reporting the range of source contributions that can be expected at a receptor, via the IQR source contribution data, provides a more meaningful basis for comparison with other source apportionment results (Watson et al, 2008; Reff et al, 2007) and permits further research inquiries (e.g., Do some factors have greater range in their of source apportionment estimate and, if so, can this be reconciled with a priori expectations of their interpreted source type?). 


\section{E.3 CMB protocol}

\section{Modeling strategy}

CMB (i.e., US EPA CMB 8.2) was applied to the intraurban sampling campaign dataset (i.e., field data: Hamilton) to quantify source type contributions to ambient PAH and PAH toxicity across the urban area, permitting spatial analysis of differences in 'source mix' and provide better understanding of previously identified 'hot spots' of elevated PAH or PAH toxicity (Anastasopoulos et al, 2012).

\section{Model input - intraurban ambient data}

$\mathrm{CMB}$ model input requires the measured concentrations of species at receptors (i.e., ambient data, AD) and associated uncertainty. Species screening of the intraurban field sampling data for $\mathrm{CMB}$ modeling retained 13 species (Table E.3-1). Naphthalene (NAP) and Acenaphthylene (ACY) were not retained for PMF-CMB modeling due to incompatibility with NAPS data (i.e., NAP sampled as VOC rather than PAH) and known volatility-related sampling issues (i.e., negative sampling artifacts; US EPA, 1999); NAP and ACY were also sampled at concentrations below the MDL at nearly all sites in the winter campaign (Anastasopoulos et al, 2012), precluding their use for analysis of intraurban differences. Since initial PMF modeling was carried out for Hamilton alongside other sites (i.e., Toronto, background) a common species suite was selected to facilitate inter-site comparisons; this also omitted dibenz(ah)anthracene (DBA) due to a large number of missing and below $\mathrm{DL}_{\mathrm{j}}$ samples at the background site (US EPA, 2008; Sonoma, 2008; Reff et al, 2007). ${ }^{39}$

The winter campaign intraurban field sampling data proved highly amenable to CMB modeling. Most sampled species, from light to heavy MW, showed concentrations above $\mathrm{DL}_{\mathrm{j}}$ at majority of sites and sampled concentrations showed good intraurban variation (i.e. coefficient of variation, $\mathrm{CV}=0.52,0.57$ for $\Sigma_{\text {light }} \mathrm{PAH}, \Sigma_{\text {heavy }} \mathrm{PAH}$; Anastasopoulos et al, 2012). The winter period was also expected to offer good representation of local and seasonal source types due to inclusion of seasonal source emissions from space heating and more frequent southwesterly winds favouring transport of proximal expressway traffic sources (Anastasopoulos et al, 2012). These considerations were in addition to the

\footnotetext{
${ }^{39}$ There is health effects interest in DBA due to its high relative toxicity, which is greater than that of BAP. The Hamilton central site dataset had DBA in sufficient data quality to permit its inclusion in the PMF model; in the intraurban field dataset, however, DBA was sampled at levels below detection limit at all sites.
} 
inherent amenability of winter season PAH data to receptor modeling, including increased source-receptor conservation due to decreased atmospheric reactivity at lower temperatures and increased PM-associated heavy MW PAH concentrations due to seasonal meteorological factors such as reduced mixing height and higher wind speeds (Anastasopoulos et al, 2012; Sun et al, 2006).

By comparison, the summer period intraurban field dataset was not readily amenable to modeling due to concentrations being sampled below $\mathrm{DL}_{\mathrm{j}}$ at majority of sites for many heavy MW species (Anastasopoulos et al, 2012). The absence of sufficient sampled mass to capture intraurban variability in heavy MW PAH is a significant limitation since these species are required to adequately characterize traffic exhaust emissions (Park, 2011; Ravindra, 2008; Larsen and Baker, 2003) as well as to adequately estimate PAH toxicity (i.e., heavier MW species exhibit greater toxicity; Nisbet and Lagoy, 1992).

Table E.3-1. Field-sampled PAH species and detection limits.

\begin{tabular}{|c|c|c|c|c|}
\hline PAH Species & Label & $\begin{array}{r}\text { MW } \\
\text { group }\end{array}$ & $\begin{array}{r}\text { 14-day } \\
D L \\
{\left[\mathrm{ng} / \mathrm{m}^{3}\right]}\end{array}$ & $\begin{array}{r}\text { PMF- } \\
\text { CMB } \\
\text { modeling }\end{array}$ \\
\hline naphthalene & NAP & light & 0.124 & no \\
\hline acenaphthylene & $\mathrm{ACY}$ & light & 0.142 & no \\
\hline acenapthene & ACE & light & 0.134 & yes \\
\hline fluorene & FLU & light & 0.102 & yes \\
\hline anthracene & ANT & light & 0.099 & yes \\
\hline phenanthrene & PHE & light & 0.076 & yes \\
\hline fluoranthene & FLT & light & 0.068 & yes \\
\hline pyrene & PYR & light & 0.054 & yes \\
\hline chrysene & $\mathrm{CHR}$ & heavy & 0.110 & yes \\
\hline benzo(a)anthracene & BAA & heavy & 0.112 & yes \\
\hline benzo(a)pyrene & BAP & heavy & 0.189 & yes \\
\hline benzo(b)fluoranthene & $\mathrm{BbFLT}$ & heavy & 0.181 & yes \\
\hline benzo(k)fluoranthene & BkFLT & heavy & 0.178 & yes \\
\hline dibenz(a,h)anthracene & DBA & heavy & 0.215 & no \\
\hline benzo(g,h,i)perylene & BghiP & heavy & 0.207 & yes \\
\hline indeno(1,2,3-cd)pyrene & IPYR & heavy & 0.229 & yes \\
\hline
\end{tabular}

Note:

1. Detection Limit (DL) for intraurban field sampling dataset as reported by analyzing lab (i.e., 14day field sampling period at nominal flow rate).

The CMB AD input file used lab-reported concentrations $\left(\mathrm{ng} / \mathrm{m}^{3}\right)$ identical to prior published analysis (Anastasopoulos et al, 2012) with data treatment consistent between PMF and CMB receptor model (Table E.2-1). Species concentrations greater than their 
respective method detection limit were used as reported while species concentrations below detection limit were replaced with one-half their detection limit; the treatment of below detection limit sampled concentrations was done to avoid introducing 'false intraurban variability' for species with low concentrations, since inter-site differences in concentration could not be meaningfully distinguished below detection limits.

For all species concentrations greater than the method detection limit, CMB input uncertainties were calculated by applying Equation E.3-1. It can be clearly seen that the $\mathrm{CMB}$ uncertainty equation is sample- and species-specific and consistent with the general form of the sample uncertainty equation summarized by Reff et al (2007); namely $\mathrm{s}_{\mathrm{ij}}=f$ $\left(\mathrm{u}_{\mathrm{ij}}, \mathrm{DL}_{\mathrm{ij}}\right)$ with the first term estimating the analytical uncertainty on the sampled concentration $\left(\mathrm{u}_{\mathrm{ij}}=\mathrm{k}_{\mathrm{j}} * \mathrm{x}_{\mathrm{ij}}\right)$ and the second term representing uncertainty associated with the method detection limit (i.e., see also Appendix A.4). Species concentrations sampled below the method detection limit were assigned comparatively greater modeling uncertainty and set equal to the detection limit.

Equation E.3-1. Species uncertainty for concentrations above detection limit in CMB ambient data.

$$
\begin{aligned}
& \qquad u_{i j}=k_{j} * x_{i j}+\frac{D L_{j}}{3} \\
& \mathrm{u}_{i j}=\text { uncertainty on concentration of species } j \text { in receptor sample } i\left(\mathrm{ng} / \mathrm{m}^{3}\right) \\
& x_{i j}=\text { concentration of species } j \text { in receptor sample } i\left(\mathrm{ng} / \mathrm{m}^{3}\right) \\
& k_{j}=\text { error fraction of species } j \text { (fraction) } \\
& M D L_{j}=\text { lab detection limit for species } j\left(\mathrm{ng} / \mathrm{m}^{3}\right)
\end{aligned}
$$

Species detection limits $\left(\mathrm{DL}_{\mathrm{j}}\right)$ for the 14-day field samples were obtained from the analyzing laboratory and are shown in Table E.3-1. Species error fractions $\left(\mathrm{k}_{\mathrm{j}}\right)$ were again obtained from the appropriate laboratory methods document (Environment Canada, 2011f) and varied so that highly volatile and more reactive species were assigned greater relative uncertainty than more conservative species, as seen in Table E.2-2 (i.e., error fractions identical to those used in prior PMF receptor modeling of the NAPS central site data). Analogous to the sensitivity analysis investigated in PMF receptor modeling, a variant of the CMB model was run using a single fixed error fraction value for estimating input data uncertainty; this model variant confirmed generally reduced goodness of fit versus the more considered uncertainty estimate using species-specific error fraction values.

As a practical matter, AD input files ordered species by increasing MW for more meaningful review of factor profiles and analysis of model results. AD input files were prepared in Microsoft Excel and exported to text file format (i.e., txt) for input to US EPA CMB 8.2.

Appendix E: Detailed modeling methodology 
Model input - PMF-generated local source profiles

$\mathrm{CMB}$ model input requires profile data (PR), comprising a species mass fraction and an associated uncertainty on the species mass fraction, for source types expected to be contributing to the ambient data (AD) observations at the receptor.

During early iterations of PMF-CMB modeling, three variants of PMF factor concentration profile data, converted appropriately to mass fractions, were examined for suitability as CMB input source profiles. First, the CMB input PR was constructed from species factor concentrations taken from the 'best' PMF base run (i.e., lowest Q). The second CMB input PR variant was constructed from species factor concentrations again taken from the 'best' base run but with an emphasis of the concentrations of characteristic species (i.e., species that were enriched in the profile) versus other profile species; this was accomplished by applying a positive magnitude rotation to the PMF 'best' base run solution using the PMF model's F-peak function while ensuring that the resulting deviation of the rotated solution from the un-rotated solution, dQ, remained within tens of units and that rotational ambiguity in the G-space scatter factor pair plots was also maintained, following PMF best practice guidelines (US EPA, 2008; Sonoma, 2008). Last, the third PR variant was constructed from species factor concentrations taken from the median of bootstrap runs.

Of the three PR variants, the bootstrap median variant was considered a priori to be constructed from the most statistically representative and probable PMF solution (i.e., solution statistics are generated for repeated model runs, each run fitting factors to a bootstrap variant of the original time series dataset), and thus is generally recommended for 'best practice' interpretation and reporting of PMF results (Watson et al, 2008; Reff et al, 2007). Constructing the CMB PR from PMF bootstrap output also permitted estimating the source profile uncertainties required by CMB by referencing the analogous bootstrap IQR statistics, applying an appropriate uncertainty formula as presented in Equation E.3-2. Consistent with uncertainty reported for PMF results, Equation E.3-2 distinguished 'characteristic' from 'non-characteristic' profile species (i.e., 'noncharacteristic species' defined as first quartile explained variance value less than $5 \%$; $\mathrm{Q}_{1}$ $\mathrm{EV}<5 \%$ ) to ensure meaningful uncertainty estimates for non-characteristic species (i.e., species with very low EV can be assigned near-zero factor profile concentrations in some bootstrap runs, possibly skewing $\mathrm{Q}_{1}$ concentration unrealistically below $\mathrm{DL}_{\mathrm{j}}$ and thus exaggerating the uncertainty estimate).

In addition to these a priori expectations, preliminary PMF-CMB modeling was able to confirm that the PMF bootstrap model results yielded meaningful local source profiles for use in $\mathrm{CMB}$, showing consistently superior model fit via goodness of fit metrics and 
lower uncertainty on source contribution estimates. Thus, the third PR variant was retained for $\mathrm{CMB}$ modeling of the intraurban $\mathrm{PAH}$ dataset, converting the PMF bootstrap factor median concentrations $\left(\mathrm{ng} / \mathrm{m}^{3}\right)$ and their corresponding uncertainties (Equation E.3-2; $\mathrm{ng} / \mathrm{m}^{3}$ ) to mass fractions (i.e., dividing each factor's species mass concentration by the sum total of species mass concentrations in the factor).

Missing data in the CMB PR input file (i.e., species not modeled in CMB) were populated with "-99.000". As for preparation of the AD input files, PR input files ordered species by increasing MW for more meaningful review of factor profiles and analysis of model results (i.e., prepared in Microsoft Excel and exported to text file format).

Equation E.3-2. Species uncertainty for CMB source profiles.

$$
\begin{aligned}
& u_{j}=\frac{\left(Q_{3_{j}}-Q_{1_{j}}\right)}{2} \text { where } E V_{Q 1_{j}} \geq 5 \% \\
& u_{j}=\frac{\left(Q_{3_{j}}-Q_{2_{j}}\right)}{2} \text { where } E V_{Q 1_{j}}<5 \%
\end{aligned}
$$

\footnotetext{
$u_{j}=$ uncertainty for species $\mathrm{j}$ in PMF bootstrap concentration profile $\left(\mathrm{ng} / \mathrm{m}^{3}\right)$

$Q_{1 j}, Q_{2 j}, Q_{3 j}=$ first, second, third quartile for species $j$ in PMF bootstrap concentration profile (ng/m $/ \mathrm{m}^{3}$ )

$E V_{Q 1 j}=$ explained variance first quartile for species $j$ (PMF bootstrap profile; \%)
}

\section{Model parameters and performance criteria}

CMB model parameters were consistent with model guidelines (US EPA, 2004; Watson et al, 2004) and were as follows: iteration delta was set to 20, maximum source uncertainty was set to $20 \%$, the minimum source projection was set to 0.95 , the source elimination option was used, and the Britt and Luecke algorithm was not used.

The quality of model solutions was first characterized using goodness of fit metrics, with comparison to model guidelines (US EPA, 2004), as follows: $\mathrm{r}^{2} \geq 0.8$; chi $^{2}<2$; percent mass $80 \%-120 \%$. As an aggregate metric of fit, the model's "best fit" fitting measure (FM) function was used, defined as the weighted sum of squares of the individual $\mathrm{r}^{2}, \mathrm{chi}^{2}$, and percent mass goodness of fit metrics along with the number of estimable sources (US EPA, 2004); an equal weighting (1.0) was used for all four FM components. The FM metric permitted a convenient first point of comparison of model performance for competing model variants (e.g., PR variants) alongside comparison of factor source contribution estimates and their relative error (i.e., T-stat values; SCE/UNC). 
At sites with high $\operatorname{chi}^{2}(>2)$, the individual species concentrations calculated by the CMB model were reviewed (i.e., "Species concentration display" in "Main Report" model output) to verify how well individual AD species concentrations were modeled. This was done by reviewing residual/uncertainty ratio (RES/UNC) values for indications of a particular species consistently modeling poorly (i.e., poor RES/UNC at many sites); large positive RES/UNC values ( $>>2$ ) indicated the species was being over-estimated by one or more profiles contributing excess mass to the species while large negative RES/UNC values $(<<-2)$ indicated the species was being under-estimated and a relevant source may be missing. (Watson et al, 2004)

Additional CMB performance metrics (i.e., "Contribution by Species"; Modified Pseudo Inverse Matrix, MPIN) were reviewed to verify that the primary species driving each estimable factor in CMB correctly corresponded to that factor's characteristic species, as previously identified in PMF and used to guide interpretation of the factor as a PAH source type. (US EPA, 2004; Watson et al, 2004)

At each site, factor contribution estimates ("source contribution estimates", SCE) were reviewed against their associated uncertainty estimates by reviewing their corresponding T-stat values (i.e., T-stat $=\mathrm{SCE} /$ standard error where standard error $=$ one standard deviation), which reflect uncertainty in the ambient data, the source profiles as well as any collinearities among profiles. T-stat values greater than 2.0 are generally considered preferable (US EPA, 2004); however, since uncertainty estimates on PMF-derived local source profiles and the field-sampled ambient data were conservative (i.e., uncertainty on some PR species approaching or greater than one-half the magnitude of the concentration; AD referencing species-specific error estimates ranging from approximately $10 \%$ to $50 \%$, Table $2-1$ ), SCE were considered well resolved for T-stat $>1.5$ (i.e., $\mathrm{SCE}>1.5 * \mathrm{UNC}$ ), consistent with the upper limit of the uncertainty estimates on the local source profiles. Conversely, T-stat $<1.5$ were considered poor and SCE results at such sites were treated with appropriate care when inferring spatial trends in source contributions (i.e., T-stat of 1.0 indicates SCE could not be reliably distinguished from zero after applying uncertainty estimate).

Collinearities between CMB fitting factor profiles are generally not desired but manifest only in relation to a specific ambient data set (i.e., could arise at one sampling site but not at another); thus, CMB factor collinearity could not be reliably predicted a priori by using tests of factor similitude (e.g., such as by Pearson's r or otherwise; Watson et al, 2004). Instead, collinearities between non-estimable sources (i.e., SCE value calculated but reported by the model as being outside the estimable space) were reviewed for frequency of occurrence; source profile pairs appearing as a linear combination at many sites indicated the need for more distinct input source profiles. 


\section{References}

Allen JO, Dookeran NM, Smith KA, Sarofim AF, Taghizadeh K, Lafleur AL. (1996) Measurement of polycyclic aromatic hydrocarbons associated with size-segregated atmospheric aerosols in Massachusetts. Environmental Science and Technology, 30, 1023-1031.

American Iron and Steel Institute (2012). "Coal utilization in the steel industry - coke making”. Available online:

http://www.steel.org/en/Making\%20Steel/How\%20Its\%20Made/Processes/Processes\%20 Info/Coal\%20Utilization\%20in\%20the\%20Steel\%20Industry.aspx

Amodio M, Andriani E, de Gennaro G, Di Gilio A, Ielpo P, Placentino CM, Tutino M. (2013) How a steel plant affects air quality of a nearby urban area: a study on metals and PAH concentrations. Aerosol and Air Quality Research, 13, 497-508.

Anastasopoulos AT, Wheeler AJ, Karman D, Kulka RH. (2012) Intraurban concentrations, spatial variability and correlation of ambient polycyclic aromatic hydrocarbons (PAH) and PM2.5. Atmospheric Environment, 59, 272-283.

Anastassopoulos A, Nguyen S, Xu X. (2004) On the use of the HYSPLIT model to study air quality in Windsor, Ontario, Canada. Environmental Informatics Archives, 2, 375-383.

Agency for Toxic Substances and Disease Registry (ATSDR). (1995). Toxicological Profile for Polycyclic Aromatic Hydrocarbons. US Department of Health and Human Services, Public Health Service.

Agency for Toxic Substances and Disease Registry (ATSDR). (1996) ToxFAQ on polycyclic aromatic hydrocarbons. US Department of Health and Human Services, Public Health Service.Available at: http://www .atsdr.cdc.gov/tfacts69.pdf. (accessed 10 October 2007)

Aries, E., D. Ciaparra, M.J. Schofield, D.R. Anderson, N. Schofield, and R. Fisher. (2007) Fugitive and stationary emissions from coke plants and impact on the local ambient air quality. In The Year-Book of the Coke Oven Manager's Association, 2007, 136-197. London, UK: Coke Oven Manager's Association.

Aries E, Ciaparra D, Harrad SJ, Fisher R, Anderson DR. (2009) Characterisation of PAHs in the ambient air of steelworks. Conference presentation. Birmingham, POPs Network conference, 23rd April 2009, 19 slides.

Baek S.O., Field A., Goldstone M.E., Kirk P.W., Lester J.N., Perry R., (1991a). A review of atmospheric polycyclic aromatic hydrocarbons: sources, fate and behavior. Water, Air and Soil Pollution, 60, 279-300.

Baek SO, Goldstone ME, Kirk PWW, Lester JN, Perry R. (1991b) Methodological aspects of measuring polycyclic aromatic hydrocarbons in the urban atmosphere. Environmental Technology, 12, 107-129.

Baek, SO, Goldstone ME, Kirk PWW, Lester JN, Perry R. (1991c) Phase distribution and particle size dependency of polycyclic aromatic hydrocarbons in the urban atmosphere. Chemosphere, 22 (5/6), 503-520.

Battelle. (2007) Final report on source apportionment of data from four IADN and nearby speciated PM sites. Report to Great Lakes Commission, Ann Arbor, Michigan. July 31, 2007. 
Baxter MJ, Beardah CC, Cool HEM, Jackson CM. (2005) Mathematical Geology, 37 (2).

Baxter MJ, Freestone IC. (2006) Log-ratio compositional data analysis in archaeometry. Archaeometry, 48 (3), 511-531.

Baxter MJ. Mathematics, statistics and archaeometry: the past 50 years or so. (2008) Archaeometry, 50 (6), 968-982.

Benner BA Jr, Wise SA, Currie LA, Klouda GA, Klinedinst DB, Zweidinger RB, Stevens RK, Lewis CW. (1995) Distinguishing the contributions of residential wood combustion and mobile source emissions using relative concentrations of dimethylphenanthrene isomers. Environmental Science and Technology, 29 (9), 2382-2389.

Berg T, Royset O, Steinnes E. (1994) Principal component analysis for trace elements and main components in precipitation falling on Norway. Environmental Monitoring and Assessment, 31, 259-273.

Blanchard P, Brook JR, Brazal P. (2002) Chemical characterization of the organic fraction of atmospheric aerosol at two sites in Ontario, Canada. Journal of Geophysical ResearchAtmospheres, 107, 8348-8358.

Borm PJA, Donaldson K. (2007) An introduction to particle toxicology: from coal mining to nanotechnology. In: Particle Toxicology. Editors: Donaldson K, Borm P. Boca Raton, FL, USA: CRC Press.

Brinkman G, Vance G, Hannigan MP, Milford JB. (2006) Use of synthetic data to evaluate positive matrix factorization as a source apportionment tool for PM2.5 exposure data, Environmental Science and Technology, 40 (6), 1892-1901, March 15, 2006.

Brook JR, Vega E, Watson JG. (2004) In: Particulate Matter Science for Policy Makers-a NARSTO Assessment, Part 1. Hales JM, Hidy GM (Editors). Cambridge University: London, U.K., 235-281

Brook JR, Graham L, Charland JP, Cheng Y, Fan X, Lu G, Li SM, Lillyman C, MacDonald P, Caravaggio G, MacPhee JA. (2007a) Investigation of the motor vehicle exhaust contribution to primary fine particle organic carbon in urban air. Atmospheric Environment, 41, 119-135.

Brook JR, Poirot RL, Dann TF, Lee PKH, Lillyman CD, Ip T. (2007b) Assessing Sources of PM2.5 in Cities Influenced by Regional Transport. Journal of Toxicology and Environmental Health, 70 (3), 191-199.

Bzdusek PA, Christensen ER, Li A, Zou Q (2004) Source apportionment of sediment PAHs in Lake Calumet, Chicago: application of factor analysis with nonnegative constraints. Environmental Science and Technology, 38, 97-103.

Cai C, Zhang Y, Reid BJ, Nunes LM. (2012) Carcinogenic potential of soils contaminated with Polycyclic Aromatic Hydrocarbons (PAHs) in Xiamen metropolis, China. J. Environ. Monit., 14, 3111-3117.

California Office of Environmental Health Hazard Assessment (Cal/EPA). (1993) Benzo(a)pyrene as a toxic air contaminant. Part B. Health effects of benzo(a)pyrene. Air Toxicology and Epidemiology Section, Berkeley, CA.

California Office of Environmental Health Hazard Assessment (Cal/EPA). (2002) Air Toxics Hot Spots Program risk assessment guidelines - Part II: Technical support document for describing available cancer potency factors. 
Cao, R. (1999) An overview of bootstrap methods for estimating and predicting in time series. Test , 8 (1), 95-116.

Cavalcante RM, Sousa FW, Nascimento RF, Silveira d ER, Viana RB. (2012) Influence of urban activities on polycyclic aromatic hydrocarbons in precipitation: Distribution, sources and depositional flux in a developing metropolis, Fortaleza, Brazil. Science of the Total Environment, 414, 287-292.

Canadian Environmental Protection Act (CEPA). (1999) Priority substances list assessment report - polycyclic aromatic hydrocarbons. Environment Canada, Health Canada.

Ciaparra D, Aries E, Booth M-J, Anderson DR, Almeida SM, Harrad S. (2009) Characterisation of volatile organic compounds and polycyclic aromatic hydrocarbons in the ambient air of steelworks. Atmospheric Environment, 43, 2070-2079.

Clean Air Hamilton. (2011) Air quality progress report 2010. Available online: http://cleanair.hamilton.ca/downloads/CAH\%20Report\%202010\%20Final\%20Copy.pdf (accessed February 2012)

Collins JF, Brown JP, Alexeeff GV, Salmon AG. (1998) Potency Equivalency Factors for some Polycyclic Aromatic Hydrocarbons and Polycyclic Aromatic Hydrocarbon derivatives. Regulatory Toxicology and Pharmacology 28, 45-54.

Daisey JM, Leyko MA, Kneip TJ. (1979). Source identification and allocation of polynuclear aromatic hydrocarbon compounds in the New York City aerosol: methods and applications. In: Jones, P.W., Leber, P. (Eds.), Polynuclear Aromatic Hydrocarbons. Ann Arbor Science: Ann Arbor, 201-215.

Daisey JM, Cheney JL, Lioy PJ. (1986) Profiles of organic particulate emissions from air pollution sources: Status and needs for receptor source apportionment modeling. Journal of the Air Pollution Control Association, 36 (1), 17-33.

Dann T. (1997) Ambient air measurements of Polycyclic Aromatic Hydrocarbons (PAH), Polychlorinated Dibenzo-p-Dioxins (PCDD) and Polychlorinated Dibenzofurans in Canada (1987-1997). Report AAQD 97-3.

Dickhut RM, Canuel EA, Gustafson KE, Liu K, Arzayus KM, Walker SE, Edgecombe G, Gaylor MO, Macdonald EH. (2000) Automotive Sources of Carcinogenic Polycyclic Aromatic Hydrocarbons Associated with Particulate Matter in the Chesapeake Bay Region. Environmental Science and Technology, 34, 4635-4640.

Dimashki M, Lim L, Harrison R, Harrad S. (2001) Temporal trends, temperature dependence, and relative reactivity of atmospheric polycyclic aromatic hydrocarbons. Environmental Science and Technology, 35, 2264-2267.

Dockery DW, Pop CA, Xu X, Spengler JD, Ware JH, Fay ME, Ferris BG, Speizer FE. (1993) An association between air pollution and mortality in six U.S. cities. N. Engl. J. Med., 329, 1753-1759.

Duvall R, Olson D, Norris G, McDow S, Williams R. (2007) Source apportionment - Preliminary ambient findings. Presentation made to DEARS Stakeholder Meeting, October 24, 2007, Detroit, MI, 26 slides.

Dvorska A, Lammel G, Klanova J. (2011) Use of diagnostic ratios for studying source apportionment and reactivity of ambient polycyclic aromatic hydrocarbons over Central Europe. Atmospheric Environment, 45, 420-427. 
Environment Canada. (2000) (Authored by Cianciarelli D and Morcos R.) Characterization of organic compounds from selected residential wood stoves and fuels. Emissions Research and Measurement Division, Environmental Technology Advancement Directorate. Environment Technology Centre, Environment Canada. ERMD Report 2000-01.

Environment Canada. (2004) National Air Pollution Surveillance (NAPS) Network, air quality in Canada: 2001 summary and 1990-2001 trend analysis. Report EPS 7/AP/36 (May 2004).

Environment Canada. (2005) Quality assurance quality control (QAQC) dataset with replicate analysis using US EPA Method TO-13A of NAPS PAH samples for purposes of calculating species detection limits. Received from Claire Austin in hard copy in April 2012.

Environment Canada. (2006) Toxic substances list - updated Schedule 1 as of December 27, 2006. Available online at http://www.ec.gc.ca/CEPARegistry/subs_list/Toxicupdate.cfm. (accessed 20 October 2007)

Environment Canada. (2007a) NAPS annual raw data. Available online at: http://www.etccte.ec.gc.ca/NapsAnnualRawData. (login access arranged with Environment Canada, Environmental Technology Centre)

Environment Canada. (2007b) Management of toxic substances - substance detail - PAHs. (document on the internet) Available at: http://www.ec.gc.ca/TOXICS/EN/detail.cfm?par_substanceID=14\&par_actn=s1

Environment Canada. NPRI online data search. (2007c) (searchable database available on the internet) Available at: http://www.ec.gc.ca/pdb/querysite/query_e.cfm

Environment Canada. (2007d) NPRI-listed CEPA-toxic substances in Canada. (document on the internet, accessed 15 October 2007) Available at:

http://www.ec.gc.ca/pdb/npri/2003N_Overview/Summary2001/NO_SumFinal2001_4_e. $\mathrm{cfm}$

Environment Canada. (2007e) NPRI - the four new groupings of ATH substances - substancespecific context polycyclic aromatic hydrocarbons. (document on the internet, accessed 20 October 2007) Available at: http://www.ec.gc.ca/pdb/npri/npri_pah_e.cfm

Environment Canada. (2007g) Tour of Environmental Technology Centre NAPS labs and conversation with technician, 7 June 2007.

Environment Canada. (2010) Criteria Air Contaminants (CACs) Technical Source Guide for Reporting to the National Pollutant Release Inventory. Online at: http://www.ec.gc.ca/inrp-npri/default.asp?lang=En\&n=3B695DF5$1 \&$ offset $=10 \&$ toc $=$ show

Environment Canada. (2011a) Toxic Substances List - Schedule 1. Available online: http://www.ec.gc.ca/lcpe-cepa (accessed September 2011)

Environment Canada. (2011b) National Pollutant Release Inventory (NPRI). Ottawa, Ontario. Available online: http://www.ec.gc.ca/pdb/websol/querysite/query_e.cfm (accessed February 2012)

Environment Canada. (2011c) National Air Pollutant Surveillance (NAPS) Network, annual raw data. Ottawa, Ontario. Available online: http://www .etccte.ec.gc.ca/NapsAnnualRawData (accessed June 2011) 
Environment Canada, 2011d. Canadian climate normals, 1971-2000. Available online (searchable database): http://www.climate.weatheroffice.gc.ca/climate_normals (accessed May 2011)

Environment Canada. (2011e) Historical Climate Data, 2000-2010. Environment Canada, 20002010. Available online (searchable database): http://climate.weather.gc.ca/advanceSearch/searchHistoricData_e.html [accessed: 2011]

Environment Canada. (2011f) Method 3.03/5.1, Appendix B, "Uncertainty estimates by species, not recovery corrected".

Environment Canada. (2011g) Detailed Substance Report for BaP at U.S. Steel Canada Inc. Hamilton Works (NPRI ID 2984) "Reporter comments (releases): Ironmaking (blast Furance) and Steelmaking (Basic Oxygen Furnace) were not operating in 2011." Available online: http://www.ec.gc.ca/inrp-npri/ (accessed 16 July 2013)

Environment Canada. (2012) National Pollutant Release Inventory (NPRI) Toolbox. Online at: http://www.ec.gc.ca/inrp\%2Dnpri/default.asp?lang=En\&n=65A75CDF-1

Environmental Working Group (EWG). (2007) Human toxome project. (document on the internet, accessed 15 October 2007) Available at:

http://www.bodyburden.org/chemicals/chemical_classes.php?class=Polyaromatic+hydroc arbons+(PAHs)

Equilibrium Environmental Inc. (2006) Potency equivalency factors for carcinogenic polycyclic aromatic hydrocarbons. Prepared under contract to: URS Canada Inc., Final Report to Health Canada. March 31, 2006. Contract \#4500112430.

Fang G-C, Wu Y-S, Chen J-C, Chang C-N, Ho T-T. (2006) Characteristic of polycyclic aromatic hydrocarbon concentrations and source identification for fine and coarse particulates at Taichung Harbor near Taiwan Strait during 2004-2005. Science of the Total Environment, 366, 729-738.

Fine PM, Cass GR, Simoneit BRT. (2001) Chemical Characterization of Fine Particle Emissions from Fireplace Combustion of Woods Grown in the Northeastern United States. Environmental Science and Technolology, 35, 2665-2675.

Fine PM, Cass GR, Simoneit BRT. (2002) Chemical Characterization of Fine Particle Emissions from the Fireplace Combustion of Woods Grown in the Southern United States. Environmental Science and Technology, 36, 1442-1451.

Fischer PH, Hoek G, van Reeuwijk H, Briggs DJ, Lebret E, van Wijnen JH, Kingham S, Elliott PE. (2000) Traffic-related differences in outdoor and indoor concentrations of particles and volatile organic compounds in Amsterdam. Atmospheric Environment, 34, 37133722 .

Fraser MP, Buzcu B, Yue ZW, McGaughey GR, Desai NR, Allen DT, Seila RL, Lonneman WA, Harley RA. (2003a) Separation of fine particulate matter emitted from gasoline and diesel vehicles using chemical mass balancing techniques. Environmental Science and Technology, 37 (17, Sep 1), 3904-3909.

Fraser MP, Yue ZW, Buzcu B. (2003b) Source apportionment of fine particulate matter in Houston, TX, using organic molecular markers, Atmospheric Environment, 37 (15, May), 2117-2123. 
Fraser MP, Cass GR, Simoneit BRT. (1998) Gas-phase and particle-phase organic compounds emitted from motor vehicle traffic in a Los Angeles roadway tunnel. Environmental Science and Technology, 32, 2051-2060.

Fujita EM, Zielinska B, Arnott WP, Campbell DE, Reinhart L, Sagebiel JC, Chow JC. (2005) Gasoline/Diesel PM Split Study: Source and Ambient Sampling, Chemical Analysis, and Apportionment Phase. Desert Research Institute, prepared for National Renewable Energy Laboratory.

Gaga EO. (2004) Investigation of Polycyclic Aromatic Hydrocarbon (PAH) deposition in Ankara. $\mathrm{Ph} . \mathrm{D}$. thesis, Middle East Technical University. Ankara, Turkey.

Galarneau E, Makar PA, Mourad S, Diamond ML. (2007) Estimation of atmospheric emissions of six semi-volatile polycyclic aromatic hydrocarbons (PAHs) in southern Canada and the USA by use of an emissions processing system. Environmental Science Technology, 41, 4205-4213.

Galarneau E. (2008) Source specificity and atmospheric processing of airborne PAHs: Implications for source apportionment. Atmospheric Environment, 42, 8139-8149.

Gannon University. (2007) Evaluation of the extent and transport capabilities of atmospheric polycyclic aromatic hydrocarbons in the Lake Erie watershed, Final Report. Prepared for: The Great Lakes Commission Great Lakes Air Deposition Program.

Gertler AW, Gillies JA, Pierson WR. (2000) An assessment of the mobile source contribution to PM10 and PM2.5 in the United States. Water, Air, and Soil Pollution, 123, 203-214.

Gertler AW, Gillies JA, Pierson WR, Rogers CF, Sagebiel JC, Abu-Allaban M, Coulombe W, Tarnay L, Cahill TA. (2002) Real-World Particulate Matter and Gaseous Emissions from Motor Vehicles in a Highway Tunnel. In: Emissions from Diesel and Gasoline Engines Measured in Highway Tunnels. Health Effects Institute Research Report, Number 107, January.

Gigliotti CL, Dachs J, Nelson ED, Brunciak PA, Eisenreich SJ. (2000) Polycyclic aromatic hydrocarbons in the New Jersey coastal atmosphere. Environmental Science and Technology, 34, 3547-3554.

Gold DR, Litonjua AA, Zanobetti A, Coull BA, Schwartz J, MacCallum G, Verrier RL, Nearing BD, Canner MJ, Suh H, Stone PH. (2005) Air pollution and ST-segment depression in elderly subjects. Environmental Health Perspectives, 113, 883-887.

Grahame TJ, Schlesinger RB. (2007) Health effects of airborne particulate matter: do we know enough to consider regulating specific particle types or sources? Inhalation Toxicology, $19,457-481$.

Gullett BK, Touati A, Hays MD. (2003) PCDD/F, PCB, HxCBz, PAH, and PM Emission Factors for Fireplace and Woodstove Combustion in the San Francisco Bay Region. Environmental Science and Technology, 37, 1758-1765.

Guo H, Lee S, Ho K, Wang X, Zou S. (2003) Particle-associated polycyclic aromatic hydrocarbons in urban air of Hong Kong. Atmospheric Environment, 37 (38), 5307-5317.

Guo H, Wang T, Simpson IJ, Blake DR, Yu XM, Kwok YH, Li YS. (2004) Source contributions to ambient VOCs and $\mathrm{CO}$ at a rural site in eastern China. Atmospheric Environment, 38, 4551-4560. 
Hanedar A, Alp K, Kaynak B, Avsar E. (2014) Toxicity evaluation and source apportionment of Polycyclic Aromatic Hydrocarbons (PAHs) at three stations in Istanbul, Turkey. Science of the Total Environment, 488-489, 437-446.

Harrison RM, Smith DJT, Luhana L. (1996) Source Apportionment of Atmospheric Polycyclic Aromatic Hydrocarbons Collected from an Urban Location in Birmingham, U.K. Environmental Science and Technology, 30, 825-832.

Harrison RM, Tilling R, Callen Romero MS, Harrad S, Jarvis K. (2003) A study of trace metals and polycyclic aromatic hydrocarbons in the roadside environment. Atmospheric Environment, 37, 2391-2402.

Hays MD, Smith ND, Kinsey J, Dong Y, Kariher P. (2003) Polycyclic aromatic hydrocarbon size distributions in aerosols from appliances of residential wood combustion as determined by direct thermal desorption - GC/MS. Journal of Aerosol Science, 34, 1061-1084.

Health Canada. (2006a) Health effects of air pollution. (document on the internet, updated 2006 May 16, accessed 2008 January 31) Available from: http://www.hc-sc.gc.ca/ewhsemt/air/out-ext/effe/health_effects-effets_sante_e.html

Henderson SB, Beckerman B, Jerrett M, Brauer M. (2007) Application of land use regression to estimate long-term concentrations of traffic-related nitrogen oxides and fine particulate matter. Environmental Science and Technology, 41, 2422-2428.

Heo, J-B, Hopke PK, Yi S-M. (2009) Source apportionment of $\mathrm{PM}_{2.5}$ in Seoul, Korea. Atmos. Chem. Phys., 9, 4957-4971.

Hinds WC. (1999) Aerosol technology: properties, behaviour, and measurement of airborne particles. Wiley-Interscience, 2nd edition.

Hopke PK. (1982) Comments on "Trace Element Concentrations in Summer Aerosols at Rural Sites in New York State and Their Possible Sources' by P. Parekh and L. Husain and 'Seasonal Variations in the Composition of Ambient Sulfur-Containing Aerosols" by R. Tanner and B. Leaderer, Atmospheric Environment, 16, 1279-1280.

Hopke PK. (2000) Workshop on UNMIX and PMF as applied to PM2.5. 14-16 February 2000, U.S. EPA, RTP, NC. Final Report. June 2000.

Hopke PK. (2003a) Recent developments in receptor modeling. J. Chemometrics, 17, 255-265.

Hopke PK, Ramadan Z, Paatero P, Norris GA, Landis MS, Williams RW, Lewis CW. (2003b) Receptor modelling of ambient and personal exposure samples: 1998 Baltimore Particulate Matter Epidemiology-Exposure Study. Atmospheric Environment, 37, 32893302.

Hopke PK. (2004) A Guide to Positive Matrix Factorization. Center for Air Resources Engineering and Science, Clarkson University. Potsdam, NY.

Hopke, PK. (2006) Utilizing meteorological data to identify local sources. Center for Air Resources Engineering and Science, Clarkson University. Potsdam, NY.

Hopke PK, Ito K, Mar T, Christensen WF, Eatough DJ, Henry RC, Kim E, Laden F, Lall R, Larson TV, Liu H, Neas L, Pinto J, Stolzel M, Suh H, Paatero P, Thurston GD. (2006) PM source apportionment and health effects: 1. Intercomparison of source apportionment results. Journal of Exposure Science and Environmental Epidemiology, 16, 275-286. 
Integaire. (2005) Case study submission form - how to measure PAH? (document on the internet, accessed 18 January 2007) Available at:

http://www.integaire.org/databasenew/topic_templates/uploaded/view_topic.php?id=418 $\& c=\& m=0$

Ioannidis JPA (2005) Why Most Published Research Findings Are False. PLoS Med, 2 (8), 124. doi: 10.1371/journal.pmed.0020124

Ito K, Xue N, Thurston G. (2004) Spatial variation of PM2.5 chemical species and sourceapportioned mass concentrations in New York City. Atmospheric Environment, 38, $5269-5282$.

Jaeckels JM, Bae M-S, Schauer JJ. (2007) Positive matrix factorization (PMF) analysis of molecular marker measurements to quantify the sources of organic aerosols. Environmental Science and Technology, 41 (16), 5763-5769.

Jang E, Alam MS, Harrison RM. (2013) Source apportionment of polycyclic aromatic hydrocarbons in urban air using positive matrix factorization and spatial distribution analysis. Atmospheric Environment, 79, 271-285.

Jenkins BM, Jones AD, Turn SQ, Williams RB. (1996) Emission factors for polycyclic aromatic hydrocarbons from biomass burning. Environmental Science and Technology, 30, 24622469.

Jerrett M, Burnett RT, Kanaroglou P, Eyles J, Finkelstein N, Giovis C, Brook JR. (2001) A GIS environmental justice analysis of particulate air pollution in Hamilton, Canada. Environment and Planning A., 33 (6), 955-973.

Jerrett M, Finkelstein M. (2005) Geographies of risk in studies linking chronic air pollution exposure to health outcomes. J. Toxicol. Environ. Health A., 68, 1207-1242.

Jerrett M, Buzzelli M, Burnett RT, DeLuca PF. (2005) Particulate air pollution, social confounders, and mortality in small areas of an industrial city. Social Sci. Med., 60, $2845-2863$.

Jerrett M, Burnett RT, Ma R, Pope III CA, Krewski D, Newbold KB, Thurston G, Shi Y, Finkelstein N, Calle EE, Thun MJ. (2005a). Spatial analysis of air pollution and mortality in Los Angeles. Epidemiology, 16, 727-736.

Jerrett M, Buzzelli M, Burnett RT, DelLuca PF. (2005b). Particulate air pollution, social confounders, and mortality in small areas of an industrial city. Social Science \& Medicine, 60, 2845-2863.

Joliffe IT. (2002) Principal component analysis, Second edition. Springer, New York.

Jung HJ, Yan B, Chillrud SN, Perera FP, Whyatt R, Camann D, Kinney PL, Miller RL. (2010) Assessment of benzo(a)pyrene-equivalent carcinogenicity and mutagenicity of residential indoor versus outdoor polycyclic aromatic hydrocarbons exposing young children in New York City. Int. J. Environ. Res. Public Health, 7, 1889-1900.

Kam W, Liacos JW, Schauer JJ, Delfino RJ, Sioutas C. (2012) On-road emission factors of PM pollutants for light-duty vehicles (LDVs) based on urban street driving conditions. Atmospheric Environment, 61, 378-386.

Kanaroglou PS, Jerrett M, Morrison J, Beckerman B, Arain MA, Gilbert NL, Brook JR. (2005) Establishing an air pollution monitoring network for intra-urban population exposure assessment: a location-allocation approach. Atmospheric Environment, 39, 2399-2409. 
Karman D, Anastassopoulos A. (2010). Analysis of intraurban variability of particulate matter and polycyclic aromatic hydrocarbons (PAH) for Health Canada's "Hamilton spatial study" - Final Report Parts 1, 2. Submitted to: Amanda Wheeler, Air Health Science Division, Health Canada.

Karman D, Shen J. (2006) Mobile 6C Modelling and Emissions of Selected Toxic Substances from On-Road Motor Vehicles in Ontario, submitted to the Toxics Prevention Division, Environmental Protection Branch-Ontario Region, Environment Canada.

Karman D, Anastassopoulos A. (2009) Source Apportionment of PAHs at Ontario Sites Using Receptor Modelling Techniques - Final Report. Submitted to: Toxics Prevention Division, Environmental Protection Branch-Ontario Region, Environment Canada, September 2009.

Karman D, Anastassopoulos A, Dogan G. (2009) "Source Apportionment of PAHs at Ontario Sites Using Receptor Modeling Techniques", Submitted to: Toxics Prevention Division Environmental Protection Branch-Ontario Region Environment Canada, September 2009.

Kavouras IG, Koutrakis P, Tsapakis M, Lagoudaki E, Stephanou EG, Von Baer D, Oyola P. (2001) Source apportionment of urban particulate aliphatic and polynuclear aromatic hydrocarbons (PAHs) using multivariate methods. Environmental Science and Technology, 35, 2288-2294.

Khalili NR, Scheff PA, Holsen TM. (1995) PAH source fingerprints for coke ovens, diesel and gasoline engines, highway tunnels, and wood combustion emissions. Atmospheric Environment, 29, 533-542.

Kim E, Hopke PK, Edgerton ES. (2003) Source identification of Atlanta aerosol by Positive Matrix Factorization. J. Air \& Waste Manage. Assoc. 53, 731-739.

Kindig DA. (2007) Understanding Population Health Terminology. Milbank Quarterly, 85 (1), $139-161$.

Kinney P, Aggarwal M, Northridge ME, Janssen NA, Shepard P. (2000) Airborne Concentrations of PM2.5 and Diesel Exhaust Particles on Harlem Sidewalks: A Community-Based Pilot Study. Environ. Health Perspect., 108 (3), 213-218.

Kinney P, Spengler J, Brown K. (2006) Assessing population exposures in studies of human health effects of PM2.5. EM: The Magazine For Environmental Managers, 9, 16-21.

Kirton P, Ellis J, Crisp PT. (1991) The analysis of organic matter in coke oven emissions. Fuel, 70 , December.

Kjellstrom T, Friel S, Dixon J, Corvalan C, Rehfuess E, Campbell-Lendrum D, Gore F, Bartram J. (2007) Urban environmental health hazards and health equity. Journal of Urban Health, 84, 86-97.

Kong S, Shi J, Lu B, Qiu W, Zhang B, Peng Y, Zhang B, Bai Z. (2011) Characterization of PAHs within PM10 fraction for ashes from coke production, iron smelt, heating station and power plant stacks in Liaoning Province, China. Atmospheric Environment, 45, 37773785 .

Kristensson A, Johansson C, Westerholm R, Swietlicki E, Gidhagen L, Wideqvist U, Vesely V. (2004) Real-world traffic emission factors of gases and particles measured in a road tunnel in Stockholm, Sweden. Atmospheric Environment, 38, 657-673. 
Kuntasal UO. (2005) Temporal variations and sources of organic pollutants in two urban atmospheres: Ankara and Ottawa. Ph.D. thesis, Middle East Technical University. Ankara, Turkey.

Larsen III RK, Baker JE. (2003) Source apportionment of polycyclic aromatic hydrocarbons in the urban atmosphere: A comparison of three methods. Environmental Science and Technology, 37, 1873-1881.

Larsen JC, Larsen PB. (1998) Chemical carcinogens. In: Air Pollution and Health (R.E. Hester and R.M. Harrison, eds.). Cambridge, UK: The Royal Society of Chemistry, 33-56.

Lee JY, Kim YP. (2007) Source apportionment of the particulate PAHs at Seoul, Korea: impact of long range transport to a megacity. Atmos. Chem. Phys. Discuss., 7, 1479-1506.

Lee JH, Gigliotti CL, Offenberg JH, Eisenreich SJ, Turpin BJ. (2004) Sources of polycyclic aromatic hydrocarbons to the Hudson River Airshed. Atmos. Environ., 38, 5971-81.

Lee PK, Brook JR, Dabek-Zlotorzynska E, Mabury SA. (2003) Identification of the major sources contributing to PM2.5 observed in Toronto. Environ. Sci. Technol., 37 (21), 4831-4840.

Legzdins AE, McCarry BE, Bryant DW. (1994) Polycyclic aromatic compounds in Hamilton air: their mutagenicity, ambient concentrations and relationships with atmospheric pollutants. Polycyclic Aromatic Compounds, 5, 157-165.

Li A, Jang J, Scheff PA. (2003) Application of EPA CMB8.2 model for source apportionment of sediment PAHS in lake calumet, Chicago. Environmental Science and Technology, 37, 2958-2965.

Li A, Schoonover TM, Zou Q, Norlock F, Conroy LM, Scheff PA, Wadden RA. (2005a) Polycyclic aromatic hydrocarbons in residential air of ten Chicago area homes : concentrations and influencing factors. Atmospheric Environment, 39, 3491-3501.

Li C, Fu J, Sheng G, Bi X, Hao Y, Wang X, Mai B. (2005b) Vertical distribution of PAHs in the indoor and outdoor PM2.5 in Guangzhou, China. Building and Environment, 40, 329341 .

Li N, Kim S, Wang M, Froines J, Sioutas C, Nel A. (2002) Use of a stratified oxidative stress model to study the biological effects of ambient concentrated and diesel exhaust particulate matter. Inhal. Toxicol., 14, 459-486.

Liacos J, Kam W, Delfino R, Schauer J, Sioutas C. (2012) Characterization of organic, metal and trace element PM2.5 species and derivation of freeway-based emission rates in Los Angeles, CA. Science of the Total Environment, 435-436:159-166.

Lima ALC, Farrington JW, Reddy CM. (2005) Combustion-Derived Polycyclic Aromatic Hydrocarbons in the Environment-A Review. Environmental Forensics, 6, 109-131.

Liu Y, Liu L, Lin J, Tang N, Hayakawa K. (2006) Distribution and characterization of polycyclic aromatic hydrocarbon compounds in airborne particulates of east Asia. China Particuology, 4 (6), 283-292.

Lough GC, Schauer JJ. (2007) Sensitivity of Source Apportionment of Urban Particulate Matter to Uncertainty in Motor Vehicle Emissions Profiles, J. of the Air and Waste Management Association, 57 (10), 1200-1213. 
Lough GC, Christensen CG, Schauer JJ, Tortorelli J, Mani E, Lawson DR, Clark NN. (2007) Development of Molecular Marker Source Profiles for Emissions from On-Road Gasoline and Diesel Vehicle Fleets, J. of the Air and Waste Management Association, 57 (10), 1190-1199.

Lu H, Zhu L, Chen S. (2008) Pollution level, phase distribution and health risk of polycyclic aromatic hydrocarbons in indoor air at public places of Hangzhou, China. Environmental Pollution, 152, 569-575.

Maciejczyk PB, Offenberg JH, Clemente J, Blaustein M, Thurston GD, Chen LC. (2004) Ambient pollutant concentrations measured by a mobile laboratory in South Bronx, NY. Atmospheric Environment, 38, 5283-5294.

Mackay D, Shi WY, Ma J-C, Lee SC. (2006) Handbook of physical-chemical properties and environmental fate for organic chemicals. $2^{\text {nd }}$ edition. CRC Press, Taylor \& Francis Group, Boca Raton, FL, USA.

Malcolm HM, Dobson S. (1994) The calculation of an environmental assessment level (EAL) for atmospheric PAHs using relative potencies. Department of the Environment, London, UK, 34-46.

Manchester-Neesvig JB, Schauer JJ, Cass GR. (2003) The distribution of particle-phase organic compounds in the atmosphere and their use for source apportionment during the Southern California children's health study, J. of the Air and Waste Management Association, 53 (9, Sep 1), 1065-1079.

Mantis J, Chaloulakou A, Samara C. (2005) PM10-bound polycyclic aromatic hydrocarbons (PAHs) in the Greater Area of Athens, Greece. Chemosphere, 59, 593-604.

Mari M, Harrison RM, Schuhmacher M, Domingo JL, Pongpiachan S. (2010) Inferences over the sources and processes affecting polycyclic aromatic hydrocarbons in the atmosphere derived from measured data. Science of the Total Environment, 408, 2387-2393.

Marr LC, Kirchstetter TW, Harley RA, Miguel AH, Hering SV, Hammond SK. (1999) Characterization of Polycyclic Aromatic Hydrocarbons in Motor Vehicle Fuels and Exhaust Emissions. Environ. Sci. Technol., 33, 3091-3099.

McDonald J, Zielinska B, Fujita E, Sagebiel J, Chow J, Watson J. (2000) Fine Particle and Gaseous Emission Rates from Residential Wood Combustion. Environ. Sci. Technol., 34, 2080-2091.

Mckay JC. (2006) "Iron and steel industry". Canadian Encyclopedia. Available online (accessed 5 March 2013): http://www.thecanadianencyclopedia.com/articles/iron-and-steel-industry

Menezes HC, Cardeal ZL. (2012) Study of polycyclic aromatic hydrocarbons in atmospheric particulate matter of an urban area with iron and steel mills. Environmental Toxicology and Chemistry, 31 (7), 1470-1477.

Miller L, Lemke LD, Xu X, Molaroni SM, You H, Wheeler AJ, Booza J, Grgicak-Mannion A, Krajenta R, Graniero P, Krouse H, Lamerato L, Raymond D, Reiners J, Weglicki L. (2010) Intra-urban correlation and spatial variability of air toxics across an international airshed in Detroit, Michigan (USA) and Windsor, Ontario (Canada). Atmospheric Environment, 44, 1162-1174. 
Miller RL, Garfinkel R, Horton M, Camann D, Perera FP, Whyatt RM, Kinney PL. (2004) Polycyclic aromatic hydrocarbons, environmental tobacco smoke, and respiratory symptoms in an inner-city birth cohort. Chest, 126 (4), 1071-1078.

Moonesinghe R, Khoury MJ, Janssens ACJW. (2007) Most Published Research Findings Are False-But a Little Replication Goes a Long Way. PLoS Med., 4 (2), e28.

Mudge SM. (2007) Multivariate Statistical Methods in Environmental Forensics. Environmental Forensics, 8, 155-163.

Muller P. (1997) Scientific Criteria Document for Multimedia Standards Development Polycyclic Aromatic Hydrocarbons (PAH); Part 1: Hazard Identification and Dose-Response Assessment. Standard Development Branch, Ontario Ministry of Environment and Energy.

Nadon L, Siemiatycki J, Dewar R, Krewski D, Gerin M. (2007) Cancer risk due to occupational exposure to polycyclic aromatic hydrocarbons. Am J and Med., 28 (3), 303-324.

Narvaez R, Hoepner L, Chillrud S, Yan B, Garfinkel R, Whyatt R, Camann D, Perera F, Kinney P, Miller R. (2008) Spatial and temporal trends of polycyclic aromatic hydrocarbons and other traffic-related airborne pollutants in New York City. Environmental Science and Technology, 42, 7330-7335.

National Research Council. (2001) Research priorities for airborne particulate matter - III - early research progress. National Academy Press, Washington, DC, USA.

Natural Resources Canada. (2007) Survey of household energy use, detailed statistical report. Cat. No. M144-120/2-2007E. available online: http://oee.nrcan.gc.ca/publications/statistics/sheu07/index.cfm

Nielsen T, Jørgensen HE, Larsen JC, Poulsen M. (1996) City air pollution of polycyclic aromatic hydrocarbons and other mutagens: occurrence, sources and health effects. The Science of Total Environment, 189/190, 41-49.

Nielsen T, Feilberg A, Binderup M-L. (1999) The variation of street air levels of PAH and other mutagenic PAC in relation to regulations of traffic emissions and the impact of atmospheric processes. Environmental Science and Pollution Research, 6 (3), 133-137.

Ning Z, Polidori A, Schauer JJ, Sioutas C. (2008) Emission factors of PM species based on freeway measurements and comparison with tunnel and dynamometer studies. Atmospheric Environment, 42, 3099-3114.

Nisbet I, Lagoy P. (1992) Toxic equivalency factors (TEFs) for polycyclic aromatic hydrocarbons (PAHs). Regul. Toxicol. Pharmacol., 16, 290-300.

Ogulei D, Hopke PK, Zhou L, Paatero P, Park S-S, Ondov JM. (2005) Receptor modeling for multiple time resolved species: the Baltimore supersite. Atmospheric Environment 39, 3751-3762.

Ohura T, Amagai T, Sugiyama T, Fusaya M, Matsushita H. (2004) Characteristics of particle matter and associated polycyclic aromatic hydrocarbons in indoor and outdoor air in two cities in Shizuoka, Japan. Atmospheric Environment, 38, 2045-2054.

Okuda T, Okamoto K, Tanaka S, Shen Z, Han Y, Huo Z. (2010) Measurement and source identification of polycyclic aromatic hydrocarbons (PAHs) in the aerosol in Xi'an, China, by using automated column chromatography and applying positive matrix factorization (PMF). Science of the Total Environment, 408, 1909-1914. 
Olson DA, McDow SR. (2009) Near roadway concentrations of organic source markers. Atmospheric Environment, 43, 2862-2867.

Ontario Ministry of Environment. (2013) Air quality in Ontario (annual reports by year). Available online (accessed 4 November 2013): http://www.airqualityontario.com/press/publications.php

Oros DR, Simoneit BRT. (2000) Identification and emission rates of molecular tracers in coal smoke particulate matter. Fuel, 79, 515-536.

Oros DR, Simoneit BRT. (2001a) Identification and emission factors of molecular tracers in organic aerosols from biomass burning: part 1. temperate climate conifers . Applied Geochemistry, 16, 1513-1544.

Oros DR, Simoneit BRT. (2001b) Identification and emission factors of molecular tracers in organic aerosols from biomass burning: part 2. deciduous trees. Applied Geochemistry, $16,1545-1565$.

Oros DR, Abas MR, Omar NYMJ, Rahman NA, Simoneit BRT. (2006) Identification and emission factors of molecular tracers in organic aerosols from biomass burning: part 3. grasses. Applied Geochemistry, 21, 919- 940.

Paatero P, Hopke PK. (2003) Discarding or downweighting high-noise variables in factor analytic models. Analytica Chimica Acta, 490, 277-289.

Paatero P, Tapper U. (1994) Positive matrix factorization: a non-negative factor model with optimal utilization of error estimates of data values. Environmetrics, 5, 111-126.

Paatero P. (1997) Least squares formulation of robust non-negative factor analysis. Chemometrics and Intelligent Laboratory Systems, 37, 23-35.

Park SS, Kim YJ. (2005) Source contributions to fine particulate matter in an urban atmosphere. Chemosphere, 59, 217-226.

Park SS, Kim YJ, Kang CH. (2002) Atmospheric polycyclic aromatic hydrocarbons in Seoul, Korea. Atmospheric Environment, 36, 2917-2924.

Park S-U, Kim J-G, Jeong M-J, Song B-J. (2011) Source identification of atmospheric polycyclic aromatic hydrocarbons in industrial complex using diagnostic ratios and multivariate factor analysis. Archives of Environmental Contaminant Toxicology, 60, 576-589.

Perera FP, Rauh V, Whyatt RM, Tang D, Tsai D, Tsai WY, Bernert JT, Tu YH, Andrews H, Barr DB, Camann DE, Diaz D, Dietrich J, Reyes A, Kinney PL. (2005) A summary of recent findings on birth outcomes and developmental effects of prenatal ETS, PAH, and pesticide exposures. Neurotoxicology, 26 (4), 573-587.

Peters A, Wichmann HE, Tuch T, Heinrich J, Heyder J. (1997) Respiratory effects are associated with the number of ultrafine particles. Am. J. Respir. Crit. Care Med., 155, 1376-1383.

Phuleria HC, Geller MD, Fine PM, Sioutas C. (2006) Size-resolved emissions of organic tracers from light- and heavy-duty vehicles measured in a California roadway tunnel. Environmental Science and Technology, 40, 4109-4118.

Phuleria HC, RJ Sheesley, JJ Schauer, PM Fine, C Sioutas. (2007) Roadside measurements of size-segregated particulate organic compounds near gasoline and diesel-dominated freeways in Los Angeles, CA. Atmospheric Environment, 41, 4653-4671. 
Pinto JP, Lefohn AS, Shadwick DS. (2004) Spatial variability of PM2.5 in urban areas in the United States. Journal of the Air \& Waste Management Association, 54, 440-449.

Politis DN. (2003) The impact of bootstrap methods on time series analysis. Statistical Science, 18 (2), 219-230.

Politis DN and White H. (2004) Automatic Block-Length Selection for the Dependent Bootstrap. Economotric Reviews, 23 (1), 53-70.

Praveena SM, Kwan OW, Ari AZ. (2012) Effect of data pre-treatment procedures on principal component analysis: a case study for mangrove surface sediment datasets. Environ. Monit. Assess., 184, 6855-6868.

Ramdahl T. (1983) Nature, 306, 580-582.

Ravindra K, Bencs L, Wauters E, Dehoog J, Deutsch F, Roekens E, Bleux N, Berghmans P, Vangrieken R. (2006) Seasonal and site-specific variation in vapor and aerosol phase PAHs over Flanders (Belgium) and their relation with anthropogenic activities. Atmospheric Environment, 40 (4), 771-785.

Ravindra K, Sokhi R, van Grieken R. (2008) Atmospheric polycyclic aromatic hydrocarbons: Source attribution, emission factors and regulation. Atmospheric Environment, 42, 28952921.

Reff A, Eberly SI, Bhave PV. (2007) Receptor modeling of ambient particulate matter data using positive matrix factorization: review of existing methods. Journal of the Air \& Waste Management Association, 57, 146-154.

Rehwagen M, Muller A, Massolo L, Herbarth O, Ronco A. (2005) Polycyclic aromatic hydrocarbons associated with particles in ambient air from urban and industrial areas. Sci. of The Total Environ., 348 (1-3), 199-210.

Reid MK, Spencer KL. (2009) Use of principal components analysis (PCA) on estuarine sediment datasets: The effect of data pre-treatment. Environmental Pollution, 157, 22752281.

Reyment R, Joreskog KG. (1993) Applied factor analysis in the natural sciences. 2nd edition. Cambridge University Press. 371 pages.

Rogge WF, Hildemann LM, Mazurek MA, Cass CR. (1993a) Sources of fine organic aerosol. 2. noncatalyst and catalyst- equipped automobiles and heavy-duty diesel trucks. Environ. Sci. Technol., 27, 636-651.

Rogge WF, Hildemann LM, Mazurek MA, Cass CR. (1993b) Sources of fine organic aerosol. 5. natural gas home appliances. Environ. Sci. Technol., 27, 2736-2744.

Rogge WF, Hildemann LM, Mazurek MA, Cass CR. (1997a ) Sources of fine organic aerosol. 7. hot asphalt roofing tar pot fumes. Environ. Sci. Technol., 31, 2726-2730.

Rogge WF, Hildemann LM, Mazurek MA, Cass CR. (1998) Sources of fine organic aerosol. 9. pine, oak, and synthetic log combustion in residential fireplaces. Environ. Sci. Technol. $32: 13-22$.

Ropkins K, Beebe J, Li H, Daham B, Tate J, Bell M, Andrews G. (2009) Real-World Vehicle Exhaust Emissions Monitoring: Review and Critical Discussion. Critical Reviews in Environmental Science and Technology, 39, 79-152. 
Samet JM, Pope AC III. (2003) Epidemiologic research needs for particulate air pollution. Journal of Toxicology and Environmental Health, Part A, 66, 1873-1876.

Samet JM, Dominici F, Curriero FC, Coursac I, Zeger LS. (2000) Fine particulate air pollution and mortality in 20 U.S. cities. N. Engl. J. Med., 242, 1742-1749.

Sanderson EG, Raqbi A, Vyskocil A, Farant J-P. (2004) Comparison of particulate polycyclic aromatic hydrocarbon profiles in different regions of Canada. Atmospheric Environment, $38,3417-3429$.

Schauer JJ, Cass GR. (2000) Source apportionment of wintertime Gas-Phase and Particle Phase Air Pollutants Using Organic Compounds as Tracers. Environmental Science and Technology, 34, 1821-1832.

Schauer JJ, Glynis CL. (2006) Gasoline/Diesel PM Split Study, University of WisconsinMadison, prepared for National Renewable Energy Laboratory.

Schauer JJ, Rogge WF, Hildemann LM, Mazurek MA, Cass GR. (1996) Source apportionment of airborne particulate matter using organic compounds as tracers. Atmospheric Environment, 30, 22, 3837-3855.

Schauer J, Kleeman M, Cass R, Simoneit B. (2001) Measurement of Emissions from Air Pollution Sources. 3. C1-C29 Organic Compounds from Fireplace Combustion of Wood. Environ. Sci. Technol., 35, 1716-1728.

Schauer JJ, Fraser MP, Simoneit BRT. (2002a) Source Reconciliation of Atmospheric Gas-Phase and Particle Phase Pollutants during a Severe Photochemical Smog Episode, Environmental Science and Technology, 36, 3806-3814.

Schnelle-Kreis J, Gebefugi I, Welzl G, Jaensch T, Kettrup A. (2001) Occurrence of particleassociated polycyclic aromatic compounds in ambient air of the city of Munich. Atmospheric Environment, 35 (S1), S71-S81.

Schwartz J, Litonjua A, Suh H, Verrier M, Zanobetti A, Syring M, Nearing B, Verrier R, Stone P, MacCallum G, Speizer FE, Gold DE. (2005) Traffic related pollution and heart rate variability in a panel of elderly subjects. Thorax, 60, 455-461.

Scott JA. (2009) The mechanism of retene toxicity in the early life stages of fish. Ph.D. thesis, Queen's University. Kingston, Ontario, Canada.

Seinfeld JF, Pandis SN. (2006) Atmospheric Chemistry and Physics: from air pollution to climate change. Wiley Blackwell, $2^{\text {nd }}$ Edition.

Shah SD, Ogunyoku A, Miller JW, Cocker DR. (2005) On-Road Emission Rates of PAH and nAlkane Compounds from Heavy-Duty Diesel Vehicles. Environ. Sci. Technol., 39, 52765284 .

Shen G, Tao S, Wei S, Zhang Y, Wang R, Wang B, Li W, Shen H, Huang Y, Yang Y, Wang W, Wang X, Massey Simonich SL. (2012) Retene Emission from Residential Solid Fuels in China and Evaluation of Retene as a Unique Marker for Soft Wood Combustion. Environ. Sci. Technol., 46, 4666-4672.

Shi G-L, Li X, Feng Y-C, Wang Y-Q, Wu J-H, Li J, Zhu T. (2009) Combined source apportionment using positive matrix factorizaiton-chemical mass balance and principal component analysis/multiple linear regression-chemical mass balance models. Atmospheric Environment, 43 (18), 2929-2937. 
Shi J, Peng Y, Li W, Qiu W, Bai Z, Kong S, Jin T. (2010) Characterization and source identification of PM10-bound polycyclic aromatic hydrocarbons in urban air of Tianjin, China. Aerosol and Air Quality Research, 10, 507-518.

Simcik MF, Eisenreich SJ, Lioy PJ. (1999) Source apportionment and source/sink relationships of PAHs in the coastal atmosphere of Chicago and lake Michigan. Atmospheric Environment, 33, 5071-5079.

Sisovic A, Fugas M, Sega K. (1996) Assessment of human inhalation exposure to polycyclic aromatic hydrocarbons. Journal of Exposure Analysis and Environmental Epidemiology, $6,439-447$.

Sisovic A, Skrbec A, Vadjic V, Kalinic N, Hrsak J. (2002) PAH levels and profiles in the suspended particulate matter in Zagreb through four seasons. Environmental Monitoring and Assessment, 74, 217-224.

Sofowote UM, McCarry BE, Marvin CH. (2008) Source Apportionment of PAH in Hamilton Harbour Suspended Sediments: Comparison of Two Factor Analysis Methods. Environ. Sci. Technol., 42, 6007-6014.

Sofowote UM, Allan LM, McCarry BE. (2010a) Evaluation of PAH diagnostic ratios as source apportionment tools for air particulates collected in an urban-industrial environment. Journal of Environmental Monitoring 12, 417-424.

Sofowote UM, Allan LM, McCarry BE. (2010b) A comparative study of two factor analytic models applied to PAH data from inhalable air particulate collected in an urban-industrial environment. Journal of Environmental Monitoring, 12, 425-433.

Sofowote UM, Hung H, Rastogi AK, Westgate JN, Deluca PF, Su Y, McCarry BE. (2011) Assessing the long-range transport of PAH to a sub-Arctic site using positive matrix factorization and potential source contribution function. Atmospheric Environment, 45, 967-976.

Somers CM, McCarry BE, Malek F, Quinn JS. (2004) Reduction of particulate air pollution lowers the risk of heritable mutations in mice. Science, 304, 1008-1010.

Sonoma. (2008) Workbook on source apportionment using Positive Matrix Factorization. Workshop and workbook materials presented to Environment Canada November 9-10, 2004, Vancouver, British Columbia.

Spengler J, Lwebuga-Mukasa J, Vallarino J, Melly S, Chillrud S, Baker J, Minegishi T. (2011) Air Toxics Exposure from Vehicle Emissions at a U.S. Border Crossing: Buffalo Peace Bridge Study. Health Effects Institute Research, Report 158, July 2011.

Statistics Canada. (2011) Households and the environment: energy use. Catalogue no. 11-526-S.

Statistics Canada. (2012a) Toronto, Ontario (Code 535) and Ontario (Code 35) (table). Census Profile. 2011 Census. Statistics Canada Catalogue no. 98-316-XWE. Ottawa. Released October 24, 2012. http://www12.statcan.gc.ca/census-recensement/2011/dp$\mathrm{pd} /$ prof/index.cfm (accessed October 24, 2014).

Statistics Canada. (2012b) Hamilton, Ontario (Code 537) and Ontario (Code 35) (table). Census Profile. 2011 Census. Statistics Canada Catalogue no. 98-316-XWE. Ottawa. Released October 24, 2012. http://www12.statcan.gc.ca/census-recensement/2011/dppd/prof/index.cfm (accessed October 24, 2014). 
Stella A, Piccardo MT, Pala M, Balducci D, Cipolla M, Ceppi M, Valerio F. (2012) Temporal and spatial variations of polycyclic aromatic hydrocarbon concentrations around a coke oven plant. Journal of the Air \& Waste Management Association, 62, 9, 1003-1011.

Stieb DM, Burnett RT, Smith-Doiron M, Brion O, SHin HH, Economou V. (2008) A New Multipollutant, No-Threshold Air Quality Health Index Based on Short-Term Associations Observed in Daily Time- Series Analyses. J. Air \& Waste Manage. Assoc., $58,435-450$.

Sun P, Blanchard P, Brice KA, Hites RA. (2006) Trends in polycyclic aromatic hydrocarbon concentrations in the Great Lakes atmosphere. Environmental Science and Technology, 40, 6221-6227.

Tobiszewski M, Namiesnik J. (2012) PAH diagnostic ratios for the identification of pollution emission sources. Environmental Pollution, 162, 110-119.

Tolbert. (2007) Invited commentary: Heterogeneity of particulate matter health risks. American Journal of Epidemiology, 166 (8), 889-891.

Tsai J-H, Lin K-H, Chen C-Y, Ding J-Y, Choa C-G, Chiang H-L. (2007) Chemical constituents in particulate emissions from an integrated iron and steel facility. J Hazard Mater., 147, 111-119.

Tsapakis M, Stephanou EG. (2005) Occurrence of gaseous and particulate polycyclic aromatic hydrocarbons in the urban atmosphere: study of sources and ambient temperature effect on the gas/particle concentration and distribution. Environmental Pollution, 133, 147156.

United States Environmental Protection Agency (US EPA). (1989) AP-42 (draft 5th Edition), Volume II: Mobile sources. US EPA, Washington, DC. Online at:

http://www.epa.gov/ttn/chief/ap42/index.html

United States Environmental Protection Agency (US EPA). (1998a) Quality assurance guidance document 2.12: Monitoring PM2.5 in ambient air using designated reference or Class 1 equivalent methods. Human Exposure and Atmospheric Sciences Division, Research Triangle Park, NC.

United States Environmental Protection Agency (US EPA). (1998b) Locating and estimating air emissions from sources of polycyclic organic matter. Report No. EPA-454/R-98-014, US EPA, OAQPS, Washington, DC.

United States Environmental Protection Agency (US EPA). (1999) Compendium of methods for the determination of toxic organic compounds in ambient air, second edition, Compendium Method TO-13A: Determination of polycyclic aromatic hydrocarbons (PAHs) in ambient air using gas chromatography/mass spectrometry (GC/MS). EPA/625/R-96/010b, Center for Environmental Research Information, Office of Research and Development, Cincinnati, $\mathrm{OH}$.

United States Environmental Protection Agency (US EPA). (2000a) Air toxics - napthalene. (document on the internet, accessed 25 October 2007) Available at:

http://www .epa.gov/ttn/uatw/hlthef/naphthal.html

United States Environmental Protection Agency (US EPA). (2000b) Guidance for Data Quality Assessment - Practical Methods for Data Analysis, EPA QA/G-9 QA00 Update (EPA/600/R-96/084, July 2000), Office of Environmental Information, Washington, DC 20460. 
United States Environmental Protection Agency (US EPA). (2003) 1999 National emissions inventory. US EPA, Washington, DC. Available at:

ftp://ftp.epa.gov/EmisInventory/finalnei99ver3/haps/.864

United States Environmental Protection Agency (US EPA). (2004) EPA-CMB8.2 Users Manual EPA-452/R-04-011, C. Thomas Coulter, Air Quality Modeling Group, Emissions, Monitoring \& Analysis Division, Office of Air Quality Planning \& Standards, Research Triangle Park, NC, December 2004.

United States Environmental Protection Agency (US EPA). (2004) Factor Information REtrieval (FIRE) v6.25. US EPA, Washington, DC. Available at: http://www.epa.gov/ttn/chief/software/fire

United States Environmental Protection Agency (US EPA). (2007) Air toxics - polycyclic organic matter. Available at http://www.epa.gov/ttn/atw/hlthef/polycycl.html. (accessed 15 October 2007)

United States Environmental Protection Agency (US EPA). (2008) EPA Positive Matrix Factorization (PMF) 3.0 Fundamentals \& User Guide. EPA 600/R-08/108, July 2008, US EPA, Washington, DC. Available at: http://www .epa.gov/heasd/products/pmf/pmf.html

United States Environmental Protection Agency (US EPA). NMIM. (2008) US EPA, Washington, DC. Online at: http://www.epa.gov/otaq/nmim.htm

United States Environmental Protection Agency (US EPA). SPECIATE v4.2. (2009) US EPA, Washington, DC. Available at: http://www .epa.gov/ttnchie1/software/speciate/index.html

United States Environmental Protection Agency (US EPA). MOVES. (2010) US EPA, Washington, DC. Online at: http://www.epa.gov/otaq/models/moves/index.htm

United States Environmental Protection Agency (US EPA). (2011a) SPECIATE v4.3. US EPA, Washington, DC. Available at: http://www.epa.gov/ttnchie1/software/speciate/index.html

United States Environmental Protection Agency (US EPA). AP-42 (5th Edition), Volume I: Stationary point and area sources. (2011b). US EPA, Washington, DC. Online at: http://www.epa.gov/ttn/chief/ap42/index.html

United States Environmental Protection Agency (US EPA). (2012a) WebFIRE - online searchable database of Factor Information Retrieval System (FIRE). US EPA, Washington, DC. Online at: http://cfpub.epa.gov/webfire/

United States Environmental Protection Agency (US EPA). (2012b) Download of FIRE database in CSV format. US EPA, Washington, DC. Online at: http://cfpub.epa.gov/webfire/

Van Noort PCM, Wondergem E. (1985) Scavenging of airborne polycyclic aromatic hydrocarbons by rain. Environmental Science and Technology, 19, 1044-1048.

Venkataraman C, Lyons JM, Friedlander SK. (1994) Size distributions of Polycyclic Aromatic Hydrocarbons and Elemental Carbon. 1. Sampling, measurement methods, and source characterization. Environ. Sci. Technol. 1994, 28, 555-562.

Venkataraman C, Friedlander SK. (1994) Size distributions of polycyclic aromatic hydrocarbons and elemental carbon: 2. Ambient measurements and effects of atmospheric processes. Environmental Science and Technology, 28, 563-572. 
Viana M, Pandolfi M, Minguillon MC, Querol X, Alastuey A, Monfort E, Celades I. (2008) Intercomparison of receptor models for PM source apportionment: Case study in an industrial area. Atmospheric Environment, 42, 3820-3832.

Waldman JM, Lioy PJ, Greenberg A, Butler JP. (1991) Analysis of human exposure to benzo(a)pyrene via inhalation and food ingestion in the total human environmental exposure study (THEES). Journal of Exposure Analysis and Environmental Epidemiology, 1, 193-225.

Wallace J, Corr D, Kanaroglou P. (2010) Topographic and spatial impacts of temperature inversions on air quality using mobile air pollution surveys. Science of the Total Environment, 408, 5086-5096.

Wang D, Tian F, Yang M, Liu C, Li Y-F. (2009) Application of positive matrix factorization to identify potential sources of PAHs in soil of Dalian, China. Environmental Pollution, $157,1559-1564$.

Wang H-S, Cheng Z, Liang P, Shao D-D, Kang Y, Wu S-C, Wong CKC, Wong MH. (2010) Characterization of PAHs in surface sediments of aquaculture farms around the Pearl River Delta. Ecotoxicology and Environmental Safety, 73, 900-906.

Ward T, Smith G. (2004) High-Volume PUF versus Low-Volume PUF Sampling Comparison for Collecting Gas Plus Particulate Polycyclic Aromatic Hydrocarbons, Aerosol Science and Technology, 38, 10, 972-979.

Watson JD, Fujita E, Chow JC, Zielinska B. (1998) Northern Front Range Air Quality Study Final Report. Desert Research Institute.

Watson et al, (2004) Protocol for Applying and Validating the CMB Model for PM2.5 and VOC. EPA-451/R-04-001.

Watson JG, Zhu T, Chow JC, Engelbrecht JP, Fujita EM, Wilson WE. (2002) Receptor Modeling Application Framework for Particle Source Apportionment; Chemosphere, 49, 10931136.

Watson JG, Chen L-WA, Chow JC, Doraiswamy P, Lowenthal DH. (2008) Source apportionment: findings from the US Supersites Program. J. Air \& Waste Manage. Assoc., 58, 265-288.

Wheeler AJ, Xu X, Kulka R, You H, Wallace L, Mallach G, Van Ryswyk K, MacNeill M, Kearney J, Rasmussen PE, Dabek-Zlotorzynska E, Wang D, Poon R, Williams R, Stocco C, Anastassopoulos A, Miller JD, Dales R, Brook JR. (2011) Windsor, Ontario exposure assessment study: design and methods validation of personal, indoor, and outdoor air pollution monitoring. Journal of the Air \& Waste Management Association, 61, 324-338.

Wheeler AJ, Xu X, Smith-Doiron M, Gilbert N, Brook JR. (2006) Intra-urban variability of air pollution - monitoring and modelling of PM2.5, PM2.5-10, and NO2. (poster presented at ISEE ISEA Conference, 2006)

Wheeler AJ, Smith-Doiron M, Xu X, Gilbert NL, Brook JR. (2008) Intra-urban variability of air pollution in Windsor, Ontario- Measurement and modeling for human exposure assessment. Environmental Research, 106, 7-16.

Wilson R, Spengler JD. (1996) Emissions, dispersion, and concentration of particles. In: Particles in our air - concentrations and health effects. Editors: Wilson R, Spengler JD, Harvard University Press. 
Wingfors H, Sjodin A, Haglund P, Brorstrom-Lunden E. (2001) Characterisation and determination of profiles of polycyclic aromatic hydrocarbons in a traffic tunnel in Gothenburg, Sweden. Atmospheric Environment, 35, 6361-6369.

World Coal Institute. (2007) "Coal \& Steel”, 7 pages. Available online (accessed 5 March 2013): http://www.worldcoal.org/resources/wca-publications/

WHO (World Health Organization). (1998) Environmental Health Criteria 202, Selected Nonheterocyclic Polycyclic Aromatic Hydrocarbons. International Programme on Chemical Safety, World Health Organization, Geneva.

Yang HH, Lee WJ, Chen SJ, Lai SO. (1998) PAH emission from various industrial stacks. Journal of Hazardous Materials, 60 (2), 159-174.

Yang HH, Lai SO, Hsieh LT, Hsueh HJ, Chi TW. (2002) Profiles of PAH emission from steel and iron industries. Chemosphere, 48(10), 1061-1074.

Yunker MB, Macdonald RW, Vingarzan R, Mitchell RH, Goyette D, Sylvestre S. (2002) PAHs in the Fraser River basin: a critical appraisal of PAH ratios as indicators of PAH source and composition. Organic Geochemistry, 33, 489-515.

Zhao W, Hopke PK, Norris G, Williams R, Paatero P. (2006) Source apportionment and analysis on ambient and personal exposure samples with a combined receptor model and an adaptive blank estimation strategy. Atmos. Environ., 40, 3788-3801.

Zhao J, Zhang F, Chen J, Xu Y. (2010) Characterization of polycyclic aromatic hydrocarbons and gas/particle partitioning in a coastal city, Xiamen, Southeast China. Journal of Environmental Science, 22, 7, 1014-1022.

Zhao W, Hopke PK, Karl T. (2004) Source Identification of Volatile Organic Compounds in Houston, Texas. Environ. Sci. Technol., 38, 1338-1347.

Zheng M, Cass GR, Ke L, Wang F, Schauer JJ, Edgerton ES, Russell AG. (2007) Source apportionment of daily fine particulate matter at Jefferson Street, Atlanta, GA, during summer and winter. Journal of the Air and Waste Management Association, 57 (2), 228242. 